\title{
Forward Sound Propagation around Seamounts: Application of Acoustic Models to the Kermit-Roosevelt and Elvis seamounts
}

By

Hyun Joe Kim

B.S., Seoul National University, 1993

M.S., Seoul National University, 1995

Submitted in partial fulfillment of the requirements for the degree of

Doctor of Philosophy

at the

MASSACHUSETTS INSTITUTE OF TECHNOLOGY

and the

WOODS HOLE OCEANOGRAPHIC INSTITUTION

June 2009

(C) 2009 Hyun Joe Kim

All rights reserved.

The author hereby grants to MIT and WHOI permission to reproduce and to distribute publicly paper and electronic copies of this thesis document in whole or in part in any medium now known or hereafter created.

Signature of Author

Joint Program in Oceanography/Applied Ocean Science and Engineering Massachusetts Institute of Technology and Woods Hole Oceanographic Institution May 27, 2009

Certified by

Arthur B. Baggeroer

Thesis Supervisor

Accepted by

James C. Preisig Chair, Joint Committee for Applied Ocean Science and Engineering Woods Hole Oceanographic Institution

Accepted by

David E. Hardt

Chairman, Committee on Graduate Students Massachusetts Institute of Technology 

Forward Sound Propagation around Seamounts:

Application of Acoustic Models to the Kermit-Roosevelt and Elvis seamounts

by

Hyun Joe Kim

Submitted to the Department of Mechanical Engineering

on May 27, 2009 in partial fulfillment of the requirements of the degree of

Doctor of Philosophy in Mechanical Engineering

\begin{abstract}
The Basin Acoustic Seamount Scattering Experiment (BASSEX) of 2004 was conducted to measure forward-scattering around the Kermit-Roosevelt Seamount Complex in the Northeast Pacific. The BASSEX experiment was focused on the bathymetric effects on acoustic propagation, in particular, on direct blockage, horizontal refraction, diffraction, and scattering by the seamounts. A towed hydrophone array, with 64 sensors cut for $250 \mathrm{~Hz}$ (3m spacing), was used to measure the signals transmitted from the aforementioned broadband sources at many locations around the Kermit-Roosevelt and Elvis seamounts. Utilizing the measured broadband signals from the towed array, the size of the shadow zone was obtained. The measured data in the BASSEX experiment strongly support the understanding of the complicated phenomena of sound propagation around the seamounts. In addition, the experimental data could be used to validate current 2D and 3D theoretical models and develop new models to properly realize the sound propagation with such complicated phenomena.

In this thesis, the reconciliation between the measured pulse arrivals from the BASSEX experiment and various two-dimensional (2D) and three-dimensional (3D) theoretical models is carried out to investigate the physical characteristics of the sound propagation around seamounts: First, the 2D Parabolic Equation (PE) model and the 2D ray tracing model are used to reconcile each ray arrival with the BASSEX experiment in terms of arrival time and grazing angle. We construct a sound speed field database based on the sound speed profiles from the BASSEX experiment, World Ocean Atlas (WOA) 2005, and CTD casts using the objective analysis.
\end{abstract}

Second, 3D broadband sound propagation around a conical seamount is 
investigated numerically using the 3D spectral coupled-mode model (W. Luo, PhD Thesis, MIT, 2007). Since the calculation of 3D broadband pulses with the spectral coupledmode model requires extensive computation time, a parallel program is developed with a clustered computing system to obtain results in reasonable time. The validation of the 3D spectral coupled-mode model is performed by a series of comparisons between the various 2D and 3D models for a shallow-water waveguide. The Kermit-Roosevelt seamount is modeled by a simple conical seamount for the 3D model. The computed 3D broadband pulses for the modeled conical seamount are compared with those from the BASSEX experiment and the 2D PE simulation.

Through this analysis, we examine the limit of the application of the sound propagation models and improve the efficiency of the 3D sound propagation model using parallel computing to obtain a broadband pulse in a reasonable amount of time.

Thesis Supervisor: Arthur B. Baggeroer

Title: Ford Professor of Engineering

Secretary of Navy / Chief of Naval Operations Chair for Ocean Sciences

Henrik Schmidt

Title: Professor of Ocean Engineering

Massachusetts Institute of Technology

James F. Lynch

Title: Senior Scientist / Department Chair of Applied Ocean Physics and Engineering Massachusetts Institute of Technology / Woods Hole Oceanographic Institution

Kevin D. Heaney

Ocean Acoustical Services and Instrumentation Services, Inc.

Pierre Lermusiaux

Title: Professor of Mechanical Engineering

Massachusetts Institute of Technology 


\section{Acknowledgements}

I would like to thank my advisor, Prof. Arthur Baggeroer, for his support and encouragement to complete this work. He gave me an opportunity to study ocean acoustics, and guided me with extreme patience.

I would like to express my gratitude to the members of my thesis committee. Prof. Henrik Schmidt always gave me valuable advices and inspiration. Thanks are due to Dr. James F. Lynch at WHOI and Dr. Kevin D. Heaney, at Ocean Acoustical Services and Instrumentation Services, Inc., for their review and valuable comments.

Prof. Pierre Lermusiaux gave me a chance to use his enormous computer, without which I would never have obtained any results. In his group, I would like to thank Wayne G. Leslie for his valuable data of the sound speeds, and thanks are also due to Patrick Haley for helping me a lot whenever I was in trouble with the computer system.

Thanks are due to Prof. Nicholas C. Makris for his excellent teaching at my first ocean acoustics class.

I am deeply indebted to Joseph Sikora for his hard work on the data analysis. It was my great pleasure working with him on the same problem. Thanks are due to Wenyu Luo for his program, valuable suggestions and comments. I would like to thank Julie Mullen at the Lincoln Laboratory; she showed me the new world of parallel computing.

I am indebted to the Acoustics Group members, Konstantinos Pelenakis, Andrew Poulsen, Tianrun Chen, Jinshan Xu, Deanelle Symonds, Ding Wang, Ioannis Bertsatos, Srinivasan Jagannathan, Kevin Cockrell, Hadi Tavakoli Nia, Raymond Lum for their helpful comments. Thanks are also due to Harish Mukundan for his kindness as a neighbor.

I would like thank Geoff Fox for his wonderful and perfect administrative work. Thanks are due to Thalia Rubio for her reviews for the English grammar and style.

I would like to thank Korean colleagues, Sunwoong Lee, Kwang Hyun Lee and 
Sungho Lee.

Most of all, my family has always stood by me. Dad shows me his endless support. Especially, I would like to give my special thanks to my wife, Seok Joo, whose endless love enabled me to complete this work. My sons, Sa Joon and Sa Yoon, always gave me great pleasure in the tough times. 


\section{Contents}

1 Introduction

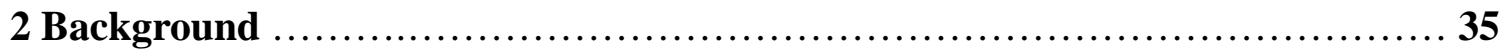

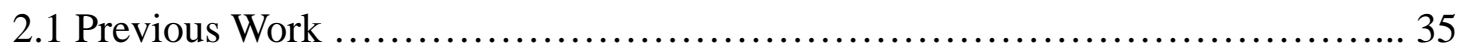

2.1.1 Experimental approach to the acoustic shadowing by seamounts ............ 36

2.1.2 Theoretical approach ....................................................

2.2 BASSEX (Basin Acoustics Seamount Scattering Experiment) ................... 42

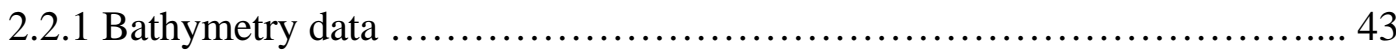

2.2.2 Acoustic sources ..............................................................

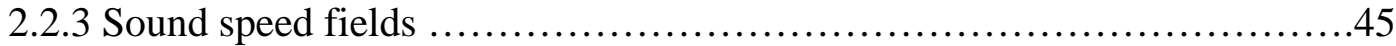

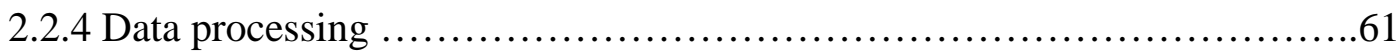

2.3 Sound propagation around a seamount .....................................62

3 Sound Propagation Models ......................................................... 73

3.1 Two-dimensional, Nx2D, and three-dimensional sound propagation ............73

3.2 Normal modes in a range-dependent waveguide and adiabatic approximation ....74

3.3 Three-dimensional ray theory and horizontal ray theory with the adiabatic mode

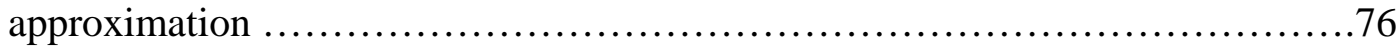

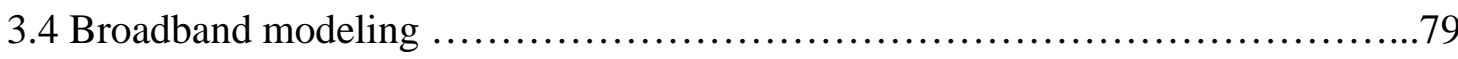

3.5 Three-dimensional spectral coupled-mode model around a conical seamount .....80

3.6 Three-dimensional spectral coupled-mode model vs. three-dimensional parabolic

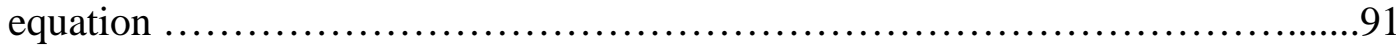

3.7 Parallel computing and the approximations ….............................95

3.7.1 Parallel computing .................................................100

3.7.2 Azimuth modal cut-off .............................................104

3.7.3 Approximations with truncation of normal modes and sub-sampling of

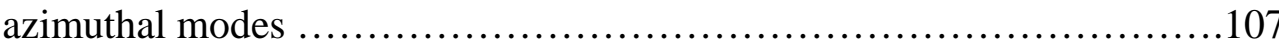


3.7.4 Real-value argument approximation of the Hankel and Bessel function ...112

\section{Reconciliation of arrival pulses between the BASSEX experiment and two-} dimensional sound propagation model ................................. 115

4.1 Parabolic equation .......................................................116

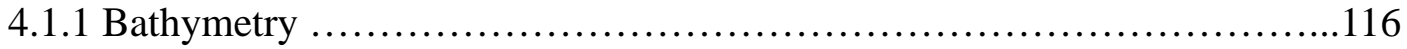

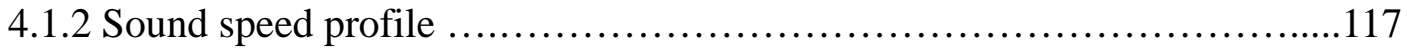

4.1.3 Bottom properties .............................................117

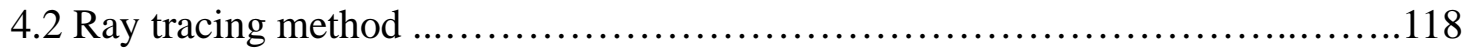

4.3 Behind the Elvis seamount: day 268 with XBT sound speeds .................118

4.4 Behind the Kermit-Roosevelt seamount: day 267 with the sound speed fields from

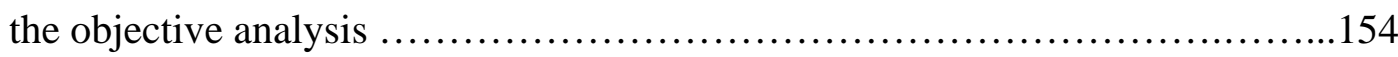

4.5 Behind the Kermit-Roosevelt seamount: day 266 with the sound speed fields from

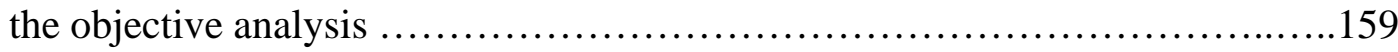

4.6 Open sea case: day 271 with the sound speed fields from the objective analysis 164

4.7 Nx2D computation with a simplified conical seamount approximation and a range-averaged sound speed profile

5 Three-dimensional sound propagation around a seamount

5.1 Comparison of two-dimensional and three-dimensional sound propagation models for a shallow-water waveguide with a conical seamount $\ldots \ldots \ldots \ldots \ldots \ldots \ldots \ldots . . .180$

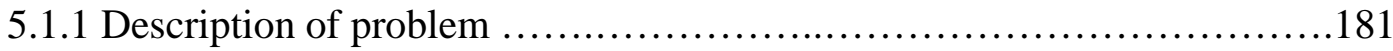

5.1.2 Comparison of transmission loss for a CW source $(40 \mathrm{~Hz})$................181

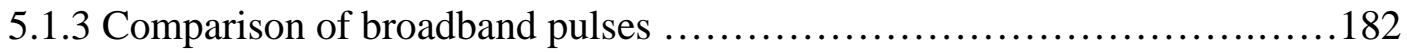

5.1.4 Comparison of transfer function between the two-dimensional and three-

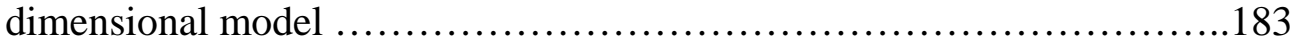

5.2 Broadband pulse modeling for a benchmark problem in deep-water waveguide with

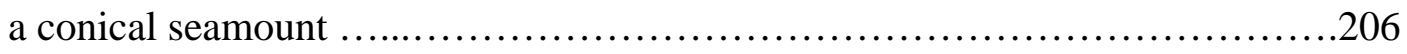

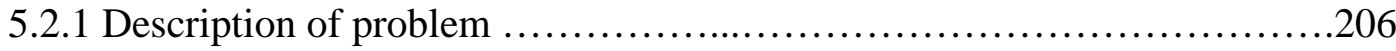


5.2.2 Transmission loss for a $10 \mathrm{~Hz}$ CW source

207

5.2.3 Comparison of pulse arrivals between the 3D spectral coupled-mode model and the ray tracing method without seamount ..........................208

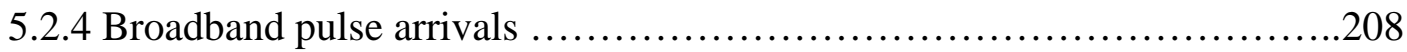

5.3 Broadband pulse modeling for the LOAPEX source .........................236

5.3.1 Approximation of the Kermit-Roosevelt seamount with a conical seamount .236

5.3.2 Comparison of Transmission loss from two-dimensional and threedimensional model ...................................................... 236

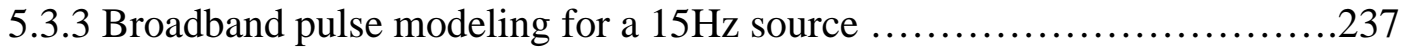

5.3.4 Broadband pulse modeling for a $68 \mathrm{~Hz}$ source ..........................238

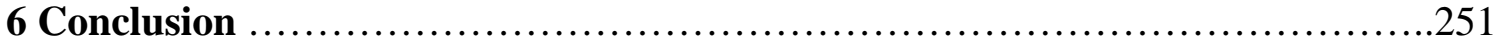

A Specifications of acoustic sources in the BASSEX experiment ..................255

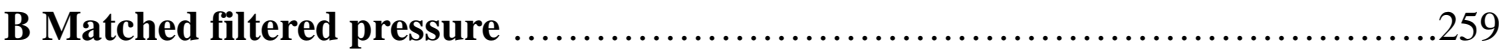

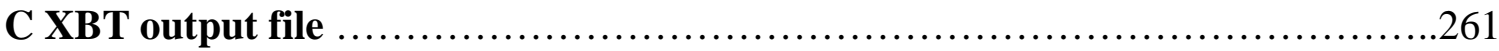

D Modal decomposition method: modal spectrum of the Parabolic Equation

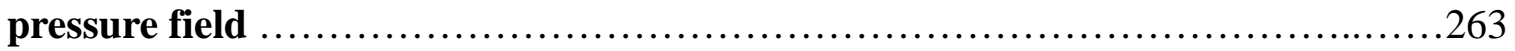

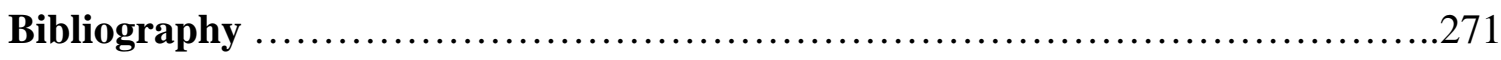




\section{List of Figures}

1-1 Bathymetry around the Kermit-Roosevelt and Elvis seamounts with the source locations (S1 \& S2 for the SPICEX sources, LOAPEX (T1000) for the LOAPEX

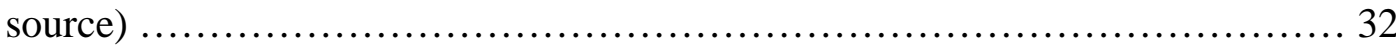

1-2 Measured peak sound levels received from the SPICEX source S1 (left panel) and S2

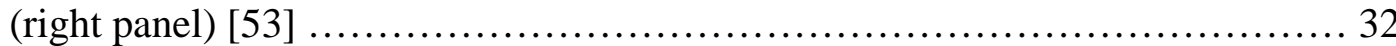

2-1 Experimental transmission loss collected by Chapman and Ebbeson [4] ........... 37

2-2 Kermit-Roosevelt and Elvis seamount; the minimum water depths of the seamounts are $980 \mathrm{~m}$ and $1380 \mathrm{~m}$, respectively [53]

2-3 Locations of acoustic sources in the BASSEX experiment: (S) SPICEX moored sources, (T) LOAPEX stations, (Kauai) NPAL Kauai Source [53]

2-4 (a) XBT casts locations around the Kermit-Roosevelt and Elvis seamounts; the circles denote the locations where the XBT casts were conducted. Two SPICEX sources (S1 and S2) are shown with the LOAPEX source (T1000). Two locations where the CTD casts were carried out in the LOAPEX experiment are shown with CTD 1 and CTD 2

2-4 (b) XBT cast locations around the Kermit-Roosevel and Elvis seamounts 47

2-5 Comparison of sound speed profiles from the WOA05 and XBT for two selected locations. 'XBT' denotes the terminal depth of XBT casts. The right panels shows the compared sound speed profiles, and the left panels show the difference in sound speed profiles between the WOA05 and the XBT casts. The two locations are chosen near the SPICEX 2 source (top panels) and the top of the Kermit-Roosevelt

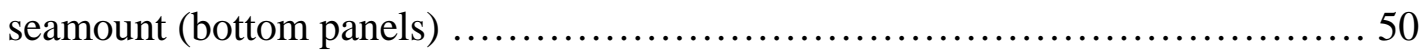


2-6 Comparison of the horizontal variation of sound speed from the XBT casts and WOA05 at the water depth 0 and 10m; the four lower panels show sound speeds specifically around the seamounts 51

2-7 Comparison of the horizontal variation of sound speed from the XBT casts and WOA05 at the water depth 800 and $900 \mathrm{~m}$; the four lower panels show sound speeds specifically around the seamounts 52

2-8 Comparison of the sound speed profile from the XBT and WOA05 along an acoustic path for day 268 over the Elvis seamount; the top panel shows the comparison of range-averaged sound speed as a function of depth, the middle (XBT) and bottom (WOA05) panels show variability of sound speed along the acoustic path 53

2-9 Sound speed fields from the objective analysis for various depths. The circle corresponds to the peak of the Kermit-Roosevelt seamount, and the square denotes

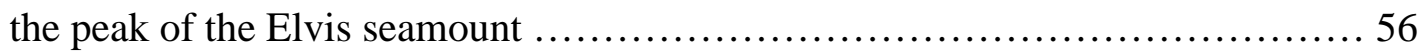

2-10 Comparison of sound speed fields from the WOA05 (top panels), XBT data (middle panels), and objective analysis (bottom panels)

2-11 Comparison of sound speed fields for an acoustic path of day 268 from the XBT data (top panel), WOA05 (middle panel), and objective analysis (bottom panel) .. 58

2-12 Comparison of sound speed profiles at locations where CTD casts were carried out

2-13 Comparison of pulse arrivals from the experimental measurement (red) and the 2D PE simulated pulse arrivals with truncated XBT data at 1500m (run 51, blue). 'OAG16' denotes the simulated pulse arrivals with the XBT casts without the truncation, which gives additional early arrivals before the main arrivals (lower panel). In 'OAG16+ctd', the sound speed below 1500m was replaced by the sound speeds from CTD data, and the additional arrivals with 'OAG16' disappeared (top panel) 
2-14 Ray propagation with a seamount made by RAY [40] program. The rays were generated using the range-dependent sound speeds from the XBT casts nearest the acoustic path (Fig. 4-2 for day 268) 63

2-15 Range-stacked pulses arrivals at a receiver depth, 250m. The red dashed line denotes the blocked refracted wave by the seamount. This result is made by the PE simulation for the Elvis seamount

2-16 Strong mode coupling with seamount bathymetry. This is obtained from PE simulation at a frequency, $250 \mathrm{~Hz}$, and the modal amplitude can be estimated by the modal decomposition method (Appendix D). In higher modes, their energy is dissipated by the seamount, and also lower mode amplitude is disturbed by the seamount. Transfer of energy between modes is clearly visible in the figure. After the seamount, energy transfer to higher modes from lower modes, i.e., mode repopulation, occurs 65

2-17 Modal shapes for $1-4^{\text {th }}$ and $100^{\text {th }}, 200^{\text {th }}, 300^{\text {th }}, 400^{\text {th }}$ modes versus range. Bathymetry and SVP (top left panel) as well as the number of propagating modes (top right panel) are shown 66

2-18 Turning depths of the water trapped modes at three locations: around the acoustic source, a small seamount, and the peak of the Elvis seamount

2-19 Sum of amplitude squared along the bathymetry, which provides an idea of energy dissipation by the blockage of the seamount

2-20 (a) Modal pulse arrivals (top panel), (b) group speed of each mode (bottom panel)

2-21 Horizontal refracted rays for the $1^{\text {st }} \sim 151^{\text {st }}$ modes for an acoustic path (top panel), and $1^{\text {st }}$ (black), $51^{\text {st }}$ (blue), $101^{\text {st }}$ (red) modes for three different acoustic paths (bottom panel). The circles denote the locations where a horizontal refracted ray is detected in the BASSEX experiment. The refracted angles between a geodesic and a $101^{\text {st }}$ mode ray 
are 21.35 and 5.75 degrees for the rays passing left and right side with respect to the peak of the seamount

2-22 Measured pulse arrivals for LOAPEX source, $68 \mathrm{~Hz}$ at $350 \mathrm{~m}$ depth, behind the Kermit-Roosevelt seamount, showing pulse arrivals with a different arrival angle (adapted from [53], and reproduced) .................................... 72

3-1 Description of problem with stepwise conical seamount $[10]$................... 80

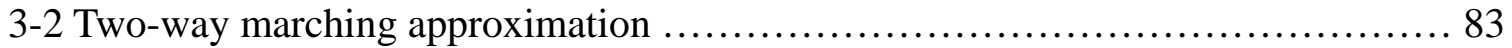

3-3 Single-scatter approximation between two neighboring rings $\ldots \ldots \ldots \ldots \ldots \ldots \ldots \ldots . . .63$

3-4 One-way approximation between two neighboring rings $\ldots \ldots \ldots \ldots \ldots \ldots \ldots \ldots . . . . \ldots 7$

3-5 Structure of 3D spectral coupled-mode model program ........................ 88

3-6 Do-loops associated with azimuth modes and range steps $\ldots \ldots \ldots \ldots \ldots \ldots \ldots \ldots \ldots . .90$

3-7 Comparison of computational domain for the 3D spectral coupled-mode model with an approximated conical seamount and the 3D PE with real range-dependent

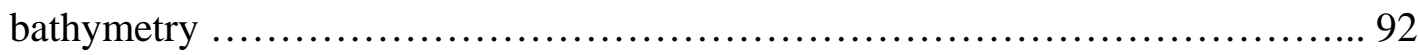

3-8 Proposed 3D PE model with high computational efficiency for a long-range and highly range-dependent problem ...................................... 94

3-9 $\left|a_{m n}^{j}\right|$ at the outermost ring after inward/outward marching with different number of sections, 1024 and 2048. In the left panel, the artifacts from the stepwise approximation are shown in the top right corner; however, the artifacts disappear with 2048 sections in the right panel .................................... 96

3-10 Description of problem with a $10 \mathrm{~Hz}$ CW source (see Section 5.2 for details) ..... 99

3-11 Computation time of CPUs with equally spaced azimuth modes ............... 102

3-12 Computation time of CPUs with linearly increasing number of azimuth modes. The program adds some additional higher azimuth modes at the beginning of the 
computation; therefore, the jobs for these higher azimuth modes were performed very quickly, which explains the abrupt change in the workload ratio at the highest number of CPUs 103

3-13 Amplitudes of incoming coupling coefficients, $\left|b_{m n}^{j}\right|$, at the fifth normal mode in terms of azimuth modes and sections 104

3-14 Amplitudes of outgoing and incoming coupling coefficients, $\left|a_{m n}^{j}\right|$ and $\left|b_{m n}^{j}\right|$, respectively, in four different sections in terms of normal and azimuth modes ... 106

3-15 Transmission losses for various number of normal modes along the centerline (0 degree, top panel) and 7.5 degrees off centerline (bottom panel) ................. 109

3-16 Transmission losses with various sub-samplings of azimuth modes: 1/2 sampling (top left), 1/5 sampling (top right) and 1/7 sampling (bottom right). The transmission losses with the sub-samplings are obtained only in the region outside the seamount 110

3-17 Interpolation of the real part of the $P_{m}^{J}$ at each azimuth mode at $150 \mathrm{~km}$ for $1 / 2$ (top panel) and 1/5 (bottom panel) samplings. The blue triangles denote the correct coefficients from the computation; the red circles denote the azimuth modes chosen for the sub-sampling. The cyan circles are the interpolated coefficients based on the sub-sampled coefficients (red circles) 111

3-18 TL with the real argument approximation of the Hankel and Bessel function compared to the TL without the approximation for a $10 \mathrm{~Hz}$ CW source ........... 113

4-1 Acoustic path (geodesic) that passes over the peak of Elvis seamount. The depth of the peak of Elvis seamount is $1369 \mathrm{~m}$ from the sea surface ....................... 119

4-2 Receiver locations for the reference acoustic path that passes over the peak of Elvis seamount; this acoustic path is designed to pass over the apex of Elvis seamount 120

4-3 Bathymetry and sound speed profiles along the acoustic path. The sound speed 
profiles are obtained from the XBT casts in the BASSEX experiment, and the XBT cast locations nearest the acoustic path were used. The sound speed profiles are linearly interpolated in ranges along the acoustic path to reduce the abrupt change of sound speed in the simulations. In the left panel, the range-averaged sound speed profile is shown, and the right panel shows the perturbed sound speed profiles along the acoustic path 121

4-4 Sound speeds derived from XBT casts of BASSEX experiment along the acoustic path (Figure 4-1); the left panel shows range-averaged sound speed profile, and the right panel shows the difference from the range-averaged sound speed ..... 122

4-5 Range-stacked arrival pulses for the range of $325 \sim 625 \mathrm{~km}$. Although receiver depths vary in the range of $240 \sim 300 \mathrm{~m}$, the pulse arrivals at the depth $270 \mathrm{~m}$ are given in this figure

4-6 Compared range-stacked arrival pulses for the range of $325 \sim 625 \mathrm{~km}$ with (left panel) and without (right panel) seamount ..................................... 128

4-7 Comparison of arrival pulses from experiment and PE simulation for day 268. For each panel, the amplitudes of pulses are normalized by the maximum values of all pulses. B and $\mathrm{C}$ are located in the transition region between the refracted wave arrival zone (convergence zone) and shadow zone. This implies that the size of the shadow zone might be underestimated by the PE simulation. Another significant discrepancy can be found at A, which is located above the Elvis seamount. As shown in this figure, the early arrival refracted waves in the PE simulation were not measured in the experiment

4-8 Comparison of pulse arrivals by the ray tracing method, 2D PE, and the BASSEX experiment for 26802 130

4-9 Ray arrivals from ray tracing method for 26802. Ray arrivals are grouped into 4 groups: only refracted wave (R) without bottom interactions, surface-reflectedbottom-reflected wave (SRBR), only surface-reflected wave (SR), and only bottom 
reflected wave (BR). (+) and (-) signs denote up and down rays, respectively. The number before the sign means the total number of turns

4-10 Comparison of pulse arrivals by the ray tracing method, 2D PE, and the BASSEX

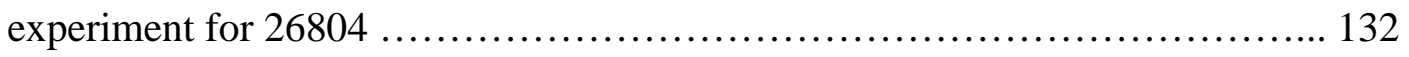

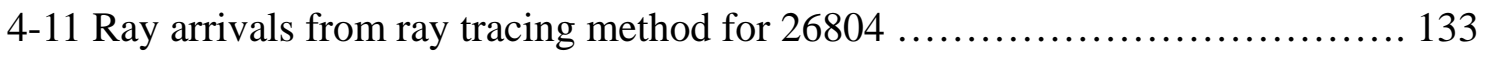

4-12 Comparison of pulse arrivals by the ray tracing method, 2D PE, and the BASSEX

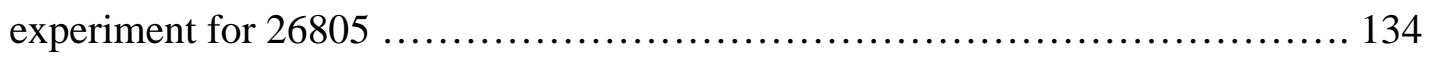

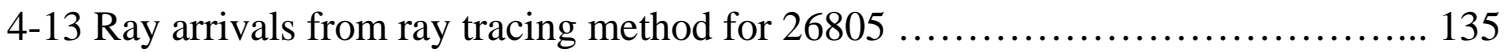

4-14 Comparison of pulse arrivals by the ray tracing method, 2D PE, and the BASSEX

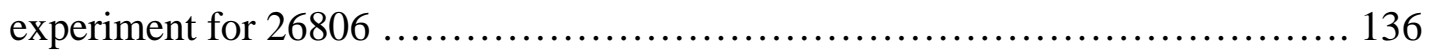

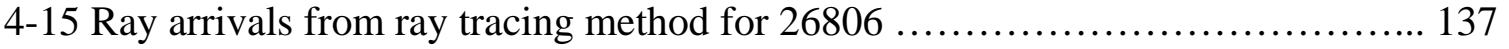

4-16 Comparison of pulse arrivals by the ray tracing method, 2D PE, and the BASSEX

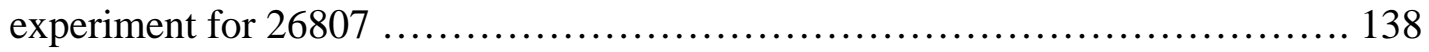

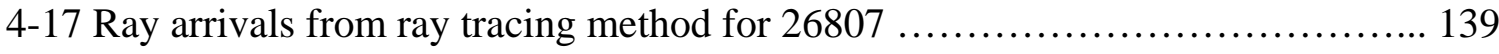

4-18 Comparison of pulse arrivals by the ray tracing method, 2D PE, and the BASSEX

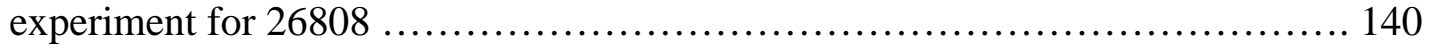

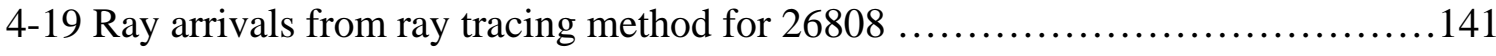

4-20 Comparison of pulse arrivals by the ray tracing method, 2D PE, and the BASSEX

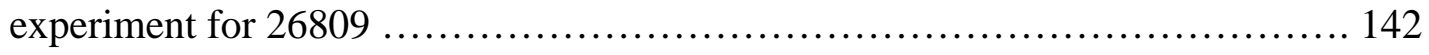

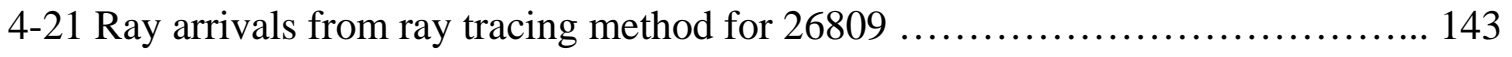

4-22 Comparison of pulse arrivals by the ray tracing method, 2D PE, and the BASSEX

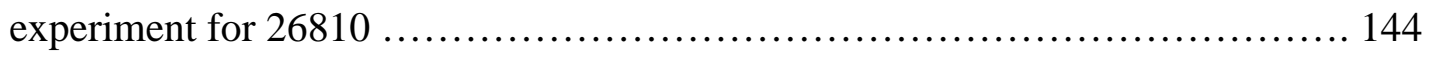

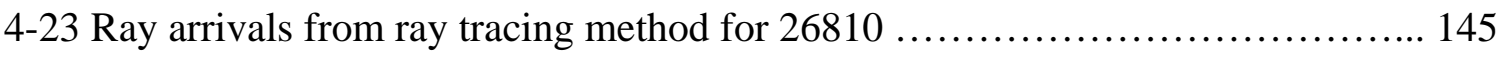


4-24 Comparison of pulse arrivals by the ray tracing method, 2D PE, and the BASSEX

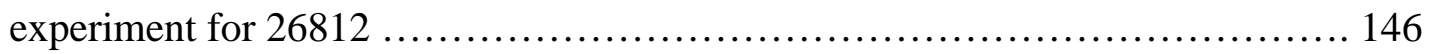

4-25 Ray arrivals from ray tracing method for 26812 .......................... 147

4-26 Comparison of pulse arrivals by the ray tracing method, 2D PE, and the BASSEX

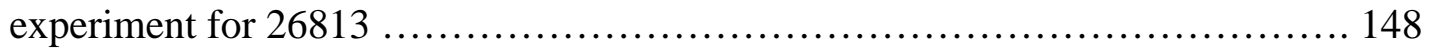

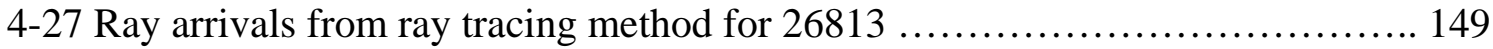

4-28 Comparison of pulse arrivals by the ray tracing method, 2D PE, and the BASSEX

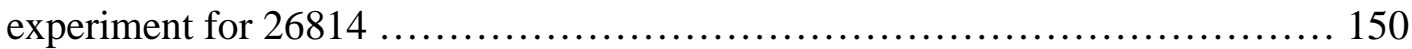

4-29 Ray arrivals from ray tracing method for $26814 \ldots \ldots \ldots \ldots \ldots \ldots \ldots \ldots \ldots \ldots \ldots \ldots \ldots \ldots$

4-30 Comparison of pulse arrivals by the ray tracing method, 2D PE, and the BASSEX

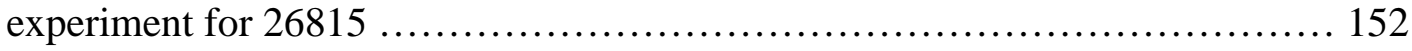

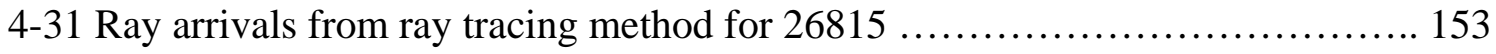

4-32 Receiver locations for day 267, as well as an acoustic path passing over the KermitRoosevelt seamount from the SPICEX source 2 .......................... 155

4-33 Comparison of the peak signal levels from the measurement and the $2 \mathrm{D} P E$

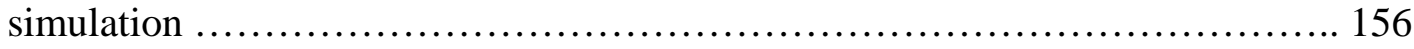

4-34 Comparison of pulse arrivals from the ray tracing method, the 2D PE, and the experiment for 26708 157

4-35 Comparison of pulse arrivals by the ray tracing method, the $2 \mathrm{D} \mathrm{PE}$, and the

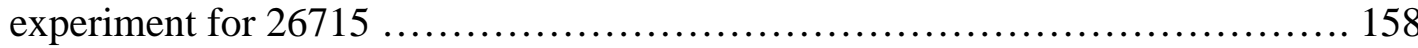

4-36 Receiver locations for day 264, as well as an acoustic path passing over the KermitRoosevelt seamount .................................................. 160

4-37 Comparison of peak signal levels from the measurement and 2D PE simulation . 161 
4-38 Comparison of pulse arrivals by the ray tracing method, 2D PE, and the experiment for 26414 162

4-39 Comparison of pulse arrivals by the ray tracing method, 2D PE, and the experiment for 26420 163

4-40 Receiver locations for day 271; two acoustic paths are shown for 27100 and 27108 166

4-41 Comparison of arrival pulses from experiment (left panel) and PE simulation (right panel) for day 271, SPICEX source 1

4-42 Comparison of arrival pulses from experiment (left panel) and PE simulation (right panel) for day 271, SPICEX source 2 168

4-43 Comparison of arrival pulses from the experiment (black) and PE simulation (red) for day271, SPICEX source 1 (top panel) and SPICEX source 2 (bottom panel). Arrival time errors were not corrected. Amplitudes are normalized by the maximum amplitude

4-44 Acoustic paths for the four acoustic paths: day 264 from the SPICEX source 1 to the apex of the Kermit-Roosevelt seamount, day 265 from the SPICEX source 1 to the apex of the Elvis seamount, day 267 from the SPICEX source 2 to the apex of the Kermit-Roosevelt seamount and day 268, from the SPICEX source 2 to the apex of the Elvis seamount 172

4-45 Approximated conical seamounts for the four acoustic paths 174

4-46 Range-averaged sound speed fields for the four acoustic paths; sound speeds are obtained from the XBT casts 174

4-47 Range-stacked pulse arrivals for the acoustic paths from the centerline to the outermost ring 175

4-48 Conical seamounts with range-averaged sound speed fields 176 


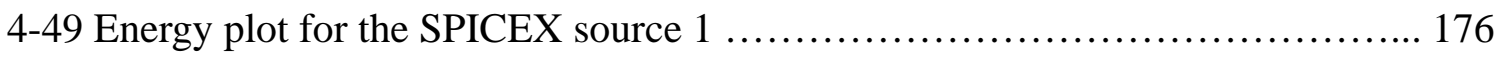

4-50 Conical seamounts with range-averaged sound speed fields ................. 177

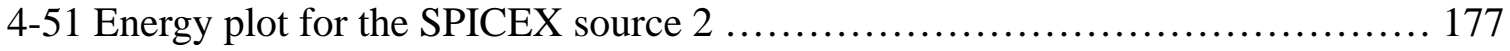

4-52 Comparison of the arrival energy between the Nx2D simulation and measurement (the measurements are adapted from [53]) ............................. 178

5-1 Schematic of a shallow-water waveguide with a conical seamount .............. 184

5-2 Time series and spectrum of the broadband source .......................... 186

5-3 Convergence test for the range and depth step size of the RAM and FOR3D along the centerline (through the peak of the seamount, $z=100 \mathrm{~m}$ ) with a $40 \mathrm{~Hz} \mathrm{CW}$ source 187

5-4 Convergence test for the range step size of the CSNAP along the centerline through

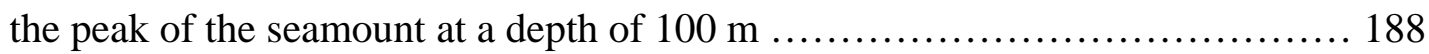

5-5 Comparison of TL from the RAM, CSNAP, and FOR3D (2D) along the centerline through the peak of the seamount at a depth of $100 \mathrm{~m}$........................ 188

5-6 Comparison of TL from the FOR3D for two different angular step sizes on a horizontal plane (top panel) and along the centerline (bottom panel) ............ 189

5-7 Comparison of TL from the FOR3D for the Nx2D and 3D problem with a $40 \mathrm{~Hz}$ CW source at a depth of $25 \mathrm{~m}$. The TL is shown on a horizontal plane (top panel) and along the centerline through the peak of the seamount (bottom panel) 190

5-8 Comparison of TL from the FOR3D for the Nx2D and 3D problem with a $40 \mathrm{~Hz} \mathrm{CW}$ source at a depth of $100 \mathrm{~m}$. The TL is shown on a horizontal plane (top panel) and along the centerline through the peak of the seamount (bottom panel) 191

5-9 Comparison of TL from the 3D spectral coupled-mode model and FOR3D for a 3D problem with a $40 \mathrm{~Hz}$ CW source at a depth of $25 \mathrm{~m}$. The TL is shown on a horizontal plane (top panel) and along the centerline through the peak of the 
seamount (bottom panel)

5-10 Comparison of TL from the 3D spectral coupled-mode mode and FOR3D for a 3D problem with a $40 \mathrm{~Hz} \mathrm{CW}$ source at a depth of $100 \mathrm{~m}$. The TL is shown on a horizontal plane (top panel) and along the centerline through the peak of the seamount (bottom panel) 193

5-11 Comparison of broadband pulses at a depth of 25m, 0.0 degrees off the centerline: RAM (2D, upper panel) and CSNAP (2D, lower panel) 194

5-12 Comparison of broadband pulses at a depth of $25 \mathrm{~m}, 0.0$ degrees off the centerline: FOR3D (3D, upper panel) and 3D-CSNAP (3D, lower panel) 195

5-13 Comparison of broadband pulses at a depth of $100 \mathrm{~m}, 0.0$ degrees off the centerline: RAM (2D, upper panel) and CSNAP (2D, lower panel) ..................... 196

5-14 Comparison of broadband pulses at a depth of $100 \mathrm{~m}, 0.0$ degrees off the centerline: FOR3D (3D, upper panel) and 3D-CSNAP (3D, lower panel) 197

5-15 Comparison of broadband pulses at a depth of 25 m, 1.0 degrees off the centerline: RAM (2D, upper panel), FOR3D (3D, lower left panel) and 3D-CSNAP (3D, lower right panel) 198

5-16 Comparison of broadband pulses at a depth of 100 m, 1.0 degree off the centerline: RAM (2D, upper panel), FOR3D (3D, lower left panel) and 3D-CSNAP (3D, lower right panel) 199

5-17 Comparison of broadband pulses at a depth of $25 \mathrm{~m}, 2.0$ degrees off the centerline: RAM (2D, upper panel), FOR3D (3D, lower left panel) and 3D-CSNAP (3D, lower right panel) 200

5-18 Comparison of broadband pulses at a depth of $100 \mathrm{~m}, 2.0$ degrees off the centerline: RAM (2D, upper panel), FOR3D (3D, lower left panel) and 3D-CSNAP (3D, lower 
right panel) 201

5-19 Comparison of broadband pulses at a depth of $25 \mathrm{~m}, 3.0$ degrees off the centerline: RAM (2D, upper panel), FOR3D (3D, lower left panel) and 3D-CSNAP (3D, lower right panel)

5-20 Comparison of broadband pulses at a depth of 100 m, 3.0 degrees off the centerline: RAM (2D, upper panel), FOR3D (3D, lower left panel) and 3D-CSNAP (3D, lower right panel) 203

5-21 Comparison of the transfer function between RAM (2D), FOR3D (3D), and 3D spectral coupled-mode model (3D) before the seamount $(r=3.5 \mathrm{~km}$, depth $=25 \mathrm{~m}$, azimuth angle $=0.0$ degrees): amplitude (top panel) and phase (bottom panel) ... 204

5-22 Comparison of the transfer function between RAM (2D), FOR3D (3D), and 3D spectral coupled-mode model (3D) behind the seamount $(\mathrm{r}=7.0 \mathrm{~km}$, depth $=25 \mathrm{~m}$, azimuth angle $=0.0$ degrees): amplitude (top panel) and phase (bottom panel) ... 205

5-23 Schematic diagram of a benchmark problem with a conical seamount at a range of $100 \mathrm{~km}$ from the acoustic source, and the angle between acoustic paths passing through the centerline and outermost ring becomes 11.31 degrees. The source is located at the depth of $100 \mathrm{~m}$

5-24 Source spectrum at a center frequency of $15 \mathrm{~Hz}$ with $10 \mathrm{~Hz}$ bandwidth 213

5-25 Number of vertical modes at each frequency for $10 \mathrm{~Hz}$ bandwidth from 10 to $20 \mathrm{~Hz}$; waterborne and propagation modes are compared in terms of frequency. No waterborne mode exists for this frequency band at the top of the seamount, i.e., there is no ray passing over the seamount without bottom-bouncing 213

5-26 TL for a $10 \mathrm{~Hz}$ CW source at a depth of $300 \mathrm{~m}$; the upper panel shows a discrepancy of the TL between the 2D and 3D model. The TL from the 3D model (3D spectral coupled-mode model) shows greater value than those from 2D model (CSNAP) by 5 10dB. The lower panel shows the TL on a horizontal plane, which shows clear 
5-27 Ray representation without a seamount. The convergence zones consist in the refracted rays, shown in black, without bottom-bouncing. The ray family at the convergence zone is entirely blocked by the seamount that is located at a range of $100 \mathrm{~km}$ from the acoustic source 215

5-28 Computed pulse arrivals from the 3D spectral coupled-mode model (lower panel) compared with the ray tracing method (upper panel). In the upper panel, the eigenrays are shown in terms of reduced time; the rays are comparable to the computed pulse arrivals, far outside the seamount, 30 degrees off the centerline, with no diffraction by the seamount. At this depth, significant convergence zone arrivals are followed by bottom-bounced rays. The numbers in the upper panel denote the number of bottom-bounces for each ray family

5-29 Comparison of arrival time between the ray tracing method and 3D model at a range of $130 \mathrm{~km}$. The computed pulse arrivals from the 3D spectral coupled-mode mode, along an acoustic path of the 30 degrees off the centerline, have no $3 \mathrm{D}$ effect by the seamount. The $A$ ray family forms strong convergence zones without bottombouncing; the arrival times of this ray family in the ray tracing method and the 3D spectral coupled-mode mode are quite close to each other. The ray families $B, C$, and $D$ correspond to the bottom-bouncing ray families with 2, 3, and 4 bottom-bounces, respectively 217

5-30 Computed pulse arrivals at a depth of $200 \mathrm{~m}$. The waterborne modes at the outermost ring are used for the mode coupling. The pulse arrivals are shown in the reduced time with a reference time of $1520 \mathrm{~m} / \mathrm{s}$. The angle denotes an acoustic path running off the centerline. The convergence zone arrivals are all blocked after the seamount up to 10 degrees, and some reflections are shown in the shadow zone ..218

5-31 Computed pulse arrivals at a depth of $200 \mathrm{~m}$; the pulse arrivals on a horizontal plane are shown in term of the reduced time with a reference speed of $1520 \mathrm{~m} / \mathrm{s}(0.6 \sim 1.3$ seconds). The first convergence zone arrivals appear at the reduced time of $0.7 \sim 0.8$ 
seconds, at a range of $60 \mathrm{~km}$; the first convergence zone arrivals are followed by the weak reflections. The second convergence zone appears at $1.3 \mathrm{sec}, 120 \mathrm{~km}$..... 219

5-31 (continued) Computed pulse arrivals at a depth of 200m (1.4 2.1 seconds); the third convergence zone arrivals appear at 1.8 1.9 seconds, 180 190km from the source. The reflections are not significant because only waterborne modes are used

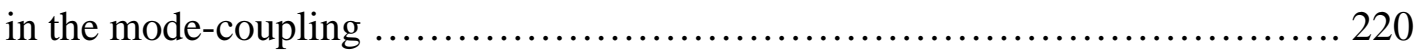

5-32 Computed pulse arrivals at a depth of $1000 \mathrm{~m}$; the waterborne modes at the outermost ring are used for the mode coupling. The pulse arrivals are shown in the reduced time with a reference time of $1520 \mathrm{~m} / \mathrm{s}$. The angle denotes an acoustic path running off the centerline. The two strong ray family arrivals appear in higher angle; however, those are all blocked in lower angle after the seamount up to 10 degrees. Weak reflections are shown in the shadow zone 221

5-33 Computed pulse arrivals at a depth of $1000 \mathrm{~m}$; the pulse arrivals on a horizontal plane are shown in terms of the reduced time with reference speed of $1520 \mathrm{~m} / \mathrm{s}(0.6$ $\sim 1.3$ seconds) 222

5-33: (continued) Computed pulse arrivals at a depth of 1000m (1.4 2.1 seconds) ...223

5-34 Computed pulse arrivals at a depth of $200 \mathrm{~m}$; the propagation modes at the outermost ring are used for the mode coupling, which provides full ray arrivals, including bottom bounces. The pulse arrivals are shown in the reduced time with a reference time of $1520 \mathrm{~m} / \mathrm{s}$. The angle denotes an acoustic path running off the centerline. The convergence zone arrivals are all blocked after the seamount up to 10 degrees and the strong reflections are shown in the perturbed zone. However, disturbances of bottom bouncing rays due to the seamount appear in much higher angles up to 16 degrees, compared to the cases with the waterborne modes 224

5-35 Computed pulse arrivals at a depth of 200m; the pulse arrivals on a horizontal plane are shown in terms of the reduced time with a reference speed of $1520 \mathrm{~m} / \mathrm{s}(0.75 \sim$ 
2.5 seconds). The first convergence zone arrivals appear before the seamount at 0.75 seconds, $60 \mathrm{~km}$ from the source, which are followed by the strong reflections. The pulse arrivals appear to be moving toward the acoustic source because of the higher propagation speed relative to the reference speed. The second convergence zone appears at 1.25 seconds, $120 \mathrm{~km}$, which clearly shows the shadowing cast of the seamount. The third and fourth convergence zone appear at 1.75 2.0, 2.5 seconds, $190 \mathrm{~km}$ and $250 \mathrm{~km}$ from the source, respectively. At around 2.25 seconds, between the third and fourth convergence zone appearances, a convergence is formed by the reflected-refracted rays within the perturbation zone. Reflections due to the seamount generate quite complicated patterns of pulse arrivals in and around the shadow zone, including waves going away form the seamount to higher angles ...225

5-36 (continued) Computed pulse arrivals at a depth of 200m (2.75 4.5 seconds) ... 226

5-37 (continued) Computed pulse arrivals at a depth of 200m (4.75 6.5 seconds) ... 227

5-38 Computed pulse arrivals at a depth of $1000 \mathrm{~m}$; the propagation modes at the outermost ring are used for the mode coupling, which gives full ray arrivals including bottom- bouncing. The pulse arrivals are shown in the reduced time with a reference time of $1520 \mathrm{~m} / \mathrm{s}$. The angle denotes an acoustic path running off the centerline. The two strong ray families arrives in higher angles without blockage; however, these are all blocked in lower angle after the seamount up to 10 degrees and the strong reflections appear in shadow zone. Disturbances of bottom bouncing waves due to the seamount are shown in much higher angle up to 16 degrees .... 228

5-39 Computed pulse arrivals at a depth of $1000 \mathrm{~m}$; the pulse arrivals on a horizontal plane are shown in term of the reduced time with a reference speed of $1520 \mathrm{~m} / \mathrm{s}$

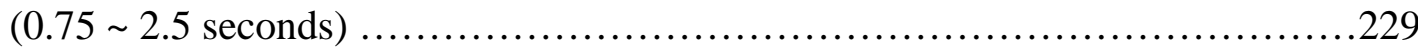

5-39 (continued) Computed pulse arrivals at the depth of 1000m (2.75 4.5 seconds) 230

5-39 (continued) Computed pulse arrivals at the depth of 1000m (4.75 6.5 seconds) 231 
5-40 Comparison of amplitude of computed pulses between waterborne modes (black), propagation modes (blue), and full modes (with leaky modes, red) at a depth of 200 $\mathrm{m}$. The ranges are $90 \mathrm{~km}$ (top panel) and 100km (bottom panel) ................. 232

5-41 Comparison of amplitude of computed pulses between waterborne modes (black), propagation modes (blue) and full modes (w/ leaky modes, red) at a depth of $200 \mathrm{~m}$. The ranges are 110km (top panel) and 150km (bottom panel) ................... 233

5-42 Comparison of amplitude of computed pulses between waterborne modes (black), propagation modes (blue) and full modes (w/ leaky modes, red) at a depth of $1000 \mathrm{~m}$. The ranges are $90 \mathrm{~km}$ (top panel) and 100km (bottom panel) .................... 234

5-43 Comparison of amplitude of computed pulses between waterborne modes (black), propagation modes (blue) and full modes (w/ leaky modes, red) at a depth of $1000 \mathrm{~m}$. The ranges are 110km (top panel) and 150km (bottom panel) ................... 235

5-44 Bathymetry around Kermit-Roosevelt seamount complex ...................... 239

5-45 Shapes of seamount at the selected depths with circular sections of approximated cone. The blue line was chosen for the conical shape of the seamount ............ 240

5-46 Comparison of the real bathymetry (left panel) and an approximated conical seamount (right panel) ................................................... 240

5-47 A problem with a conical seamount approximation of the Kermit-Roosevelt seamount 241

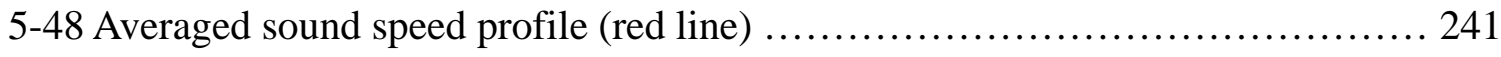

5-49 Compared TL from the CSNAP and 2D PE (RAM) .......................... 242

5-50 TL on a horizontal plane at a depth of 250m from the Nx2D (RAM, top panel) and the 3D model (3D spectral coupled-mode model, bottom panel) .................. 243

5-51 TL using the 3D spectral coupled-mode model with various range steps (or the 
number of sections)

5-52 Computed pulse arrivals for a broadband source with a center frequency of $15 \mathrm{~Hz}$ source at a depth of $200 \mathrm{~m}$ (7.0 8.4 seconds)

5-52 (continued) Computed pulse arrivals for a broadband source with a center frequency of $15 \mathrm{~Hz}$ source at a depth of $200 \mathrm{~m}$ (10.2 11.6 seconds) 246

5-53 Computed pulse arrivals for a broadband source with a center frequency of $15 \mathrm{~Hz}$ source at a depth of $500 \mathrm{~m}$ (7.0 8.4 seconds)

5-53 (continued) Computed pulse arrivals for a broadband source with a center frequency of $15 \mathrm{~Hz}$ source at a depth of $500 \mathrm{~m}$ (10.2 11.6 seconds)

5-54 Comparison of pulse arrivals between two broadband pulses with a center frequency of $68.2 \mathrm{~Hz}$ LOAPEX source (with water-borne modes only) and $15 \mathrm{~Hz}$ source (with propagation modes) ) for 26601. The angles denote the location of the 26601 with respect to the peak of the seamount. 249

5-55 Kermit-Roosevelt seamount and the location of receiver for 26601 (Longitude: 146.329506, Latitude: 39.484648)

5-56 Pulse arrivals for 26601 (LOAPEX) using 2D PE. Range-dependent bathymetry and the sound speed fields from objective analysis are used for the simulation. Beamformed pulse arrivals at a depth of $300 \mathrm{~m}$ (left panel) and the depth stacked wave fronts (right panel) are shown

250

5-57 Measured pulse arrivals in the BASSEX experiment for 26601. E appears to be a horizontal refracted ray by the Kermit-Roosevelt seamount 250 


\section{List of Tables}

2-1 Correlation parameters for the objective analysis of sound speed fields

3-1 Comparison between 3D spectral coupled-mode model and 3D PE method

3-2 Computation time for various no. range step and no. normal modes in hours

4-1 Bottom properties for the PE simulation

118

4-2 Receiver locations and simulation results for day268

4-3 Receiver locations in day 267 experiment for SPICEX source 2 .155

4-4 Receiver locations in day 264 experiment for SPICEX source 1 .160

4-5 Receiver locations in day 271 experiment for SPICEX source 1 and 2 .165

5-1 Definition of problem for the shallow water case with a seamount 185 


\section{Chapter 1}

\section{Introduction}

Although long-range ocean acoustics has advanced to predicting the arrival time within a millisecond at megameter ranges, the application of acoustic models to long-range acoustic propagation has been limited to two-dimensional (2D) or Nx2D models due to the complexity of the problem as well as computational efficiency. Therefore, ocean waveguides with strong azimuthal coupling still remain highly challenging because a full three-dimensional (3D) modeling is required.

Acoustic propagation around seamounts is a good example of a problem which has strong mode coupling and horizontal refraction by the sloped bathymetry of the seamount. In addition, uncertainties from oceanographic variability, e.g., sound speed variability due to the internal wave, and the geoacoustic property of seabottom increase the complexity of modeling acoustic propagation.

Physical experiments [1-4] and theoretical approaches [5-7, 15-16, 23] have been explored for over 30 years; however, due to the complexity of the problem, the phenomena of acoustic propagation around seamounts are not well understood.

In 2004, the Basin Acoustic Seamount Scattering Experiment (BASSEX) was conducted in the North Pacific as a part of long-range ocean acoustic propagation experiments of NPAL04 (North Pacific Acoustic Laboratory 2004) with two other experiments, called LOAPEX (Long-range Ocean Acoustic Program Experiment) and SPICE04. The BASSEX experiment was focused on the bathymetric effects on acoustic propagation, in particular, on direct blockage, refraction, diffraction, and scattering by seamounts. Moored and ship-deployed acoustic sources transmitted m-sequence signals at about $192 \mathrm{~dB}$ re $1 \mu \mathrm{Pa}$, including two SPICEX sources which transmitted eleven 12.3 
second sequences every hour at $250 \mathrm{~Hz}$ carrier frequency ( $83 \mathrm{~Hz}$ bandwidth), and a LOAPEX source which transmitted forty-one 30 second sequences at $68.2 \mathrm{~Hz}$ carrier frequency (35Hz bandwidth). The distances between the SPICEX sources and the Kermit-Roosevelt seamount are 617 and 504km.

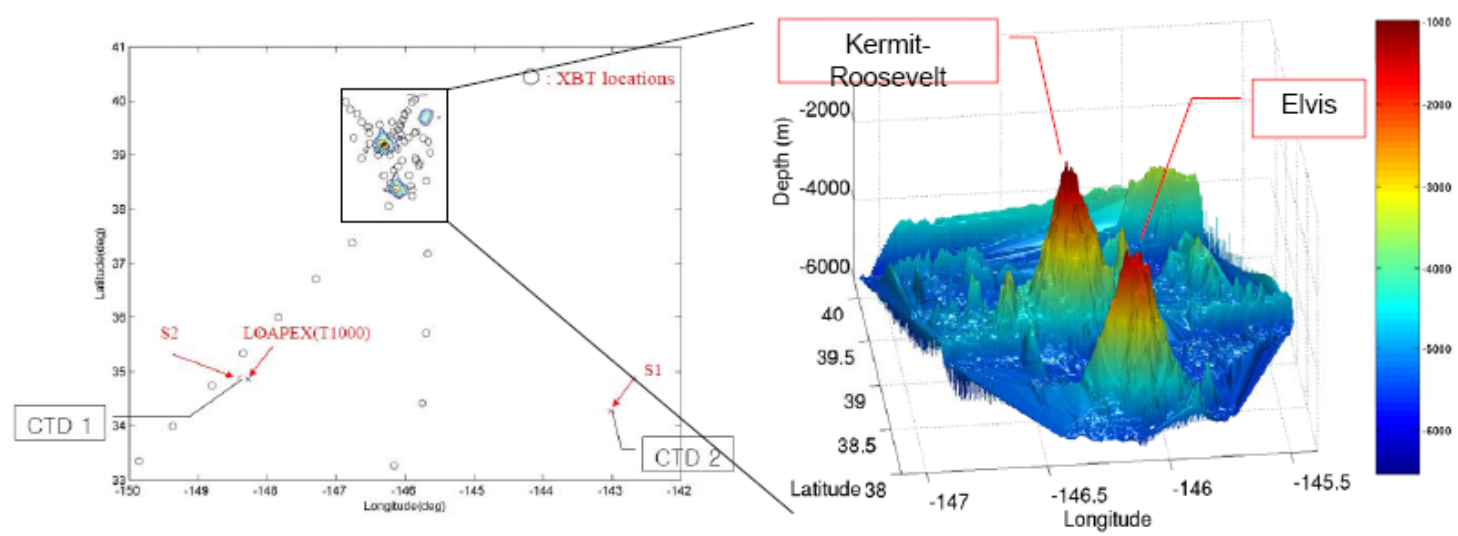

Figure 1-1: Bathymetry around the Kermit-Roosevelt and Elvis seamounts with the source locations (S1 \& S2 for the SPICEX sources, LOAPEX (T1000) for the LOAPEX source).
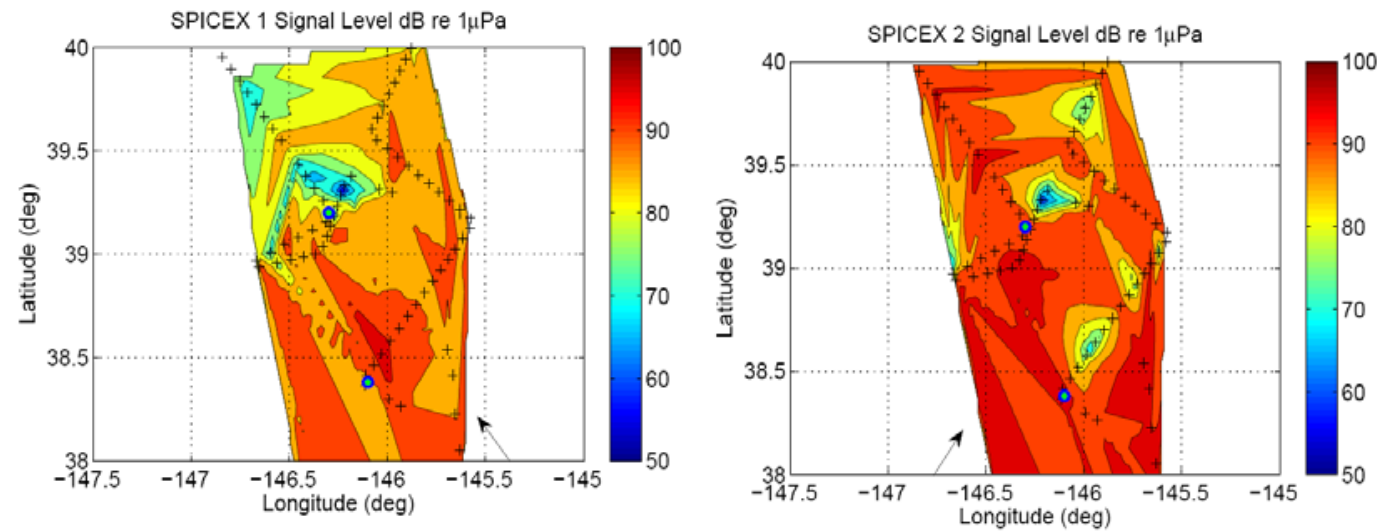

Figure 1-2: Measured peak sound levels received from the SPICEX source S1 (left panel) and S2 (right panel) [53].

The Five Octave Research Array (FORA), which is a towed hydrophone array with 64 sensors cut for $250 \mathrm{~Hz}$ (3m spacing), was used to measure the signals transmitted from the aforementioned broadband sources at many locations around the Kermit-Roosevelt 
and Elvis seamounts. Utilizing the measured broadband signals from the towed array, the size of the shadow zone was obtained and is shown in Fig. 1-2. Figure 1-2 shows the measured peak sound levels from the SPICEX sources; deep shadow zones as well as the formation of convergence zones are clearly visible behind the seamounts.

The measured data in the BASSEX experiment strongly support the understanding of the complicated phenomena of sound propagation around the seamounts. In addition, the experimental data could be used to validate current 2D and 3D theoretical models and develop new models to properly realize the sound propagation with such complicated phenomena.

In this thesis, the reconciliation between the measured pulse arrivals from the BASSEX experiment and various 2D and 3D theoretical models is carried out to explain the physical characteristics of the sound propagation around seamounts. Through this analysis, we examine the limit of the application of the models, and improved the efficiency of the 3D sound propagation model using parallel computing to generate a broadband pulse in a reasonable amount of time.

In Chapter 2, discussions are presented concerning previous efforts to explore the acoustic propagation around seamounts by experimental and theoretical approaches. A detailed description of the BASSEX experiment is addressed concerning bathymetry, acoustic sources, and sound speed fields. In this chapter, we also introduce an objective analysis for reducing uncertainties from the limited coverage of sound speed measurements. The objective analysis was performed with measured sound speeds in the BASSEX experiment and with climatological data, as well as with two CTD measurements in the LOAPEX experiment. The smooth sound speed fields were obtained from the objective analysis and used in parts of the 2D PE simulation.

In Chapter 3, various 3D sound propagation models are presented; specifically, the 3D spectral coupled-mode model is discussed in detail. Since the 3D coupled-mode model requires extensive computational demand, parallel computing is essential to obtain results in a reasonable amount of computation time. Therefore, more detailed discussions of the promising ways to increase computational efficiency and parallel computing are 
presented.

The simulation results are composed of two parts. First, in Chapter 4, the measured pulse arrivals in the BASSEX experiment are reconciled using 2D sound propagation models. The 2D Parabolic Equation (PE) and 2D ray tracing model were used to identify each ray arrival in terms of arrival time and grazing angle. The comparisons are carried out for SPICEX 1 and 2 sources with $250 \mathrm{~Hz}$ of center frequency. The simulations are performed for the acoustic paths which pass over the Kermit-Roosevelt and Elvis seamounts. Also, 2D simulations are carried out for open sea cases. The correlation of clear shadow zone and reappearance of convergence zone behind seamount is presented, and individual arrivals are matched with each other from BASSEX data and simulation.

Second, in Chapter 5, 3D broadband sound propagation around a conical seamount is investigated numerically using the 3D spectral coupled-mode model. The computed pulse arrivals are compared with the measurement in the BASSEX experiment for the LOAPEX ship-deployed acoustic source, which is centered at $68.2 \mathrm{~Hz}$. The broadband pulses are generated by the Fourier synthesis technique based on the frequency-domain solutions, which are calculated by the 3D spectral coupled-mode model. Since the calculation of the 3D broadband pulses with the 3D spectral coupled-mode model requires extensive computation time, which depends on the number of frequencies, normal modes, azimuth modes, and range steps, a parallel program is developed with a cluster computing system to obtain results in a reasonable time. The Kermit seamount is modeled by a simple conical seamount: the water depth at the peak is $954 \mathrm{~m}$ with a flat bottom at $5750 \mathrm{~m}$ depth. Two acoustic sources were examined with center frequencies of $15 \mathrm{~Hz}$ and $68.2 \mathrm{~Hz}$ : the sources are located $300 \mathrm{~km}$ and $510 \mathrm{~km}$ from the peak of the seamount. For the $68.2 \mathrm{~Hz}$ source, only the water trapped modes are used due to the limited computational ability. The computed 3D broadband pulses show the perturbed sound fields by the seamount, which are compared with results from the BASSEX experiment and from the 2D simulation using the 2D PE.

This research is supported by the United States Navy, Office of Naval Research (ONR), contact number N00014-04-1-0124. 


\section{Chapter 2}

\section{Background}

This chapter reviews previous experimental work and computational models concerning sound propagation around seamounts. A detailed description of the BASSEX experiment is given in terms of bathymetry, acoustics sources, sound speeds, and data processing. Finally, the basic phenomena on the sound propagation around a seamount are briefly presented.

\subsection{Previous work}

Physical experiments [1-4] and theoretical approaches [5-7, 15-16, 23] to the sound propagation around seamounts have been explored for over 30 years. The experiments were conducted in relatively short ranges and focused on measuring additional transmission loss by the blockage of a seamount. A full 3D sound propagation model with coupled normal mode theory was proposed by Buckingham[21] and Athanassoulis and Prospathopoulous[7], but the application has been limited due to the low computational efficiency of the sound propagation models as well as the limited computational ability. A more stable and numerically effective 3D spectral coupled-mode model was proposed by Luo [10] using the superposition representation of the external fields of the seamount and the two-way marching approximation. However, this model still requires improvement in computational efficiency to realize the broadband pulse simulation. 


\subsubsection{Experimental approach to the acoustic shadowing by seamounts}

Northrop [1] measured sound signals from 300 small underwater explosions at various depths in the Northeastern Pacific ocean at the SOFAR depth; hydrophones were located near Midway and Wake Islands. Explosions were detonated at the azimuth of $38^{\circ}$ to $78^{\circ}$ relative to Wake Island; shots were fired both on the sound channel axis and above the axis. Travel paths to Wake Islands crossed the Hawaiian Arch while those to Midway Island were unobstructed. The recordings were made for the peak signal level; the difference in peak signal level between the level at Wake Island and the level at Midway Island was measured. The measurement showed main shadow zones by the Hawaiian Arch as a function of the azimuth angle. In this work, Northrop explained some receptions through the Hawaiian Arch by passing over the seamount with bottomreflection-surface-reflection for steeper rays. In addition, the spectral energy density ratio between Wake and Midway Islands showed frequency independence.

Nutile and Guthrie[2] examined the acoustic shadowing produced by seamounts by using shot signals and CW signals. Explosive acoustic sources were dropped from a ship traversing a course approximately perpendicular to a line from the ship to a hydrophone of the Pacific Missile Impact Locating System (P-MILS) near Midway Island, and the shots were detonated at the nominal depth of $200 \mathrm{~m}$. The distance from the sources to the receiver varied between $1552 \mathrm{~km}$ and $1646 \mathrm{~km}$. The rectified shot signatures were aligned for identification of arrivals and assignment to ray paths. The signals traveled across an area of seamounts located north of the Hawaiian Ridge, and the missing arrivals were related to the ray-path blocking effect by the seamounts; the missing arrivals could be determined by comparison of shot signatures and predicted by carrying out the ray-trace calculations. This study showed that the acoustic shadowing for $14.65 \mathrm{~Hz} \mathrm{CW}$ signals was correlated with the blockage of the shot signals by the seamounts.

Ebbeson and Turner[3] examined the acoustic shadowing for a $230 \mathrm{~Hz}$ CW source over the Dickins Seamount in the Northeast Pacific. In their experiment, the source was towed at depths of 18 and $184 \mathrm{~m}$; the hydrophones were located relatively close to the 
seamount at a range of $60 \mathrm{~km}$ from the seamount peak in depths ranging from 329 to $633 \mathrm{~m}$. The minimum depth of the near peak of the Dickins Seamount was $420 \mathrm{~m}$, and the sound channel axis was at a depth of 350m, slightly above the peak. The results showed increased transmission loss of up to $15 \mathrm{~dB}$ for the shallow source in which all deep refracted waves could be blocked by the seamount. However, acoustic shadowing and reflection effects were minimal for the deep source because most of the source energy propagated along the sound-channel axis was located above the seamount peak. The analysis to identify reflected waves was performed by the ray tracing method. In their work, back reflections were separated from directly received energy by using the opposite Doppler shift, which is significant if the source is on the same side of the seamount and within $15 \mathrm{~km}$ of the seamount peak. The back reflections from the seamount were at the level of 6 to $13 \mathrm{~dB}$ below the direct signal level.

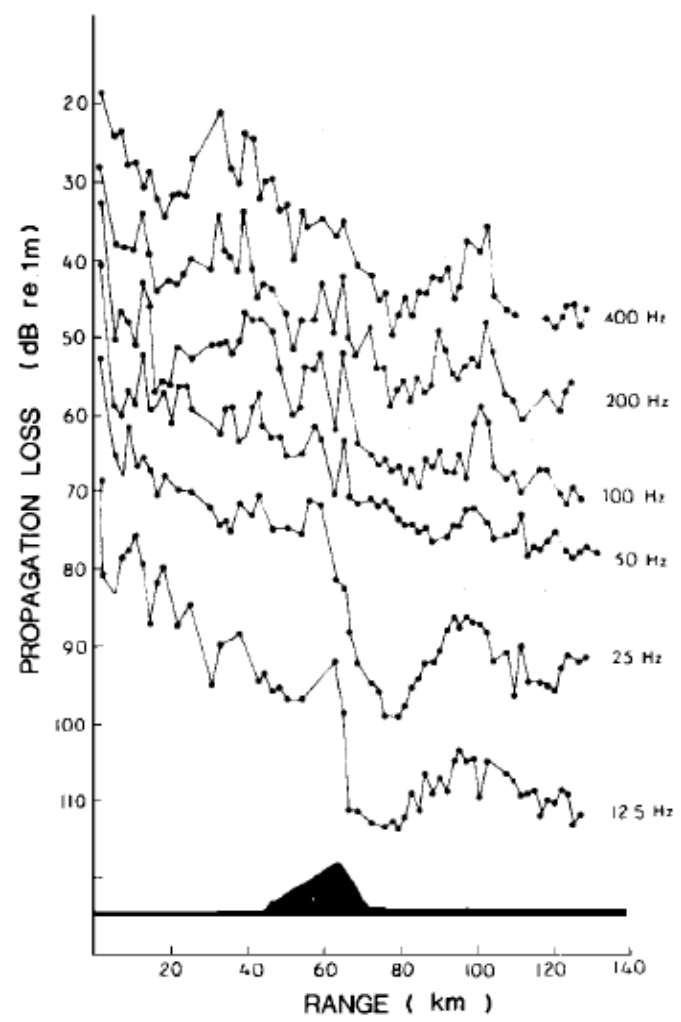

Figure 2-1: Experimental transmission loss collected by Chapman and Ebbeson [4]. 
Chapman and Ebbeson[4] presented the results of the multi-path propagation over the Dickins Seamount for small explosive shots; two charges were deployed at the depths of 24 and 196m. The data were presented in 1/3-octave bands from 12.5 to $400 \mathrm{~Hz}$; the propagation loss for the shallow shots was increased by 10 15dB over the loss expected in the absence of the seamount, as shown in Fig. 2-1. Since the receiver was located in the acoustic shadowing zone, all the refracted waves were blocked by the seamount. In this work, the first and dominant pulse consisted of a diffracted wave which passed over the seamount by rough-surface forward scattering and diffraction for high frequencies over $50 \mathrm{~Hz}$. It was followed by a series of surface-bottom interacted waves with a weaker level. To interpret these pulses, Chapman and Ebbeson proposed to use both the ray tracing method and the Medwin-Spaulding model [5]. This model is based on the theory of diffraction over a wedge, and on laboratory experiments with a scale model of Dickins Seamount. In this model, the ray tracing method was used to predict the propagation loss, and was applied separately, from the source to the seamount, and from the seamount to the receiver. The Medwin-Spaulding model of seamount shadowing predicts an $f^{1 / 2}$ dependence of the shadowing loss, which was in good agreement with the experimental data only at frequencies greater than $50 \mathrm{~Hz}$. The cause for the discrepancy could be a change in the scattering behavior from rough-surface forward scattering for higher frequencies to smooth-surface specular reflection for lower frequencies.

\subsubsection{Theoretical approach}

Computation time has been a large obstacle to solving range-dependent long-range acoustic propagation problems with three-dimensional (3D) effects.

Kuperman et al. [6] explored rapid three-dimensional acoustic field computations for a complex ocean environment using the adiabatic and coupled-mode theory. The precalculations of both vertical and horizontal quantities by the adiabatic and coupled-mode theory are used as an input into "spreadsheet" type manipulations. By doing the precalculations, the complex three dimensional field computations are reduced to 
manipulations of partial solutions to the wave equation. Kuperman et al. applied this method to a Gulf Stream environment near the continental shelf with a goal of validating the results from the adiabatic and coupled-mode computations by comparison with those from the Nx2D parabolic equation method.

\section{A. Full 3-D models}

Buckingham[21] proposed a theoretical model of acoustic propagation based on the normal mode theory in the ocean around a conical seamount, in which the seamount is assumed to be rigid, with the apex of the seamount just touching the surface of the ocean. This work showed the shadow zone lying behind the seamount using the mode amplitude function.

Athanassoulis and Prospathopoulous[7] gave an analytical solution to the threedimensional (3-D) problem of acoustic scattering from a nonpenetrable cylindrical island in shallow water. In this work, the ocean environment around the island is considered as range independent, and the bottom is assumed to be hard or soft. The presented analytical solution can be applied to a relatively low frequency problem, which can serve as a 3-D benchmark solution.

Taroukadis[8] decomposed a conical seamount into superposed rings and set up a coupled-mode formulation for the Helmholtz equation. The pressure field at each ring is expressed as a series expansion of normal modes and cosine functions in the azimuthal direction. The coupled coefficients are obtained by the continuity conditions at the interfaces of the rings. However, this method has a numerical instability with higher order of Hankel functions.

Eskenzai[9] used the Direct Global Matrix (DGM) approach to overcome the numerical instability of Taroudakis' method, and obtained a perturbation zone, or shadow zone, behind the seamount. However, in his work, the dimension of the resultant linear system was too large to be solved effectively.

Luo[10] proposed a more stable and effective 3D spectral coupled-mode model 
based on the well-known Coupled SACLANTCEN normal mode propagation loss model (C-SNAP) [63] and on the works of Taroukadis and Eskenzai. To obtain numerical stability, $J_{m}\left(k_{r n} r\right)$ and $H_{m}^{(1)}\left(k_{r n} r\right)$ are used with the advantage of linear independence of the two functions for both large and small arguments, and the improvement of efficiency is achieved by the introduction of the superposition representation of the external field with respect to the seamount.

Lee and Schultz [15] developed a 3D parabolic equation (PE) model that handles wide propagation angles in depth and in azimuth. The code, FOR3D, by Lee et al., used the finite difference solution, and applications were made to an Atlantic Ocean shelfslope environment with realistic bottom topographic variations and sound-speed profiles [16]. The 3D PE developed by Collins [14] handles wide angle propagation in depth as well as narrow angle propagation in azimuth with the effects of rough boundaries at the ocean surface. Collins et al. [13] compared the finite difference, split-step Fourier and split-step Padé algorithms in terms of efficiency and capability. The efficiency of the split-step Padé algorithm over the finite difference algorithm can be up to two orders of magnitude or more on a parallel-processing computer because the finite difference algorithm requires a relatively large number of range steps. Brooke et al. [73] developed a Canadian Parabolic Equation model (PECan). In the model, the split-step Padé algorithm was employed for sound propagation in range, and a finite-difference approximation with a low-order Padé approximation to the square-root operator was used for the 3D azimuthal coupling.

Fawcett [17] applied the 3D PE model to a penetrable ocean wedge, and the results were compared with those from the ray diagrams using the 3D adiabatic mode theory.

Another study using the 3D PE model, 3DWAPE, on a CW source with higherorder finite difference schemes in azimuth was performed by Sturm and Fawcett [52]. To consider 3D effects in the 3D PE model properly, the arc length between two adjacent vertical sections should be less than a quarter of the wavelength. To meet this requirement, the number of vertical sections increases as the desired range increases and hence the computational demand can be extensive. The higher-order finite difference schemes 
reduce the required number of vertical sections, which leads to lower computational demand. Sturm [52] applied the developed full 3D PE model, 3DWAPE, to the propagation of a broadband sound pulse in 3D shallow water waveguides. In this study, a Padé series azimuthal expansion was used to obtain a very wide angle capability in azimuth.

\section{B. Horizontal refraction}

After the introduction of the term "horizontal refraction" by Weston [25], many researchers have tried to explain the horizontal refracted wave for the coastal wedge and seamounts, which have a strong refraction effect due to the sloping bathymetry. Harrison $[18,19]$ derived analytical ray paths caused by repeated reflections at a sloping sea bed, and showed shadow zone boundaries for a seamount. Munk and Zachariasen [26] also analyzed the refraction of acoustic energy by seamounts and islands with a simplified assumption of a purely conical shape based on adiabatic mode theory.

Doolittle et al. [20] observed the horizontal refraction due to multiple reflections from a sloping bottom. Smith et al. [24] applied a 3-D ray code, a hybrid ray-mode code, and a 3-D parabolic equation model to a study of significant azimuthal coupling which exists in the vicinity of the shelf break. Although this study included up-slope, slant-slope, and cross-slope propagation, no significant azimuthal coupling was found.

Heaney et al. [22] calculated very long-range horizontal propagation ray paths for a 1960 experiment which measured sound near Bermuda from three underwater explosions near Perth, Australia. The ray paths were calculated based on the adiabatic mode theory (see Section 3.3) in which a horizontal refraction is determined by the gradient of a local phase speed for each vertical mode. The mode coupling effects are neglected with an assumption that the ocean waveguide varies slowly in the horizontal plane with respect to other relevant scales. A horizontally refracted ray for a vertical mode reflects horizontal changes in the entire sound speed profile and bottom interaction. McDonald et al. [23] also applied the adiabatic mode theory to the examination of the propagation paths for the 
Heard Island Feasibility Test.

\subsection{Basin Acoustics Seamount Scattering Experiment (BASSEX)}

In 2004, the Basin Acoustic Seamount Scattering Experiment (BASSEX) was conducted in the North Pacific. The BASSEX experiment was performed at the same time as two other experiments, LOAPEX (Long-range Ocean Acoustic Program Experiment) and SPICE04, as parts of long-range ocean acoustic propagation experiment of NPAL04 (North Pacific Acoustic Laboratory).

The objective of the SPICE04 was to explore the role of ocean spiciness in longrange sound propagation, i.e., temperature and salinity fluctuations. The goal of the LOAPEX experiment was the investigation of range dependence of resolved acoustic rays, ray arrivals in a geometric shadow zone, and frequency dependence of sound propagation, as well as large-scale oceanography including the basin-scale observations of heat content and temperature.

The BASSEX experiment was focused on the bathymetric effects on acoustic propagation, in particular, on direct blockage, refraction, diffraction, and scattering by seamounts. Moored and ship deployed acoustic sources transmitted m-sequence signals at about $192 \mathrm{~dB}$ re $1 \mu \mathrm{Pa}$, including two SPICEX sources which transmitted eleven 12.3 second sequences every hour at $250 \mathrm{~Hz}$ carrier frequency ( $83 \mathrm{~Hz}$ bandwidth), and a LOAPEX source which transmitted forty-one 30 second sequences at $68.2 \mathrm{~Hz}$ carrier frequency ( $35 \mathrm{~Hz}$ bandwidth). The distances between the SPICEX sources and the Kermit-Roosevelt seamount are 617 and 504km. The Five Octave Research Array (FORA), a towed hydrophone array with 64 sensors cut for $250 \mathrm{~Hz}$ (3m spacing), was used to measure the signals transmitted from the aforementioned broadband sources at many locations around the Kermit-Roosevelt and Elvis seamounts. During the experiment, 
the bathymetry around the seamounts and the sound speed profile were measured. More detailed discussion of the experiment can be found in [31, 32, 33, and 34]. In the following sections, the bathymetry, sound speed profiles, and acoustic sources used in the BASSEX experiment are addressed briefly.

\subsubsection{Bathymetry data}

Bathymetry data around the Kermit-Roosevelt and Elvis seamounts were taken during the BASSEX experiment using the $R / V$ Roger Revell's EM120 Multibeam Swath bathymetry Echo Sounder [53]. The high resolution multi-beam data around the Kermit-Roosevelt and Elvis seamounts were taken; the bathymetry data around the seamounts are shown in Fig. 2-2.

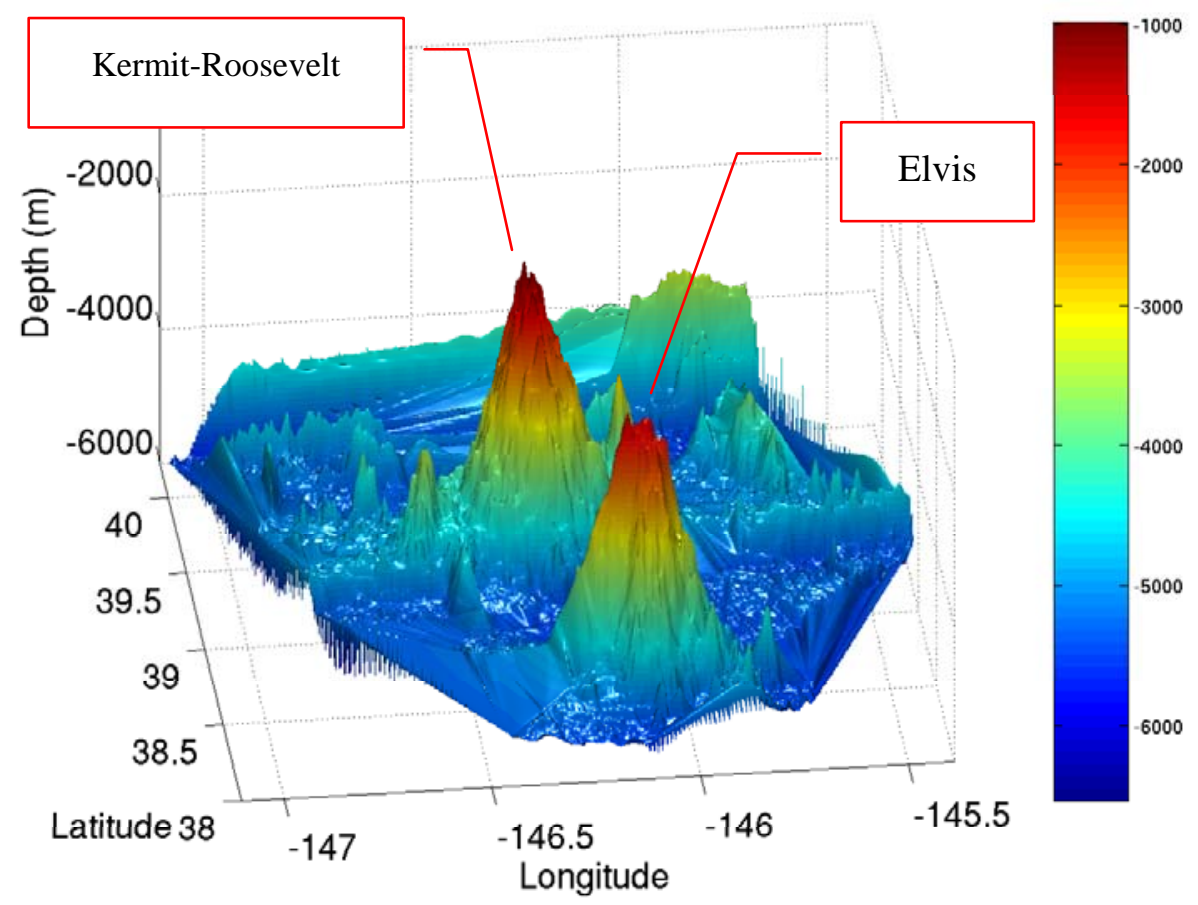

Figure 2-2: Kermit-Roosevelt and Elvis seamount; the minimum water depths of the seamounts are $980 \mathrm{~m}$ and 1380m, respectively [53]. 


\subsubsection{Acoustic sources}

In the BASSEX experiment, four acoustic sources were used: SPICEX source 1 and 2 , LOAPEX, and NPAL Kauai source, as shown in Fig. 2-3. The parameters of the acoustic sources are described in Appendix A.

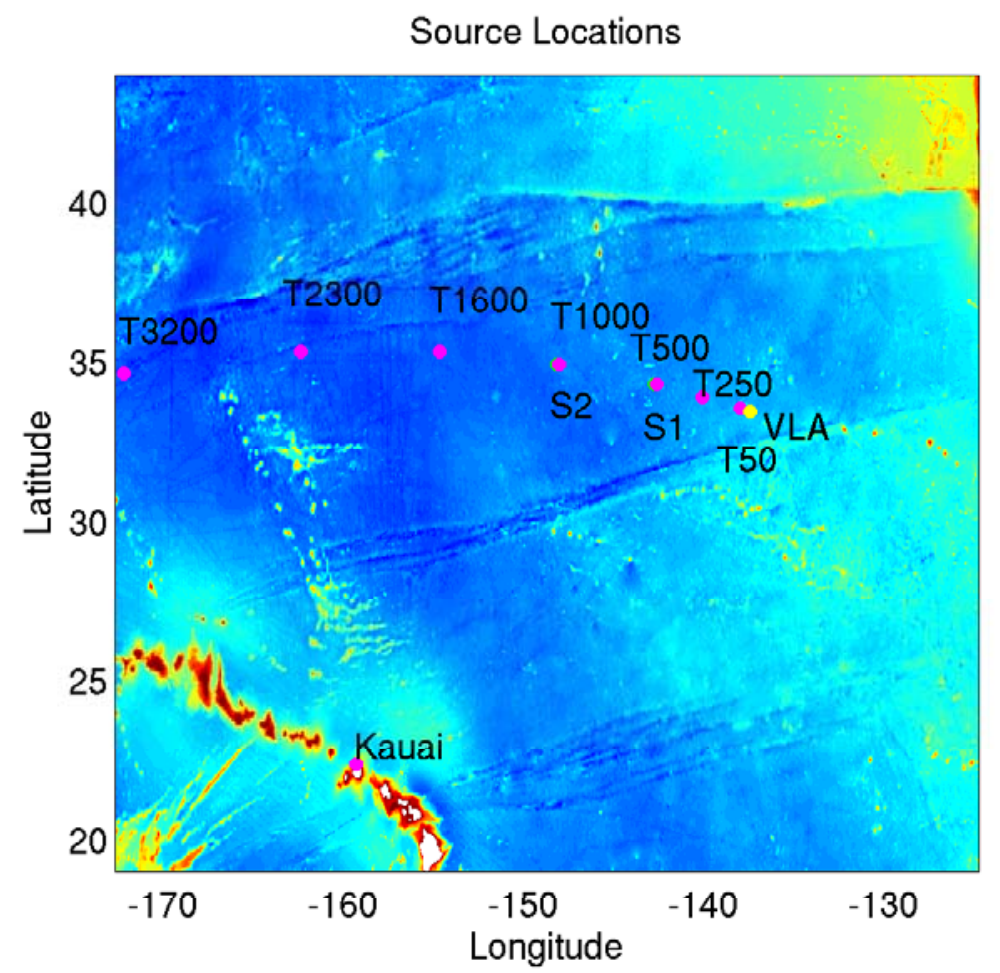

Figure 2-3: Locations of acoustic sources in the BASSEX experiment: (S) SPICEX moored sources, (T) LOAPEX stations, (Kauai) NPAL Kauai Source [53].

Two SPICEX sources were moored, and transmitted eleven 12.3 second sequences every hour at $250 \mathrm{~Hz}$ carrier frequency with $83 \mathrm{~Hz}$ bandwidth. The SPICEX acoustic sources [31] have m-sequence signals and were located around the sound channel axis at a water depth of 750m. A LOAPEX source was deployed at various locations around SPICE arrays. The distances between the SPICEX 1 and 2 sources and the KermitRoosevelt seamount are 617 and 504km, respectively.

The LOAPEX acoustic source had three types of signals: m-sequence, continuous 
wave (CW), and pulsed frequency modulated (PFM) signals. The m-sequence is the preferred signal in this study. The m-sequence signals consisted of forty-one 30 second sequences at $68.2 \mathrm{~Hz}$ carrier frequency with $35 \mathrm{~Hz}$ bandwidth at a depth of $350 \mathrm{~m}$. Among the LOAPEX stations, the T1000 was chosen because the deployed location was close to the SPICEX 2 source.

The NPAL Kauai source was located at a depth of $807 \mathrm{~m}$, approximately $14.8 \mathrm{~km}$ north of Kauai island. The Kauai source was not considered in this work.

\subsubsection{Sound speed fields}

Sound speed is the important environmental input for a sound propagation model. The horizontal array at a shallow depth can only detect early-arrival steep rays that sample the sound speed fields of the entire ocean column; therefore, the error in the prediction of sound speed fields results in significant error in the prediction of the rays of the simultation. The discrepancy between real and predicted sound speed gives two important effects on the arrival rays: one is a shift in arrival time, and the other is the diffusion or fluctuation of wave fronts.

The shift in arrival time between predicted and measured rays mainly comes from the difference in the temperature predicted by the climatology, which is the basis of acoustic thermometry. Some of this difference might be from the error of the sound speed equation [38, 39, 42]. The intenal ocean wave causes the fluctuation of wave fronts, including a rapid variation that is called a broadband fluctuation. This broadband fluctuation is associated with multiple arrivals, and internal wave seems responsible for this fluctuation [47].

The diffused wave fronts can result in missing or additional ray arrivals in the measurement. In addition, the broadband fluctuation, or multiple arrivals, greatly increase the difficulty in identifying individual rays. 
In the BASSEX experiment, sound speed profiles were measured by an expendable bathythermograph (XBT). The locations where the XBT casts were conducted are shown in Fig. 2-4 (a).

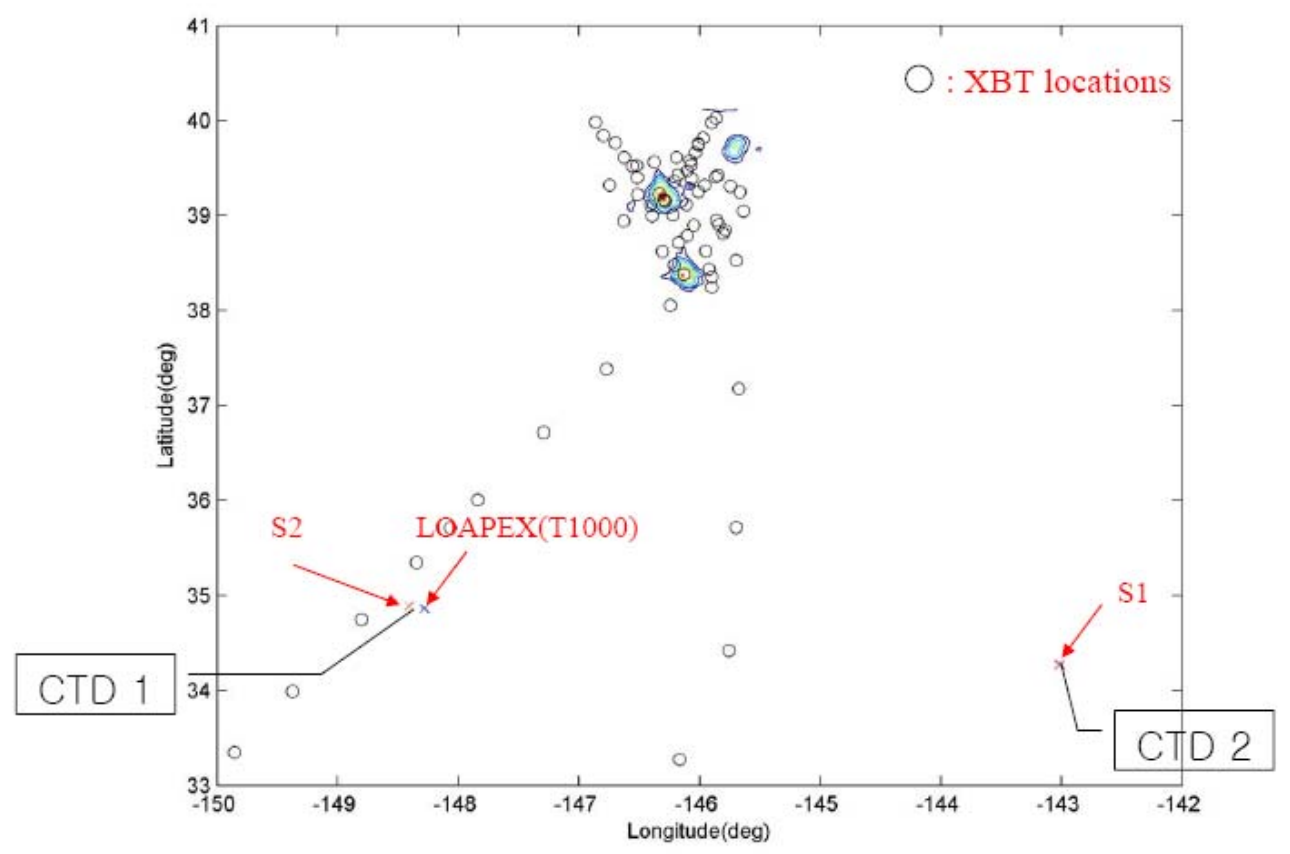

Figure 2-4: (a) XBT casts locations around the Kermit-Roosevelt and Elvis seamounts; the circles denote the locations where the XBT casts were conducted. Two SPICEX sources (S1 and S2) are shown with the LOAPEX source (T1000). Two locations where the CTD casts were carried out in the LOAPEX experiment are shown with CTD 1 and CTD 2.

The XBT provides the temperature data of the water column, and sound speed can be computed from the temperature data with the given salinity table. Examples of the sound speed profile from the XBT casts are shown in Fig. 2-5. The XBT operates in a water depth that is limited by the type of sensor; for example, T-5 has $1830 \mathrm{~m}$ of the maximum operating depth, and the maximum depth of 'Fast Deep' is $1000 \mathrm{~m}$. Beyond the terminal depth, sound speed or temperature should be extended based on available measurements or database. In this work, the sound speed beyond the terminal depth of the XBT casts were extended by the Carter table ( see Appedix C ). 


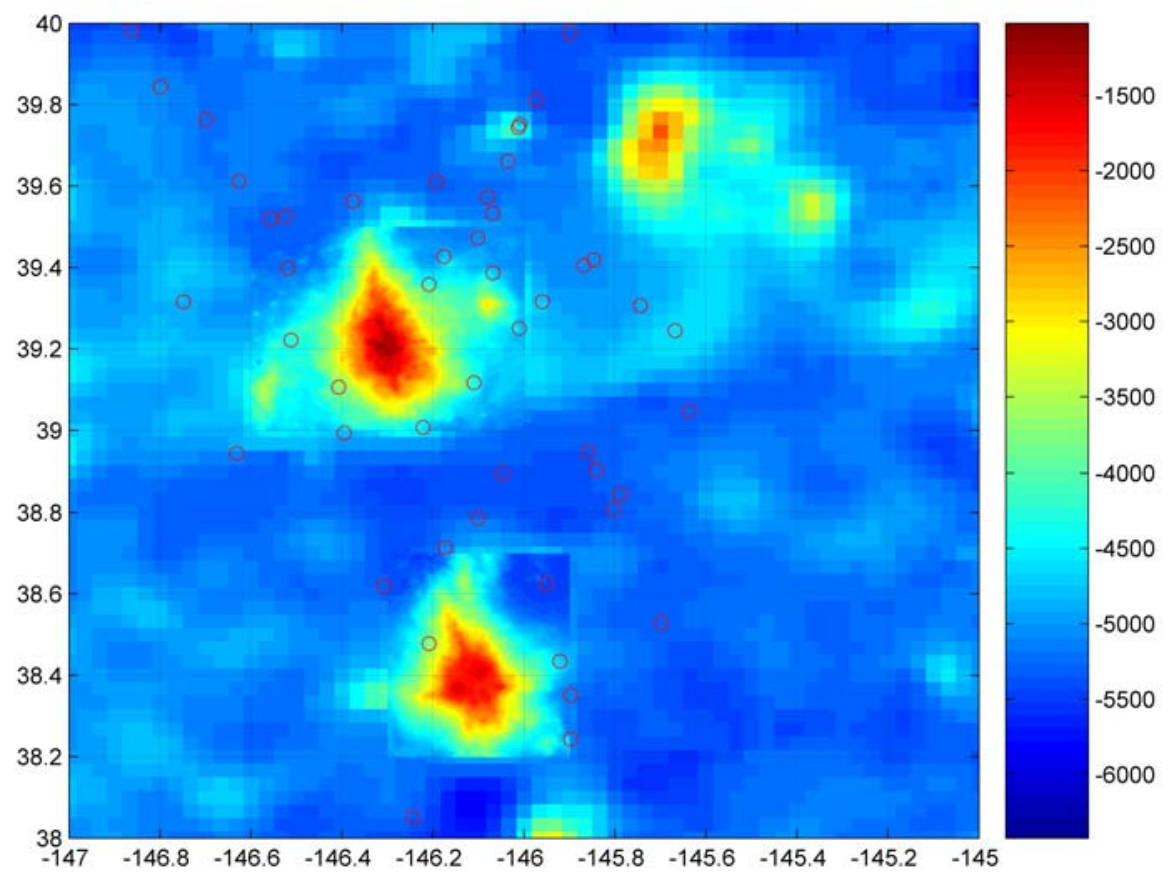

Figure 2-4: (b) XBT cast locations around the Kermit-Roosevel and Elvis seamounts.

As shown in Fig. 2-4(b), the XBT locations were covered well around the KermitRoosevelt and Elvis seamounts; however, in the area between the SPICEX sources and the seamounts, limited measurement of sound speeds was carried out. In particular, no XBT cast was conducted around the SPICEX 1 source or along the acoustic path to the seamounts from the source. This sparse and gappy sound speed measurement can be quite common in any experiment because the measurement of the sound speed for the whole area is difficult and costly to cover the whole area. Specifically, since 3D sound propagation is the main topic of this work, sound speeds of the whole area, including around the seamounts, and acoustic sources, as well as acoustic paths between the seamounts and acoustic asources, are more essential than the 2D sound propagation problem. Generally, the 2D sound propagation problem needs sound speeds only along an acoustics path between a source and receiver. 
The limited coverage of raw measurement increases uncertainties in the sound propagation model. To reduce the uncertainties from the missing sound speeds, various kinds of sound speed data can be used: raw measurement from other experiments, climatological data, and dynamic models [61].

In this work, climatological data from World Ocean Atlas 2005 [36, 37] were used; these sound speed profiles will be referred to hereafter as the WOA05. The sound speeds were computed using the sound speed equation of Del Grosso [35]. Since WOA05 gives the monthly climatology for $0 \sim 1500 \mathrm{~m}$, sound speed profiles are extended to $5500 \mathrm{~m}$ using seasonal climatology and nearest-neighbor values. Sound speed at a certain location can be obtained by linear interpolation [37].

The sound speed profiles given by the XBT casts at two locations are compared with those from WOA05 in Fig. 2-5. The two locations are chosen around the SPICEX 2 source (top panels) and the top of the Kermit-Roosevelt seamount (bottom panels). The right panels give the difference in sound speed profiles between the WOA05 and the XBT casts. The differences are quite large at the mixed layer, up to $6 \mathrm{~m} / \mathrm{s}$.

Figure 2-6 and 2-7 show the horizontal variation of sound speed fields from the XBT casts and WOA05 for the depths of $0 \mathrm{~m}, 10 \mathrm{~m}, 800 \mathrm{~m}$ and $900 \mathrm{~m}$, showing clear discrepancies between the two sound speed fields on a horizontal plane. First, the sound speed fields from the WOA05 are smooth, while the measured sound speed fields show a sharp change in the sound speed. Second, overall, the measured sound speed fields give a higher sound speed than those from the WOA05. In addition, there are irregular isolated spots, which have a much higher sound speed locally, specifically around the seamounts. In the measured sound speed fields of Fig. 2-6, a significant isolated region with a higher sound speed than the WOA05 is shown around the sound channel axis, $-146^{\circ} \mathrm{E} \sim-147^{\circ} \mathrm{E}$, and $36{ }^{\circ} \mathrm{N} \sim 38^{\circ} \mathrm{N}$.

Figure 2-8 shows the comparisons of the measured sound speed fields and the WOA05 along an acoustic path between the SPICEX 2 source and the apex of the Elvis seamount (day 268). Since the XBT casts were carried out along this acoustic path, the comparison clearly shows the difference between these sound speed fields. In the top 
panel, the range-averaged sound speed profiles are close to each other except for above the sound channel axis. The XBT casts show higher sound speeds above the sound channel axis than the WOA05. However, in the two lower panels, the variation of sound speed field along the acoustic path is quite different. A large difference exists in the smoothness of the sound speed fields along the acoustic path, showing locally isolated regions, which have much higher or lower sound speeds, up to $10 \mathrm{~m} / \mathrm{s}$ compared to the nearby field.

From the comparisons, we explored the horizontal difference between sound fields from the XBT casts and a database, WOA05, and the difference along a sound path for day 268 (Section 4.3). In addition, we confirmed that the measured sound speed fields require an interpolation scheme to construct smooth and statistically correct sound speed fields based on the various data of sound speed, raw measurements, and climatological data to reduce the uncertainty due to the limited coverage of XBT casts.

Several approaches for smoothed sound speed fields can be found in [38, 43, 48, 49] by using the objective analysis. In [38], the objective analysis was used to obtain sound speed fields from the XBT and CTD data, which give travel time error based on the error map associated with the sound speed fields. In [43], the objective analysis was used to meld upper ocean perturbations derived from the XBT/CTD data into historical data (WOA04) smoothly. Newhall et. al. [49] suggested a way to interpolate sparse, irregularly spaced, and gappy data on a regular grid in a horizontal plane using the objective analysis for improving the 3D ray-tracing model. More detailed discussion of the method of objective analysis in the ocean prediction system and data assimilation can be found in [50,58, and 60]. 

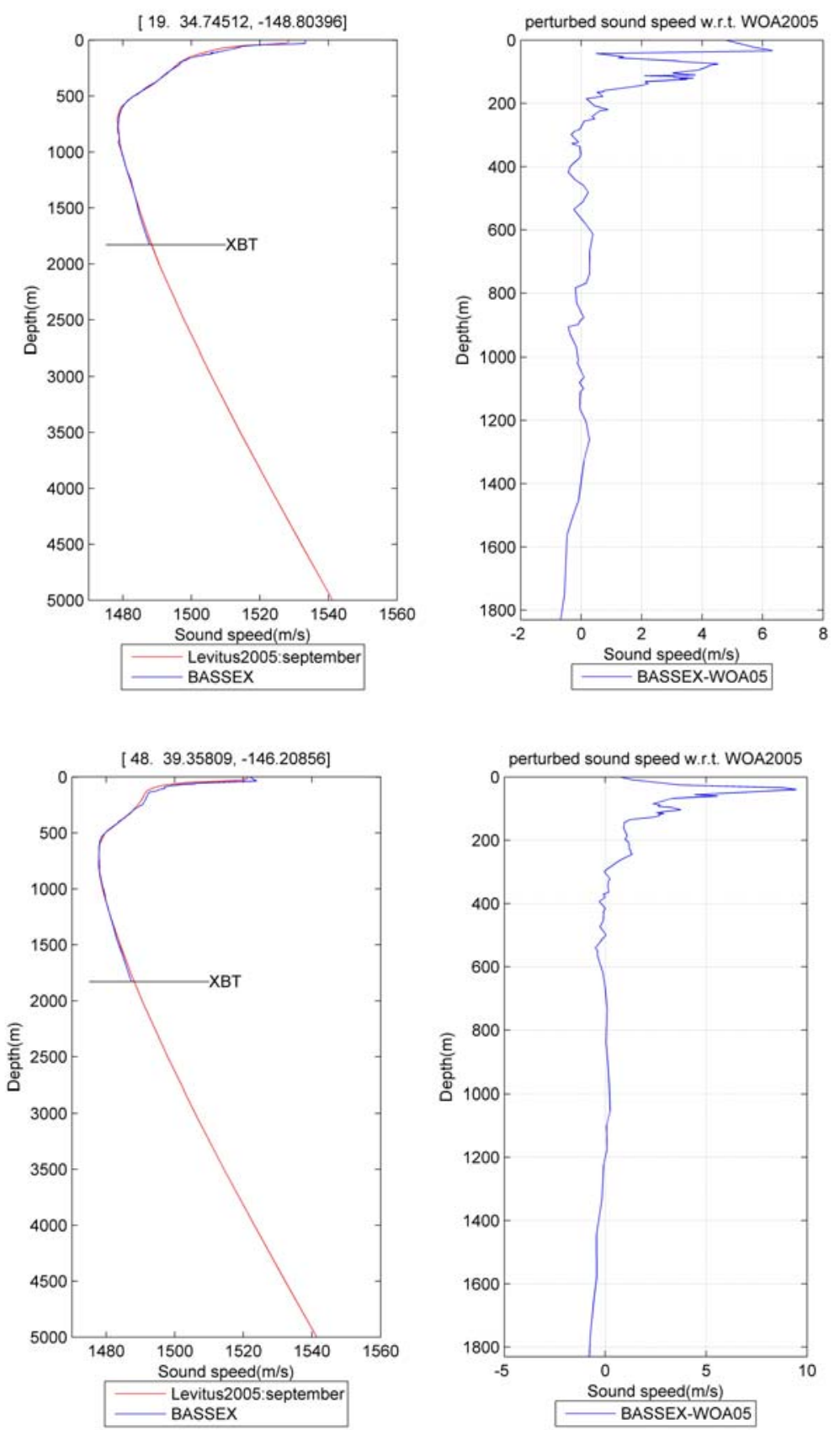

Figure 2-5: Comparison of sound speed profiles from the WOA05 and XBT for two selected locations. 'XBT' denotes the terminal depth of XBT casts. The right panels shows the compared sound speed profiles, and the left panels show the difference in sound speed profiles between the WOA05 and the XBT casts. The two locations are chosen near the SPICEX 2 source (top panels) and the top of the Kermit-Roosevelt seamount (bottom panels). 

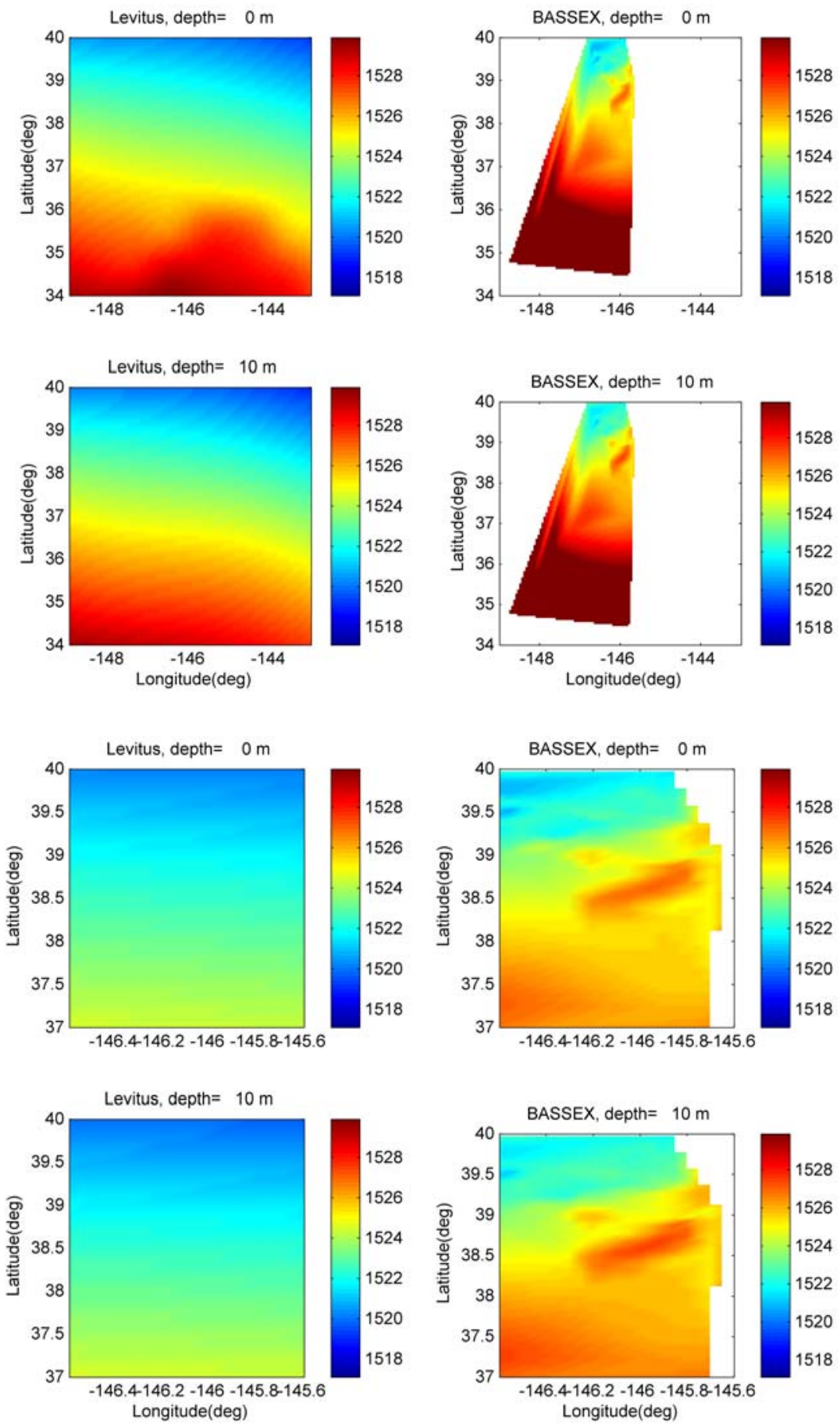

Figure 2-6: Comparison of the horizontal variation of sound speed from the XBT casts and WOA05 at the water depth 0 and $10 \mathrm{~m}$; the four lower panels show sound speeds specifically around the seamounts. 

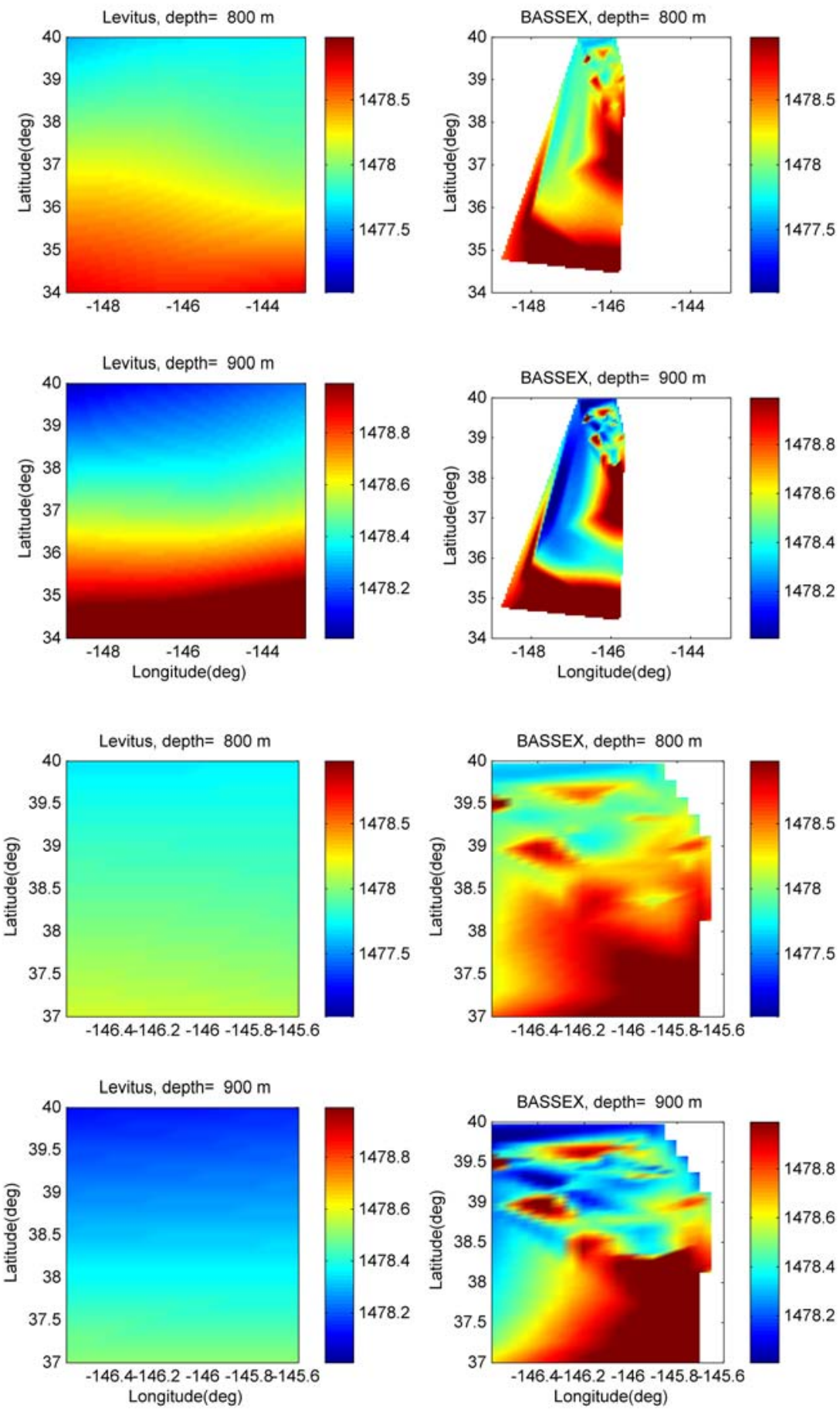

Figure 2-7: Comparison of the horizontal variation of sound speed from the XBT casts and WOA05 at the water depth 800 and $900 \mathrm{~m}$; the four lower panels show sound speeds specifically around the seamounts. 

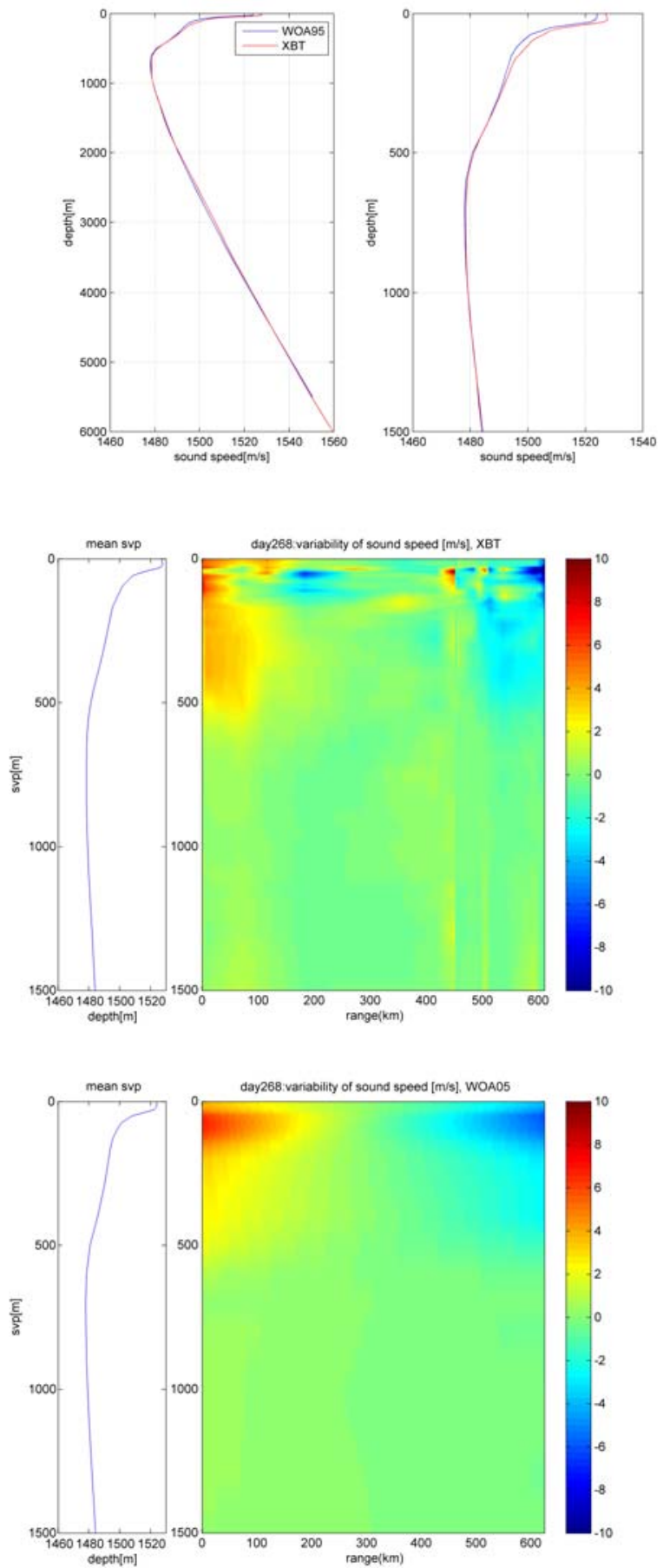

Figure 2-8: Comparison of the sound speed profile from the XBT and WOA05 along an acoustic path for day 268 over the Elvis seamount; the top panel shows the comparison of range-averaged sound speed as a function of depth, the middle (XBT) and bottom (WOA05) panels show variability of sound speed along the acoustic path. 
In this work, we constructed a smooth sound speed database using the objective analysis based on the XBT data from the BASSEX experiment and the climatological data, called WOA05, for the area around seamounts and acoustic sources as well as along acoustic paths between them. The sound speed database constructed by the objective analysis enables us to apply 2D and 3D sound propagation models for any acoustic path. The objective analysis was carried out by Pierre F. J. Lermusiaux and Wayne G. Lelie of MIT following the HOPS (Harvard Ocean Prediction System) optimal interpolation(OI) [Appendix of 58].

In addition to the XBT data from the BASSEX experiment and WOA05, available CTD data from the LOAPEX experiment were added to the objective analysis. The locations where the CTD casts were carried out are only around the SPICEX 1, 2 and LOAPEX (T1000) sources, as shown in Fig. 2-4 (a). Since the CTD cast can measure sound speeds up to $5000 \mathrm{~m}$ deep and the sound speeds at deep water are not sensitive to the area, these CTD data can be used for sound speeds at deep sea beyond the terminal depth of XBT casts.

Fig. 2-12 shows the differences between various sound speed profiles from XBT/CTD, WOA05, and objetive analysis at the locations where CTD casts were carried out. As shown in the right panel in the figure, the sound speed profiles from XBT below $1500 \mathrm{~m}$ deep, which was extended based on the Carter table, are different from other sound speed profiles. As a result, the inclusion of CTD cast in objective analysis resulted in increased correlation in pulse arrivals, in particular, at the open water case of day 271; an example of pulse arrivals is given in Fig. 2-13, and more cases are available in Chapter 4-6.

For the objective analysis, various correlation parameters were examined, 180 540 km for the zero-crossing length and 60 180 km for the e-folding decay length. Sound speed fields were tested with the parameters using the 2D PE to estimate the impact of the correlation parameters on the pulse arrivals. Significant difference was not found within the limited tests, but more tests would be needed to estimate the impact correctly, which might be beyond the scope of this work. 
In Table 2-1, the correlation parameters used in the objective analysis are shown, and the resultant sound speed fields are given in Fig. 2-9. Run 51 used all three data, the XBT data, WOA05, and CTD data, for the background mean field as well as for the synoptic measurement field. The XBT data were truncated below $1500 \mathrm{~m}$ to reduce the uncertainties from the extension of the sound speed based on the Carter table.

In Fig. 2-10, the sound speed fields from objective analysis, Run 51, were compared with linearly interpolated XBT data and WOA05; this comparison clearly shows that WOA05 is fit in to smoothed XBT data. Figure 2-11 shows the comparison of sound speed fields from XBT data, WOA05, and objective analysis. The sound speed fields from the objective analysis are very close to those from XBT data, but more smoothed sound fields can be obtained by the objective analysis.

Table 2-1: Correlation parameters for the objective analysis of sound speed fields

\begin{tabular}{|c|c|c|}
\hline \multicolumn{2}{|l|}{ Run no. } & 51 \\
\hline \multicolumn{2}{|c|}{ Synoptic field } & complete_short $^{\mathrm{A}}$ \\
\hline \multicolumn{2}{|c|}{ Mean field } & complete_short \\
\hline \multirow[t]{3}{*}{ Synoptic } & zonal/meridional zero crossing length $[\mathrm{km}]$ & 540 \\
\hline & zonal/meridional decorrelation length[km] & 180 \\
\hline & decorrelation time scale [day] & 30 \\
\hline \multirow[t]{3}{*}{ Mean } & zonal/meridional zero crossing length $[\mathrm{km}]$ & 540 \\
\hline & zonal/meridional decorrelation length[km] & 180 \\
\hline & decorrelation time scale [day] & 10000 \\
\hline
\end{tabular}

${ }^{A}$ complete_short used the WOA05, CTD and XBT ( truncated at 1500m ) for the objective analysis. 

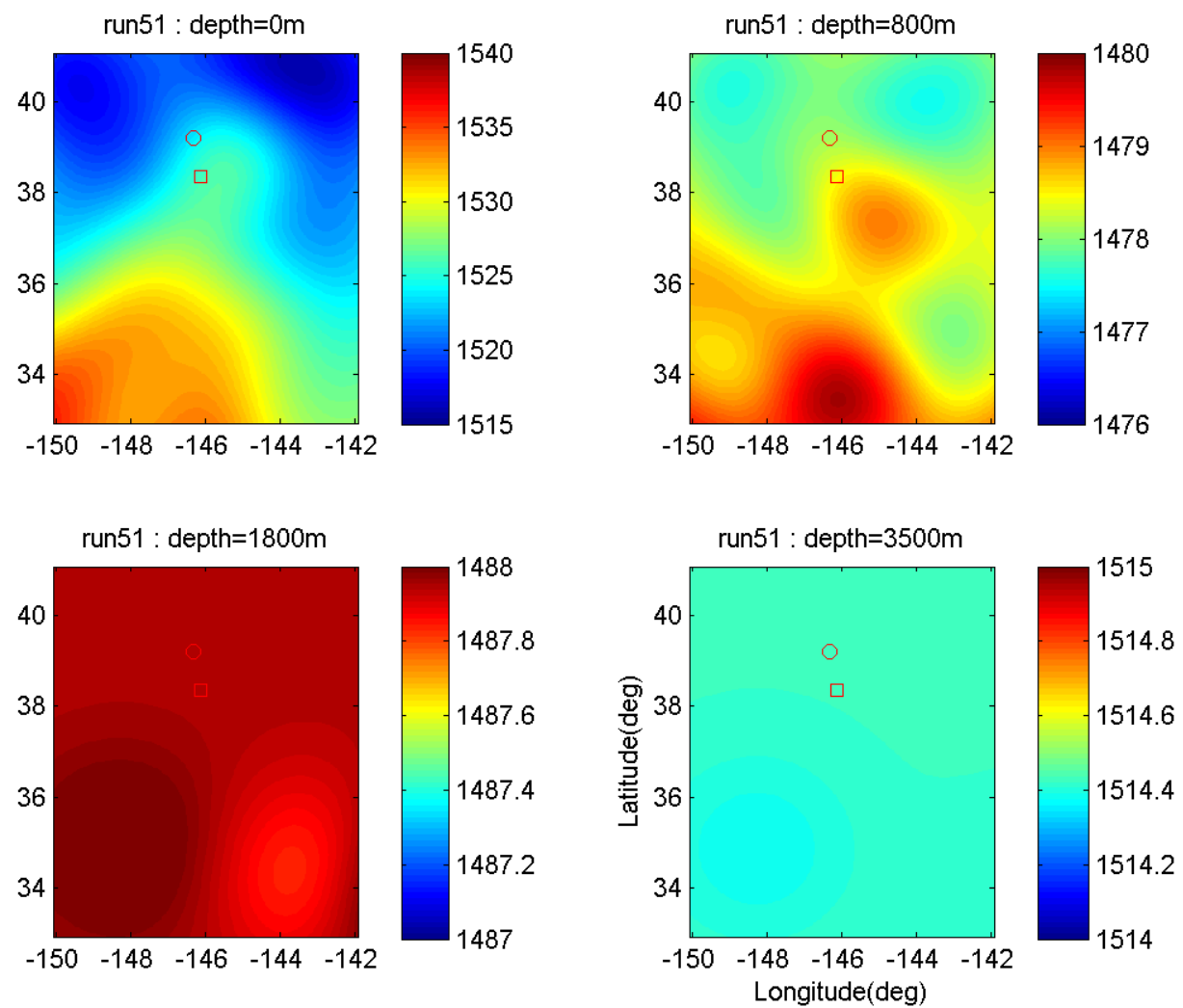

Figure 2-9: Sound speed fields from the objective analysis for various depths. The circle corresponds to the peak of the Kermit-Roosevelt seamount, and the square denotes the peak of the Elvis seamount. 

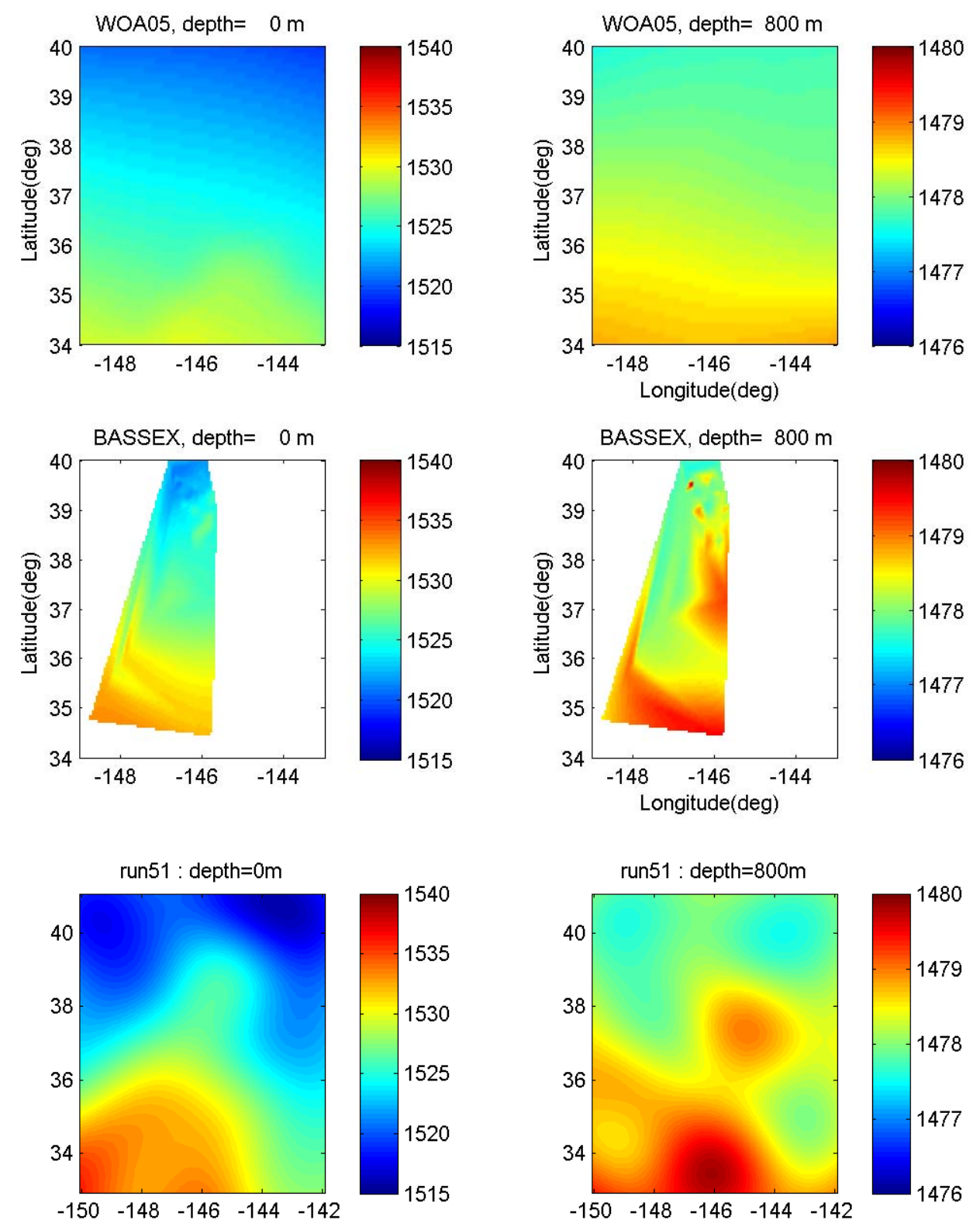

Figure 2-10: Comparison of sound speed fields from the WOA05 (top panels), XBT data (middle panels), and objective analysis (bottom panels). 

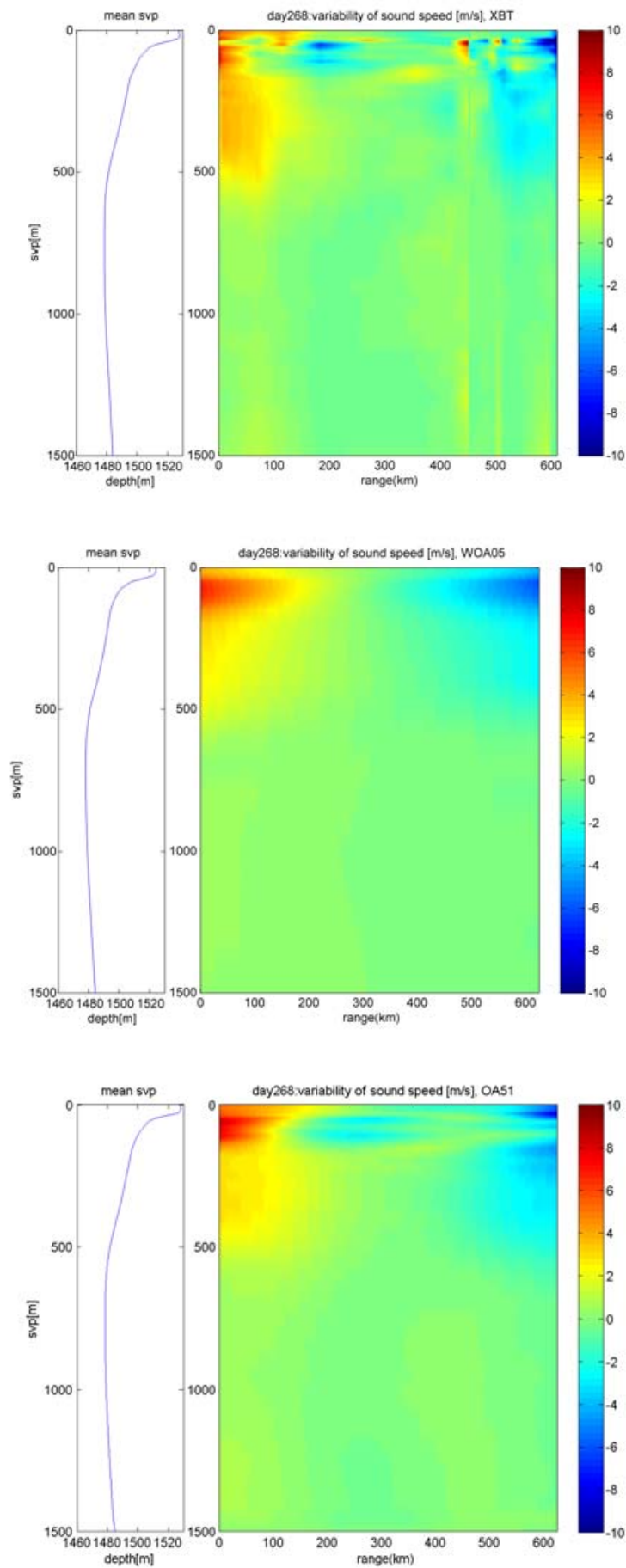

Figure 2-11: Comparison of sound speed fields for an acoustic path of day 268 from the XBT data (top panel), WOA05 (middle panel), and objective analysis (bottom panel). 

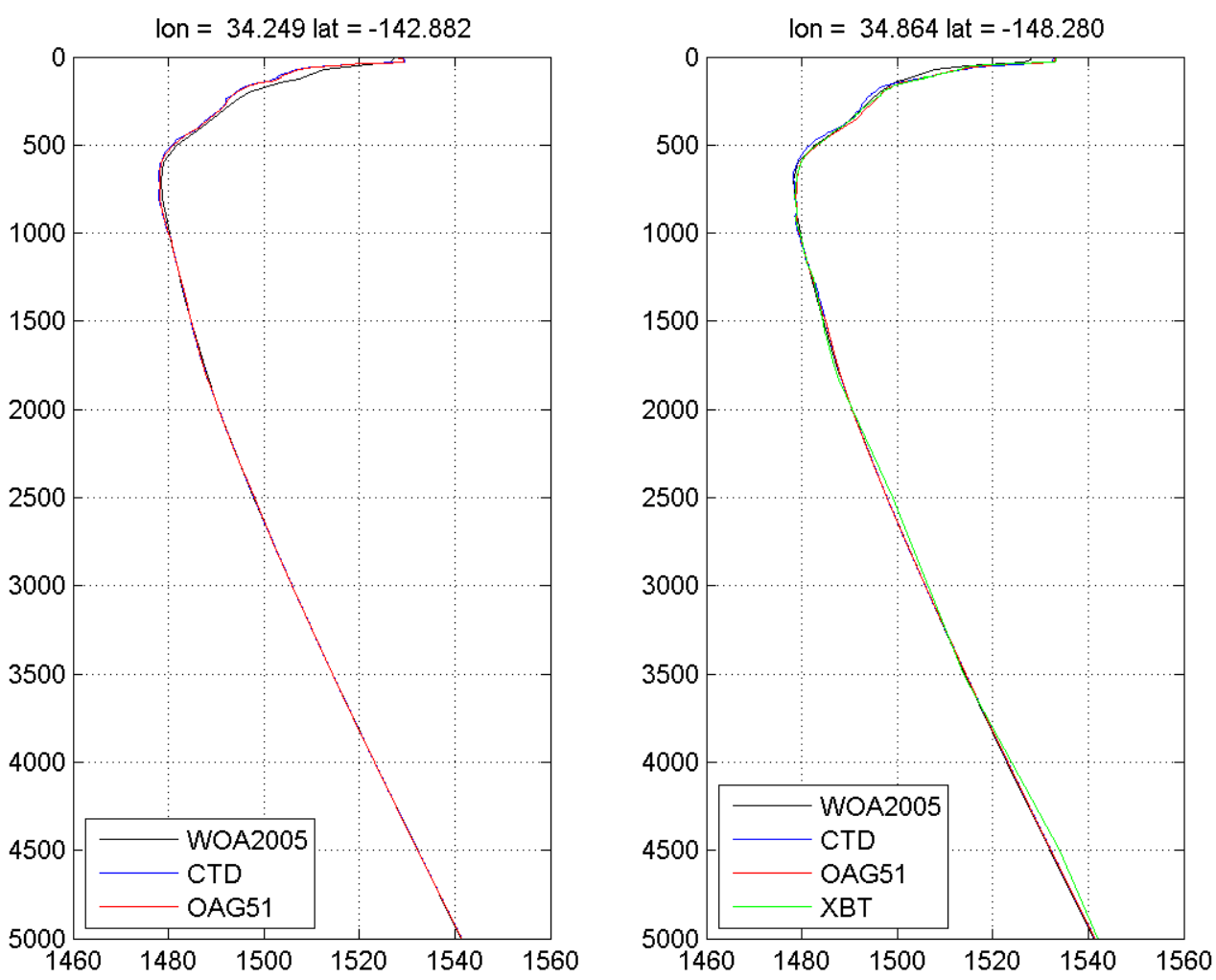

Figure 2-12: Comparison of sound speed profiles at locations where CTD casts were carried out. 
As a result, the sound speed extension below the terminal depth in the XBT casts based on the database can provide some errors to the sound-speed gradient below the sound channel axis. Since a deep-down and high angle ray samples the sound speeds of the entire water column, the sound-speed gradient below the sound channel axis has a large effect on the pulse arrivals with a horizontal array located at a shallow depth.

As an example, in Fig. 2-13, the arrival pulses with the sound speeds which are extended based on the database, i.e., the Carter table, in the lower panel (blue), shows two additional pulses along with the measurement in the upper panel (red). The additional pulses disappear with the sound speeds extended with the CTD data in the upper panel (blue) increasing the correlation between the measuremnt and simulation. This increased correlation implies that a CTD cast can be used to provide precise sound-speed gradient below the sound channel axis if we have limited sound speed data in depth such as XBT casts. Since the sound-speed gradient is not sensitive to area because it depends on the pressure-gradient effect, only one or two CTD casts over the entire area can be enough for the correction of the sound-speed gradient.
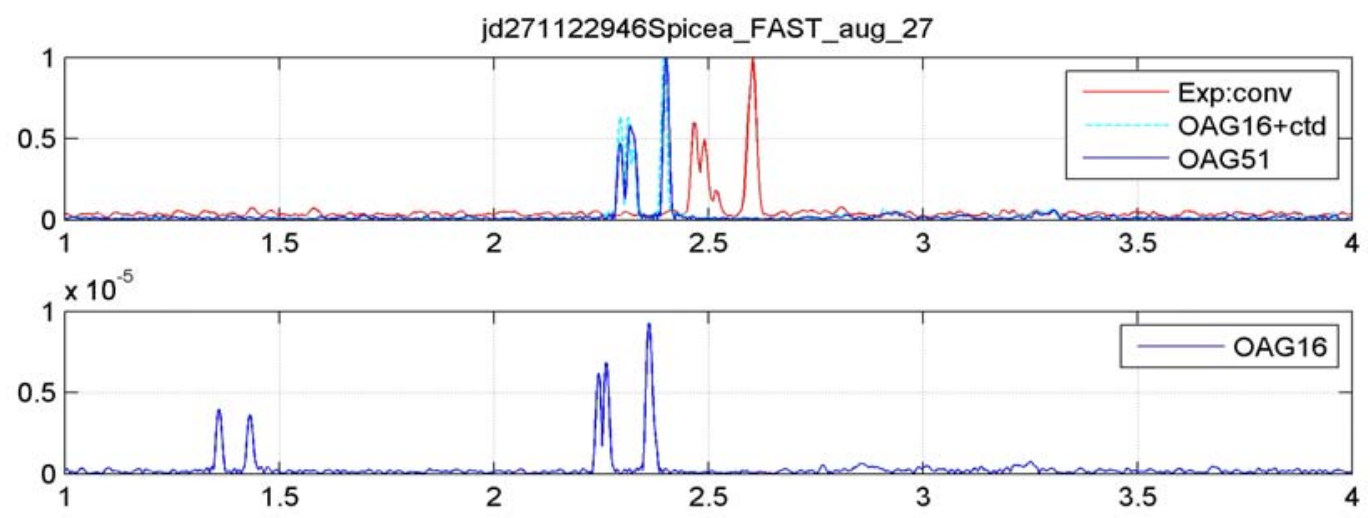

Figure 2-13: Comparison of pulse arrivals from the experimental measurement (red) and the pulse arrivals using 2D PE with truncated XBT data at 1500m (run 51, blue) in the top panel. 'OAG16', in the lower panel, denotes the simulated pulse arrivals with the XBT casts without the truncation, showing additional early arrivals before the main arrivals. In 'OAG16+ctd', the sound speeds below 1500m were replaced by the sound speeds from CTD data, and the additional arrivals with 'OAG16’ disappear (top panel). 


\subsubsection{Data processing}

Measured raw data from the Five Octave Research Array (FORA) were processed by Joseph Sikora [53] into the arrival time, direction of ray arrival, and signal levels.

At first, the data were demodulated down to base-band and decimated for computational efficiency. In the case of the SPICEX sources, the signals have approximately $83 \mathrm{~Hz}$ bandwidth at $250 \mathrm{~Hz}$ carrier frequency. The demodulated and decimated signals were beamformed. In this study, two kinds of beamformer were used: conventional beamformer and Minimum Power Distortionless Response (MPDR) beamformer. The MPDR beamformer gives higher angular resolution of the direction of ray arrivals.

The beamformed time series was matched filtered, or pulse-compressed (Appendix B), with the synthetic Doppler-corrected m-sequences. The matched filter is an optimal estimator of the signal amplitude and time delay with the assumption of Guassian noise and linear, non-dispersive propagation along the different sound paths [29].

The arrival angles off from the source were transformed into those with respect to the end-fire array to identify the individual rays. The heading of the array to the source was determined by the GPS location of the ship and source, and the WGS 1984 ellipsoid model of the Earth. The heading of the array was averaged between the two magnetic sensors output. The pitch data of the array were used for the angular transformation [53]. 


\subsection{Sound propagation around a seamount}

Sound propagation around a seamount is known as a typical range-dependent problem, in which the variability of environments strongly influences the sound field. The variability is mainly due to the varying bathymetry, but the variability of sound speed profiles cannot be neglected in the long-range propagation. Fig. 2-14 shows a typical ray diagram in the presence of a seamount. The ray diagram up to $400 \mathrm{~km}$ before the seamount shows well known convergence zone propagation in deep ocean. The convergence zone propagation creates repetitive convergence zones of high sound intensity at the distance of $50 \mathrm{~km}$. Since the convergence-zone width increases as the range increases, eventually, after several hundred kilometers, the zones overlap and become indistinguishable [28]. This convergence zone propagation enables long-range sound propagation of high intensity and without low distortion.

As rays meet the upslope of the seamount, steeper angle arrivals are blocked by the seamount. The fact that ray angles are steepened by twice the bottom slope per reflection results in the redirecting of rays toward the source (backscattering), or the passing over the seamount with several surface and bottom reflections. However, both cases suffer high reflection loss. Therefore, arrivals can be missing, or diffused and weakened. Moreover, the 3D refraction effect will be introduced; reflections from sloping bottoms produce the refracted arrivals diverting from the acoustic path. So only lower angle rays, less than +/-10 degrees, propagate over the seamount without being disturbed by the seamount.

Fig. 2-15 shows range-stacked broadband pulse arrivals by the PE simulation (Section 4.1), showing clearly the blocked refracted rays denoted by red-dashed lines (A). In addition, bottom-reflected higher angle rays are blocked by the seamount, and a series of scattered waves by the seamount appears after refracted wave arrivals (C). 


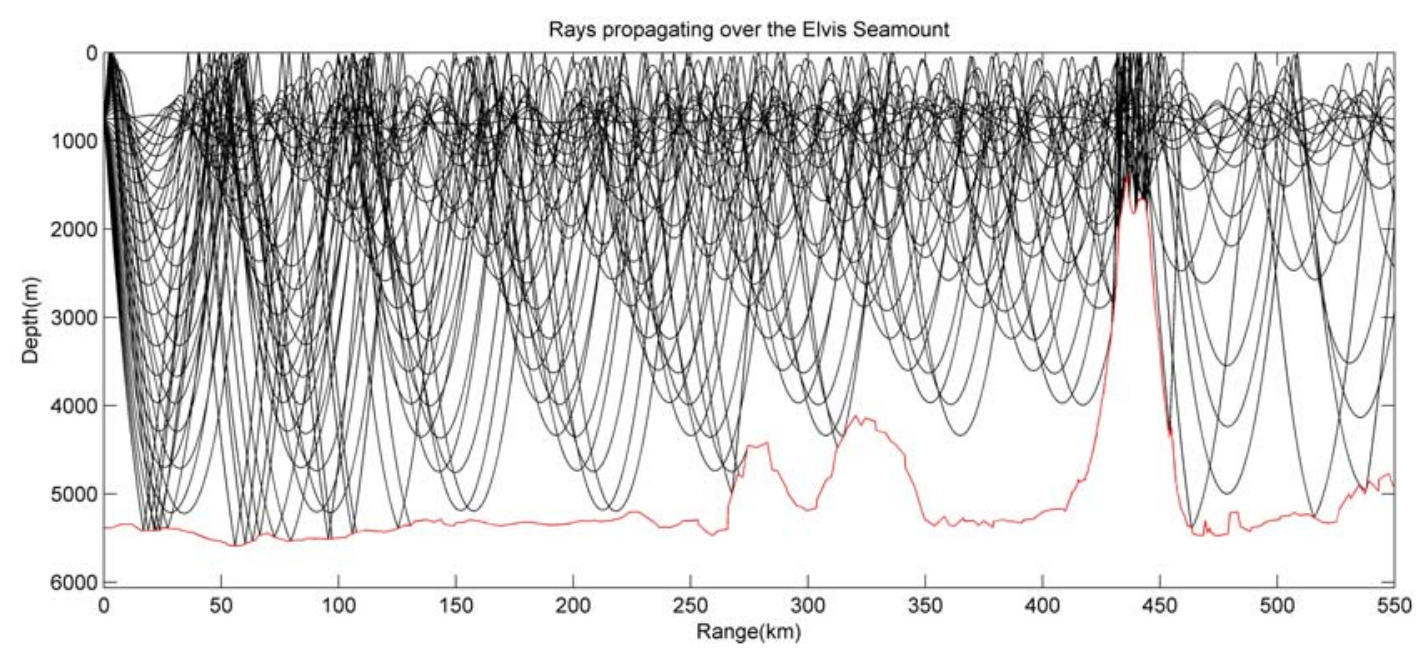

Figure 2-14: Ray propagation with a seamount made by RAY [40] program. The rays were generated using the range-dependent sound speeds from the XBT casts nearest the acoustic path (Fig. 4-2 for day 268).

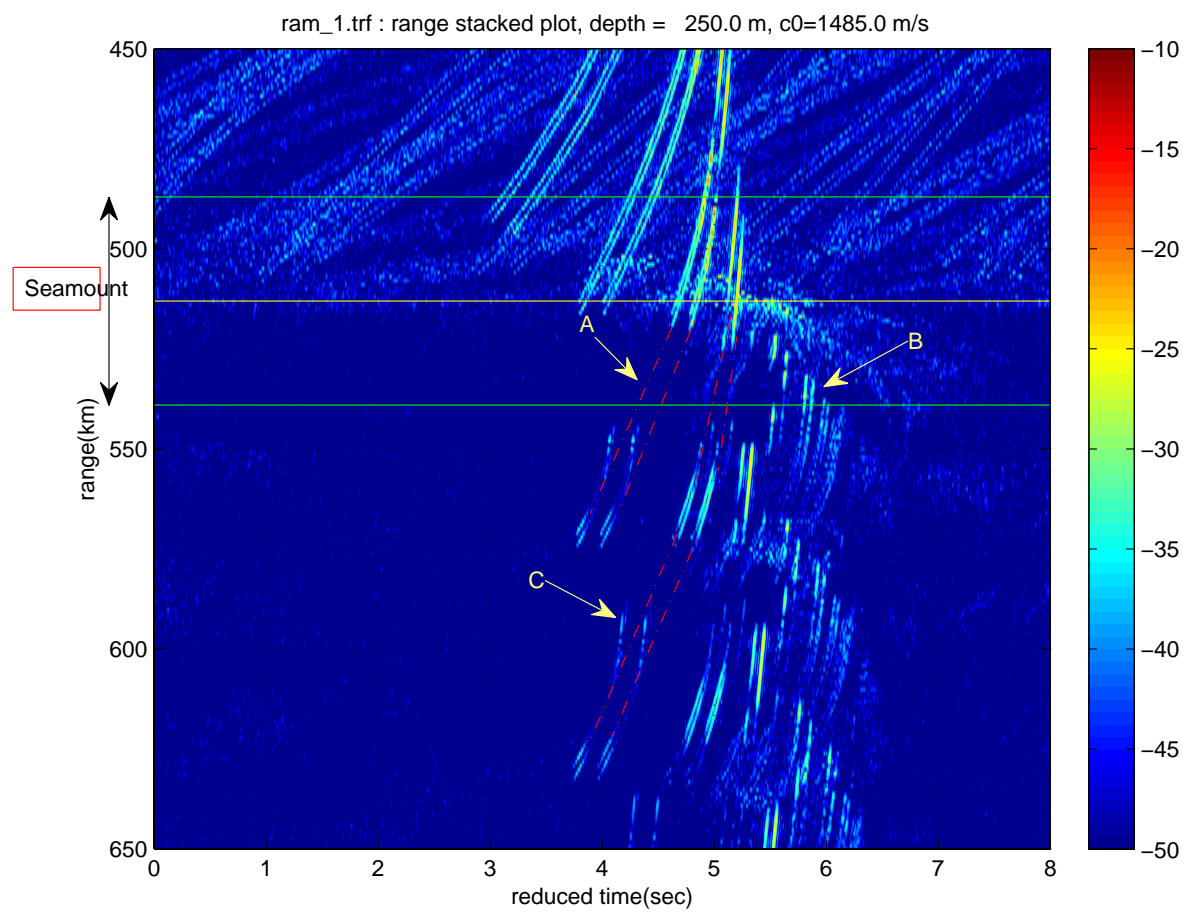

Figure 2-15: Range-stacked pulses arrivals at a receiver depth, 250m. The red dashed line denotes the blocked refracted wave by the seamount. This result is made by the PE simulation for the Elvis seamount. 
The bathymetric change of the seamount induces strong mode coupling, which results in redistribution of energy between modes. To examine the strong mode coupling, the 2D PE simulation for a frequency $(250 \mathrm{~Hz})$ was performed for range-dependent bathymetry and a range-averaged sound speed field (see Fig. 2-17), and the result can be presented by the modal amplitude using the modal decomposition method [44, 45, 46, and Appendix D].

From (D.6) the PE modal amplitude can be expressed as the following equation:

$$
A_{m}(r ; \omega)=\int_{0}^{D} \Psi_{P E}(r, z ; \omega) \frac{\Phi_{m}(r, z ; \omega)}{\rho(z)} d z,
$$

where $\Phi_{m}(r, z ; \omega)$ is the local modal shape, and $\Psi_{P E}$ is the factorized pressure fields from (D.1), which can be obtained by the product of the PE pressure fields, $P_{P E}(r, z ; \varpi)$ and $\sqrt{r} e^{-j k_{0} r}$.

Figure 2-16 shows the absolute value of the resultant modal amplitude, $\left|A_{m}(r ; \omega)\right|$, along the acoustic path over the seamount. Figure 2-17 shows the modal shapes of the selected modes along the acoustic path, and Fig. 2-18 shows the turning depths of the trapped modes in the waveguide for the three selected locations, around the acoustic source, a small seamount, and the peak of the Elvis seamount.

Around the acoustic source, modes below 391 are trapped in water, which correspond to the refracted-refracted waves. Higher modes than the trapped modes start to feel the bottom interaction and result in quite perturbed patterns of the modal amplitudes. As the range increases, the higher modes are blocked by the two small seamounts.

Before the seamount, modes below 255 are trapped in water, corresponding to the refracted-refracted wave. The modes lower than 35 can only pass the seamount without any interaction with the seamount. However, modes higher than 100 are disturbed and blocked by the seamount at the peak of the seamount; this is called the modal cut-off. 
After the seamount, energy transfer from lower modes to higher modes occurs, which is called the mode repopulation.

Fig. 2-19 shows the sum of amplitude squared along the bathymetry, which is equivalent to energy. This figure gives an idea of energy dissipation from the blockage of the seamount. As the range increases, the energy decreases from the dissipation through the sea bottom, and then there is an abrupt drop of the energy with the two small seamounts. Another large drop in the energy takes place just after the big seamount.

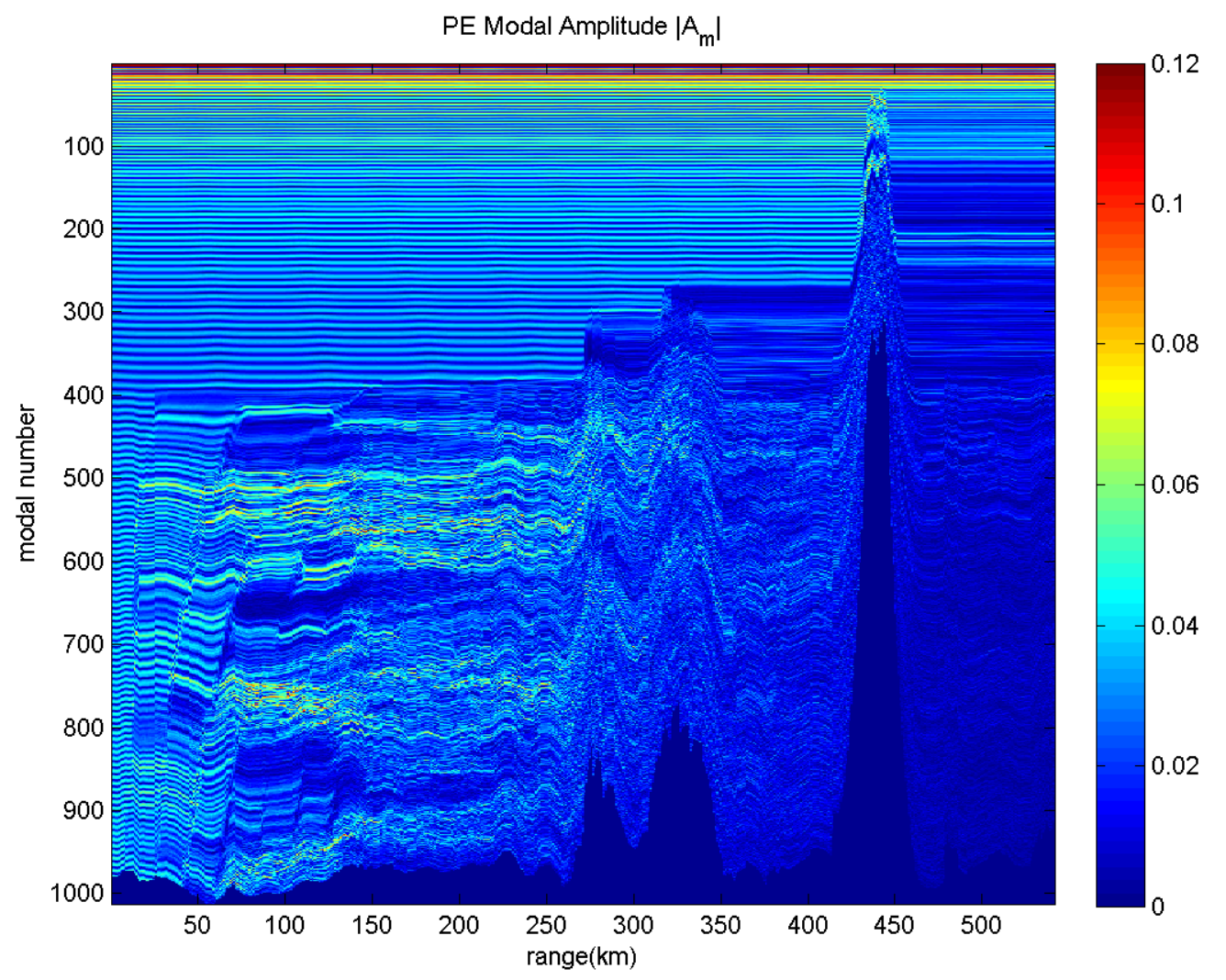

Figure 2-16: Strong mode coupling with seamount bathymetry. This is obtained from PE simulation at a frequency, $250 \mathrm{~Hz}$, and the modal amplitude can be estimated by the modal decomposition method (Appendix D). In higher modes, their energy is dissipated by the seamount, and also lower mode amplitude is disturbed by the seamount. Transfer of energy between modes is clearly visible in the figure. After the seamount, energy transfer to higher modes from lower modes, i.e., mode repopulation, occurs. 

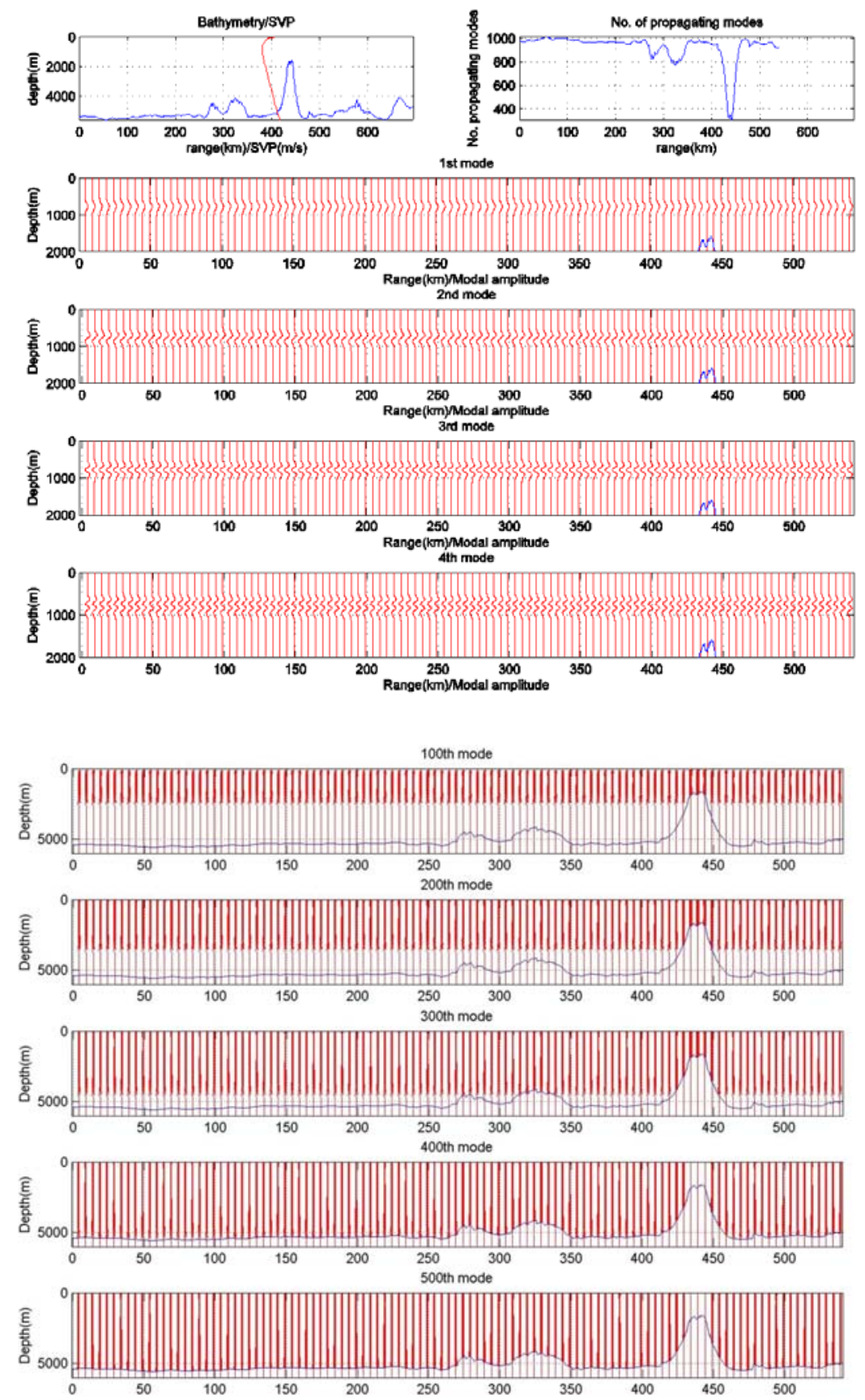

Figure 2-17: Modal shapes for $1-4^{\text {th }}$ and $100^{\text {th }}, 200^{\text {th }}, 300^{\text {th }}, 400^{\text {th }}$ modes versus range. Bathymetry and SVP (top left panel) as well as the number of propagating modes (top right panel) are shown. 

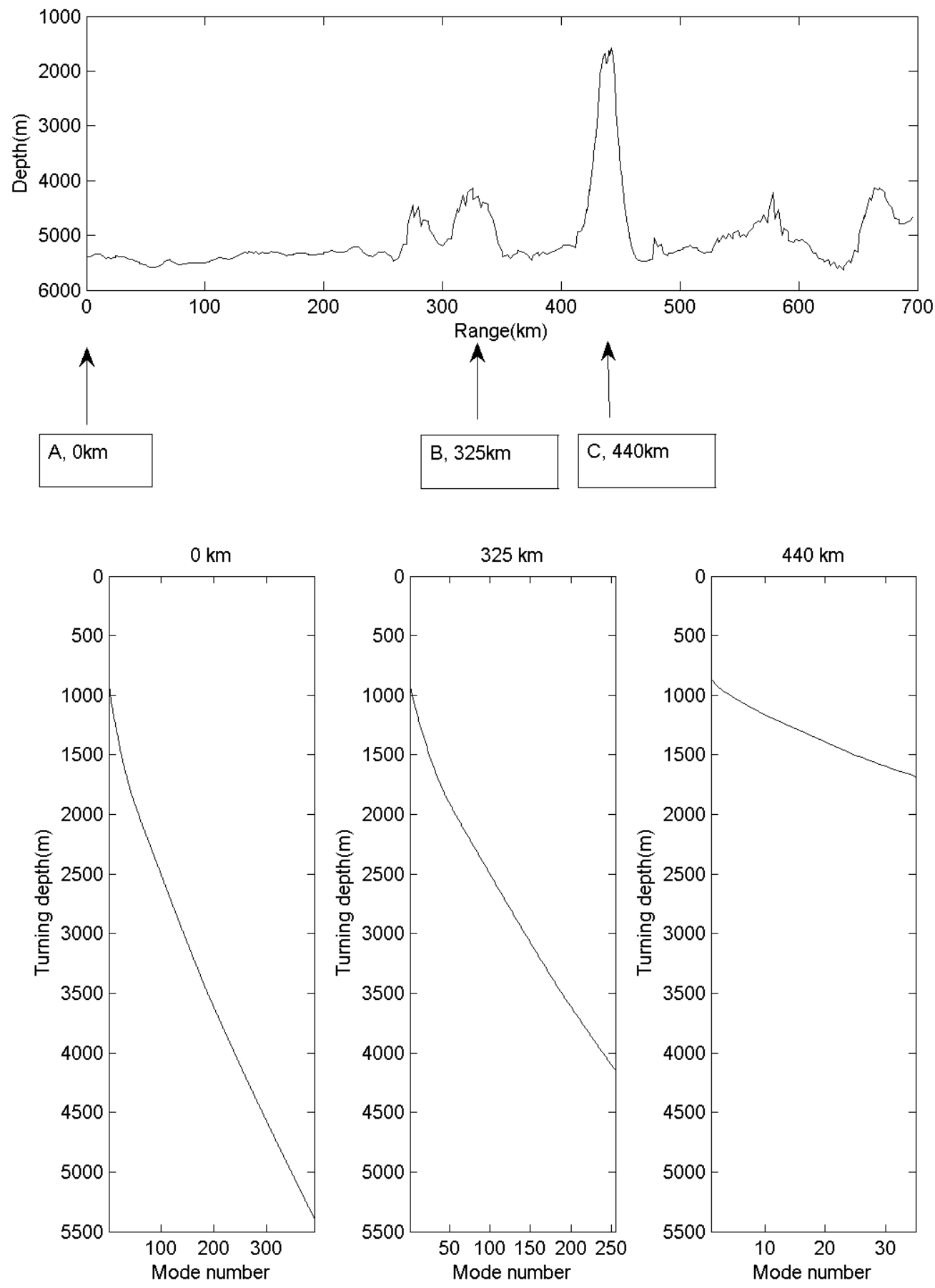

Figure 2-18: Turning depths of the water trapped modes at three locations: around the acoustic source, a small seamount, and the peak of the Elvis seamount. 

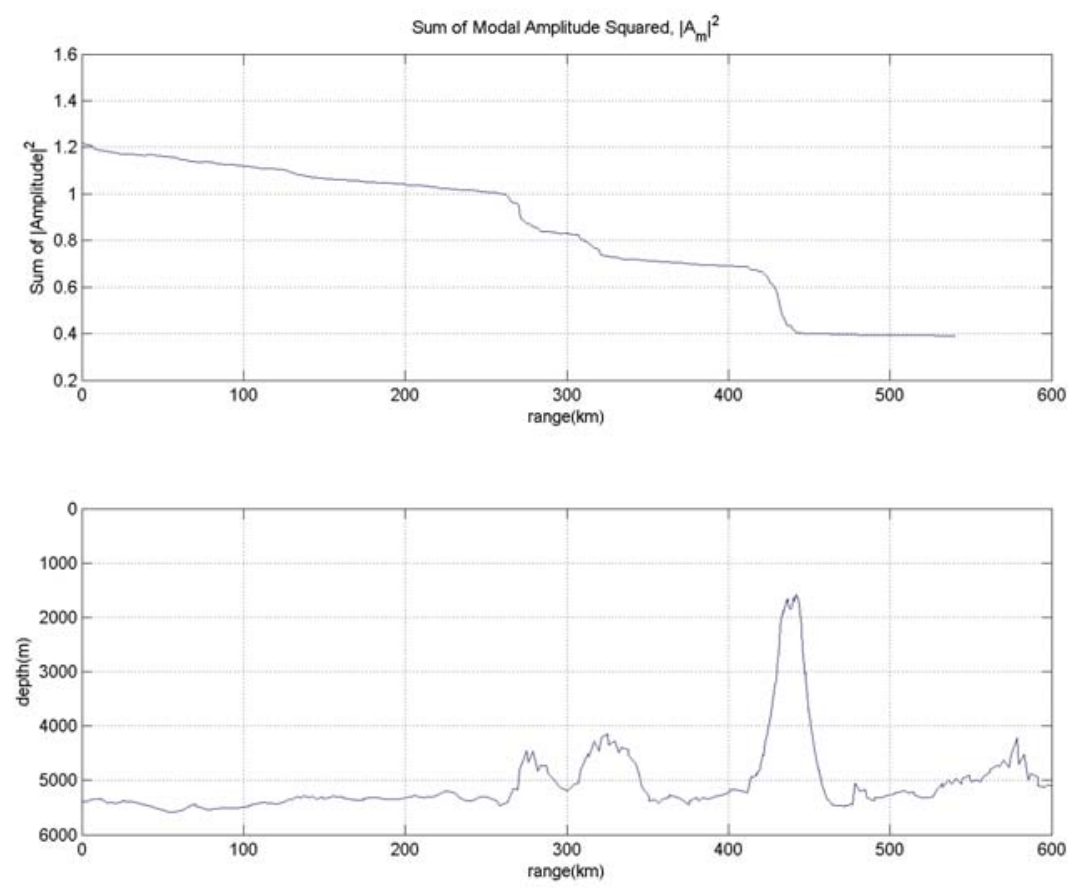

Figure 2-19: Sum of amplitude squared along the bathymetry, which provides an idea of energy dissipation by the blockage of the seamount.

Figure 2-20 shows modal pulse arrivals in terms of the absolute value of the pulse amplitude, at the distance of $400 \mathrm{~km}$ from the source with a flat bottom: this result is constructed by the 2D PE simulation over the source bandwidth $(50 \mathrm{~Hz})$ and the modal decomposition method. The $1-400^{\text {th }}$ modal pulses are trapped in the water column corresponding to refracted-refracted waves. In water trapped modes, higher angle rays arrive faster than lower ones because of higher group speed (see lower panel in Fig. 2-20), which can be explained by the fact that steeper rays sample higher sound speed. However, modes higher than 400 touch the bottom, which are not trapped in the water column but start to generate bottom-reflected waves that arrive later with lower group speed. The resultant pulse can be constructed by the interference of the modal pulses with different phases. 

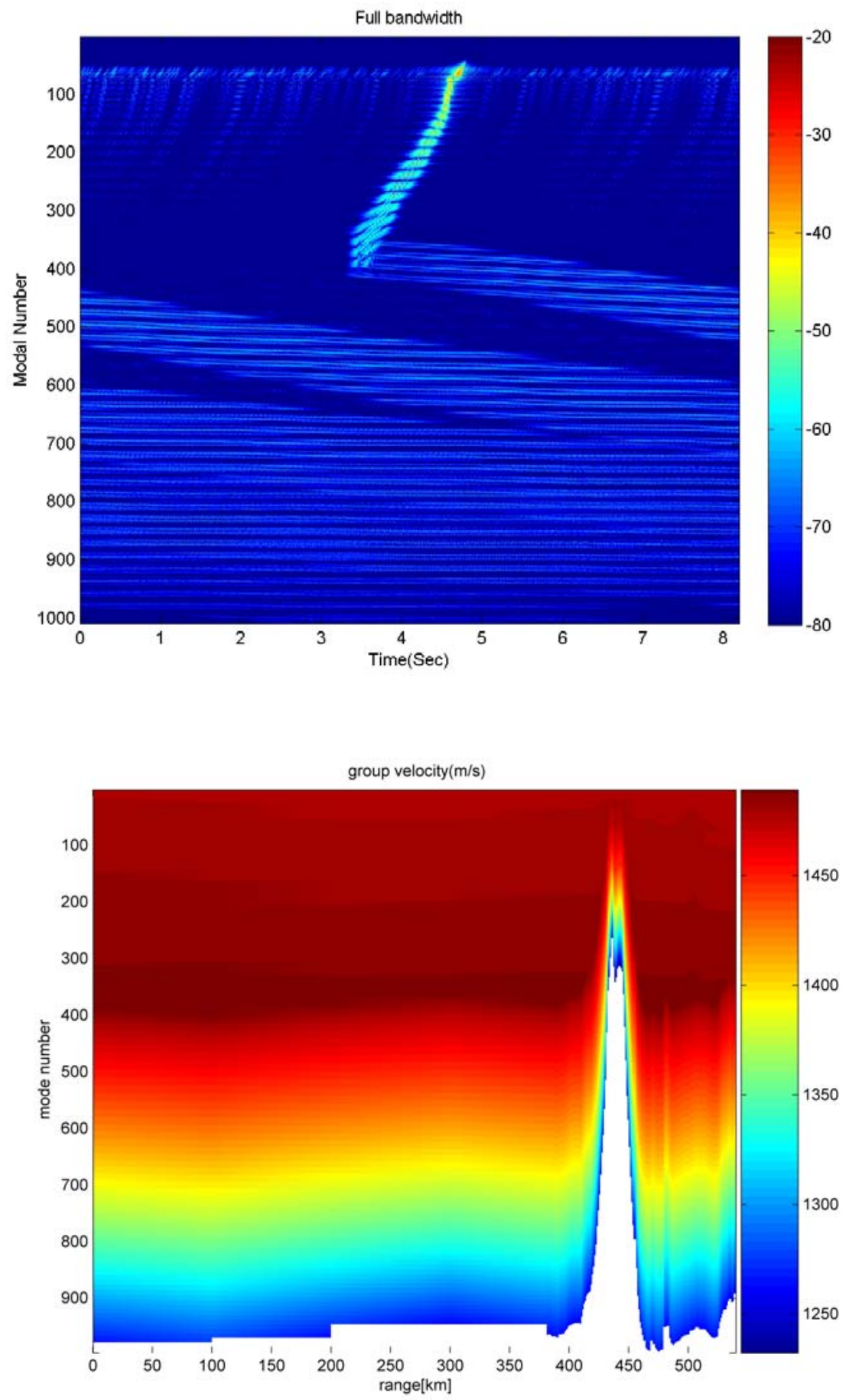

Figure 2-20: (a) Modal pulse arrivals (top panel), (b) group speed of each mode (bottom panel). 
As previously mentioned, with the 3D refraction effect, multiple reflections from sloping bottoms produce refracted arrivals diverting from the acoustic path. In addition, if we presume the adiabatic approximation (Section 3-3), rays for each mode can be traced with refraction by the gradient of the horizontal wavenumber, $k_{n}$, or local phase speed. The horizontal refraction reflects horizontal changes in the entire sound speed profile and bottom interaction through the vertical acoustic mode structure.

Figure 2-22 shows measured arrival pulses in the BASSEX experiment [53], for the LOAPEX source with lower center frequency $(68.2 \mathrm{~Hz})$. Most pulses are measured at broadside, which coincides with a direct acoustic path (geodesic) between the source and a receiver, but there is an additional pulse coming from a different arrival angle, which could be the horizontally refracted wave.

Figure 2-21 shows the horizontally refracted wave for the $1^{\text {st }} \sim 151^{\text {st }}$ modes for an acoustic path in the top panel and $1^{\text {st }}$ (black), $51^{\text {st }}$ (blue), $101^{\text {st }}$ (red) modes for three different acoustic paths in the bottom panel using the adiabatic mode theory, in which the horizontal refraction is caused by the effect of strong bathymetric change around the seamount. The lowest mode runs along the geodesic; higher modes experience higher refracted angles, and divert more from the geodesic. Note that, for the higher modes, the angles between a horizontal refracted ray and a geodesic on the left and right sides with respect to an acoustic path passing over the peak of the seamount are not symmetric. This axis-asymmetric of the horizontal refracted angles suggests that we have to consider the axis-asymmetric of the environments.

Strictly speaking, since the varying bathymetry of the seamount induces strong mode coupling, the assumption of the adiabatic approximation breaks down in this problem. However, the result provides a possible explanation for a measured horizontal refracted wave in the BASSEX experiment. 

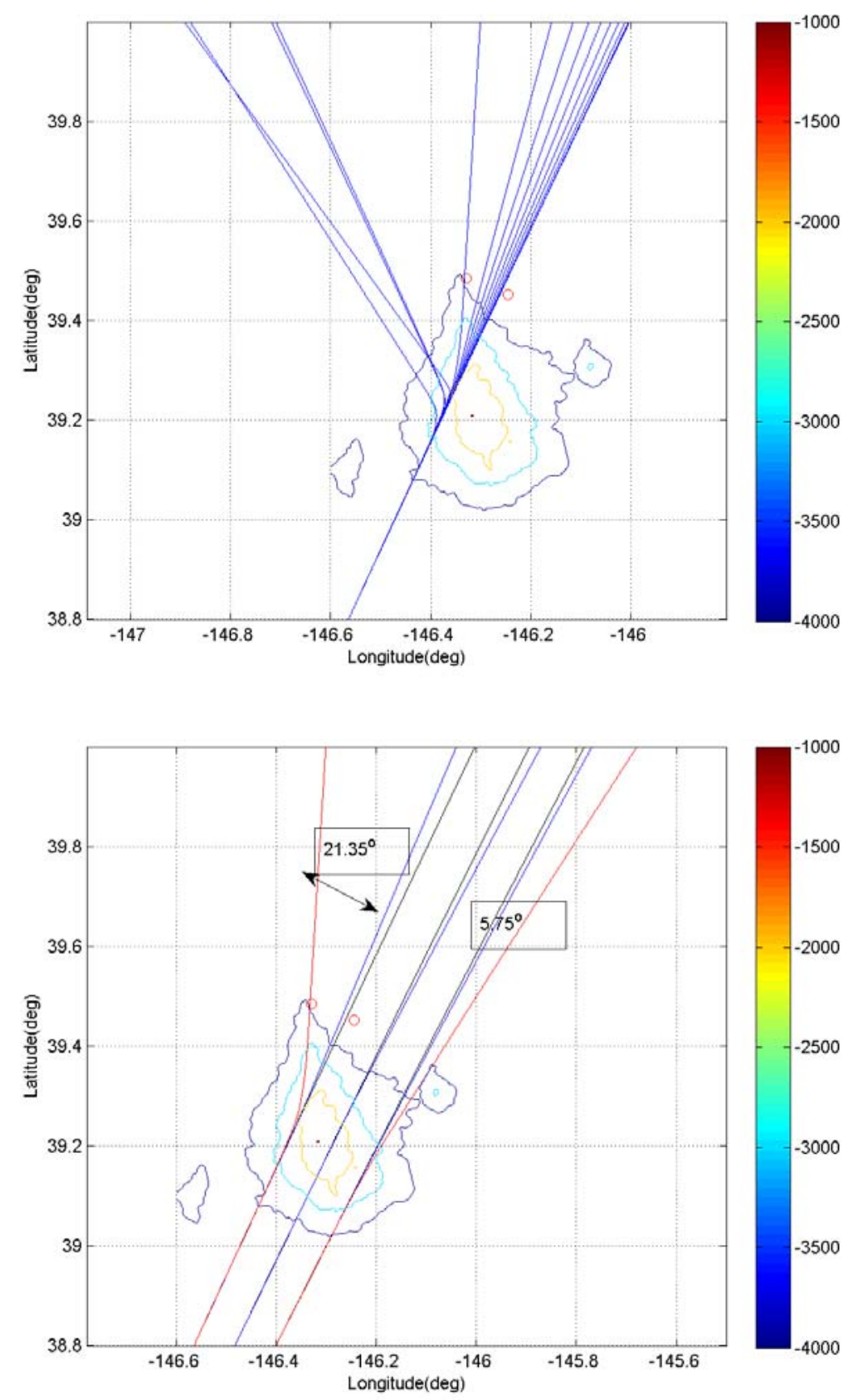

Figure 2-21: Horizontally refracted rays for the $1^{\text {st }} \sim 151^{\text {st }}$ modes for an acoustic path (top panel), and $1^{\text {st }}$ (black), $51^{\text {st }}$ (blue), $101^{\text {st }}$ (red) modes for three different acoustic paths (bottom panel). The circles denote the locations where a horizontal refracted ray is detected in the BASSEX experiment. The refracted angles between a geodesic and a $101^{\text {st }}$ mode ray are 21.35 and 5.75 degrees for the rays passing the left and right sides with respect to an acoustic path passing the peak of the seamount. 

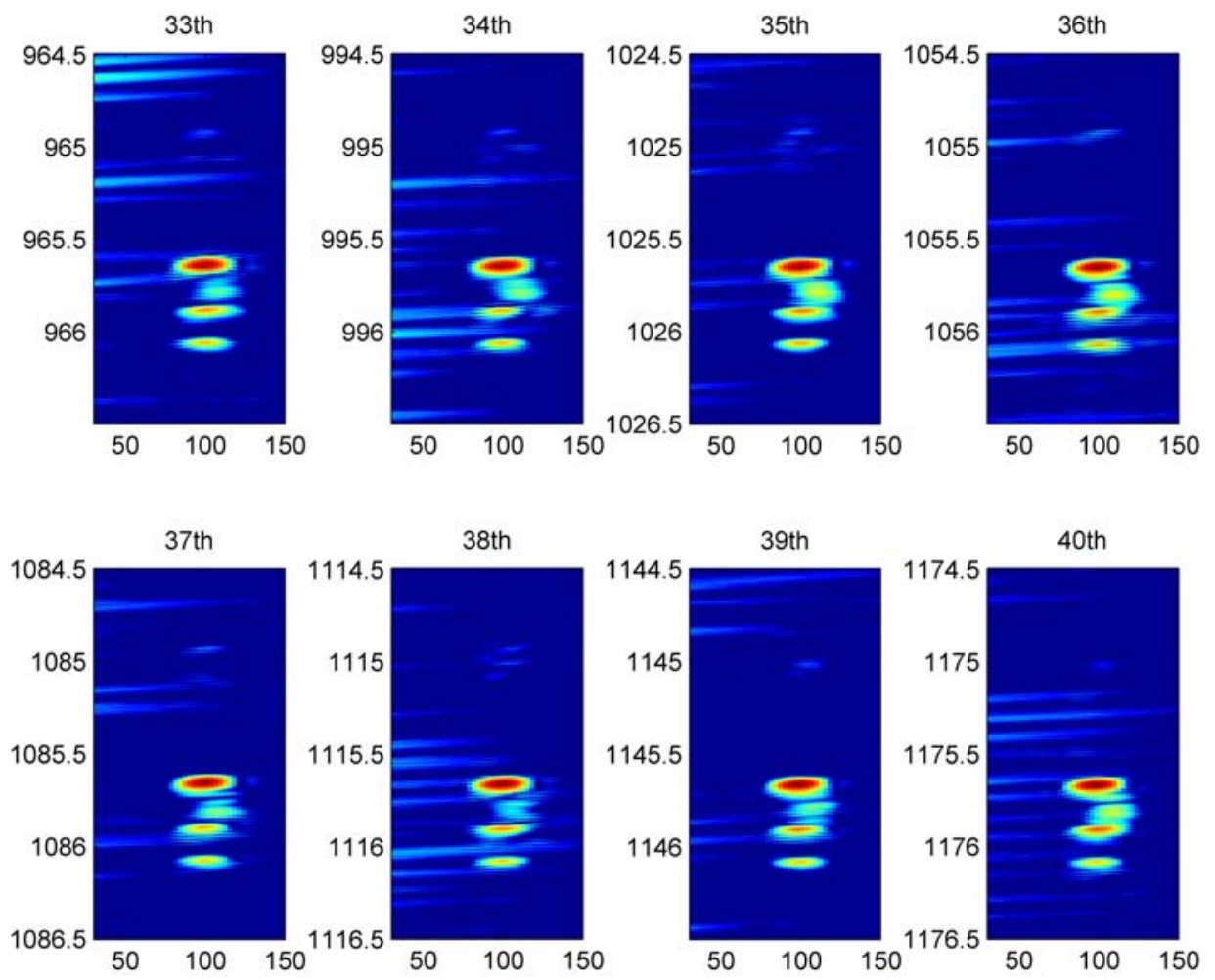

Figure 2-22: Measured pulse arrivals for LOAPEX source, $68 \mathrm{~Hz}$ at $350 \mathrm{~m}$ depth, behind the Kermit-Roosevelt seamount, showing pulse arrivals with a different arrival angle (adapted from [53], and reproduced). 


\section{Chapter 3}

\section{Sound propagation models}

In this chapter, the two-dimensional (2D) and three-dimensional (3D) sound propagation models used in this thesis are outlined. First, 2D, Nx2D, and 3D concepts in sound propagation model are presented in Section 3.1. The coupled normal mode theory is outlined in Section 3.2, and the 3D horizontal ray theory is described in Section 3.3. In Section 3.4, the broadband pulse modeling by the Fourier synthesis is presented. The 3D spectral coupled-mode model is outlined in Section 3.5, and a comparison between the 3D spectral coupled-mode model and the 3D parabolic equation (PE) is discussed in Section 3.6. A discussion of parallelization and approximation to increase computational efficiency follows in Section 3.7.

\subsection{Two-dimensional, Nx2D, and three-dimensional sound propagation}

The three-dimensional far-field equation in cylindrical coordinates takes the form [15]:

$$
u_{r r}+2 i k_{0} u_{r}+u_{z z}+\frac{1}{r^{2}} u_{\theta \theta}+k_{0}^{2}\left[n^{2}(r, \theta, z)-1\right] u=0
$$

The Nx2D concept was introduced by Perkins and Baer [27], and the proposed algorithm is: solve 2D problems in $\mathrm{N}$ vertical planes and combine the results to form an approximate 3D problem. This Nx2D method is valid, provided redirection of energy in 
azimuth due to boundary interaction is negligible. Equation (3.1) becomes

$$
u_{r r}+2 i k_{0} u_{r}+u_{z z} \quad+k_{0}^{2}\left[n^{2}(r, \theta, z)-1\right] u=0 .
$$

As we can see, the azimuthal coupled term $\left(\frac{1}{r^{2}} u_{\theta \theta}\right)$ has been dropped, but the index of refraction $(n(r, \theta, z))$ is still dependent on the azimuth angle. If azimuthal coupling is weak or absent, the Nx2D method can greatly reduce the computation time using the 3D calculation.

In the 2D case, we assume that there is no variation in azimuthal direction, i.e., no horizontal refraction. Therefore, the index of refraction $(n(r, z))$ no longer depends on the azimuth angle.

$$
u_{r r}+2 i k_{0} u_{r}+u_{z z} \quad+k_{0}^{2}\left[n^{2}(r, z)-1\right] u=0 .
$$

\subsection{Normal modes in a range-dependent waveguide and adiabatic approximation [30]}

If we consider that the acoustic waveguide in which the sound speed varies in depth as well as in range, but, is much slower in depth, then the 3D Helmholtz equation in cylindrical coordinates can be expressed as:

$$
\nabla^{2} p(r, z)+\frac{\omega^{2}}{c^{2}(r, z)} p=0
$$

Suppose for an arbitrary range $r$, eigenfunctions $\psi_{l}(r, z)$ satisfy the equation with proper boundary conditions at the bottom and surface 


$$
\frac{d^{2} \psi_{l}}{d z^{2}}+\left[k^{2}(r, z)-k_{r l}^{2}\right] \psi_{l}=0
$$

where $k_{r l}$ are horizontal wave number of $l^{\text {th }}$ mode. Then the acoustic field can be represented as an expansion in terms of eigenfunctions, $\psi_{l}(r, z)$, and the expansion coefficients, $\Psi_{l}(r)$ :

$$
p(r, z)=\sum_{l} \Psi_{l}(r) \psi_{l}(r, z)
$$

The expansion coefficients are given by $\Psi_{l}(r)=C_{l} H_{o}^{(1)}\left(k_{r l} r\right)$, in which $C_{l}$ is a slowly varying function of $r$. Substituting Eq. (3.6) into Eq. (3.4), and using the property of orthonormality of eigenfunctions, $\psi_{l}(r, z)$, i.e.,

$$
\int_{0}^{h} \frac{\psi_{l} \psi_{m}(r, z)}{\rho} d z=\delta_{l m}
$$

a set of coupled differential equations can be obtained as follows:

$$
\left[\nabla_{r}^{2}+k_{r m}^{2}(r)\right] \Psi_{m}=-2 \sum_{l} \nabla_{r} \Psi_{l} \int \psi_{m} \nabla_{r} \psi_{l} d z-\sum_{l} \Psi_{l} \int \psi_{m} \nabla_{r}^{2} \psi_{l} d z
$$

The coupled terms on the right hand side of Eq. (3.8) are small if the properties of the waveguide vary slowly in the range. If the right-hand side equals to zero, the equations of the normal modes become uncoupled, which means that each normal mode propagates in the waveguide independently, or energy is not transferred to the other modes. This approximation is called adiabatic approximation, which neglects the interaction between normal modes.

With the adiabatic approximation, Eq. (3.8) becomes a horizontal wave equation for modal amplitudes, 


$$
\frac{1}{r} \frac{\partial}{\partial r}\left(r \frac{\partial \Psi_{m}}{\partial r}\right)+k_{r m}^{2}(r) \Psi_{m}=0
$$

Let us introduce a new function $F_{m}(r)=r^{1 / 2} \Psi_{m}(r)$. Then Eq. (3.9) becomes

$$
F_{m}^{\prime \prime}=-\left(k_{r m}^{2}+\frac{1}{4 r^{2}}\right) F_{m}
$$

For $k_{r m} r \gg 1$, the solution of Eq. (3.10) is the same as that from the WenzelKramers-Brillouin-Jeffreys (WKBJ) approximation.

Finally, the solution becomes

$$
p(r, z)=\sqrt{\frac{2}{\pi}} e^{-j \frac{\pi}{4}} \sum_{l} \psi_{l}\left(r_{s}, z\right) \psi_{l}(r, z) \frac{e^{j \int_{0}^{r} k_{r l} d r}}{\sqrt{k_{r l} r}}
$$

\subsection{Three-dimensional ray theory and horizontal ray theory with the adiabatic approximation}

A set of three-dimensional ray equations in ellipsoidal coordinates was derived by Yan and Yen [57] as follows:

$$
\begin{aligned}
\frac{d \phi}{d s} & =\frac{\cos \theta \cos \alpha}{\mu(\phi)-r}, \\
\frac{d \lambda}{d s} & =\frac{\cos \theta \sin \alpha}{(v(\phi)-r) \cos (\phi)}, \\
\frac{d r}{d s} & =\sin \theta,
\end{aligned}
$$




$$
\begin{aligned}
\frac{d \alpha}{d s}= & \frac{\cos \theta \tan \phi \sin \alpha}{v(\phi)-r}+\sin \theta \sin \alpha \cos \alpha\left(\frac{1}{v-r}-\frac{1}{\mu-r}\right), \\
& +\left(-\frac{\sin \alpha}{\mu(\phi)-r} \frac{\partial}{\partial \phi}+\frac{\cos \alpha}{(v(\phi)-r) \cos \phi} \frac{\partial}{\partial \lambda}\right) \frac{\ln N}{\cos \theta} \\
\frac{d \theta}{d s}= & -\cos \theta\left(\frac{\sin ^{2} \alpha}{v-r}+\frac{\cos ^{2} \alpha}{\mu-r}\right) \\
& +\left(-\frac{\sin \theta \cos \alpha}{\mu(\phi)-r} \frac{\partial}{\partial \phi}-\frac{\sin \theta \sin \alpha}{(v(\phi)-r) \cos \phi} \frac{\partial}{\partial \lambda}+\cos \theta \frac{\partial}{\partial r}\right) \ln N \\
\mu(\phi) & =r_{e q}\left(1-\varepsilon^{2}\right) /\left(1-\varepsilon^{2} \sin ^{2} \phi\right)^{3 / 2}, \\
v(\phi) & =r_{e q} /\left(1-\varepsilon^{2} \sin ^{2} \phi\right)^{1 / 2},
\end{aligned}
$$

where $\phi$ is geographic latitude (the angle between the surface normal and the equatorial plane), $r$ is ocean depth with downward positive, $\lambda$ is longitude, $\theta$ is grazing angle, and $\alpha$ is azimuth, i.e., the ray heading measured clockwise from north. Coordinates on the surface of ellipsoid are adapted, and $k_{n}$ is the horizontal wave number. Eccentricity, $\varepsilon$ ( $=0.081819191$ for the earth), is defined such that $r_{p}=r_{e q} \sqrt{1-\varepsilon^{2}}$ with $r_{p}$, polar radius, and $r_{e q}$, equatorial radius. $N=1 / C(\phi, \lambda, r)$ is slowness, where $C$ denotes sound speed as a function of position.

To compute travel time and propagation range, Eq. (3.19) and Eq. (3.20) can be used.

$$
\begin{aligned}
& \frac{d t}{d s}=\frac{1}{C} \\
& \frac{d R}{d s}=\cos \theta \sqrt{\left(\frac{\mu}{\mu-r} \cos \alpha\right)^{2}+\left(\frac{v}{v-r} \sin \alpha\right)^{2}} .
\end{aligned}
$$


The horizontal ray equation is a special case of the 3D ray equation derived above, which can be obtained by setting $\theta$ (grazing angle) and $r$ (ray depth) to be zero. This approximation makes a ray lie on the surface of the earth. The slowness, $N$, can be replaced by the horizontal wavenumber, $k_{n}$. With the adiabatic approximation, the horizontal wave equation (3.9) leads to horizontal rays for each mode, which are refracted by the gradient of the horizontal wavenumber, $k_{n}$. The horizontal ray equation can be obtained as follows [23, 24]:

$$
\begin{aligned}
& \dot{\phi}=\cos \alpha / \mu(\phi), \\
& \dot{\lambda}=\sin \alpha /(v(\phi) \cos \phi), \\
& \dot{\alpha}=\frac{\sin \alpha}{v(\phi)} \tan \phi-\left(\frac{\sin \alpha}{\mu(\phi)} \frac{\partial}{\partial \phi}-\frac{\cos \alpha}{v(\phi) \cos \phi} \frac{\partial}{\partial \lambda}\right) \ln k_{n}, \\
& \mu(\phi)=r_{e q}\left(1-\varepsilon^{2}\right) /\left(1-\varepsilon^{2} \sin ^{2} \phi\right)^{3 / 2}, \\
& v(\phi)=r_{e q} /\left(1-\varepsilon^{2} \sin ^{2} \phi\right)^{1 / 2},
\end{aligned}
$$

where $\phi$ is geographic latitude (the angle between the surface normal and the equatorial plane), $\lambda$ is longitude, and $\alpha$ is the ray heading measured clockwise from north. Coordinates on the surface of ellipsoid are adapted, and $k_{n}$ is the horizontal wave number. Eccentricity, $\varepsilon\left(=0.081819191\right.$ for the earth), is defined such that $r_{p}=r_{e q} \sqrt{1-\varepsilon^{2}}$ with $r_{p}$, polar radius, and $r_{e q}$, equatorial radius.

The horizontal ray theory may not be adequate for the sound propagation around a seamount because of strong mode coupling from the large change of bathymetry, while the 3D ray theory can overcome the shortcoming.

However, the 3D ray theory also has a limitation to realization of the reflected rays by a seamount with complicated bathymetry. A reflection at the sea bottom is very sensitive to the local slope of the bathymetry if we use a simple geometric reflection condition. In this work, the horizontal ray theory was used to demonstrate the existence 
of the refracted rays at the higher modes due to the bathymetry effect qualitatively (Fig. 2-19).

\subsection{Broadband modeling}

A broadband signal can be obtained from a Fourier transform of the frequency-domain solutions over the source bandwidth as follows:

$$
p(r, z, t)=\operatorname{Re}\left\{\frac{1}{\pi} \int_{0}^{\omega_{\max }} p(r, z, \omega) e^{-j \omega t} d \omega\right\}=\operatorname{Re}\left\{\frac{1}{\pi} \int_{0}^{\omega_{\max }} S(\omega) g(r, z, \omega) e^{-j \omega t} d \omega\right\},
$$

where $S(\varpi)$ is the source spectrum, and $g(r, z, \omega)$ is the spatial transfer function [28]. 


\subsection{Three-dimensional spectral coupled-mode model around a conical seamount}

Luo[10] proposed a more stable and numerically effective three-dimensional (3D) spectral coupled-mode model based on the well-known Coupled SACLANTCEN normal mode propagation loss model (C-SNAP) [63].

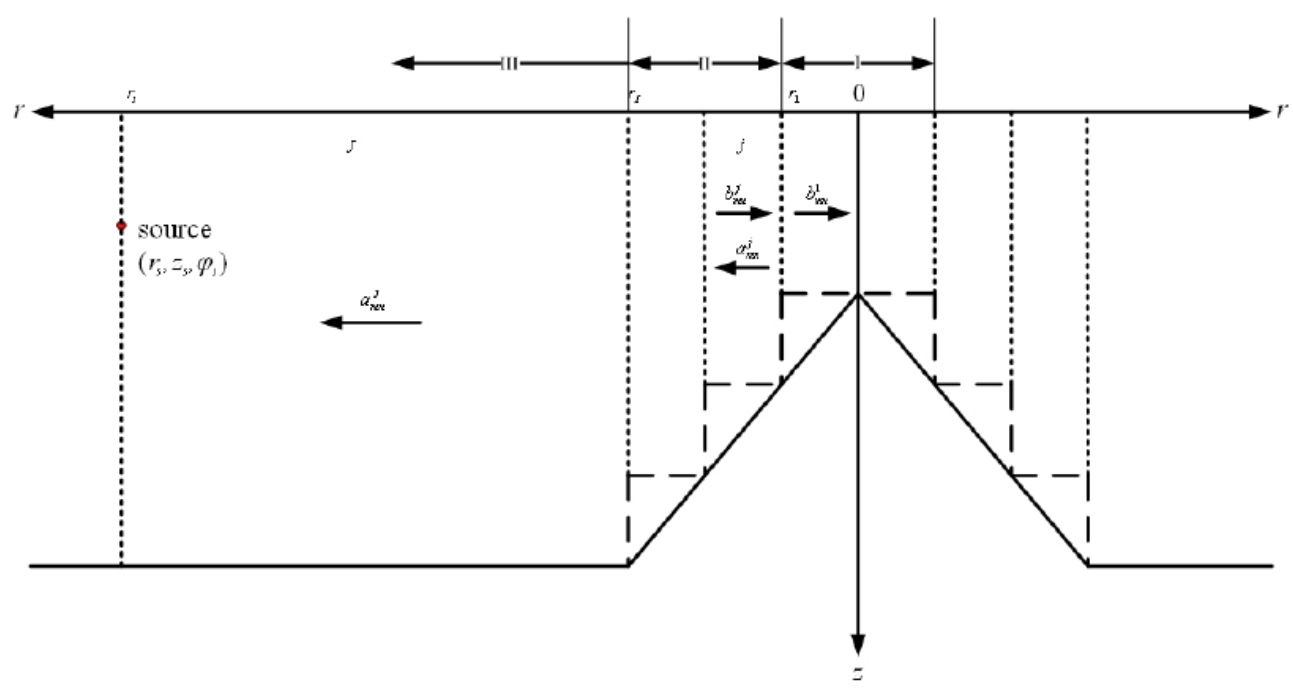

Figure 3-1: Description of problem with stepwise conical seamount [10].

In the spectral coupled-mode model, the pressure field can be expressed as follows:

1) $r \leq r_{1}$ : the innermost ring,

$$
p^{1}(r, z, \phi)=\sum_{m=0}^{\infty} \sum_{n=1}^{\infty} b_{m n}^{1} \hat{J}_{m n}^{1}(r) \Psi_{n}^{1}(z) \Phi_{m}(\phi),
$$

2) $r^{j-1}<r \leq r^{j}$ : ring $\mathrm{j}$, 


$$
p^{j}(r, z, \phi)=\sum_{m=0}^{\infty} \sum_{n=1}^{\infty}\left[a_{m n}^{j} \hat{H}_{m n}^{j}(r)+b_{m n}^{j} \hat{J}_{m n}^{j}(r)\right] \Psi_{n}^{j}(z) \Phi_{m}(\phi)
$$

3) $r>r_{I}$ : outside of the base of the seamount

$$
p(r, z, \phi)=p_{i}\left(r^{\prime}, z\right)+\sum_{m=0}^{\infty} \sum_{n=1}^{\infty} a_{m n}^{J} \hat{H}_{m n}^{J}(r) \Psi_{n}^{J}(z) \Phi_{m}(\phi),
$$

where $r^{\prime}$ is the range of a field point from the source, and $p_{i}\left(r^{\prime}, z\right)$ is the pressure by the incident wave as shown here:

$$
\begin{aligned}
& r^{\prime}(r, \phi)=\sqrt{r^{2}+r_{s}^{2}-2 r r_{s} \cos \left(\phi_{s}-\phi\right)}, \\
& p_{i}\left(r^{\prime}, z\right)=\frac{i}{4} \frac{1}{\rho\left(z_{s}\right)} \sum_{n=1}^{\infty} \Psi_{n}^{J}\left(z_{s}\right) \Psi_{n}^{J}(z) H_{0}^{(1)}\left(k_{r n}^{J} r^{\prime}\right) .
\end{aligned}
$$

The pressure fields outside the base of the seamount can be expressed as the superposition of the pressure fields by the incident wave and by the outgoing wave from the seamount. This superposition reduces the minimum number of azimuthal orders to $k_{0} r_{I}$, where $k_{0}$ is the medium wave number at the source $\left(=\omega / c_{0}\right), r_{I}$ is the radius of the base of the conical seamount. For a fixed source frequency, the number of azimuthal orders depends only on the size of the seamount, not the source distance.

The $\Psi_{n}^{j}(z)$ denotes the depth-dependent eigenfunctions at ring $\mathrm{j}$, and the azimuthal eigenfunctions, $\Phi_{m}(\phi)$, are defined as:

$$
\Phi_{m}(\phi)=e_{m} \cos m\left(\phi-\phi_{s}\right), m=0,1,2, \ldots
$$

where $\phi_{s}$ is the direction of an acoustic source with respect to the peak of seamount, and 


$$
e_{m}=\left\{\begin{array}{ll}
\frac{1}{\sqrt{2 \pi}}, & m=0 \\
\frac{1}{\sqrt{\pi}}, & m \neq 0
\end{array}\right. \text {. }
$$

The normalized Hankel and Bessel functions and derivatives used above are defined as follows:

$$
\begin{aligned}
& \hat{H}_{m n}^{j}(r) \triangleq \frac{H_{m}^{(1)}\left(k_{r n}^{j} r\right)}{H_{m}^{(1)}\left(k_{r n}^{j} r^{j-1}\right)}, \\
& D \hat{H}_{m n}^{j}(r) \triangleq \frac{d H_{m}^{(1)}\left(k_{r n}^{j} r\right) / d\left(k_{r n}^{j} r\right)}{H_{m}^{(1)}\left(k_{r n}^{j} r^{j-1}\right)}, \\
& \hat{J}_{m n}^{j}(r) \triangleq J_{m}\left(k_{r n}^{j} r\right) H_{m}^{(1)}\left(k_{r n}^{j} r^{j}\right), \\
& D \hat{J}_{m n}^{j}(r) \triangleq \frac{d J_{m}\left(k_{r n}^{j} r\right)}{d\left(k_{r n}^{j} r\right)} H_{m}^{(1)}\left(k_{r n}^{j} r^{j}\right) .
\end{aligned}
$$

$a_{m n}^{j}$ and $b_{m n}^{j}$ are coupling coefficients for outgoing and ingoing waves with respect to the peak of a seamount, respectively. The coupling coefficients can be computed with the boundary conditions at the leftmost and rightmost interfaces. To compute the coupling coefficients, $a_{m n}^{j}$ and $b_{m n}^{j}$, we need to construct and solve a large matrix for all rings for a azimuth mode using the direct global matrix approach [9]. However, this approach requires extensive physical memory size and computational ability.

Luo [10] suggested a two-way marching as an approximation of the whole coupling in the direct global matrix approach. The two-way marching consists of the inward marching coupling with the single-scatter approximation and the outward marching with the approximate single-scatter method (or one-way approximation) [54]. This two-way marching enables splitting of the large matrix in the direct global matrix approach into small multiple linear systems which can be solved with much lower computational ability. 


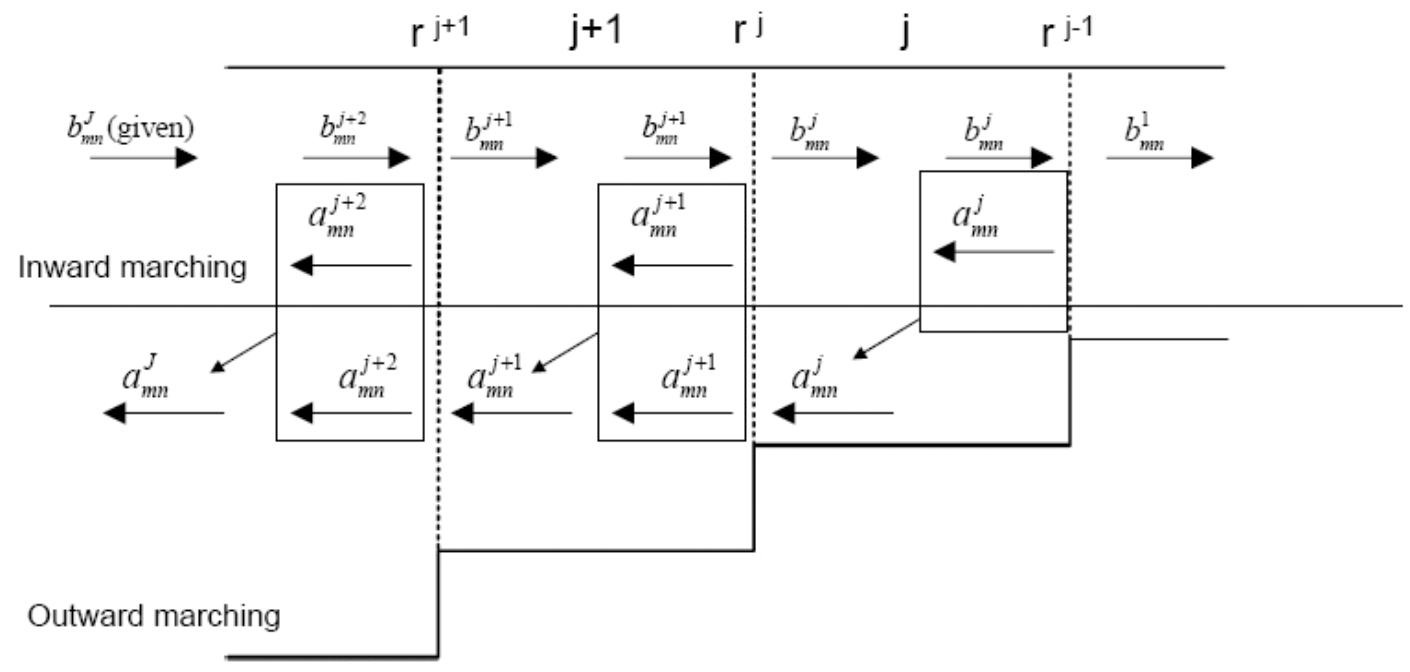

Figure 3-2: Two-way marching approximation.

For the inward marching coupling with the single-scatter approximation, an incoming wave, $b_{m n}^{j+1}$, is given at the left ring $j+1$, with a purely outgoing wave at the right ring, $b_{m n}^{j}$ as shown in Fig. 3-3. Since the incoming wave, $b_{m n}^{j+1}$, at the left ring is given by the incoming wave from an acoustic source, the remaining two unknowns, $a_{m n}^{j+1}$ and $b_{m n}^{j}$, can be expressed as $b_{m n}^{j+1}$.

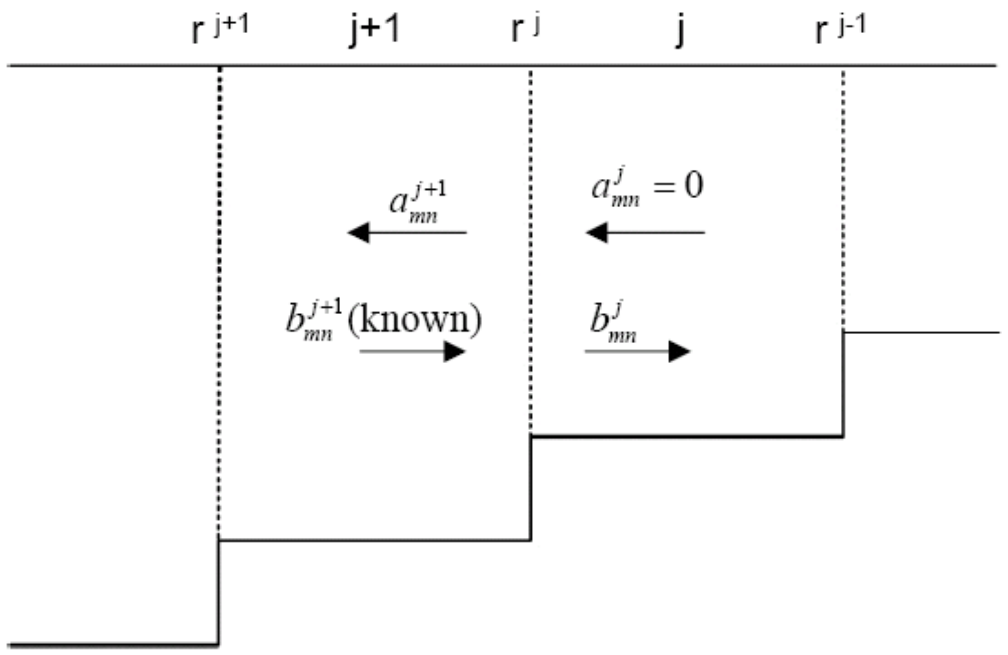

Figure 3-3: Single-scatter approximation between two neighboring rings. 
We apply the boundary conditions at the interface, $r=r^{j}$, between ring $j+1$ and ring $j$.

1) Continuity of pressure at $r=r^{j}$

To satisfy the continuity of pressure at the boundary $r=r^{j}$ between ring $j+1$ and ring $j$ :

$$
\begin{aligned}
& p^{j}\left(r^{j}, z, \phi\right)=p^{j+1}\left(r^{j}, z, \phi\right), \\
& \sum_{m=0}^{\infty} \sum_{n=1}^{\infty}\left[a_{m n}^{j} \hat{H}_{m n}^{j}\left(r^{j}\right)+b_{m n}^{j} \hat{J}_{m n}^{j}\left(r^{j}\right)\right] \Psi_{n}^{j}(z) \Phi_{m}(\phi)= \\
& \sum_{m=0}^{\infty} \sum_{n=1}^{\infty}\left[a_{m n}^{j+1} \hat{H}_{m n}^{j+1}\left(r^{j}\right)+b_{m n}^{j+1} \hat{J}_{m n}^{j+1}\left(r^{j}\right)\right] \Psi_{n}^{j+1}(z) \Phi_{m}(\phi)
\end{aligned}
$$

for the $m^{\text {th }}$ azimuthal mode, we have

$$
\sum_{n=1}^{\infty}\left[a_{m n}^{j} \hat{H}_{m n}^{j}\left(r^{j}\right)+b_{m n}^{j} \hat{J}_{m n}^{j}\left(r^{j}\right)\right] \Psi_{n}^{j}(z)=\sum_{n=1}^{\infty}\left[a_{m n}^{j+1} \hat{H}_{m n}^{j+1}\left(r^{j}\right)+b_{m n}^{j+1} \hat{J}_{m n}^{j+1}\left(r^{j}\right)\right] \Psi_{n}^{j+1}(z)
$$

By applying the operator $\int_{0}^{\infty} \frac{1}{\rho^{j}(z)} \Psi_{v}^{j}(z)(\bullet) d z$ to Eq. (3.39), we obtain

$$
\left[a_{m v}^{j} \hat{H}_{m \nu}^{j}\left(r^{j}\right)+b_{m v}^{j} \hat{J}_{m v}^{j}\left(r^{j}\right)\right]=\sum_{n=1}^{\infty}\left[a_{m n}^{j+1} \hat{H}_{m n}^{j+1}\left(r^{j}\right)+b_{m n}^{j+1} \hat{J}_{m n}^{j+1}\left(r^{j}\right)\right] C_{a v n}^{j+1},
$$

where $C_{a v n}^{j+1} \triangleq \int_{0}^{\infty} \frac{1}{\rho^{j}(z)} \Psi_{\nu}^{j}(z) \Psi_{n}^{j+1}(z) d z$.

2) Continuity of normal particle velocity at $r=r^{j}$

To satisfy the continuity of normal particle velocity at the boundary $r=r^{j}$ 
between ring $j+1$ and ring $j$ :

$$
\left.\frac{1}{\rho^{j}} \frac{\partial p^{j}}{\partial r}\right|_{r=r^{j}}=\left.\frac{1}{\rho^{j+1}} \frac{\partial p^{j+1}}{\partial r}\right|_{r=r^{j}}
$$

for the $m^{\text {th }}$ azimuthal mode, we have

$$
\begin{aligned}
\frac{1}{\rho^{j}} \sum_{n=1}^{\infty}\left[a_{m n}^{j} k_{r n}^{j} D \hat{H}_{m n}^{j}\left(r^{j}\right)+b_{m n}^{j} k_{r n}^{j} \hat{J}_{m n}^{j}\left(r^{j}\right)\right] \Psi_{n}^{j}(z)= \\
\frac{1}{\rho^{j+1}} \sum_{n=1}^{\infty}\left[a_{m n}^{j+1} k_{r n}^{j+1} D \hat{H}_{m n}^{j+1}\left(r^{j}\right)+b_{m n}^{j+1} k_{r n}^{j+1} \hat{J}_{m n}^{j+1}\left(r^{j}\right)\right] \Psi_{n}^{j+1}(z)
\end{aligned}
$$

By applying the operator $\int_{0}^{\infty} \Psi_{v}^{j}(z)(\cdot) d z$ to Eq. (3.43), we obtain

$\left[a_{m v}^{j} D \hat{H}_{m \nu}^{j}\left(r^{j}\right)+b_{m \nu}^{j} D \hat{J}_{m \nu}^{j}\left(r^{j}\right)\right]=\sum_{n=1}^{\infty}\left[a_{m n}^{j+1} D \hat{H}_{m n}^{j+1}\left(r^{j}\right)+b_{m n}^{j+1} D \hat{J}_{m n}^{j+1}\left(r^{j}\right)\right] C_{b v n}^{j+1}$,

where $C_{b v n}^{j+1} \triangleq \frac{k_{r n}^{j+1}}{k_{r v}^{j}} \int_{0}^{\infty} \frac{1}{\rho^{j+1}(z)} \Psi_{v}^{j}(z) \Psi_{n}^{j+1}(z) d z$

Eq. (3.43) and Eq.(3.44) can be rewritten in the matrix form,

$$
\begin{aligned}
& \hat{\mathbf{H}}_{m}^{j} \mathbf{a}_{m}^{j}+\hat{\mathbf{J}}_{m}^{j} \mathbf{b}_{m}^{j}=\mathbf{C}_{a}^{j+1}\left(\hat{\mathbf{H}}_{m}^{j+1} \mathbf{a}_{m}^{j+1}+\hat{\mathbf{J}}_{m}^{j+1} \mathbf{b}_{m}^{j+1}\right), \\
& \mathbf{D} \hat{\mathbf{H}}_{m}^{j} \mathbf{a}_{m}^{j}+\mathbf{D} \hat{\mathbf{J}}_{m}^{j} \mathbf{b}_{m}^{j}=\mathbf{C}_{b}^{j+1}\left(\mathbf{D} \hat{\mathbf{H}}_{m}^{j+1} \mathbf{a}_{m}^{j+1}+\mathbf{D} \hat{\mathbf{J}}_{m}^{j+1} \mathbf{b}_{m}^{j+1}\right),
\end{aligned}
$$

where $\hat{\mathbf{H}}_{\mathrm{m}}^{\mathrm{j}}, \mathbf{D} \hat{\mathbf{H}}_{\mathrm{m}}^{\mathrm{j}}, \hat{\mathbf{J}}_{\mathrm{m}}^{\mathrm{j}}, \mathbf{D} \hat{\mathbf{J}}_{\mathrm{m}}^{\mathrm{j}}, \hat{\mathbf{H}}_{\mathrm{m}}^{\mathrm{j}+1}, \mathbf{D} \hat{\mathbf{H}}_{\mathrm{m}}^{\mathrm{j}+1}, \hat{\mathbf{J}}_{\mathrm{m}}^{\mathrm{j}+1}, \mathbf{D} \hat{\mathbf{J}}_{\mathrm{m}}^{\mathrm{j}+1}$ are diagonal matrices. For example, 


$$
\hat{\mathbf{H}}_{\mathbf{m}}^{\mathbf{j}}=\operatorname{diag}\left(\hat{H}_{m n}^{j}\left(r^{j}\right)\right)_{n=1,2,3, \ldots, N}=\operatorname{diag}\left(\frac{H_{m}^{(1)}\left(k_{r n}^{j} r^{j}\right)}{H_{m}^{(1)}\left(k_{r n}^{j} r^{j-1}\right)}\right)_{n=1,2,3, \ldots, N},
$$

where $N$ is the number of normal modes. $\mathbf{a}_{\mathrm{m}}^{\mathbf{j}}, \mathbf{b}_{\mathrm{m}}^{\mathbf{j}}, \mathbf{a}_{\mathrm{m}}^{\mathbf{j}+1}$, and $\mathbf{b}_{\mathrm{m}}^{\mathbf{j}+1}$ are column vectors such as:

$$
\mathbf{a}_{\mathbf{m}}^{\mathbf{j}}=\left[\begin{array}{c}
a_{m 1}^{j} \\
a_{m 2}^{j} \\
\vdots \\
a_{m N}^{j}
\end{array}\right] .
$$

With the single-scatter approximation, $\mathbf{a}_{\mathbf{m}}^{\mathbf{j}}=\mathbf{0}$ and $\hat{\mathbf{H}}_{\mathbf{m}}^{\mathbf{j}+1}=\mathbf{I}$, which comes from the definition of the normalized Hankel function, the two unknowns, $\mathbf{a}_{\mathbf{m}}^{\mathbf{j}+1}$ and $\mathbf{b}_{\mathbf{m}}^{\mathbf{j}}$ can be obtained from the Eq. (3.46) and Eq. (3.47) in terms of $\mathbf{b}_{\mathbf{m}}^{\mathbf{j}}$ :

$$
\begin{aligned}
& \mathbf{a}_{\mathrm{m}}^{\mathrm{j}+1}=-\left(\mathbf{D} \hat{\mathbf{J}}_{\mathrm{m}}^{\mathrm{j}} \mathbf{C}_{\mathrm{a}}^{\mathrm{j}+1} \hat{\mathbf{H}}_{\mathrm{m}}^{\mathrm{j}+1}-\hat{\mathbf{J}}_{\mathrm{m}}^{\mathrm{j}} \mathbf{C}_{\mathrm{b}}^{\mathrm{j}+1} \mathbf{D} \hat{\mathbf{H}}_{\mathrm{m}}^{\mathrm{j}+1}\right)^{-1}\left(\mathbf{D} \hat{\mathbf{J}}_{\mathrm{m}}^{\mathrm{j}} \mathbf{C}_{\mathrm{a}}^{\mathrm{j}+1} \hat{\mathbf{J}}_{\mathrm{m}}^{\mathrm{j}+1}-\hat{\mathbf{J}}_{\mathrm{m}}^{\mathrm{j}} \mathbf{C}_{\mathrm{b}}^{\mathrm{j}+1} \mathbf{D} \hat{\mathbf{J}}_{\mathrm{m}}^{\mathrm{j}+1}\right) \mathbf{b}_{\mathrm{m}}^{\mathrm{j}+1}, \\
& \mathbf{b}_{\mathbf{m}}^{\mathbf{j}}=\left(\hat{\mathbf{J}}_{\mathbf{m}}^{\mathbf{j}}\right)^{-1} \mathbf{C}_{\mathbf{a}}^{\mathbf{j + 1}}\left(\mathbf{I} \mathbf{a}_{\mathbf{m}}^{\mathbf{j}+1}+\hat{\mathbf{J}}_{\mathbf{m}}^{\mathbf{j + 1}} \mathbf{b}_{\mathbf{m}}^{\mathbf{j}+1}\right) .
\end{aligned}
$$




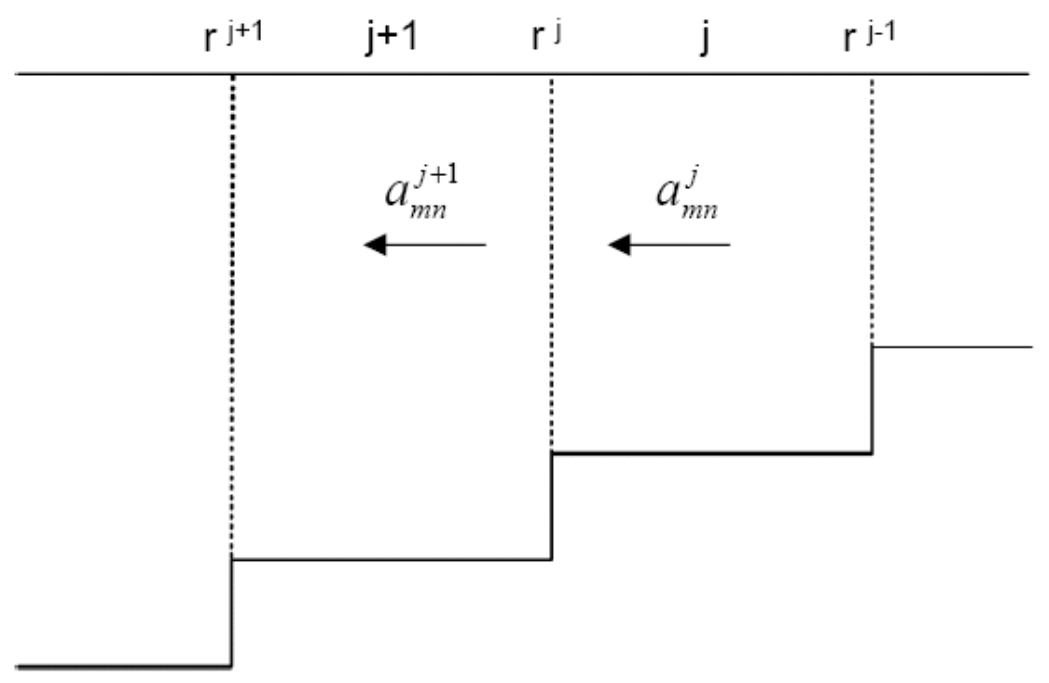

Figure 3-4: One-way approximation between two neighboring rings.

For the outward marching coupling, the one-way approximation is used as shown in Fig. 3-4. In the one-way approximation, we assume that no back-scattering is allowed, i.e., a purely incoming wave at the right ring $j$ is assumed with a purely outgoing wave at the left ring $j+1, b_{m n}^{j}=0$ and $b_{m n}^{j+1}=0$, respectively. Then the outgoing wave at ring $j, a_{m n}^{j}$ can be expressed in terms of $a_{m n}^{j+1}$ with the boundary condition between two neighboring rings, ring $j+1$ and $j$ as follows:

$$
\mathbf{a}_{m}^{j+1}=\left(G_{a}^{j+1}\right)^{-1}\left(D \hat{J}_{m}^{j+1} C_{c}^{j} \hat{H}_{m}^{j}-\hat{J}_{m}^{j+1} C_{d}^{j} D \hat{H}_{m}^{j}\right) a_{m}^{j}
$$

where,

$$
\begin{aligned}
& C_{c v n}^{j} \triangleq \int_{0}^{\infty} \frac{1}{\rho^{j+1}(z)} \Psi_{v}^{j}(z) \Psi_{n}^{j+1}(z) d z=\frac{k_{r n}^{j}}{k_{r v}^{j+1}} C_{b v n}^{j+1}, \\
& C_{d v n}^{j} \triangleq \frac{k_{r n}^{j}}{k_{r v}^{j+1}} \int_{0}^{\infty} \frac{1}{\rho^{j}(z)} \Psi_{v}^{j+1}(z) \Psi_{n}^{j}(z) d z=\frac{k_{r n}^{j}}{k_{r v}^{j+1}} C_{a v n}^{j+1}, \\
& \left(\mathbf{G}_{\mathbf{a}}^{j+1}\right)^{-1}=i \frac{\pi r^{j}}{2} \operatorname{diag}\left(k_{r v}^{j+1} \frac{H_{m}^{(1)}\left(k_{r v}^{j+1} r^{j}\right)}{H_{m}^{(1)}\left(k_{r v}^{j+1} r^{j+1}\right)}\right) .
\end{aligned}
$$


As shown in Fig. 3-2, $a_{m n}^{j}$ from the outward marching is added to the $a_{m n}^{j}$ from the inward marching before marching to the next range step.

Note that the coupling coefficients, $a_{m n}^{j}$ and $b_{m n}^{j}$ are computed at each azimuthal mode independently.

After the inward and outward marching, the total pressure fields can be computed by Eq. (3.27) (3.30) with the computed coupling coefficients, $a_{m n}^{j}$ and $b_{m n}^{j}$.

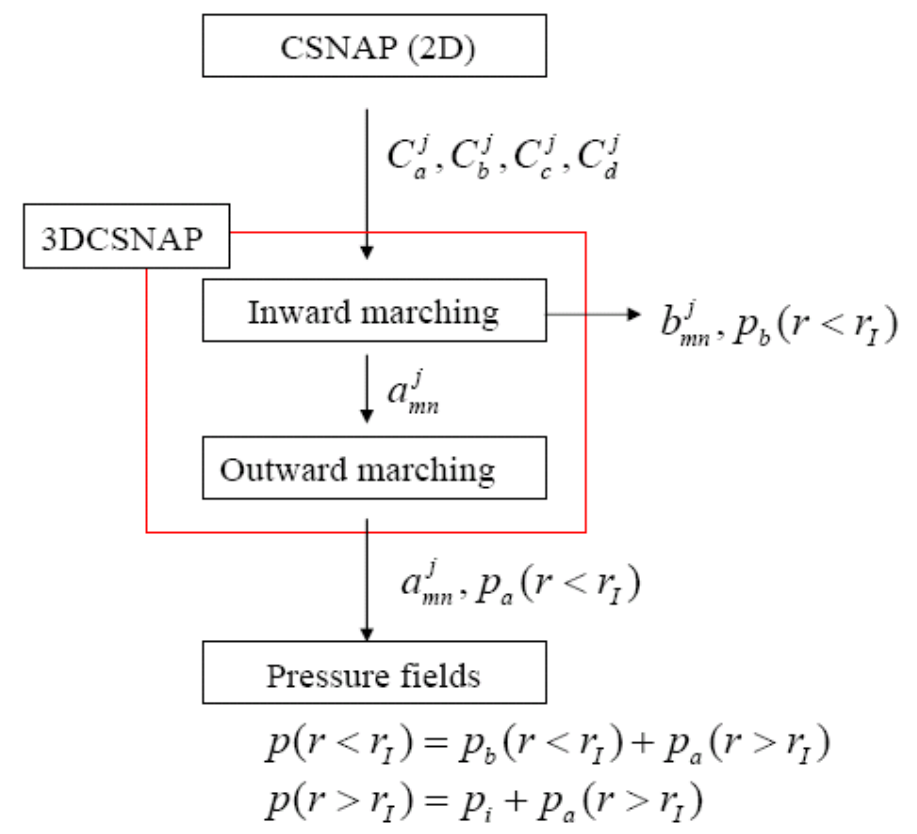

Figure 3-5: Structure of 3D spectral coupled-mode model program.

Fig. 3-5 shows the structure of 3D spectral coupled-mode model program. The coupled matrices, $C_{a}^{j}, C_{b}^{j}$, in Eq. (3-41), (3-45), (3-53), and (3-54) are computed at every range step using the 2D C-SNAP program [63]. The same coupled matrices are required for all the azimuthal modes. In addition, the coupled matrices are required for both the inward and outward marching. Therefore, it is essential to avoid solving the depthseparated wave equation at every azimuthal mode; the coupled matrices are precalculated and saved to a file to reduce computation time. 
At higher frequency, the size of the coupled matrices, $C_{a}^{j}, C_{b}^{j}$, is proportional to $N^{2} * N r^{*}$, where $N$ is the number of modes and $N r$ is the number of range steps. The size of the file, including the coupled matrices, may be so large that it is a bottleneck of the whole computation because of the slow file I/O, compared to fast operation by the CPU. In particular, this bottleneck becomes significant when we carry out the inward/outward marching at each azimuth mode, as shown in left panel of method I in Fig. 3-6. In this method, the input of the coupling matrices, $C_{a}^{j}, C_{b}^{j}$, should be repeated $N r^{*} M$ times, which can slow down the entire computation significantly.

To reduce the number of reading files for the coupling matrices, a do-loop for the azimuth modes can be moved into the inward/outward marching, as shown in the right panel of Fig. 3-6 (method II). At each section, the computation for all azimuth modes is performed in the inward/outward marching. This method can reduce the entire computation time by $1 / \mathrm{N}$ compared to method I.

However, another issue arises associated with the coupling coefficients, $a_{m n}^{j}$ and $b_{m n}^{j}$. As shown in Fig. 3-2, $a_{m n}^{j}$ from the inward marching should be added to the outward marching; therefore, $a_{m n}^{j}$ from the inward marching should be saved for the outward marching in method II. In method I, it is not necessary to save $a_{m n}^{j}$ in the inward marching. The size of $a_{m n}^{j}$ and $b_{m n}^{j}$ is proportional to $N^{*} M^{*} N r$, where $N$ is the number of modes, $M$ is the number of azimuthal modes, and $\mathrm{Nr}$ is the number of range steps. This size after the inward marching can amount to several hundred gigabytes at a higher frequency. This large size of the coupling coefficients requires a large storage capacity (hard disk drive), which limits the overall efficiency of the program. Of course, if we need to save the coupling coefficients, $a_{m n}^{j}$ and $b_{m n}^{j}$, for further purposes as well as the pressure fields, the large size of the coefficients may be an issue even with method I.

Using a perturbation of the horizontal wavenumber and modal shape [69, 70] can be a promising way with method I to overcome the problem associated with the slow file $\mathrm{I} / \mathrm{O}$ and the requirement of a large storage. 

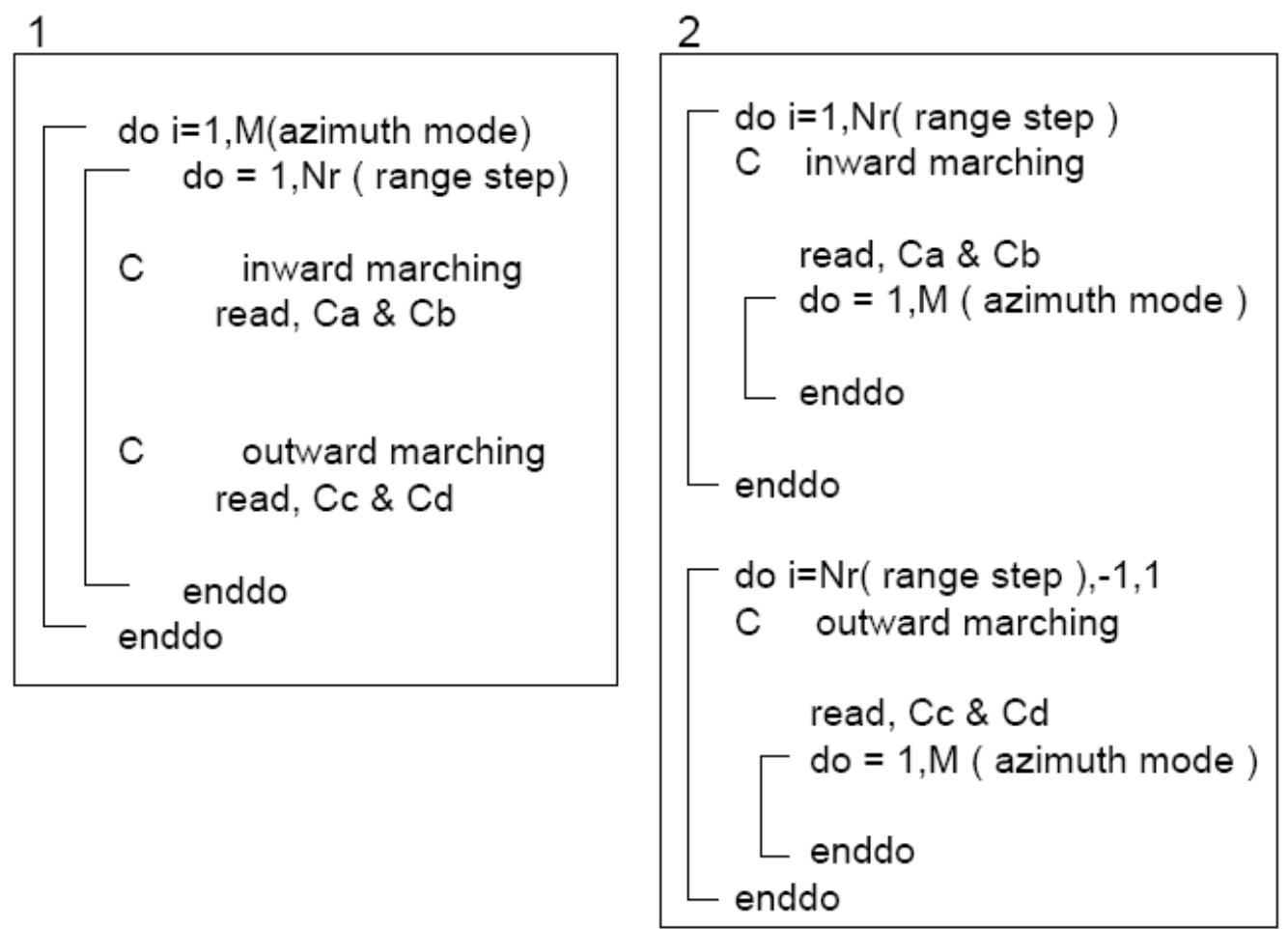

Figure 3-6: Do-loops associated with azimuth modes and range steps.

Based on the 3D spectral coupled-mode model, two programs were developed in Fortran 95 and MATLAB ${ }^{\odot}$. These programs can be run in single- or multi-processor environment using parallel computing. A detailed description on the development of the parallel computing is presented in Section 3.7.1. 


\subsection{Three-dimensional spectral coupled-mode model vs. three- dimensional parabolic equation}

The Parabolic Equation (PE) has been applied to long-range sound propagation successfully, and it has grown to be the most popular technique for range-dependent problems in acoustics [28]. Three-dimensional (3D) PE codes have also been developed [13-17]; however, the applications have been limited to shallow water and/or a short range problem because of the extensive computational demand. To consider 3D effects properly, the arc length between two adjacent vertical sections should be less than a quarter of wavelength. This requires that the number of vertical sections increases at a longer range, which means that the computational demand can be extremely extensive.

The developed 3D spectral coupled-mode model shows higher computational efficiency to compute a broadband pulse for sound propagation around a seamount with a low frequency source or a limited number of normal modes (see Chapter 5). However, this model is only applicable to an axisymmetric bathymetry. In addition, rangedependent sound speed fields can not be used with this model. Because of the limitation with the axisymmetric bathymetry, and range-dependent sound speed fields, more realistic realization of sound propagation with $3 \mathrm{D}$ effects from complicated bathymetry can not be accomplished. In contrast, the 3D PE can consider full range-dependent environments, bathymetry, and sound speed fields. Figure 3-7 demonstrates the large difference between the computational domain with a conical seamount and real rangedependent complicated bathymetry for both models. However, the backscattering wave can not be realized with the 3D PE that uses an approximated model with a one-way wave equation ${ }^{1}$.

\footnotetext{
${ }^{1}$ Collins and Evans [74] proposed a two-way PE method to handle backscattered acoustic energy in the ocean. The two-way PE is implemented for a two-dimensional problem, which is based on the single-scattering approximation with a sequence of range-independent regions.
} 
After all, the 3D coupled-mode model and 3D PE are mutually complementary to realize 3D sound propagation; the choice of model can be decided based on the complexity of the environments. In addition, an efficient 3D PE method is essential, and the comparisons between the two models are required in various problems.
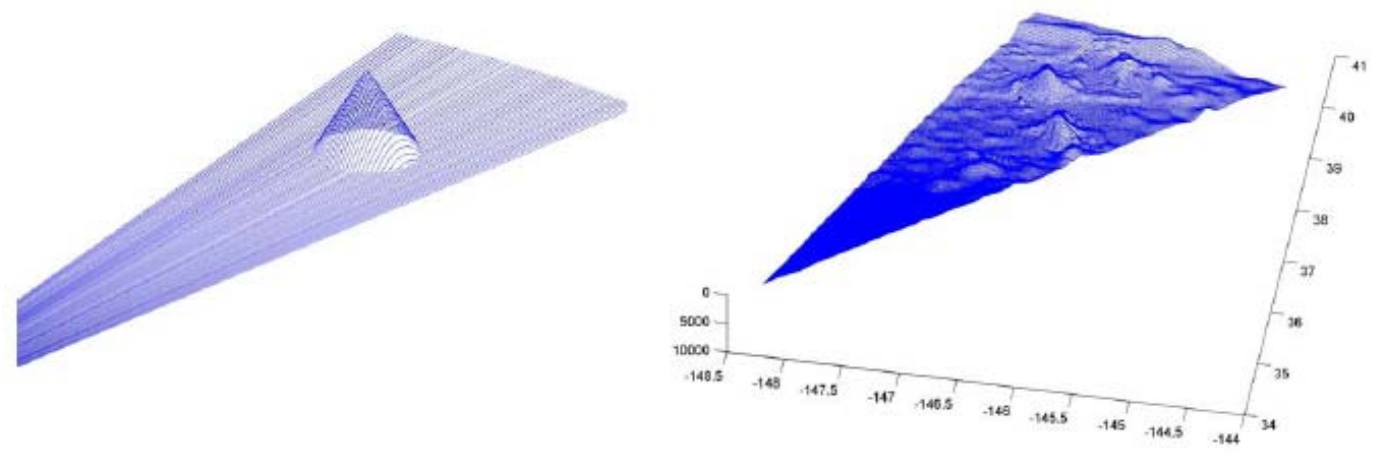

Figure 3-7: Comparison of computational domain for the 3D spectral coupled-mode model with an approximated conical seamount and the 3D PE with real range-dependent bathymetry.

Table 3-1: Comparison between 3D spectral coupled-mode model and 3D PE method

\begin{tabular}{|l|l|}
\hline \multicolumn{1}{|c|}{ 3D spectral coupled-mode model } & \multicolumn{1}{c|}{ 3D PE method } \\
\hline Exact solution to full wave equation & One-way wave approximation \\
\hline Applicable to axisymmetric bathymetry & Range dependent bathymetry \\
\hline Range independent sound speed fields & Range dependent sound speed fields \\
\hline Easy for parallel computing in azimuth modes & Difficult for parallel computing \\
\hline Computation time $\sim\left(N^{3}+I / O\right) * M * N_{r}{ }^{A}$ & Computation time $\sim N_{z} * N_{r} * N_{t}^{B}$ \\
\hline$M \sim\left[k_{r} r_{I}\right]$, depends on frequency ${ }^{\mathrm{C}}$ & $N_{t} \sim$ range of receiver \\
\hline
\end{tabular}

${ }^{\text {A }} N$ : number of normal modes, $\mathrm{I} / \mathrm{O}$ : file I/O overhead, $M$ : number of azimuth modes, $N_{r}$ : number of sections,

${ }^{\text {B }} N_{z}$ : no. of vertical segments, $N_{r}$ : no. range steps, $N_{t}$ : no. of segments in azimuth,

${ }^{\mathrm{C}} k_{r}$ : wavenumber, $r_{I}$ : radius of the base of the seamount. 
For parallel computing, the 3D spectral coupled-mode model can be parallelized easily in terms of azimuth modes, called embarrassingly parallel, because the computation for azimuth modes is performed independently. In contrast, the parallel computing of the 3D PE method may be complicated with large message passing between CPUs for solving in depth and azimuth at every range step even with an efficient alternating direction method.

The computation time of the 3D spectral coupled-mode model is proportional to $\left(N^{3}+I / O\right.$ overhead) $* M * N_{r}$, where $N$ is the number of normal modes, $M$ is the number of azimuthal mode, which can be obtained from $\left[k_{r} r_{I}\right]$, and $N_{r}$ is number of range steps. The parameters are all dependent on frequency, meaning that the computation time can be extremely extensive at higher frequency. The computation time of the 3D PE seems less sensitive to the source frequency than that of 3D spectral coupled-mode model. However, the number of angular sections, which is proportional to the source frequency, becomes a significant obstacle to practical application of long-range sound propagation with higher frequency, due to the marching scheme of PE from the acoustic source.

The problem of the number of angular sections could be solved with the higher order finite difference scheme [51] and parallel computing. In addition, a 2D or Nx2D problem can be assumed before a large change of bathymetry such as a seamount. This assumption is valid if the azimuthal coupling is small enough to be ignored with a slowly changing bathymetry. Since the required number of sections at a short range is much smaller than one at a long range, the number of sections can be varied while marching in range. Instead, an interpolation scheme should be adopted for the pressure fields after regridding of the computational domain due to the increase of the number of angular sections. This interpolation scheme might be valid with slow varying of the phase of the pressure fields. This proposed efficient 3D PE model is shown in Fig. 3-8, but development of code will remain as a future work.

One more drawback that should be addressed concerning the 3D PE method is an uncertainty at side boundaries. Generally, 2D solutions are imposed on the side boundaries. This boundary condition requires a wide computational domain, enough so that the horizontal refraction due to the seamounts at the side boundaries can be neglected. 
However, a computational domain that is too wide leads to a large number of angular sections increasing the computation time.

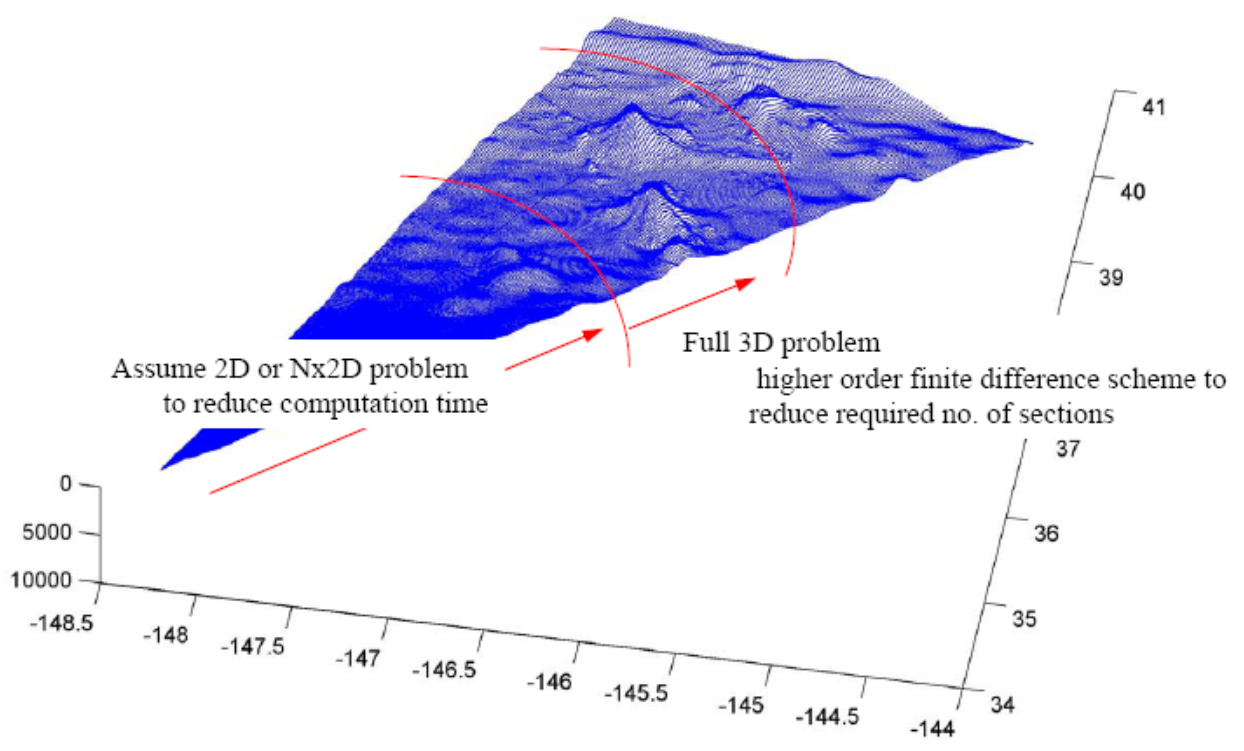

Figure 3-8: Proposed 3D PE model with high computational efficiency for a long-range and highly range-dependent problem. 


\subsection{Parallel computing and the approximation}

Although the 3D spectral coupled normal mode code can be much more efficient than earlier models [10], this model still requires huge computational demand, in particular, for a deep-sea problem at a higher frequency.

The computation time of the 3D spectral coupled-mode model is proportional to

$$
\left[N^{3}+(\text { File I/O overhead })\right] * M * N_{r}
$$

where $N$ : no. of normal modes, $M$ : no. of azimuth modes $=\left[k_{r} r_{I}\right]$, and $N_{r}$ : no. of range steps. $N, M$ and $N_{r}$ are all dependent on frequency, i.e., the computation time increases dramatically at higher frequencies. For example, consider a problem with a $68.2 \mathrm{~Hz} \mathrm{CW}$ source and the radius of the conical seamount of $28.6 \mathrm{~km}$ (see Fig. 5-47). The parameters associated with the computation time become:

$$
\begin{aligned}
& M \geq\left[k r_{I}\right]=\left[\frac{2 \pi f_{c}}{c_{0}} r_{I}\right]=8170, \\
& d r \leq \frac{\lambda}{4} \sim 5.5 m \rightarrow N_{r} \geq \frac{28600}{5.5} \sim 5200 \sim 2^{12} \text { or } 2^{13} .
\end{aligned}
$$

At the outermost ring, there are 119 water-borne modes, 378 propagation modes including bottom-bouncing modes, and 598 modes including leaky modes. These values must be satisfied as a reasonable guideline for a valid solution; otherwise, we need to perform a series of convergence tests for the parameters. In Fig. 3-9, the amplitude of outgoing wave terms, $\left|a_{m n}^{j}\right|$, are compared with two different numbers of sections at the outermost ring after the outward/inward marching. For 1024 range steps, the high amplitude artifacts are shown at the higher normal/azimuth mode where the amplitude converges to zero in the left panel of Fig. 3-9. Luo [10] pointed out that these artifacts originate with the stepwise approximation if the range step is large enough to generate a 
back-scattered wave at each stair step. Therefore, to remove these artifacts, the range step should be less than $\lambda / 2$ or meet a stricter criterion, $\lambda / 4$. Fig. 3-9, in the right panel, shows the disappearance of the artifacts with a finer step size, 2048 range steps.

$\left|a_{m n}^{j}\right|$ at the outermost ring

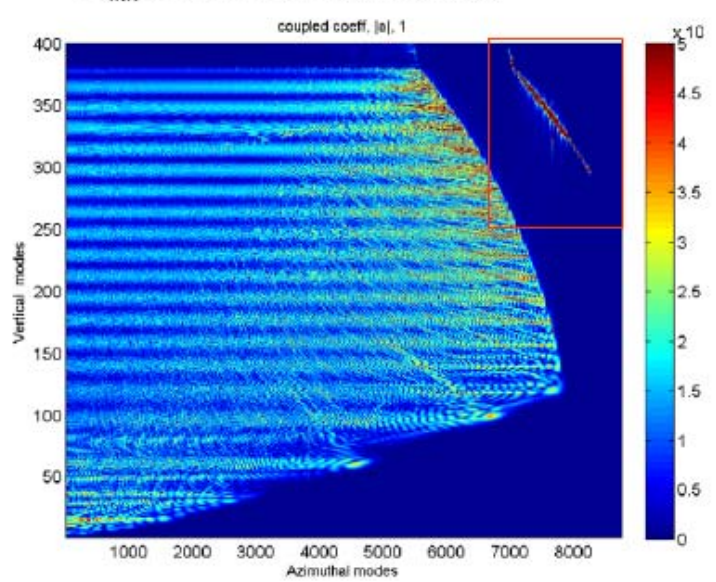

1024 sections

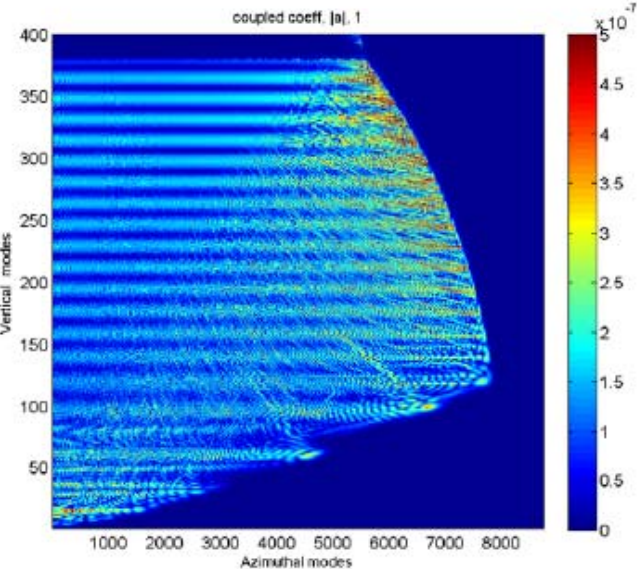

2048 sections

Figure 3-9: $\left|a_{m n}^{j}\right|$ at the outermost ring after inward/outward marching with different number of sections, 1024 and 2048. In the left panel, the artifacts from the stepwise approximation are shown in the top right corner; however, the artifacts disappear with 2048 sections in the right panel.

Table 3-2 shows the computation time with various numbers of normal modes and range steps for the $68.2 \mathrm{~Hz} \mathrm{CW}$ source. At a greater number of normal modes and higher range steps, a parallel program was used to obtain a result in a reasonable amount of computation time. The computation time was given in hours per CPU (column A), and also core hours (column B) were estimated only by the product of computation time and number of CPUs. During the test, three different systems were used: a quad-core PC, a clustered computer at MIT, and LLGRID at the MIT Lincoln Laboratory. Since the performances of the systems are different, and the test environments were not exactly the same for all the cases, it is difficult to compare the computation time directly. For 
example, the program for a single-processor is faster than the one for multi-processors in the same condition due to so-called parallel overhead. However, we could compare the computation time roughly to make a guideline. For example, it may take 100 days to obtain a result for a CW source in the case of 500 normal modes and 2048 sections with a single CPU. This means that even with 100 CPUs and parallel computing, we could obtain a broadband pulse in 240 days, if we consider a broadband pulse that is 8 seconds long with $30 \mathrm{~Hz}$ bandwidth. This large amount of computation time would not be acceptable in practical use.

Table 3-2: Computation time for various no. range step and no. normal modes in hours

\begin{tabular}{|c|c|c|c|c|c|c|}
\hline \multirow{2}{*}{$\begin{array}{c}\text { No. of } \\
\text { Normal mode step }\end{array}$} & \multicolumn{2}{|c|}{512} & \multicolumn{2}{c|}{1024} & \multicolumn{2}{c|}{2048} \\
\cline { 2 - 7 } & A & B & A & B & A & B \\
\hline 100 & $26.7 / 1$ & 26.7 & $39.5 / 1^{1}$ & 39.5 & $50.1 / 1$ & 50.1 \\
\hline 200 & - & - & $45.2 / 2$ & 90.4 & $41.6 / 8$ & 332.8 \\
\hline 300 & - & - & $70.0 / 8$ & 560.0 & - & - \\
\hline 400 & - & - & $180.0 / 8$ & 1440.0 & $17.0 / 100$ & 1700.0 \\
\hline 500 & & - & - & - & $23.47 / 100$ & 2347.0 \\
\hline
\end{tabular}

A: [Computation time (in hours) per CPU] / [number of CPUs].

B: Core hours (in hours). The core hours are estimated computation time for a single-core, i.e. computation time per CPU *number of CPUs.

${ }^{1}$ : The computer system used is different depending on the number of CPUs:

1: PC with 3.0GHz,

2 and 8: clustered computer at MIT,

100: LLGRID at the MIT Lincoln Laboratory.

In addition to the large number of normal modes, azimuth modes, and range steps, there are several problems to be addressed carefully for better computational efficiency and stable computation: 1) matrix inverse, 2) coupling matrices, $C_{a}^{j}, C_{b}^{j}$, and 3) Hankel function computation. 
At every azimuth mode, we need to solve equations (3.50) and (3.51) for the outward marching. In (3.50), a matrix inverse, $\left(\mathbf{D} \hat{\mathbf{J}}_{\mathrm{m}}^{\mathrm{j}} \mathbf{C}_{\mathbf{a}}^{\mathbf{j}+1} \hat{\mathbf{H}}_{\mathrm{m}}^{\mathrm{j}+\mathbf{1}}-\hat{\mathbf{J}}_{\mathrm{m}}^{\mathbf{j}} \mathbf{C}_{\mathbf{b}}^{\mathrm{j}+1} \mathbf{D} \hat{\mathbf{H}}_{\mathrm{m}}^{\mathrm{j}+1}\right)^{-\mathbf{1}}$, is required, which is the reason why the amount of computation time is proportional to $N^{3}$ in Eq. 3-56. We could reduce the computational demand for the matrix inverse using a more efficient matrix inverse scheme as well as a reduction in the total number of the matrix inverse. The matrix inverse is required to be carried out repeatedly $M$ (number of azimuth modes) times, which is quite large at high frequency. Therefore, the reduction of the number of azimuth modes may be a better way to reduce the total amount of computation time, which will be discussed more in Section 3.7.2.

As stated in Section 3-5, the coupling matrices, $C_{a}^{j}, C_{b}^{j}$, can increase the computational demand due to the overhead of slow file I/O. This will be discussed more in Section 3.7.1 for parallel computing.

The best way to decrease the amount of computation time is to reduce the number of normal modes, azimuth modes, and range steps in Eq. (3.56), since the amount of computation time is directly proportional to the product of the parameters. In Section 3.7.3, the truncation of normal modes and the sub-sampling of azimuth modes are examined as promising ways to reduce computation time.

The 3D spectral coupled-mode model is expressed in the Bessel and Hankel functions; therefore, it is essential to compute these functions in a fast and stable way. In Section 3.7.4, the real-valued argument approximation of the Bessel and Hankel function will be examined; this enables use of a look-up table, rather than computing the Bessel and Hankel function at every step. Another issue concerning the Hankel function is associated with the instability at higher modes with a high complex part of the argument. The stability of the Hankel function should be handled very carefully in numerical calculation.

In the following sections, 3.7.2 3.7.4, results are computed for a problem in Fig. 310 with a $10 \mathrm{~Hz}$ CW source. 


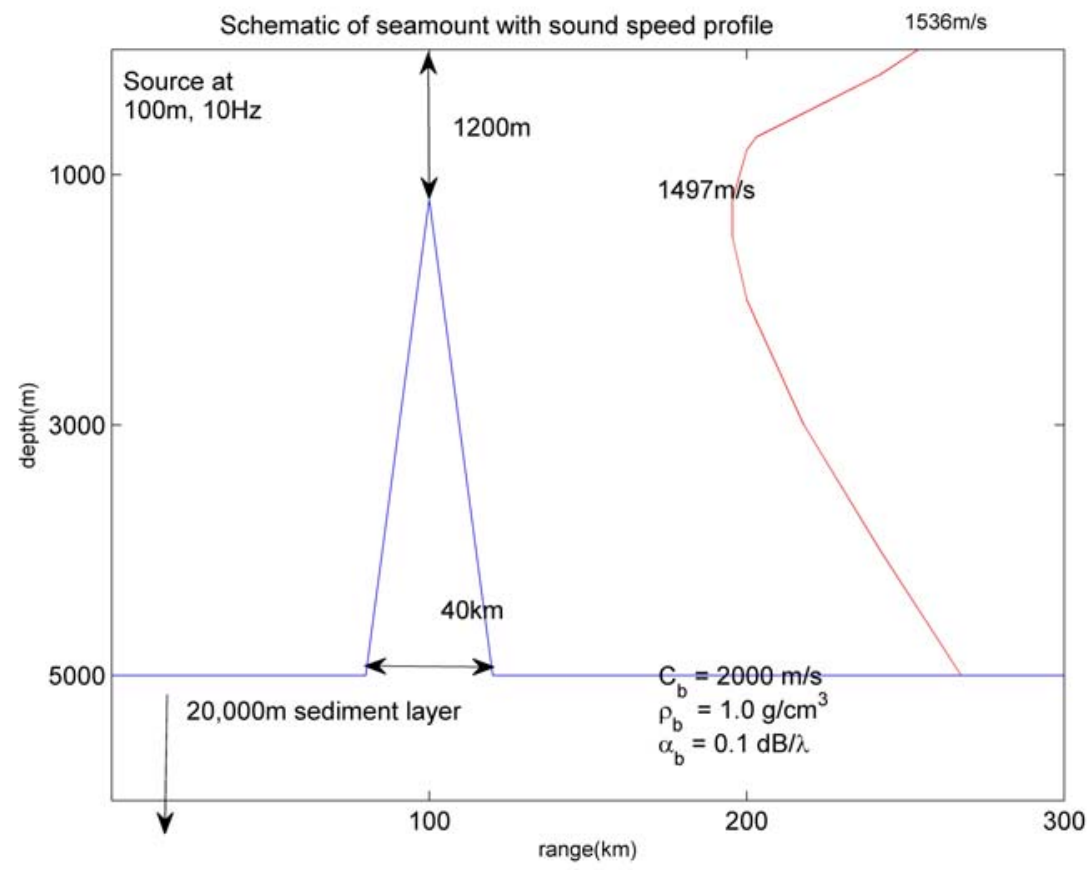

Figure 3-10: Description of problem with a $10 \mathrm{~Hz}$ CW source (see Section 5.2 for details). 


\subsubsection{Parallel computing}

Recently, the rapid advance in computer technology has enabled us to solve a complicated and realistic long-range sound propagation problem in a reasonable computation time; however, 3D sound propagation problems are beyond the current computational ability. We described, in previous sections, the large computation time of the 3D spectral coupled-mode model, in particular, for the deep-sea, high frequency sound propagation problems, even for a CW source. Therefore, parallel computing is essential to obtain a transmission loss or broadband pulse in a reasonable computation time.

If the computation time for a CW source is not significantly large, a single-core program can be used. For a broadband pulse, the single-core program computes pressure field solutions for multi-frequencies independently and simultaneously in a cluster computer. The single-core program gives much higher efficiency, avoiding the parallel overhead, which means additional computation time to coordinate parallel tasks. If the computation for a CW source, however, takes too much time, it is necessary to use a parallel program. The approach we use here is to divide the azimuth modes into a certain number of sets and distribute the jobs for the sets of azimuth modes over the available CPUs. The computations of (3-51), (3-52), and (3-53) should be done independently and repeatedly in azimuth modes.

Since the azimuth modal cut-off as well as modal cut-off of normal modes take place along the inward and outward marchings (see section 3.7.2), the inward marching for a certain azimuth mode can be stopped at a certain section before the center of the seamount. This azimuth modal cut-off requires larger computational demand at lower modes because the coupling coefficients, $a_{m n}^{j}$ and $b_{m n}^{j}$, converge to zero more quickly at a higher azimuth mode.

In Fig. 3-11, the workloads for 100 CPUs with equally spaced azimuth modes are shown; the workload can be compared by the ratio of computation time to the maximum computation time among all CPUs. The workloads linearly decrease at higher azimuth 
modes. Therefore, the total computation time is determined by the first CPU with the largest computation time, while the other CPUs are waiting with idle tasks. The ideal scenario for the highest computational efficiency might be distributing the jobs with equal computation time. To finish the distributed jobs for all CPUs at the same time, the workload for each CPU should be balanced.

As a way to balance the workload for all the CPUs, the linearly increasing number of azimuth modes was tested; i.e., more azimuth modes are assigned to higher mode. Figure 3-12 shows the workload ratio with respect to the maximum computation time with a linearly increasing number of azimuth modes. The workload ratios with the linearly varying number of azimuth modes are more balanced than the equally spaced azimuth modes. The overall workload ratios are increased to $1 \sim 0.6$, which are compared to $1 \sim 0.1$ for the equal spaced azimuth modes in Fig. 3-11. The balancing of the workloads could be improved with more careful distribution of jobs over the CPUs.

The parallel version of the MATLAB ${ }^{\odot}$ program was developed with the pMatalb [66] which is a Message Passing Interface (MPI) library for MATLAB ${ }^{\odot}$ developed by the MIT Lincoln Laboratory [67]. In addition, a parallel Frotran program was developed and is compatible with widely used MPI libraries, openMPI [64] and mpich2 [65]. The programs were tested and run on three different computer systems: a quadcore PC of 3.0GHz, a clustered system at MIT, and Lincoln Laboratory GRID (LLGRID) at the MIT Lincoln Laboratory. The MATLAB ${ }^{\odot}$ code can be easier to run and revise, but the Fortran code is much faster than the MATLAB ${ }^{\odot}$ code. The Fortran code was also tested and run in a cluster computing system with Condor, a specialized workload management system for computer-intensive jobs [68]. 

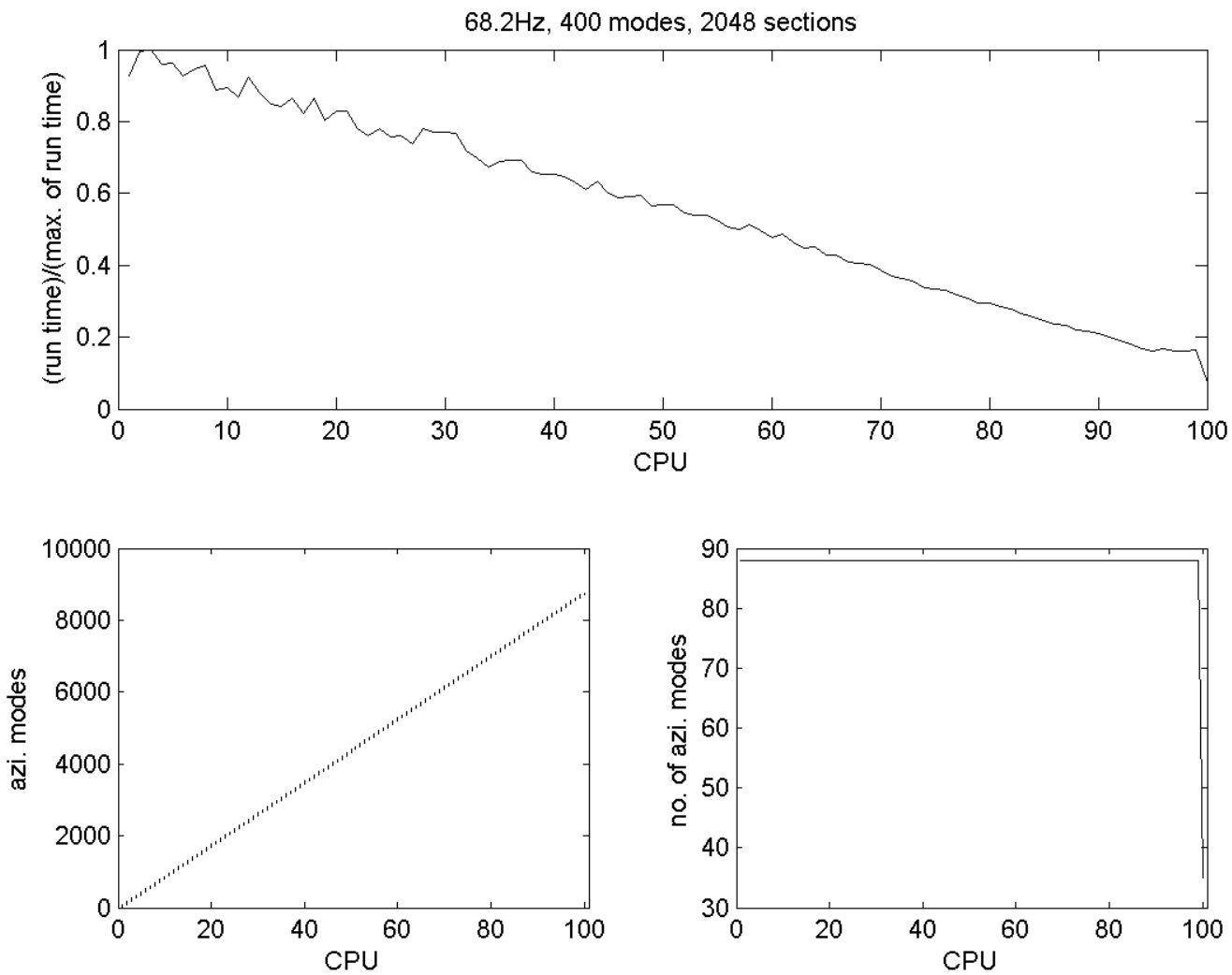

Figure 3-11: Computation time of CPUs with equally spaced azimuth modes. 

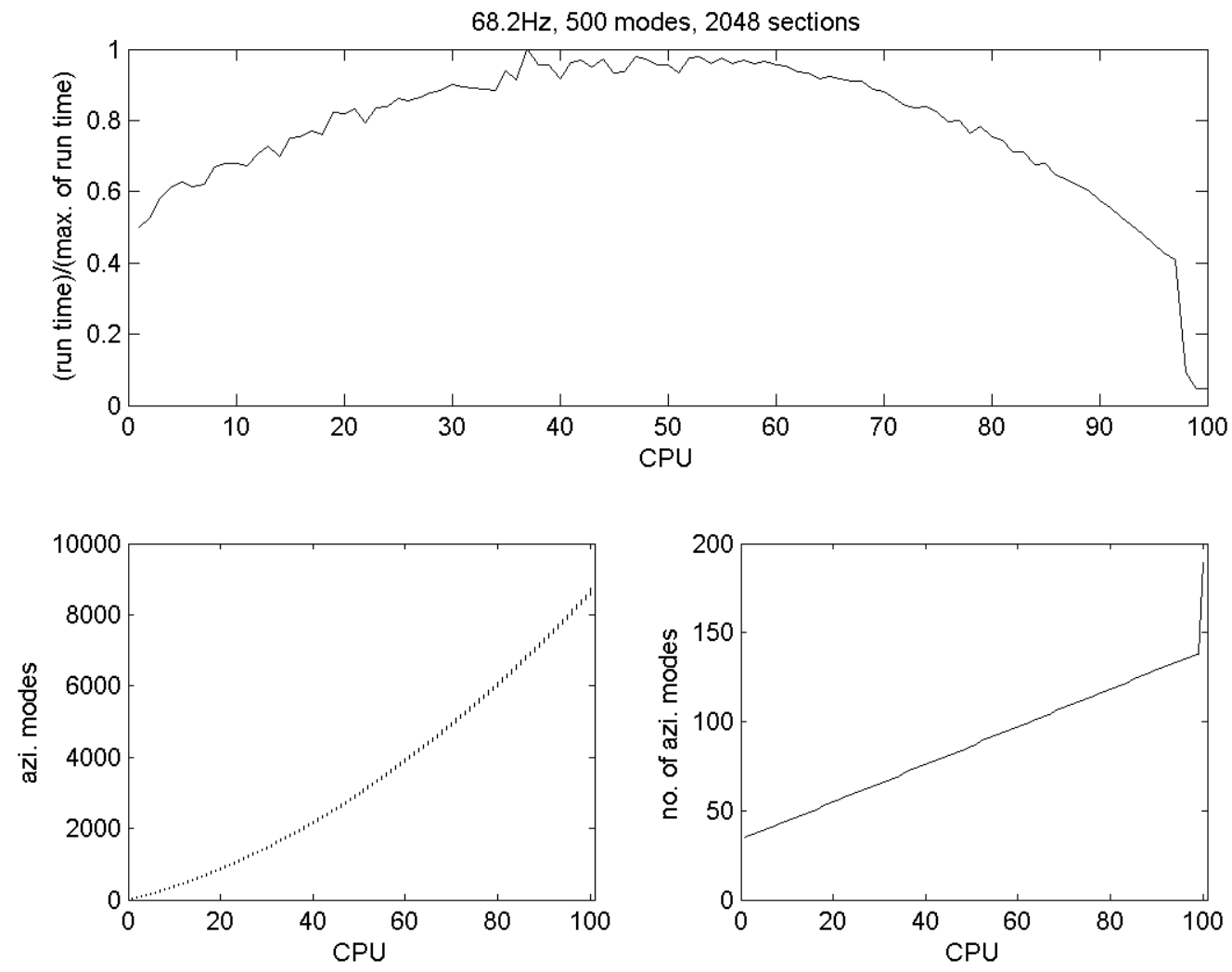

Figure 3-12: Computation time of CPUs with linearly increasing number of azimuth modes. The program adds some additional higher azimuth modes at the beginning of the computation; therefore, the jobs for these higher azimuth modes were performed very quickly, which explains the abrupt change in the workload ratio at the highest number of CPUs. 


\subsubsection{Azimuth modal cut-off}

As the inward marching heads toward the center of a seamount, the well-known 'modal cut-off' takes place due to the upslope sound propagation. The modal cut-off explains that some trapped modes are lost to continuous modes as the water depth decreases; the modal cut-off happens with significant energy transfer.

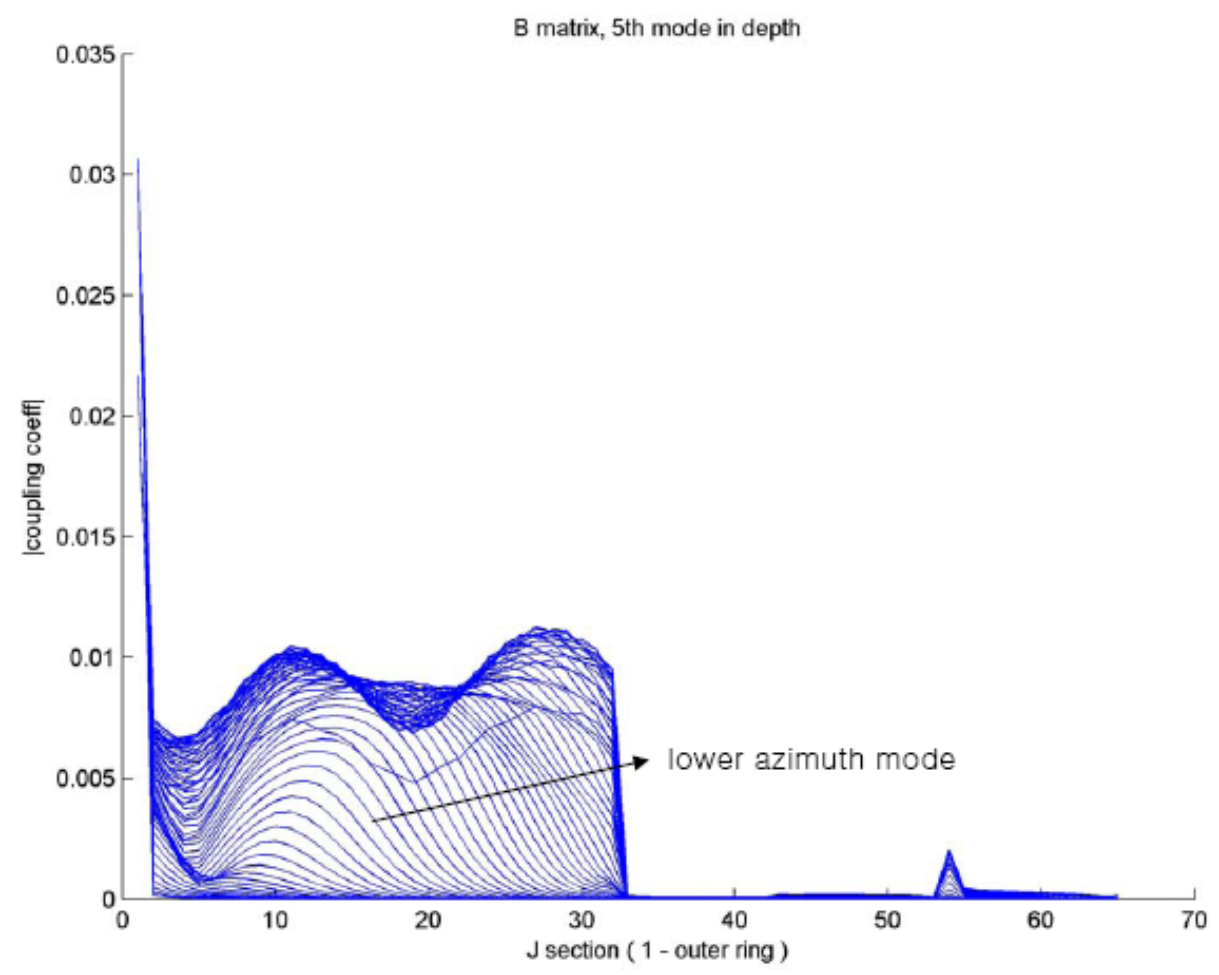

Figure 3-13: Amplitudes of incoming coupling coefficients, $\left|b_{m n}^{j}\right|$, at the fifth normal mode in terms of azimuth modes and sections.

Figure 3-13 shows the coupling coefficients, $\left|b_{m n}^{j}\right|$, for the fifth normal mode along sections in the inward marching. The modal cut-off of the fifth mode after the thirty-third section takes place with the abrupt change of the coupling coefficients, which converge to zero after the section. In addition to the modal cut-off, we can see that the coupling coefficients for the higher azimuth modes converge to zero before the modal cut-off takes 
place. As clearly shown in the figure, the coupling coefficients for the higher modes decrease to zero faster than those for lower modes. This phenomenon is hereafter called azimuth modal cut-off.

The azimuth modal cut-off is quite clearly shown in Fig. 3-14. The amplitudes of the coupling coefficients, $\left|b_{m n}^{j}\right|$, in four different sections are shown in the lower panels during inward marching to the center of the seamount. The $\left|b_{m n}^{j}\right|$ are shown in terms of the azimuth modes and the normal modes.

In the first section, the constant amplitude is given for all azimuth modes as an initial condition, but the significant parts of the amplitudes of the coupling coefficients become narrower and more limited in both the azimuth modes and the vertical modes in the subsequent sections. The azimuth modal cut-off is valid for the coupling coefficients for the outgoing wave, $\left|a_{m n}^{j}\right|$, which are shown in the top panels in Fig. 3-14.

This azimuth modal cut-off enables us to limit the number of azimuth modes in a certain section, which can be decided from the highest horizontal wavenumber and the range from the center of the seamount as shown in the following equation:

$$
M^{j} \geq\left[k_{1}^{j} r^{j}\right],
$$

where $M^{j}$ : the minimum number of azimuthal modes, $k_{1}^{j}$ : the largest horizontal wave number, and $r^{j}$ : the radius of the ring for section $j$.

The limitation of the number of azimuth modes can reduce the total computation time, which was implemented in the developed 3D spectral coupled-mode program. 


$$
\left|a_{m n}^{j}\right|
$$
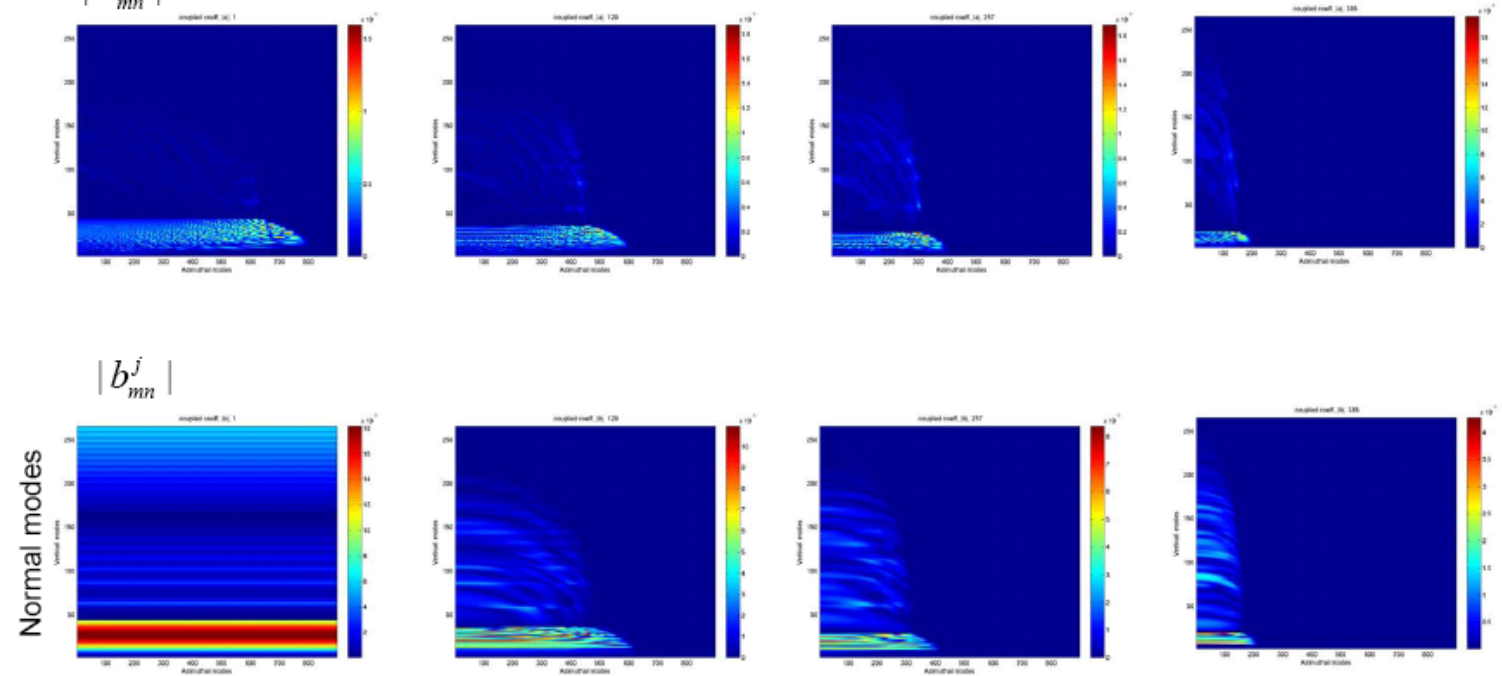

Azimuth modes

Figure 3-14: Amplitudes of outgoing and incoming coupling coefficients, $\left|a_{m n}^{j}\right|$ and $\left|b_{m n}^{j}\right|$, respectively, in four different sections in terms of normal and azimuth modes. 


\subsubsection{Approximations with the truncation of normal modes and the sub- sampling of azimuthal modes}

The best way to reduce the amount of computation time might be to use fewer normal

and azimuth modes. However, we are unable to reduce the number of sections along a conical seamount because of the artifacts in higher azimuth and normal modes due to the coarse step size, as shown in Fig. 3-9. In this chapter, some other promising ways to reduce the number of normal modes and azimuth modes will be examined.

1) Truncation of normal modes

For the problem with a $10 \mathrm{~Hz}$ CW source shown in Fig. 3-10, at the outermost ring, there are 12 water-borne modes, 43 propagation modes including 31 bottom-bouncing modes, and 221 leaky modes. In this problem, since the frequency is so low that a leaky mode plays a significant role associated with the energy dissipation at a higher mode, in particular, around the seamount. Figure 3-15 shows the transmission loss (TL) with truncation of normal modes such as 50,100, and full modes (266). The overall accuracy of the approximation with the truncation of normal modes is imprecise along the centerline (top panel), and the error of TL is quite large around the seamount. As shown in the bottom panel, however, the TL for an acoustic path with 7.5 degrees off centerline shows acceptable accuracy.

In a practical sense, we can use the truncated normal modes with reasonable accuracy. This requires a series of convergence tests to maintain the accuracy at an acceptable level.

2) Sub-sampling of azimuth modes

Here we explore the reduced number of azimuth modes due to the sub-sampling of azimuth modes. If there is a coefficient which is dependent on the azimuth mode and is 
smooth enough to be interpolated, then the full set of the coefficients can be constructed by an interpolation scheme. This enables computation of the coefficients with a subset of azimuth modes increasing the computational efficiency.

The pressure fields outside of the base of the seamount can be expressed as in Eq. (3.29),

$$
\begin{aligned}
p(r, z, \phi) & =p_{i}\left(r^{\prime}, z\right)+\sum_{m=0}^{\infty}\left[\sum_{n=1}^{\infty} a_{m n}^{J} \hat{H}_{m n}^{J}(r) \Psi_{n}^{J}(z)\right] \Phi_{m}(\phi), \\
& =p_{i}\left(r^{\prime}, z\right)+\sum_{m=0}^{\infty} P_{m}^{J}(r, z) \Phi_{m}(\phi)
\end{aligned}
$$

where $P_{m}^{J}$ is the azimuthal mode amplitude.

Since $a_{m n}^{j}$ are highly fluctuating, it is difficult to interpolate $a_{m n}^{j}$. However, $P_{m}^{J}$ is less fluctuating and slowly varying compared to $a_{m n}^{j}$. Figure 3-17 shows the real parts of the $P_{m}^{J}$ for the various azimuth modes at $150 \mathrm{~km}$ from the source. The real parts of the $P_{m}^{J}$ are compared with those obtained using the interpolation with the 1/2 and 1/5 subsampling. The blue triangles denote the correct coefficients from the computation, and the red circles denote the azimuth mode chosen for the sub-sampling. The cyan circles are the interpolated coefficients based on the sub-sampled coefficients. For the 1/2 and $1 / 5$ subsampling, the computation of coupling coefficients is performed at every 2 and 5 azimuth modes, respectively. As shown in Fig. 3-17, the accuracy of the interpolation with 1/2 sub-sampling is quite acceptable, and the transmission loss (TL), given in Fig. 3-16, shows good agreement with that of the full azimuth modes. For 1/5 sub-sampling, the error becomes bigger than the $1 / 2$ sub-sampling, and the TL shows large discrepancies, specifically around the seamount. However, the TL far outside of the seamount shows quite good accuracy. 

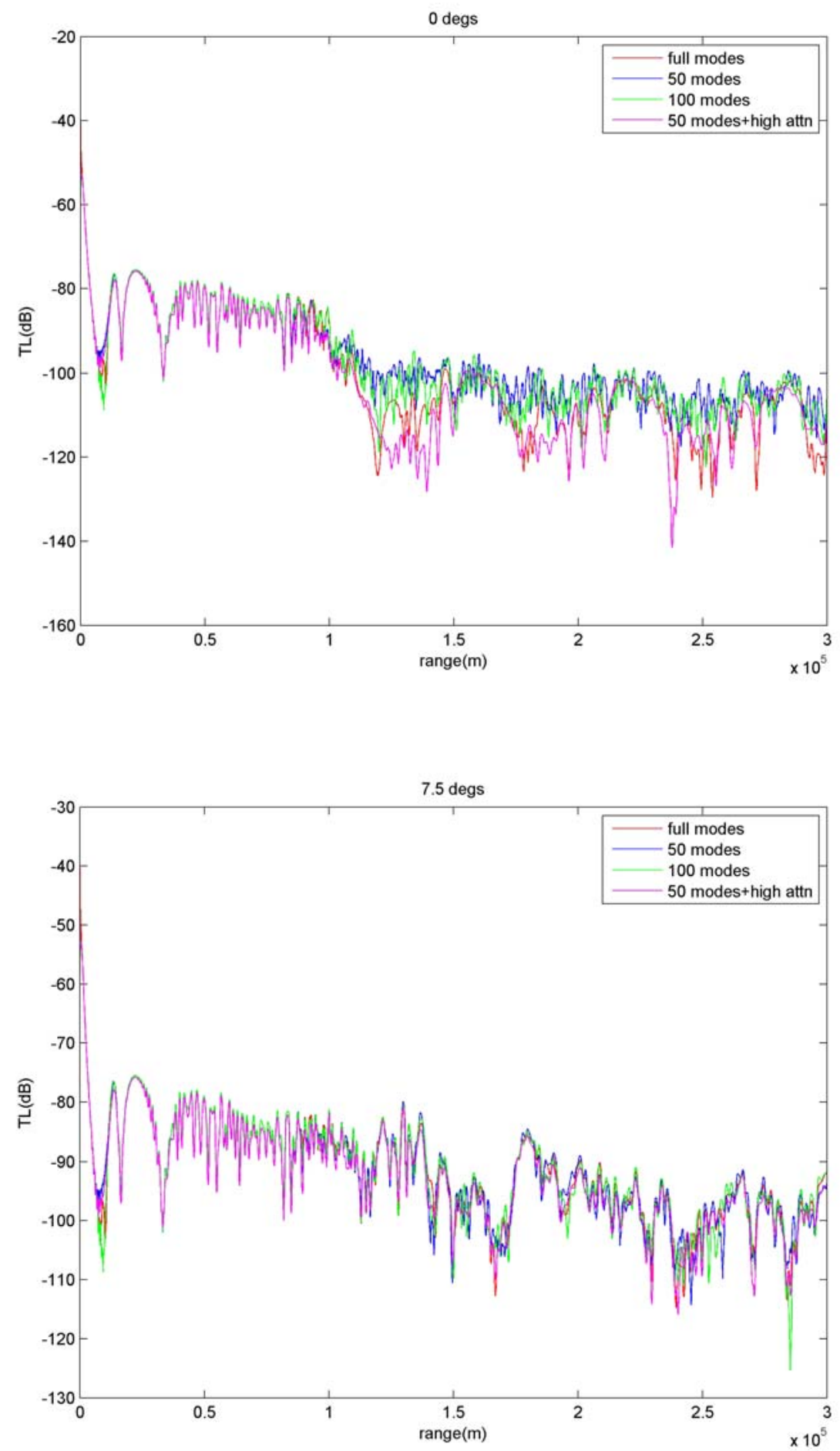

Figure 3-15: Transmission losses for various number of normal modes along the centerline (0 degree, top panel) and 7.5 degrees off centerline (bottom panel). 

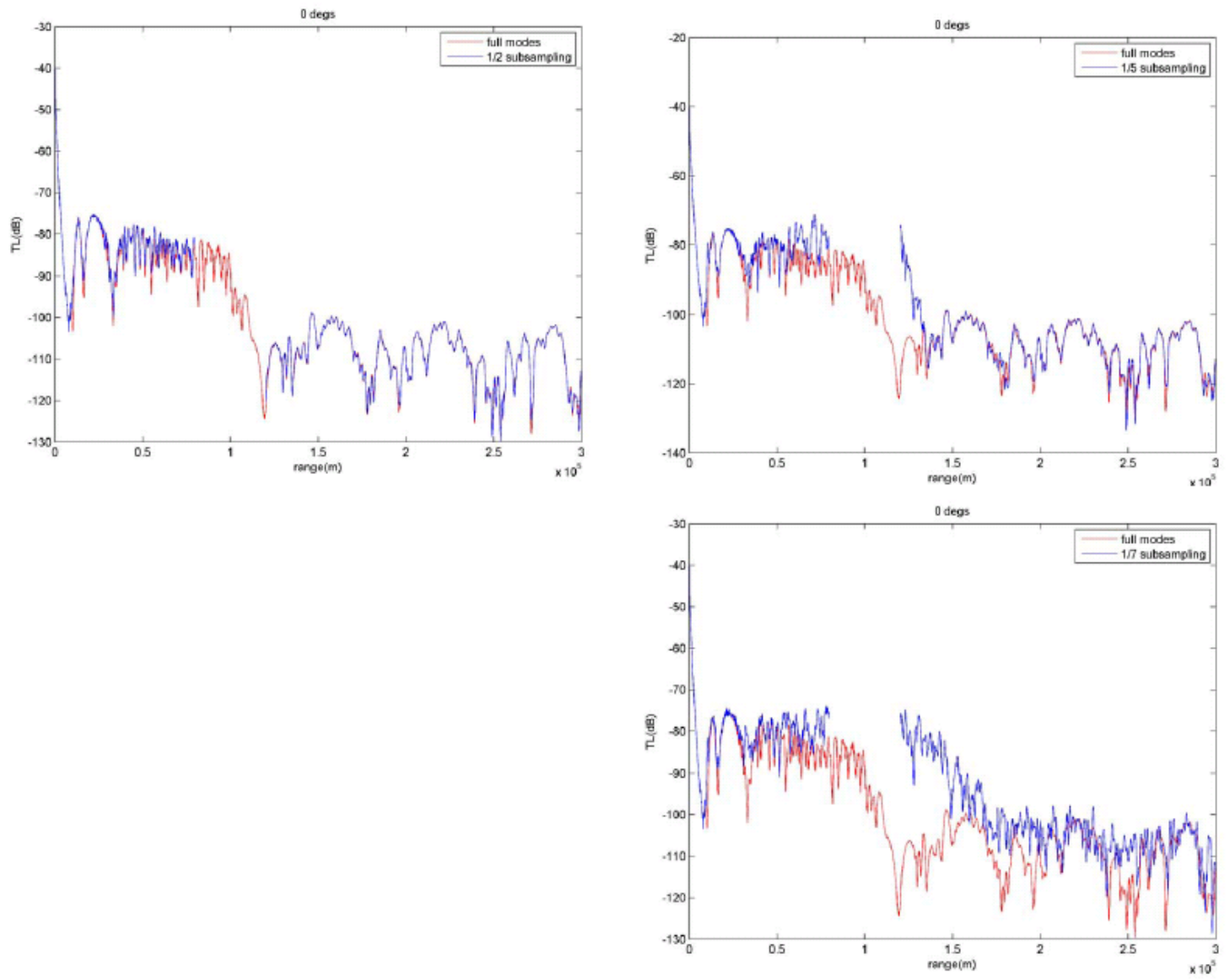

Figure 3-16: Transmission losses with various sub-samplings of azimuth modes: 1/2 sampling (top left), 1/5 sampling (top right) and 1/7 sampling (bottom right). The transmission losses with the sub-samplings are obtained only in the region outside the seamount. 

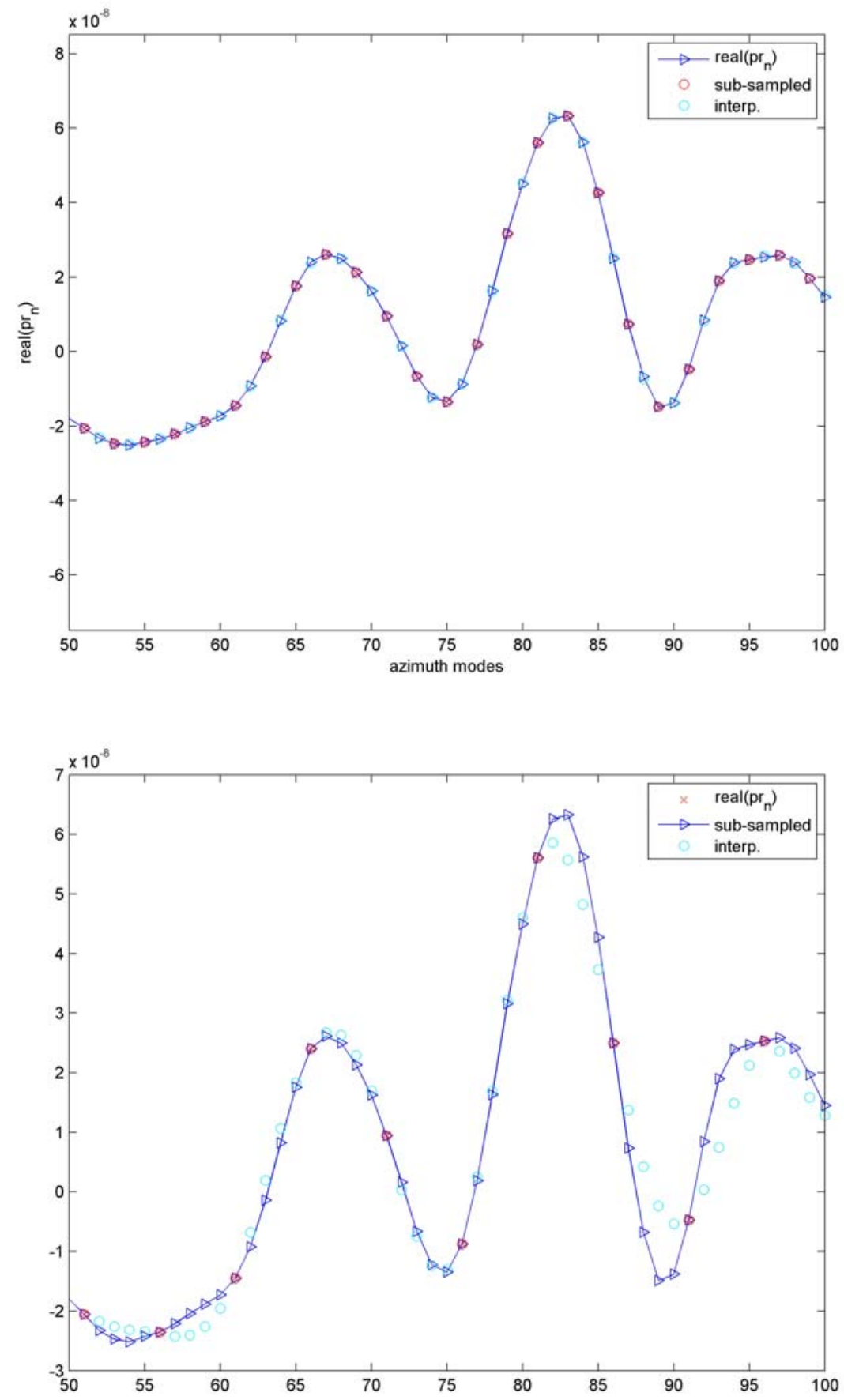

Figure 3-17: Interpolation of the real part of the $P_{m}^{J}$ at each azimuth mode at $150 \mathrm{~km}$ for $1 / 2$ (top panel) and 1/5 (bottom panel) samplings. The blue triangles denote the correct coefficients from the computation; the red circles denote the azimuth modes chosen for the sub-sampling. The cyan circles are the interpolated coefficients based on the sub-sampled coefficients (red circles). 


\subsubsection{Real-value argument approximation of the Hankel and Bessel function}

The 3D spectral coupled-mode model is expressed in the Hankel and Bessel functions. Therefore, computing these functions in a fast and stable way is essential. If we could avoid computing the Hankel and Bessel functions, currently performed at every step repeatedly, we could achieve substantial gain in computation time. As shown in Eq. (3.60), the Hankel function with the complex-value argument can be expressed as a product of an exponentially decay function and a Hankel function with the real-value argument. This approximation enables use of the look-up table of the pre-calculated Hankel function with an interpolation scheme. The look-up table can be computed and prepared before the computation. By using the look-up table, the repeated computation of the Hankel function is not necessary, thus saving computation time.

$$
H_{v}^{(1)}\left(\left(k_{r}+j \alpha\right) r\right) \approx H_{v}^{(1)}\left(k_{r} r\right) e^{-\alpha r}
$$

The Bessel function can be obtained from the approximated Hankel function. Figure 3-18 shows the transmission loss (TL) with the real argument approximation of the Hankel and Bessel function; the TL was compared to that without the approximation. The TL was calculated along the centerline between an acoustic source, a $10 \mathrm{~Hz} \mathrm{CW}$ source, and the peak of a conical seamount. The overall accuracy with the approximation seems imprecise because of the high fluctuations that originate from the error of the approximation with a high complex valued argument. The smoothed version of the approximated TL, however, shows good agreement with the TL with no approximation, except for the region just after the peak of the seamount between $10 \sim 12 \mathrm{~km}$. 


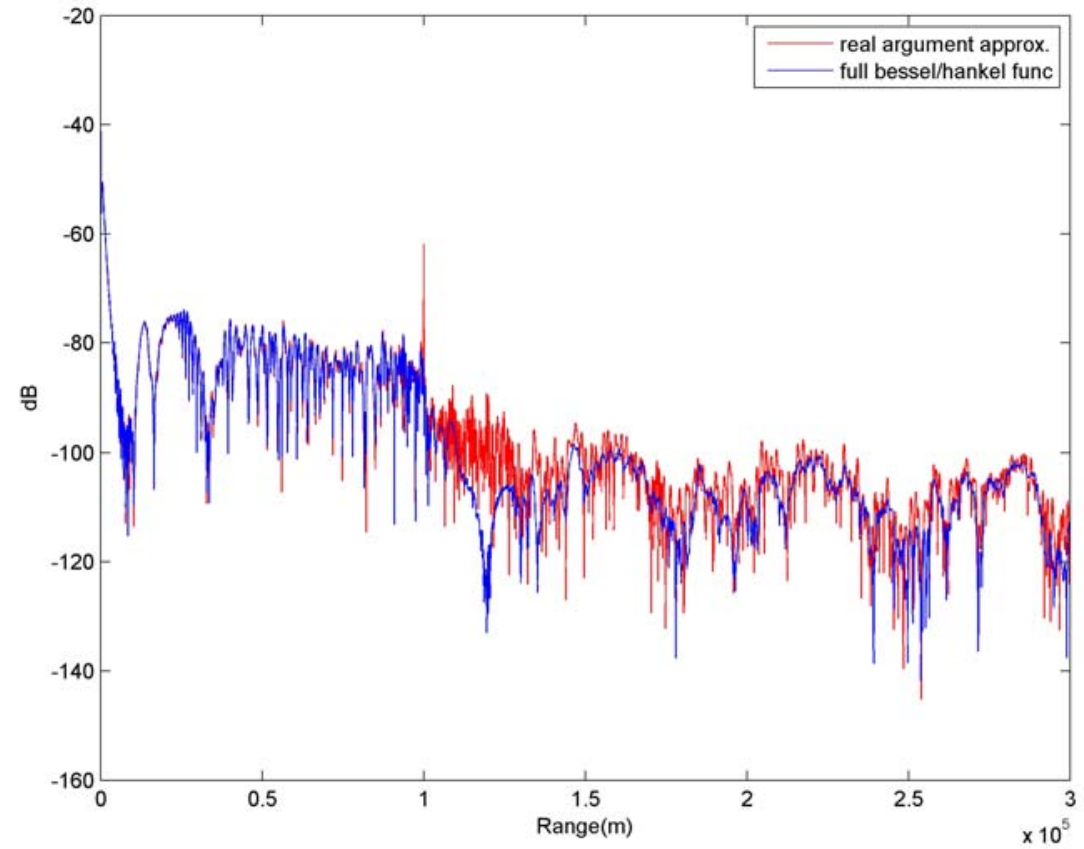

Figure 3-18: TL with the real argument approximation of the Hankel and Bessel function compared to the TL without the approximation for a $10 \mathrm{~Hz} \mathrm{CW}$ source. 


\section{Chapter 4}

\section{Reconciliation of arrival pulses between the BASSEX experiment and two-dimensional sound propagation model}

In this thesis, reconciliation of pulse arrivals between the BASSEX experiment and twodimensional simulations are carried out using the 2D Parabolic Equation (PE) and raytracing method.

In this reconciliation, various acoustic paths from the SPICEX source 1 and 2 sources to the receivers behind the Kermit-Roosevelt and Elvis seamounts as well as open sea cases are considered as follows:

1) from SPICEX source 2 over the Elvis seamount (day 268),

2) from SPICEX source 2 over the Kermit-Roosevelt seamount (day 267),

3) from SPICEX source 1 over the Kermit-Roosevelt seamount (day 264),

4) open sea cases (day 271).

Due to the limited computational efficiency of the three-dimensional (3D) model at a higher frequency, such as $250 \mathrm{~Hz}$, only 2D simulations are carried out for the SPICEX sources. Through the 2D simulations, we identify the individual ray arrivals in the BASSEX experiment by comparing the ray arrivals from the 2D simulation to those from the experiment.

Instead of using the 3D model, we perform $\mathrm{Nx} 2 \mathrm{D}$ computation using the 2D PE model. For the Nx2D PE numerical simulations, four approximated conical seamounts 
with range-averaged sound speed profiles are considered, and the locations of the shadow zone behind the seamounts can be confirmed by comparing the Nx2D computation with the BASSEX experiment in terms of the arrival energy.

\subsection{Parabolic Equation}

The RAM, a 2D Parabolic Equation (PE) model developed by Collins [12], was used in the 2D simulation for the SPICEX sources. To obtain broadband pulses by the Fourier synthesis method [28], 821 frequencies over the $100 \mathrm{~Hz}$ bandwidth with $250 \mathrm{~Hz}$ center frequency were considered. This is equivalent to an 8.192 second long simulation with a time step of 0.001 second. While the 8 second simulation is enough for refracted rays, the bottom-reflected rays give the wrap-around problem. Therefore, we introduce the high attenuation at the bottom within a $200 \mathrm{~km}$ range to reduce the bottom-reflected rays. Even in real data, no significant bottom reflected rays were found before the seamount.

Convergence tests for a single frequency $(250 \mathrm{~Hz}$, the center frequency of the SPICEX sources) were conducted with various vertical grid sizes from $0.25 \sim 1.0 \mathrm{~m}$ and $10 \sim 100 \mathrm{~m}$ range steps. Through the convergence tests, $0.5 \mathrm{~m}$ vertical grid size and $50 \mathrm{~m}$ range steps were chosen as maximum values for highest computational efficiency and consistent pressure fields.

The computation time of the 2D PE for a broadband pulse is directly dependent on the number of frequencies (or time duration), vertical grid size, and range steps; with the above conditions, the calculation requires approximately 22 hours with an single CPU of Intel CPU (Q6600, 3.24GHz).

\subsubsection{Bathymetry}

Bathymetry for an acoustic path from a source to an instantaneous array location was obtained from bathymetry measurement around the seamounts from the BASSEX 
experiment (see Section 2.2.1) and GTOPO(GDEM) database[72] for the area which is not covered by the measurement. The Voronoi interpolation, which is used in the SEALAB [41] program, and a linear 2-D interpolation were tested, but no significant difference in simulation results was found. Hence, the bathymetry was linearly interpolated.

\subsubsection{Sound speed profile}

As discussed in Section 2.2.3, sound speed fields from the XBT casts of the BASSEX experiment were limited to the covered entire area. However, for day 268, sound speed fields from the XBT casts are quite well-defined along the acoustic path over the Elvis seamount, as shown in Fig. 4-1. Therefore, the sound speed profiles from the XBT casts were used in the simulation; the nearest XBT sound speeds were chosen along the acoustic path. In contrast, for the acoustic path over the Kermit seamount (day 267, day 264) and the open sea cases (day 271), the sound speed fields from objective analysis were used, as discussed in Section 2.2.3.

\subsubsection{Bottom properties}

Since geoacoustic properties were not measured in the BASSEX experiment, two different bottom properties [42], porous basalt and loose sediment, were considered as described in Table 4-1. These bottom types are chosen to see the effect of the bottom with reflected waves behind the seamounts. If reflections by the seamount were involved, the geoacoustic properties become essential to estimate signal level and reconcile the individual rays. However, it was difficult to determine whether there was a significant difference in the simulation result with different bottom types, so most simulations were done with the porous bassalt bottom condition. In this simulation, shear effect was not included. The bottom was treated as homogeneous with an absorption layer below 7000m, 
and to reduce the bottom reflected wave, a high attenuation value $(10 d B / \lambda)$ was used for the entire bottom within a $200 \mathrm{~km}$ range from the source.

Table 4-1: Bottom properties for the PE simulation

\begin{tabular}{|c|c|c|c|c|}
\hline & $\begin{array}{c}\text { Compressional } \\
\text { Speed(m/s) }\end{array}$ & $\begin{array}{c}\text { Shear Speed } \\
(\mathrm{m} / \mathrm{s})\end{array}$ & Density $\left(\rho_{b}\right)$ & Attenuation $\left(\alpha_{p}\right)^{2}$ \\
\hline Porous Bassalt & 2200 & 1100 & $2.1 \rho_{w}$ & \multirow{2}{*}{$0.1 \mathrm{~dB} / \lambda$} \\
\cline { 1 - 3 } Loose Sediment & 1550 & 200 & $1.7 \rho_{w}$ & \\
\hline
\end{tabular}

${ }^{1}$ In this simulation, shear effect was not included.

${ }^{2}$ The bottom was considered as homogenous, while an absorbing layer with high attenuation $(10 \mathrm{~dB} / \lambda)$ was located below $7000 \mathrm{~m}$ of depth. The high attenuation value was used to reduce bottom reflected waves for $0 \sim 200 \mathrm{~km}$ range.

\subsection{Ray tracing method}

The RAY code, developed by Bowlin et al. [40], was used in ray tracing simulation. The environmental inputs, bathymetry and sound speeds, were the same as those used in the 2D PE simulation, but sound speeds from the XBT casts were smoothed well to obtain continuous sound speed fields with a bounded second derivative. Without well-smoothed sound speeds, the intrinsic smoothing function of the RAY code can lead to the incorrect ray paths.

The initial launch angles were defined by -30 to +30 degrees inclinations relative to the horizon with 0.005 degree increments, and all rays that experience five or more surface and/or bottom reflections before the seamounts are excluded from the results.

\subsection{Behind the Elvis seamount: day 268 with XBT sound speeds}

Here comparisons between the two-dimensional (2D) simulation and the experimental data for Julian day 268 (hereafter day 268) are presented. The acoustic data were gathered 
above and behind the Elvis seamount for the SPICEX 2 source. Fig. 4-1 shows an acoustic path that passes from the source over the peak of the Elvis seamount, and bathymetry and sound speed profiles along the acoustic path are given in Fig. 4-3. The sound speeds are obtained from the XBT cast measurements with the nearest neighbor rule. In Fig. 4-4, the range-averaged sound speed profile (left panel) and variability from the range-averaged sound speed profile are given. Note that there are locally isolated regions with an abrupt change of sound speeds.

Figure 4-2 gives the receiver locations obtained from GPS as well as the reference acoustic path over the apex of Elvis seamount. The location is chosen at the time when the first significant pulse of the SPICEX 2 source appears in the measurement.

Table 4-2 shows the arrival time difference between the 2D PE simulation and measurement. Compared maximum signal level in $\mathrm{dB}$ is also given in the table. There were 13 measurements in the shadow and the convergence zones along the acoustic path from the SPICEX 2 source to the peak of the Elvis seamount.

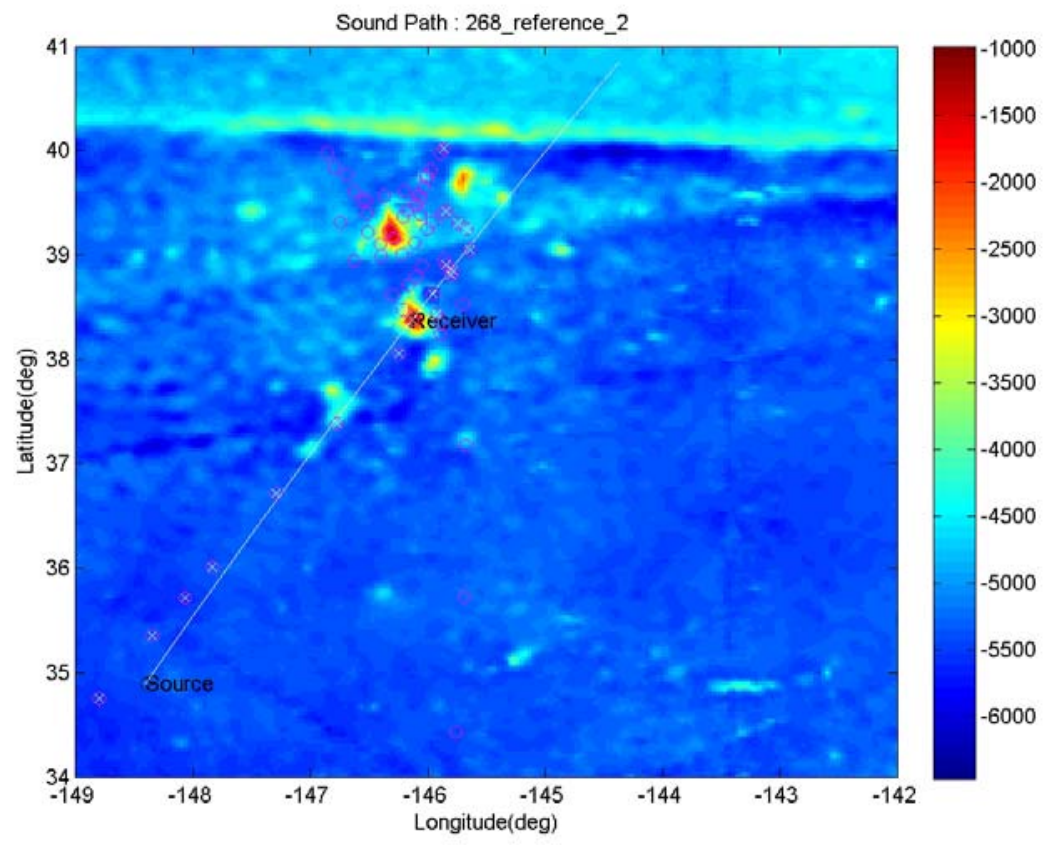

Figure 4-1: Acoustic path (geodesic) that passes over the peak of Elvis seamount. The depth of the peak of Elvis seamount is $1369 \mathrm{~m}$ from the sea surface. 


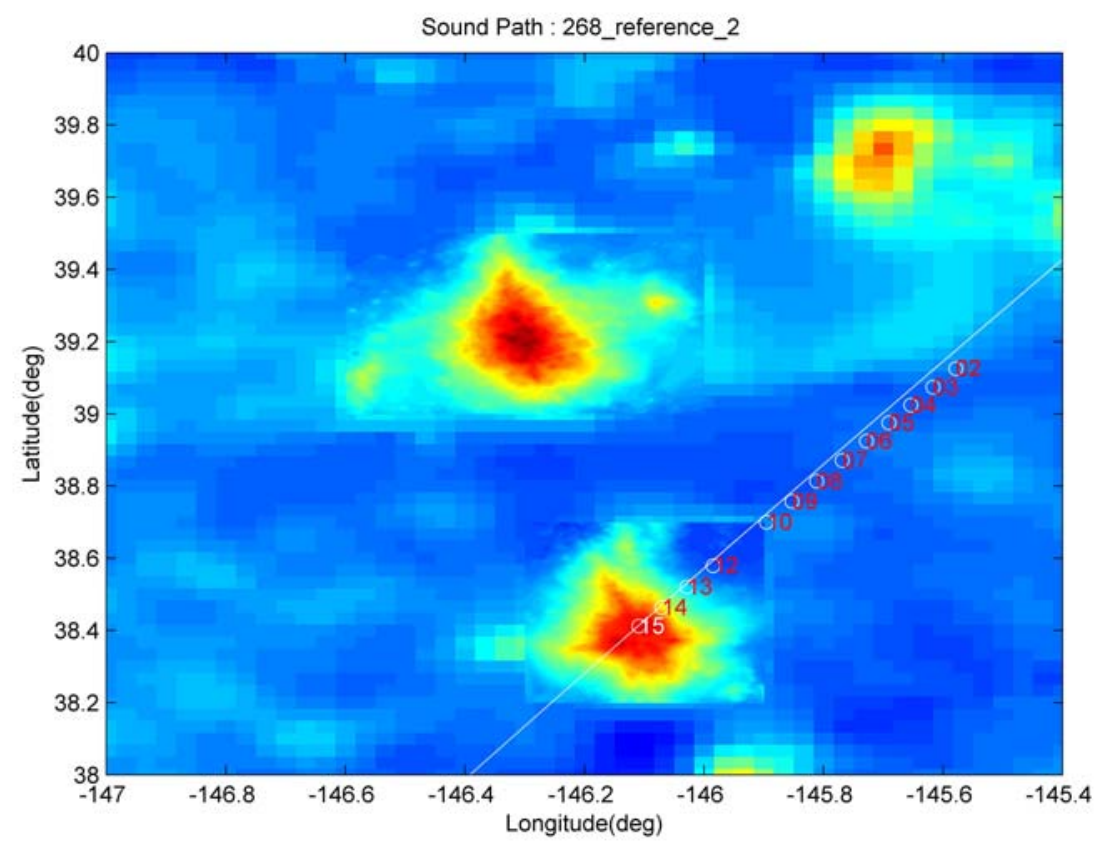

Figure 4-2: Receiver locations for the reference acoustic path that passes over the peak of Elvis seamount; this acoustic path is designed to pass over the apex of Elvis seamount. 

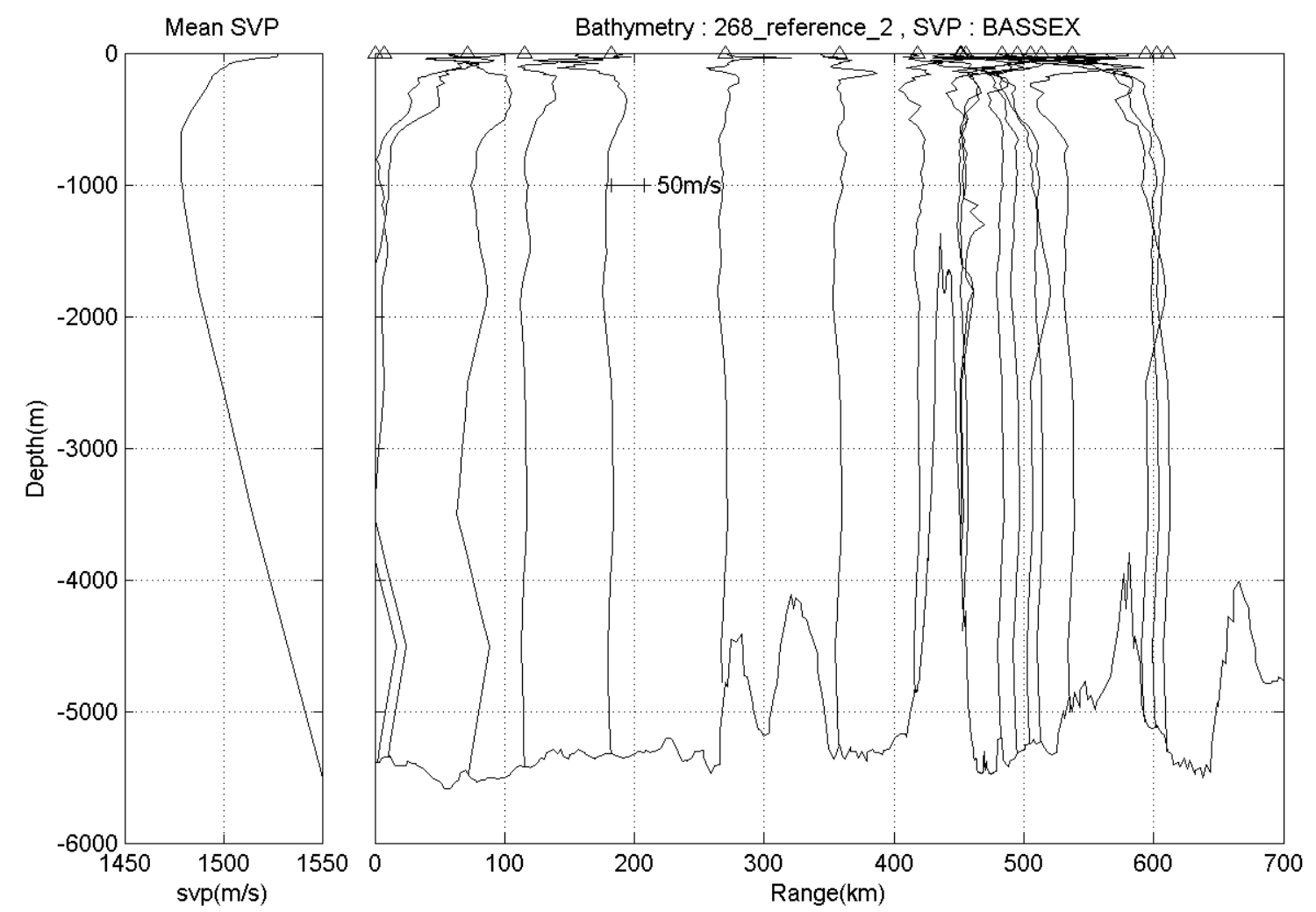

Figure 4-3: Bathymetry and sound speed profiles along the acoustic path. The sound speed profiles are obtained from the XBT casts in the BASSEX experiment, and the XBT cast locations nearest the acoustic path were used. The sound speed profiles are linearly interpolated in ranges along the acoustic path to reduce the abrupt change of sound speed in the simulations. In the left panel, the range-averaged sound speed profile is shown, and the right panel shows the perturbed sound speed profiles along the acoustic path. 


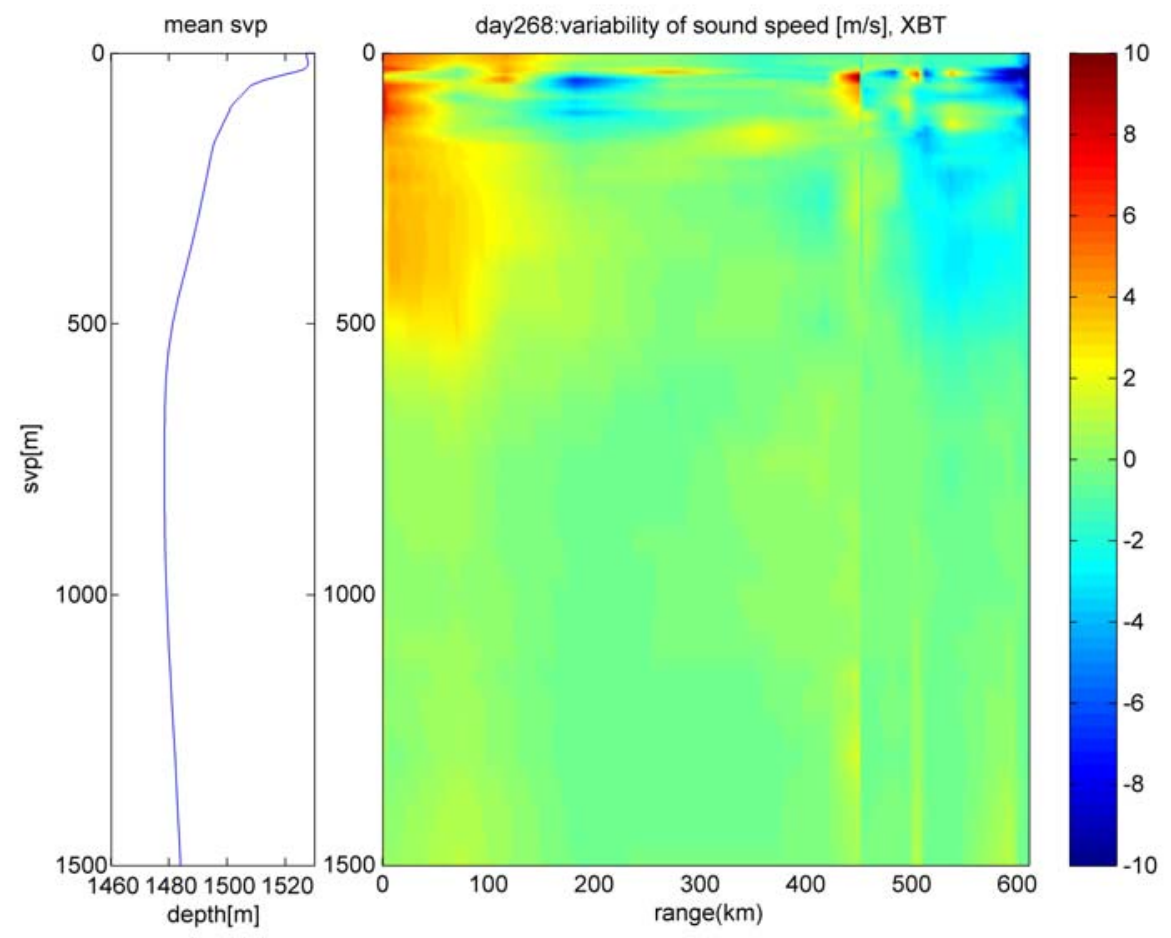

Figure 4-4: Sound speeds derived from XBT casts of BASSEX experiment along the acoustic path (Figure 4-1); the left panel shows range-averaged sound speed profile, and the right panel shows the difference from the range-averaged sound speed. 
Table 4-2: Receiver locations and simulation results for day 268

\begin{tabular}{|c|c|c|c|c|c|c|c|c|}
\hline No. & Exp. & Range $(\mathrm{km})^{1}$ & $\operatorname{Depth}(\mathrm{m})^{2}$ & $\begin{array}{l}\text { Arrival } \\
\text { time error }\end{array}$ & \multicolumn{3}{|c|}{$\begin{array}{l}\text { Maximum peak } \\
\text { amplitude }(\mathrm{dB})^{4}\end{array}$} & $\begin{array}{l}\text { Characteristics } \\
\text { of significant }\end{array}$ \\
\hline 1 & 26802 & 533.217 & 296.5 & -0.016 & $86.9^{\mathrm{A}}$ & $90.45^{\mathrm{B}}$ & $3.56^{\mathrm{C}}$ & Refracted \\
\hline 2 & 26803 & 526.664 & 275.8 & - & \multicolumn{3}{|c|}{ - } & No signal \\
\hline 3 & 26804 & 520.245 & 287.2 & -0.224 & 89.8 & 97.7 & 7.9 & Refracted \\
\hline 4 & 26805 & 513.952 & 285.2 & -0.133 & 84.9 & 92.2 & 7.3 & Refracted \\
\hline 5 & 26806 & 507.448 & 280.4 & -0.690 & 77.8 & 89.7 & 11.8 & Shadow zone \\
\hline 6 & 26807 & 500.512 & 256.1 & -0.180 & 87.0 & 67.9 & -19.1 & $\begin{array}{l}\text { Diffracted/ } \\
\text { Reflected }\end{array}$ \\
\hline 7 & 26808 & 493.152 & 251.2 & -0.261 & 83.0 & 92.7 & 9.7 & Refracted \\
\hline 8 & 26809 & 486.001 & 260.0 & -0.069 & 89.7 & 83.2 & -6.5 & Refracted \\
\hline 9 & 26810 & 478.590 & 238.3 & -0.113 & 87.1 & 61.7 & -25.5 & Boundary \\
\hline 10 & 26812 & 463.169 & 238.8 & 0.000 & 70.0 & 63.9 & -6.0 & Shadow zone \\
\hline 11 & 26813 & 455.524 & 246.6 & -0.372 & 84.8 & 83.0 & -1.8 & $\begin{array}{l}\text { Diffracted/ } \\
\text { Reflected }\end{array}$ \\
\hline 12 & 26814 & 448.268 & 272.3 & -0.018 & 79.5 & 87.3 & 7.8 & Boundary \\
\hline 13 & 26815 & 441.685 & 296.2 & -0.115 & 90.2 & 96.6 & 6.4 & Refracted \\
\hline
\end{tabular}

${ }^{1}$ The range from the SPICEX 2 source that is obtained from the GPS location when the first strong pulse arrives.

${ }^{2}$ Averaged depth.

${ }^{3}$ Arrival time of simulation minus arrival time of experiment for the reference peaks; (-) means pulses from experiment arrive later than those from simulation.

${ }^{\mathrm{A}}$ Peak amplitude of experiment in $\mathrm{dB}$ re $1 \mu \mathrm{Pa}$,

${ }^{\mathrm{B}}$ Peak amplitude of Simulation in $\mathrm{dB}$ re $1 \mu \mathrm{Pa}$,

${ }^{\mathrm{C}}$ Peak amplitude of experiment minus Peak amplitude of simulation in $\mathrm{dB}$ re $1 \mu \mathrm{Pa}$; experimental pulses are obtained by averaging over all 11 periods and grazing angles after using conventional beamformer [53]. 
The range-stacked pulse arrivals from the 2D PE simulation at the depth of $270 \mathrm{~m}$ are shown in Fig. 4-5. First, clear shadow and convergence zones can be found behind the seamount. The convergence zones consist of the refracted ray arrivals which pass over the seamount without any bottom interaction; the shadow zones are located between the convergence zones. The shadow and convergence zones are spaced approximately 50 $60 \mathrm{~km}$ apart. At the boundary of the convergence zone before the shadow zone, diffused reflected rays appear in the same pattern as the refracted rays; however, the rays arrive later than the refracted rays, which prove the rays are bottom reflected.

The appearance of shadow zones by the blockage of the refracted rays is quite clearly shown by the compared simulations with and without the seamount in Fig. 4-6. In the latter case, the seamount was removed and replaced by a straight line.

Figure 4-7 presents the comparison of arrival pulses from experiment and the 2D PE simulation. Amplitudes in each panel for the experiment and simulation are normalized by the maximum amplitude of the experiment and simulation, respectively. The comparison shows good correlation between the measurement and the PE simulation, in particular, within the convergence zones in which the refracted rays appear. However, the significant difference can be found at transition regions, i.e. the boundaries of the shadow zone. In the figure, $\mathrm{B}$ and $\mathrm{C}$ sit on the transition regions; the PE simulation shows a much higher signal level than the measurement. This implies that the span of the shadow zone might be underestimated in the simulation; i.e. the real size of the shadow zone is larger than that from the simulation. Another significant difference can be found at $\mathrm{A}$, in which data were gathered at the top of the Elvis seamount. The early arrival refracted waves were not measured in the experiment, but in the simulations they were clearly shown.

Comparison results of arrival patterns between the PE simulation, ray tracing method, and experiment in terms of arrival angle and time are shown in Fig. 4-8 4-31. Due to the uncertainty associated with the timing of FORA array by up to 0.25 seconds, it is difficult to match individual rays exactly with arrival time. The estimated errors of arrival time between the experiment and simulations are given in Table 4-2, obtained 
from considering the pattern of significant pulse arrivals. The random timing error of FORA array provides serious difficulty in analyzing the arrival time error from the uncertainty of sound speed fields.

In many cases, arrival patterns show good correlation between the results, as in 26802 (Fig. 4-8), 26804 (Fig. 4-10), 26805 (Fig. 4-12), 26808 (Fig. 4-18), 26809 (Fig. 420), 26810 (Fig. 4-21). In particular, the refracted rays from the BASSEX experiment can be reconciled with the 2D PE simulation very well, as in the above cases; however, it is difficult to match the reflected rays in the experiment, 2D PE, and ray tracing model because of the uncertainties from the geoacoustic properties. At least, we could claim that overall arrival patterns, however, seem quite close each other.

The simulation results from the ray tracing model and the 2D PE show good correlation in terms of arrival time and grazing angle. However, in some cases, since the ray tracing model is much more sensitive to the local bottom slope and sound speed fields, as in 26805, the ray tracing model can not detect a strong arrival refracted ray which is shown both in the experiment and 2D PE simulation.

Here we have compared the pulse arrivals from the BASSEX experiment with those from the 2D models, 2D PE, and ray tracing method. From now on, we discuss the main causes of the discrepancies in the comparison.

All discrepancies between the experiment and the 2D models could be explained by 1) the uncertainty in the sound speed fields, 2) geoacoustic properties, 3) diffused reflection, and 4) the 3D sound propagation effect.

In this simulation, the sound speed profiles are constructed based on the XBT casts performed in BASSEX experiment. However, we could expect fluctuations in the sound speed profiles that result in the changing of the turning depth and arrival angle of the individual ray. Whether an individual ray can pass over the seamount or can be blocked by the seamount is highly dependent on the sound speed fields along an acoustic path from source to the seamount as well as the local bathymetry of the seamount because the early arrivals or deep-down rays sample entire sound speeds in depth. Therefore, a refracted ray which passes over the seamount can be blocked by the seamount with a 
small change of sound speed fields.

If the reflection occurs on an upsloping bottom, the ray will be steepened by an angle twice that of the slope and may be converted to a bottom reflected ray. Then the signal will be attenuated rapidly by absorption and scattering at the bottom. Therefore, arrivals can be missing, or can be diffused and weakened. The uncertainty in the geoacoustic properties of the seabottom associated with the reflections might be another issue that explains the differences in the reflected waves between measurements and simulations. Moreover, the real sea-bottom is not as smooth as we assumed in the sound propagation models; the diffuse reflection from the roughness might be another source of difference.

In real measurements, the 3D horizontal refraction effect might be introduced; reflections from the sloped bottoms produce refracted arrivals diverting from the acoustic path with an angle twice the local slope of the bathymetry such as the 2D upslope sound propagation. This horizontal refraction was discussed in Section 2.4.

One more effect to increase the difficulty in identification of reflected rays might be the use of a towed horizontal array; quite diffused rays by reflection can be smoothed out by the range-averaging effect of the horizontal array. 


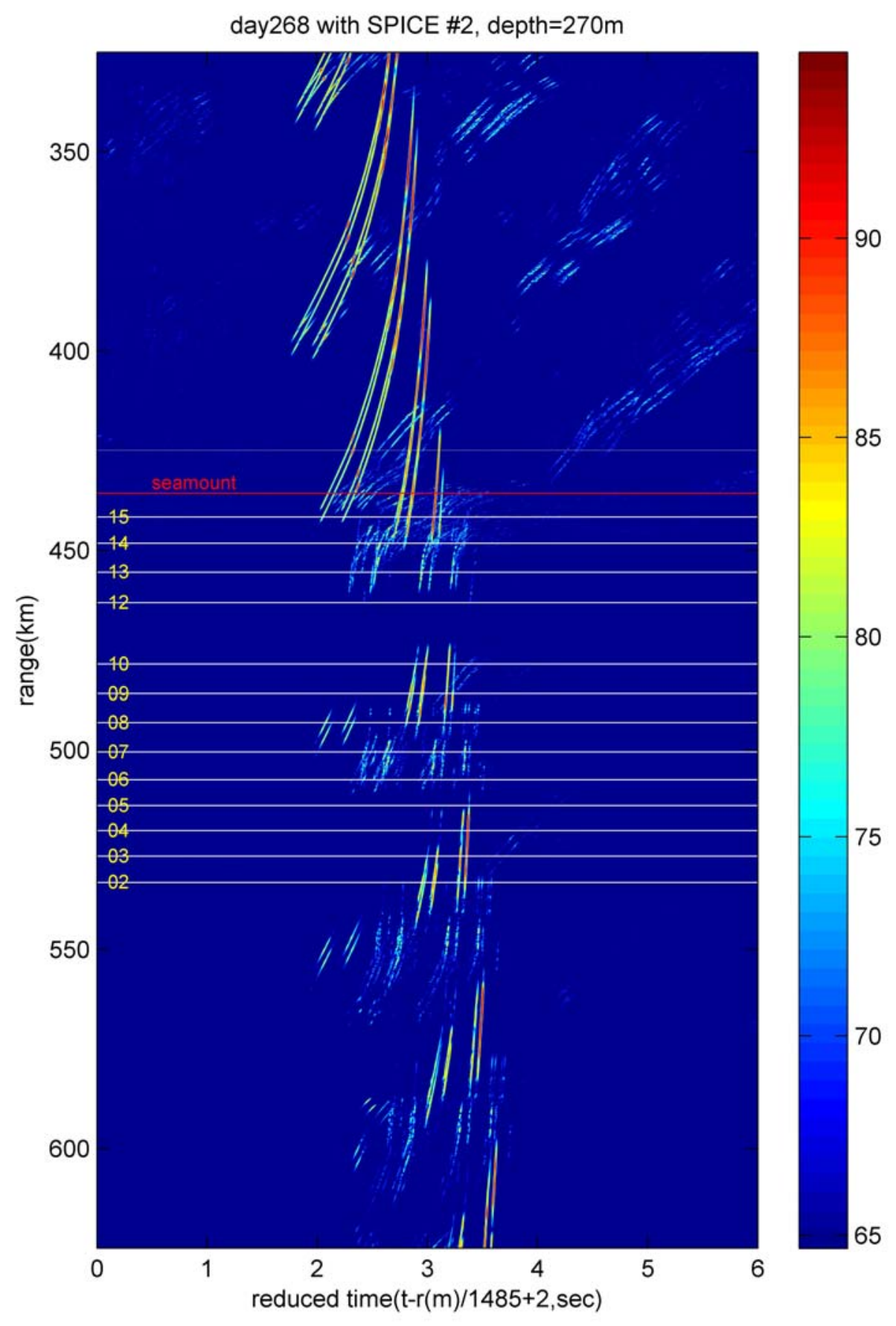

Figure 4-5: Range-stacked arrival pulses for the range of $325 \sim 625 \mathrm{~km}$. Although receiver depths vary in the range of $240 \sim 300 \mathrm{~m}$, the pulse arrivals at the depth $270 \mathrm{~m}$ are given in this figure. 

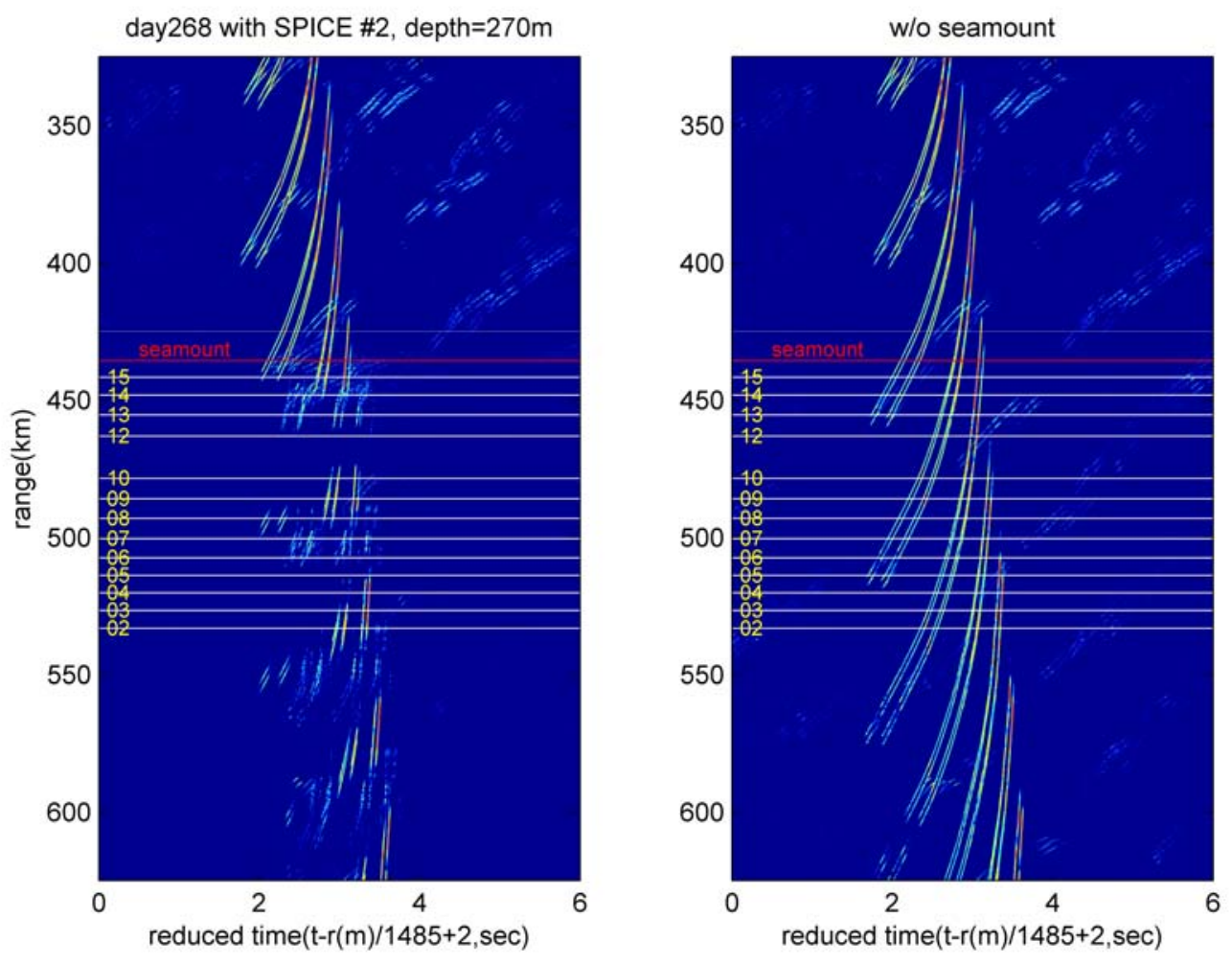

Figure 4-6: Compared range-stacked arrival pulses for the range of $325 \sim 625 \mathrm{~km}$ with (left panel) and without (right panel) seamount. 

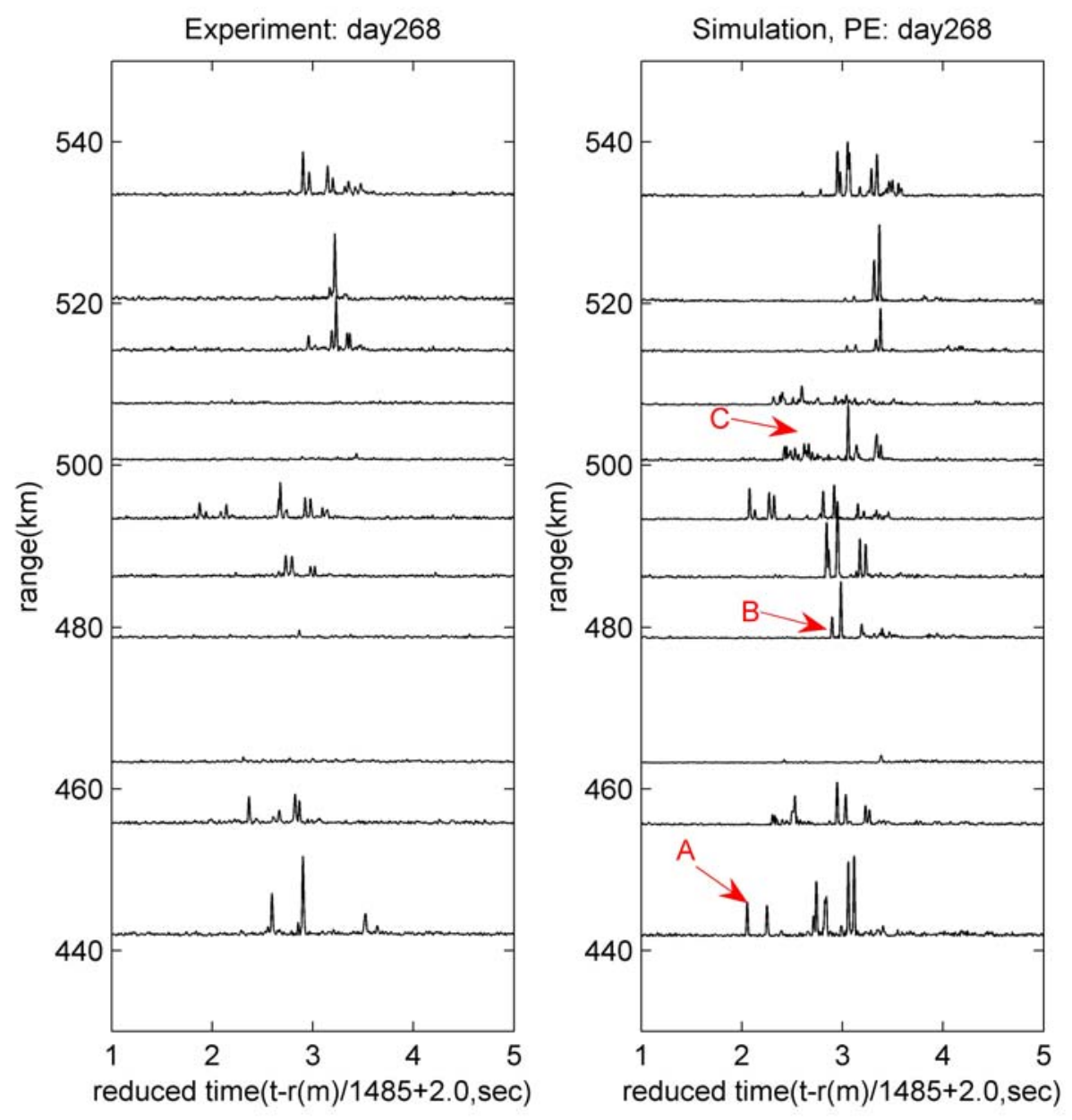

Figure 4-7: Comparison of arrival pulses from experiment and PE simulation for day 268. For each panel, the amplitudes of pulses are normalized by the maximum values of all pulses. $\mathrm{B}$ and $\mathrm{C}$ are located in the transition region between the refracted wave arrival zone (convergence zone) and shadow zone. This implies that the size of the shadow zone might be underestimated by the PE simulation. Another significant discrepancy can be found at A, which is located above the Elvis seamount. As shown in this figure, the early arrival refracted waves in the PE simulation were not measured in the experiment. 


\subsubsection{2}
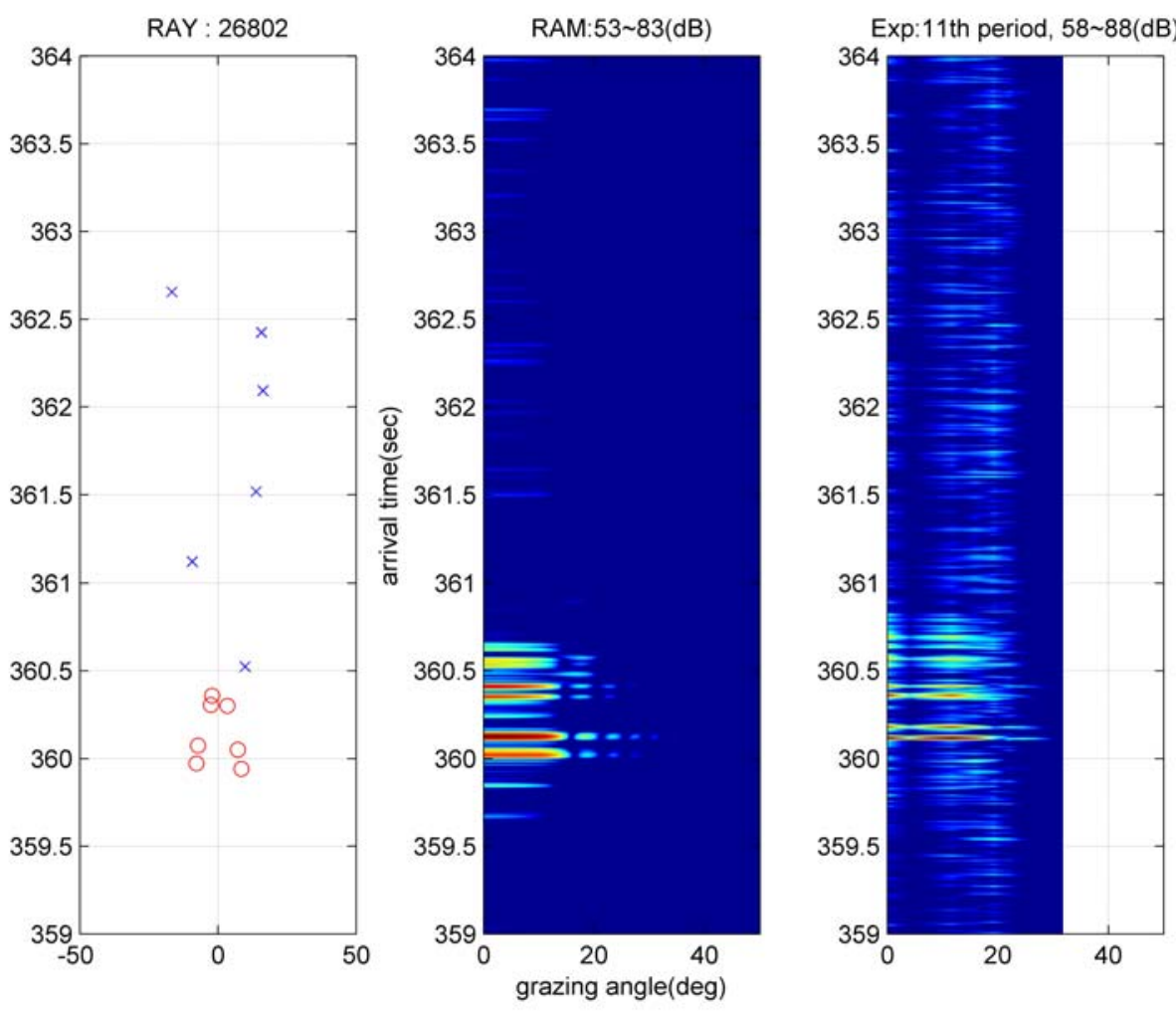

Figure 4-8: Comparison of pulse arrivals by the ray tracing method, 2D PE, and the BASSEX experiment for 26802. Ray arrivals are shown in the left panel; only refracted rays are marked with the circles, and rays that reflect at both the bottom and the surface are marked with the crosses. Results from the PE simulation and experiment are shown in the middle and the right panel with $30 \mathrm{~dB}$ dynamic range; an MPDR beamformer is used for the experiment to obtain higher angular resolution, but a conventional beamformer is used for PE simulation. Grazing angle is defined with respect to horizontal plane. The last of 11 periods of the measurement is shown in the right panel, which has the maximum amplitude. Pulse arrivals from the experiments are shifted in time by -0.016 seconds (Table 4-2), to be aligned with the other results. The significant ray arrivals are quite close to each other. The refracted rays that are shifted from higher to lower angles are followed by some lower angle ray arrivals. These low angle arrivals might be reflected-refracted rays because of the lower arrival angle and faster arrival than reflected-reflected rays. The reflectedreflected rays that correspond to higher grazing angles can be found in ray tracing and PE simulation, but no clear reception of these reflected-reflected rays was shown in the experiment. The poor reception of the reflected-reflected rays is common in all cases of day 268; this might be due to the diffuse reflection and the fact that reflection from sloping bottoms produces the horizontal refraction that diverts arrivals from the receiver. In addition, it is difficult to reconcile the reflected rays without measured geoacoustic properties. 

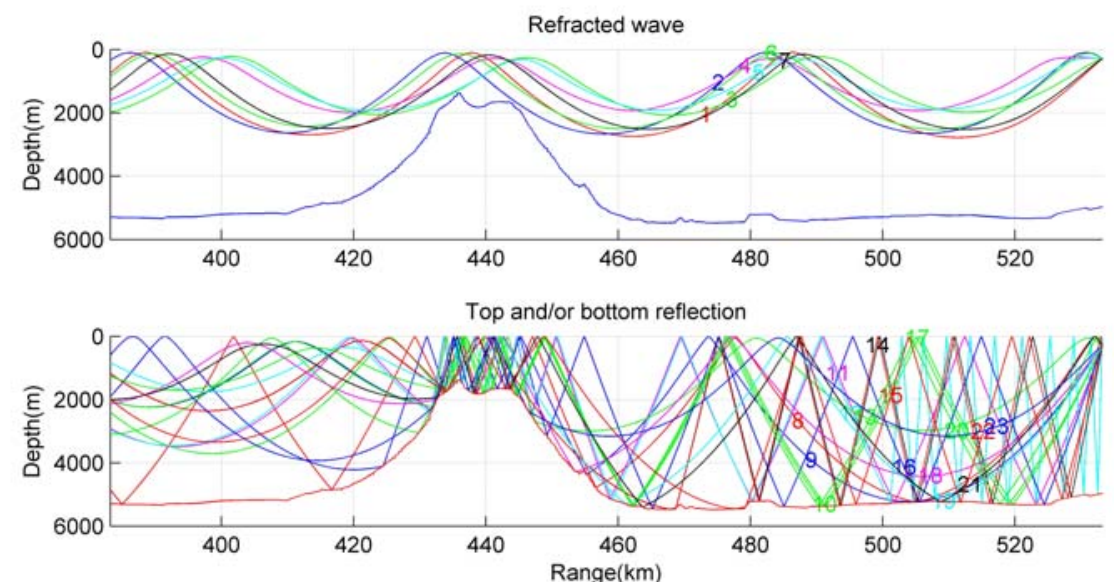

\begin{tabular}{|c|c|c|c|}
\hline \multicolumn{3}{|c|}{ ( km,296.49 m) } & $16: 35-S R B$ \\
\hline $\begin{array}{l}1: 21-R \\
2: 22-R\end{array}$ & 8:30-SRBR & 15:32-SRBR & 17:33-SRBR \\
\hline $\begin{array}{l}2: 22-R \\
3: 23-R\end{array}$ & 9:25-SRBR & 16:35-SRBR & 18:25-SRBR \\
\hline $4: 24-R$ & $\begin{array}{l}\text { 10:27-SRBR } \\
11: 35-\text { SRBR }\end{array}$ & $\begin{array}{l}\text { 17:33-SRBR } \\
18: 25-S R B R\end{array}$ & 19:27-SRBR \\
\hline $5: 25+R$ & 12:52-SRBR & $19: 27$-SRBR & 20:32-SRBF \\
\hline $6: 23+R$ & 13:24-SRBR & $20: 32$-SRBR & 21:29+SRB \\
\hline $7: 22+R$ & 14:31-SRBR & $21: 29+$ SRBR & 28+SRB \\
\hline
\end{tabular}

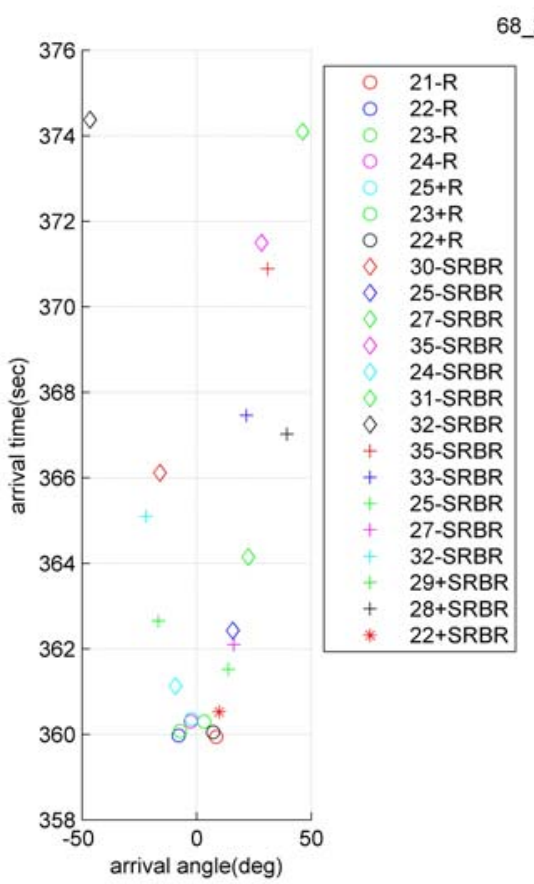

68_2A1.mat :Eigenrays at the receiver:(533.217 km,296.49 m)

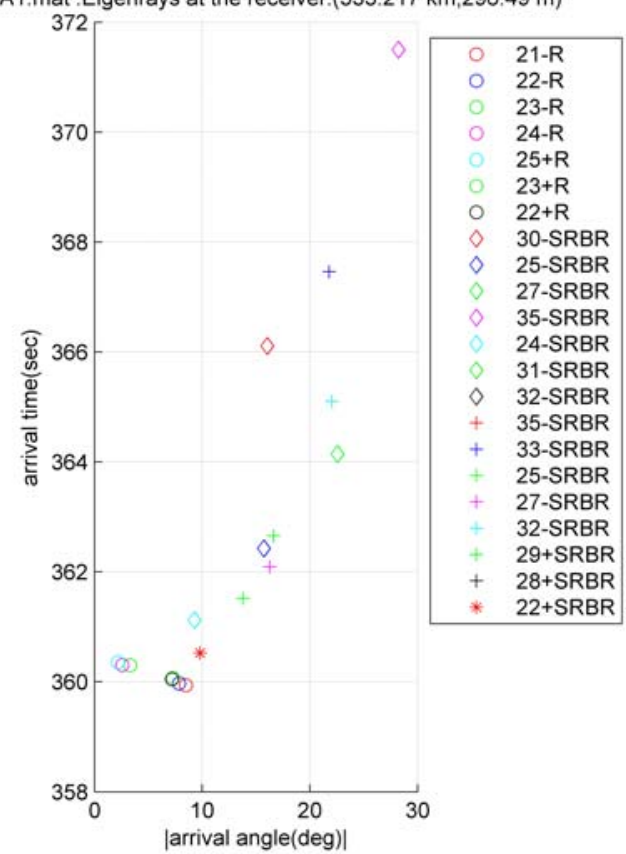

Figure 4-9: Ray arrivals from ray tracing method for 26802. Ray arrivals are grouped into 4 groups: only refracted wave (R) without bottom interactions, surface-reflected-bottom-reflected wave (SRBR), only surface-reflected wave (SR), and only bottom reflected wave (BR). (+) and (-) signs denote up and down rays, respectively. The number before the sign means the total number of turns. 


\subsubsection{4}
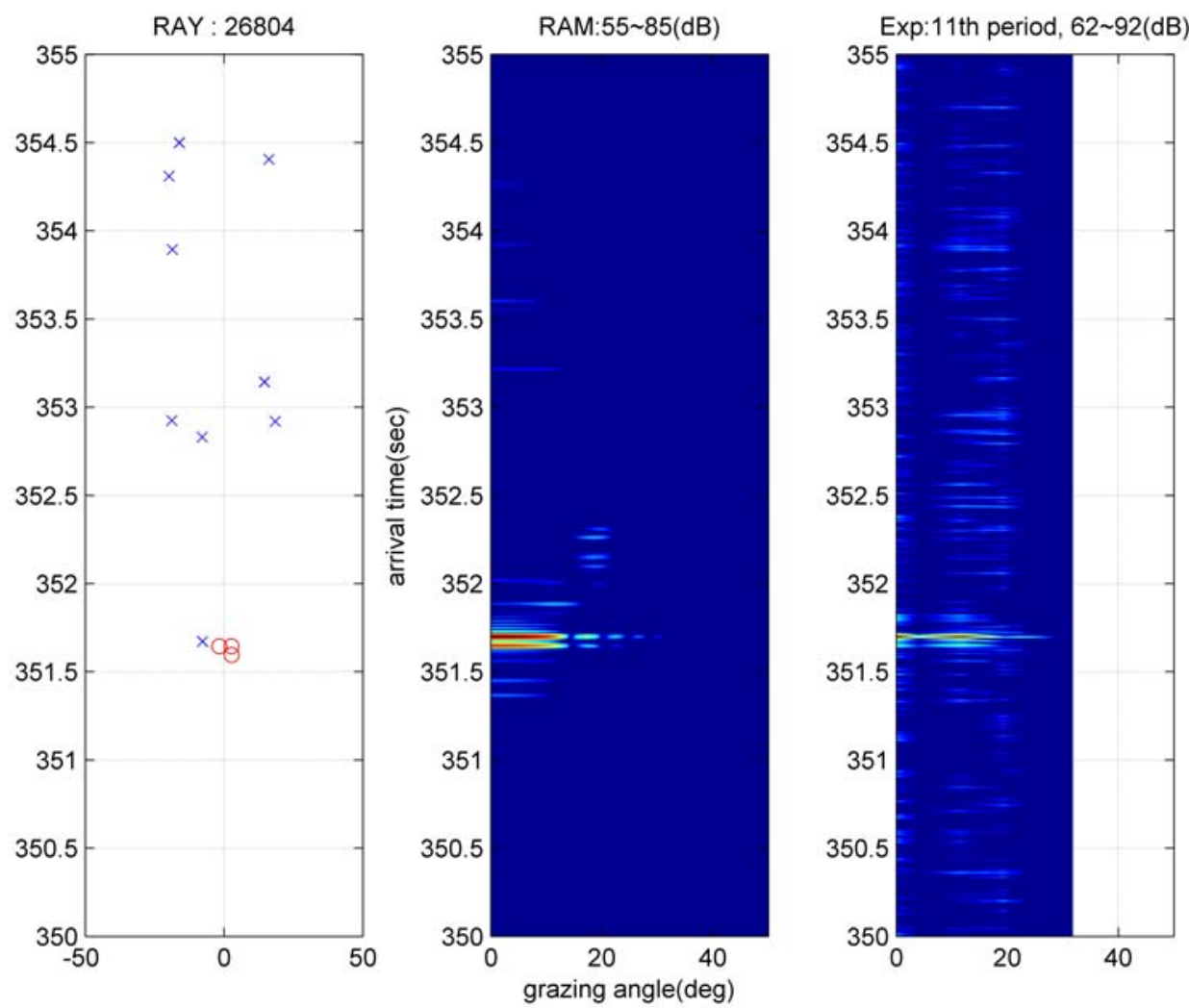

Figure 4-10: Comparison of pulse arrivals by the ray tracing method, 2D PE, and the BASSEX experiment for 26804. Ray arrivals are shown in the left panel; only refracted rays are marked with the circles, and rays that reflect at both the bottom and the surface are marked with crosses. Results from the 2D PE simulation and experiment are shown in the middle and the rightmost panel, respectively, with $30 \mathrm{~dB}$ dynamic range; an MPDR beamformer is used for the experiment to obtain higher angular resolution, but the conventional beamformer is used for the $\mathrm{PE}$ simulation. Grazing angle is defined with respect to the horizontal plane. The last of 11 periods is shown at the right panel, which has the maximum amplitude. Pulse arrivals from experiment are shifted in time by -0.224 seconds (Table $4-2$ ), to be aligned with the other results. At this receiver location, the refracted waves are almost blocked by the seamount except for a pair of refracted ones, but one wave is far stronger than the other in terms of intensity. This is consistent with the ray arrivals from the ray tracing method, as shown in the left panel. Higher angle and late arrival is not clearly shown in the experiment (rightmost panel), which is shown in the ray tracing method (leftmost panel). 

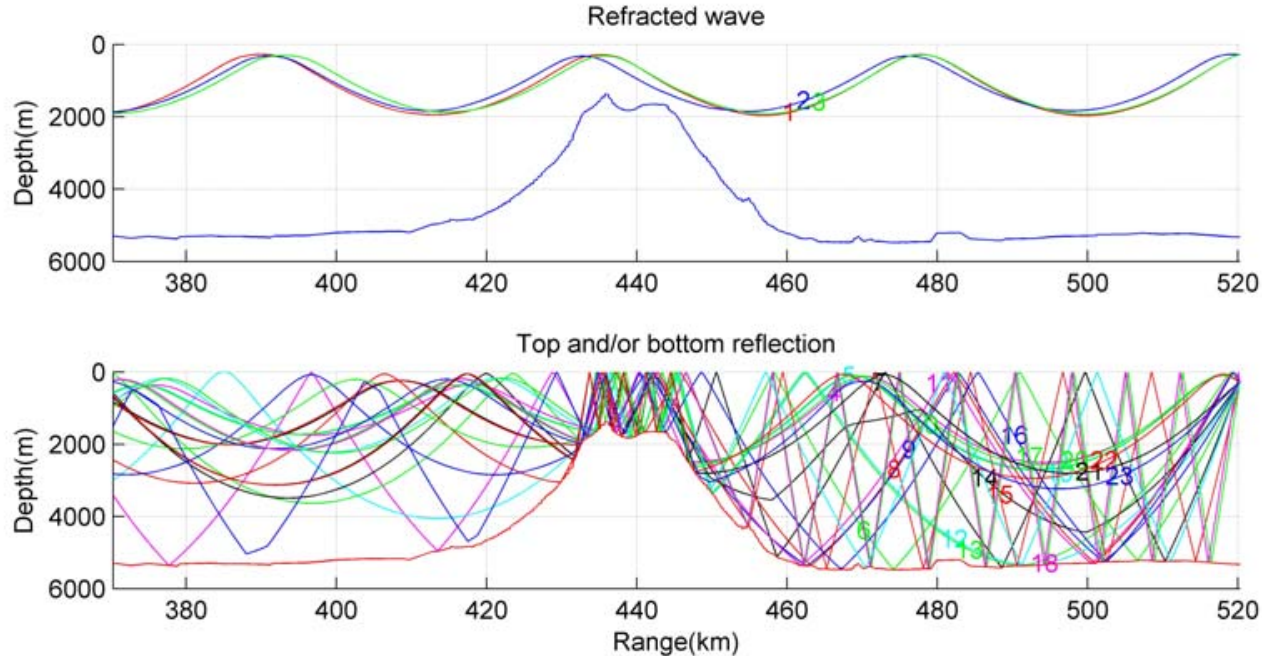

68 4A1. mat :Eigenrays at the receiver:(520.245 km,287.22 m)

$\begin{array}{lccc}1: 23-R & 8: 24-S R B R & 15: 34+\text { SRBR } & 16: 29+\text { SRBR } \\ 2: 25+R & 9: 23-\text { SRBR } & 16: 29+\text { SRBR } & 17: 42+\text { SRBR } \\ 3: 24+R & 10: 26-\text { SRBR } & 17: 42+\text { SRBR } & 18: 43+\text { SRBR } \\ 4: 28-S R B R & 11: 28-S R B R & 18: 43+\text { SRBR } & 19: 32+\text { SRBR } \\ 5: 22-S R B R & 12: 29-S R B R & 19: 32+\text { SRBR } & 20: 31+\text { SRBR } \\ 6: 29-S R B R & 13: 30-S R B R & 20: 31+\text { SRBR } & 21: 30+\text { SRBR } \\ 7: 33-S R B R & 14: 26+\text { SRBR } & 21: 30+\text { SRBR } & 22: 31+\text { SRBR } \\ & & & 23: 28+\text { SRBR }\end{array}$

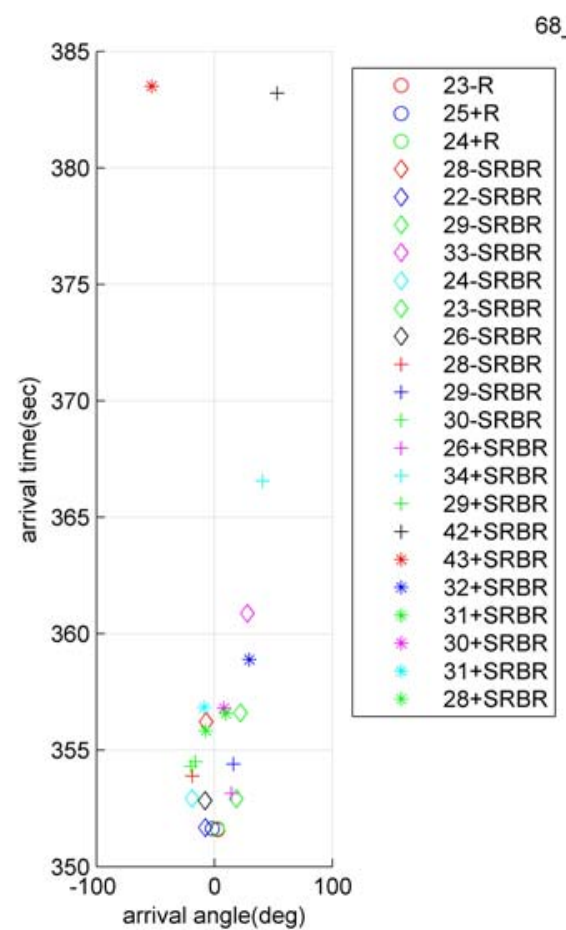

68 4A1.mat :Eigenrays at the receiver:(520.245 km,287.22 m)

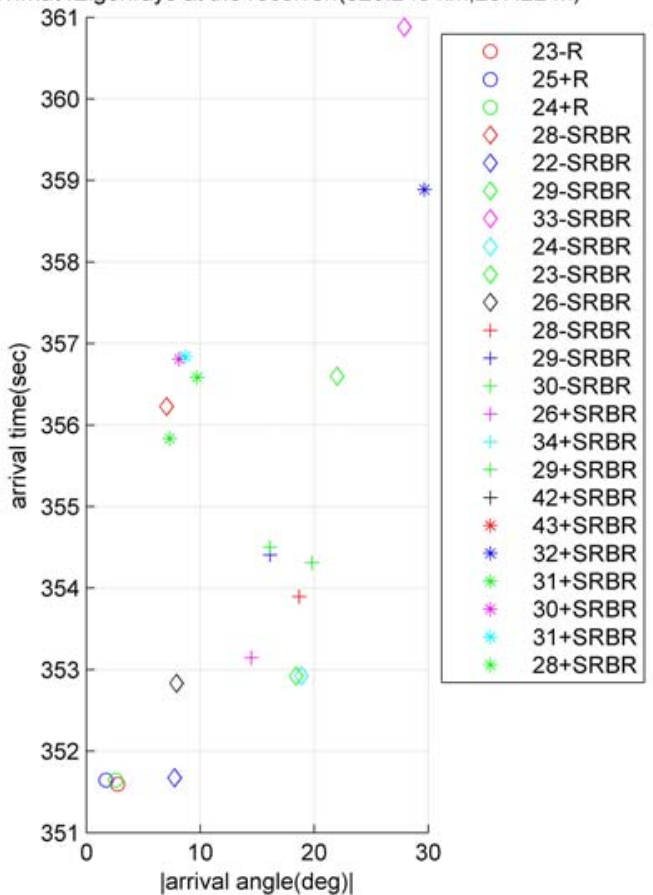

Figure 4-11: Ray arrivals from ray tracing method for 26804. Figure 4-9 provides the definition of rays. 


\subsubsection{5}
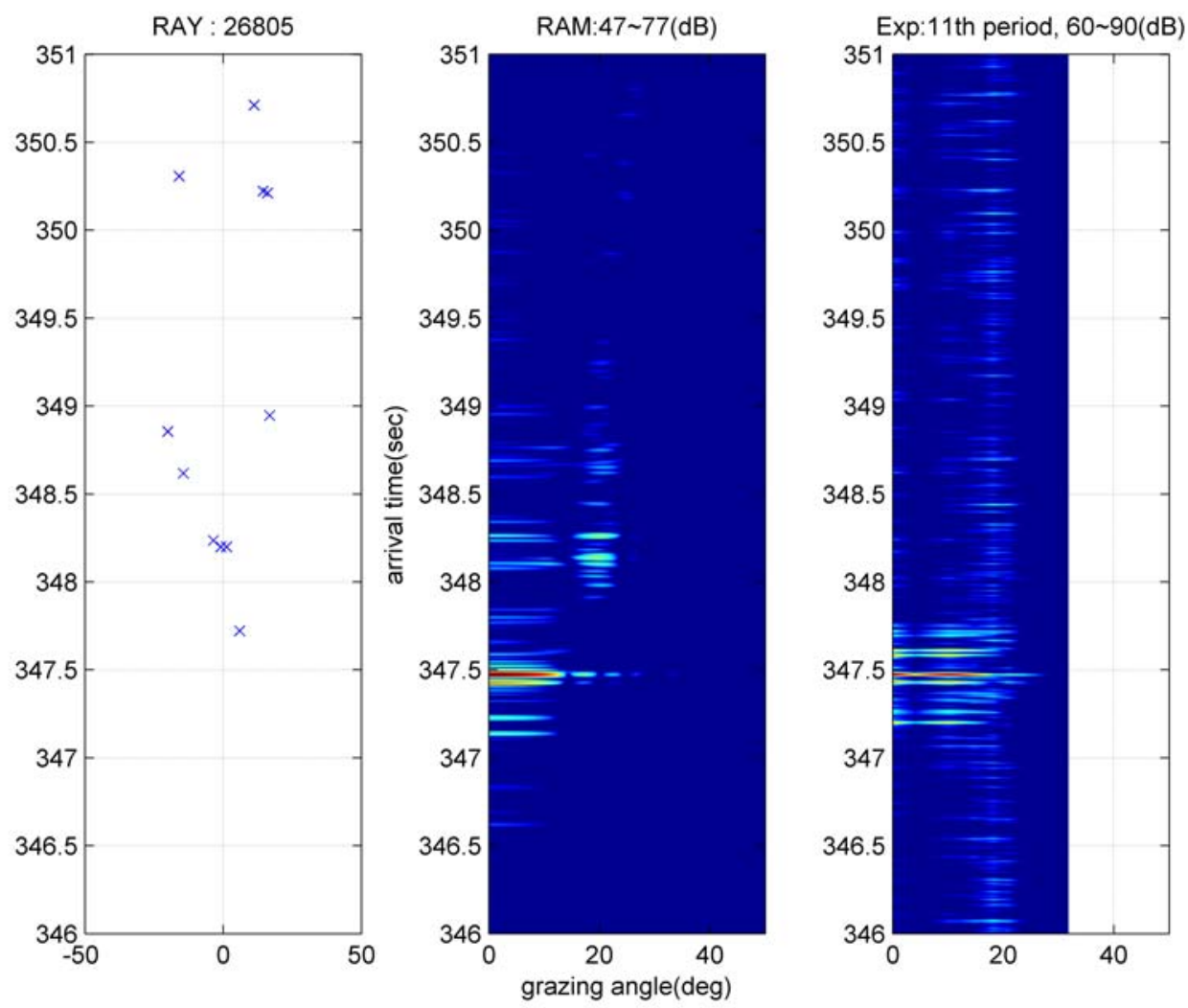

Figure 4-12: Comparison of pulse arrivals by the ray tracing method, 2D PE, and the BASSEX experiment for 26805. Pulse arrivals from the experiment are shifted in time by -0.133 seconds (Table 4-2), to be aligned with the other results. As indicated in Fig. 4-3, at this receiver location, a receiver is sitting at the boundary between shadow zone and refracted wave arrivals. A refracted ray arrival is shown in both the PE simulation and experiment, but ray tracing method does not detect any refracted ray. 

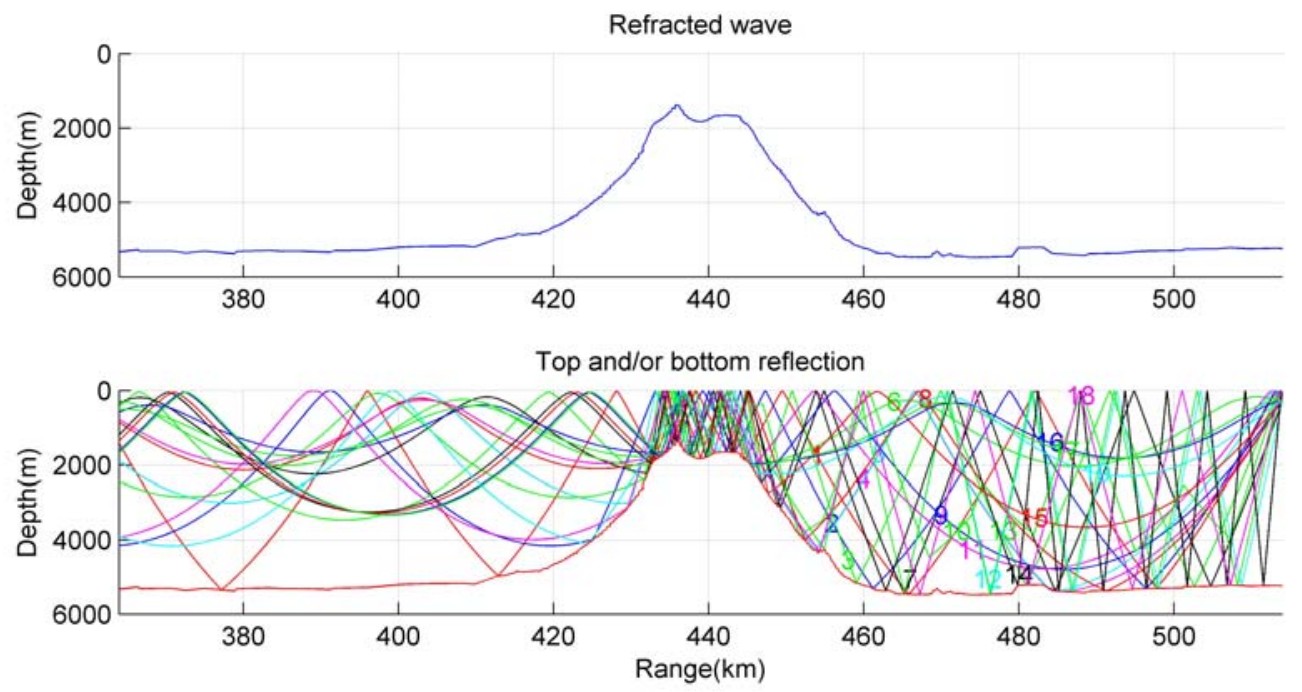

68_5A1.mat :Eigenrays at the receiver:(513.952 kmm285+3RPR

1:30-SRBR

8:25-SRBR

12:34+SRBR

2:24-SRBR

3:34-SRBR

9:29-SRBR

13:35+SRBR

4:25-SRBR

11.35+SRBR

14:43+SRBR

5:26-SRBR

12:34+SRBR

$15: 28+$ SRBR

6:24-SRBR

13:35+SRBR

16:24+SRBR

7:33-SRBR

14:43+SRBR

18.29+SRBR

19:22+SRBR

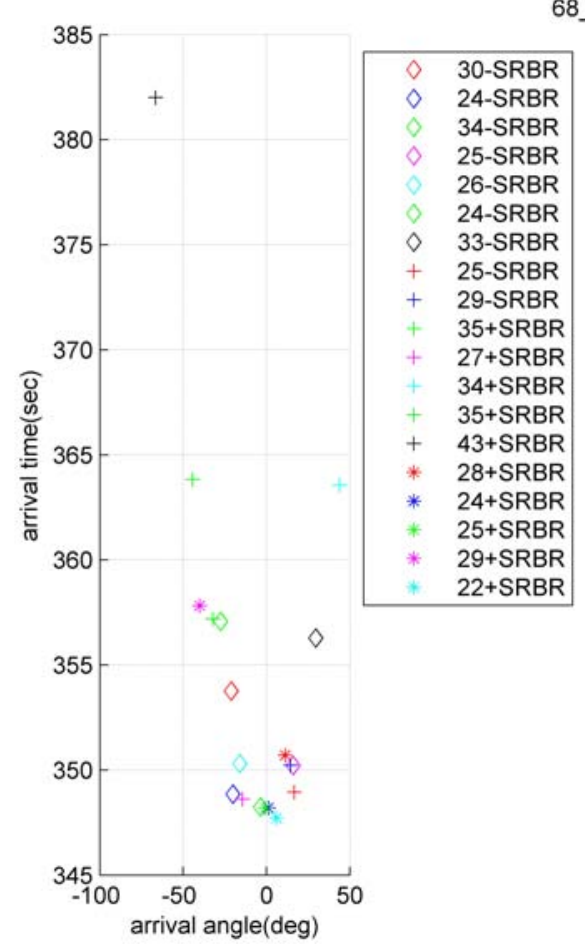

68_5A1.mat :Eigenrays at the receiver:(513.952 km,285.24 m)

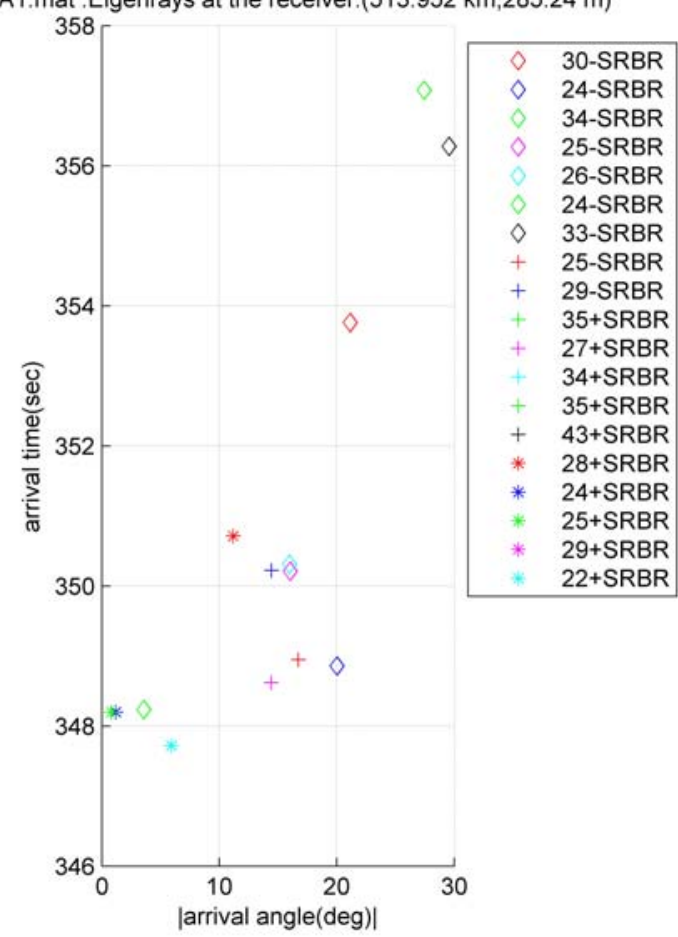

Figure 4-13: Ray arrivals from ray tracing method. Figure 4-9 provides the definition of rays. 


\subsubsection{6}
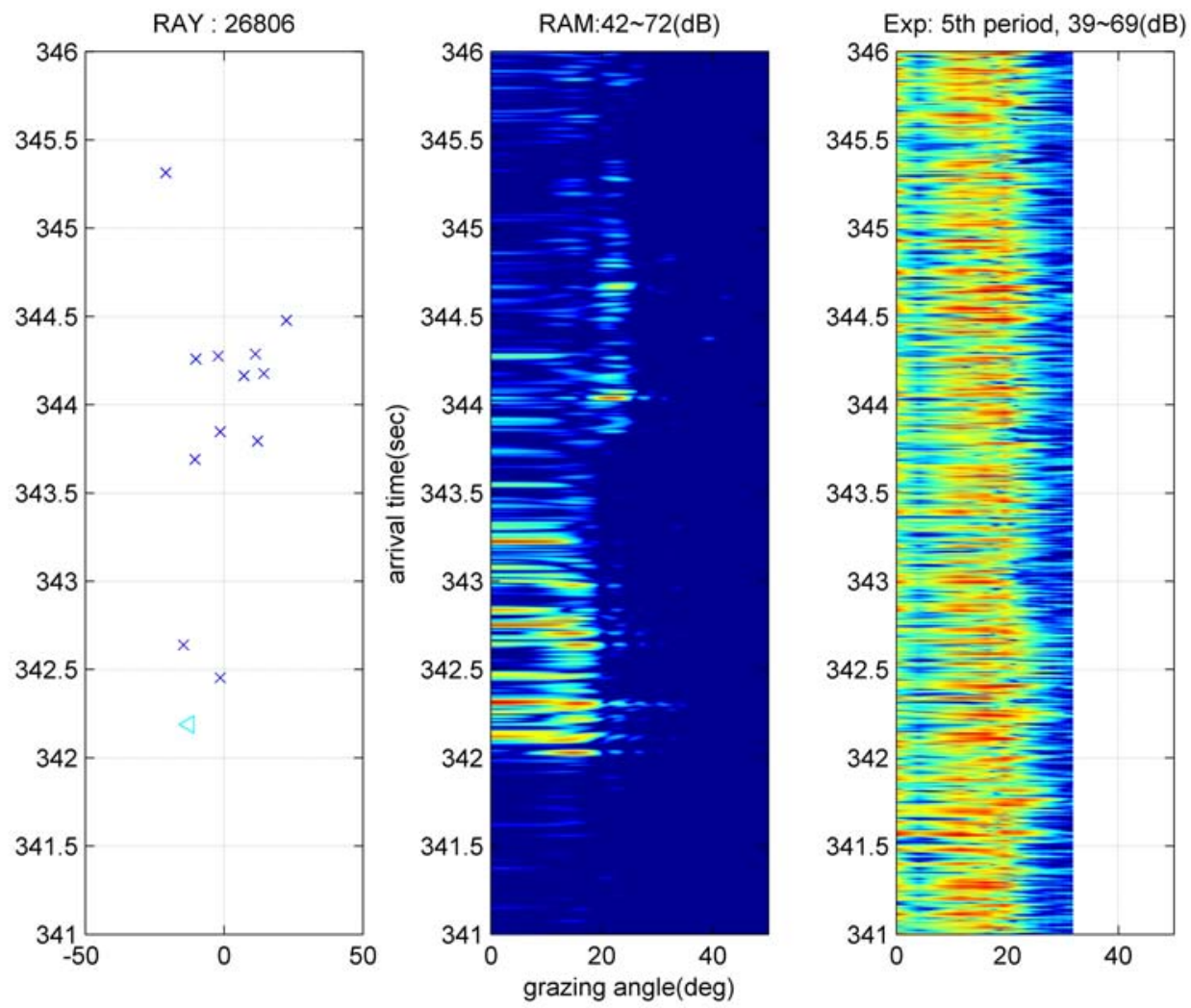

Figure 4-14: Comparison of pulse arrivals by the ray tracing method, 2D PE, and the BASSEX experiment for 26806. In the leftmost panel, the cyan triangle denotes the bottom only reflected ray. Pulse arrivals from the experiment are shifted in time by -0.690 seconds (Table $4-2$ ) to be aligned with the other results. Since at this range, a receiver is located in the shadow zone, only reflected rays appeared with low signal levels. 

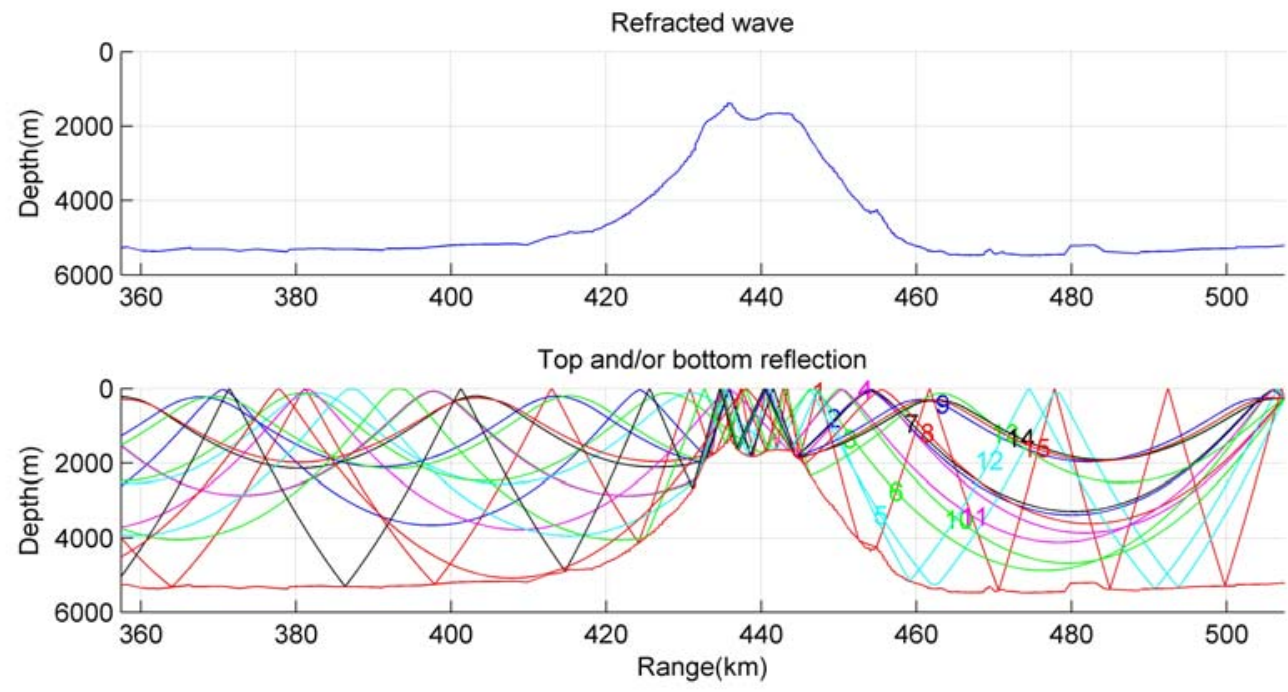

68_6A1.mat :Eigenrays at the receiver:(507.448 km,280.44 m)

1:38-SRBR

2:24-SRBR

3:24-SRBR

4:23-SRBR

5:23-SRBR

6:22-SRBR

8:26+SRBR

9:27+SRBR

10:26+SRBR

$11: 21+B R$

$11: 21+\mathrm{BR}$

$12: 25+$ SRBR

7:26-SRBR

13:24+SRBR

$14: 29+$ SRBR

$14: 29+$ SRBR

$15: 21+$ SRBR

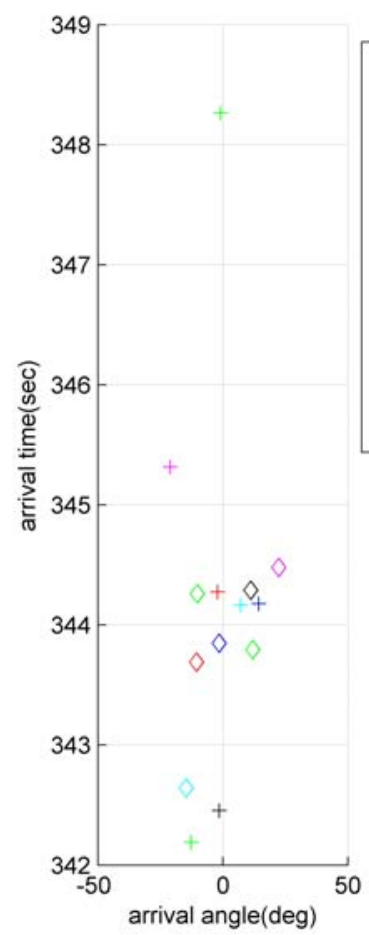

68 6A1.mat:Eigenrays at the receiver:(507.448 km,280.44 m)

\begin{tabular}{|ll|}
\hline & $24-\mathrm{SRBR}$ \\
$\diamond$ & $24-\mathrm{SRBR}$ \\
$\diamond$ & $23-\mathrm{SRBR}$ \\
$\diamond$ & $23-\mathrm{SRBR}$ \\
$\diamond$ & $22-\mathrm{SRBR}$ \\
$\diamond$ & $26-\mathrm{SRBR}$ \\
$\diamond$ & $26+\mathrm{SRBR}$ \\
+ & $27+\mathrm{SRBR}$ \\
+ & $26+\mathrm{SRBR}$ \\
+ & $21+\mathrm{BR}$ \\
+ & $25+\mathrm{SRBR}$ \\
+ & $24+\mathrm{SRBR}$ \\
+ & $29+\mathrm{SRBR}$ \\
+ & $21+$ SRBR \\
\hline
\end{tabular}

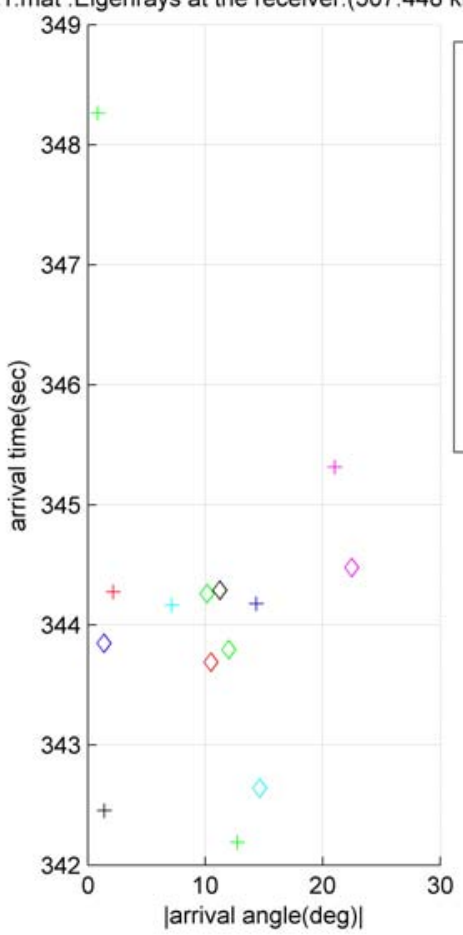

Figure 4-15: Ray arrivals from ray tracing method for 26806. Figure 4-9 provides the definition of rays. 


\subsubsection{7}
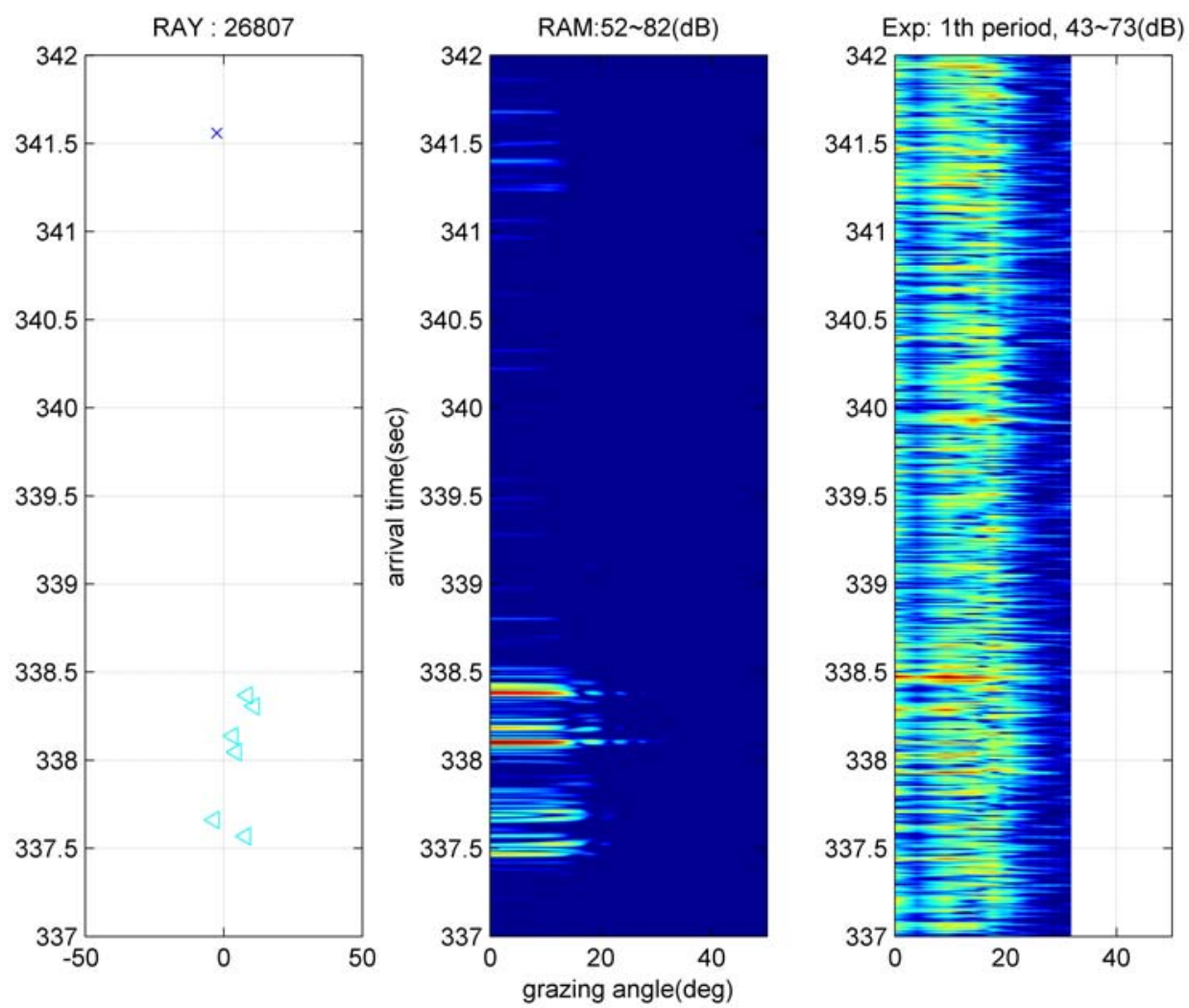

Figure 4-16: Comparison of pulse arrivals by the ray tracing method, 2D PE, and the BASSEX experiment for 26807. Pulse arrivals from the experiment are shifted in time by -0.180 seconds (Table 4-2), to be aligned with the other results. The cyan triangles denote the bottom only reflected rays in the leftmost panel. At this range, as seen in Fig.4-3, the diffused wave arrivals are dominant which are bottom-reflected by the seamount and then trapped in water column again after the reflection. Therefore, this wave has a lower arrival angle and faster arrival time than the reflected-reflected wave. These waves are clearly found in the ray tracing method (leftmost panel) and the 2D PE simulation (middle panel), but in the experiment only one relatively significant wave is measured. In the case of 26813, this kind of wave appears more clearly. 

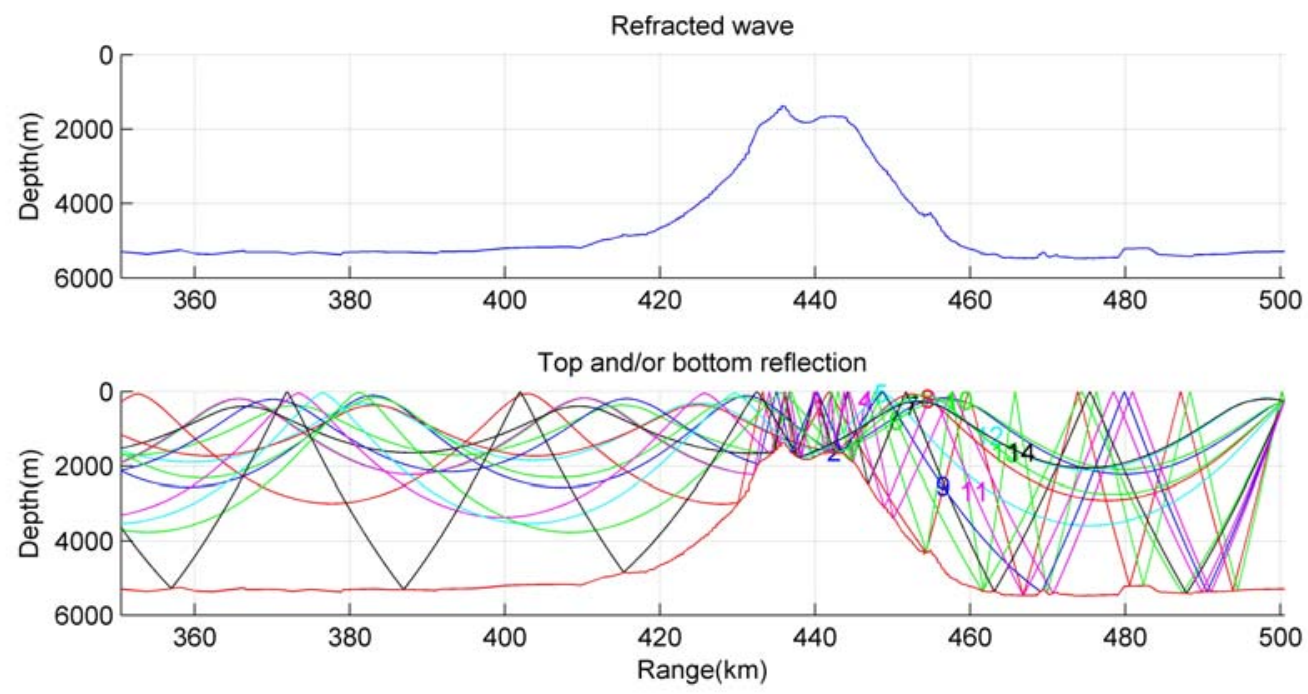

68_7A1.mat:Eigenrays at the receiver:(500.512 km,256.15 m)

$1: 30-S R B R$

2:21-BR

3:38-SRBR

$4: 35-S R B R$

5:23-BR

6:29-SRBR

8:24+BR

9:28+SRBR

$10: 22+B R$

$11: 28+$ SRBR

7:31-SRBR

$11: 28+$ SRBR $13: 20+B R$

$\begin{array}{ll}12: 21+B R & 14: 25+S R B R\end{array}$

$14: 25+S R B R$

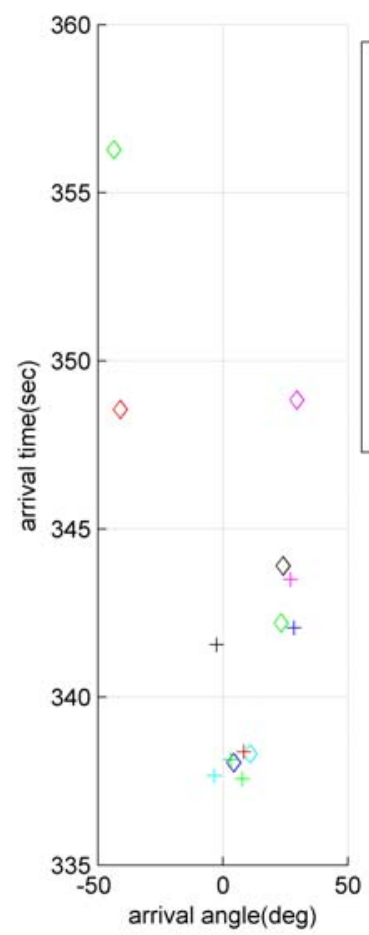

68_7A1.mat :Eigenrays at the receiver:(500.512 km,256.15 m)

\begin{tabular}{|ll|}
\hline$\diamond$ & $30-S R B R$ \\
$\diamond$ & $21-B R$ \\
$\diamond$ & $38-S R B R$ \\
$\diamond$ & $35-S R B R$ \\
& $23-B R$ \\
$\diamond$ & $29-S R B R$ \\
$\diamond$ & $31-S R B R$ \\
+ & $24+B R$ \\
+ & $28+$ SRBR \\
+ & $22+$ BR \\
+ & $28+$ SRBR \\
+ & $21+$ BR \\
+ & $20+B R$ \\
+ & $25+$ SRBR \\
\hline
\end{tabular}

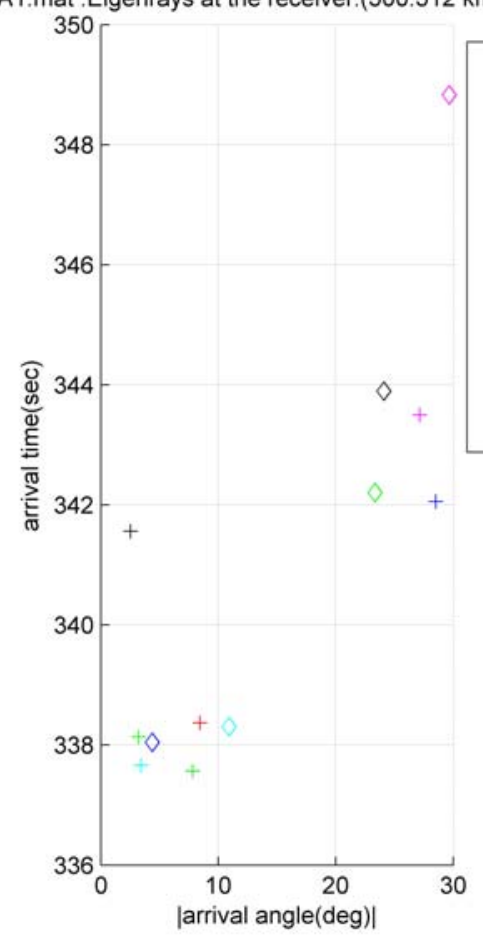

Figure 4-17: Ray arrivals from ray tracing method for 26807. Figure 4-9 provides the definition of rays. 


\subsubsection{8}
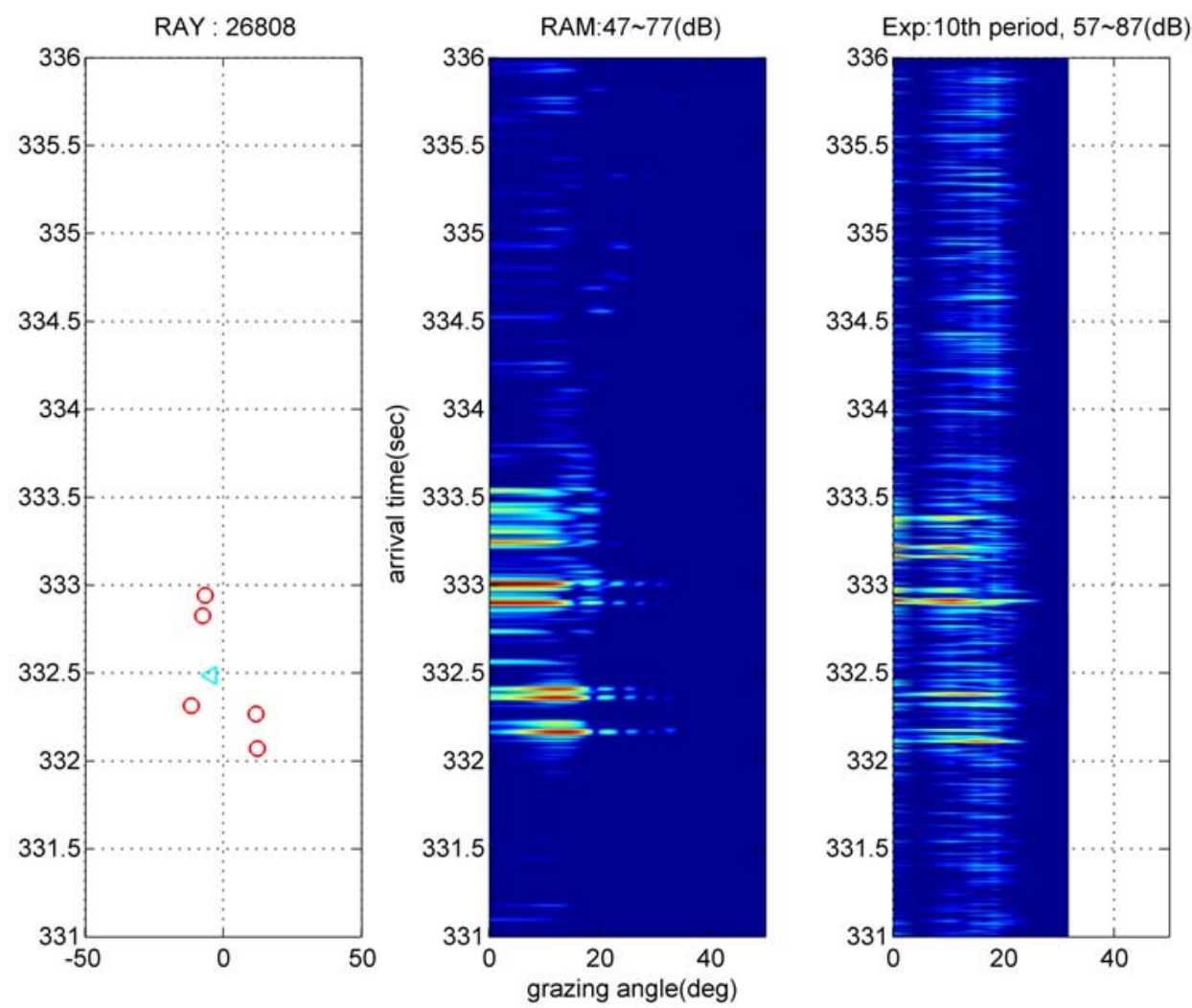

Figure 4-18: Comparison of pulse arrivals by the ray tracing method, 2D PE, and the BASSEX experiment for 26808. Pulse arrivals from the experiment are shifted in time by -0.261 seconds (Table 4-2), to be aligned with the other results. The cyan triangles denote the bottom-only reflected rays. Since horizontal array is moving to the source after passing the shadow zone, the refracted waves appear again and are dominant. The refracted waves are followed by some reflections which are consistently found in the 2D PE simulation and experiment. 

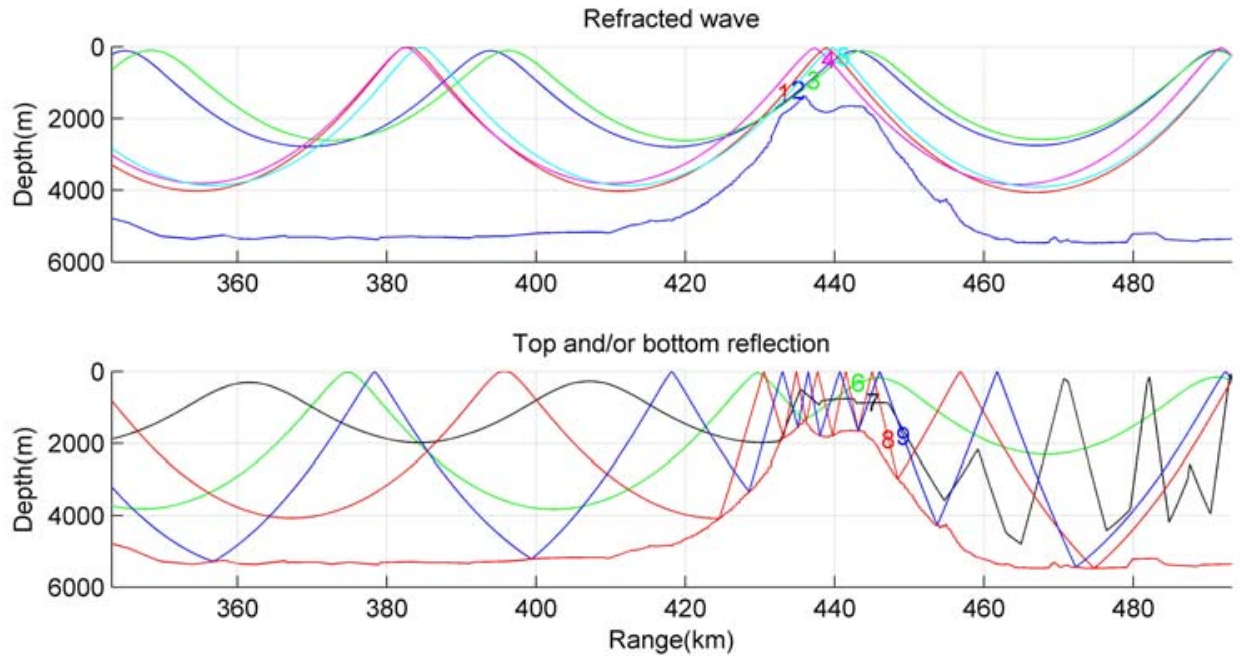

68 8A1.mat:Eigenrays at the receiver:(493.152 km,251.20 m)

1:17-R

2:20-R

$3: 21+R$

$4: 19+R$

$5: 18+R$

$6: 20-B R$

$7: 31+$ SRBR

6:20-BR

$9: 29+$ SRBR

$7: 31+$ SRBR

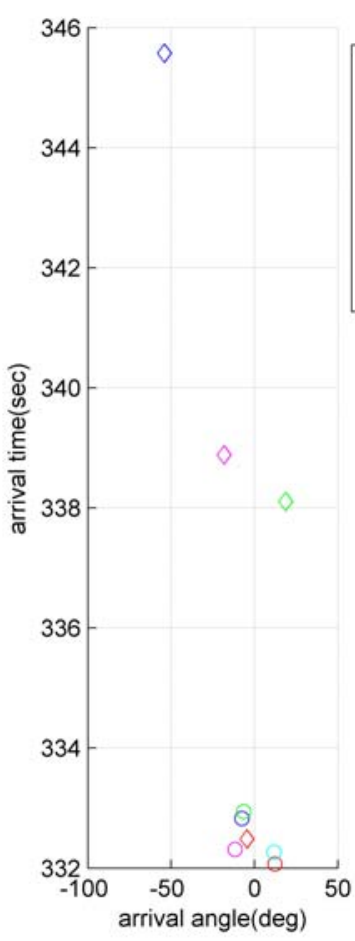

68_8A1.mat :Eigenrays at the receiver:(493.152 km,251.20 m)
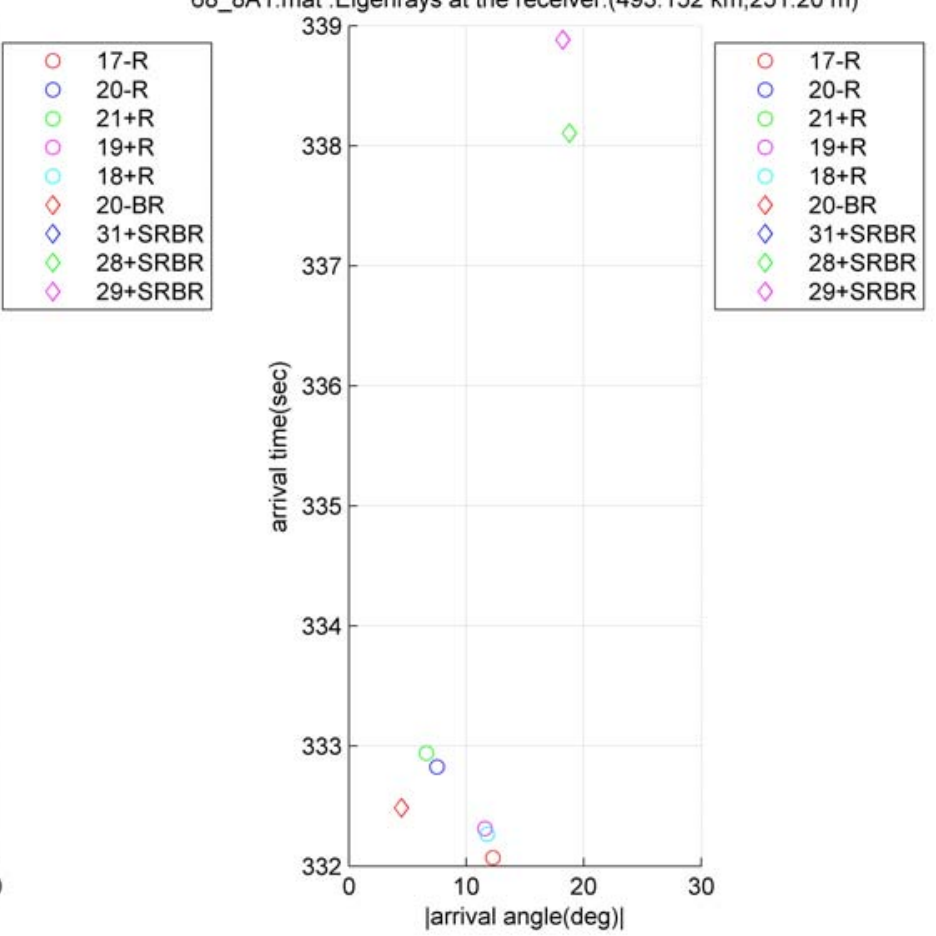

Figure 4-19: Ray arrivals from ray tracing method for 26808. Figure 4-9 provides the definition of rays. 


\subsubsection{9}
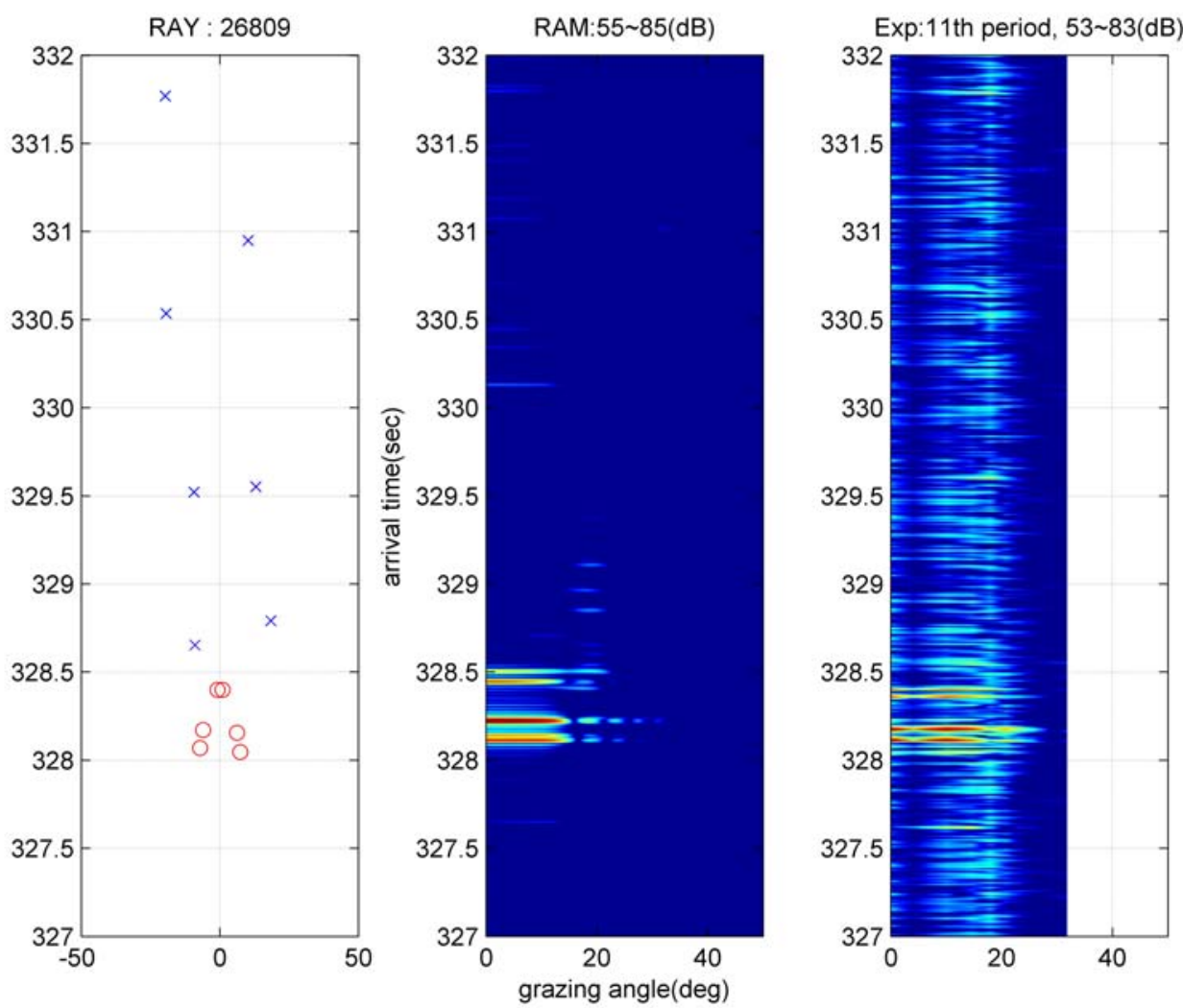

Figure 4-20: Comparison of pulse arrivals by the ray tracing method, 2D PE, and the BASSEX experiment for 26809. Pulse arrivals from the experiment are shifted in time by -0.069 seconds (Table 4-2), to be aligned with the other results. Since, at this range, a receiver is located at the middle of refracted wave zone, the clear refracted waves are detected and dominant. These waves are comparable in all the results. 

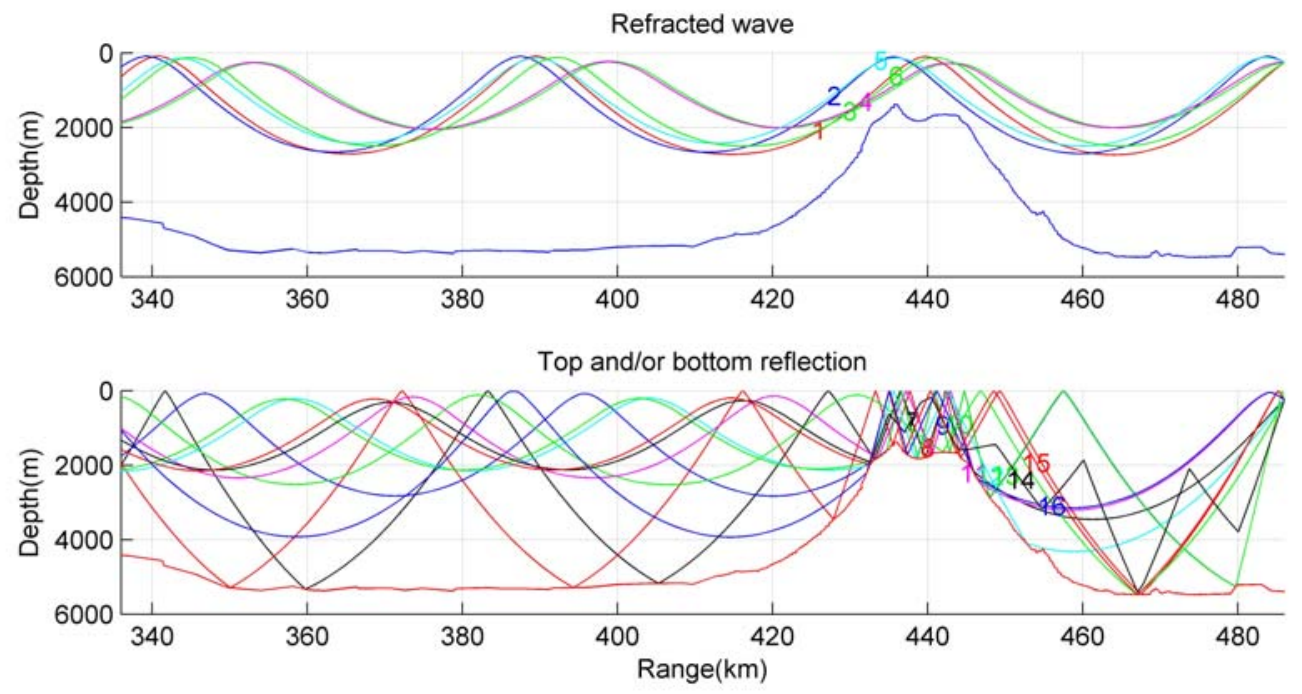

68_9A1.mat :Eigenrays at the receiver:(486.001 km,260.00 m)

1:19-R 8.26-SRBR

2:20-R 9:26-SRBR

3:21-R

$4: 22-\mathrm{R}$

$5: 21+R$

$6: 20+R$

7:23-SRBR

10:21-SRBR

11:24-SRBR

12:23-SRBR

12:23-SRBR $14: 26+$ SRBR

$15: 27+$ SRBR

$14: 26+$ SRBR

16:21+SRBR

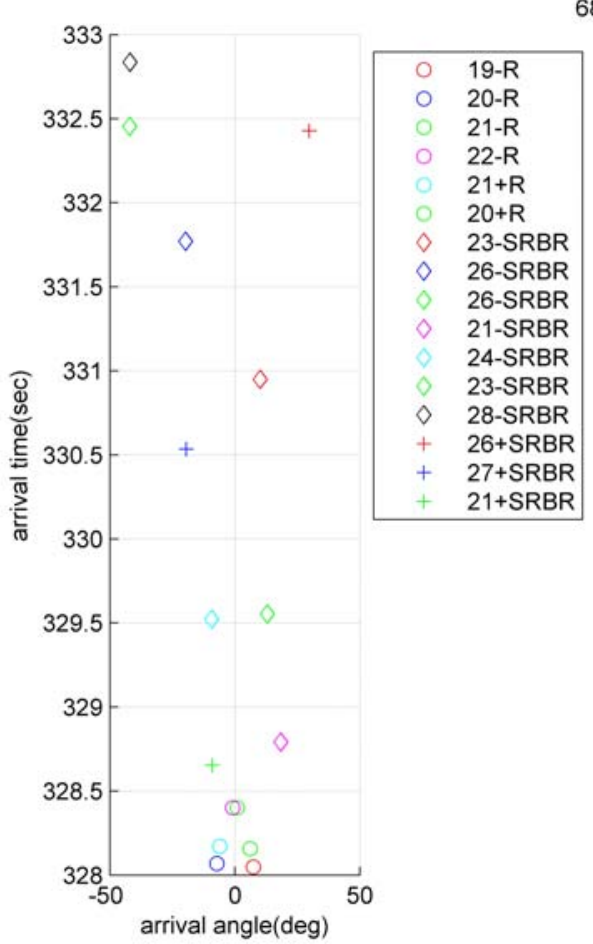

68_9A1.mat :Eigenrays at the receiver:(486.001 km,260.00 m)

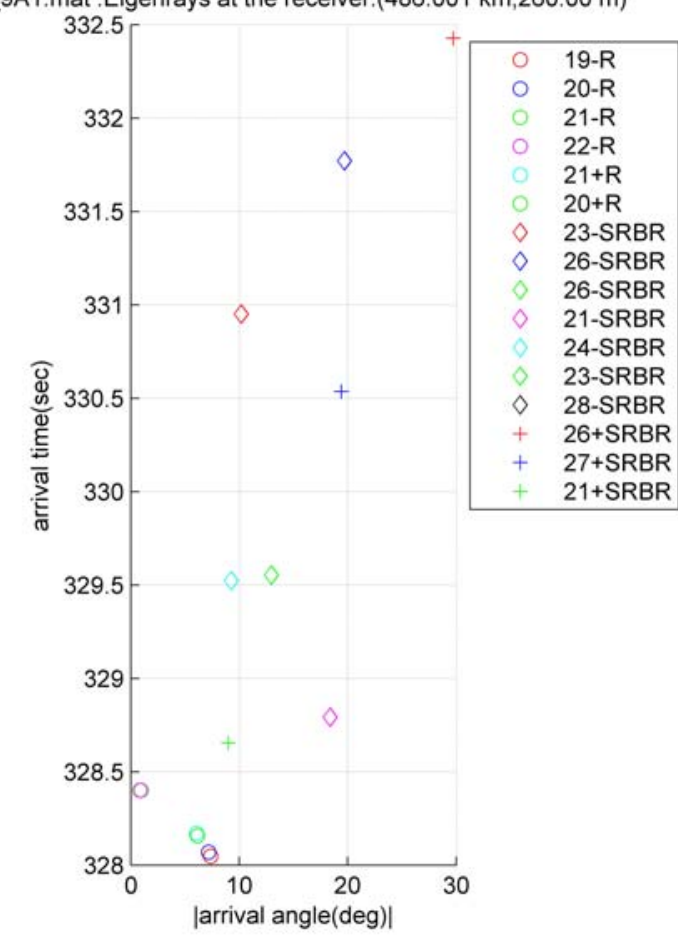

Figure 4-21: Ray arrivals from ray tracing method for 26809. Figure 4-9 provides the definition of rays. 


\subsubsection{0}
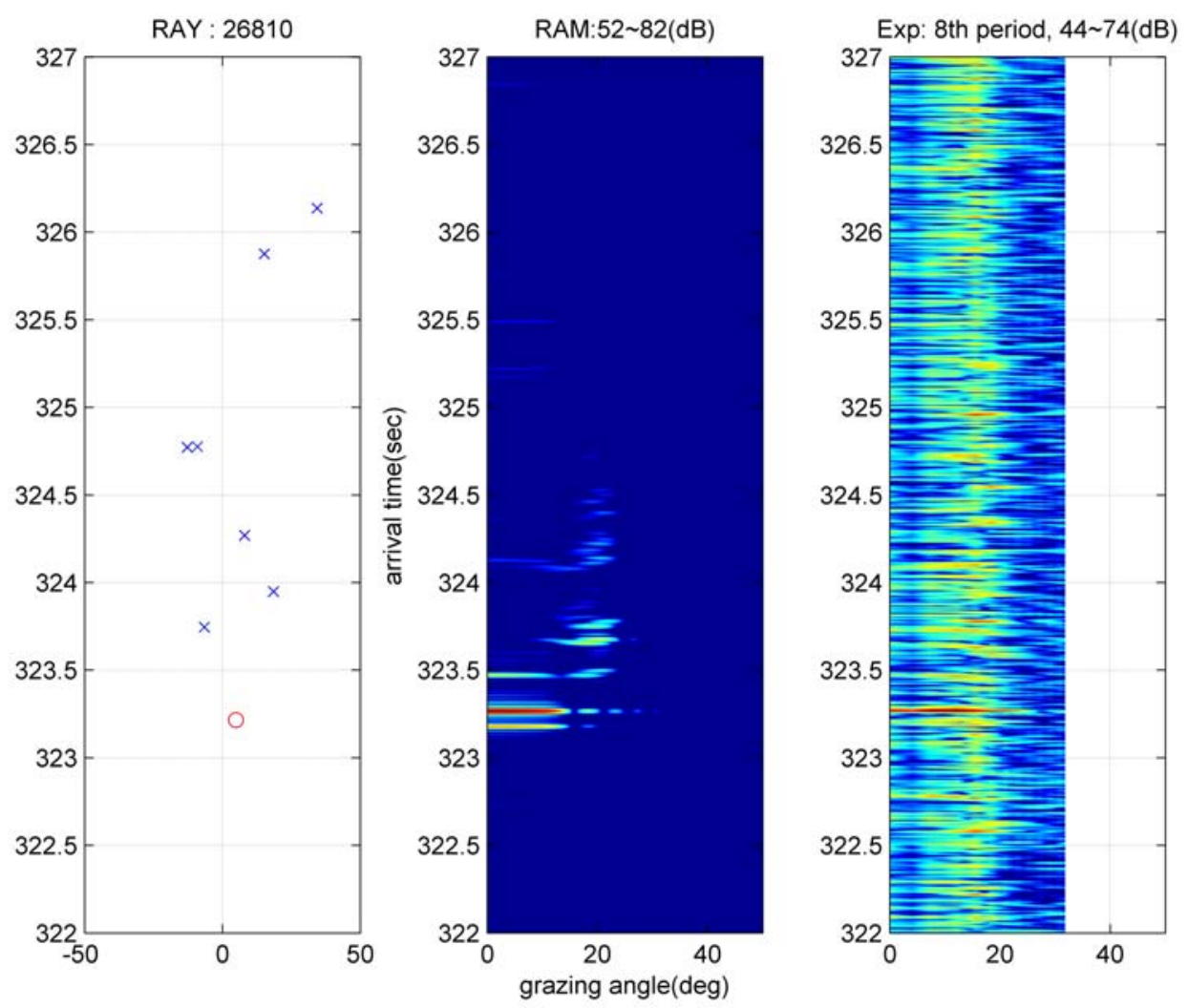

Figure 4-22: Comparison of pulse arrivals by the ray tracing method, 2D PE, and the BASSEX experiment for 26810. Pulse arrivals from the experiment are shifted in time by -0.113 seconds (Table 4-2) to be aligned with the other results. At this range, a receiver is located at the transition region between the shadow zone and refracted wave zone. The ray tracing method detects only one refracted wave rather than the 2 3 waves that appeared in the PE simulation. The MPDR beamformer (rightmost panel) detects one peak at the same arrival time with the ray tracing method and 2D PE simulation; however, the signal level is quite smaller. This corresponds to B in Fig. 4-4; due to the small amplitude, this wave cannot be shown in the experiment (left panel in Fig. 4-4). 


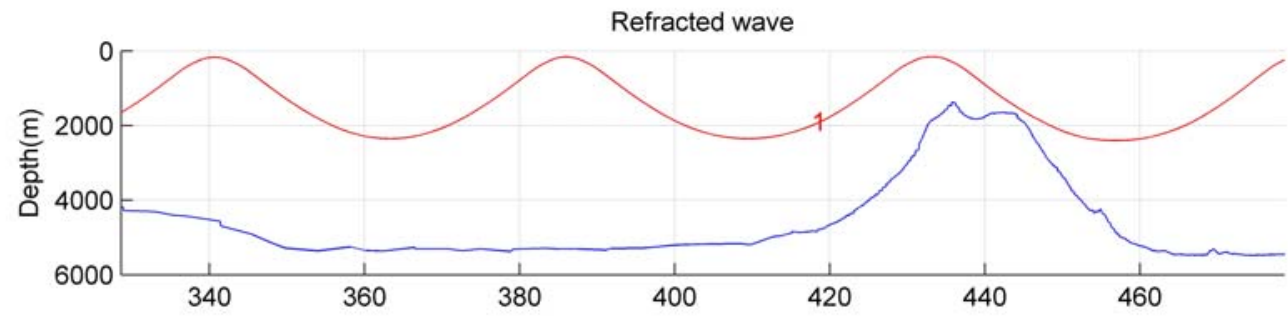

Top and/or bottom reflection

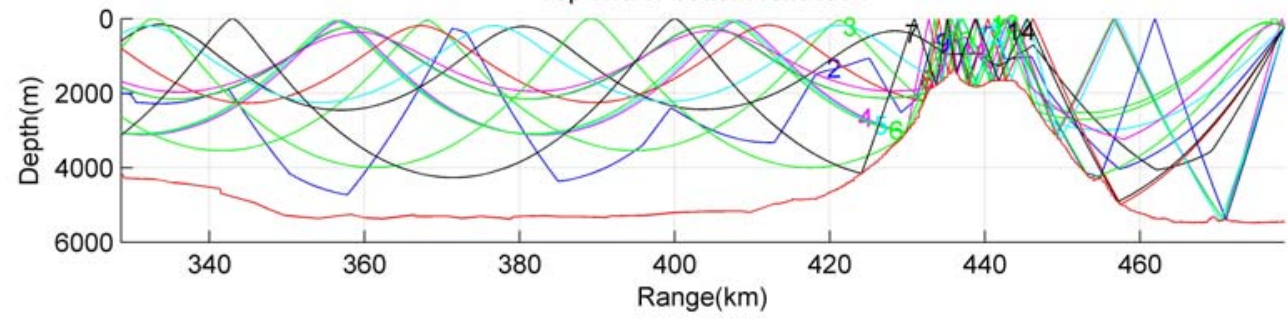

68_10A1.mat :Eigenrays at the receiver:(478.590 km,238.30 m)

$1: 20+R$

2:25-SRBR

3:30-SRBR

4:28-SRBR

5:21-SRBR

6:23-SRBR

7:21-SRBR

8:27-SRBR

9:25-SRBR

10:24-SRBR

$11: 25+$ SRBR

$12: 28+$ SRBR

$13: 21+$ SRBR

$14: 24+$ SRBR
$11: 25+S R B R$
$12: 28+S R B R$
$13: 21+$ SRBR
$14: 24+S R B R$

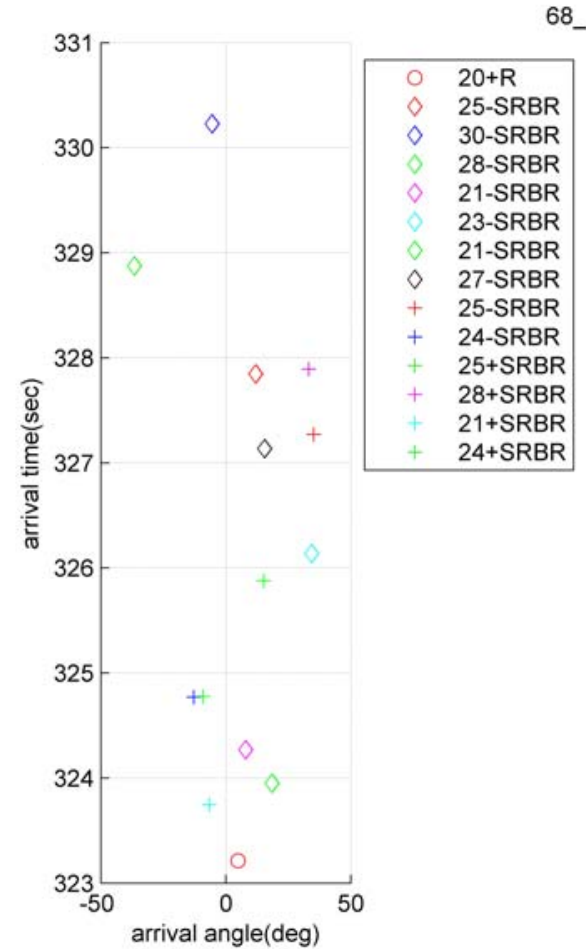

68_10A1.mat :Eigenrays at the receiver:(478.590 km,238.30 m)

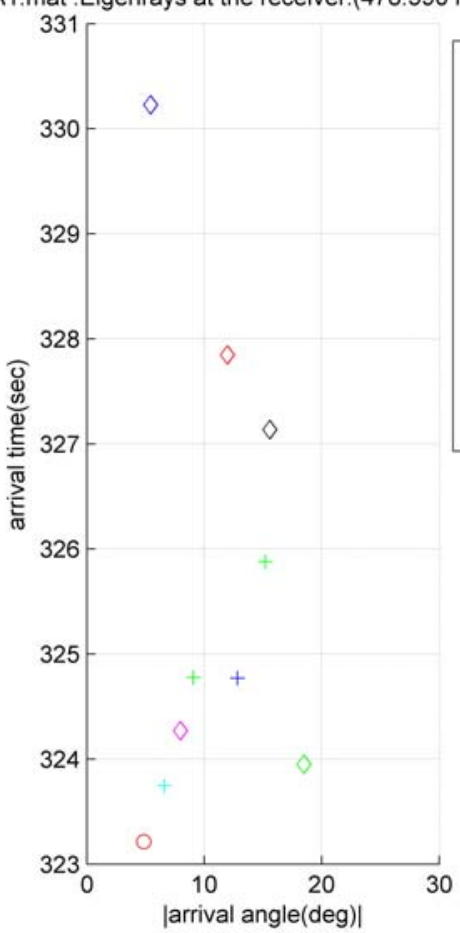

Figure 4-23: Ray arrivals from ray tracing method for 26810. Figure 4-9 provides the definition of rays. 


\subsubsection{2}
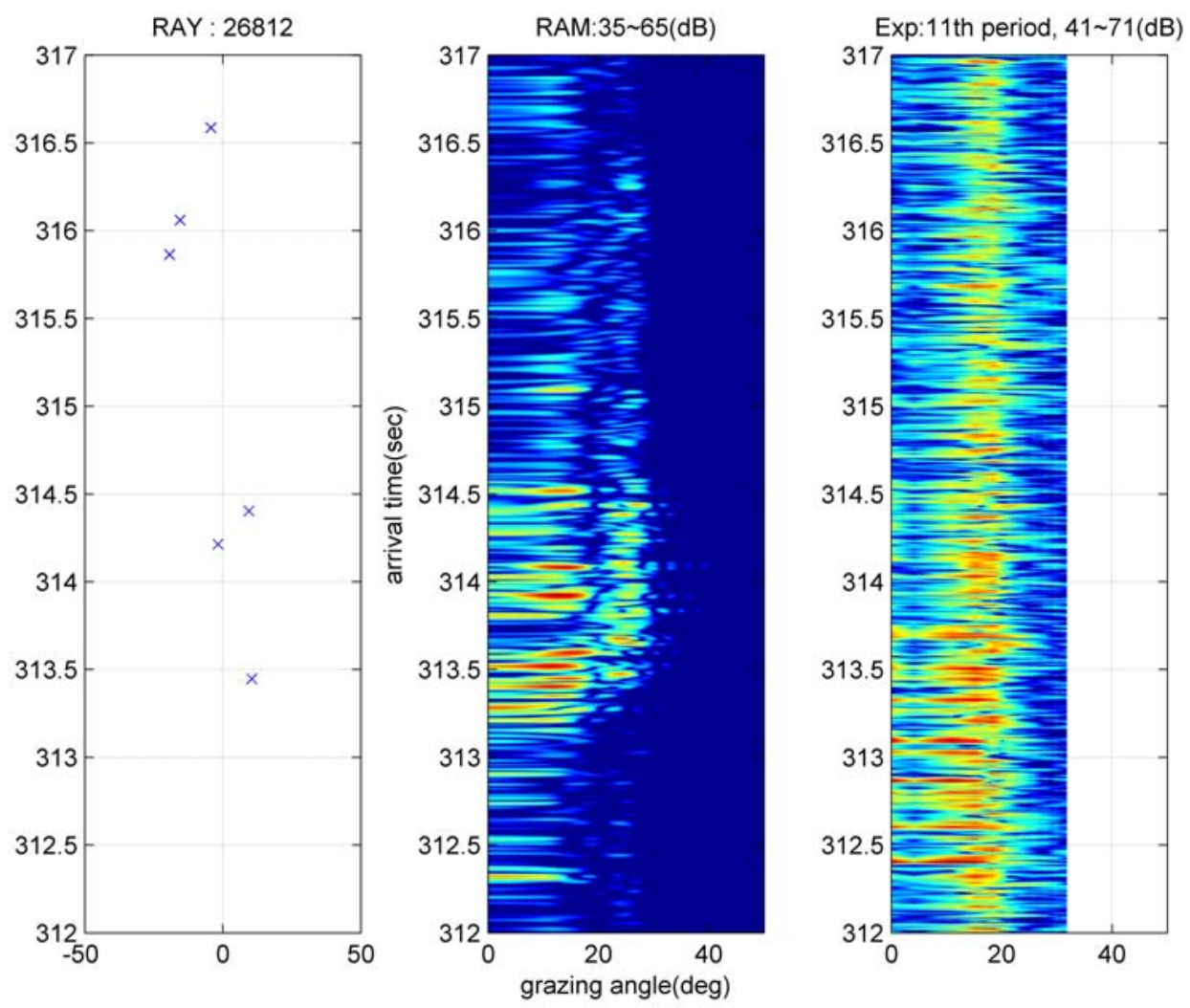

Figure 4-24: Comparison of pulse arrivals by the ray tracing method, 2D PE, and the BASSEX experiment for 26812. In the shadow zone, no refracted wave but some reflected waves with higher angles and lower signal levels can be measured in all three results. 


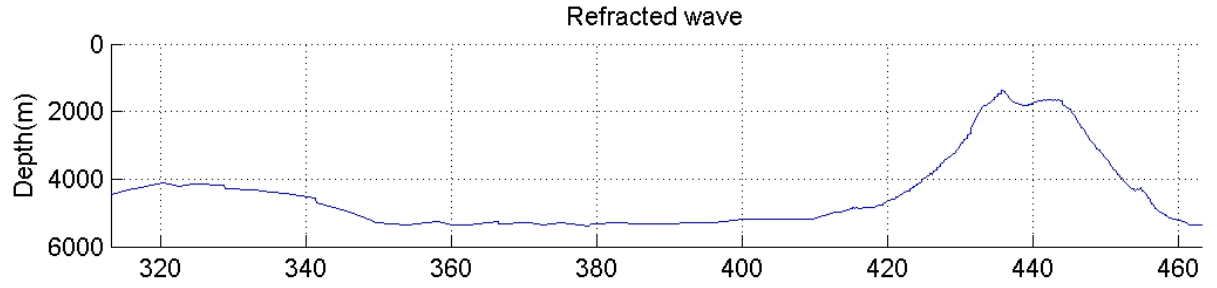

Top and/or bottom reflection

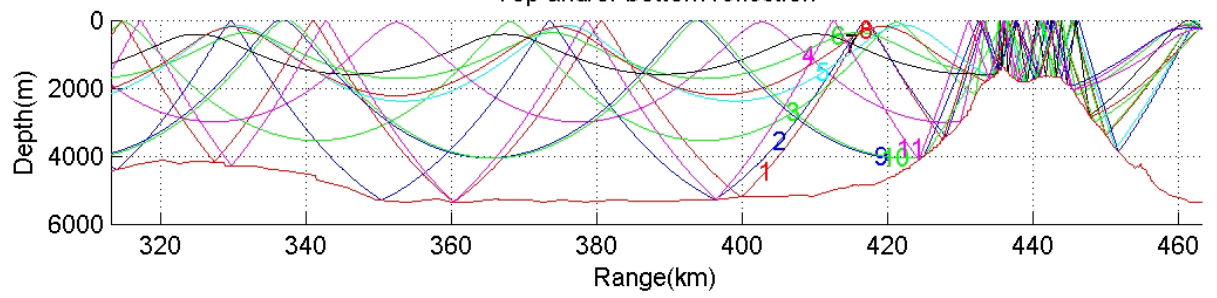

68_11A1.mat :Eigenrays at the receiver:(463.169 km,238.80 m)

1:28-SRBR 6:28-SRBR

2:26-SRBR $\quad 7: 37+$ SRBR

3:30-SRBR $\quad 8: 24+S R B R$

4:26-SRBR $\quad 9: 23+$ SRBR

$11: 26+$ SRBR
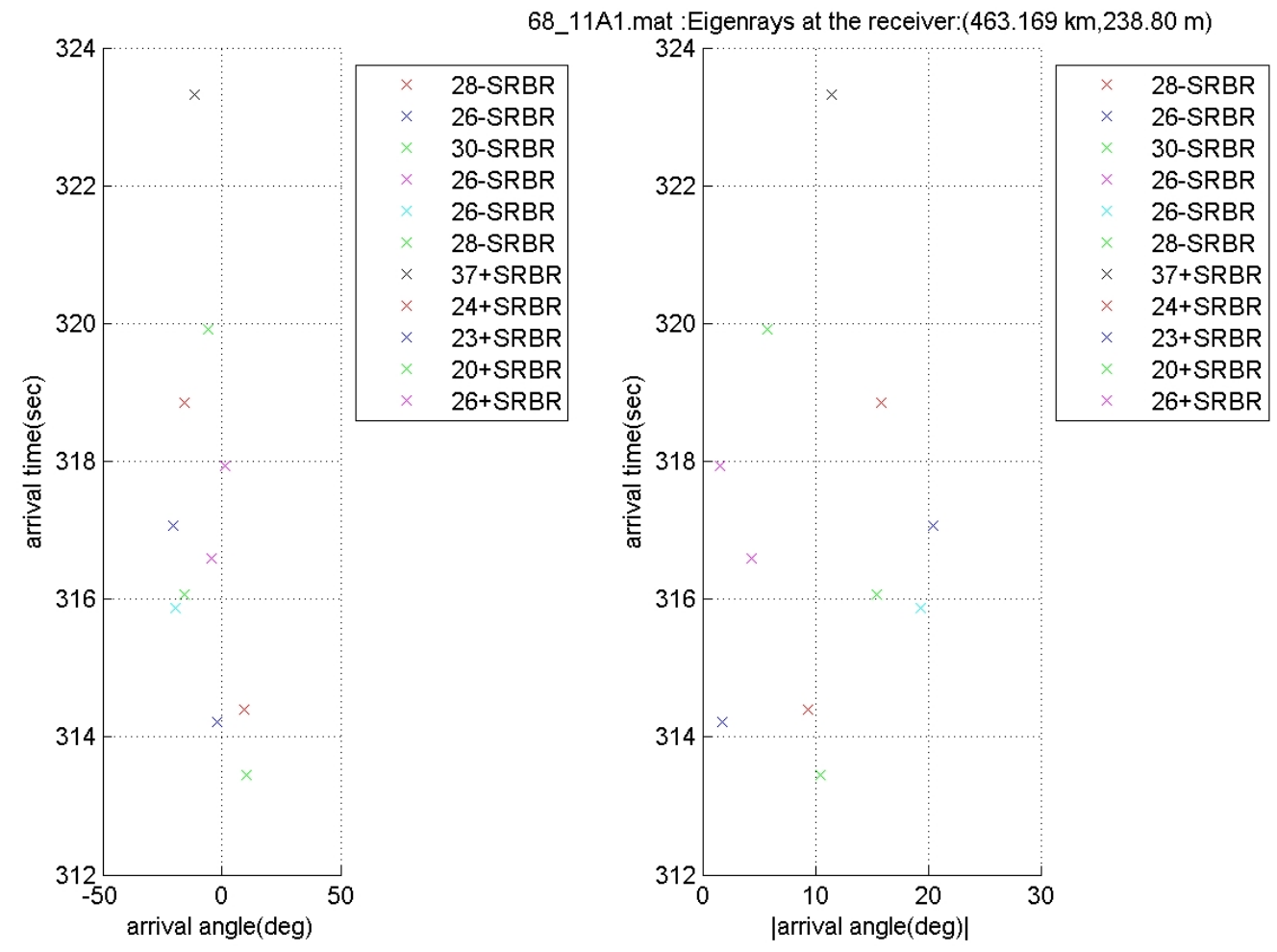

Figure 4-25: Ray arrivals from ray tracing method for 26812. Figure 4-9 provides the definition of rays. 


\subsubsection{3}
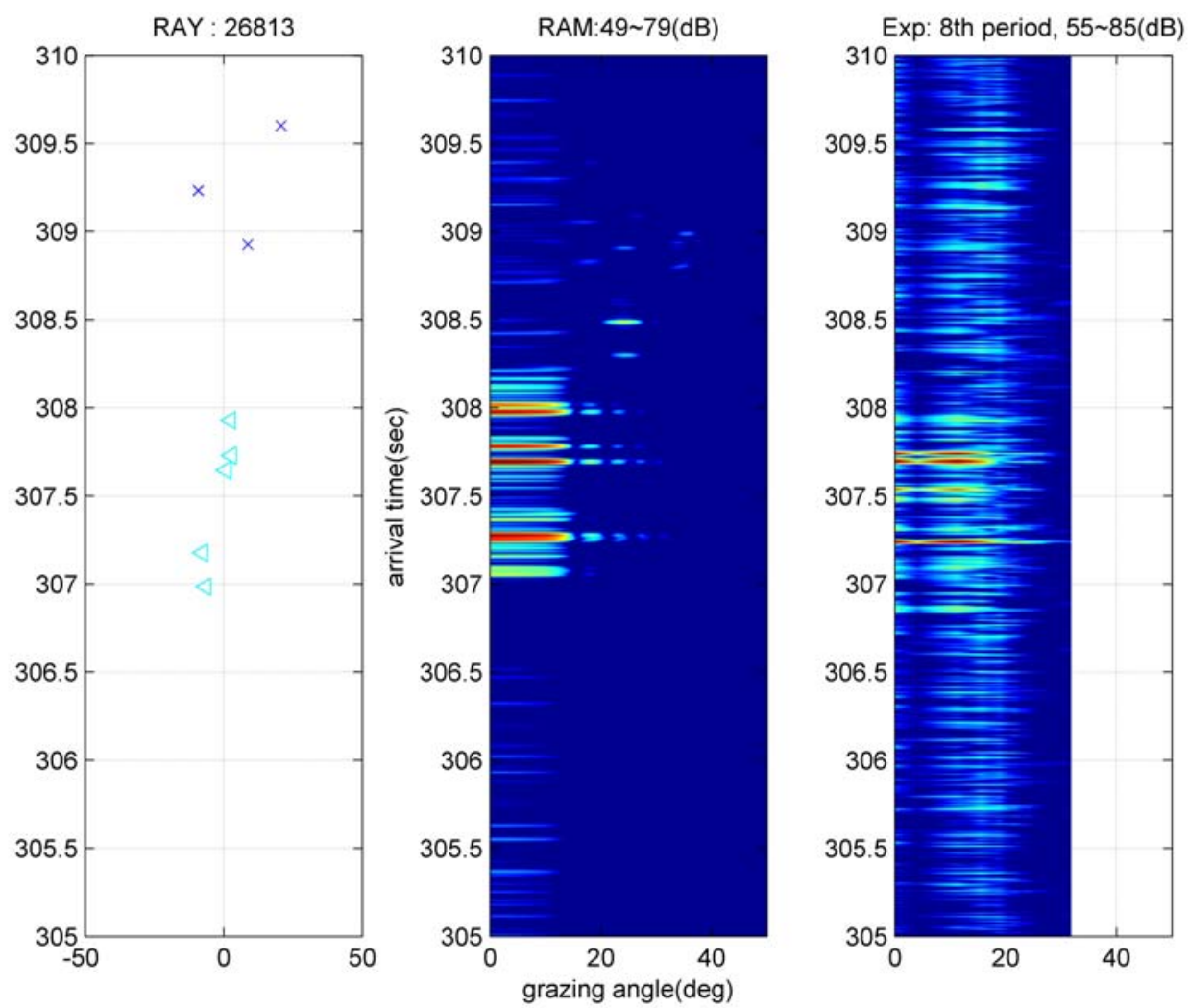

Figure 4-26: Comparison of pulse arrivals by the ray tracing method, 2D PE, and the BASSEX experiment for 26813. Pulse arrivals from the experiment are shifted in time by -0.372 seconds (Table 4-2), to be aligned with the other results. The cyan triangles denote the bottom-only reflected rays. A receiver is located in the diffraction/reflection region between the shadow zone and the refracted wave zone. Although good correlation between results from the ray tracing method and PE simulation, the results from experiment show a slightly different pulse arrival pattern. 

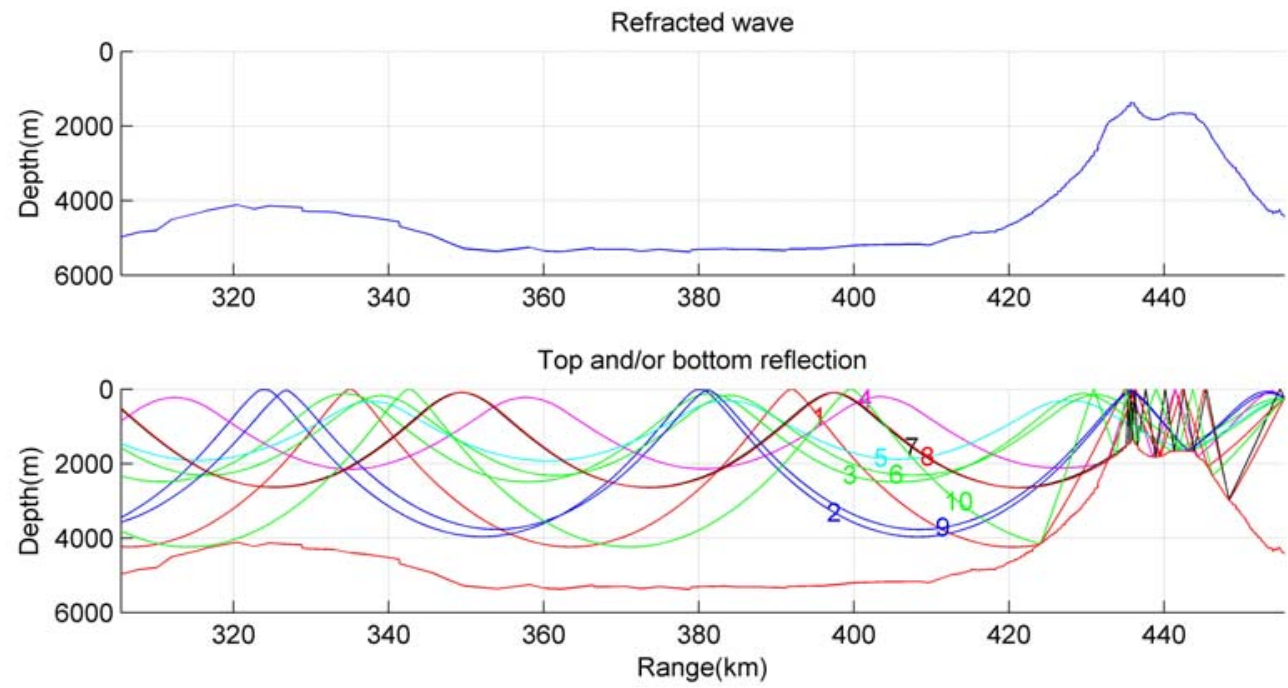

68_12A1.mat:Eigenrays at the receiver:(455.524 km,246.60 m)

1:21-SRBR

2:18-BR

3:19-BR

4:24-SRBR

5:21-BR

$6: 20+B R$

7:33+SRBR

$8: 22+$ SRBR

$6: 20+B R$

9:19+BR

$7: 33+$ SRBR

10:24+SRBR

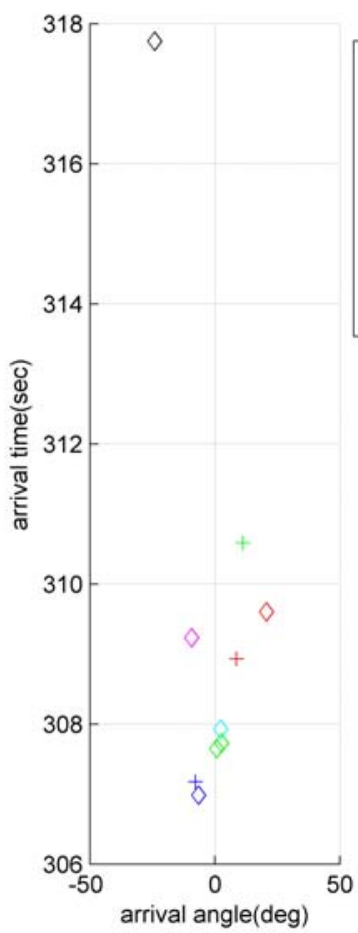

68 12A1.mat :Eigenrays at the receiver:(455.524 km,246.60 m)

\begin{tabular}{|ll|}
\hline$\diamond$ & $21-\mathrm{SRBR}$ \\
$\diamond$ & $18-\mathrm{BR}$ \\
$\diamond$ & $19-\mathrm{BR}$ \\
$\diamond$ & $24-\mathrm{SRBR}$ \\
$\diamond$ & $21-\mathrm{BR}$ \\
$\diamond$ & $20+\mathrm{BR}$ \\
$\diamond$ & $33+\mathrm{SRBR}$ \\
+ & $22+\mathrm{SRBR}$ \\
+ & $19+\mathrm{BR}$ \\
+ & $24+\mathrm{SRBR}$ \\
\hline
\end{tabular}

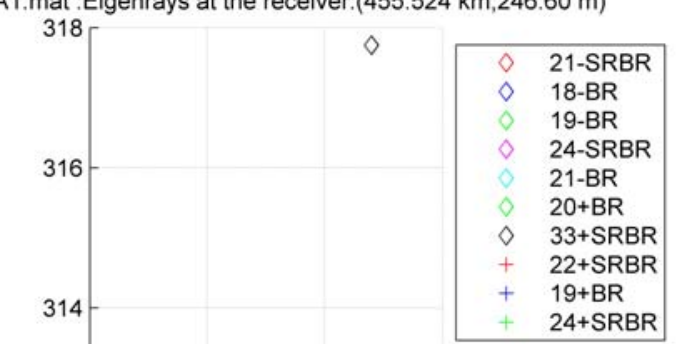

Figure 4-27: Ray arrivals from ray tracing method for 26813. Figure 4-9 provides the definition of rays. 


\subsubsection{4}
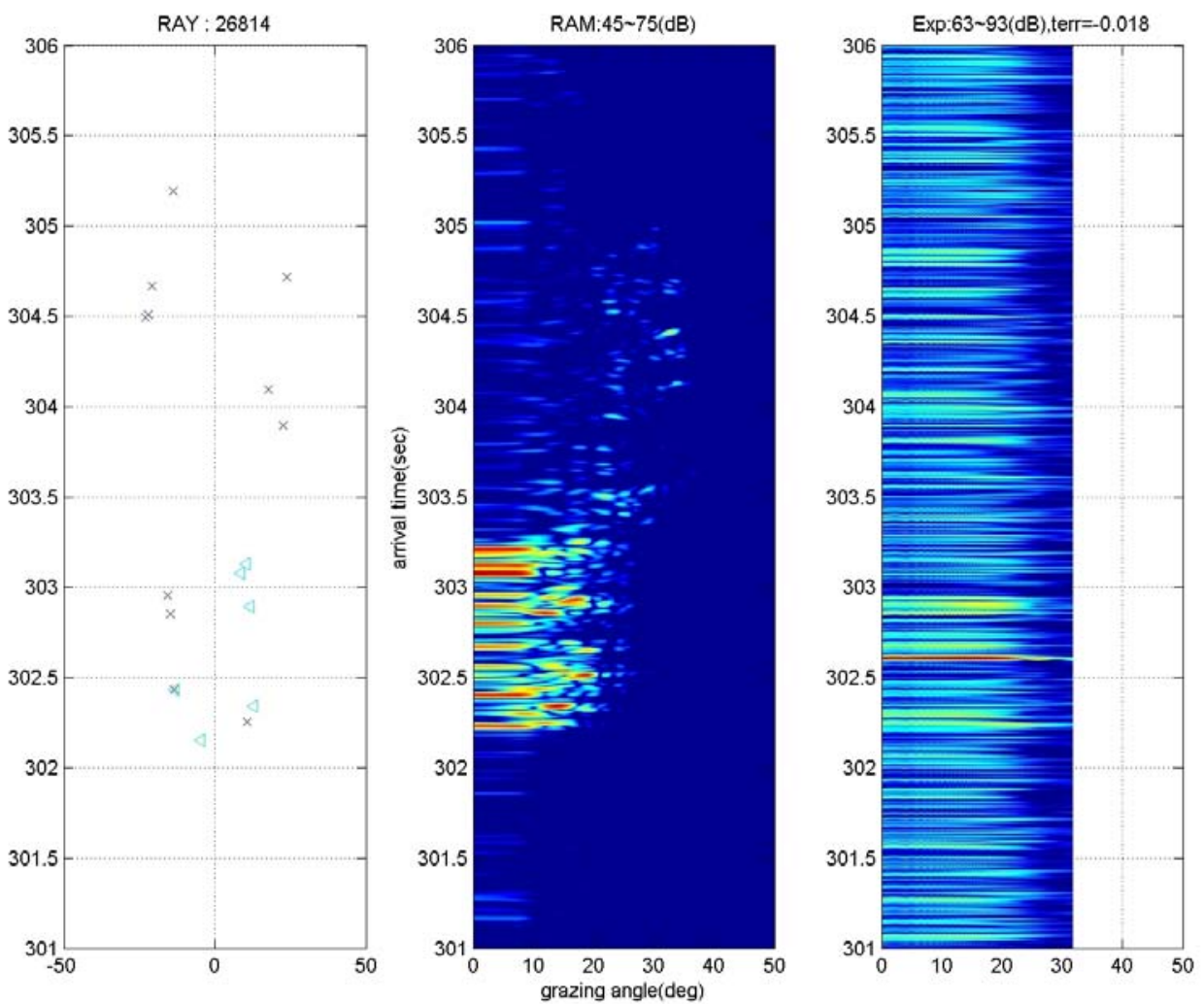

Figure 4-28: Comparison of pulse arrivals by the ray tracing method, 2D PE, and the BASSEX experiment for 26814. Pulse arrivals from the experiment are shifted in time by -0.018 seconds (Table 4-2), to be aligned with the other results. The cyan triangles denote the bottom only reflected rays. The results from the ray tracing method and PE simulation show some reflected waves, and the reflected waves are dominant. However, in the experiment, a very high intensity wave is detected. 

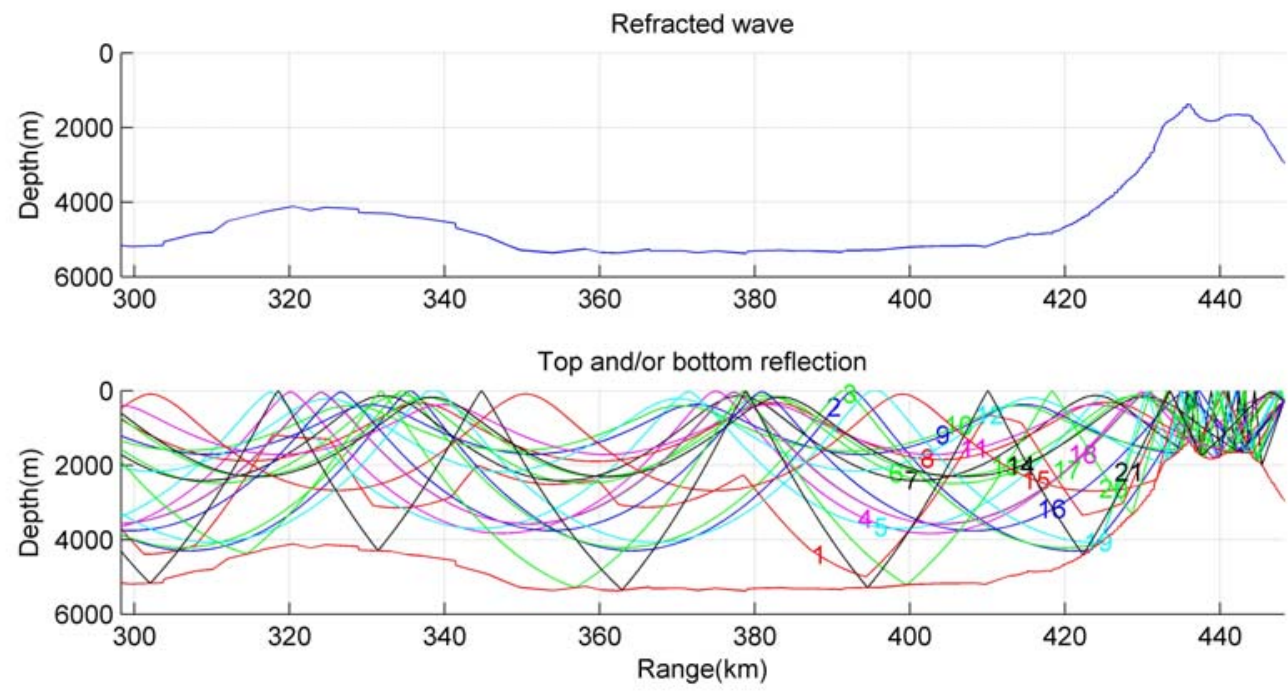

$68 \_13 \mathrm{~A} 1$.mat:Eigenrays at the receiver:(448.268 km,272.30 m)

1:22-SRBR

8:21-BR

2:17-SRBR

3:22-SRBR

9:25-SRBR

$10: 29+$ SRBR

$15: 22+$ SRBR

4:18-BR

11:22+BR

$16: 18+B R$

$17: 19+$ SRBR

$12: 25+$ SRBR

18:19+BR

13:21+SRBR

$19: 23+$ SRBR

6:20-SRBR

14:20+BR

$16: 18+B R$

17:19+SRBR

$18: 19+B R$

$19: 23+$ SRBR

7:33-SRBR

$21: 23+$ SRBR

$20: 27+$ SRBR

$21: 23+$ SRBR

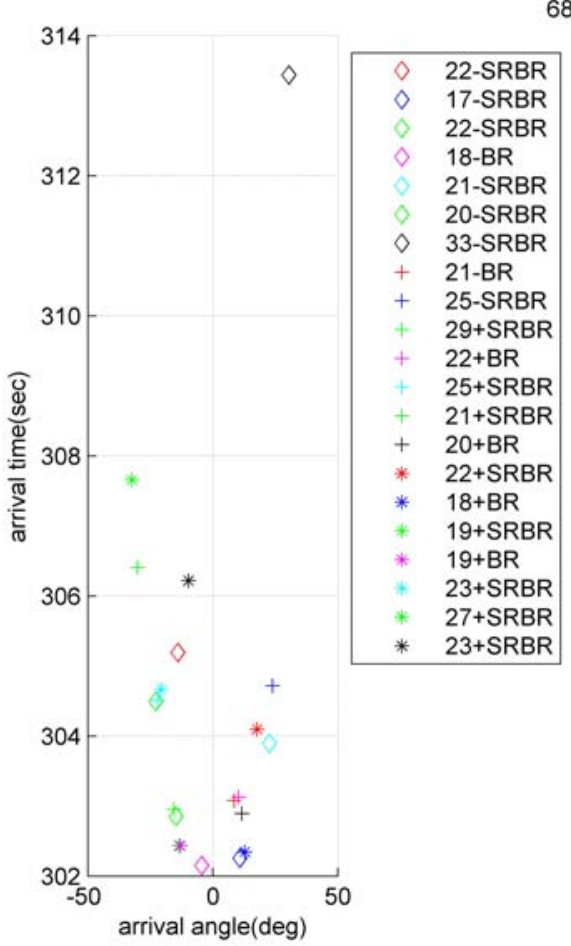

68_13A1.mat :Eigenrays at the receiver:(448.268 km,272.30 m)

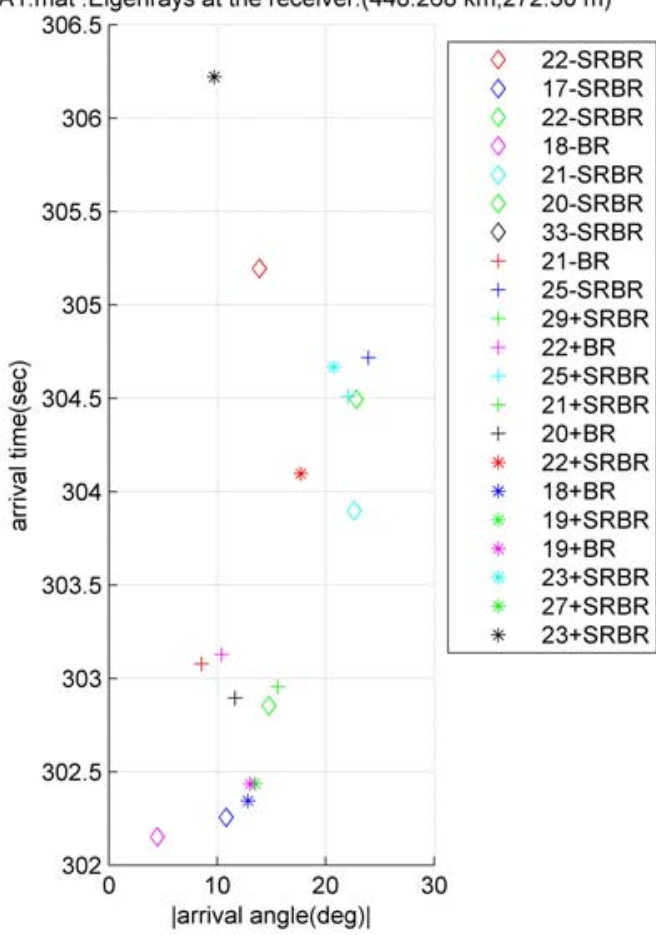

Figure 4-29: Ray arrivals from ray tracing method for 26814. Figure 4-9 provides the definition of rays. 


\subsubsection{5}
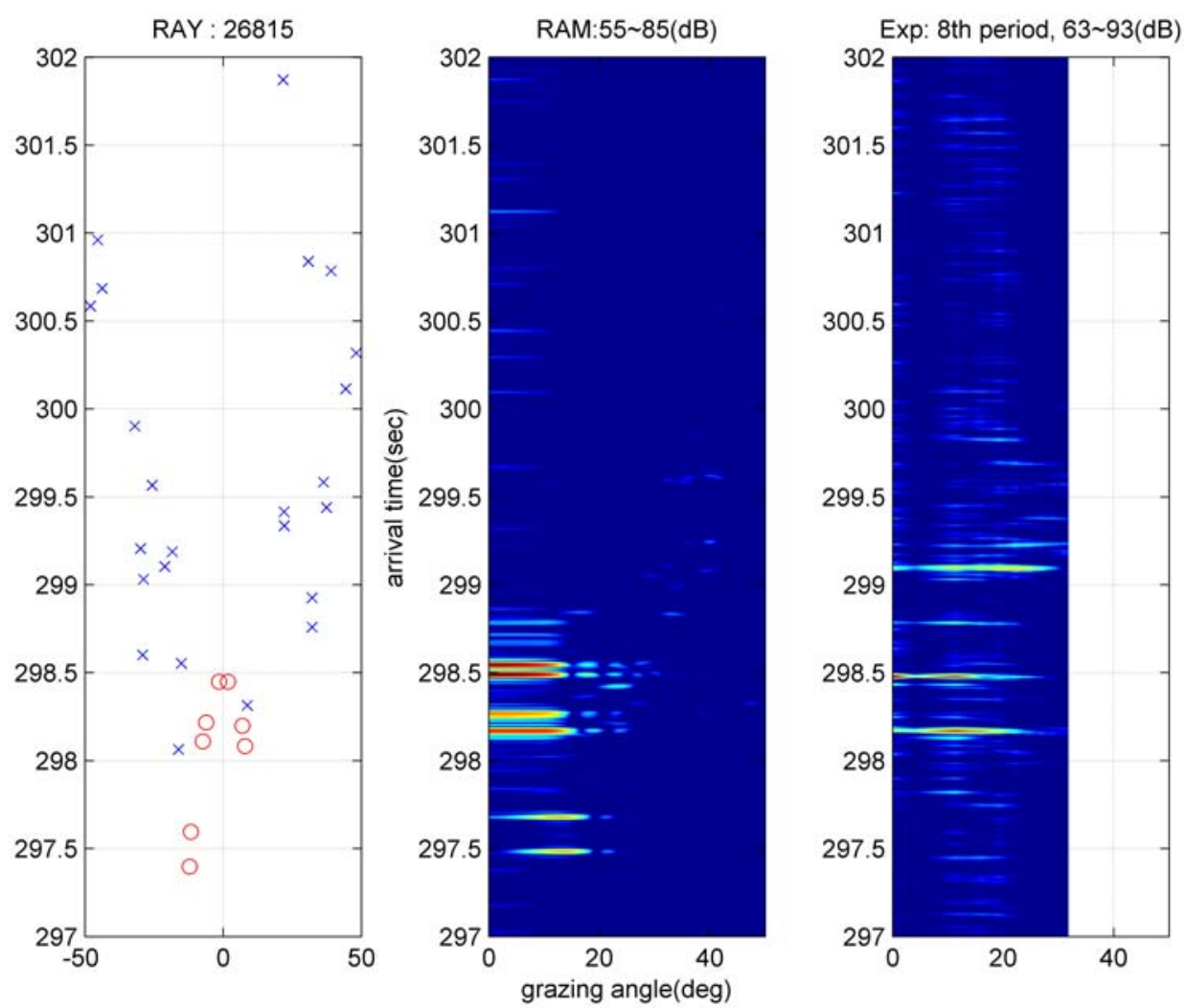

Figure 4-30: Comparison of pulse arrivals by the ray tracing method, 2D PE, and the BASSEX experiment for 26815. Pulse arrivals from the experiment are shifted in time by -0.115 seconds (Table 4-2) to be aligned with the other results. The receiver is located above the Elvis seamount, and this range also belongs to the end of the refracted wave arrival zone. However, in the experiment, two early refracted arrivals with higher angles are blocked and missing. In addition, two significant waves at 298.5 seconds are missing in the experiment. Good correlation between the ray tracing method and 2D PE is shown. 

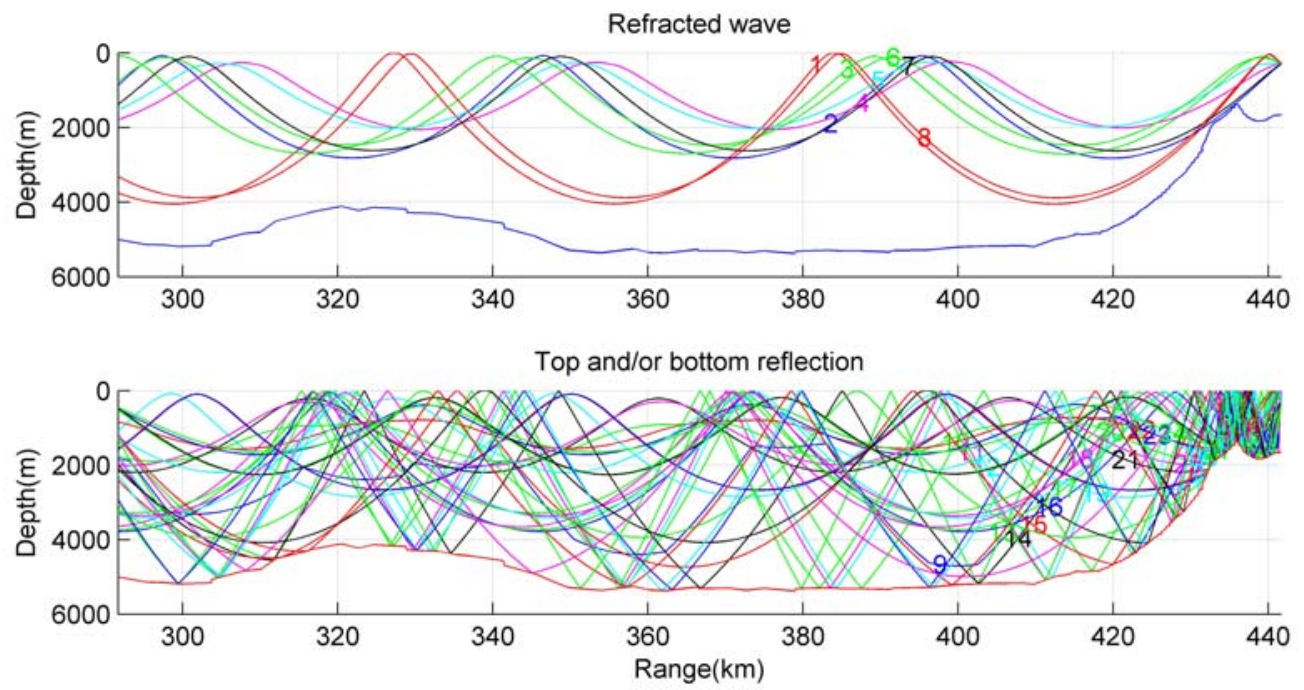

68_14A1.mat :Eigenrays at the receiver:(441.685 km,296.20 m)

$\begin{array}{lc}1: 16-R & 8: 17+R \\ 2: 17-R & 9: 21-\text { SRBR } \\ 3: 18-R & 10: 24-\text { SRBR } \\ 4: 19-R & 11: 36-\text { SRBR } \\ 5: 20-R & 12: 28-\text { SRBR } \\ 6: 19+R & 13: 26-\text { SRBR } \\ 7: 18+R & 14: 23-\text { SRBR }\end{array}$

15:18-SRBR
16:22-SRBR
17:20-SRBR
18:19-SRBR
19:20-SRBR
20:21-SRBR
21:24-SRBR

22.24-SRBR

23:23-SRBR

$24: 30+$ SRBR

25:22+SRBR

26:23+SRBR

$27: 21+$ SRBR

$28: 22+$ SRBR

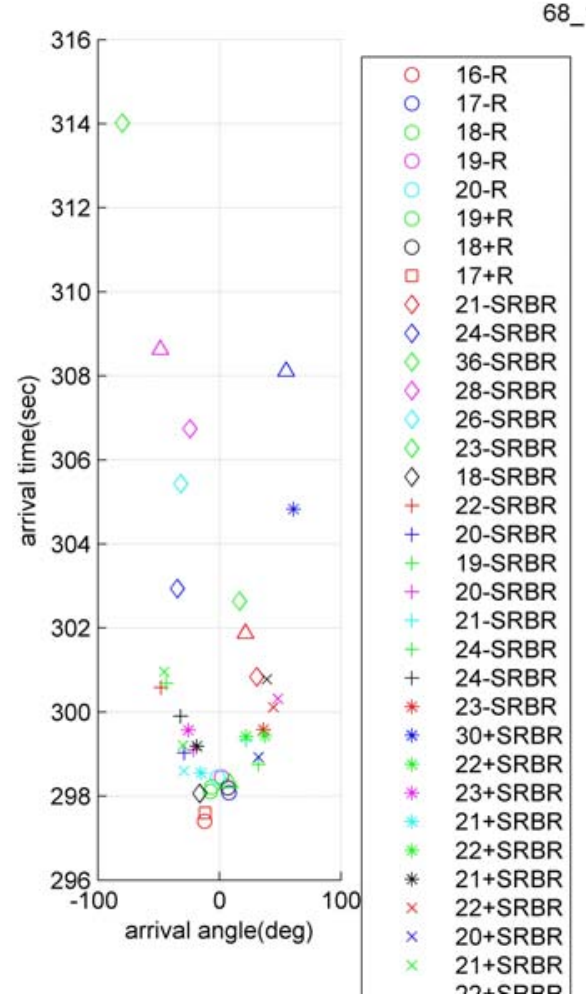

68_14A1.mat :Eigenrays at the receiver:(441.685 km,296.20 m)

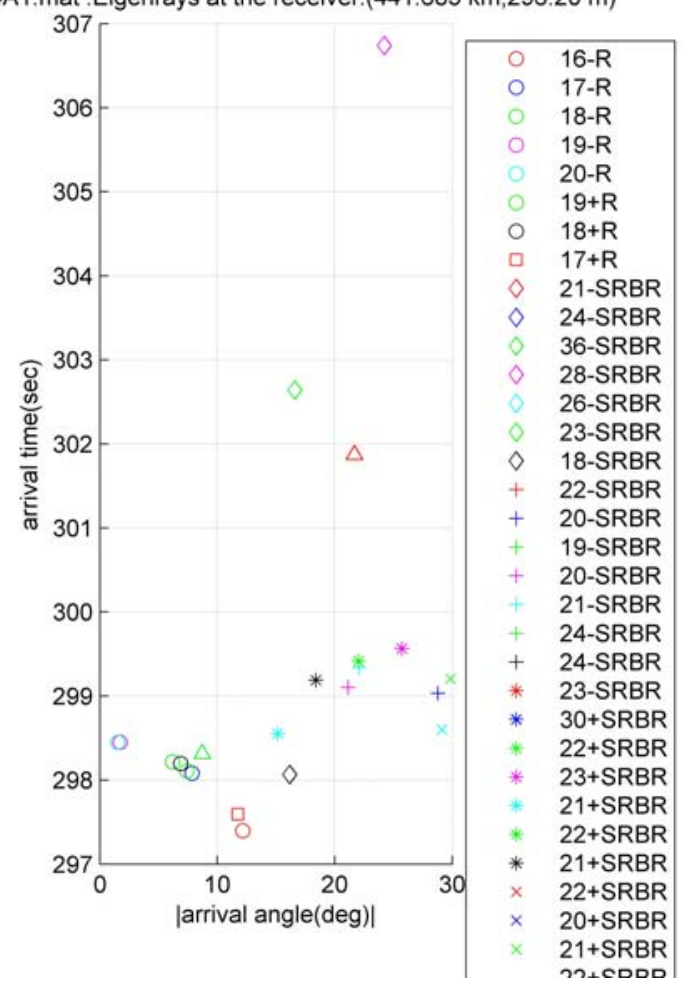

Figure 4-31: Ray arrivals from ray tracing method for 26815. Figure 4-9 provides the definition of rays. 


\subsection{Behind the Kermit-Roosevelt seamount: day 267 with the sound speed fields from the objective analysis}

In this chapter, the comparisons of the measurement and simulation are presented for the experiment performed on Julian day 267 (hereafter day 267).

Table 4-3 lists the receiver locations, which are described in Fig. 4-32. There were only eight measurements on day 267 along the acoustic path from SPICEX 2 behind the Kermit-Roosevelt seamount, and most of them were located in the shadow zone or transition region. Therefore, it is difficult to reconcile the pulse arrivals of experiments with the 2D PE simulations. Moreover, because the Kermit-Roosevelt seamount is much higher than the Elvis seamount, the perturbation by the seamount on day 267 is expected to be greater than on day 268 , which provides the larger span of the shadow zone and a more complicated arrival pattern with many reflected rays.

The sound speed fields used here are obtained from the objective analysis discussed in Section 2.2.3 because the XBT measurements are sparse along the acoustics paths between SPICEX source 2 and the receivers.

Figure 4-33 shows the comparison of peak pressures from the BASSEX experiment and the 2D PE simulation. The peak pressures from the measurement are higher than those from the 2D PE simulations, with a difference of $4 \sim 10 \mathrm{~dB}$.

Since most measurements were detected in the shadow zone or transition region, it is hard to reconcile the individual ray arrivals. Moreover, the sound speed fields from the objective analysis, with the limited measurements, could be responsible for the discrepancies in the comparisons. Figure 4-34 and Fig. 4-35 compare arrival patterns from the experiment, the 2D PE, and the ray tracing model for the selected cases, 26708 and 26715. These results show better correlation of results with higher signal levels than other cases because the receivers were located in the convergence zone. The experiment and the 2D PE simulation show good agreement in the arrival pattern of the refracted rays, while the experiment shows more reflections than the simulation. 
Table 4-3: Receiver locations in day 267 experiment for SPICEX source 2

\begin{tabular}{|c|c|c|c|}
\hline No. & Exp. & Range $(\mathrm{km})^{1}$ & ${\text { Depth }(\mathrm{m})^{2}}^{2}$ \\
\hline 1 & 26708 & 609.38 & 296.3 \\
\hline 2 & 26709 & 602.85 & 304.0 \\
\hline 3 & 26710 & 596.22 & 295.6 \\
\hline 4 & 26711 & 589.53 & 272.8 \\
\hline 5 & 26712 & 582.73 & 295.2 \\
\hline 6 & 26713 & 576.14 & 285.3 \\
\hline 7 & 26714 & 569.39 & 290.9 \\
\hline 8 & 26715 & 562.63 & 284.5 \\
\hline
\end{tabular}

${ }^{1}$ The range from the SPICEX source 2 source which is obtained from the GPS location when the first strong pulse arrives.

${ }^{2}$ Average array depth during the measurement.

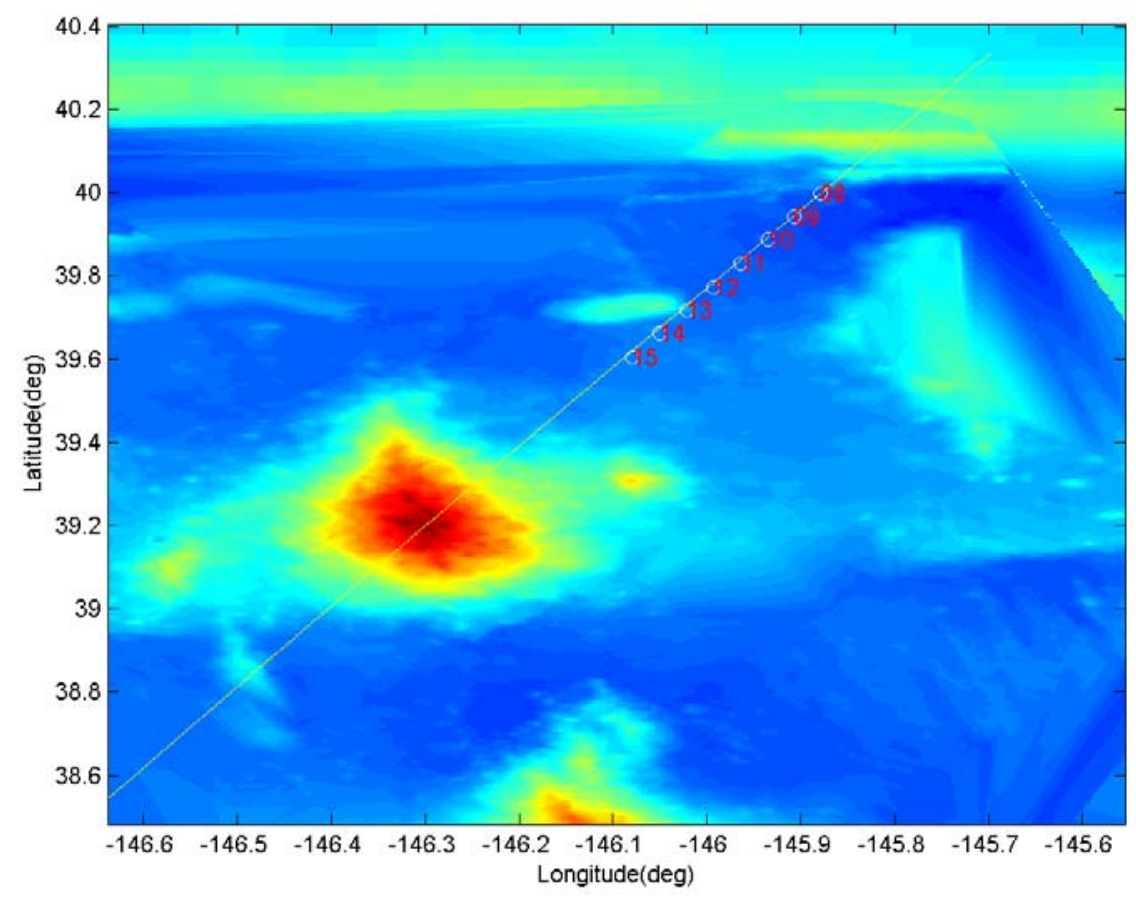

Figure 4-32: Receiver locations for day 267, as well as an acoustic path passing over the KermitRoosevelt seamount from the SPICEX source 2. 


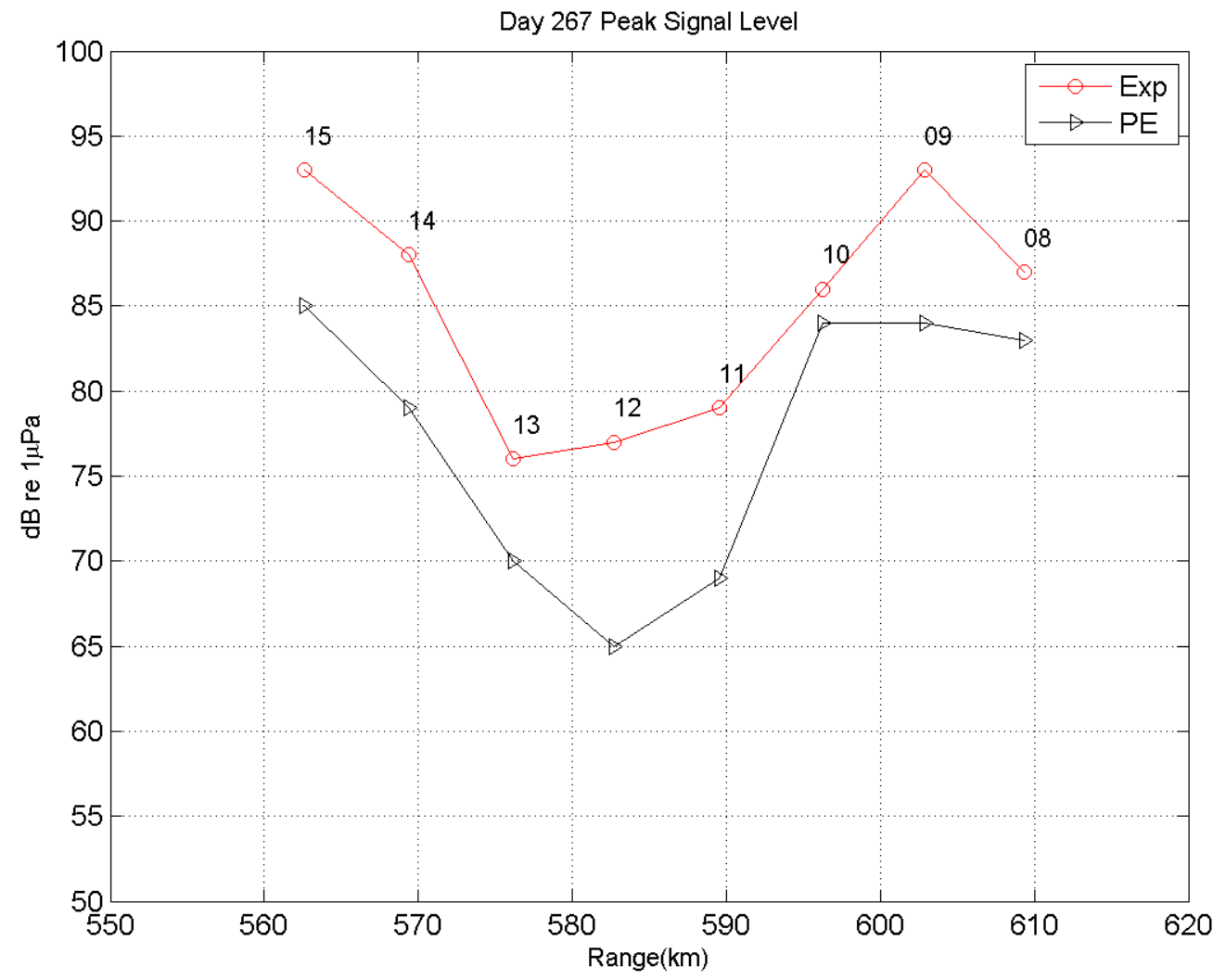

Figure 4-33: Comparison of the peak signal levels from the measurement and the 2D PE simulation. 


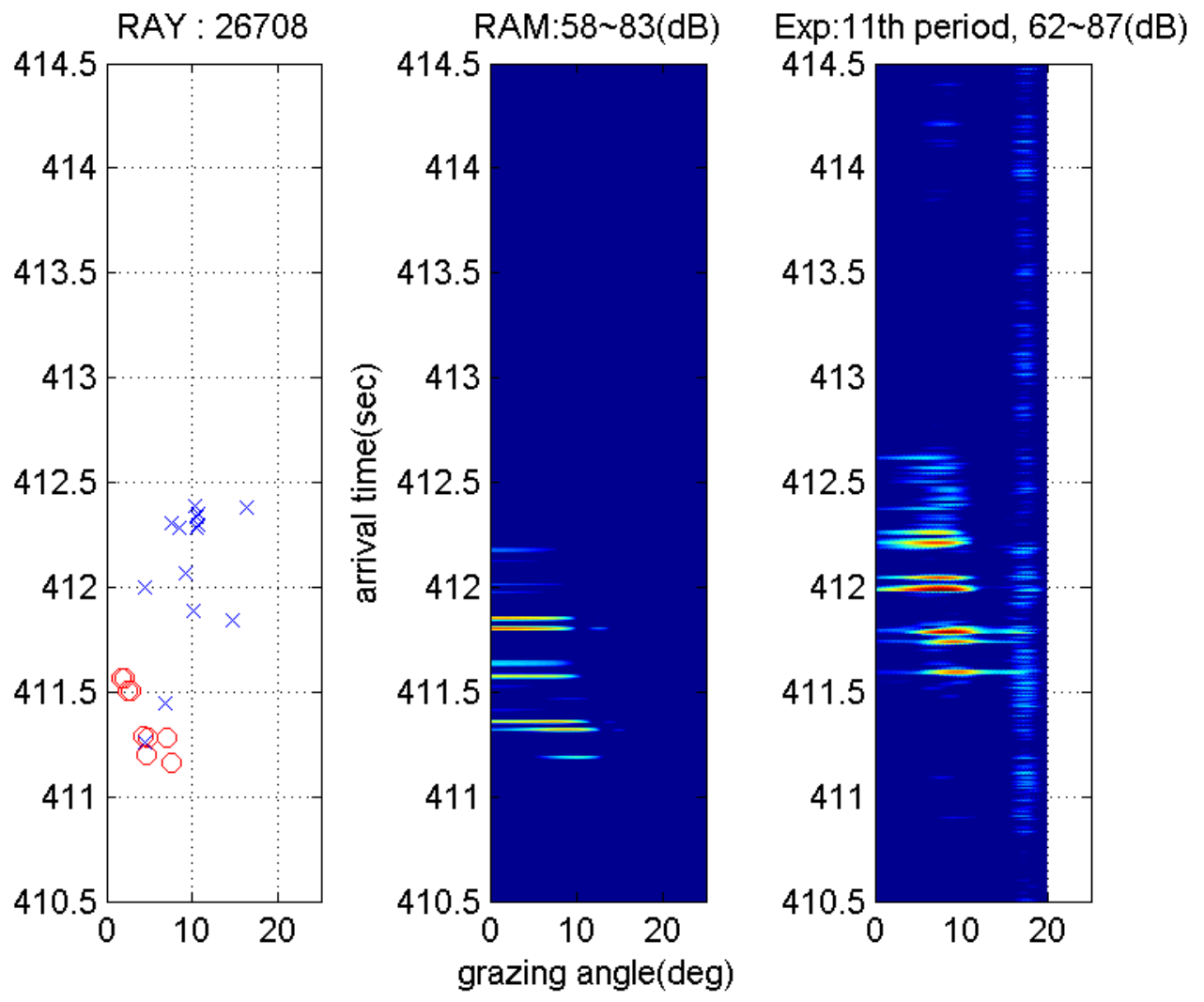

Figure 4-34: Comparison of pulse arrivals from the ray tracing method, the 2D PE, and the experiment for 26708. The ray arrivals are shown in the leftmost panel; only refracted rays are marked with circles, and the rays reflected both at the bottom and the surface are marked with crosses. Results from the 2D PE simulation and the experiment are shown in the middle and the rightmost panel with $25 \mathrm{~dB}$ dynamic range. A conventional beamformer is used for the experiment and the PE simulation. Grazing angle is defined with respect to the horizontal plane. The last of 11 periods of measurement is shown in the rightmost panel, which has the maximum amplitude among all the periods. Good correlation between pulse arrivals from the 2D PE and experiment is shown; however, the ray tracing method failed to detect some late arrivals. 

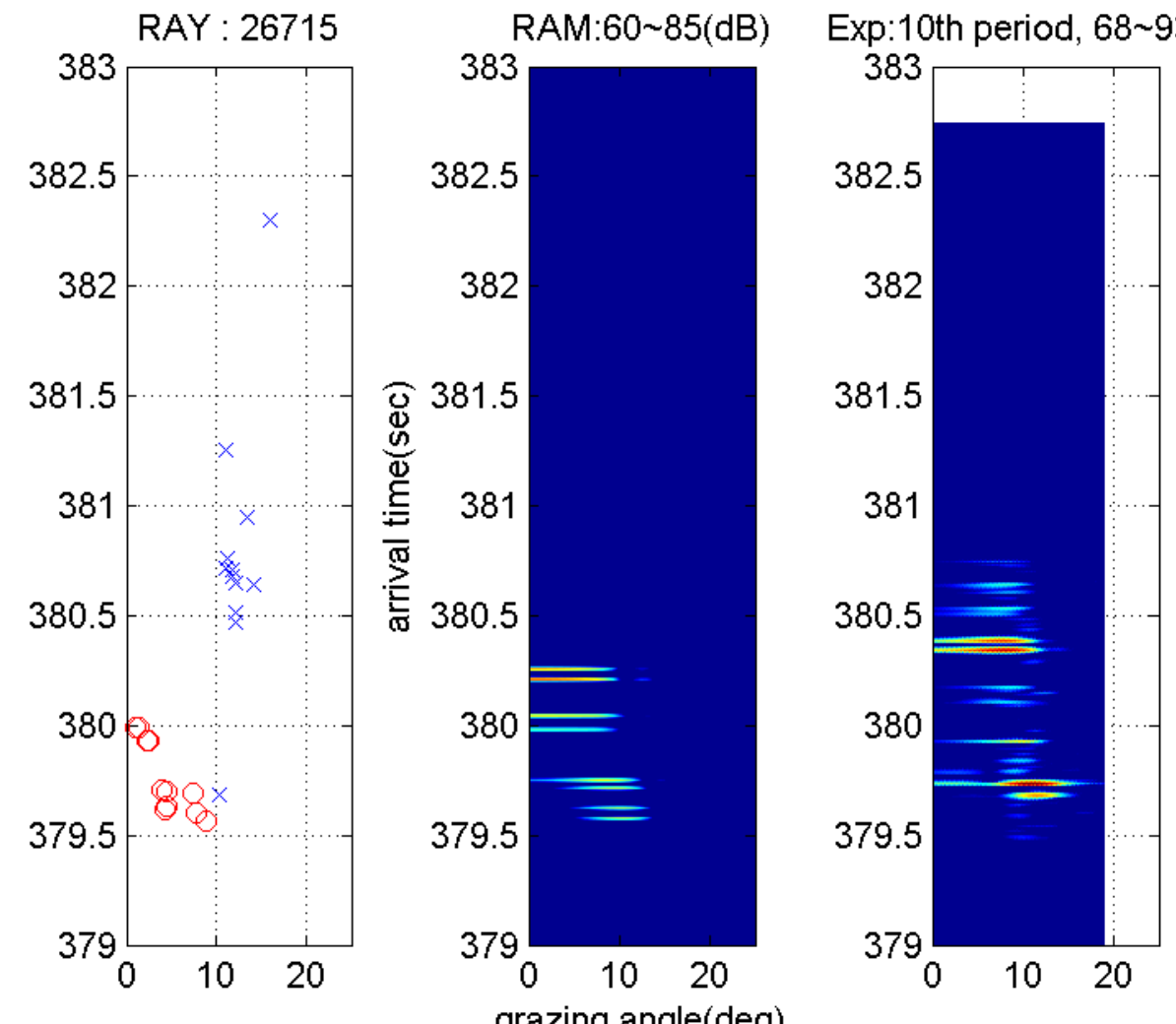

Figure 4-35: Comparison of pulse arrivals by the ray tracing method, the 2D PE, and the experiment for 26715. Good correlation between pulse arrivals from the 2D PE and experiment is shown; however, the ray tracing method fails to detect some late arrivals. 


\subsection{Behind Kermit-Roosevelt seamount: day 264 with the sound speed fields from the objective analysis}

In this chapter, the comparisons of the measurement and simulation are presented for the experiment performed on Julian day 264 (hereafter day 264).

Table 4-4 lists the receiver locations, which are described in Fig. 4-36. The receivers are located behind the Kermit-Roosevelt seamount. The sound speed fields used here are obtained from the objective analysis discussed in Section 2.2.3 because there was no sound speed measurement around SPICEX source 1.

Fig. 4-37 shows the comparison of peak pressures from the measurement and the 2D PE simulation. Note that the large drop of signal level is shown in a shadow zone just behind the seamount, around $640 \mathrm{~km}$ from the source (26417). Another shadow zone appears around 680 700 km from the source. The peak of the seamount is located at around $620 \mathrm{~km}$. The peak pressures are quite close to each other $(1 \sim 5 \mathrm{~dB})$ in most cases, with the exception of 26415 and 26417.

Figure 4-38 and Fig. 4-39 present the compared arrival patterns from the BASSEX experiment, 2D PE, and the ray tracing model. Figure 4-38 shows good correlations between results for the refracted ray arrivals; strong refracted ray arrivals are clearly shown in all the results. In the experiment, complicated reflections are detected in addition to the refracted rays. In Fig.4-39, the receivers are located above the KermitRoosevelt seamount. As expected, complicated reflections with the higher angle arrivals are shown even before the refracted ray early arrivals. The ray tracing model detects strong only-bottom reflections, which is quite consistent with the experiment. However, it is difficult to reconcile these reflected waves because geoacoustic properties and local complex bathymetry play an important role in these complicated reflections. 
Table 4-4: Receiver locations in day 264 experiment for SPICEX source 1

\begin{tabular}{|c|c|c|c|}
\hline No. & Exp. & Range $(\mathrm{km})^{1}$ & Depth(m) ${ }^{2}$ \\
\hline 1 & 26408 & 716.46 & 257.3 \\
\hline 2 & 26409 & 708.82 & 254.6 \\
\hline 3 & 26410 & 701.57 & 278.1 \\
\hline 4 & 26412 & 686.88 & 261.8 \\
\hline 5 & 26413 & 679.60 & 269.3 \\
\hline 6 & 26414 & 672.25 & 272.6 \\
\hline 7 & 26415 & 664.71 & 280.3 \\
\hline 8 & 26417 & 650.45 & 267.2 \\
\hline 9 & 26419 & 635.33 & 253.3 \\
\hline 10 & 26420 & 627.80 & 255.2 \\
\hline
\end{tabular}

${ }^{1}$ The range from the SPICEX 1 source which is obtained from the GPS location when the first strong pulse arrives.

${ }^{2}$ Average array depth during the measurement.

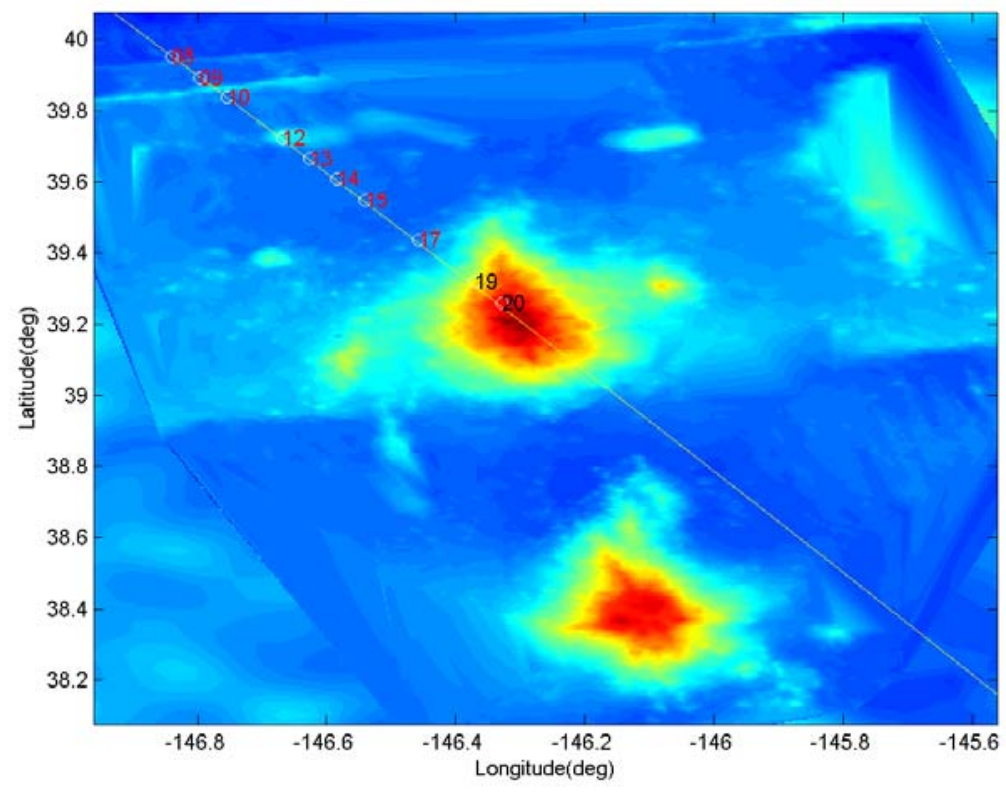

Figure 4-36: Receiver locations for day 264, as well as an acoustic path passing over the KermitRoosevelt seamount. 


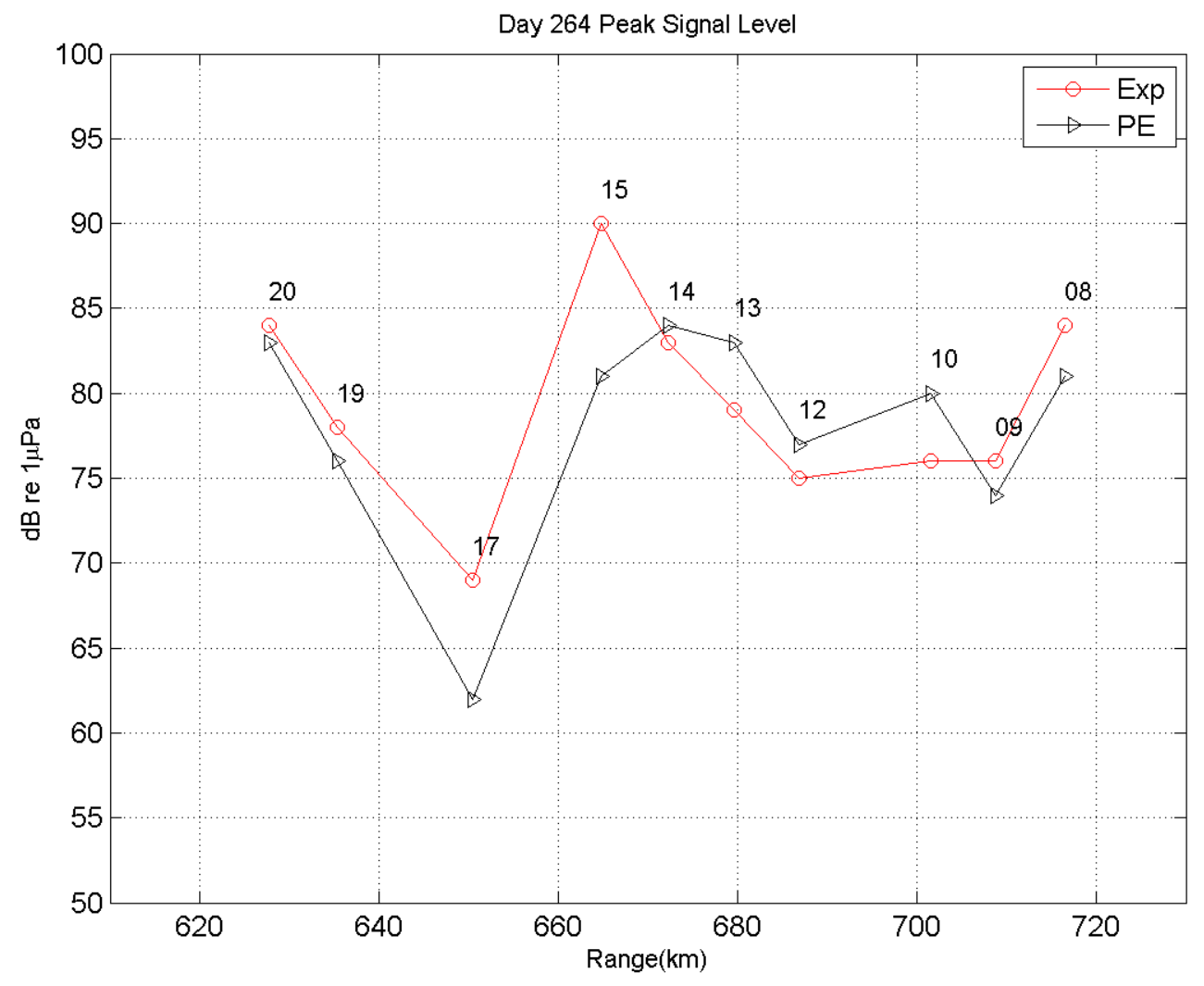

Figure 4-37: Comparison of peak signal levels from the measurement and 2D PE simulation. 

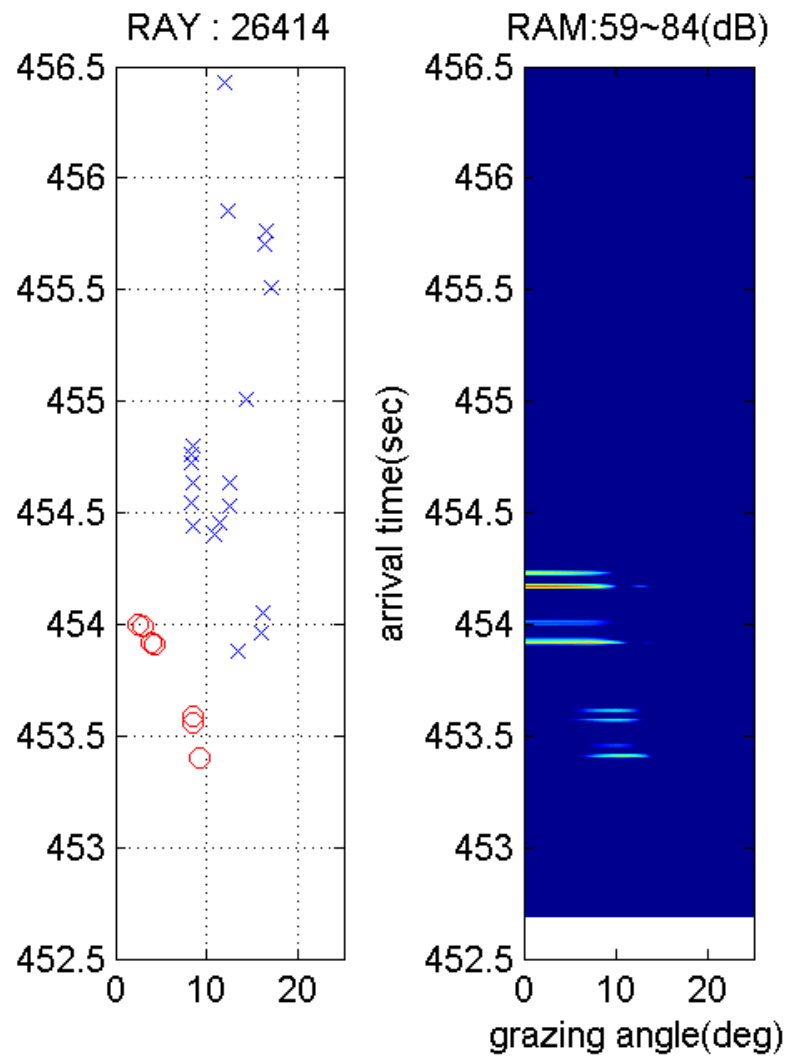

Exp: 2th period, $58 \sim 83(\mathrm{~dB})$

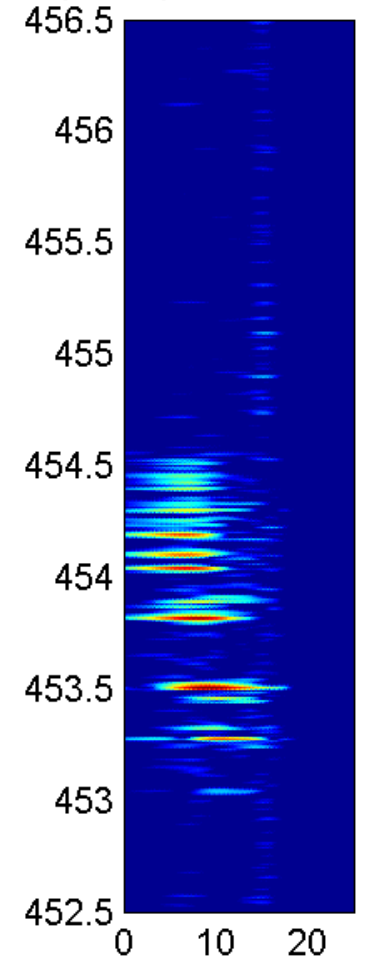

Figure 4-38: Comparison of pulse arrivals by the ray tracing method, 2D PE, and the experiment for 26414. The ray arrivals are shown in the leftmost panel; only refracted rays are marked with circles, and rays that reflect both the bottom and the surface are marked with crosses. Results from the PE simulation and the experiment are shown in the middle and the rightmost panel with $25 \mathrm{~dB}$ dynamic range. A conventional beamformer is used for the experiment and the $\mathrm{PE}$ simulation. Grazing angle is defined with respect to the horizontal plane. The second period of 11 periods of measurement is shown in the rightmost panel, which has the maximum amplitude among all periods. Strong refracted ray arrivals are clearly shown for all results. In the experiment, complicated and many reflections exist in addition to the refracted rays, which are not detected in the 2D PE simulation. The ray tracing method detects many reflected waves; however, it is difficult to match these waves with those from the experiment. 


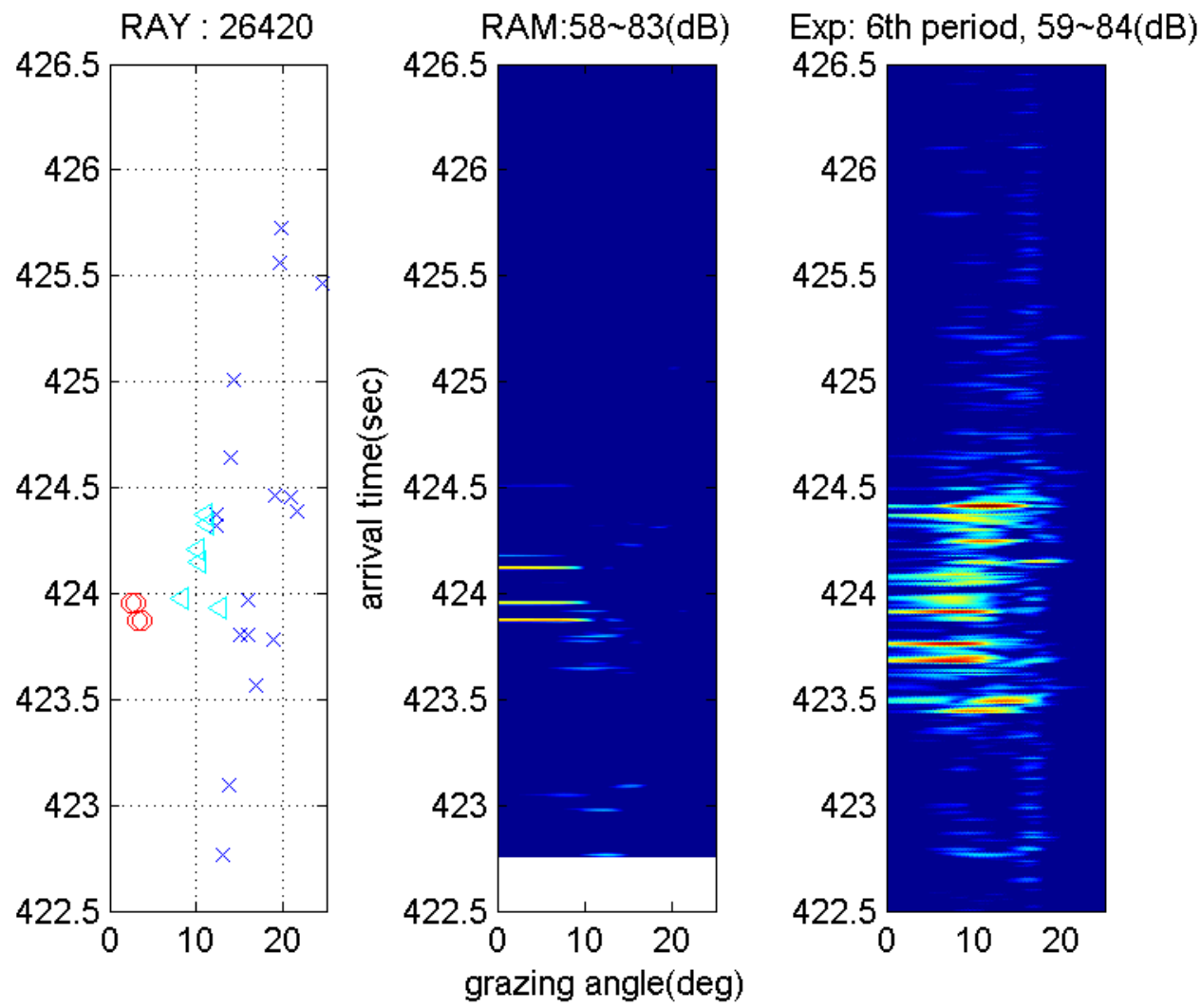

Figure 4-39: Comparison of pulse arrivals by the ray tracing method, 2D PE, and the experiment for 26420. The cyan triangles denote the only-bottom reflection rays. The receivers were located above the Kermit-Roosevelt seamount. As expected, complicated reflections with the higher angle arrivals were detected even before the refracted ray arrival. As shown in the results from the ray tracing method, the only-bottom reflections are strong, which is quite consistent with the experiment. 


\subsection{Open sea case: day 271 with the sound speed fields from the objective anlaysis}

In this chapter, the comparisons of the measurement and the 2D PE simulation are presented for the experiment performed on Julian day 271 (hereafter day 271).

Table 4-5 and Fig. 4-40 show the receiver locations. The ship was moving downward between the two SPICEX source 1 and 2 during the experiment; there are 10 measurements for the two sources. Sound speed profiles are obtained from the objective analysis discussed in Section 2.2.3.

These open sea cases are used for the calibration of the sound speed fields using the objective analysis. Sound speed fields with many parameters were tested for the best correlation to the arrival pulses. The random timing error of FORA array ( $<0.25$ seconds) leads to a serious problem in tuning of the sound speed fields; therefore, the best correlation was chosen based only on the overall pattern of the arrival pulses. The 2D PE simulation was performed with the selected sound speed fields from the calibration.

Figure 4-41 and Fig. 4-42 show the comparison of arrival pulses in terms of arrival time. The arrival pulses are quite close to each other; however, in some cases, the experiment shows quite small amplitudes, e. g., 27100.

In Fig. 4-43, the arrival pulses from the BASSEX experiment (black) and the PE simulation (red) are directly compared for the SPICEX source 1 at the top panel and SPICEX source 2 at the bottom panel. Amplitudes are normalized by the maximum value. The error of arrival time is not corrected. In addition to the random timing error of the FORA array, the uncertainty of the sound speed fields could increase the error of the arrival time; however, the arrival time error can not analyzed because of the random timing error of the FORA array. 
Table 4-5: Receiver locations in day 271 experiment for SPICEX source 1 and 2

\begin{tabular}{|c|c|c|c|c|}
\hline No. & Exp. & Range $^{1}(\mathrm{~km})$ from S1 & Range $(\mathrm{km})$ for S2 & ${\text { Depth }(\mathrm{m})^{2}}^{2}$ \\
\hline 1 & 27100 & 378.616 & 332.339 & 252.6 \\
\hline 2 & 27102 & 359.908 & 317.372 & 339.4 \\
\hline 3 & 27104 & 340.642 & 300.357 & 325.0 \\
\hline 4 & 27106 & 322.671 & 287.201 & 340.0 \\
\hline 5 & 27108 & 306.605 & 273.254 & 319.0 \\
\hline 6 & 27110 & 291.888 & 263.294 & 351.0 \\
\hline 7 & 27112 & 279.075 & 253.214 & 244.0 \\
\hline 8 & 27114 & 266.948 & 249.876 & 340.7 \\
\hline 9 & 27116 & 256.909 & 248.204 & 325.9 \\
\hline 10 & 27118 & 249.364 & 250.545 & 325.1 \\
\hline
\end{tabular}

${ }^{1}$ The range from the SPICE source 1 and 2, which is obtained from the GPS location when the first strong pulse arrives.

${ }^{2}$ Average array depth during measurement. 


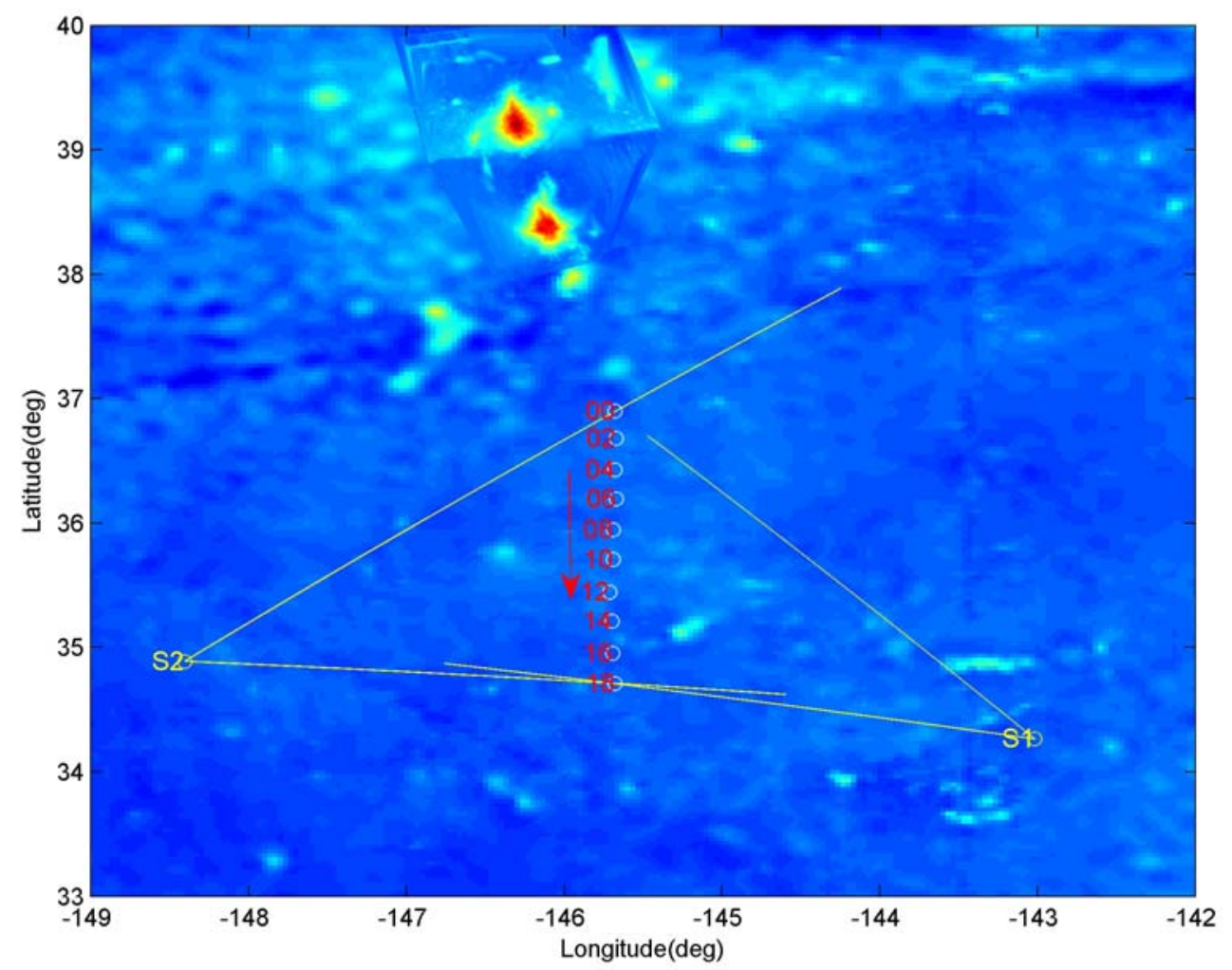

Figure 4-40: Receiver locations for day 271; two acoustic paths are shown for 27100 and 27108. 

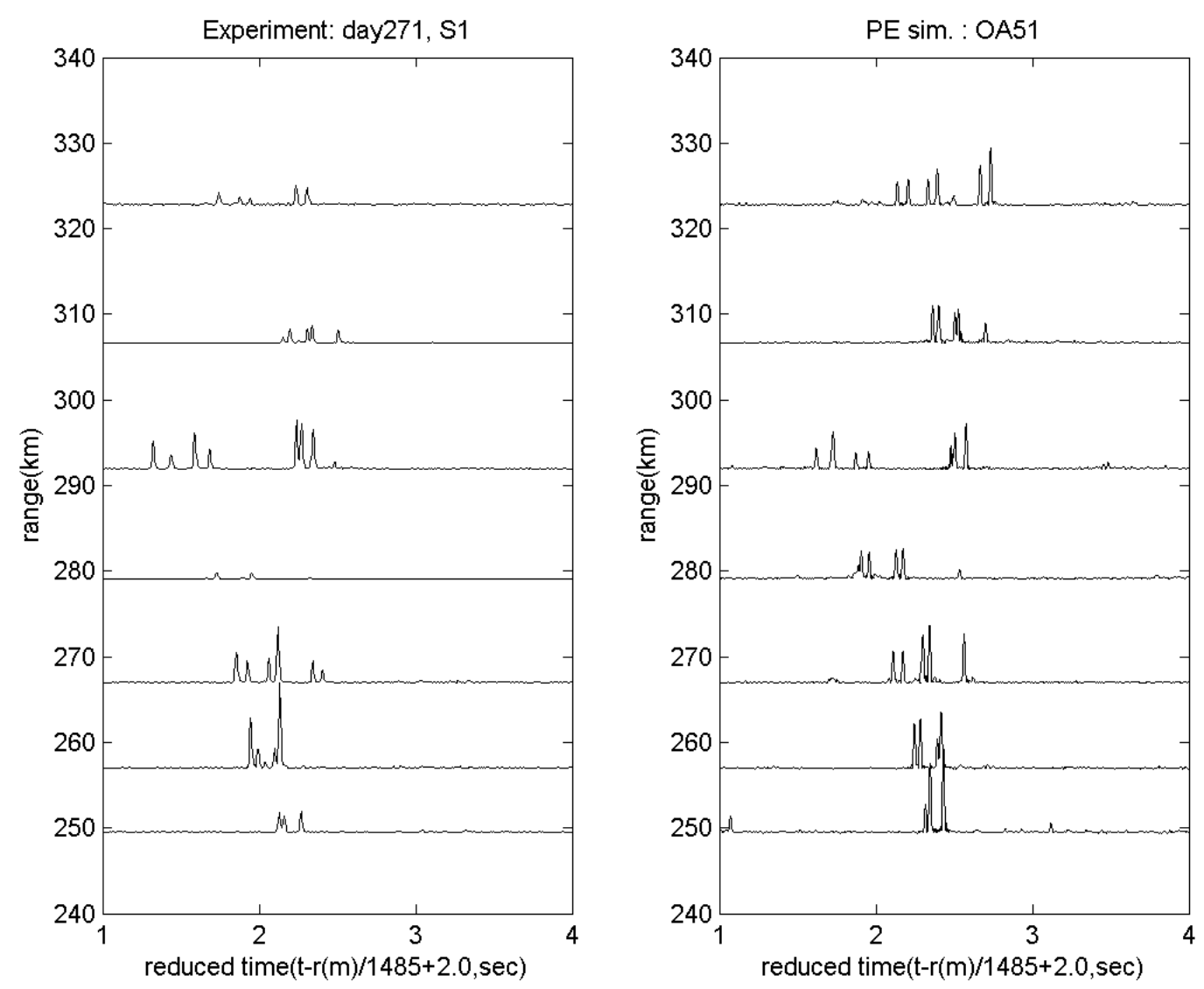

Figure 4-41: Comparison of arrival pulses from experiment (left panel) and PE simulation (right panel) for day 271, SPICEX source 1. 

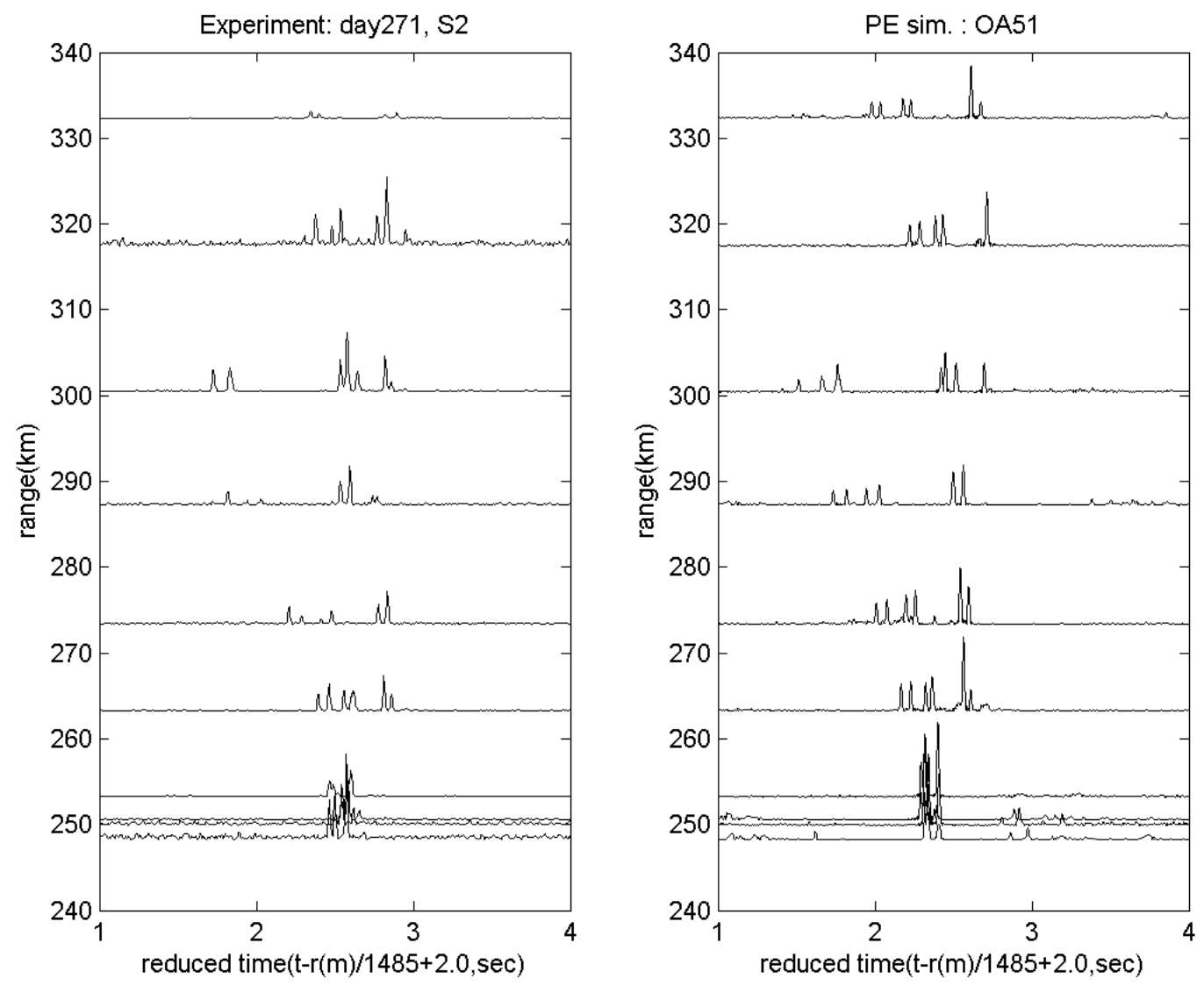

Figure 4-42: Comparison of arrival pulses from experiment (left panel) and PE simulation (right panel) for day 271, SPICEX source 2. 

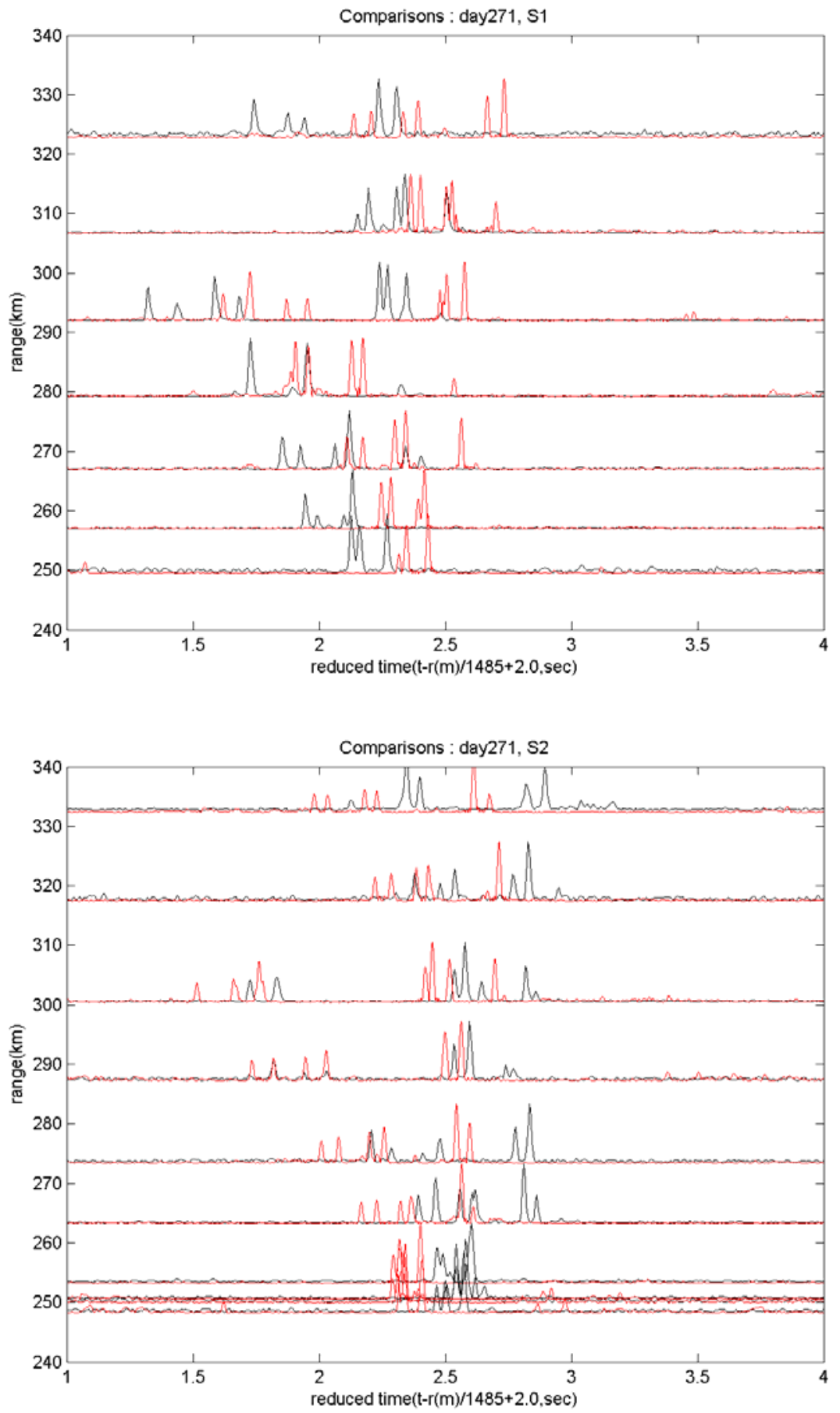

Figure 4-43: Comparison of arrival pulses from the experiment (black) and PE simulation (red) for day271, SPICEX source 1 (top panel) and SPICEX source 2 (bottom panel). Arrival time errors were not corrected. Amplitudes are normalized by the maximum amplitude. 


\subsection{Nx2D simulation with a simplified conical seamount approximation and a range-averaged sound speed profile}

In this section, the Nx2D simulation by the 2D PE for an approximated conical seamount is explored. In the Nx2D simulation, the 2D PE simulations are performed for many vertical planes, and the solutions are combined to form an approximated 3D problem without the cross-coupling between the solutions.

Figure 4-44 shows the four different acoustic paths from SPICEX source 1 and 2 to the Kermit-Roosevelt and Elvis seamounts; the acoustic paths were used for approximating the seamounts with conical shapes. The acoustic paths are as follows:

1) from the SPICEX source 1 to the apex of the Kermit-Roosevelt seamount (day 264),

2) from the SPICEX source 1 to the apex of the Elvis seamount (day 265),

3) from the SPICEX source 2 to the apex of the Kermit-Roosevelt seamount (day 267), and

4) from the SPICEX source 2 to the apex of the Elvis seamount (day 268).

Hence, Fig. 4-45 shows the range-dependent bathymetry along the acoustic paths with the conical shapes. For each approximated conical seamount, 14 acoustic paths were considered for the 2D PE simulation; the half of the conical seamount is divided into 14 vertical sections for the acoustic paths.

To examine the bathymetric effect, range-averaged sound speed profiles are considered. The range-averaged sound speed profiles for the four acoustic paths are given in Fig. 4-46; the sound speed profiles are obtained from the XBT casts in the BASSEX experiment. Figures 4-48 and 4-50 show the resultant conical seamounts and sound speed fields. Figure 4-47 shows the range-stacked arrival pulses for 7 selected vertical planes out of the 14 vertical planes. The arrival pulses describe the blockage effect of the 
seamount by showing the significant change of the disturbed arrival pulses. Note that the blocked refraction waves are clearly correlated with the height of the cross sections along the acoustic paths. This correlation can be explained by the fact that higher steep angle rays are much more blocked by the seamount than lower angle rays.

In Fig. 4-49 and 4-51, energy plots are constructed by the summation of squares of arrival pulse amplitudes at a receiver depth of $250 \mathrm{~m}$. The energy levels for each section are computed, and then the levels are linearly interpolated in azimuthal direction. Note that the discontinuity of convergence zones in the figures is explained by the discrepancy in the sound speed profiles. Although the sound speeds appear to be very close to each other, as shown in Fig. 4-46, the small discrepancy in the sound speeds results in a relatively large phase discrepancy in long-range sound propagation.

In Fig. 4-52, the computed energy is compared with the measured energy in the BASSEX experiment [53]. Although we use very simplified Nx2D model, the locations and the sizes of shadow zones show good agreement. This good agreement indicates that arrival energy is not so sensitive to the environments.

Note that, in the energy plots, some strong energy arrivals appear just before the apex of the seamount, which are not clearly shown in the measured energy. These strong energy arrivals could be explained by: 1) in the 2D model, we could not include the horizontal refraction effect by which energy can propagate out of the plane, and 2) in 2D $\mathrm{PE}$, there is no backscattering effect. Therefore, rays or energy could be trapped just before the seamount due to the 2D PE model. 

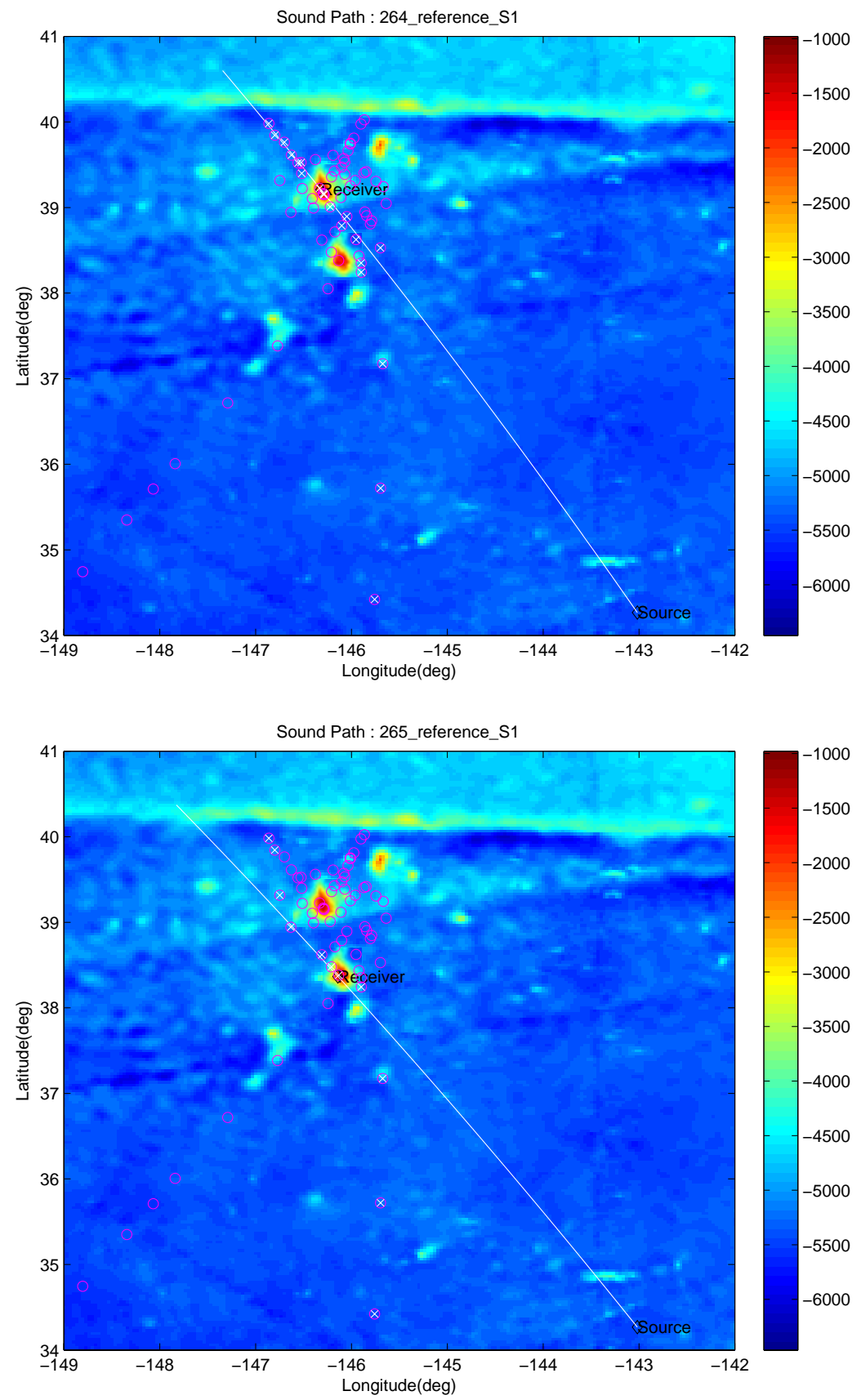

Figure 4-44: Acoustic paths for the four acoustic paths: day 264 from the SPICEX source 1 to the apex of the Kermit-Roosevelt seamount, day 265 from the SPICEX source 1 to the apex of the Elvis seamount, day 267 from the SPICEX source 2 to the apex of the Kermit-Roosevelt seamount and day 268, from the SPICEX source 2 to the apex of the Elvis seamount. 

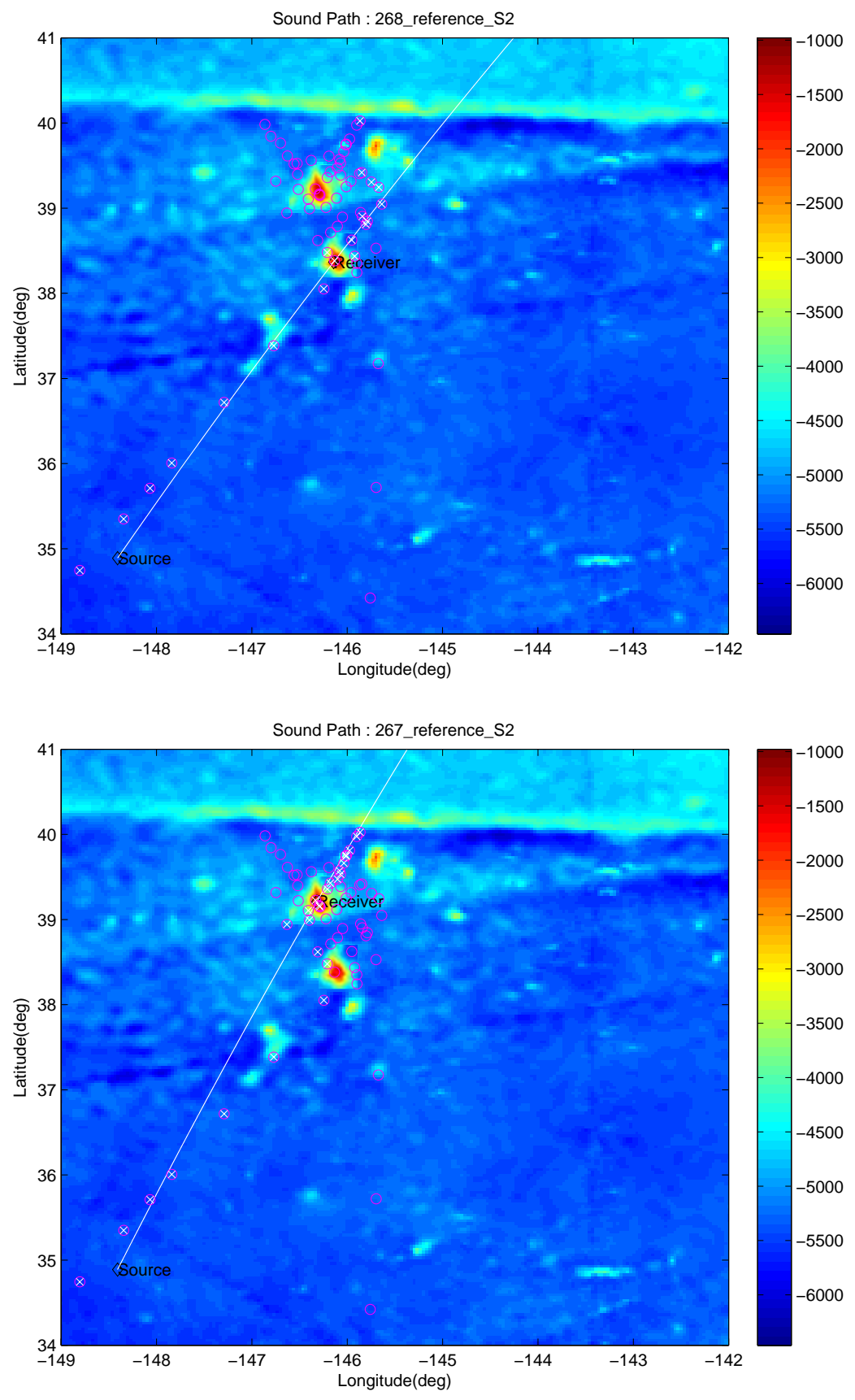

Figure 4-44: continued. 

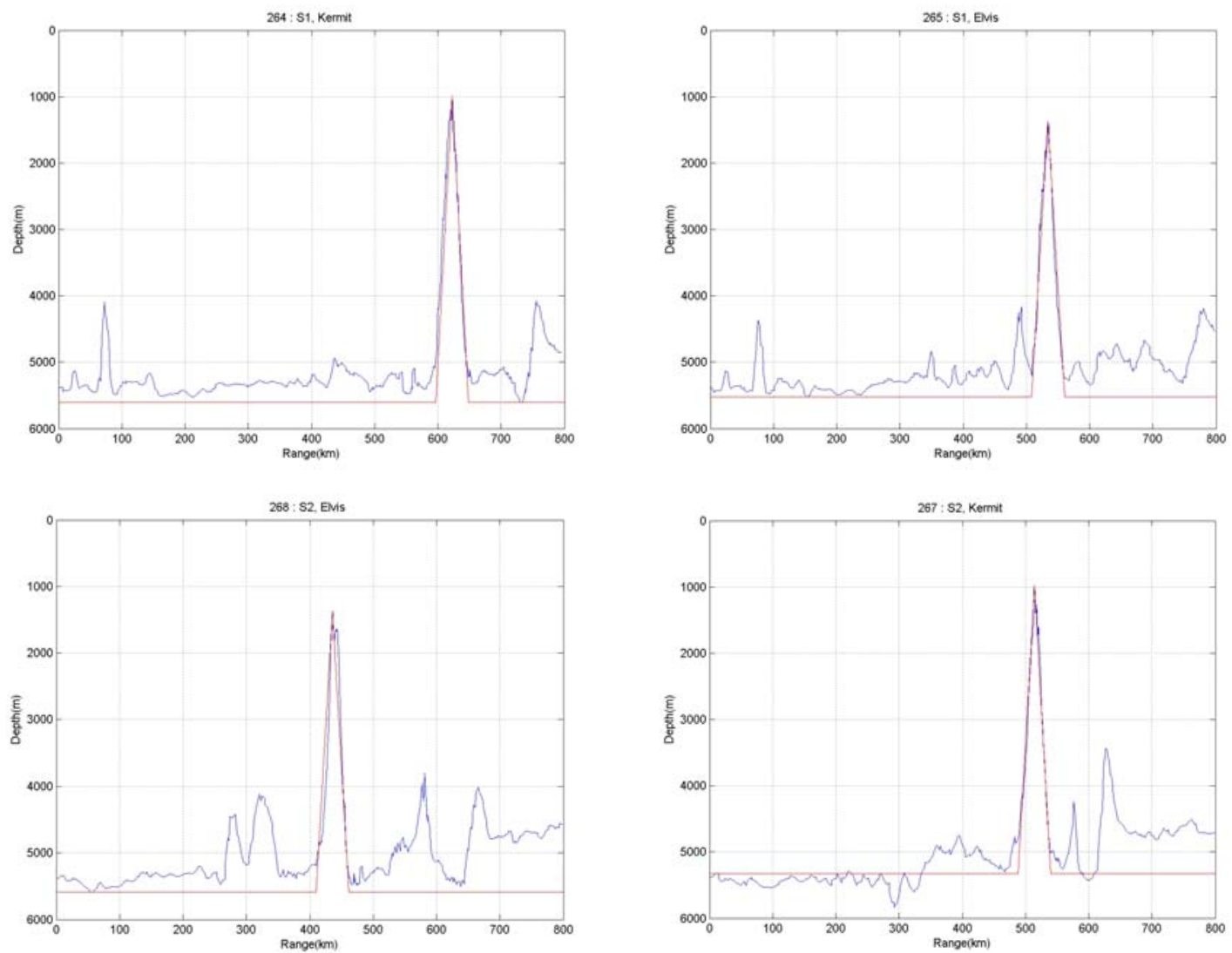

Figure 4-45: Approximated conical seamounts for the four acoustic paths.

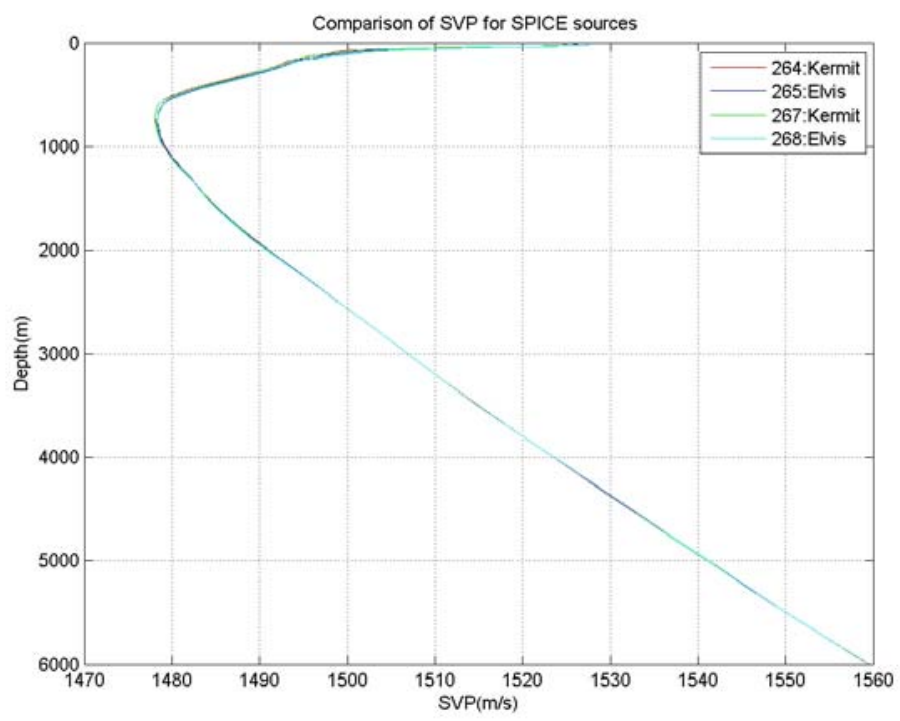

Figure 4-46: Range-averaged sound speed fields for the four acoustic paths; sound speeds are obtained from the XBT casts. 

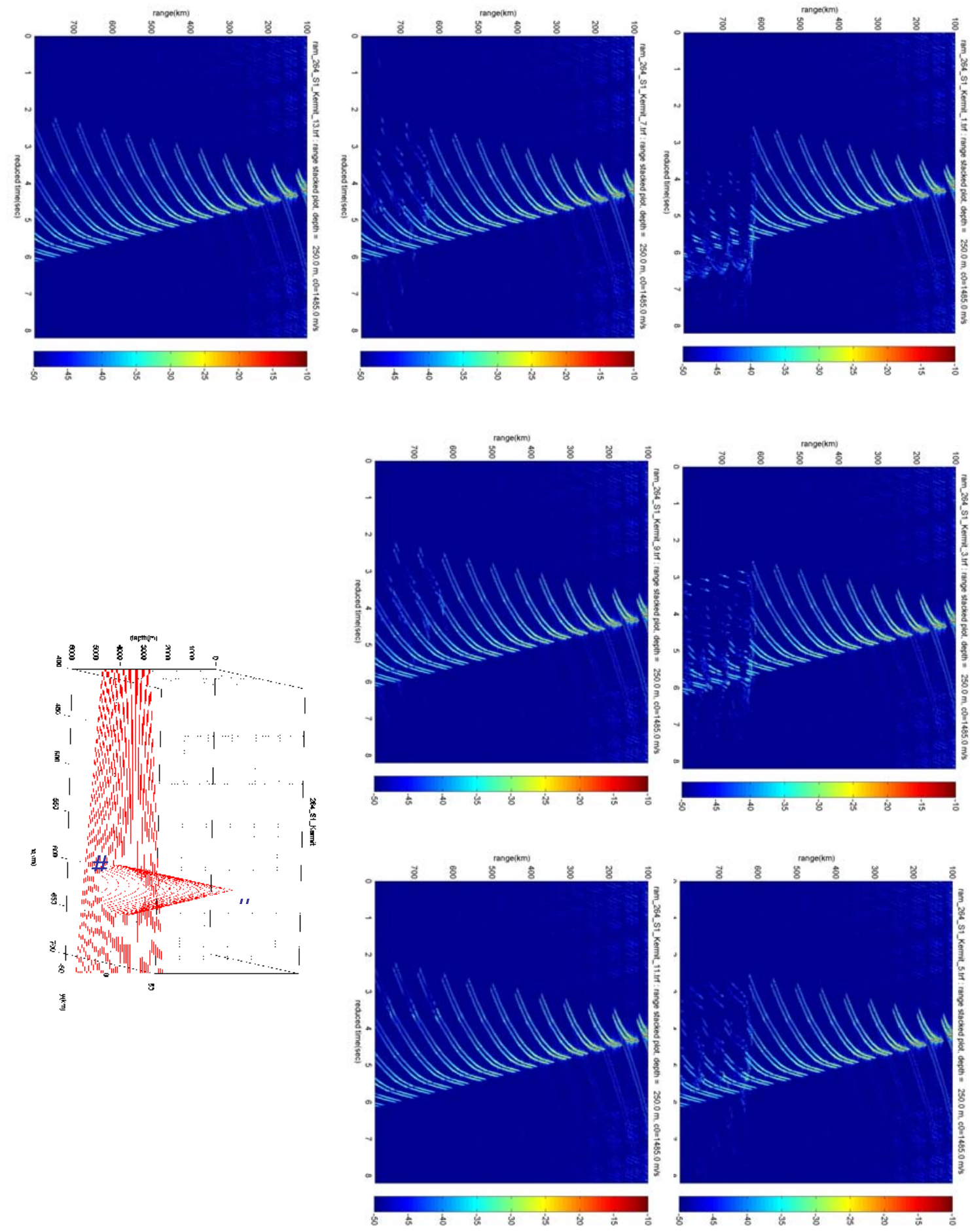

Figure 4-47: Range-stacked pulse arrivals for the acoustic paths from the centerline to the outermost ring. 

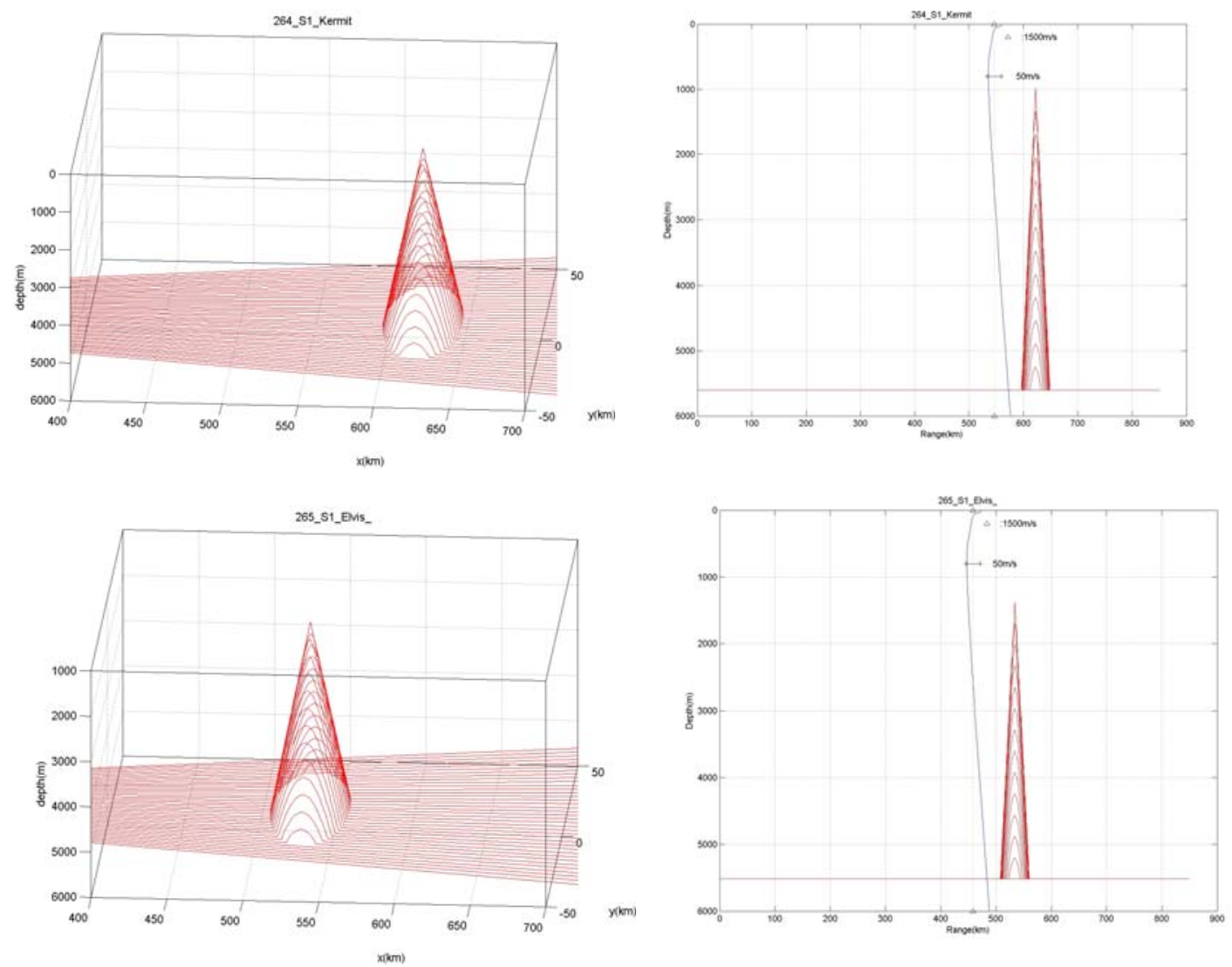

Figure 4-48: Conical seamounts with range-averaged sound speed fields.

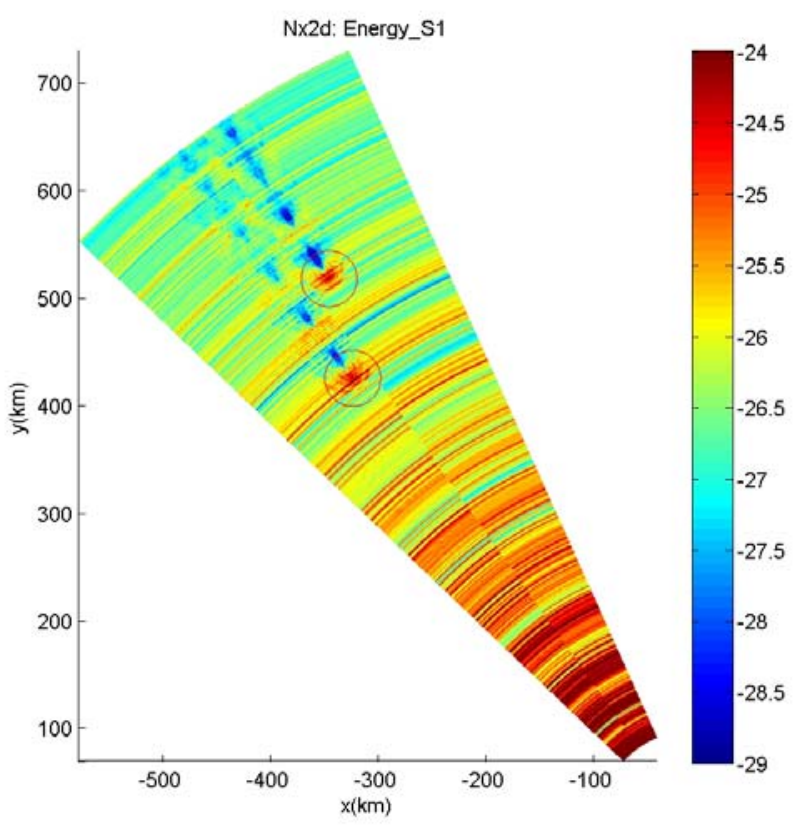

Figure 4-49: Energy plot for the SPICEX source 1. 

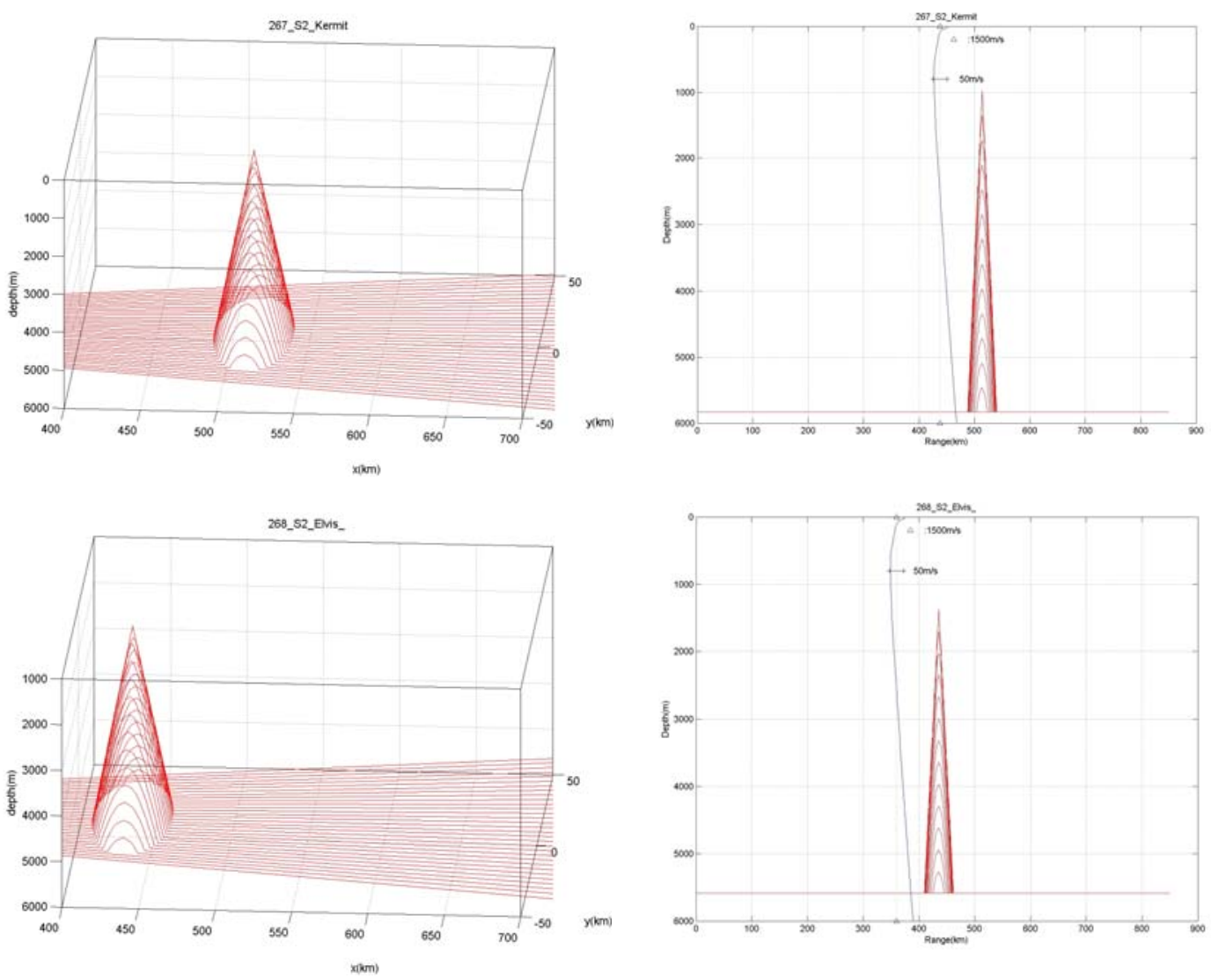

Figure 4-50: Conical seamounts with range-averaged sound speed fields.

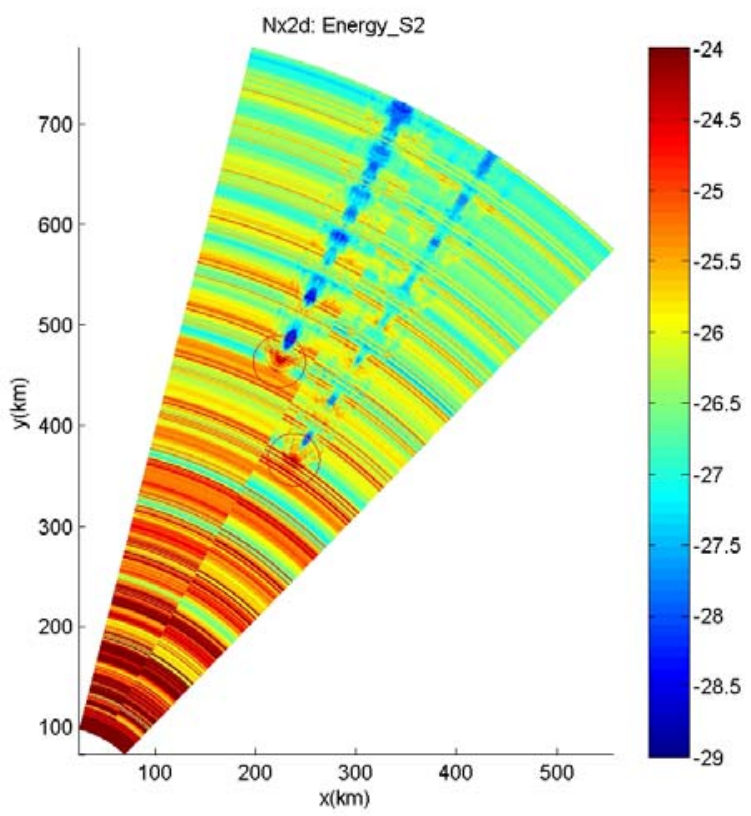

Figure 4-51: Energy plot for the SPICEX source 2. 

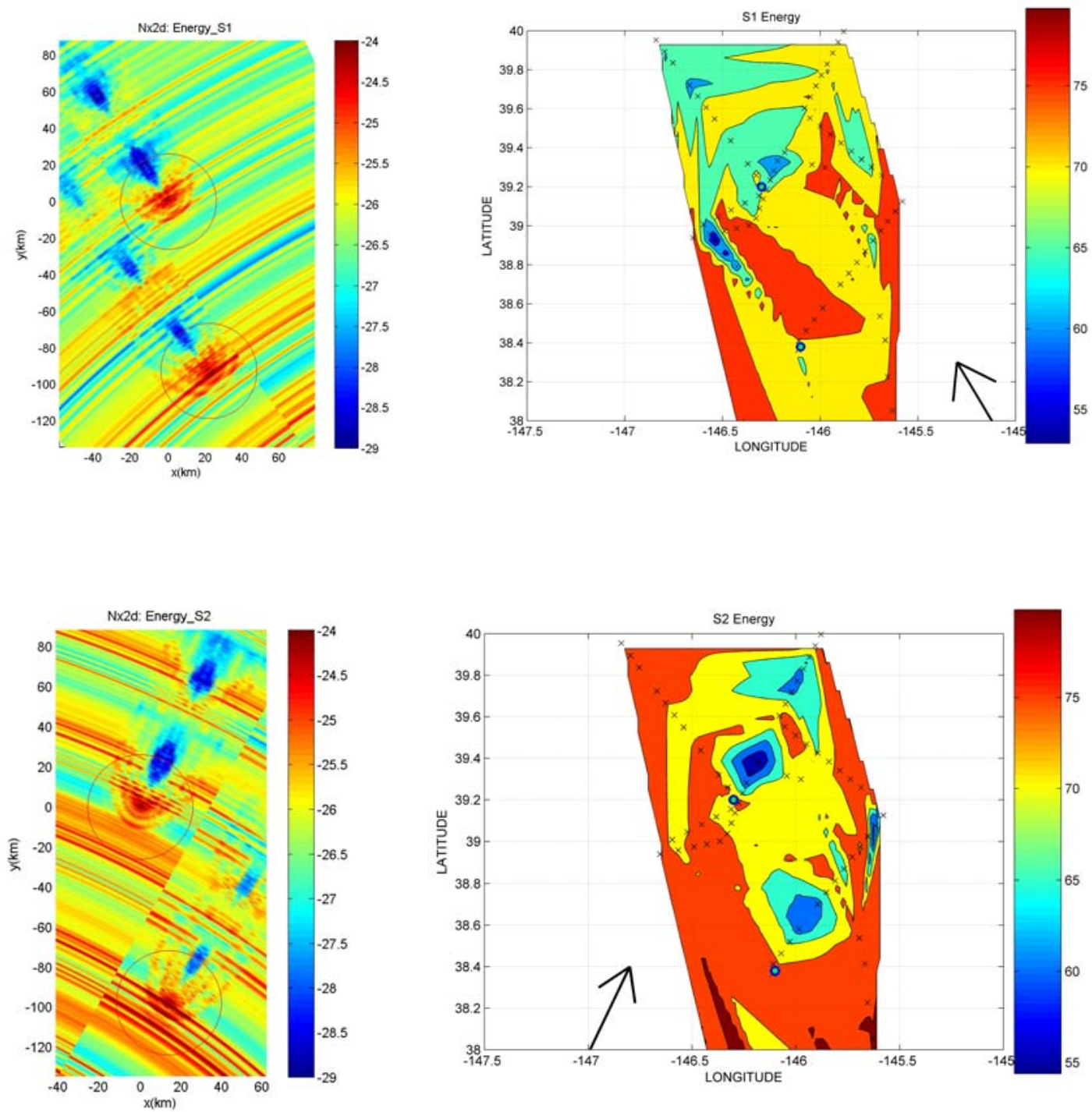

Figure 4-52: Comparison of the arrival energy between the Nx2D simulation and measurement (the measurements are adapted from [53]). 


\section{Chapter 5}

\section{Three-dimensional sound propagation around a}

\section{seamount}

In Chapter 4, we reconciled the measured broadband pulses between the BASSEX experiment and the two-dimensional (2D) sound propagation model. The 2D parabolic equation (PE) and the ray tracing method were used for the comparison. In the reconciliation of broadband pulses, we considered the range-dependent bathymetry and sound speed fields. However, in the Nx2D computation for the comparison of arrival energy, a simplified conical seamount was examined with the range-averaged sound speeds.

In this chapter, three-dimensional (3D) broadband pulses are explored around a conical seamount for the acoustic broadband sources using the 3D spectral coupled-mode model, which is described in Section 3.5. To obtain a broadband pulse, the developed 3D spectral coupled mode model with higher computational efficiency and parallel computing ability is used.

This chapter consists of three parts. First, to validate the 3D spectral coupled-mode model for sound propagation around a seamount, comparisons of transmission loss (TL) and broadband pulses are performed using various 2D and 3D sound propagation models, including the 2D PE (RAM [12]), 3D PE (FOR3D[15]), 2D coupled normal mode program (CSNAP[63]), and 3D spectral coupled-mode model. The comparison results are described in Section 5.1.

Second, a benchmark problem in a deep-sea waveguide similar to the configuration of the BASSEX experiment is considered in Section 5.2. In this work, the 3D spectral coupled-mode model is applied to the benchmark problem for an acoustic broadband source with a center frequency of $15 \mathrm{~Hz}$ (10 Hz bandwidth). 
Third, the Kermit-Roosevelt seamount is modeled with a simple conical seamount. In this work, two broadband acoustic sources are considered: a center frequency of $15 \mathrm{~Hz}$ (10 Hz bandwidth), and a center frequency of $68.2 \mathrm{~Hz}(16 \mathrm{~Hz}$ bandwidth, LOAPEX source, T1000). However, due to the limited computational ability, only water-trapped modes are computed for the $68.2 \mathrm{~Hz}$ source. The resultant broadband pulses using the 3D coupled-mode model are described in Section 5.3.

\subsection{Comparison of two-dimensional and three-dimensional sound propagation models for a shallow-water waveguide with a conical seamount}

To validate the 3D spectral coupled-mode model program, a series of comparisons are performed as follows:

a) Comparison of transmission loss between 2D models (CSNAP, RAM and FOR3D),

b) Comparison of transmission loss between Nx2D and 3D models (FOR3D),

c) Comparison of transmission loss between 3D models (FOR3D, 3D spectral coupled-mode model), and

d) Comparison of broadband pulses between 2D and 3D models (CSNAP, RAM, FOR3D, and 3D spectral coupled model).

Through these comparisons, we examine the accuracy of the widely used 2D and 3D models for sound propagation around a conical seamount. In particular, the accuracy of the 3D spectral coupled-mode model is evaluated by the comparisons. In addition, we explore the significant discrepancies between the 2D and 3D models, as well as between the PE and the coupled normal mode model. 


\subsubsection{Description of problem}

Here we consider a shallow water waveguide with a water depth of $250 \mathrm{~m}$. A conical seamount with a height of $200 \mathrm{~m}$ is located at a range of $5 \mathrm{~km}$ from the acoustic source, as shown in Fig. 5-1. The radius of the seamount at the base is $350 \mathrm{~m}$, and the slope is around $30^{\circ}$, which is quite steep. We assume a Pekeris wave guide with an isovelocity water column of $1500 \mathrm{~m} / \mathrm{s}$. The sub-bottom properties are described in Table 5-1. For RAM and FOR3D, an absorbing layer with high attenuation $(10 \mathrm{~dB} / \lambda)$ is used to prevent spurious reflections from the bottom boundary.

Figure 5-2 shows a broadband acoustic source, a center frequency of $50 \mathrm{~Hz}(50 \mathrm{~Hz}$ bandwidth) [28]:

$$
S(t)=\left\{\begin{array}{cl}
\frac{1}{2} \sin \omega_{c} t\left(1-\cos \frac{1}{4} \omega_{c} t\right), & 0 \leq t \leq 4 / f_{c} \\
0, & \text { else }
\end{array} .\right.
$$

A broadband pulse is obtained from the Fourier synthesis method described in Section 3.4.

\subsubsection{Comparison of transmission loss for a CW source $(40 \mathrm{~Hz})$}

A convergence test is performed for a CW source $(40 \mathrm{~Hz})$ to determine the adequate grid size in range and depth for the PE models, RAM and FOR3D. Figure 5-3 shows the transmission loss (TL) using the RAM (top panel) and FOR3D (bottom panel) with varying grid sizes. As a result, the FOR3D requires a smaller step size $(<10 \mathrm{~m})$ in range than the RAM $(<20 \mathrm{~m})$ for equivalent solution. In addition, the range step size was tested for the CSNAP, as shown in Fig. 5-4. In the CSNAP, the range step size is defined with a number of steps for the radius of the seamount.

Figure 5-5 shows the comparison results of TL using the RAM, CSNAP, and FOR3D with the obtained parameters from the convergence test. The TL from the RAM 
and CSNAP show quite good agreement; however, the FOR3D shows slightly different TL from the RAM and CSNAP. The overall accuracy of the FOR3D can be acceptable with the $2 \sim 3 \mathrm{~dB}$ error.

The 3D PE model, FOR3D, requires one more criterion for the angular step size, i.e., the arc length between two adjacent vertical sections. Figure 5-6 shows the comparison of TL from the FOR3D for various angular step sizes. To meet the criterion that the arc length, in general, be less than a quarter of a wavelength, the angular step size is required to be smaller than $0.054^{\circ}$ for a $40 \mathrm{~Hz} \mathrm{CW}$ source at the range of $10 \mathrm{~km}$. Here we consider $0.025^{\circ}$ and $0.0125^{\circ}$ to confirm that the solutions are converged; the TL on a horizontal plane (top panel) and along the centerline (bottom panel) show good agreement.

Figures 5-7 and 5-8 show the comparison of TL using the FOR3D for a CW source $(40 \mathrm{~Hz})$ at the depths of 25 and $100 \mathrm{~m}$. The Nx2D and 3D solutions are obtained by the FOR3D program. As shown in the figures, the TL for the Nx2D and 3D show a large discrepancy along the centerline (lower panel) as well as a disturbed pattern on a horizontal plane (upper panel). The 3D TL is higher than that of the Nx2D. On a horizontal plane, the 3D TL shows a much clearer and wider shadow zone than the Nx2D. In the Nx2D, a disturbance is confined within $4^{\circ}$, which corresponds to the angle between the centerline and an acoustic path passing over the outermost ring of the seamount; however, the disturbed region by the seamount from the 3D model is much larger than that from the Nx2D. The disturbed patterns are compared between the FOR3D and the 3D coupled-mode model in Fig. 5-9 and Fig. 5-10. Overall, the accuracy could be acceptable.

\subsubsection{Comparison of broadband pulses}

In this section, the pulse arrivals using the 2D models (RAM and CSNAP), and the 3D models (FOR3D and 3D spectral coupled-mode model) are presented. Broadband pulses are obtained from the Fourier synthesis method described in Section 3.4. For 104 
frequencies within the $50 \mathrm{~Hz}$ bandwidth, from 25 to $75 \mathrm{~Hz}$, pressure fields are computed using the 2D and 3D models, and the transfer functions are synthesized with the source spectrum, as shown in Fig. 5-2, by an inverse Fourier transform, resulting in a time series of 2.0 second period. The pulse arrivals are described in the reduced time with a reference speed of $1600 \mathrm{~m} / \mathrm{s}$. The compared results are shown in Fig. 5-11 Fig. 5-20. Note in the figures that: First, the broadband pulses from the 2D models, the RAM, and the CSNAP demonstrate no discrepancy, as shown in Fig. 5-11 and Fig. 5-13. Second, broadband pulses from the 3D models, the FOR3D and the 3D spectral coupled-mode model, show good agreement except for the strong back-scattered waves in the 3D spectral coupledmode model, as shown in Fig. 5-12 and 5-14. However, in the case of the forward scattering behind the seamount, it is valid to claim that the 3D PE, FOR3D, could be applied with good accuracy. Third, the discrepancies between broadband pulses behind the seamount are shown in Fig. 5-15 Fig. 5-20 for the selected azimuthal angles.

\subsubsection{Comparison of transfer function between the two-dimensional and three-dimensional model}

In Fig. 5-21 and 5-22, the amplitude and phase of pressure fields (transfer function) from 2D (RAM) and 3D (FOR3D and 3D-CSNAP) models are compared. Figure 5-21 compares the amplitude and phase before the seamount; there is no 3D effect due to the seamount. Therefore, as expected, no significant discrepancies are found between the amplitudes and phases. However, in Fig. 5-22, the compared amplitudes behind the seamount show large discrepancies between the 2D and 3D models. 

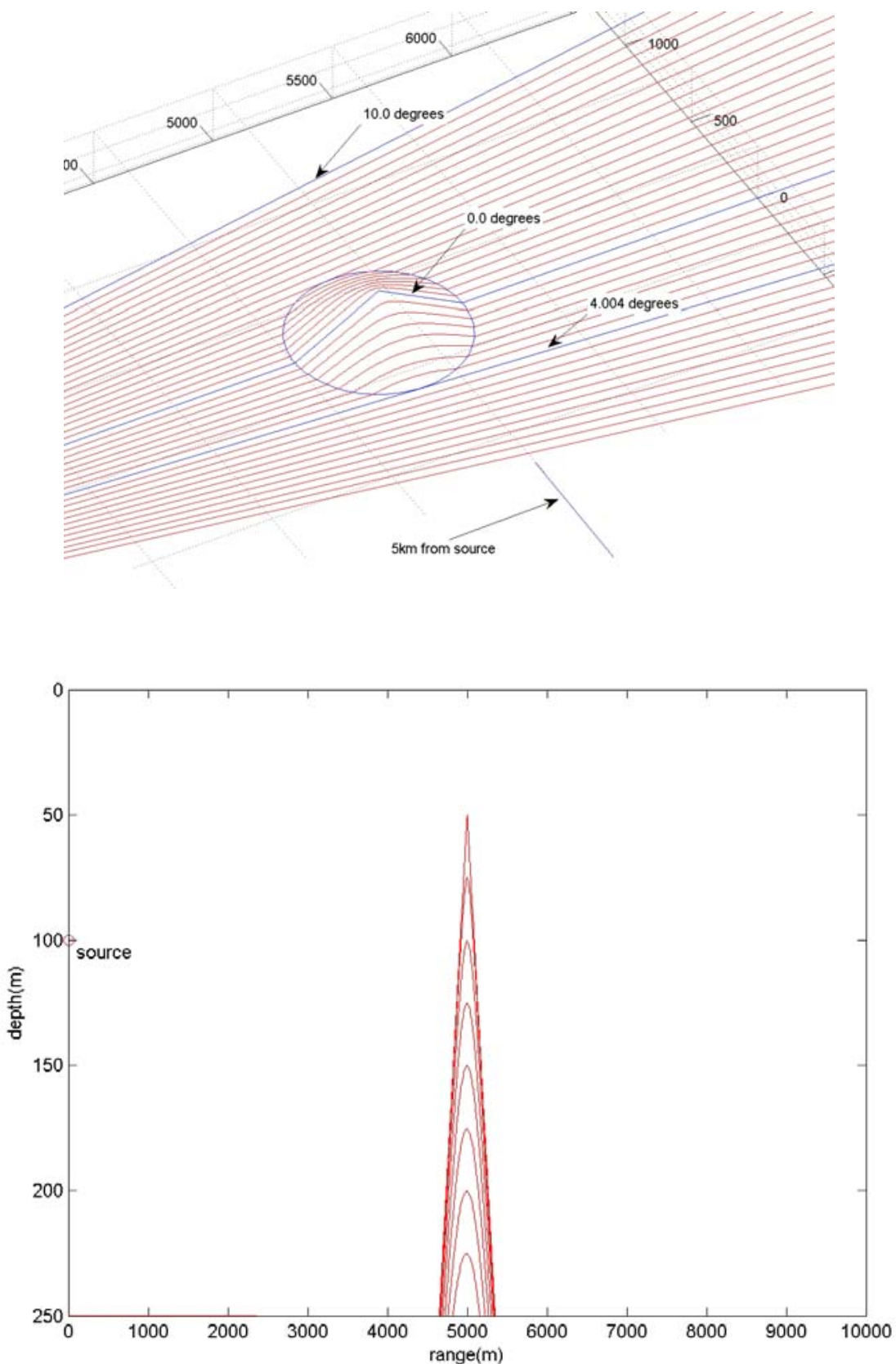

Figure 5-1: Schematic of a shallow-water waveguide with a conical seamount. 
Table 5-1: Definition of problem for the shallow water case with a seamount

\begin{tabular}{|c|c|c|}
\hline \multicolumn{3}{|c|}{ Acoustic source } \\
\hline Frequency & $40 \mathrm{~Hz}(\mathrm{CW})$ & 25 75Hz(broadband) \\
\hline Depth & $100 \mathrm{~m}$ & \\
\hline \multicolumn{3}{|c|}{ Seamount } \\
\hline Height & $200 \mathrm{~m}$ & \multirow{2}{*}{ Slope $=29.75^{\circ}$} \\
\hline Width & $700 \mathrm{~m}$ & \\
\hline Distance from the source & $5000 \mathrm{~m}$ & \\
\hline \multicolumn{3}{|c|}{ Water column } \\
\hline Sound speed $\left(c_{\omega}\right)$ & $1500 \mathrm{~m} / \mathrm{s}$ & \\
\hline \multicolumn{3}{|c|}{ Sub-bottom } \\
\hline Sound speed $\left(c_{b}\right)$ & $1800 \mathrm{~m} / \mathrm{s}$ & \\
\hline $\operatorname{Density}\left(\rho_{b}\right)$ & 2.0 & \\
\hline $\operatorname{Attenuation}(\alpha)$ & $0.1 / 10.0 * \quad \mathrm{~dB} / \lambda$ & \\
\hline
\end{tabular}

*high attenuation at 4000m depth for the RAM and FOR3D 

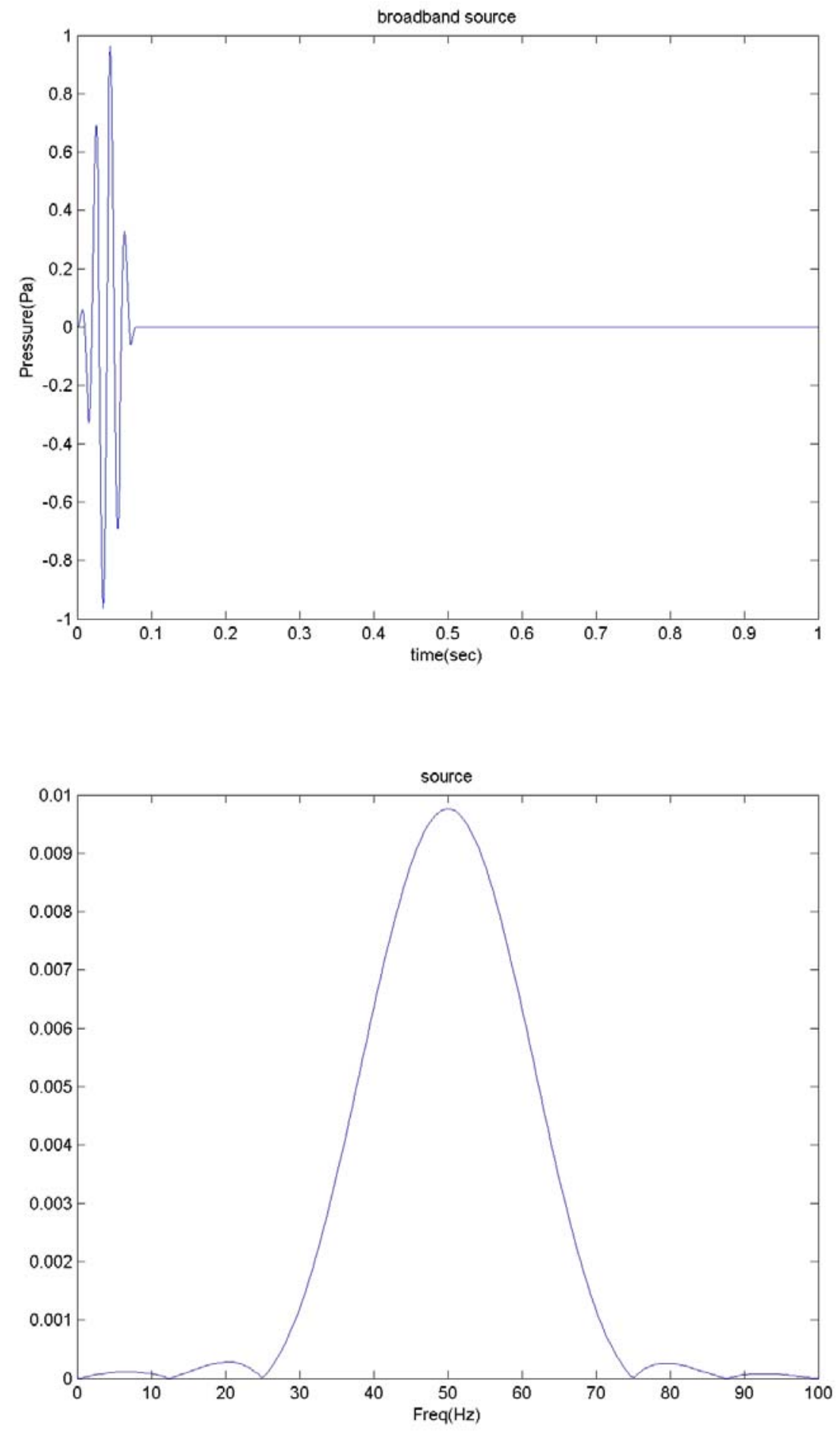

Figure 5-2: Time series and spectrum of the broadband source. 

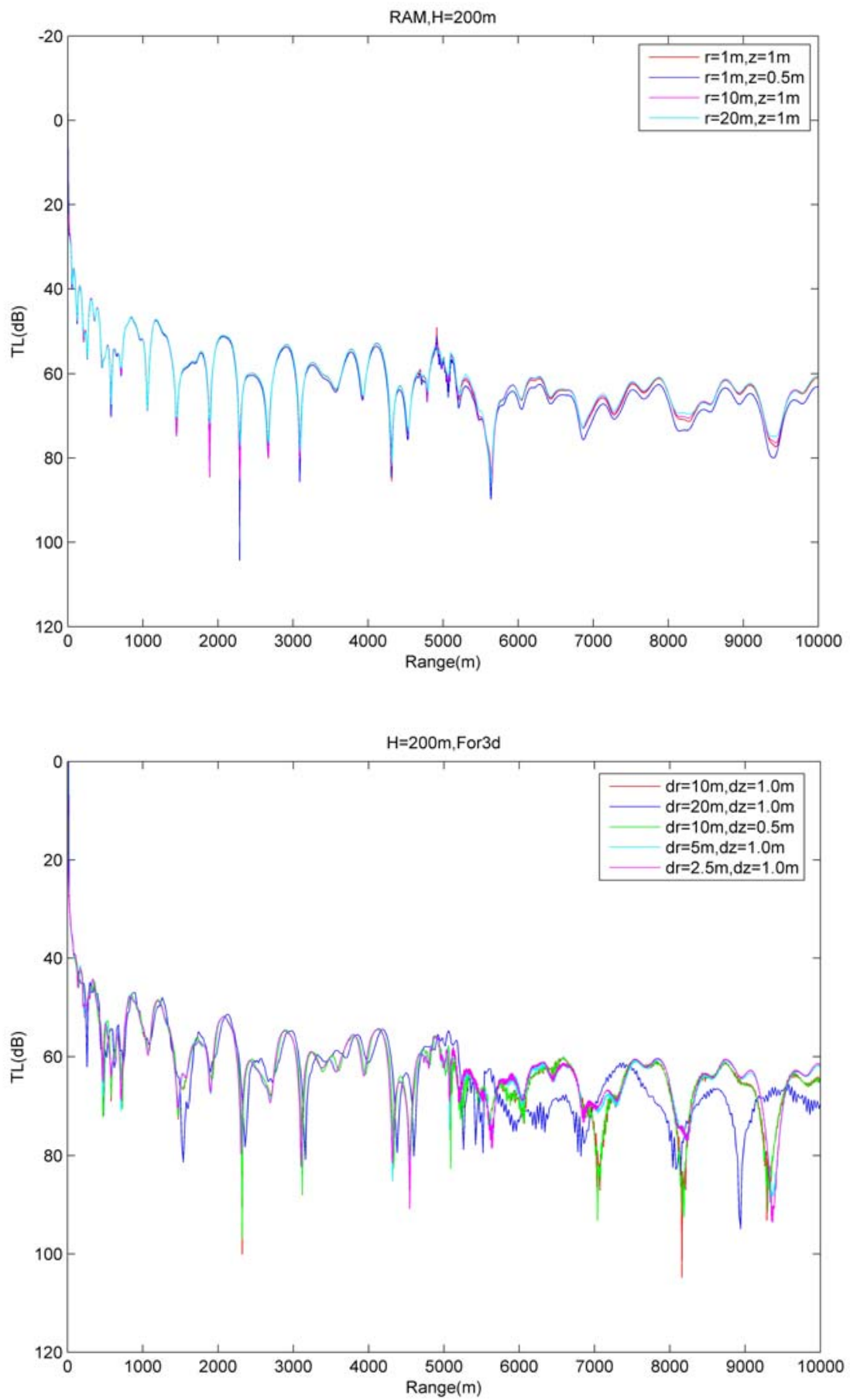

Figure 5-3: Convergence test for the range and depth step size of the RAM and FOR3D along the centerline (through the peak of the seamount, $z=100 \mathrm{~m}$ ) with a $40 \mathrm{~Hz}$ CW source. 


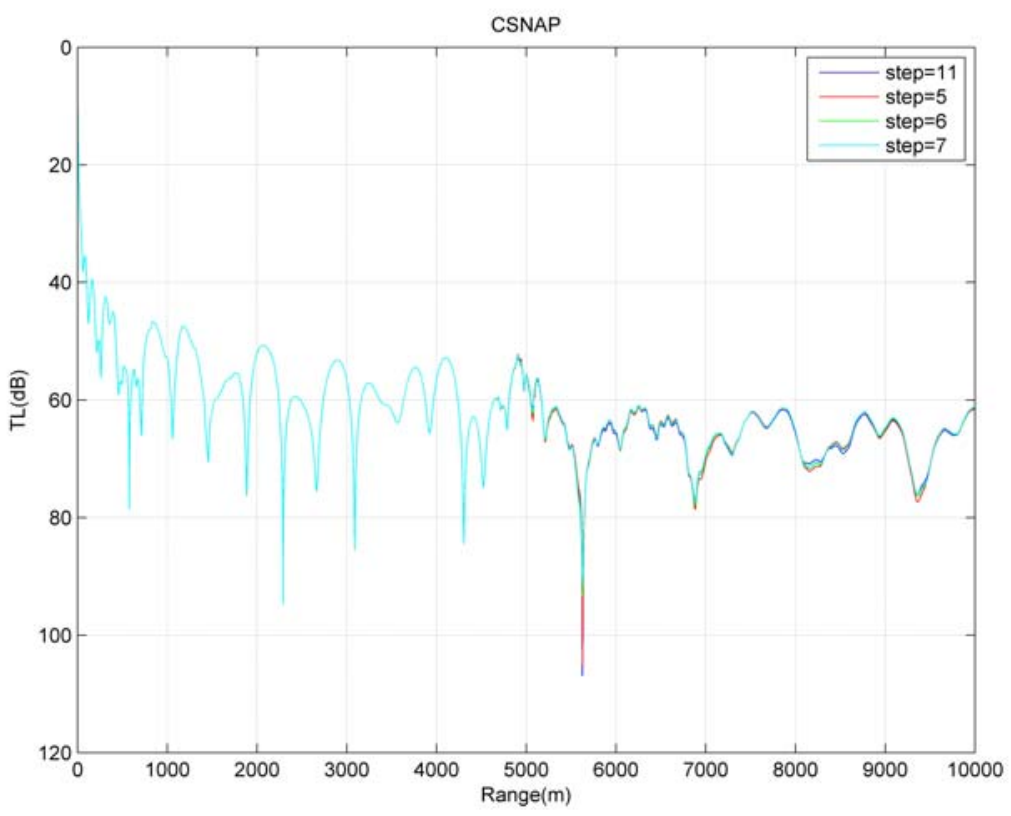

Figure 5-4: Convergence test for the range step size of the CSNAP along the centerline through the peak of the seamount at a depth of $100 \mathrm{~m}$.

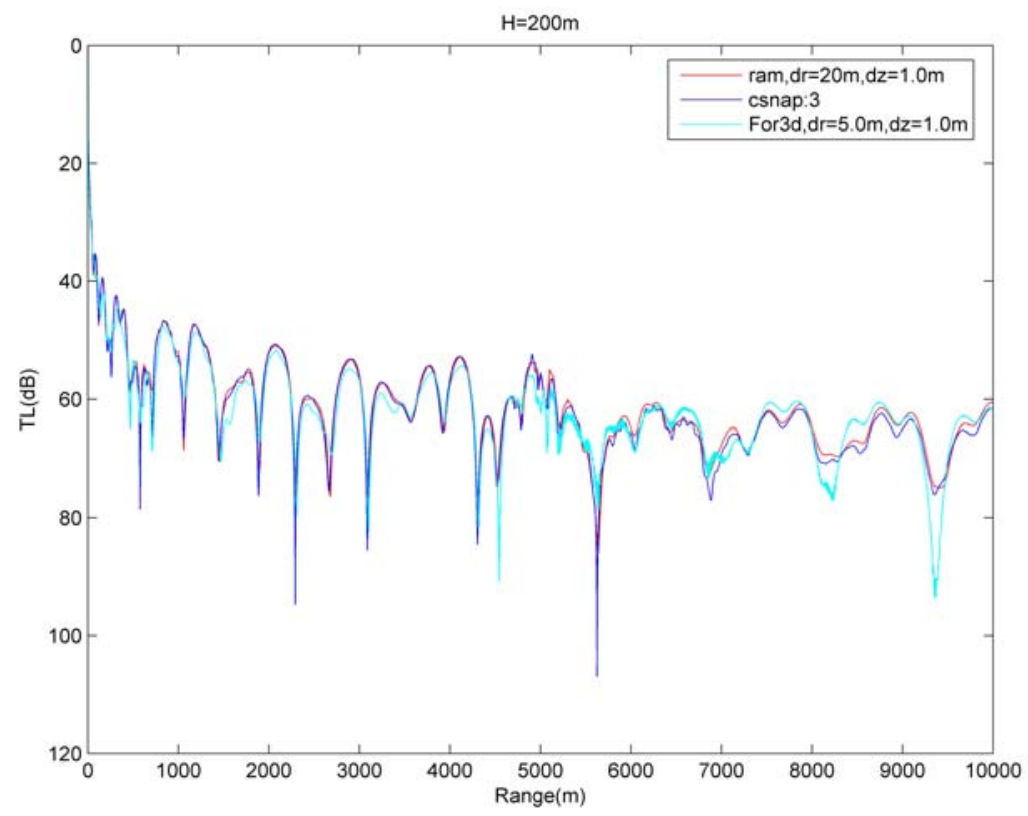

Figure 5-5: Comparison of TL from the RAM, CSNAP, and FOR3D (2D) along the centerline through the peak of the seamount at a depth of $100 \mathrm{~m}$. 

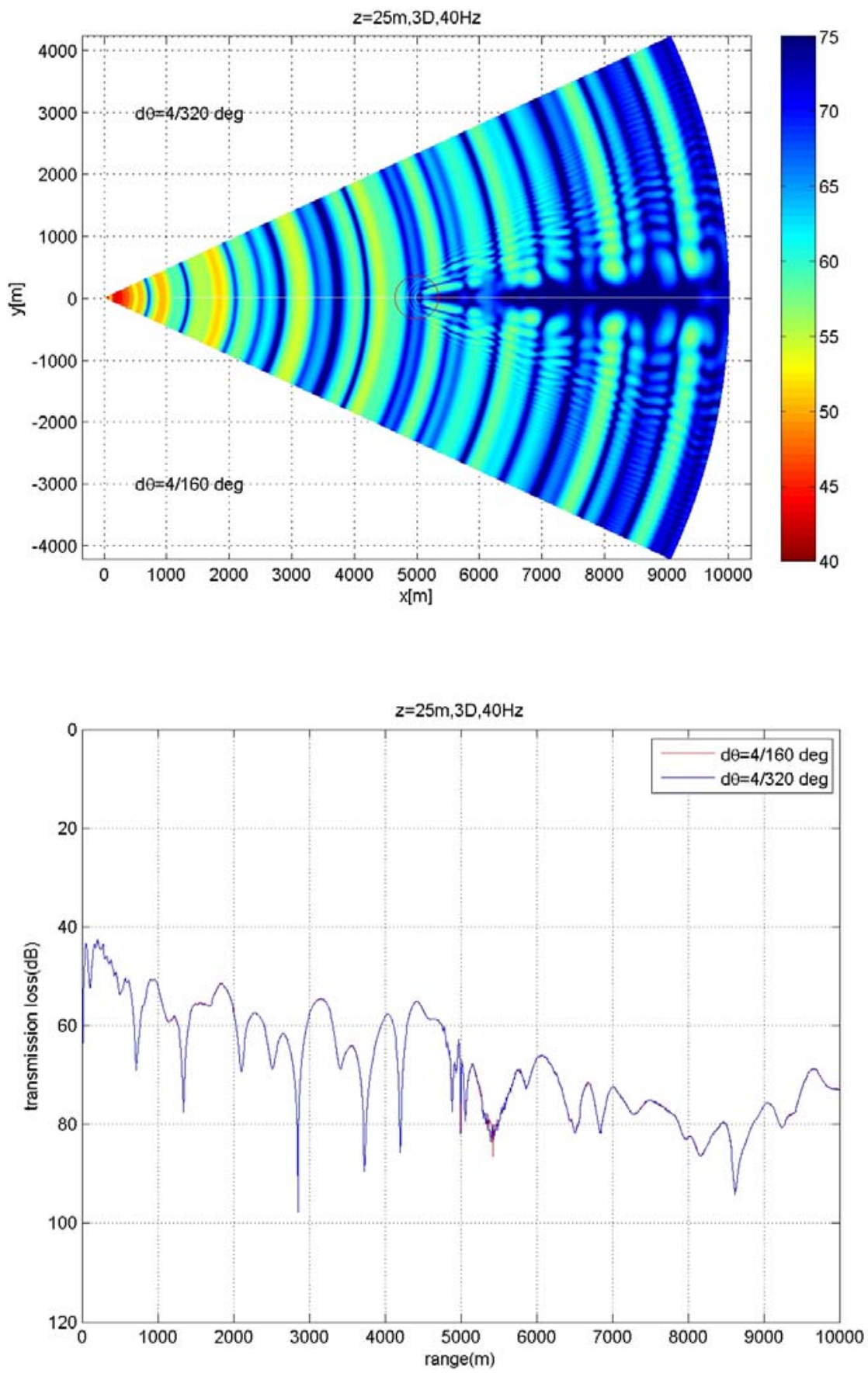

Figure 5-6: Comparison of TL from the FOR3D for two different angular step sizes on a horizontal plane (top panel) and along the centerline (bottom panel). 

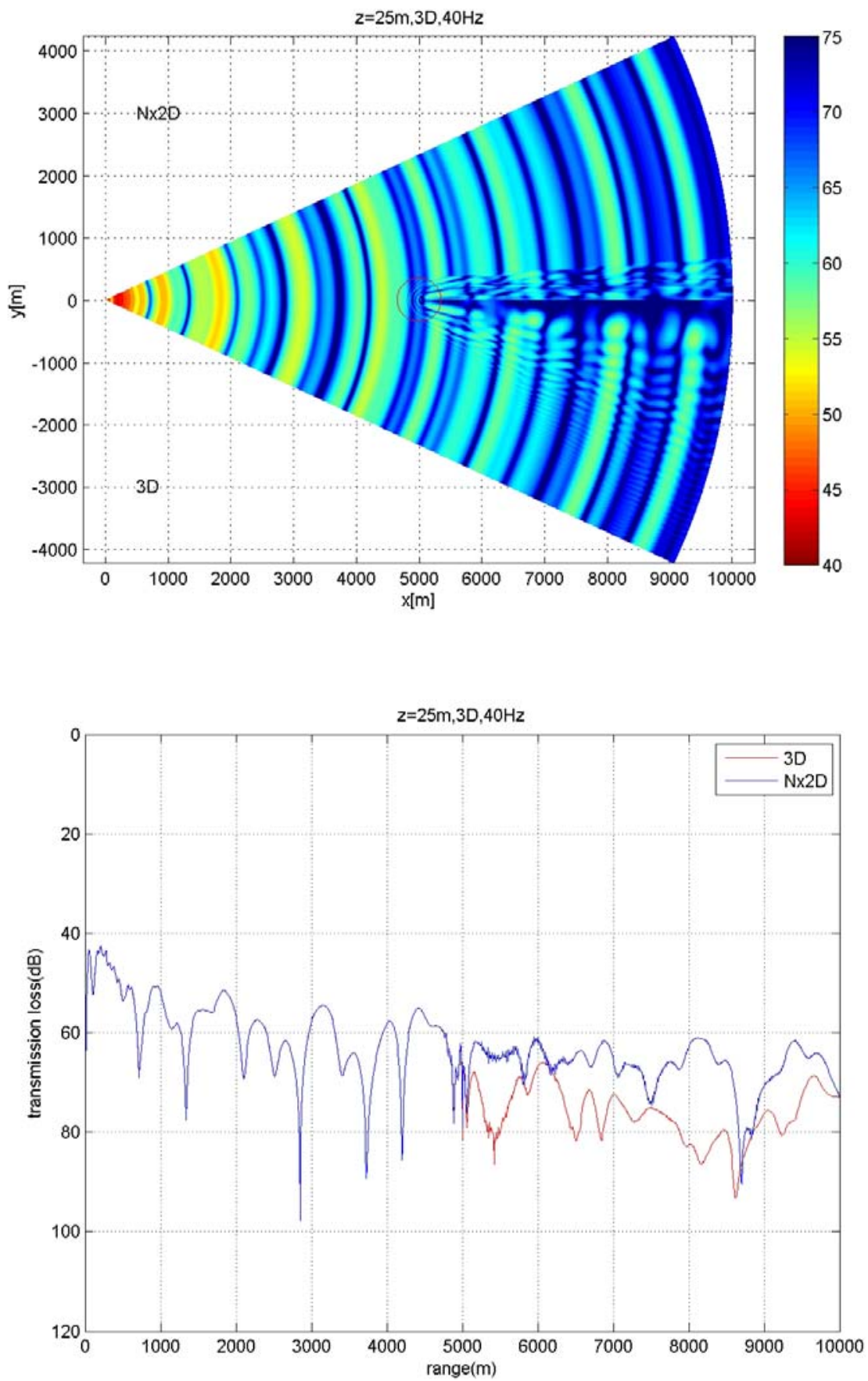

Figure 5-7: Comparison of TL from the FOR3D for the Nx2D and 3D problem with a $40 \mathrm{~Hz}$ CW source at a depth of $25 \mathrm{~m}$. The TL is shown on a horizontal plane (top panel) and along the centerline through the peak of the seamount (bottom panel). 

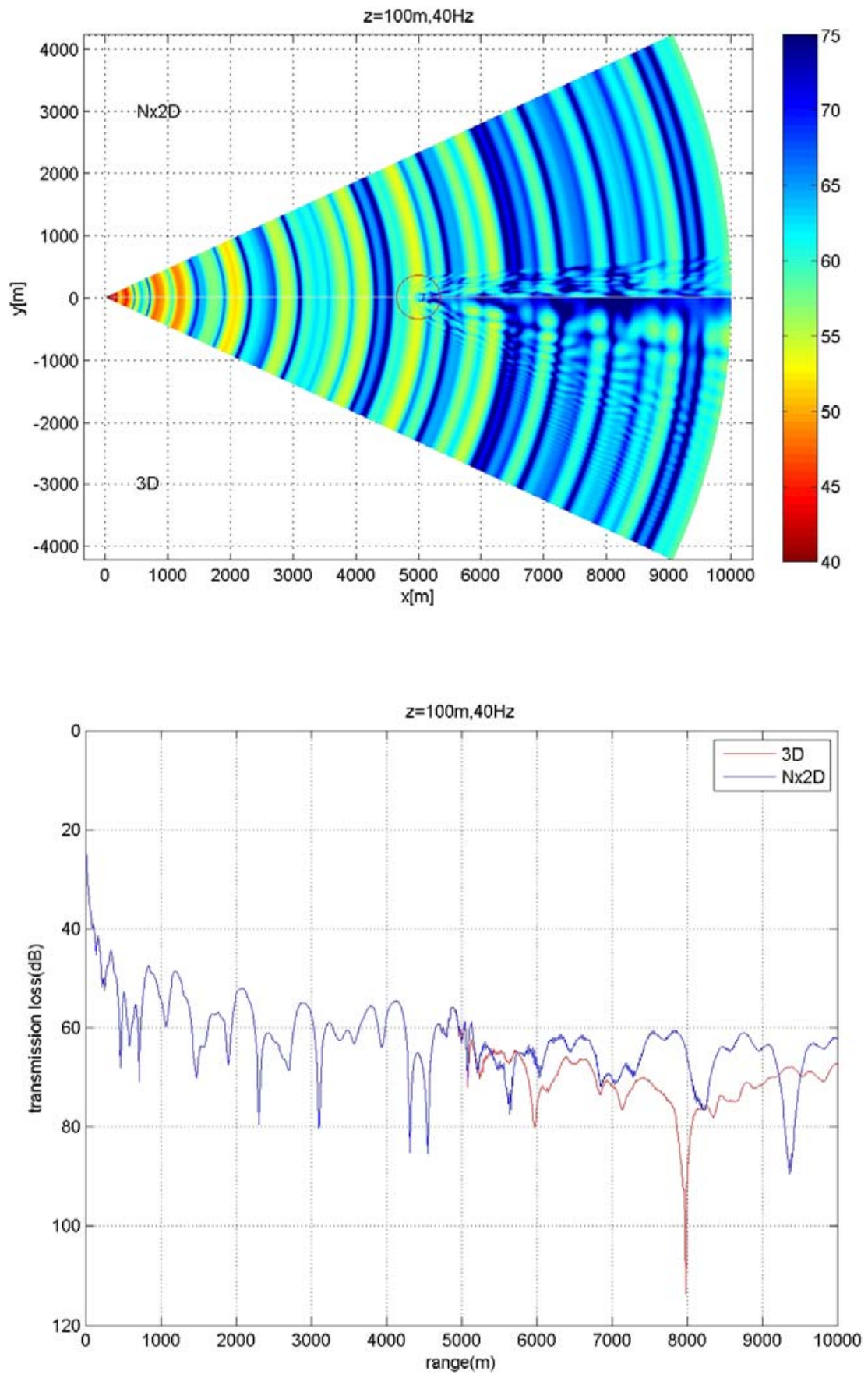

Figure 5-8: Comparison of TL from the FOR3D for the Nx2D and 3D problem with a $40 \mathrm{~Hz}$ CW source at a depth of $100 \mathrm{~m}$. The TL is shown on a horizontal plane (top panel) and along the centerline through the peak of the seamount (bottom panel). 

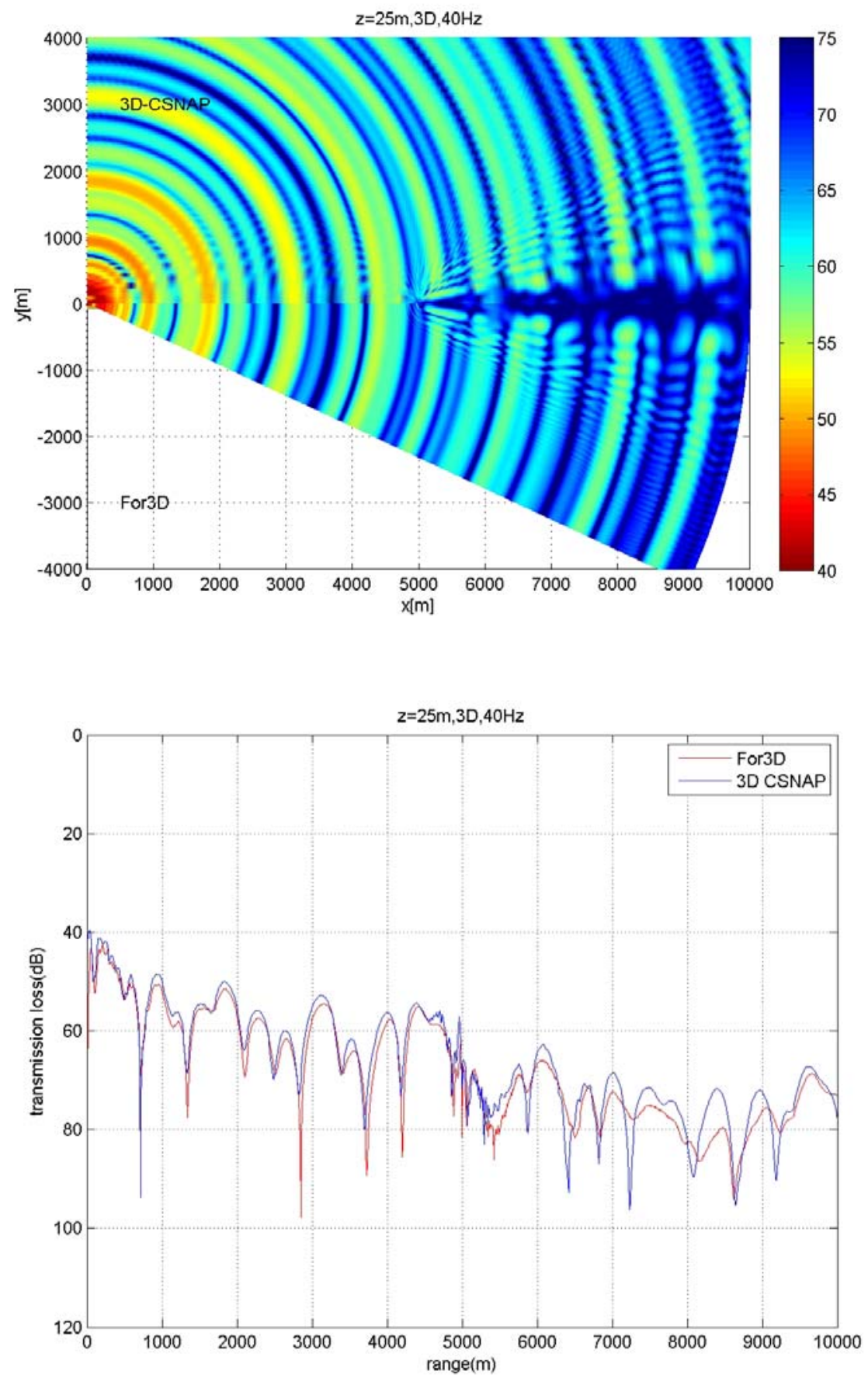

Figure 5-9: Comparison of TL from the 3D spectral coupled-mode model and FOR3D for a 3D problem with a $40 \mathrm{~Hz}$ CW source at a depth of $25 \mathrm{~m}$. The TL is shown on a horizontal plane (top panel) and along the centerline through the peak of the seamount (bottom panel). 

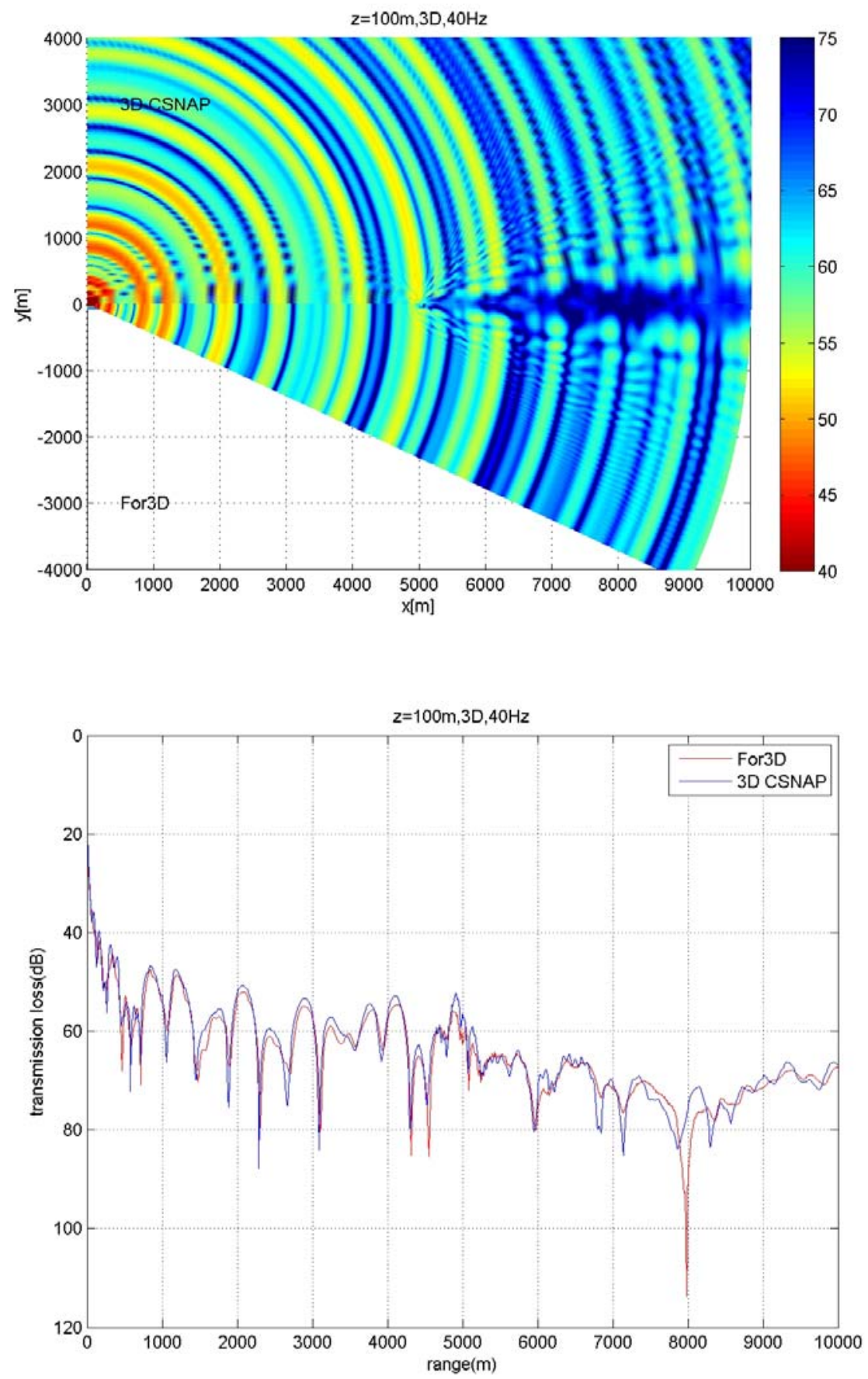

Figure 5-10: Comparison of TL from the 3D spectral coupled-mode mode and FOR3D for a 3D problem with a $40 \mathrm{~Hz}$ CW source at a depth of $100 \mathrm{~m}$. The TL is shown on a horizontal plane (top panel) and along the centerline through the peak of the seamount (bottom panel). 

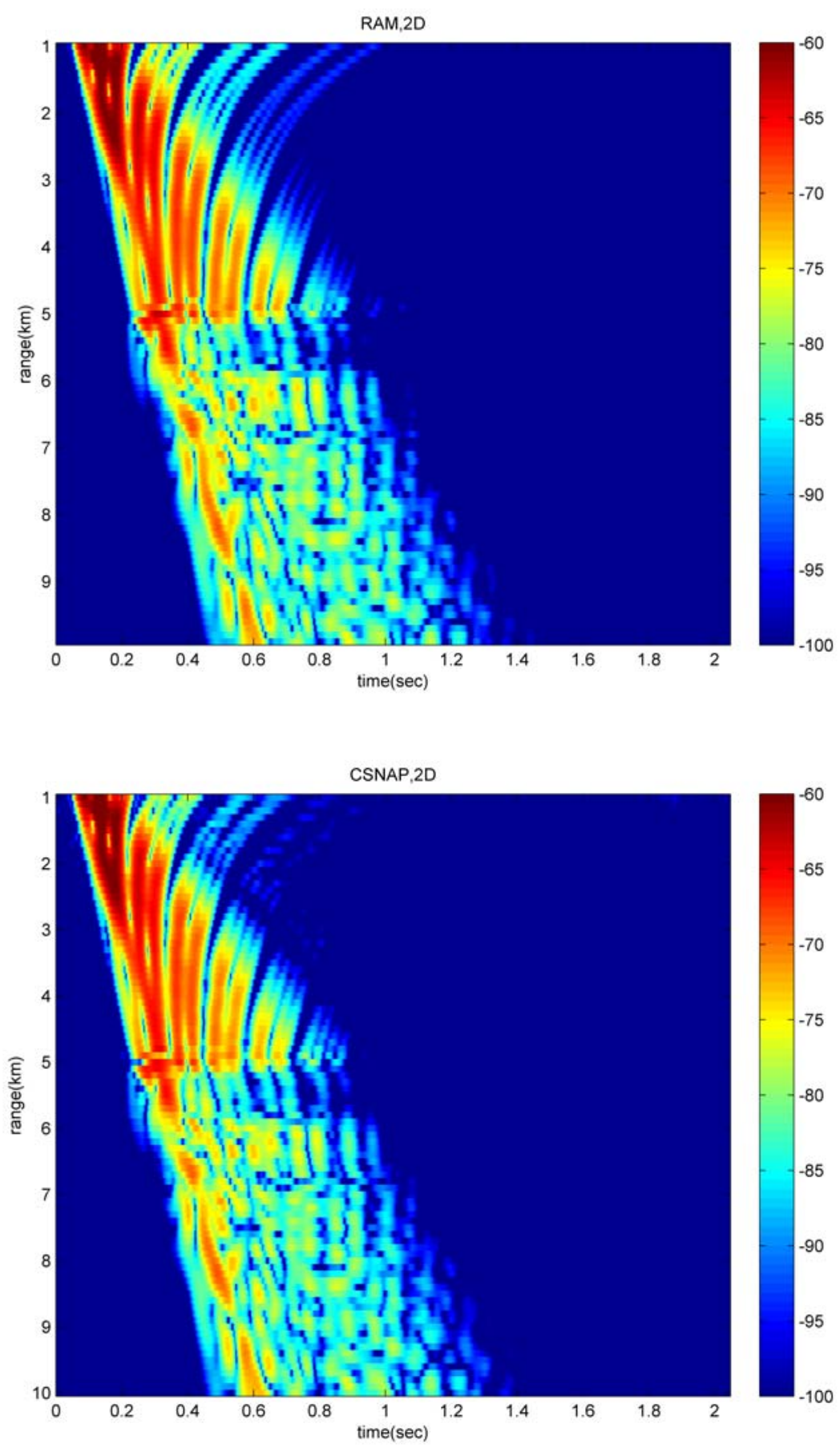

Figure 5-11: Comparison of broadband pulses at a depth of 25m, 0.0 degrees off the centerline: RAM (2D, upper panel) and CSNAP (2D, lower panel). 

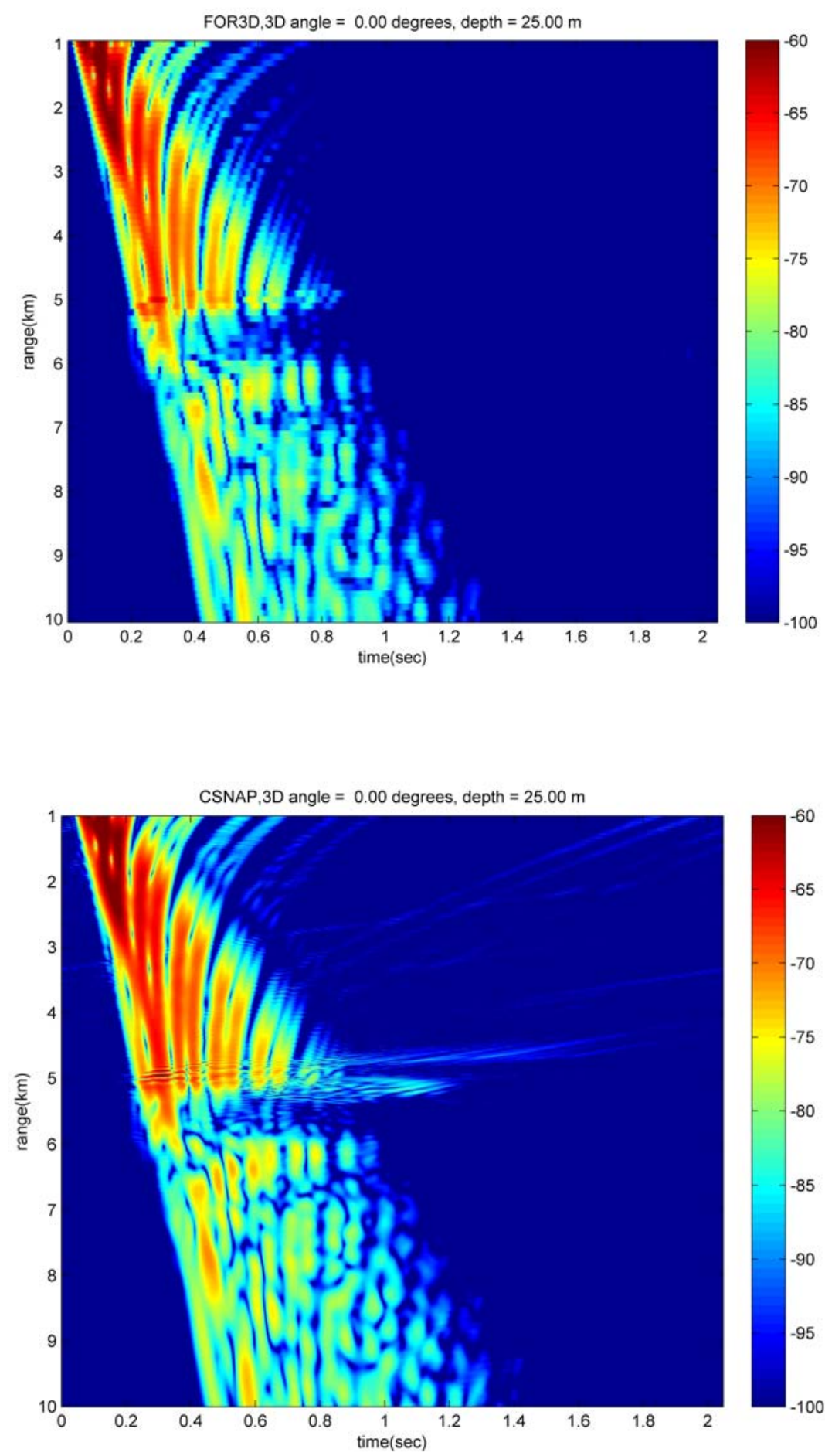

Figure 5-12: Comparison of broadband pulses at a depth of $25 \mathrm{~m}, 0.0$ degrees off the centerline: FOR3D (3D, upper panel) and 3D-CSNAP (3D, lower panel). 

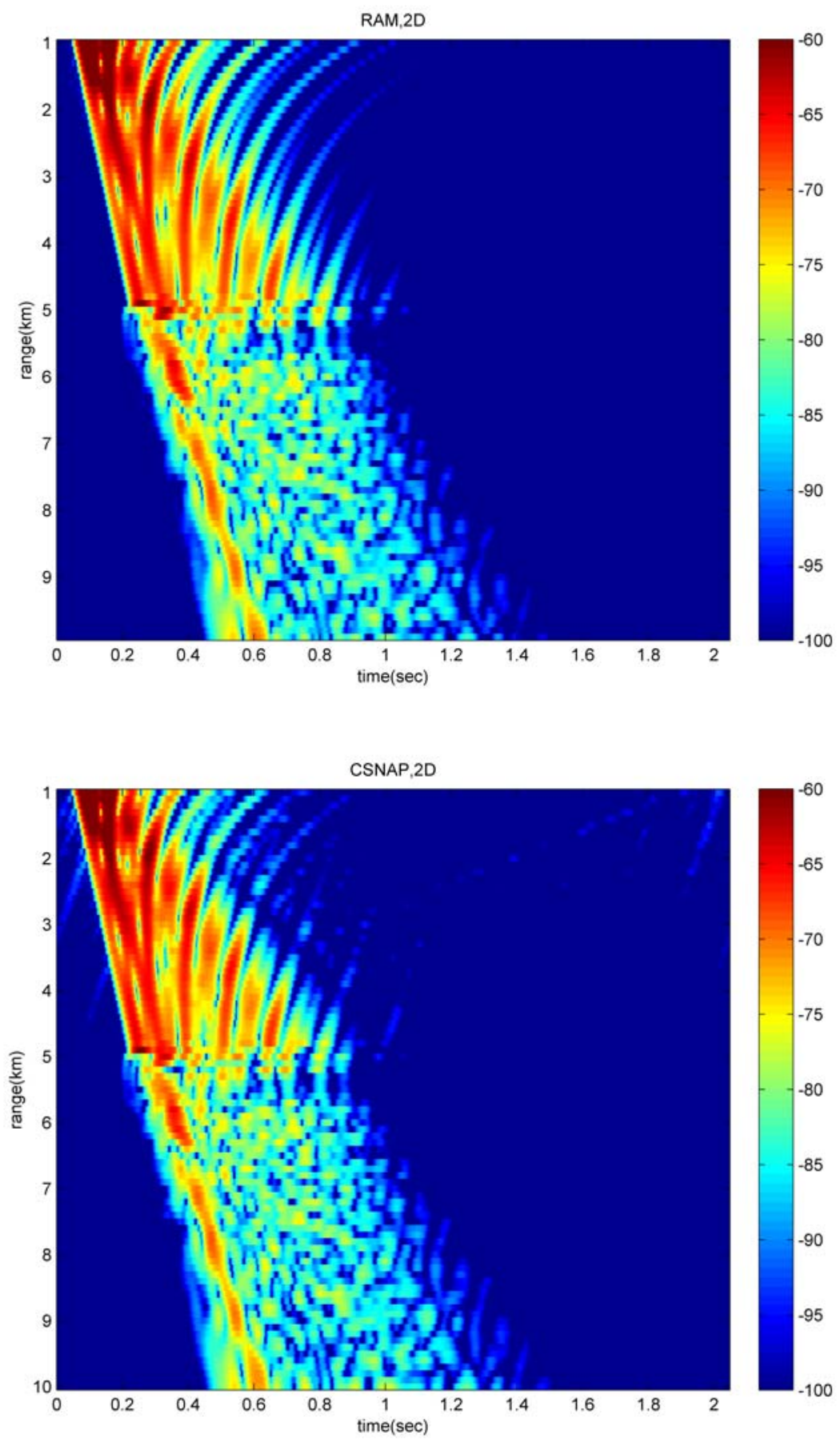

Figure 5-13: Comparison of broadband pulses at a depth of $100 \mathrm{~m}, 0.0$ degrees off the centerline: RAM (2D, upper panel) and CSNAP (2D, lower panel). 

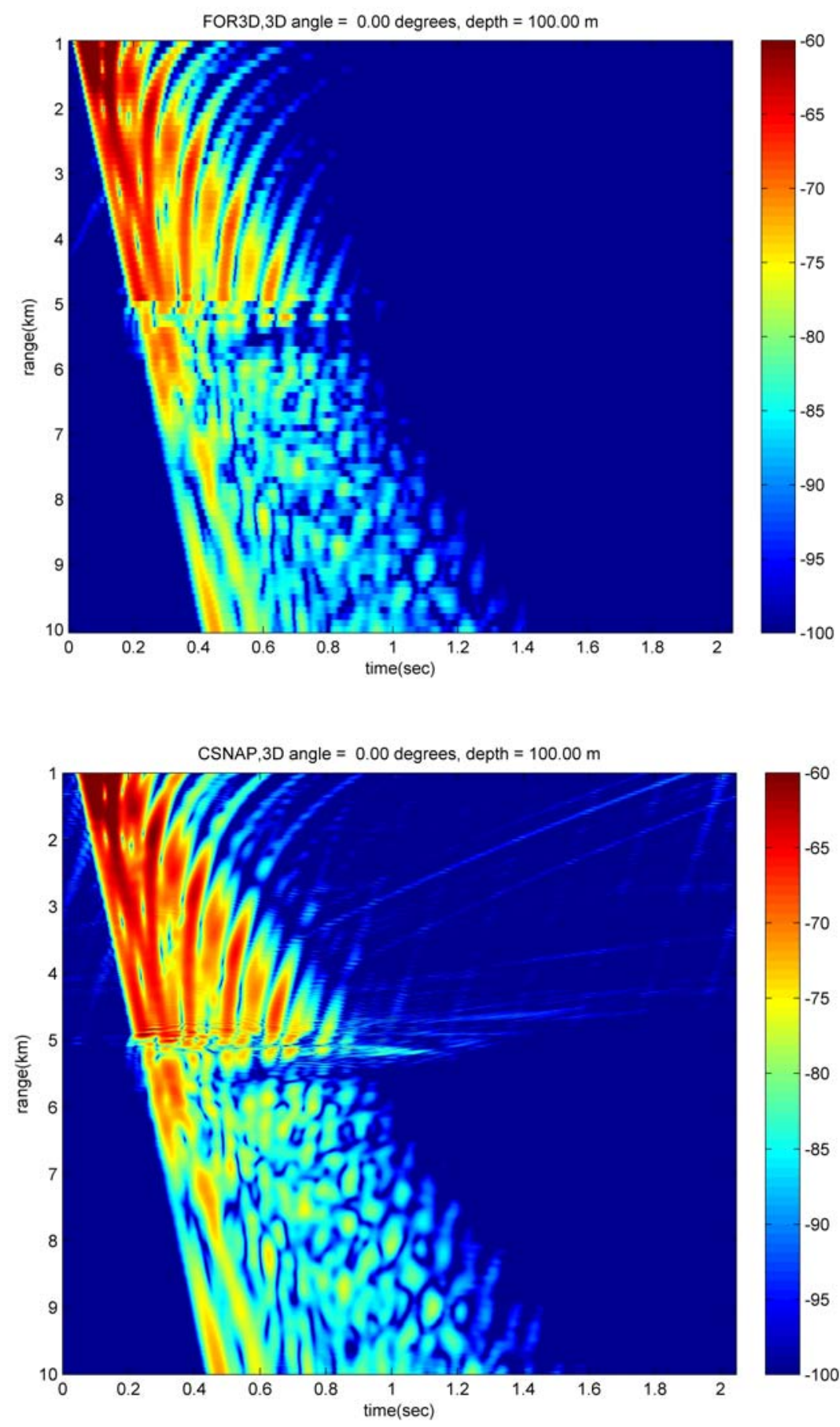

Figure 5-14: Comparison of broadband pulses at a depth of $100 \mathrm{~m}, 0.0$ degrees off the centerline: FOR3D (3D, upper panel) and 3D-CSNAP (3D, lower panel). 

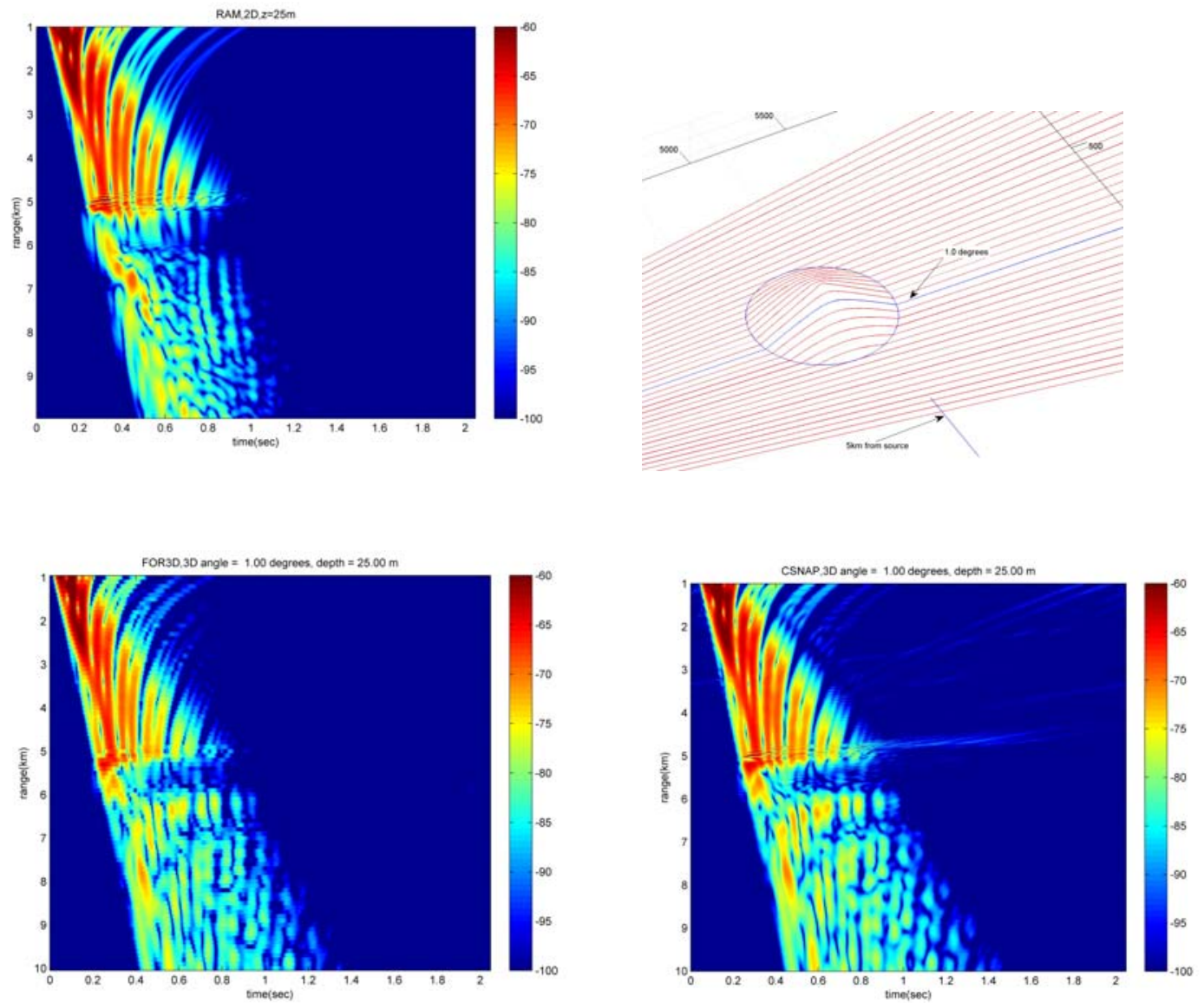

Figure 5-15: Comparison of broadband pulses at a depth of 25 m, 1.0 degrees off the centerline: RAM (2D, upper panel), FOR3D (3D, lower left panel) and 3D-CSNAP (3D, lower right panel). 

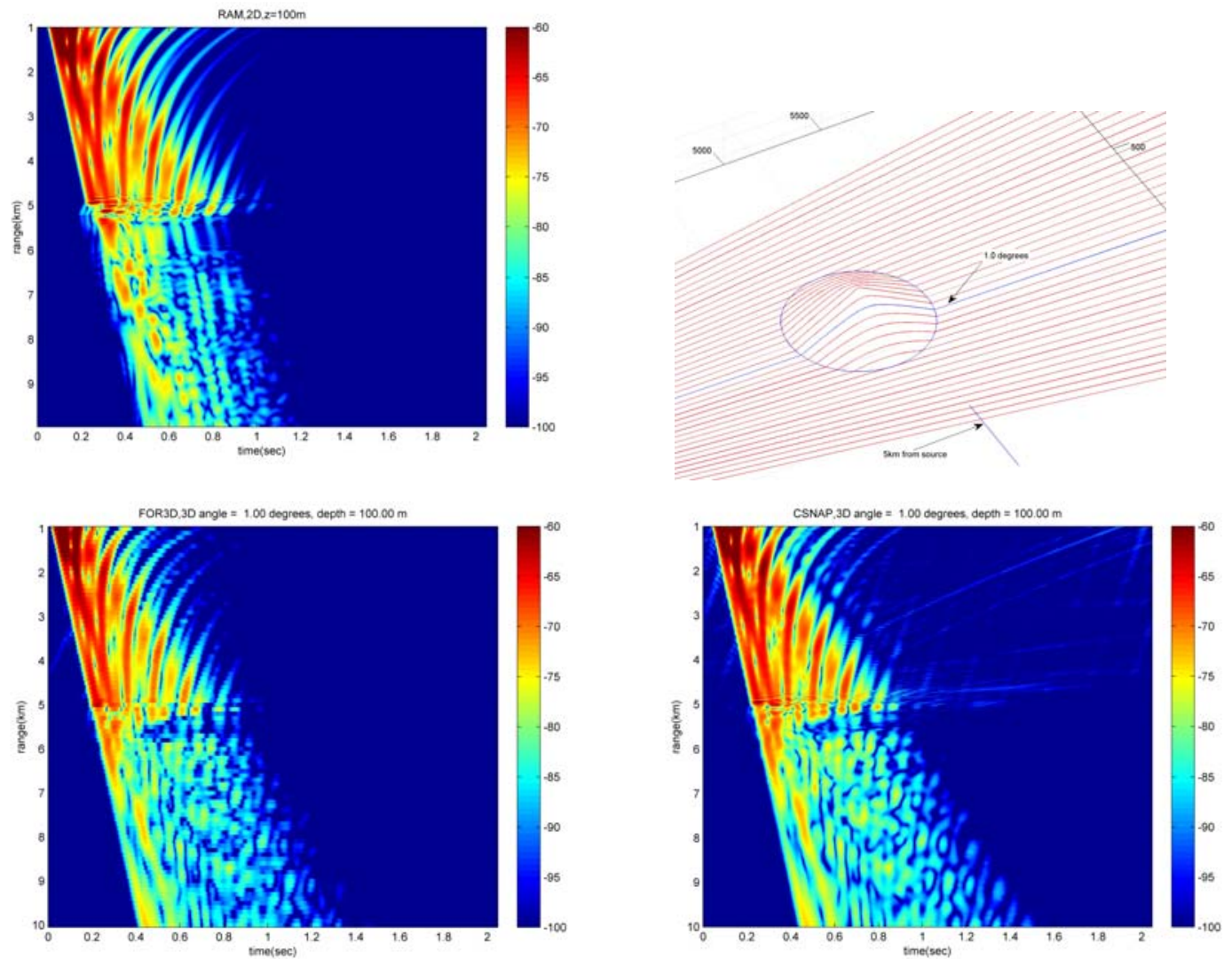

Figure 5-16: Comparison of broadband pulses at a depth of 100 m, 1.0 degrees off the centerline: RAM (2D, upper panel), FOR3D (3D, lower left panel) and 3D-CSNAP (3D, lower right panel). 

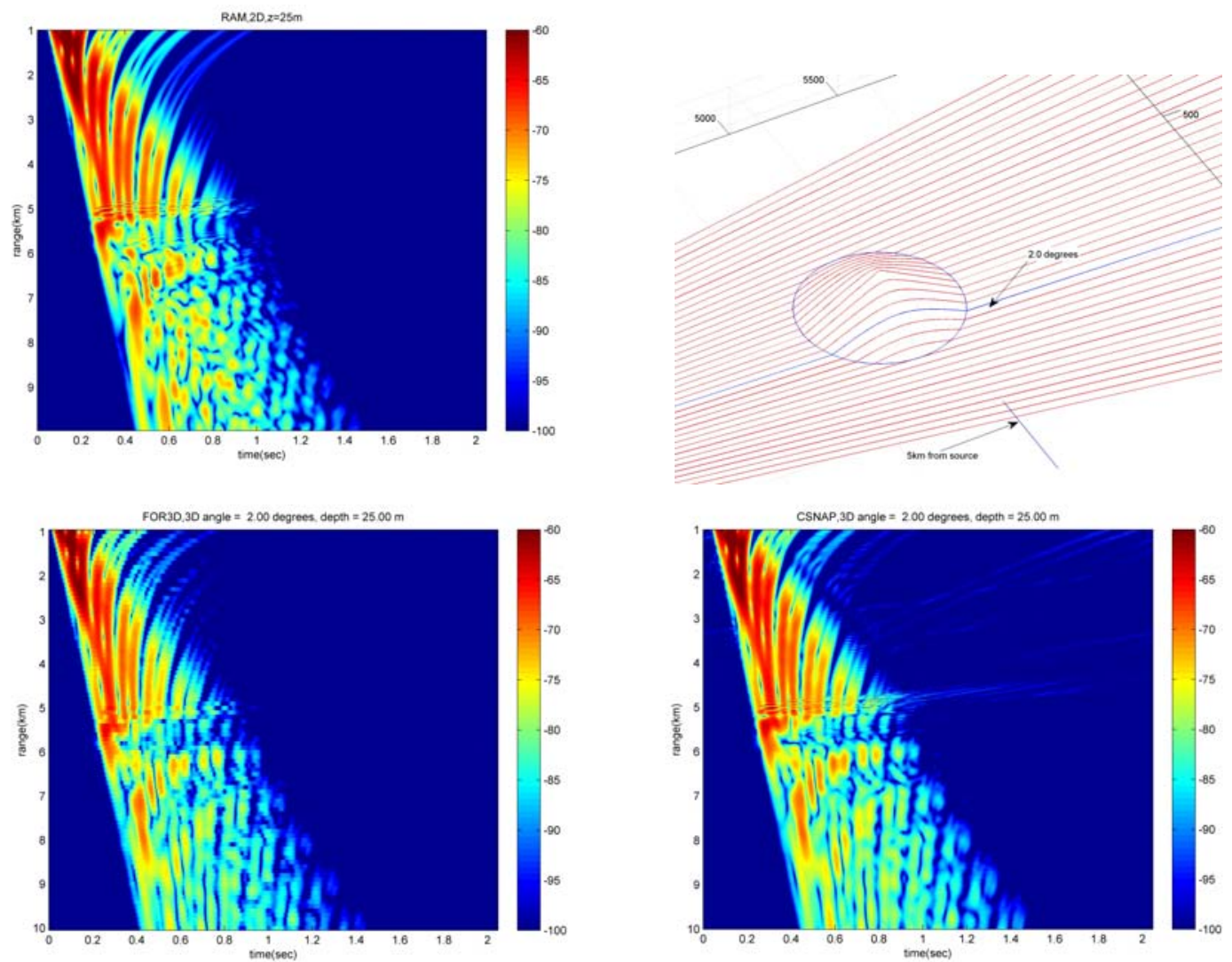

Figure 5-17: Comparison of broadband pulses at a depth of 25 m, 2.0 degrees off the centerline: RAM (2D, upper panel), FOR3D (3D, lower left panel) and 3D-CSNAP (3D, lower right panel). 

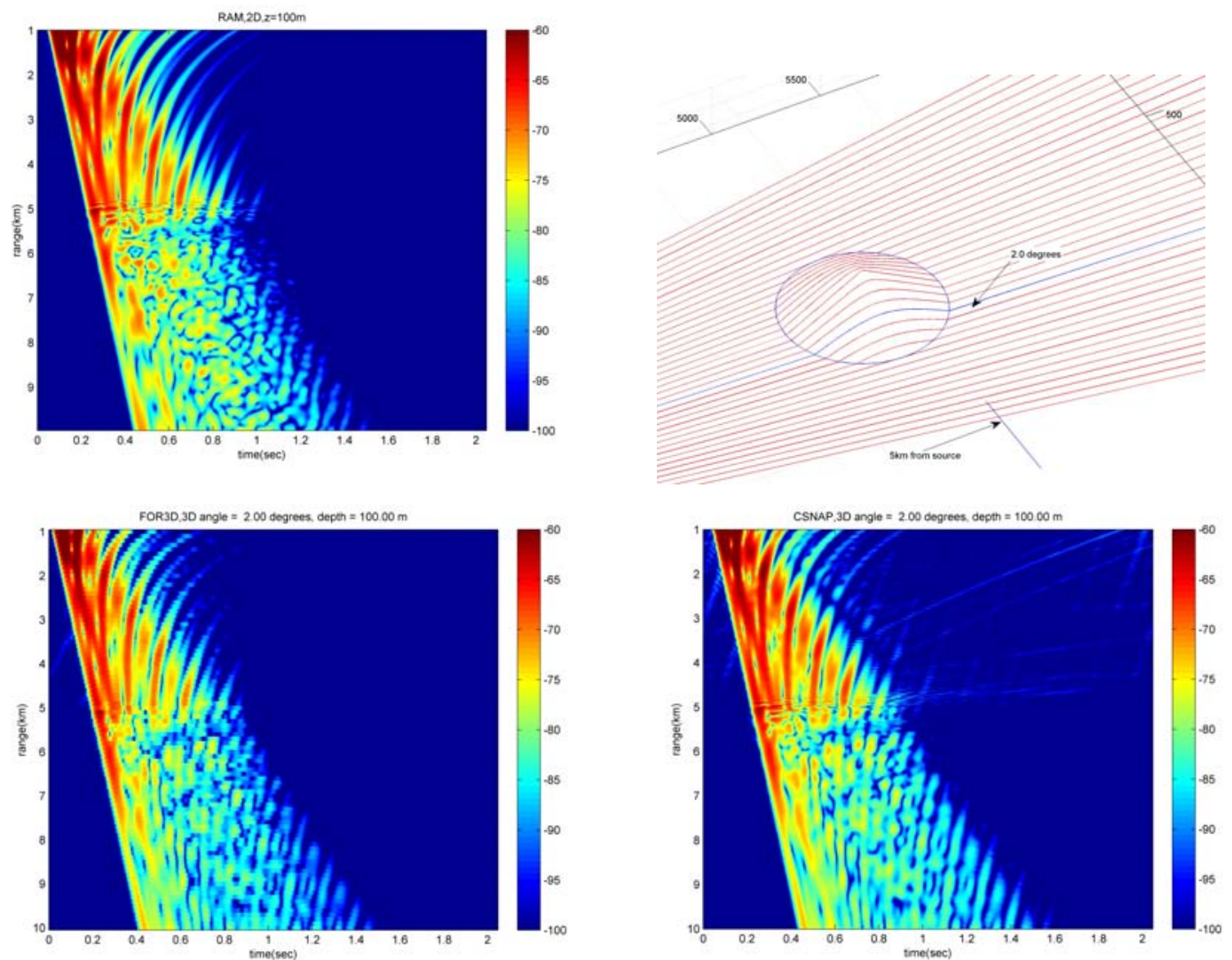

Figure 5-18: Comparison of broadband pulses at a depth of 100 m, 2.0 degrees off the centerline: RAM (2D, upper panel), FOR3D (3D, lower left panel) and 3D-CSNAP (3D, lower right panel). 

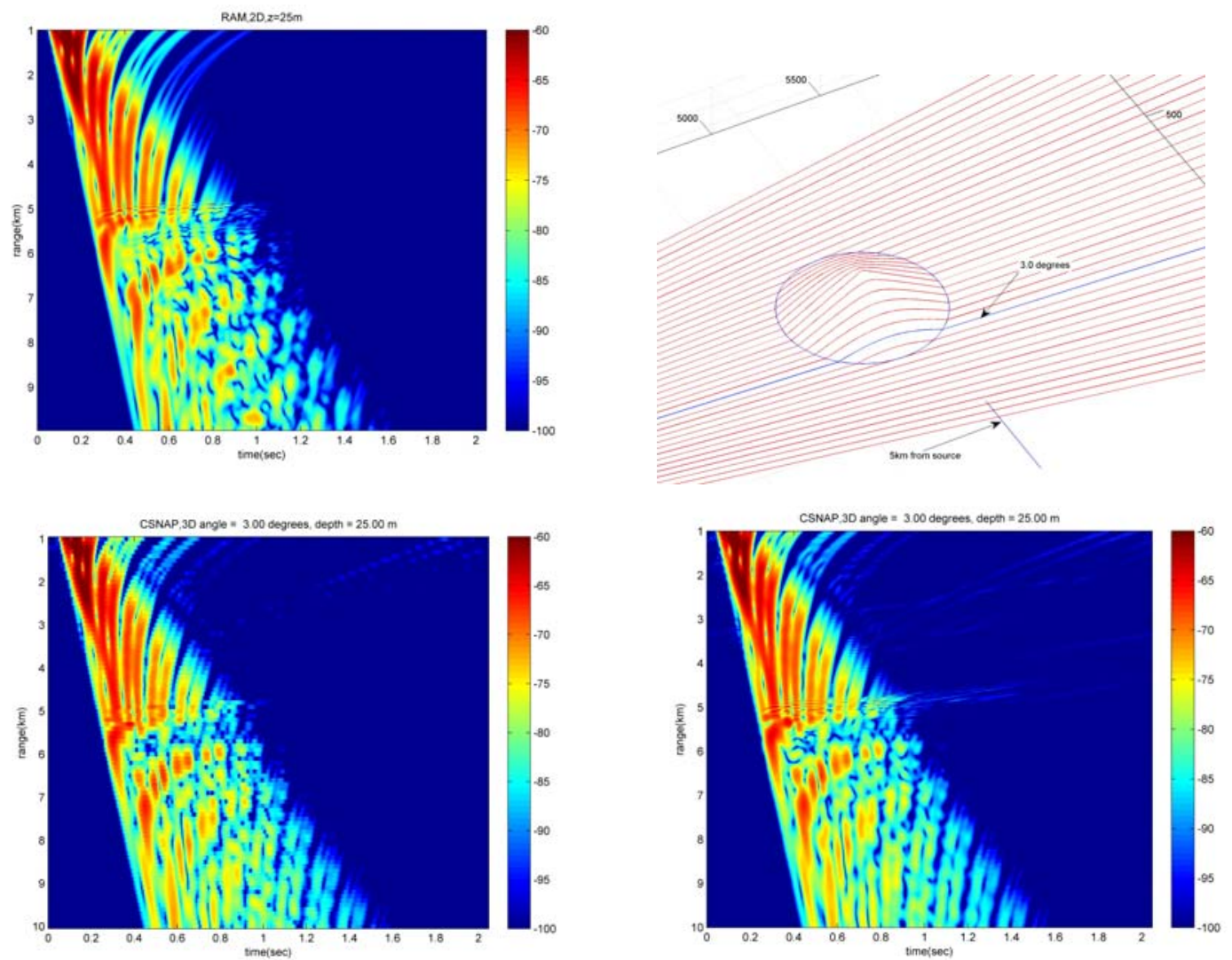

Figure 5-19: Comparison of broadband pulses at a depth of 25 m, 3.0 degrees off the centerline: RAM (2D, upper panel), FOR3D (3D, lower left panel) and 3D-CSNAP (3D, lower right panel). 

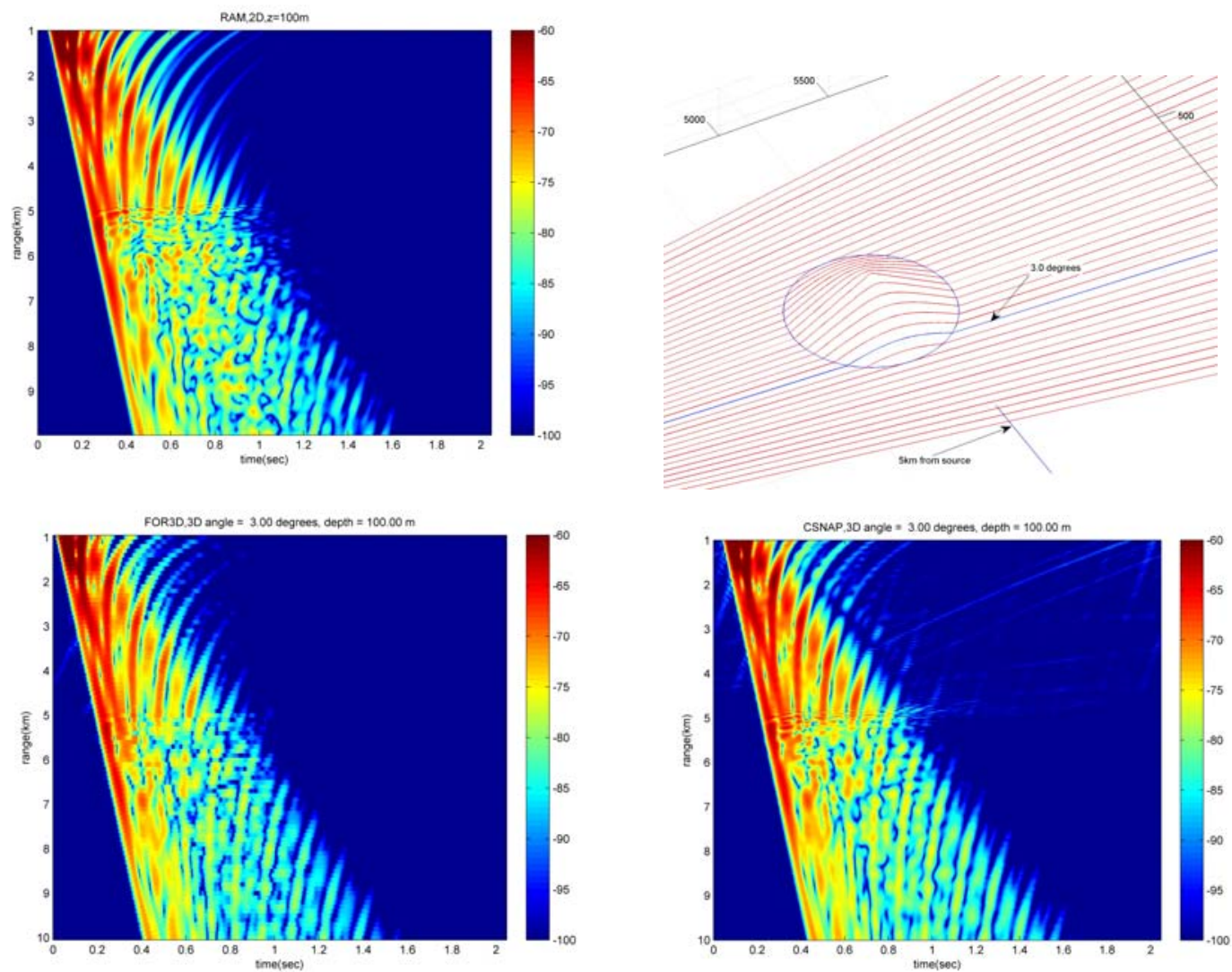

Figure 5-20: Comparison of broadband pulses at a depth of $100 \mathrm{~m}, 3.0$ degrees off the centerline: RAM (2D, upper panel), FOR3D (3D, lower left panel) and 3D-CSNAP (3D, lower right panel). 

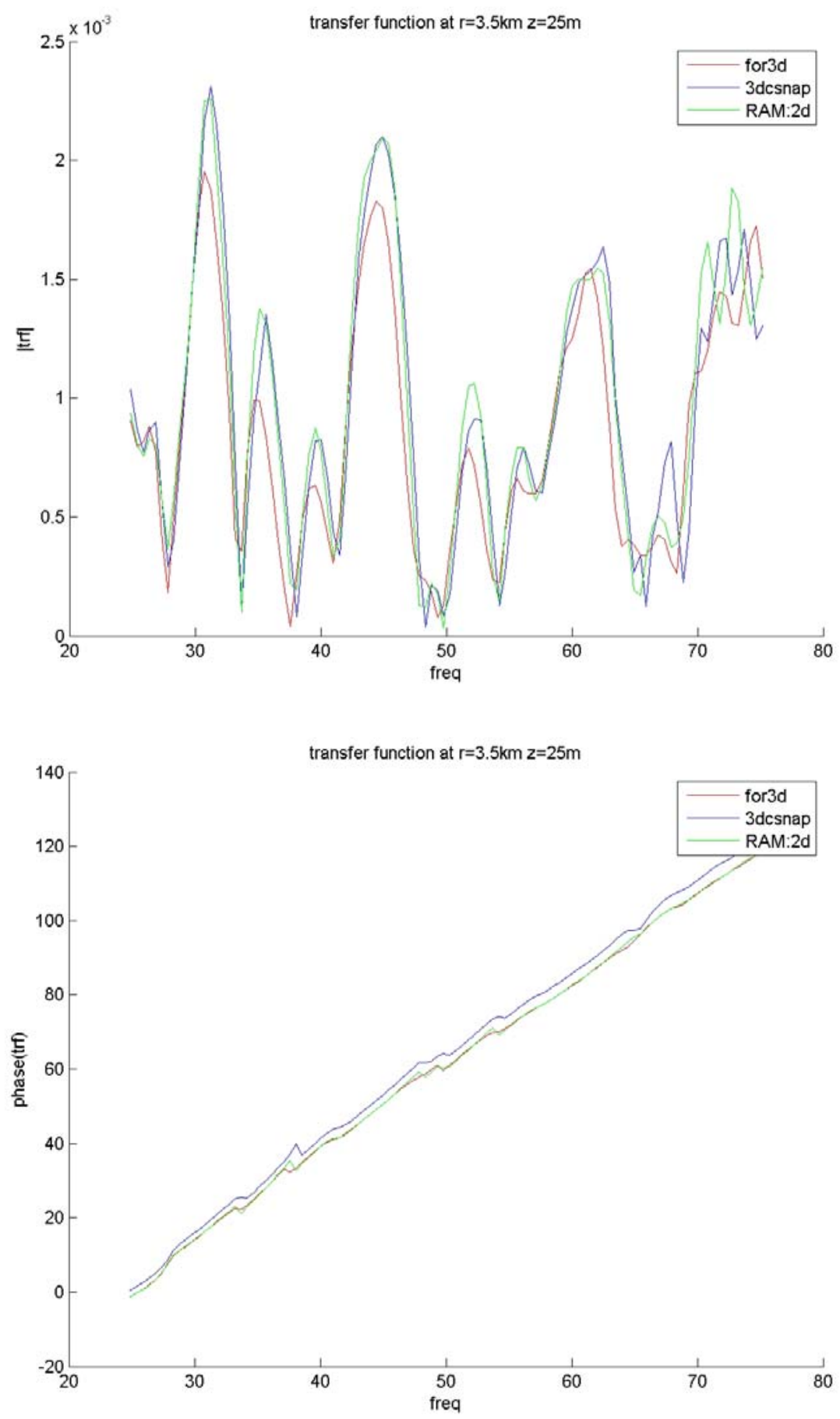

Figure 5-21: Comparison of the transfer function between RAM (2D), FOR3D (3D), and 3D spectral coupled-mode model (3D) before the seamount ( $\mathrm{r}=3.5 \mathrm{~km}$, depth $=25 \mathrm{~m}$, azimuth angle $=0.0$ degrees): amplitude (top panel) and phase (bottom panel). 

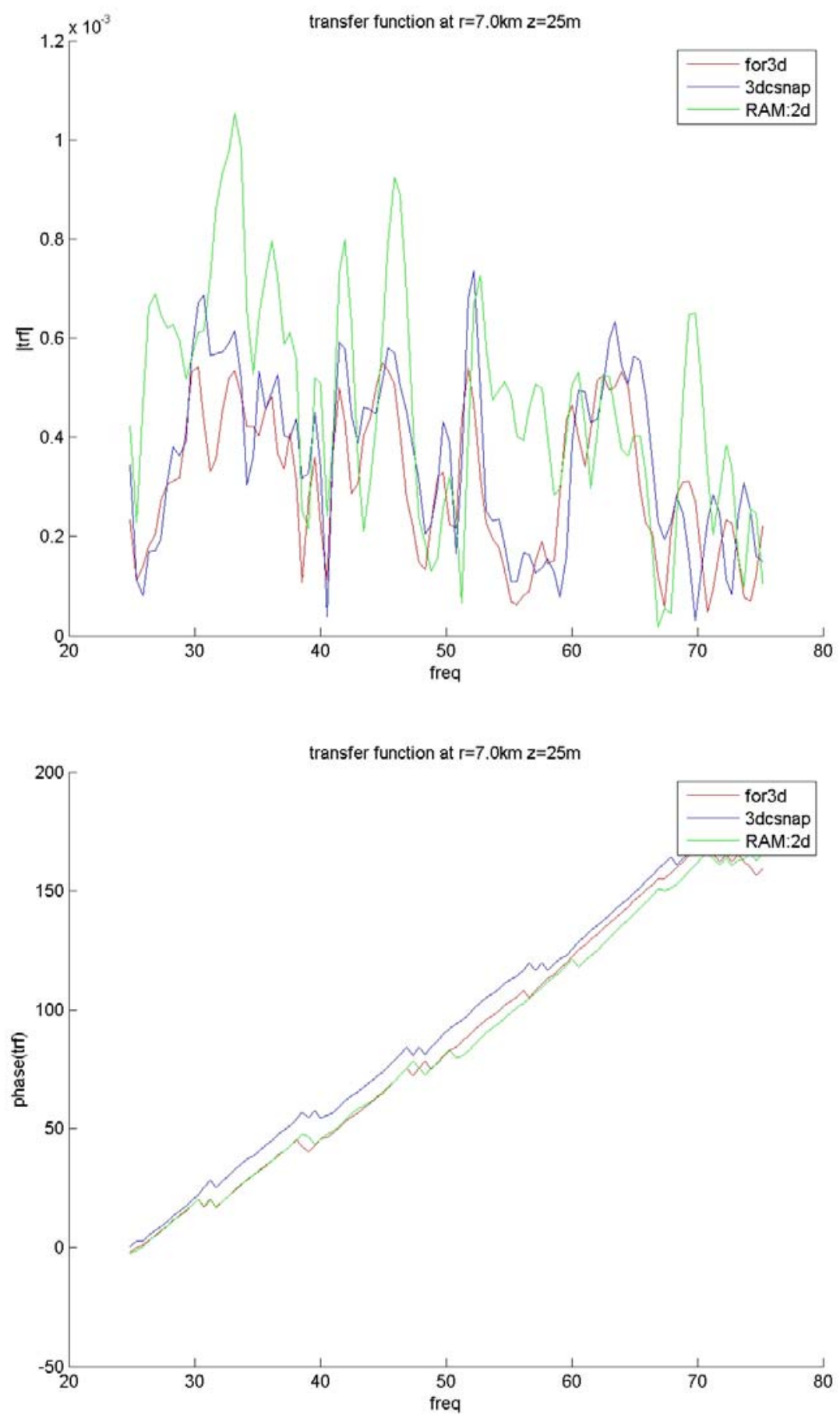

Figure 5-22: Comparison of the transfer function between RAM (2D), FOR3D (3D), and 3D spectral coupled-mode model (3D) behind the seamount $(r=7.0 \mathrm{~km}$, depth $=25 \mathrm{~m}$, azimuth angle $=0.0$ degrees): amplitude (top panel) and phase (bottom panel). 


\subsection{Broadband pulse modeling for a benchmark problem in deep-water waveguide with a conical seamount}

In the previous section, we compared the $2 \mathrm{D}$ and $3 \mathrm{D}$ models for a shallow-water waveguide problem with a conical seamount in terms of transmission loss (TL) for a continuous wave (CW) source and the broadband pulses. Although we found discrepancies of TL between the 3D PE (FOR3D) and 3D spectral coupled-mode model, the accuracy could be acceptable. In addition, we found that no significant discrepancies between the 3D PE (FOR3D) and 3D spectral coupled-mode model in the resultant broadband pulses except for the strong backscattered waves from the 3D spectral coupled-mode model. In addition,

In this chapter, we apply the 3D spectral coupled-mode model to a benchmark problem in a deep-water waveguide.

\subsubsection{Description of problem}

Here we consider a benchmark problem in a deep sea waveguide similar to the configuration of the BASSEX experiment. The schematic of the problem is given in Fig. 3. The radius of the conical seamount at the base is $20 \mathrm{~km}$ with a height of $3800 \mathrm{~m}$; the slope of the seamount is 10.76 degrees. A flat bottom is assumed at a depth of $5000 \mathrm{~m}$ for the outside of the seamount.

Range-independent sound speed is assumed, as shown in Fig. 5.23, and the geoacoustic properties of the bottom are a compressional sound speed of $2000 \mathrm{~m} / \mathrm{s}$, a density of $1.0 \mathrm{~g} / \mathrm{cm}^{3}$, and an attenuation of $0.1 \mathrm{~dB} / \lambda$. The false bottom was introduced below the sea bottom to suppress the spurious reflections from the boundary.

As shown in Fig. 5.24, an acoustic source depth is $100 \mathrm{~m}$, and the center frequency of the source is $15 \mathrm{~Hz}$ with $10 \mathrm{~Hz}$ bandwidth. Figure 5-25 shows a number of waterborne 
and propagation modes at each frequency for the $10 \mathrm{~Hz}$ bandwidth from 10 to $20 \mathrm{~Hz}$. Note that no waterborne mode exists for this frequency band at the top of the seamount, meaning that there is no ray passing over the seamount without the bottom-bouncing. The angle between the centerline and the outermost ring with respect to the source is 11.31 degrees. The seamount is located at $100 \mathrm{~km}$ from the acoustic source so that the significant refracted rays are all blocked by the seamount along the centreline, as shown in Fig. 5-27; this placement can maximize the blockage effect by the seamount.

\subsubsection{Transmission loss for a $10 \mathrm{~Hz}$ CW source}

Figure 5-26 shows the TL for a $10 \mathrm{~Hz}$ CW source at a depth of $300 \mathrm{~m}$. The upper panel compares the TL from the 2D coupled normal mode (CSNAP) and the 3D spectral coupled-mode model along the centerline. The TL from 3D is greater than that from 2D with a difference of $5 \sim 10 \mathrm{~dB}$ in most of ranges except for the convergence zones. This higher TL from the 3D model can be explained by the fact that energy dissipates outward from the centerline due to the horizontal refraction.

The lower panel shows the TL on a horizontal plane, which shows clear shadowing cast behind the seamount with weak appearance of the convergence zones. The full normal modes, including leaky modes at the outermost ring, were used for the mode coupling.

\subsubsection{Comparison of pulse arrivals between the 3D spectral coupled- mode model and the ray tracing method without seamount}

To validate pulse arrivals from the 3D spectral coupled-mode model, we compare the

pulse arrivals from the 3D spectral coupled-mode model with those from the 2D ray tracing method. In this comparison, a flat bottom is assumed in the 2D ray tracing method. The eigenrays are compared with those from the 3D spectral coupled-mode model for an acoustic path that passes far away from the seamount. Along the acoustic path, the 3D 
refraction effects could be neglected; therefore, the eigenrays from the $2 \mathrm{D}$ ray tracing method must be comparable to the pulse arrivals from the 3D spectral coupled-mode model.

Figure 5-27 shows the ray representation without a seamount. The convergence zones are composed of the refracted rays without bottom-bouncing, shown in black. The ray family at the convergence zone is all blocked by the seamount located at the $100 \mathrm{~km}$ from the acoustic source.

Figure 5-28 shows the computed arrival pulses from the 3D spectral coupled-mode model (lower panel) compared with the eigenrays from the ray tracing method (upper panel). The arrival pulses and eigenrays are shown in terms of the reduced time. The eigenrays are close to the arrival pulses far outside the seamount, 30 degrees off the centerline, with negligible 3D effects. At a depth of $200 \mathrm{~m}$, significant convergence zone arrivals are followed by the bottom-bounced rays. The numbers at the upper panel denote the number of bottom-bounces.

Figure 5-29 compares the arrival time from the 2D ray tracing method from the 3D spectral coupled-mode model at a range of $130 \mathrm{~km}$. The $A$ ray family forms a strong convergence zone without bottom-bouncing; the arrival times in this ray family are quite close to each other. $B, C$, and $D$ correspond to the bottom-bouncing ray family with 2,3 , and 4 bottom bounces, respectively.

Through these comparisons, we show the good correlation between arrival pulses modeled by the 3D spectral coupled-mode model and those from the 2D ray tracing method, with negligible 3D effects.

\subsubsection{Broadband pulse arrivals}

The pulse arrivals using the 3D spectral coupled-mode model are computed with various numbers of normal modes and at different receiver depths.

Pressure fields are computed using the 3D spectral coupled-mode model for 165 frequencies within $10 \mathrm{~Hz}$ bandwidth (10 to $20 \mathrm{~Hz}$ ), and the transfer function is 
synthesized with the source spectrum shown in Fig. 5-25 using the inverse Fourier transform, which results in 16.4 second time series. For the solution at each frequency, the waterborne modes or propagation modes in the outer-most ring are used. The number of waterborne modes varies from 11 to 22, and the number of propagation modes varies from 42 to 87 at frequencies of 10 20 Hz. The pulse arrivals are shown in the reduced time with a reference time of $1520 \mathrm{~m} / \mathrm{s}$.

\section{a) With waterborne modes only}

Here we consider the waterborne (water-trapped) modes at the outermost ring (see Fig. 525) for the mode coupling.

Figure 5-30 shows the computed arrival pulses at a depth of $200 \mathrm{~m}$ in terms of range and the reduced time for the selected angles. The angle in the figure denotes an acoustic path running off the centerline. The convergence zone arrivals are all blocked after the seamount up to 10 degrees, and weak reflections exist in shadow zone.

Figure 5-31 shows computed arrival pulses at a depth of $200 \mathrm{~m}$ on a horizontal plane; the reduced time with a reference speed of $1520 \mathrm{~m} / \mathrm{s}$ is shown at the corner of each panel. The first convergence zone arrivals appear at the reduced time of 0.7 0.8 seconds, at a range of $60 \mathrm{~km}$ from the source, followed by weak reflections. The second convergence zone appears at 1.3 seconds, $120 \mathrm{~km}$. The third convergence zone arrivals appear at 1.8 1.9 seconds, 180 190 km. The reflected waves are not significant because only waterborne modes are used.

Figure 5-32 shows computed arrival pulses at a depth of $1000 \mathrm{~m}$ in terms of range and arrival time. Two strong ray family arrivals are shown in the higher angle, but those are all blocked in the lower angle after the seamount up to 10 degrees, and weak reflections appear in the shadow zone. Computed pulse arrivals on a horizontal plane are shown in Fig. 5-33.

In the computed pulse arrivals with waterborne modes only, weak reflections are shown within the perturbed zone. 


\section{b) With propagation modes}

Here we consider the propagation modes at the outermost ring (see Fig. 5-25) for the mode coupling. The propagation modes generate stronger reflections by the seamount.

Figure 5-34 shows the computed pulse arrivals at a depth of $200 \mathrm{~m}$ in terms of range and reduced time for the selected angles. The convergence zone arrivals are all blocked after the seamount up to 10 degrees and strong reflections appear in the shadow zone. Disturbances with bottom-bouncing waves due to the seamount appear in much higher angles up to 16 degrees.

Figure 5-35 shows the computed pulse arrivals at a depth of $200 \mathrm{~m}$ on a horizontal plane; the reduced time with the reference speed of $1520 \mathrm{~m} / \mathrm{s}$ is shown at the corner of each panel. The first convergence zone arrivals appear at 0.75 seconds, $60 \mathrm{~km}$ from the source, which are followed by strong reflections. The pulse arrivals appear to be moving toward the acoustic source because of the higher propagation speed relative to the reference speed. The second convergence zone appears at 1.25 seconds, $120 \mathrm{~km}$, which clearly shows the shadowing cast of the seamount. The third and fourth convergence zones appear at 1.75 2.0, 2.5 seconds, $190 \mathrm{~km}$ and $250 \mathrm{~km}$, respectively. At around 2.25 seconds, between the third and fourth convergence zone appearances, a convergence is formed by the reflected-refracted rays within the perturbation zone. Reflections due to the seamount generate quite complicated patterns of pulse arrivals in and around the shadow zone, including waves going away from the seamount to higher angles. Figures 5-36 and 5-37 show only reflected late arrivals with the strong disturbances by the seamount.

Figure 5-38 shows the computed pulse arrivals at a depth of $1000 \mathrm{~m}$ in terms of range and reduced time for the selected angles. The two strong ray families arrive in higher angle without blockage; however, these are all blocked in lower angle after the seamount, up to 10 degrees, and strong reflections appear in the shadow zone. Disturbances in bottom-bouncing waves due to the seamount are shown in much higher angles up to 16 degrees. Figure 5-39 shows computed pulse arrivals at the depth of 
1000m on a horizontal plane.

\section{c) Comparison of pulse arrivals with various number of modes}

Comparisons of pulse arrivals are carried out to examine the effect of the number of normal modes. These comparisons are performed at the two receiver depths, $200 \mathrm{~m}$ and $1000 \mathrm{~m}$, and around seamount at $90 \mathrm{~km}, 100 \mathrm{~km}$ (peak of seamount), $110 \mathrm{~km}$, and 150 km (behind the seamount). Figures 5-40 5-43 show the comparison results of the pulse arrivals.

As we examined in Section 3.7.3, the TL is affected greatly by the truncation of normal modes especially around (or just behind) the seamount at low frequency. However, the pulse arrivals appear to be less sensitive than the TL because the pressure fields for a CW source could be smoothed in frequency, using the Fourier synthesis. As shown in the figures, pulse arrivals using the waterborne modes show limited arrivals. However, primary arrivals using the propagation modes and full modes (including leaky modes) are quite comparable to each other. This agreement indicates that we could use the waterborne modes instead of using the full modes with consistent pulse arrivals, which provides a gain in the computation time. 

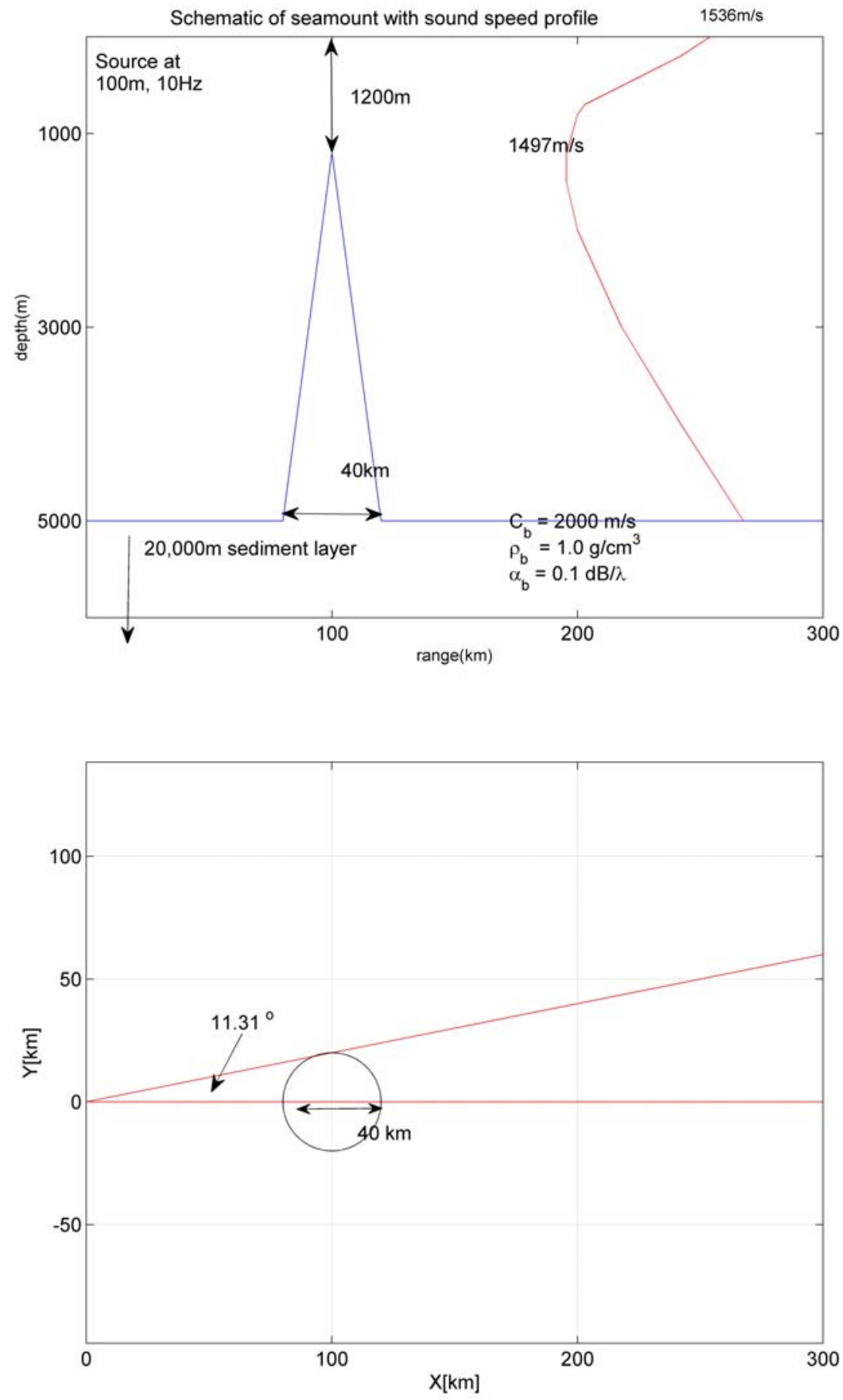

Figure 5-23: Schematic diagram of a benchmark problem with a conical seamount at a range of $100 \mathrm{~km}$ from the acoustic source, and the angle between acoustic paths passing through the centerline and outermost ring becomes 11.31 degrees. The source is located at the depth of $100 \mathrm{~m}$. 


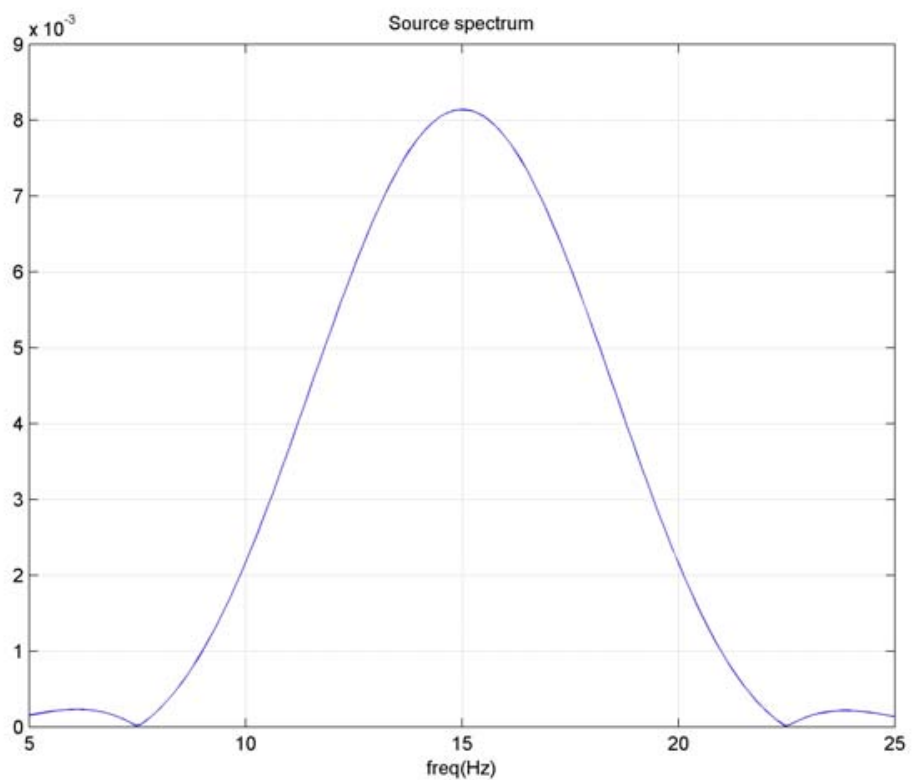

Figure 5-24: Source spectrum at a center frequency of $15 \mathrm{~Hz}$ with $10 \mathrm{~Hz}$ bandwidth.

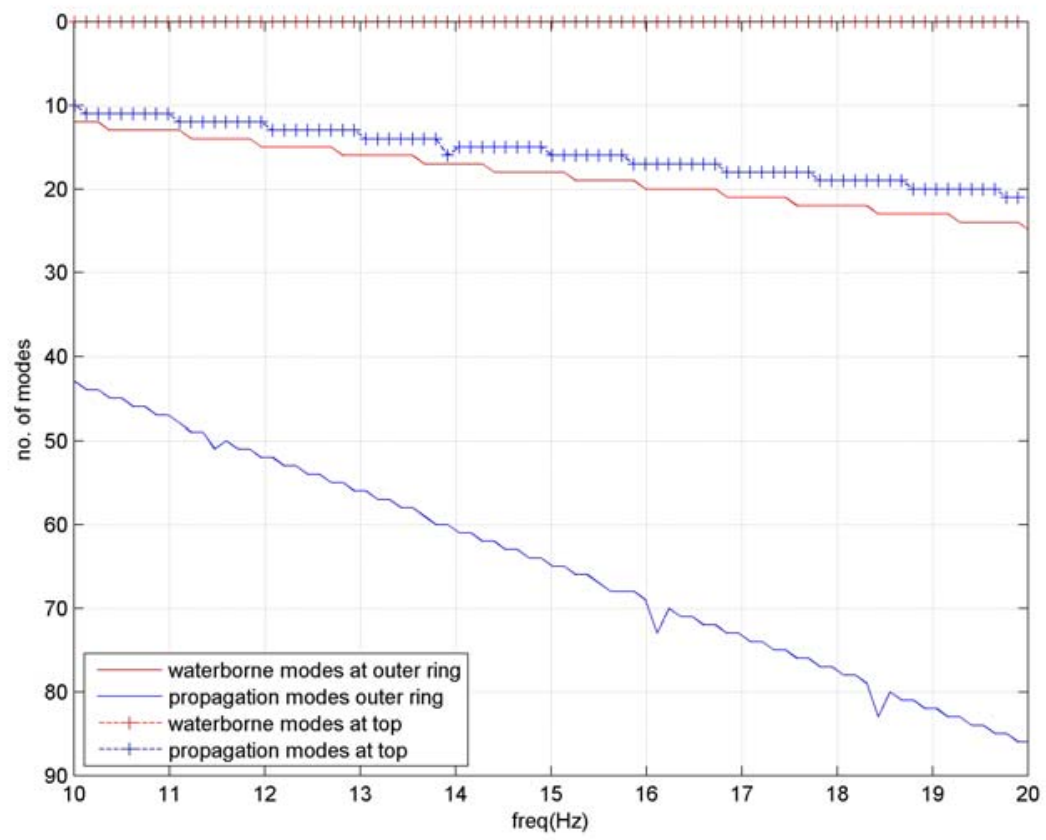

Figure 5-25: Number of vertical modes at each frequency for $10 \mathrm{~Hz}$ bandwidth from 10 to $20 \mathrm{~Hz}$; waterborne and propagation modes are compared in terms of frequency. No waterborne mode exists for this frequency band at the top of the seamount, i.e., there is no ray passing over the seamount without bottom-bouncing. 

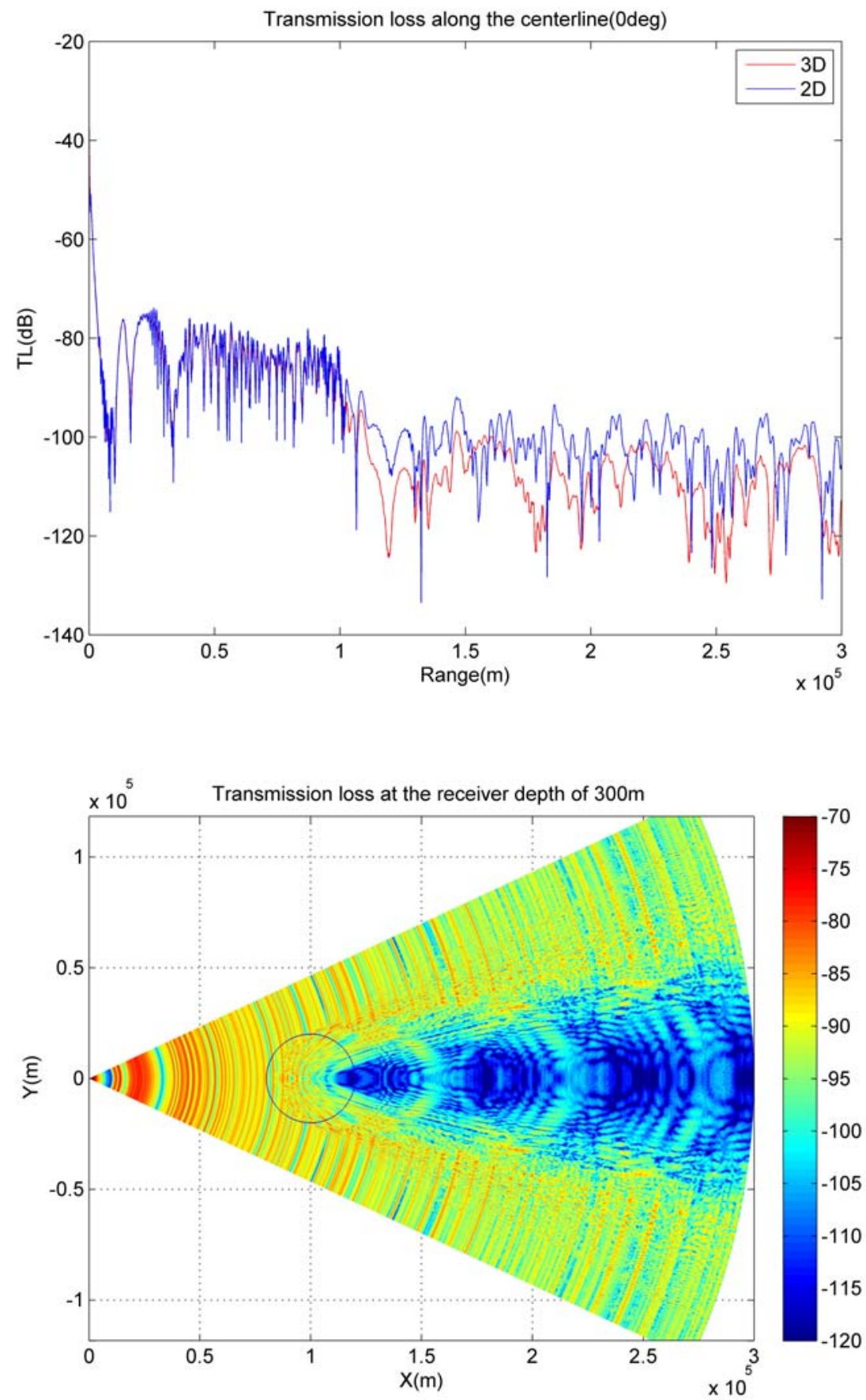

Figure 5-26: TL for a $10 \mathrm{~Hz} \mathrm{CW}$ source at a depth of $300 \mathrm{~m}$; the upper panel shows a discrepancy of the TL between the 2D and 3D model. The TL from the 3D model (3D spectral coupled-mode model) shows greater value than those from 2D model (CSNAP) by 5 10dB. The lower panel shows the TL on a horizontal plane, which shows clear shadowing cast behind the seamount. 


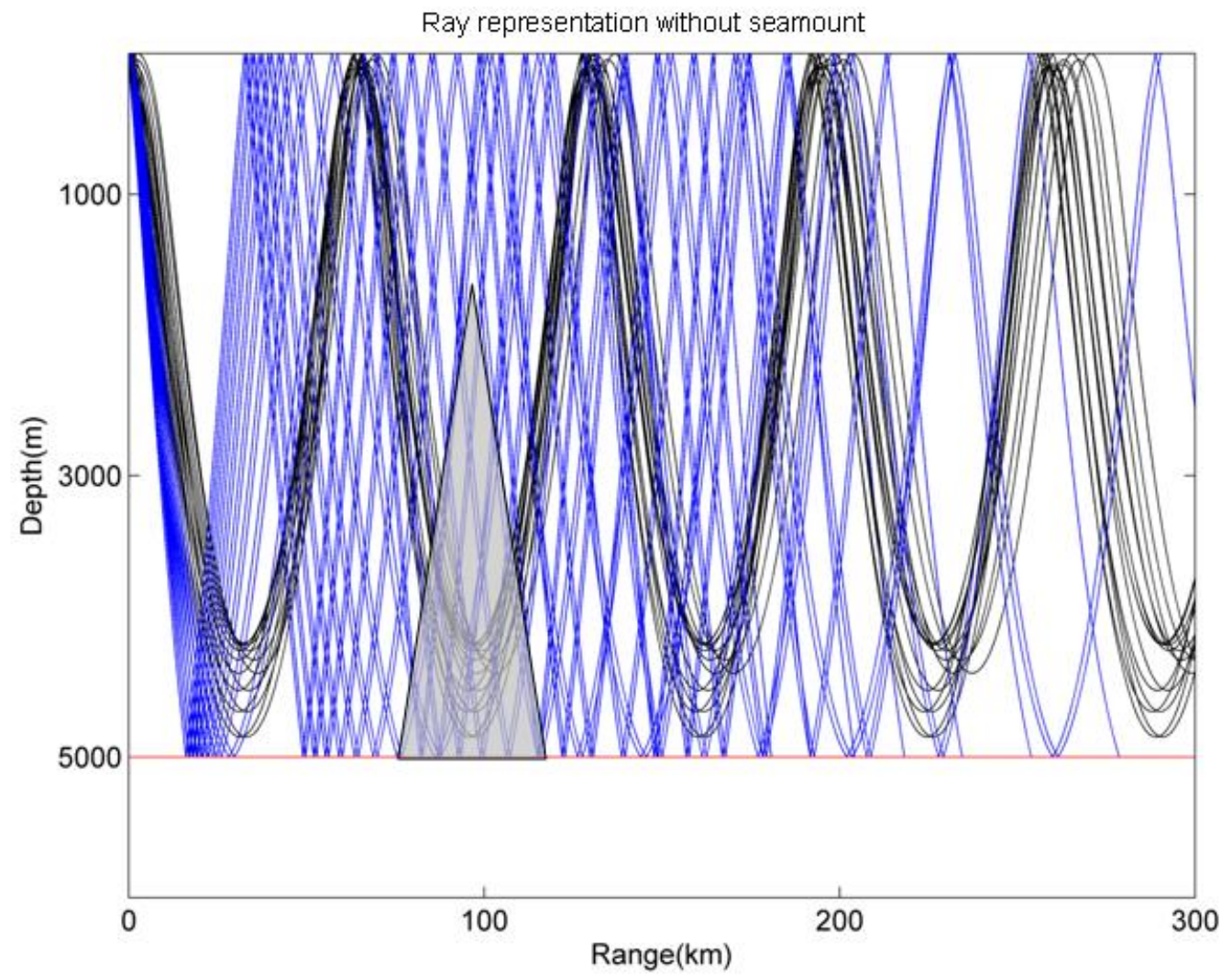

Figure 5-27: Ray representation without a seamount. The convergence zones consist in the refracted rays, shown in black, without bottom-bouncing. The ray family at the convergence zone is entirely blocked by the seamount that is located at a range of $100 \mathrm{~km}$ from the acoustic source. 

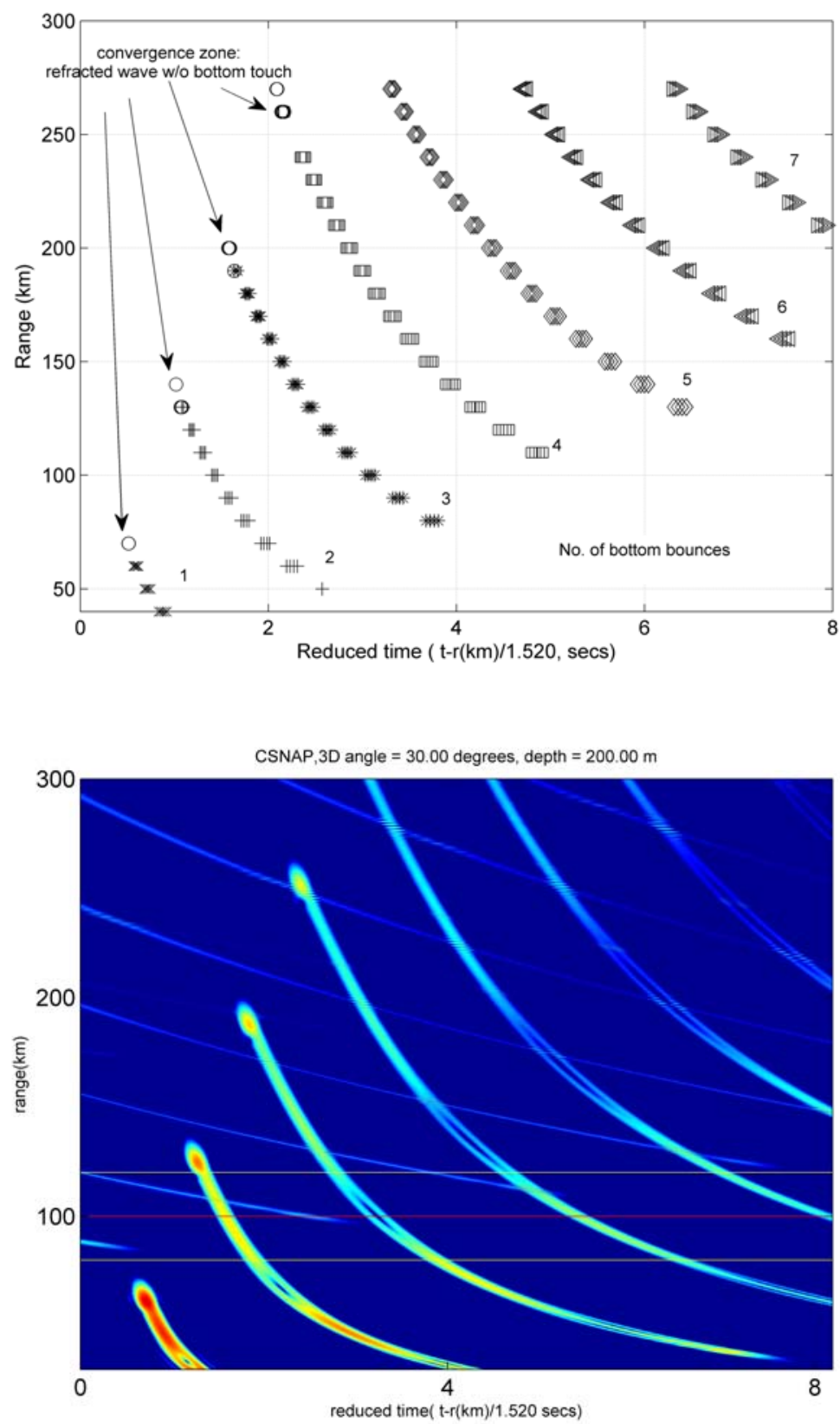

Figure 5-28: Computed pulse arrivals from the 3D spectral coupled-mode model (lower panel) compared with the ray tracing method (upper panel). In the upper panel, the eigenrays are shown in terms of reduced time; the rays are comparable to the computed pulse arrivals, far outside the seamount, 30 degrees off the centerline, with no diffraction by the seamount. At this depth, significant convergence zone arrivals are followed by bottom-bounced rays. The numbers in the upper panel denote the number of bottom-bounces for each ray family. 

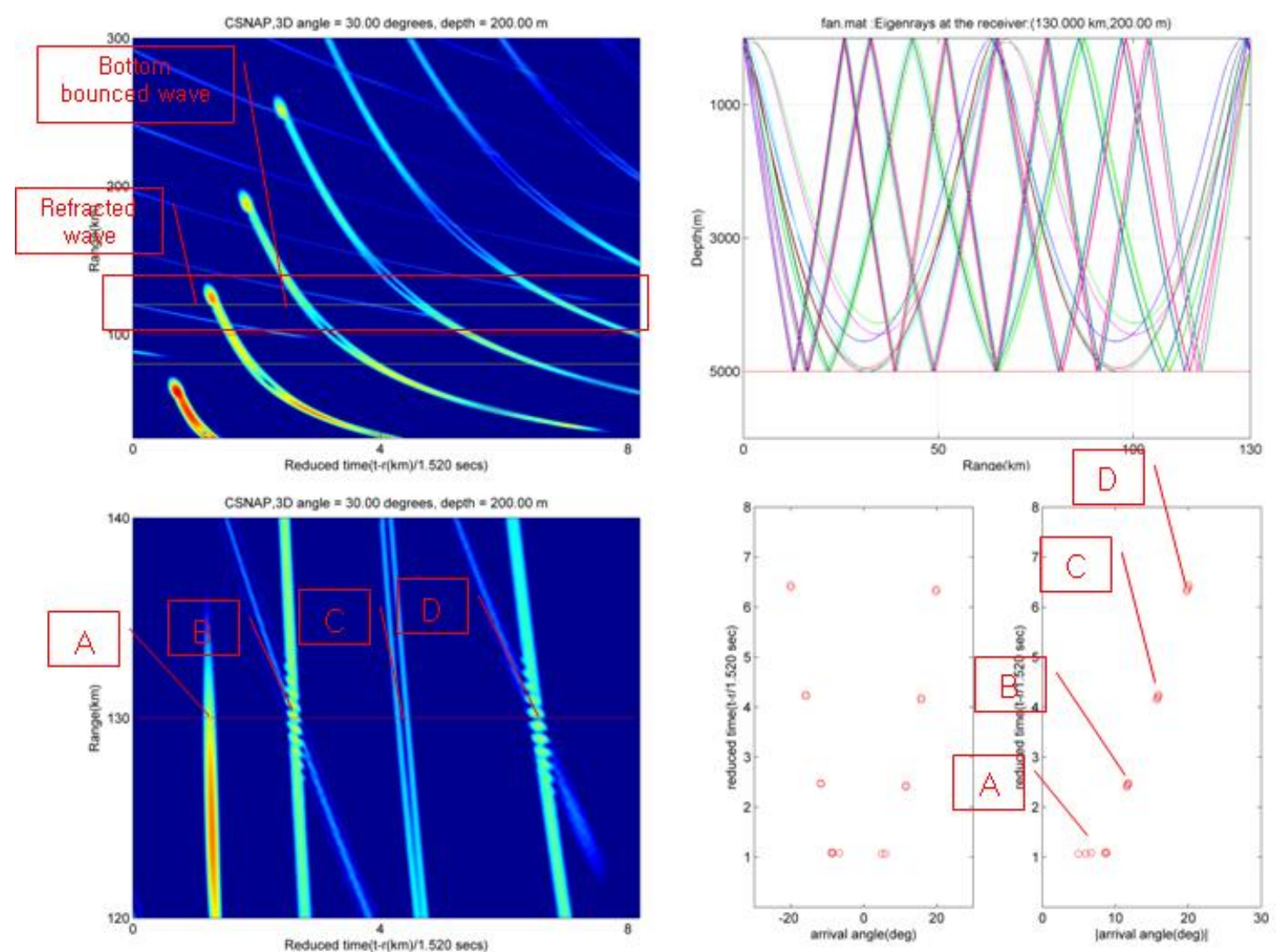

Figure 5-29: Comparison of arrival time between the ray tracing method and 3D model at a range of $130 \mathrm{~km}$. The computed pulse arrivals from the 3D spectral coupled-mode mode, along an acoustic path of the 30 degrees off the centerline, have no 3D effect by the seamount. The $A$ ray family forms strong convergence zones without bottom-bouncing; the arrival times of this ray family in the ray tracing method and the 3D spectral coupled-mode mode are quite close to each other. The ray families $B, C$, and $D$ correspond to the bottom-bouncing ray families with 2,3 , and 4 bottom-bounces, respectively. 

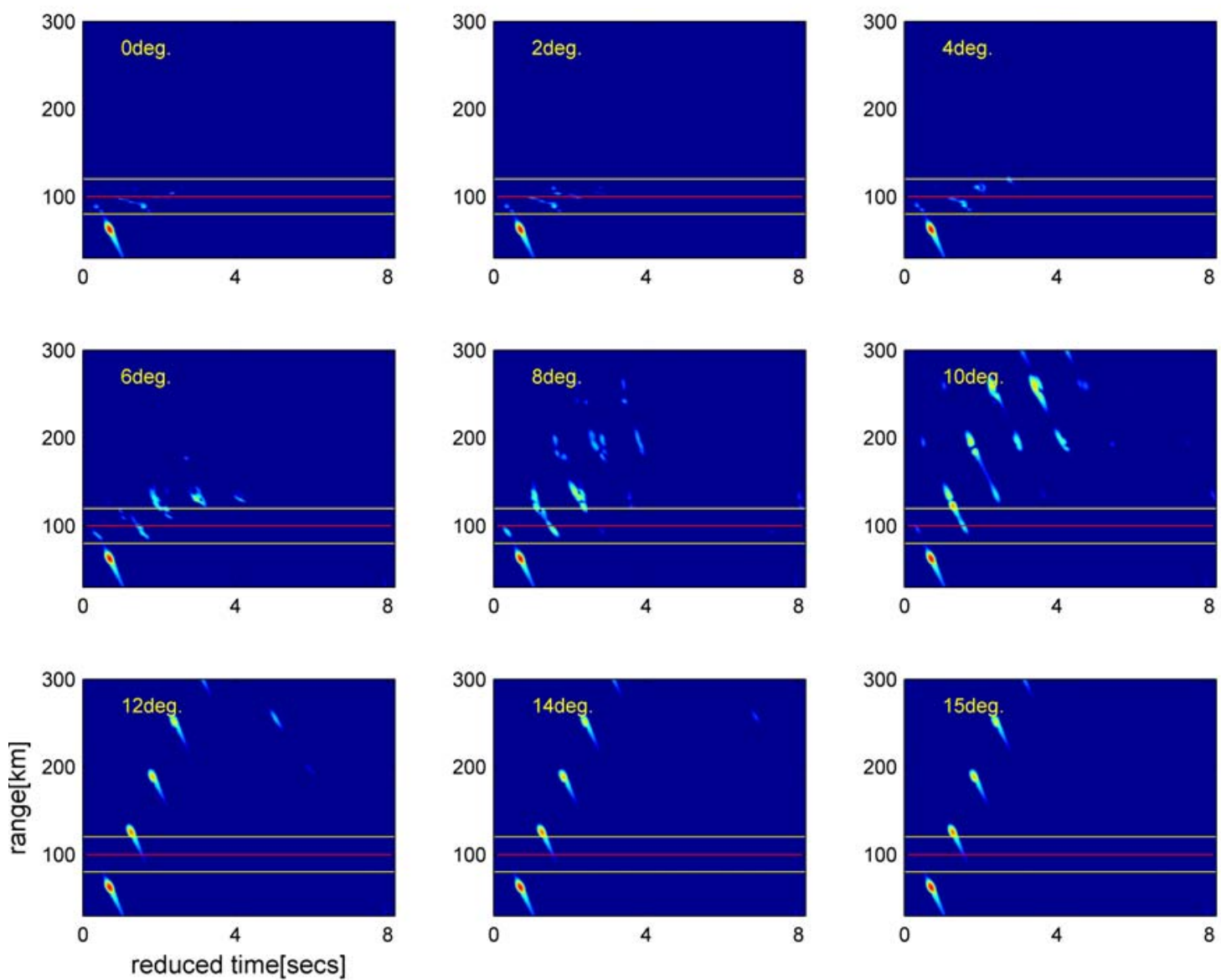

Figure 5-30: Computed pulse arrivals at a depth of $200 \mathrm{~m}$. The waterborne modes at the outermost ring are used for the mode coupling. The pulse arrivals are shown in the reduced time with a reference time of $1520 \mathrm{~m} / \mathrm{s}$. The angle denotes an acoustic path running off the centerline. The convergence zone arrivals are all blocked after the seamount up to 10 degrees, and some reflections are shown in the shadow zone. 

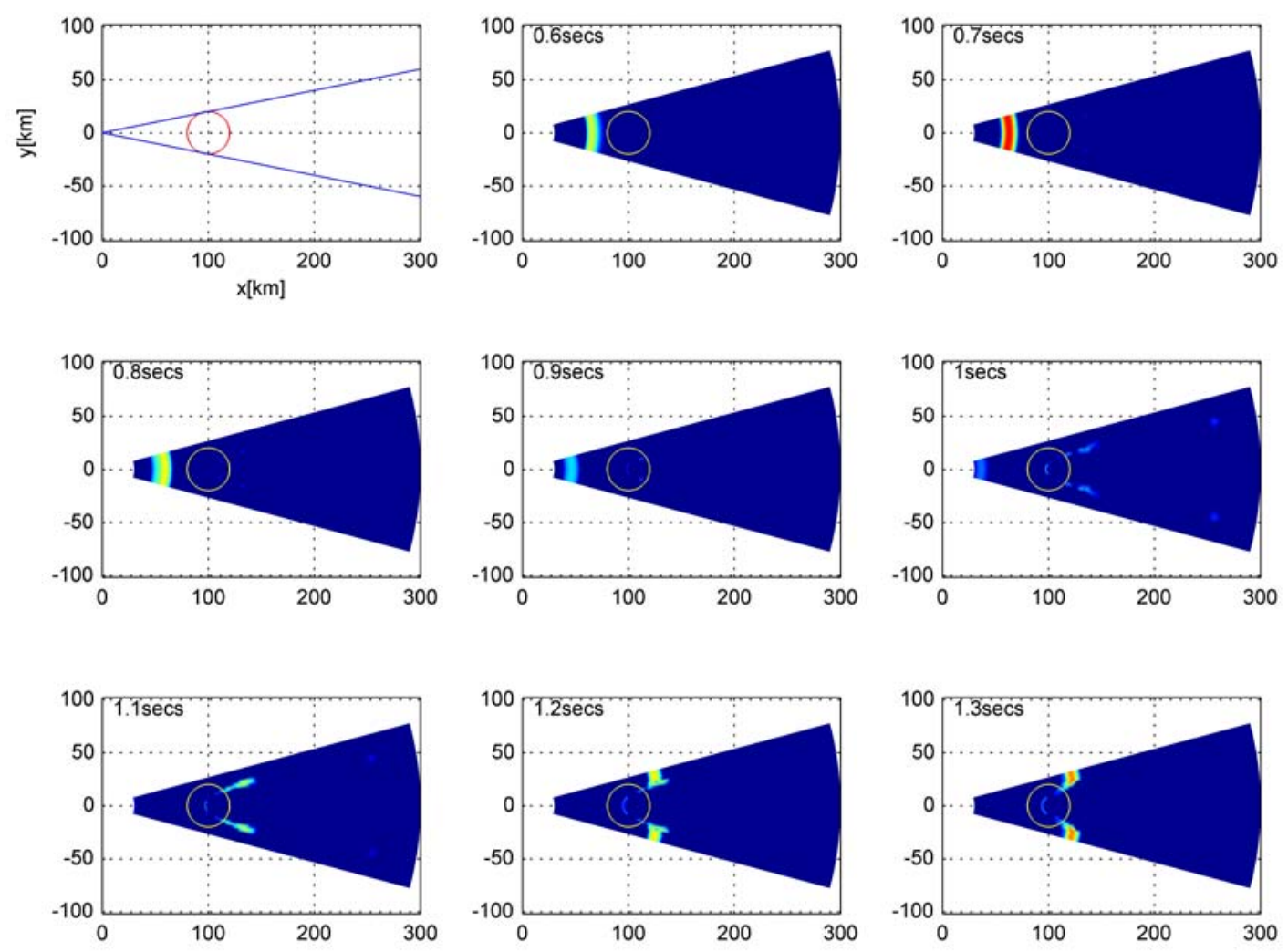

Figure 5-31: Computed pulse arrivals at a depth of $200 \mathrm{~m}$; the pulse arrivals on a horizontal plane are shown in term of the reduced time with a reference speed of $1520 \mathrm{~m} / \mathrm{s}$ ( $0.6 \sim 1.3$ seconds). The first convergence zone arrivals appear at the reduced time of $0.7 \sim 0.8$ seconds, at a range of $60 \mathrm{~km}$; the first convergence zone arrivals are followed by the weak reflections. The second convergence zone appears at $1.3 \mathrm{sec}, 120 \mathrm{~km}$. 

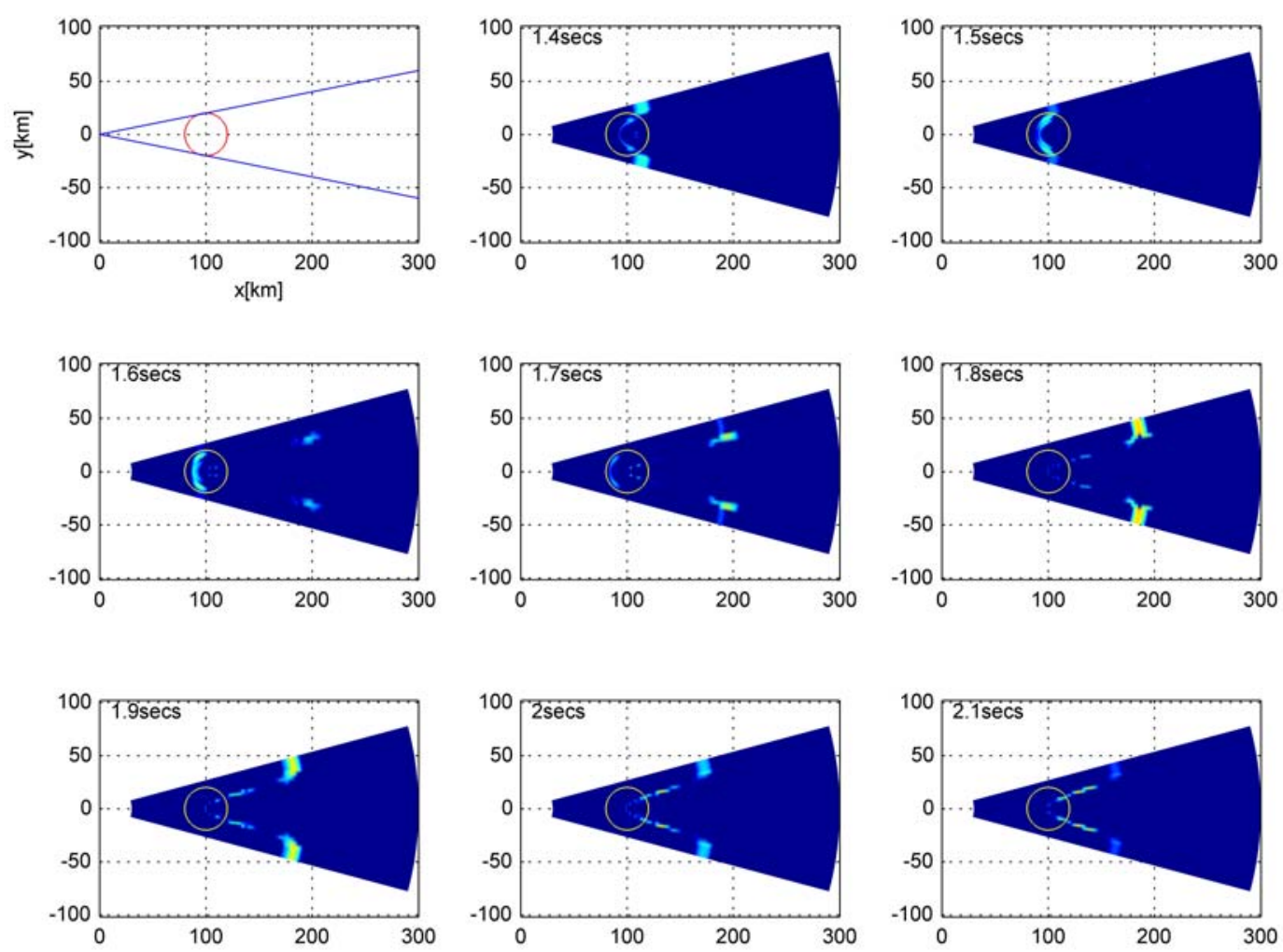

Figure 5-31: (continued) Computed pulse arrivals at a depth of 200m (1.4 2.1 seconds); the third convergence zone arrivals appear at $1.8 \sim 1.9$ seconds, $180 \sim 190 \mathrm{~km}$ from the source. The reflections are not significant because only waterborne modes are used in the mode-coupling. 

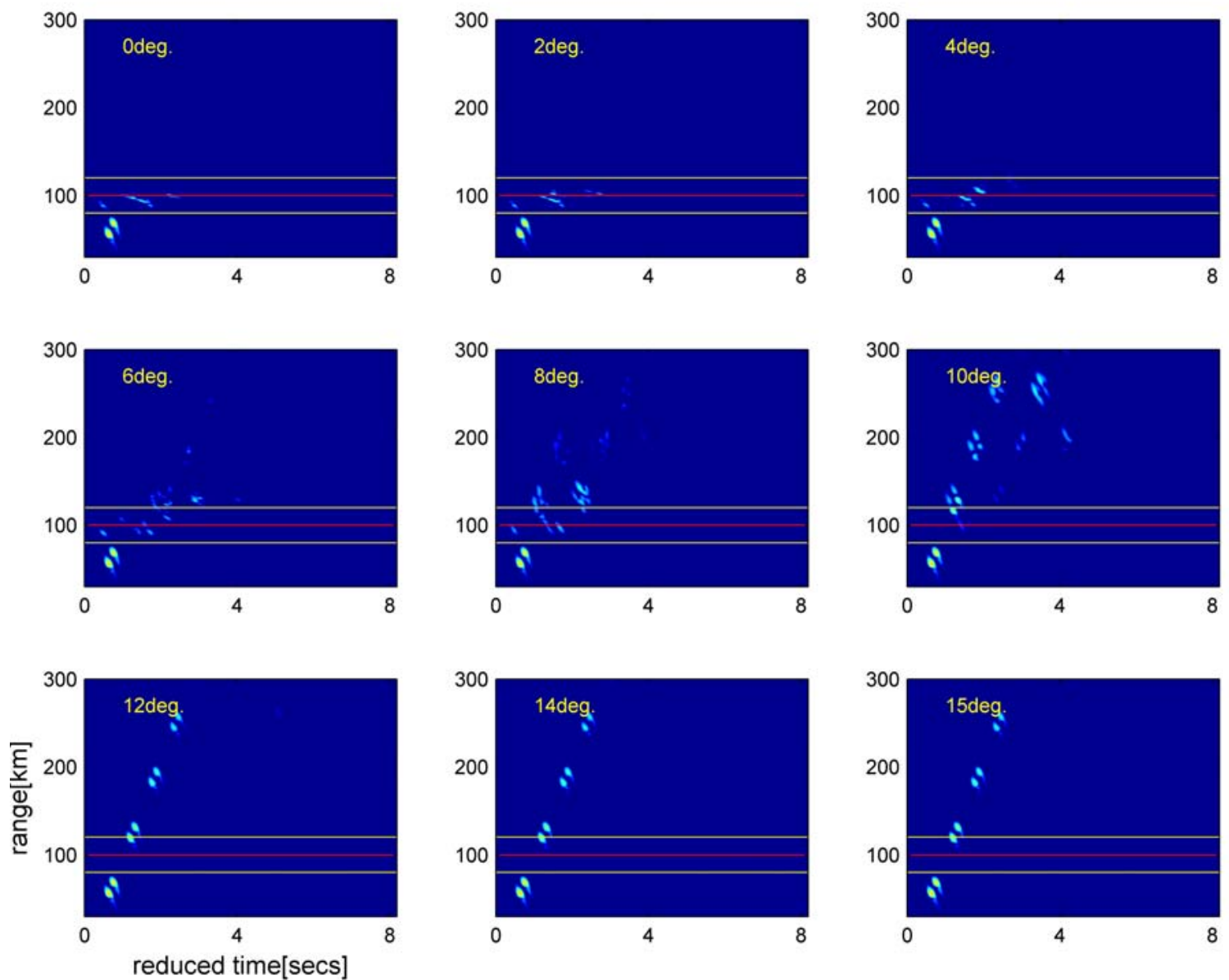

Figure 5-32: Computed pulse arrivals at a depth of $1000 \mathrm{~m}$; the waterborne modes at the outermost ring are used for the mode coupling. The pulse arrivals are shown in the reduced time with a reference time of $1520 \mathrm{~m} / \mathrm{s}$. The angle denotes an acoustic path running off the centerline. The two strong ray family arrivals appear in higher angle; however, those are all blocked in lower angle after the seamount up to 10 degrees. Weak reflections are shown in the shadow zone. 

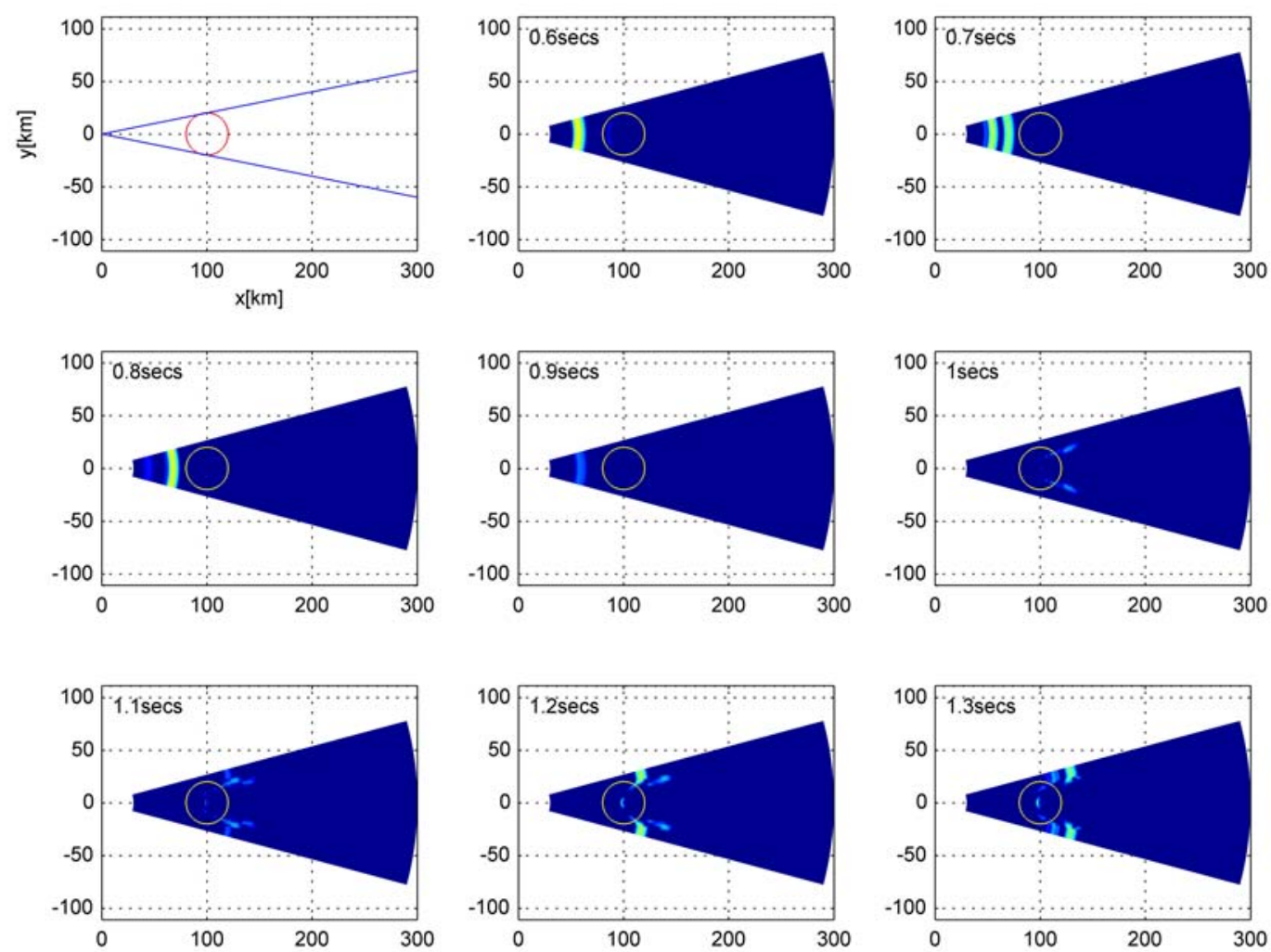

Figure 5-33: Computed pulse arrivals at a depth of $1000 \mathrm{~m}$; the pulse arrivals on a horizontal plane are shown in terms of the reduced time with reference speed of $1520 \mathrm{~m} / \mathrm{s}$ ( $0.6 \sim 1.3$ seconds). 

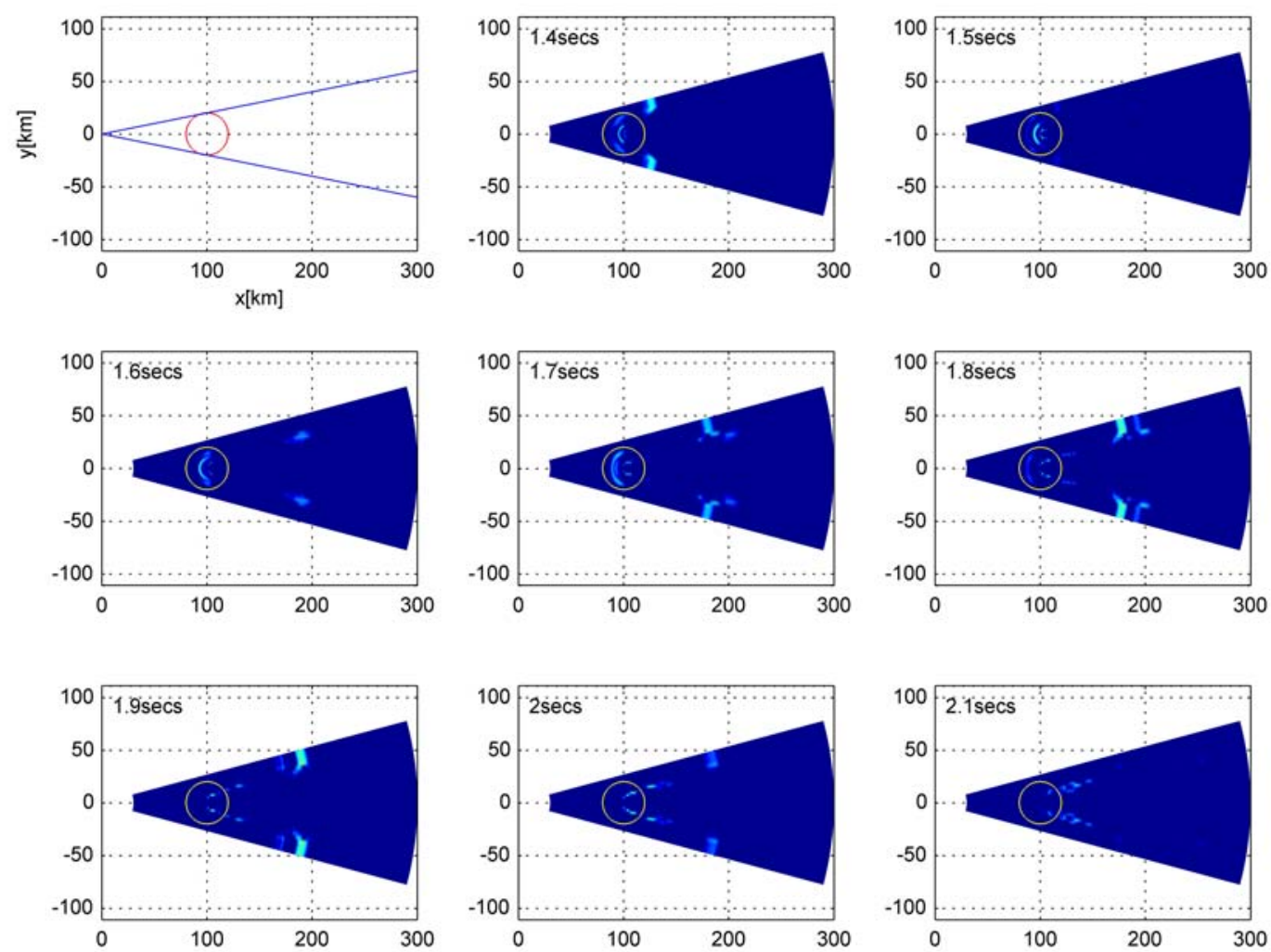

Figure 5-33: (continued) Computed pulse arrivals at a depth of 1000m (1.4 2.1 seconds). 

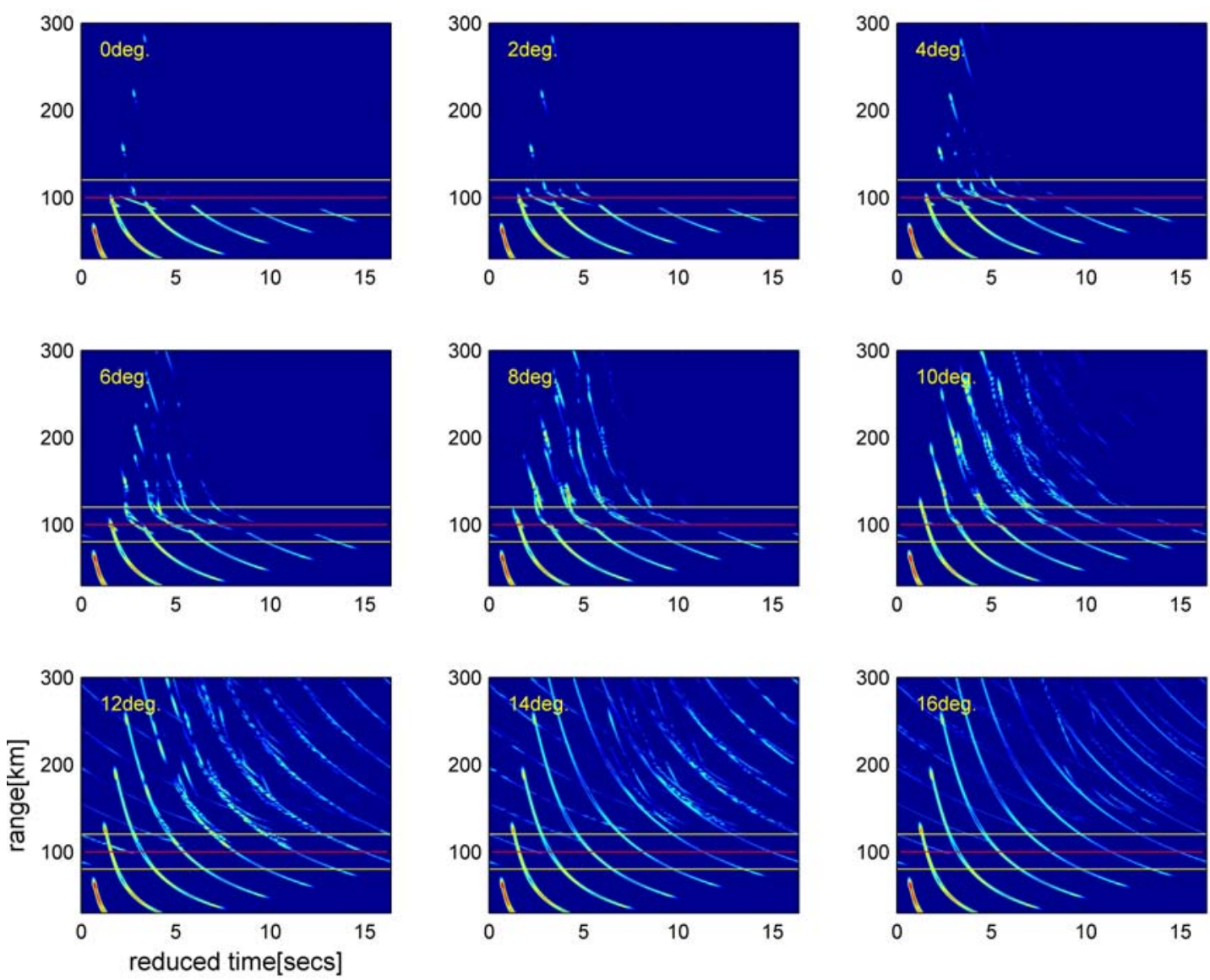

Figure 5-34: Computed pulse arrivals at a depth of $200 \mathrm{~m}$; the propagation modes at the outermost ring are used for the mode coupling, which provides full ray arrivals, including bottom bounces. The pulse arrivals are shown in the reduced time with a reference time of $1520 \mathrm{~m} / \mathrm{s}$. The angle denotes an acoustic path running off the centerline. The convergence zone arrivals are all blocked after the seamount up to 10 degrees and the strong reflections are shown in the perturbed zone. However, disturbances of bottom bouncing rays due to the seamount appear in much higher angles up to 16 degrees, compared to the cases with the waterborne modes. 

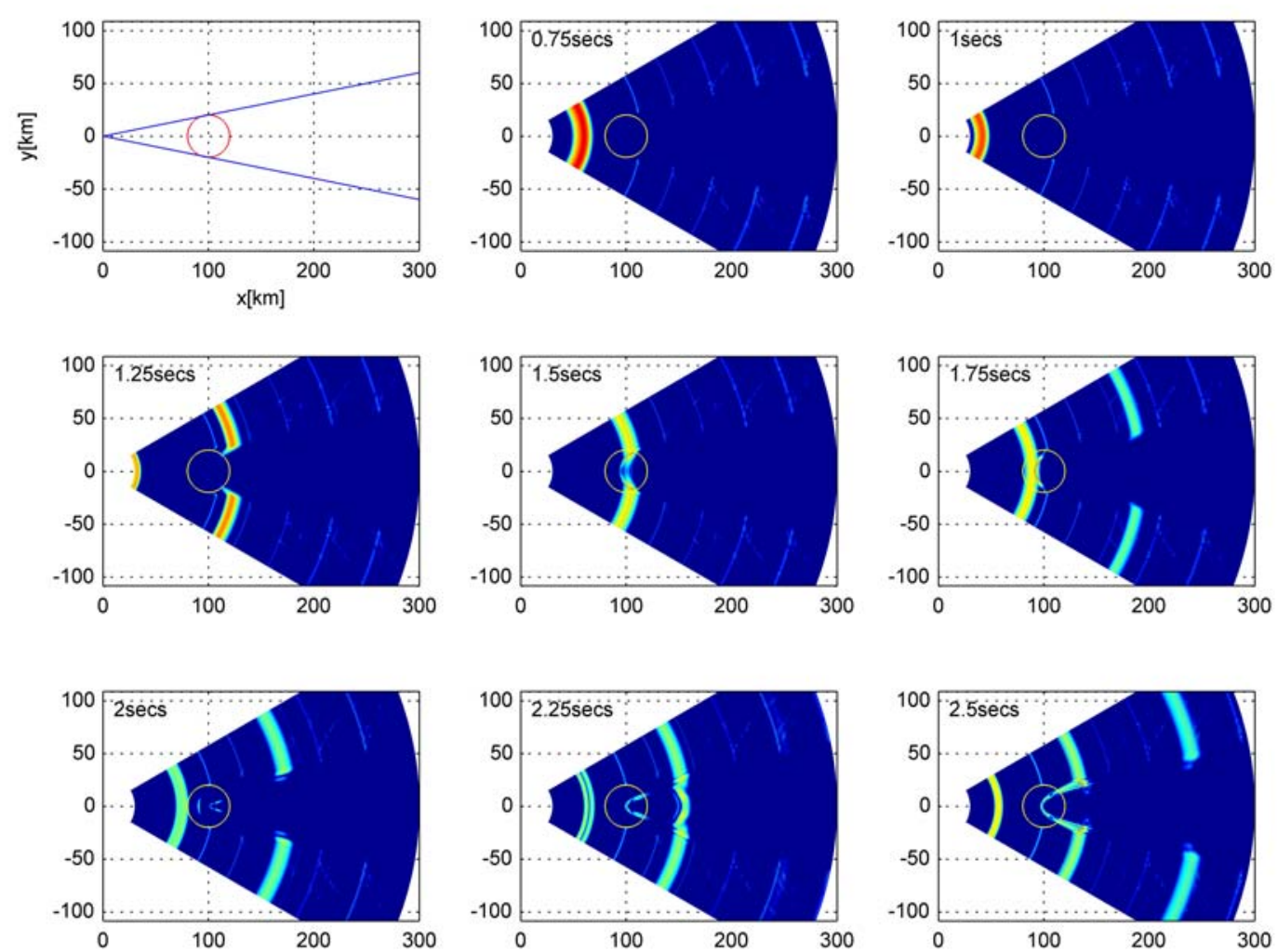

Figure 5-35: Computed pulse arrivals at a depth of 200m; the pulse arrivals on a horizontal plane are shown in terms of the reduced time with a reference speed of $1520 \mathrm{~m} / \mathrm{s}(0.75 \sim 2.5$ seconds). The first convergence zone arrivals appear before the seamount at 0.75 seconds, $60 \mathrm{~km}$ from the source, which are followed by the strong reflections. The pulse arrivals appear to be moving toward the acoustic source because of the higher propagation speed relative to the reference speed. The second convergence zone appears at 1.25 seconds, $120 \mathrm{~km}$, which clearly shows the shadowing cast of the seamount. The third and fourth convergence zone appear at 1.75 2.0, 2.5 seconds, $190 \mathrm{~km}$ and $250 \mathrm{~km}$ from the source, respectively. At around 2.25 seconds, between the third and fourth convergence zone appearances, a convergence is formed by the reflected-refracted rays within the perturbation zone. Reflections due to the seamount generate quite complicated patterns of pulse arrivals in and around the shadow zone, including waves going away form the seamount to higher angles. 

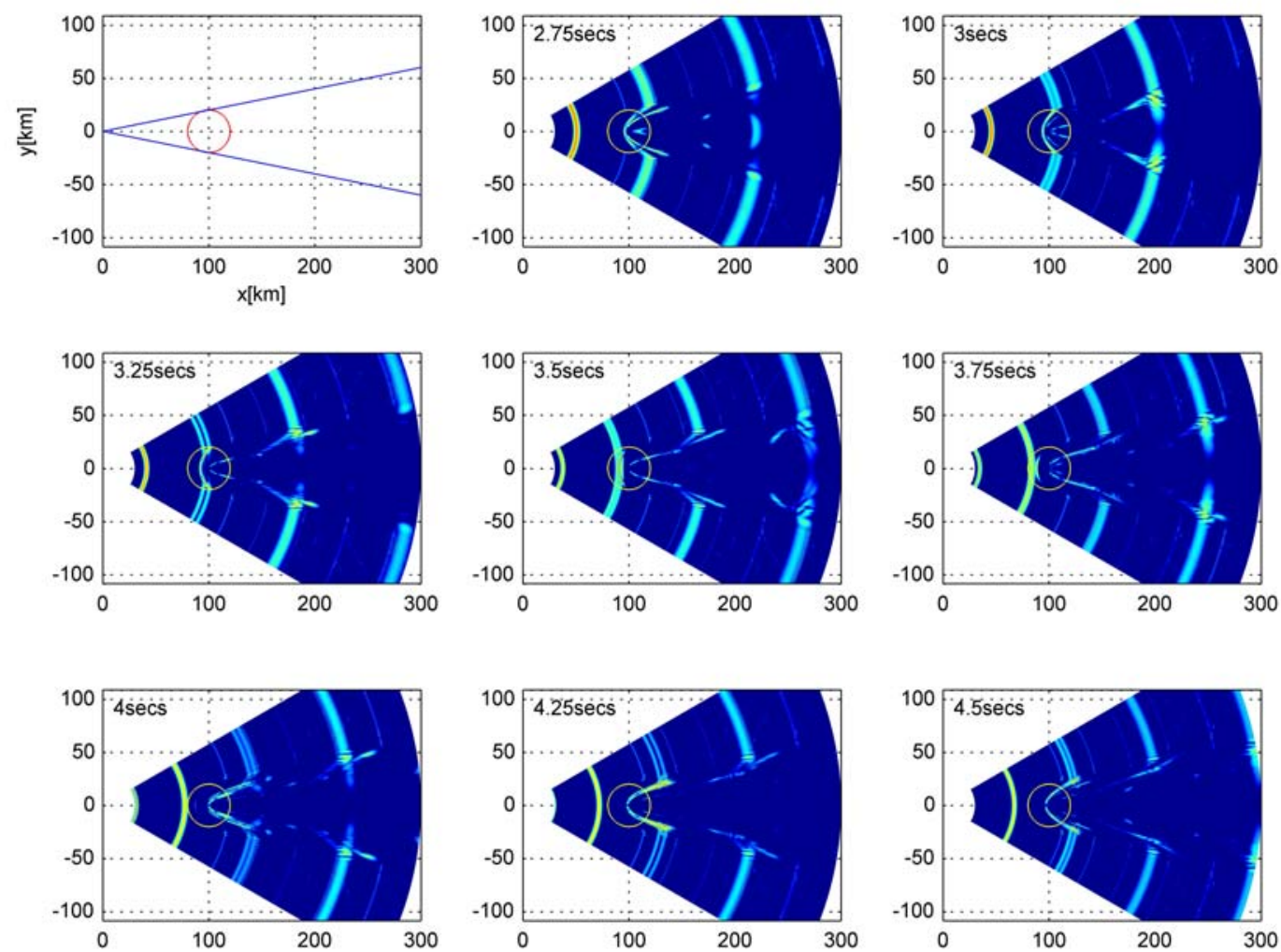

Figure 5-36: (continued) Computed pulse arrivals at a depth of 200m (2.75 4.5 seconds). 

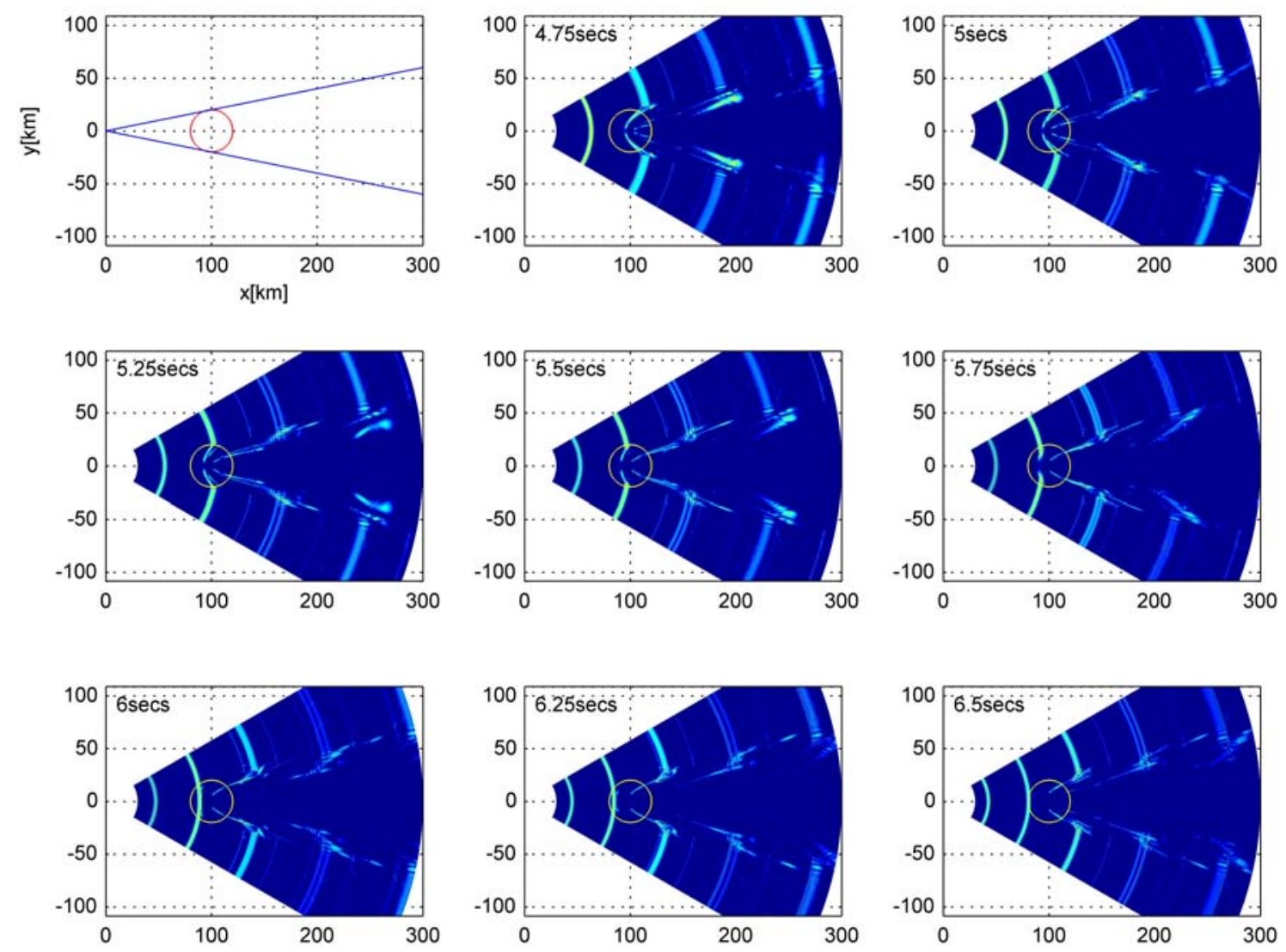

Figure 5-37: (continued) Computed pulse arrivals at a depth of 200m (4.75 6.5 seconds). 

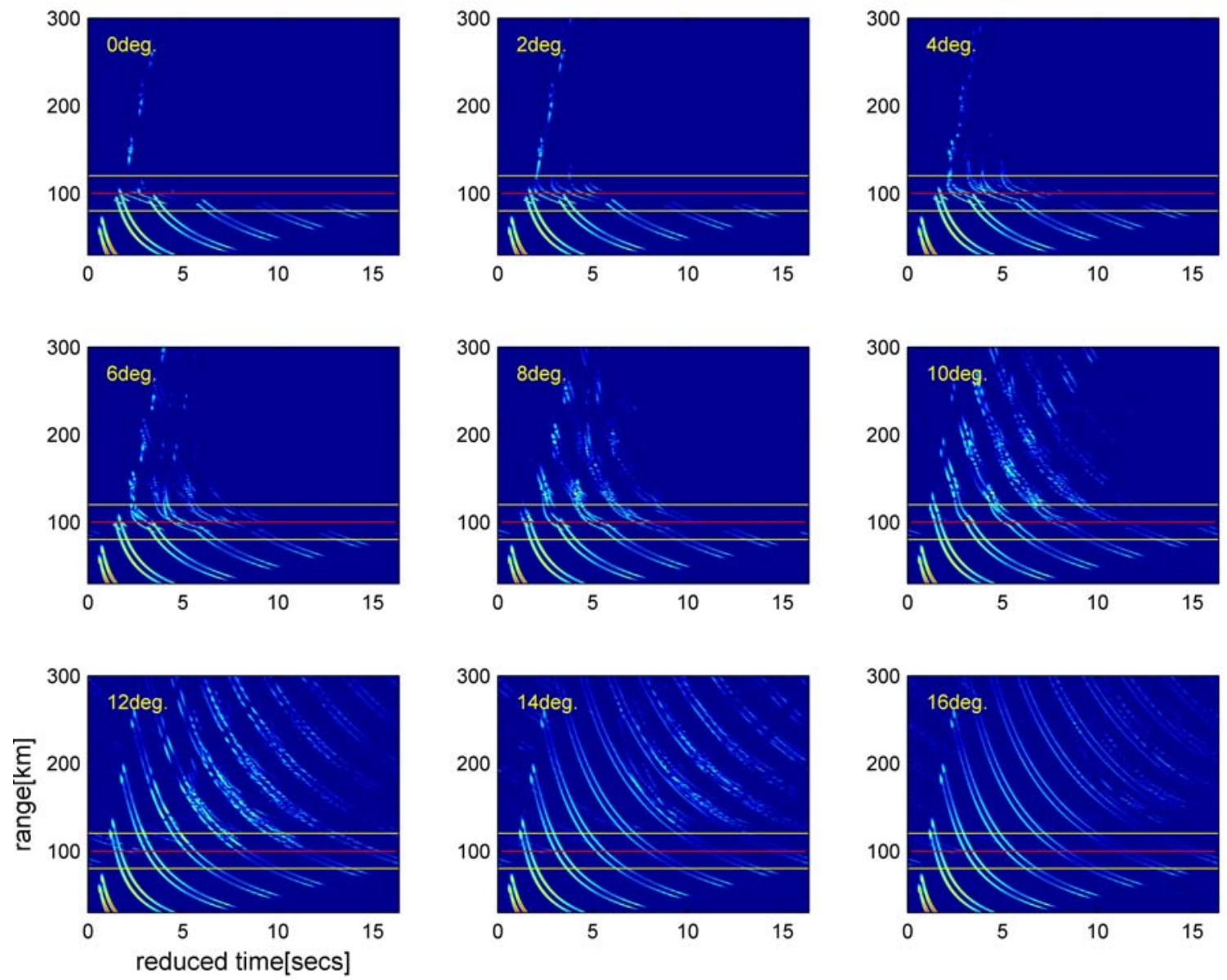

Figure 5-38: Computed pulse arrivals at a depth of 1000m; the propagation modes at the outermost ring are used for the mode coupling, which gives full ray arrivals including bottombouncing. The pulse arrivals are shown in the reduced time with a reference time of $1520 \mathrm{~m} / \mathrm{s}$. The angle denotes an acoustic path running off the centerline. The two strong ray families arrives in higher angles without blockage; however, these are all blocked in lower angle after the seamount up to 10 degrees and the strong reflections appear in shadow zone. Disturbances of bottom bouncing waves due to the seamount are shown in much higher angle up to 16 degrees. 

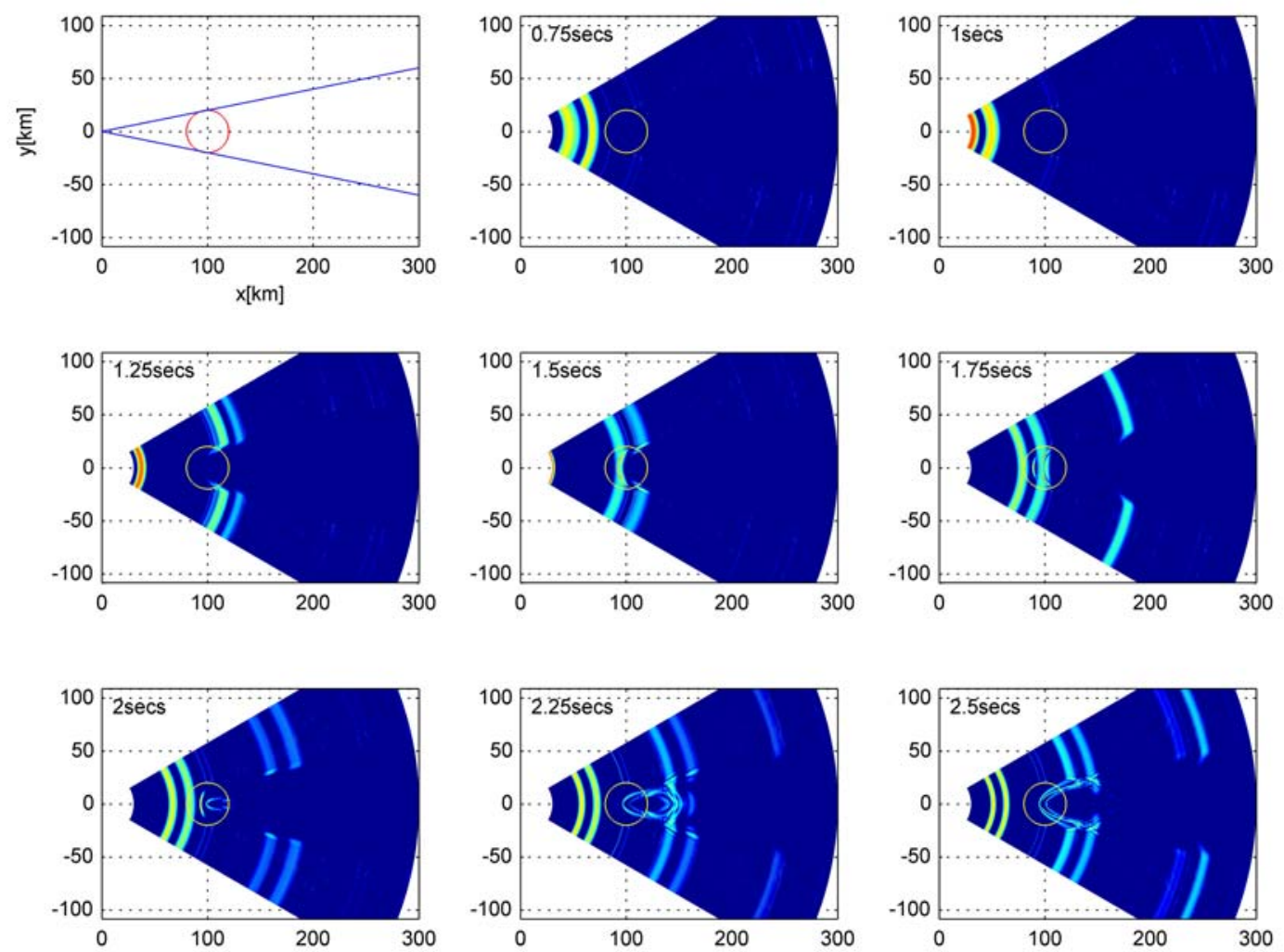

Figure 5-39: Computed pulse arrivals at a depth of $1000 \mathrm{~m}$; the pulse arrivals on a horizontal plane are shown in term of the reduced time with a reference speed of $1520 \mathrm{~m} / \mathrm{s}(0.75 \sim 2.5$ seconds). 

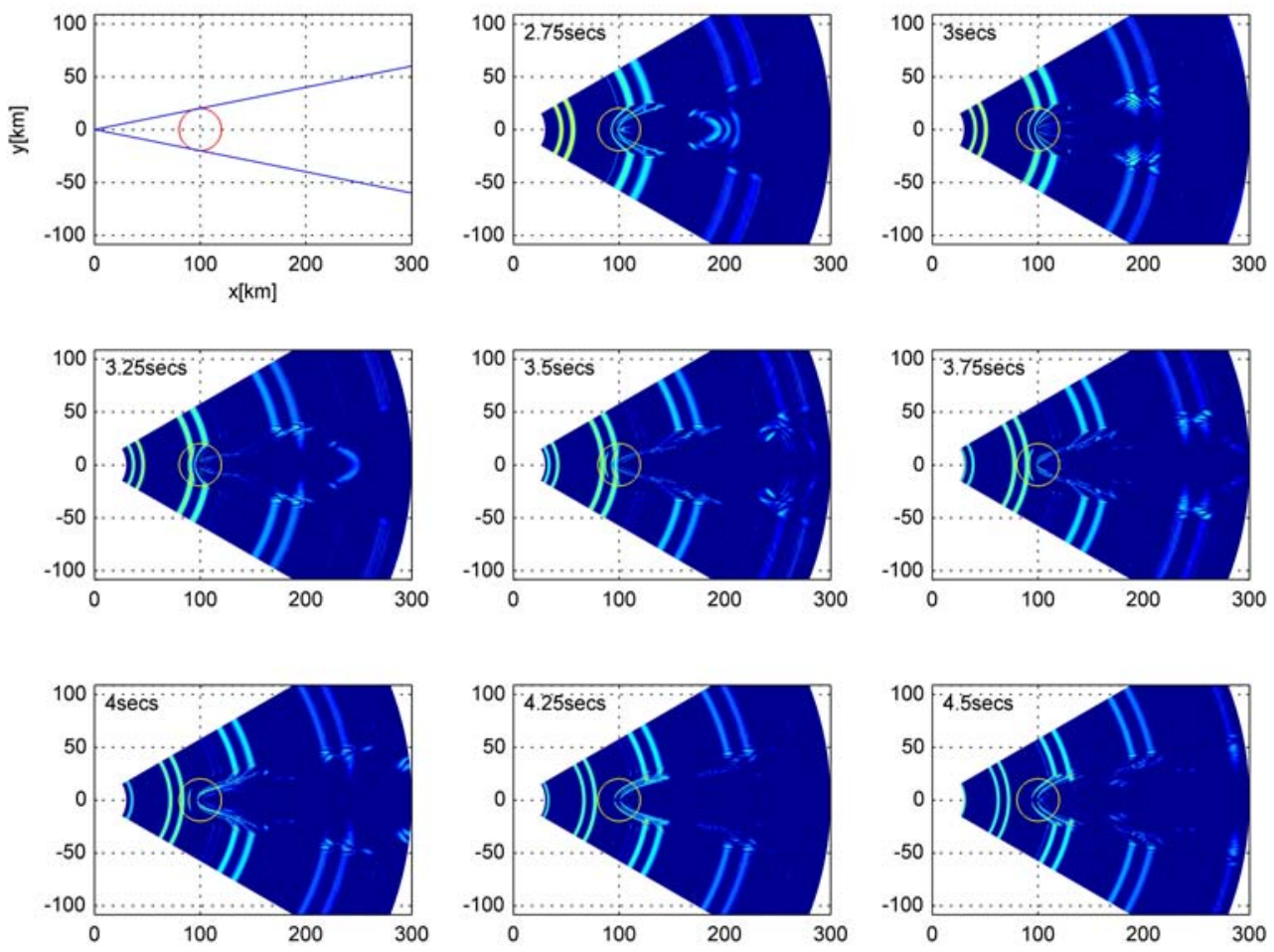

Figure 5-39: (continued) Computed pulse arrivals at the depth of 1000m (2.75 4.5 seconds). 

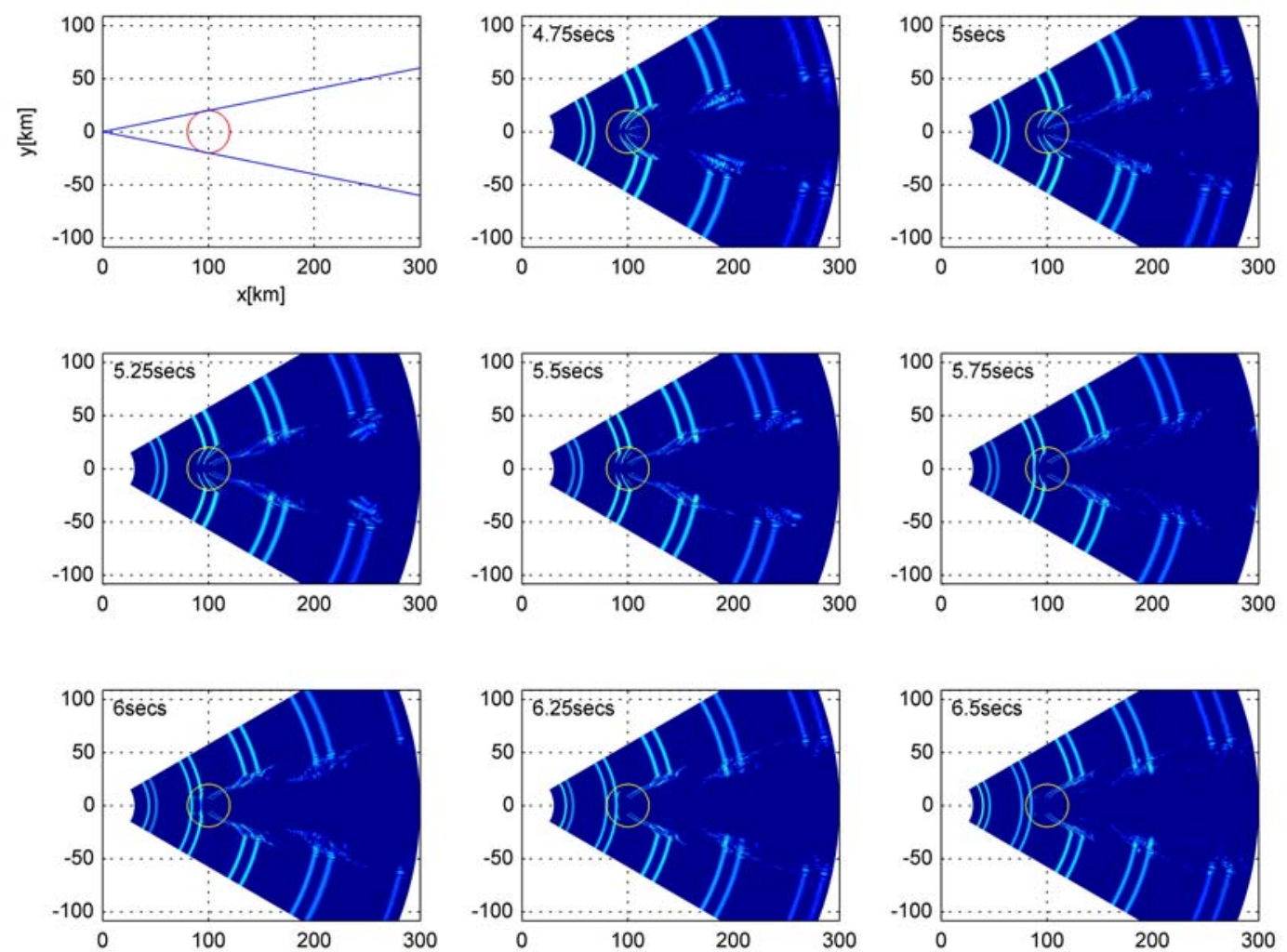

Figure 5-39: (continued) Computed pulse arrivals at the depth of 1000m (4.75 6.5 seconds). 

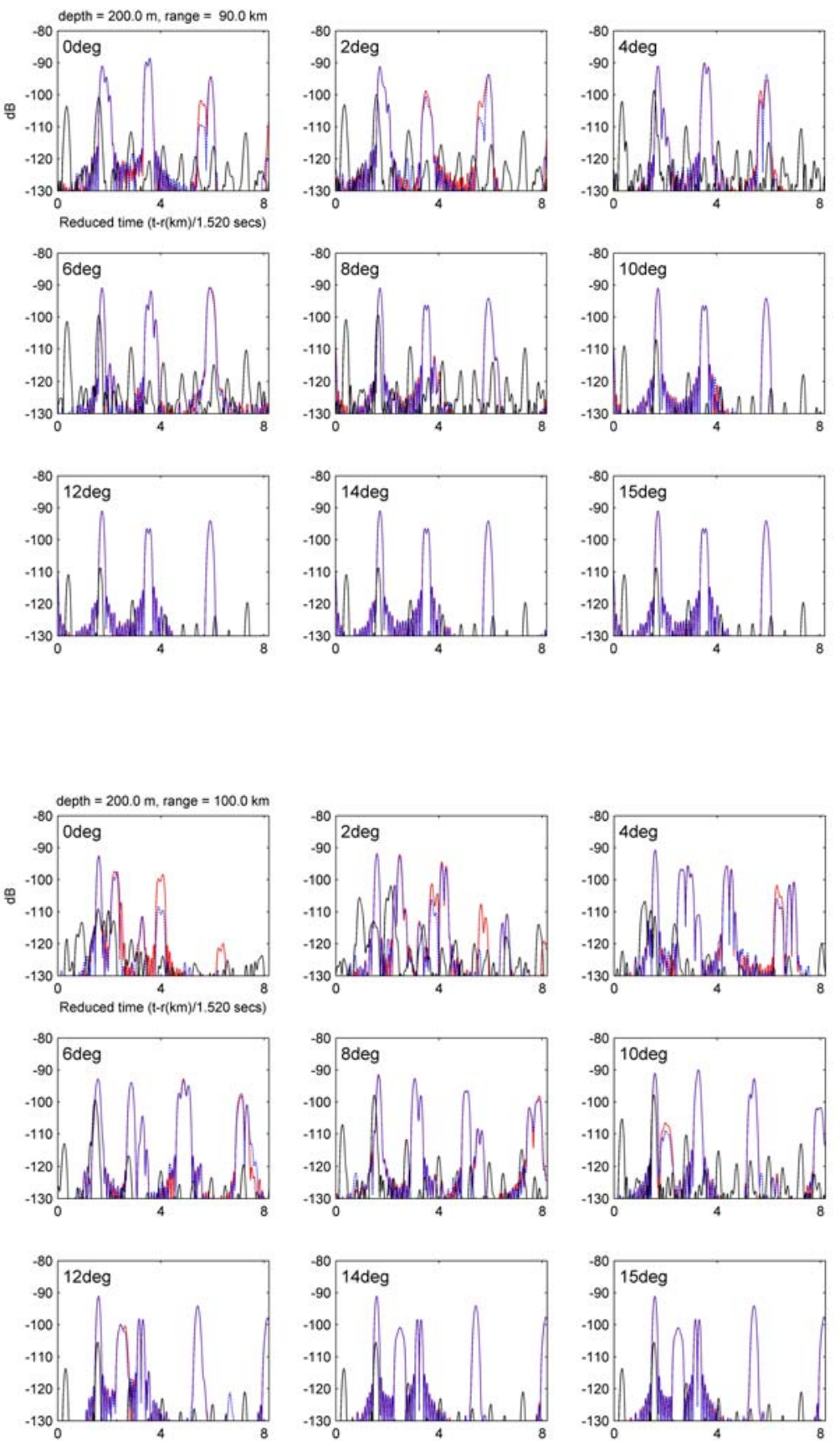

Figure 5-40: Comparison of amplitude of computed pulses between waterborne modes (black), propagation modes (blue), and full modes (with leaky modes, red) at a depth of $200 \mathrm{~m}$. The ranges are $90 \mathrm{~km}$ (top panel) and 100km (bottom panel). 

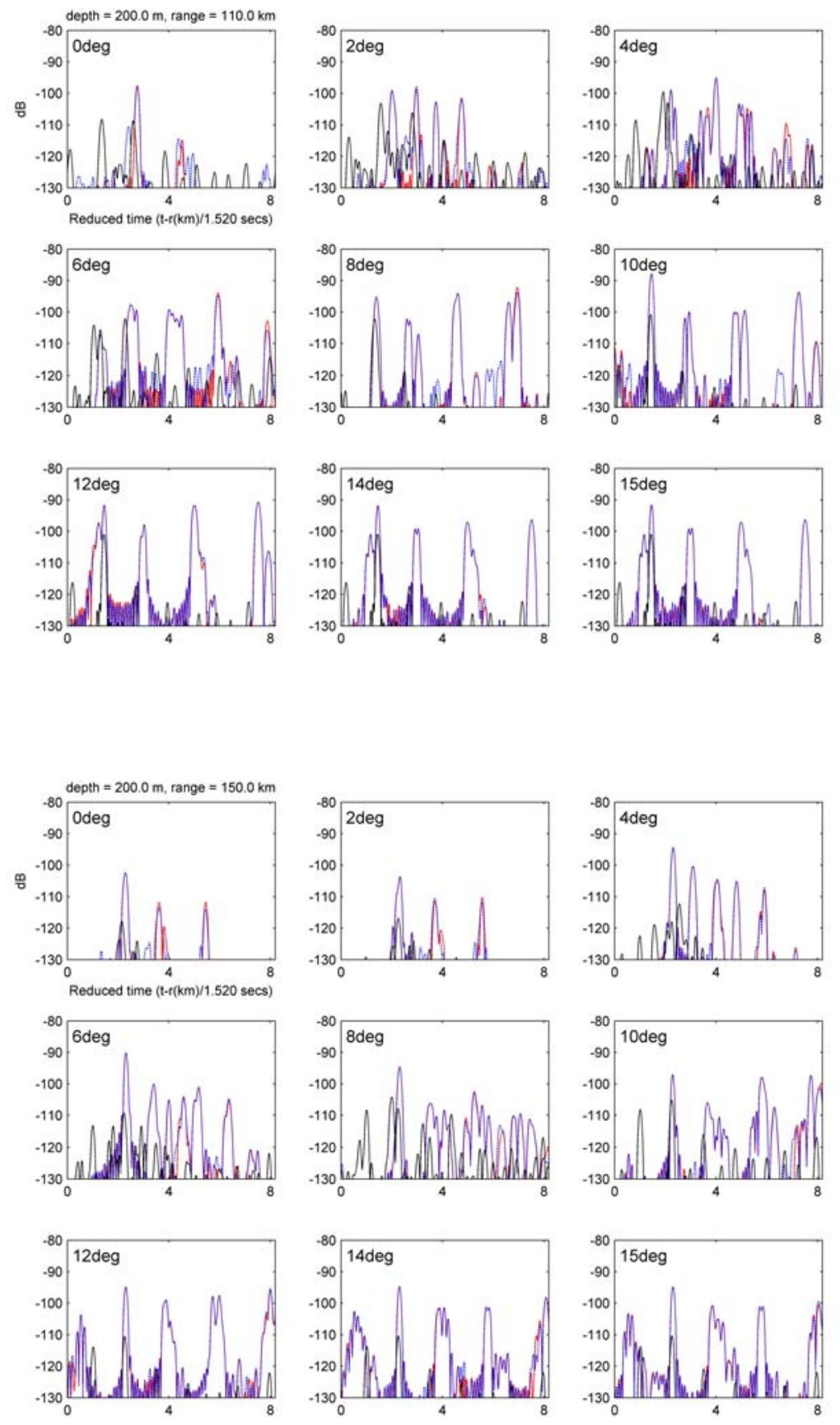

Figure 5-41: Comparison of amplitude of computed pulses between waterborne modes (black), propagation modes (blue) and full modes (w/ leaky modes, red) at a depth of $200 \mathrm{~m}$. The ranges are $110 \mathrm{~km}$ (top panel) and 150km (bottom panel). 

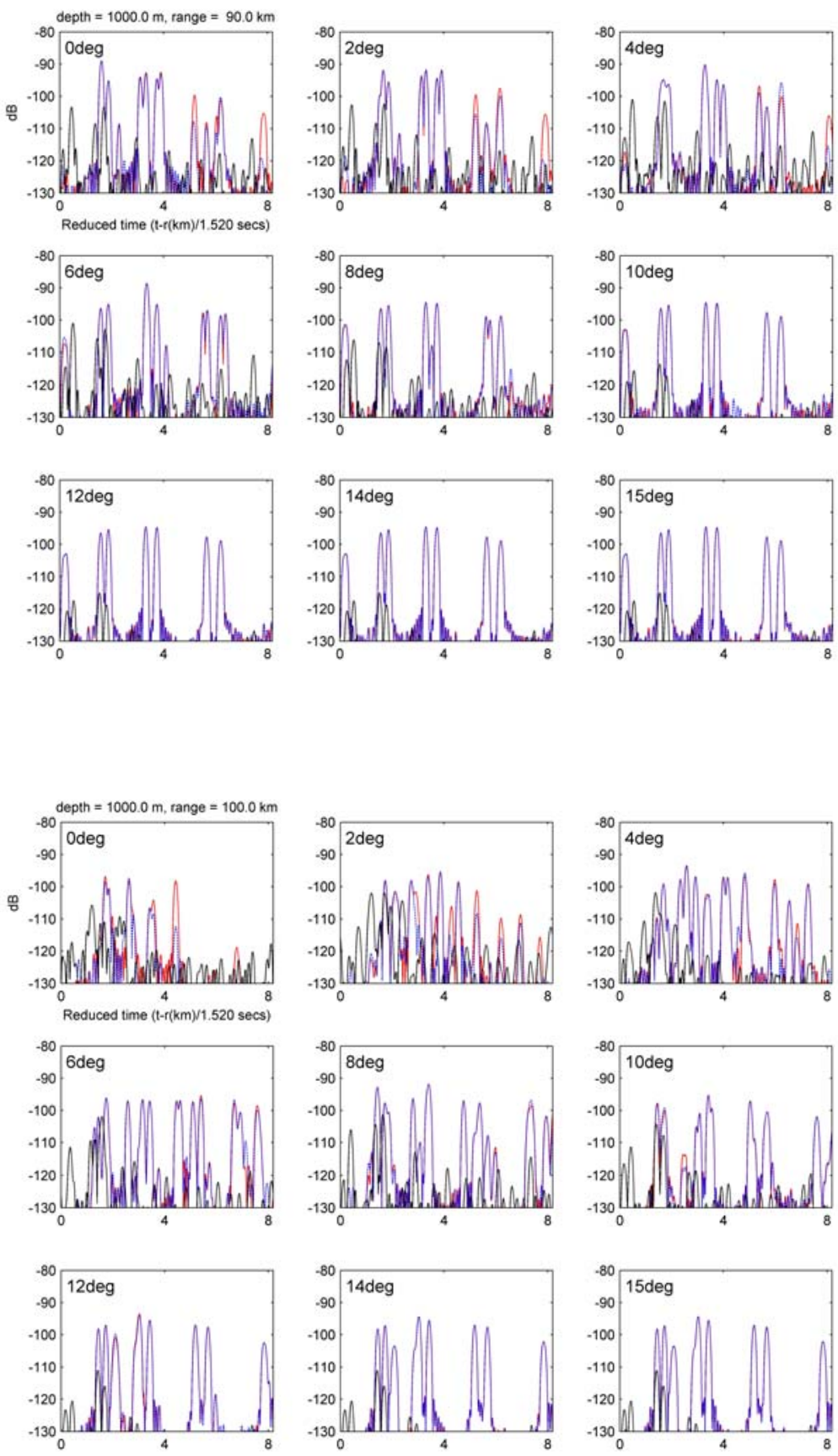

Figure 5-42: Comparison of amplitude of computed pulses between waterborne modes (black), propagation modes (blue) and full modes (w/ leaky modes, red) at a depth of $1000 \mathrm{~m}$. The ranges are $90 \mathrm{~km}$ (top panel) and 100km (bottom panel). 

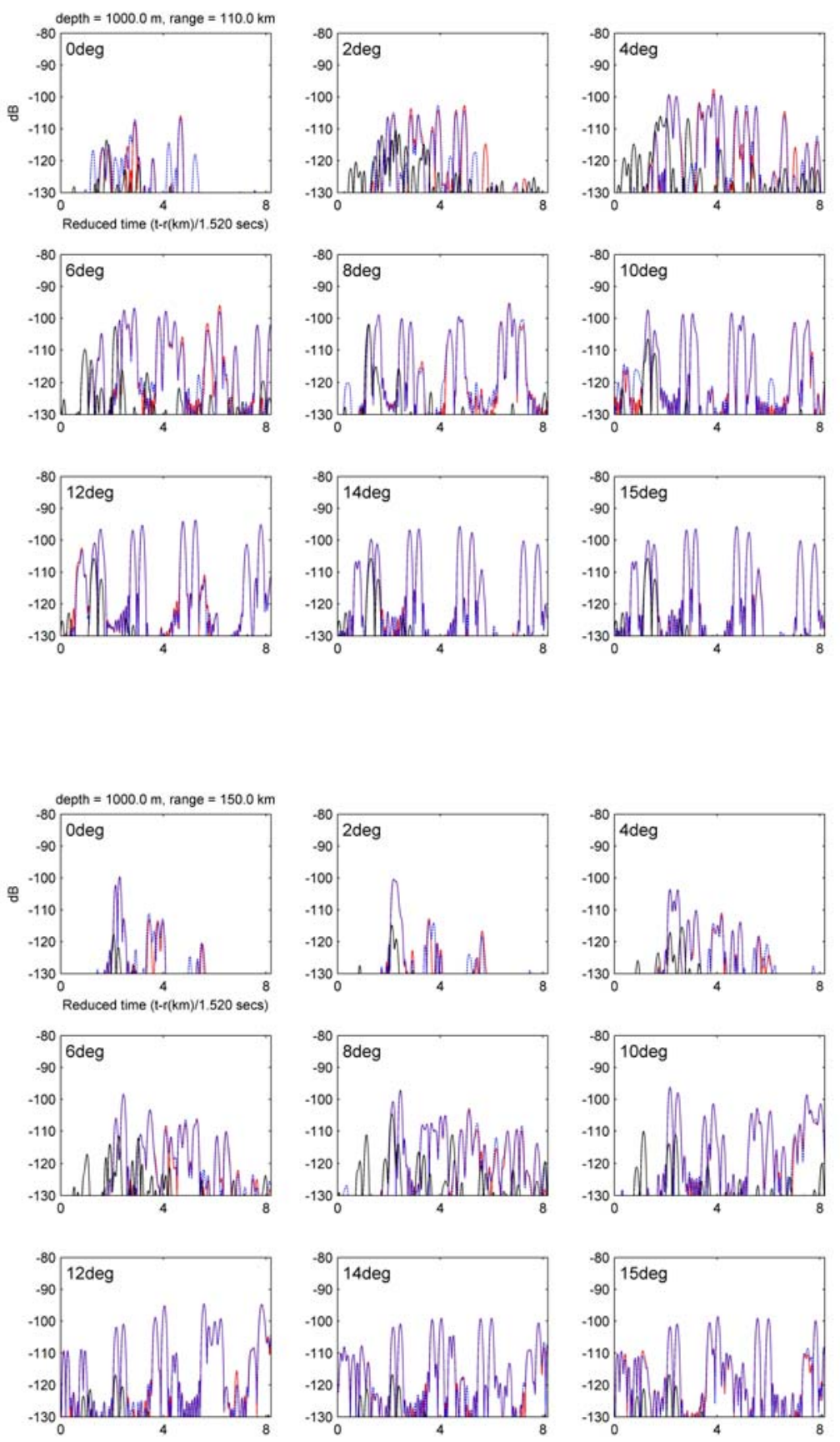

Figure 5-43: Comparison of amplitude of computed pulses between waterborne modes (black), propagation modes (blue) and full modes (w/ leaky modes, red) at a depth of $1000 \mathrm{~m}$. The ranges are $110 \mathrm{~km}$ (top panel) and 150km (bottom panel). 


\subsection{Broadband pulse modeling for the LOAPEX source}

In the previous chapter, a benchmark problem was explored with a broadband acoustic source with lower frequency at the center frequency of $15 \mathrm{~Hz}$. Resultant broadband pulses show the clear shadow zone cast and the convergence zone reappearance behind the seamount. In addition, complicated three-dimensional (3D) perturbed waves by the seamount can be found clearly in the results.

In this chapter, we apply the 3D spectral coupled-mode model to a problem with a conical seamount which models the Kermit-Roosevelt seamount; the LOAPEX source at the center frequency of $68.2 \mathrm{~Hz}$ is considered. Due to the limited computational ability, the computations were carried out with the only waterborne modes.

\subsubsection{Approximation of the Kermit-Roosevelt seamount with a conical seamount}

Since 3D spectral coupled-mode model can be applied to only axisymmetric bathymetry and range-independent sound speed fields, the Kermit-Roosevelt seamount needs to be approximated with a conical seamount.

Figure 5-45 shows the shapes of the cross sections of the Kermit-Roosevelt at selected depths compared to the circular sections of approximated conical seamount. Apparently, the shapes of the cross sections of the Kermit-Roosevelt are not perfect circles; however, the slope of the seamount can be modeled by a straight line along an acoustic path passing over the peak of the seamount from the LOAPEX source, as shown in the right bottom panel. The blue line is chosen for the slope of seamount, which corresponds to the blue circles in other panels.

Based on the approximated conical seamount, the description of the problem is shown in Fig. 5-47. The radius of the conical seamount is around $28.6 \mathrm{~km}$ with a water depth of $954 \mathrm{~m}$ at the peak of seamount; the slope of the seamount becomes $9.52^{\circ}$.

The range-independent sound speeds are obtained by averaging all sound speed 
measurements from the BASSEX experiment, as shown in Fig. 5-48. The geoacoustic properties of the sub-bottom are shown in Table 4-1, which was used in the $2 \mathrm{D}$ reconciliation of the pulse arrivals.

\subsubsection{Comparison of transmission loss from two-dimensional and three- dimensional model}

Transmission loss (TL) for a $68.2 \mathrm{~Hz}$ CW source is explored using the two-dimensional (2D) coupled normal mode method (CSNAP) and the 2D Parabolic Equation (PE, RAM), as shown in Fig. 5-49. The figure shows good agreement in TL from the CSNAP and PE. This 2D computation of the TL is performed for a convergence test, and the parameters used in the 2D computation are used in the 3D computation.

Figure 5-50 compares the 3D and Nx2D TL on a horizontal plane using the 3D spectral coupled-mode model and the 2D PE (RAM). The overall shadow zones behind the seamount are quite similar to each other.

Figure 5-51 shows the TL with various numbers of sections to examine the effect of grid size along the seamount in the 3D spectral coupled-mode model. In this case, since we use 100 vertical modes, the effect of the number of sections on the TL appears not to be significant.

\subsubsection{Broadband pulse modeling for a $15 \mathrm{~Hz}$ source}

Due to limited computational efficiency to handle a higher frequency such as at the LOAPEX source, an acoustic source with the lower frequency, $15 \mathrm{~Hz}$ of center frequency with $10 \mathrm{~Hz}$ bandwidth, is considered, and the computed arrival pulses are presented in Fig. 5-52 and 5-53. The arrival pulses are presented in the reduced time of $1520 \mathrm{~m} / \mathrm{s}$.

Compared to the benchmark problem presented in Section 5.2, the perturbation zone including the shadow zone is much smaller because the seamount is located much farther than the benchmark problem. In addition, the clear convergence zones are formed 
with the refracted rays passing over the seamount at 10.2 and 11.0 seconds. However, no significant horizontal refracted rays are found; the refracted rays were detected in the BASSEX experiment.

\subsubsection{Broadband pulse modeling for a $68 \mathrm{~Hz}$ source}

In this section, we compute the pulse arrivals for the LOAPEX source with limited modes, that is, only water-borne modes. For a $60 \mathrm{~Hz}$ source, the numbers of water-borne modes are 105 and 1 at the outermost ring and the peak of the seamount, respectively; for a 76 $\mathrm{Hz}$ source, the numbers of water-borne modes are 133 and 1 at the outermost ring and the peak of the seamount, respectively.

Figure 5-54 compares the pulse arrivals with the $68.2 \mathrm{~Hz}$ and $15 \mathrm{~Hz}$ sources. The primary pulse arrivals are quite comparable; however, the additional reflected pulse arrivals are not shown with the $68.2 \mathrm{~Hz}$ source because only water-borne modes are included in this calculation. The first three primary pulse arrivals are quite consistent with the arrival pulses from the 2D PE, as shown in Fig. 5-56. These pulses are also consistent with the detected pulses in the BASSEX experiment in Fig. 5-57; however, because of the narrow source bandwidth, and the limited normal modes for the $68.2 \mathrm{~Hz}$ source, it is difficult to resolve the higher angle arrival in the simulation, which is believed to be a horizontal refracted ray by the seamount. In addition, the approximation of the axisymmetric conical seamount can be another reason that the horizontal refracted ray is not found.

To verify this horizontal refraction, the increase in computational efficiency of the 3D spectral coupled-mode model is required to handle the higher frequency source such as $68.2 \mathrm{~Hz}$. In addition, in the experiment, the horizontal refracted rays are detected only at the left side of the seamount, which suggests that the assumption of the axisymmetry of the seamount is not valid. Therefore, a problem with the sophisticated bathymetry based on the real bathymetry with range-independent sound speed needs to be explored with a sound propagation model which can handle the sophisticated problem and is fast enough 
to obtain the broadband pulse in a reasonable time. The 3D PE can be a candidate; however, the model also needs improvement to be applied to long-range sound propagation and broadband pulses.

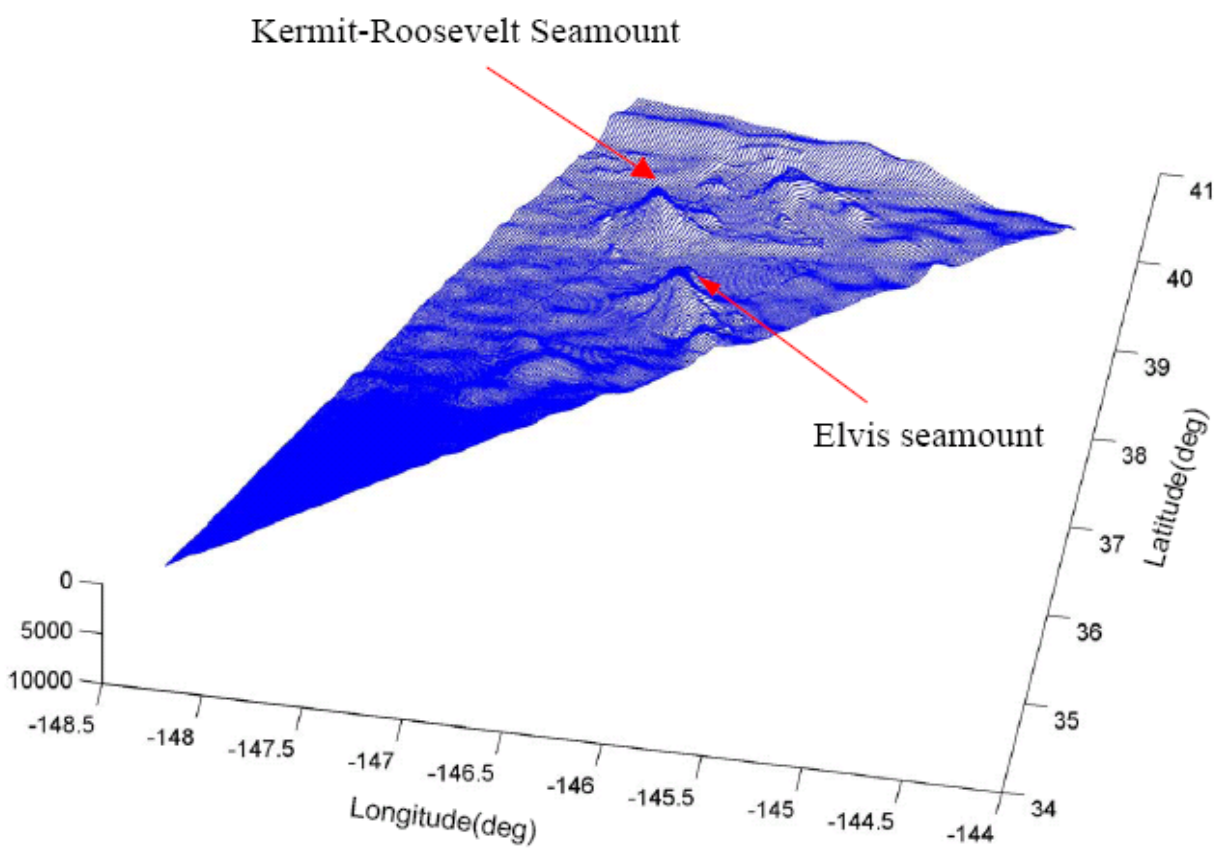

Figure 5-44: Bathymetry around Kermit-Roosevelt seamount complex. 

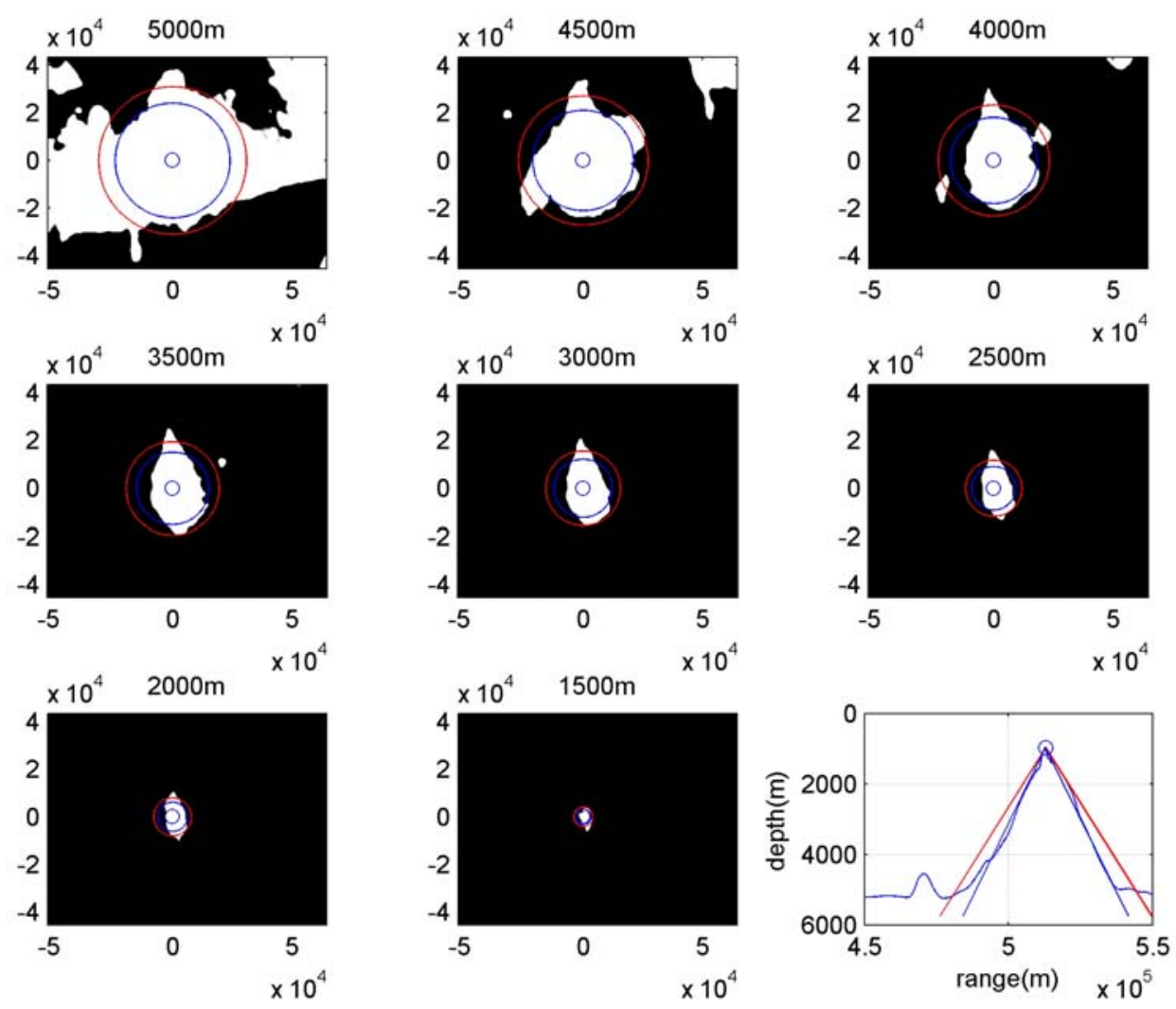

Figure 5-45: Shapes of seamount at the selected depths with circular sections of approximated cone. The blue line was chosen for the conical shape of the seamount.
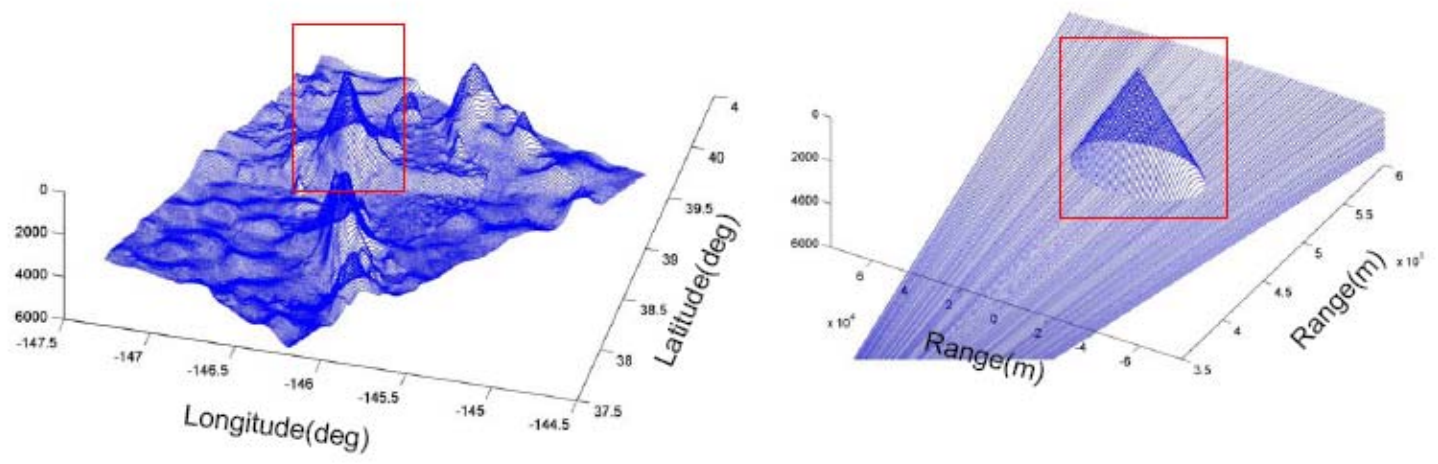

Figure 5-46: Comparison of the real bathymetry (left panel) and an approximated conical seamount (right panel). 


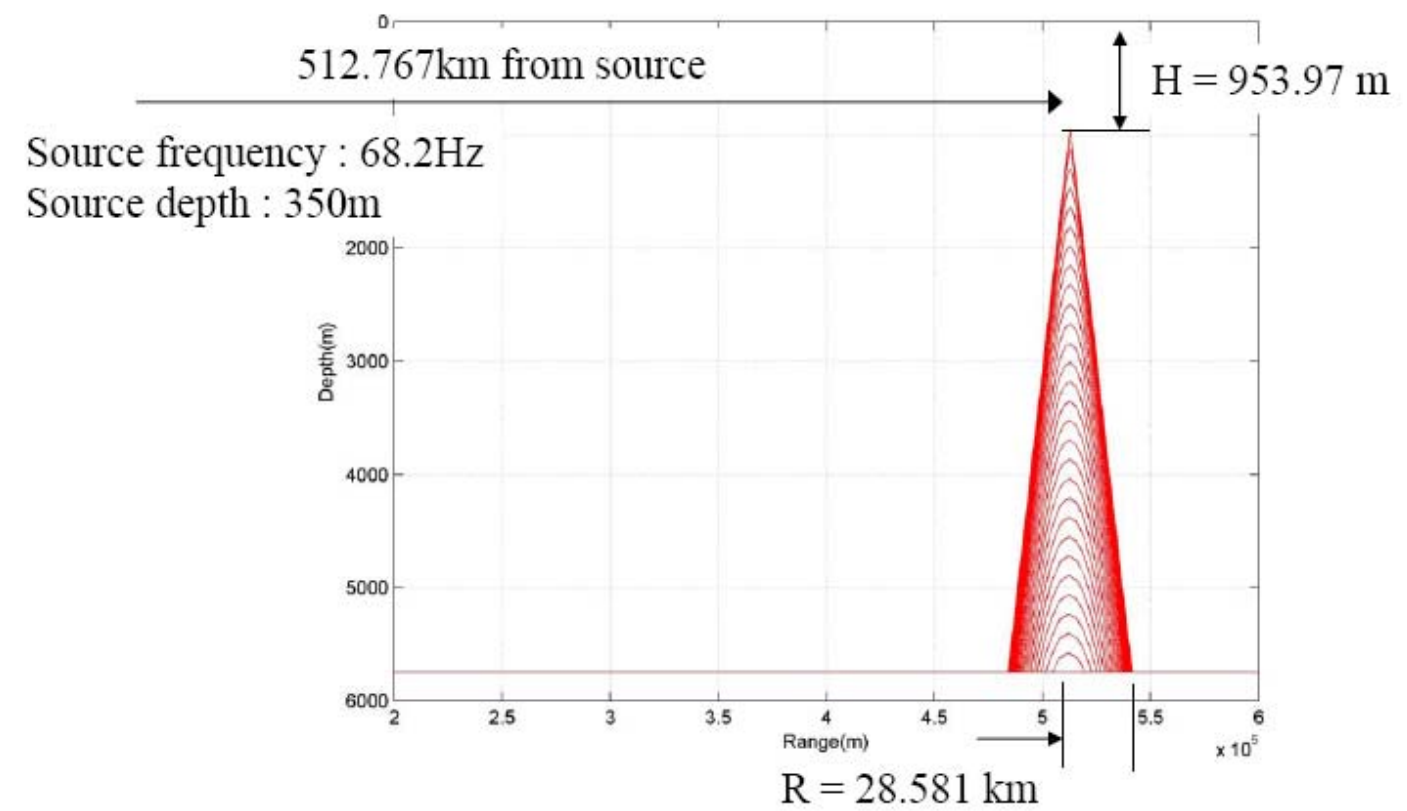

Figure 5-47: A problem with a conical seamount approximation of the Kermit-Roosevelt seamount.

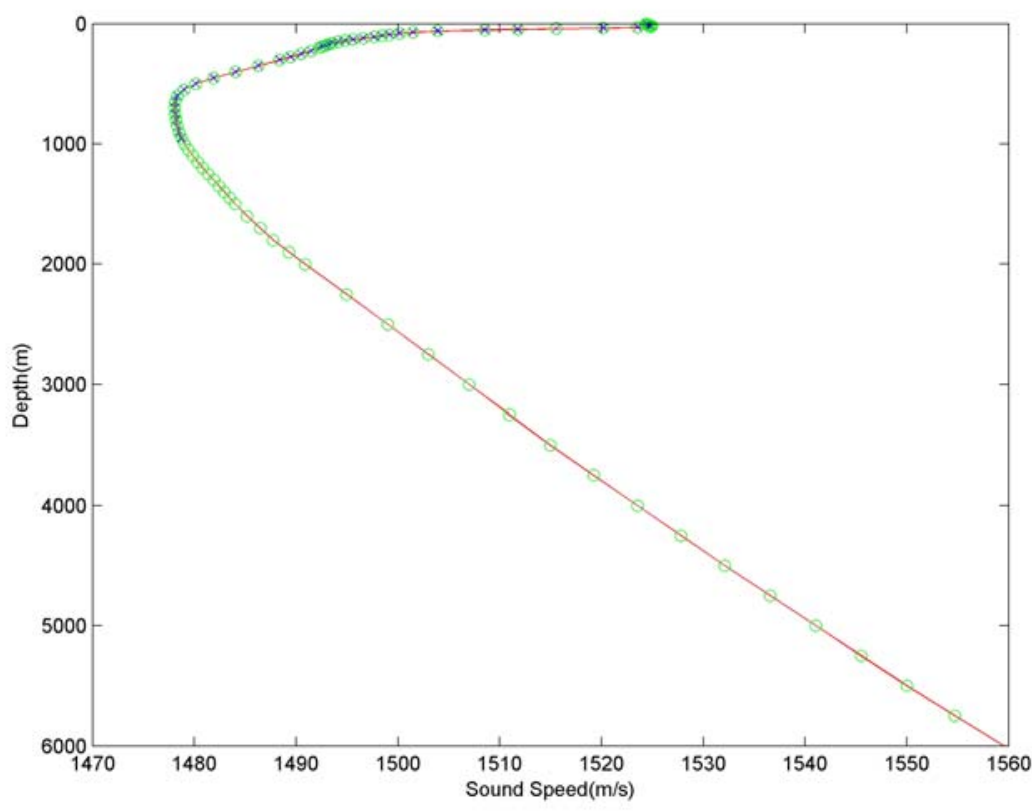

Figure 5-48: Averaged sound speed profile (red line). 

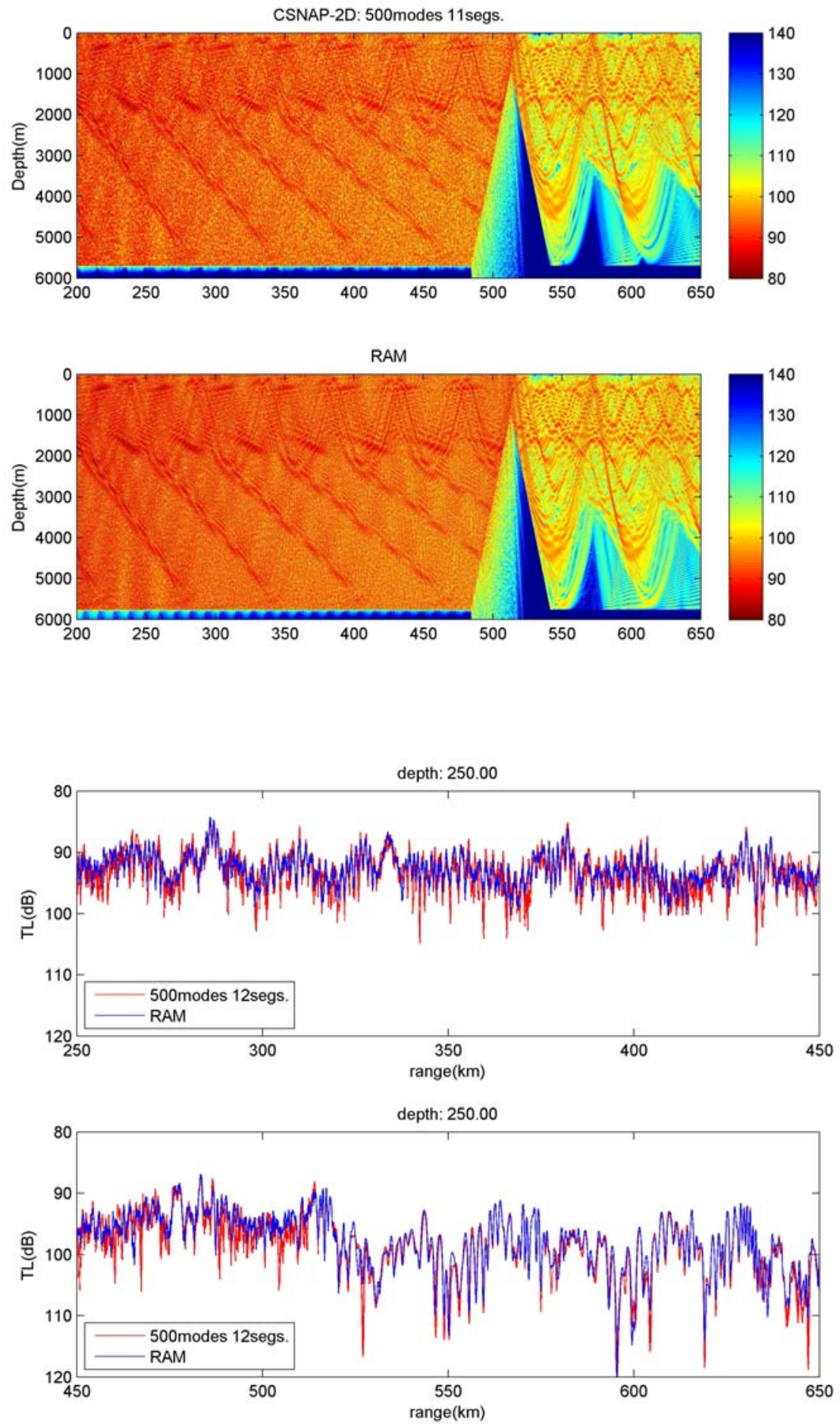

Figure 5-49: Compared TL from the CSNAP and 2D PE (RAM). 

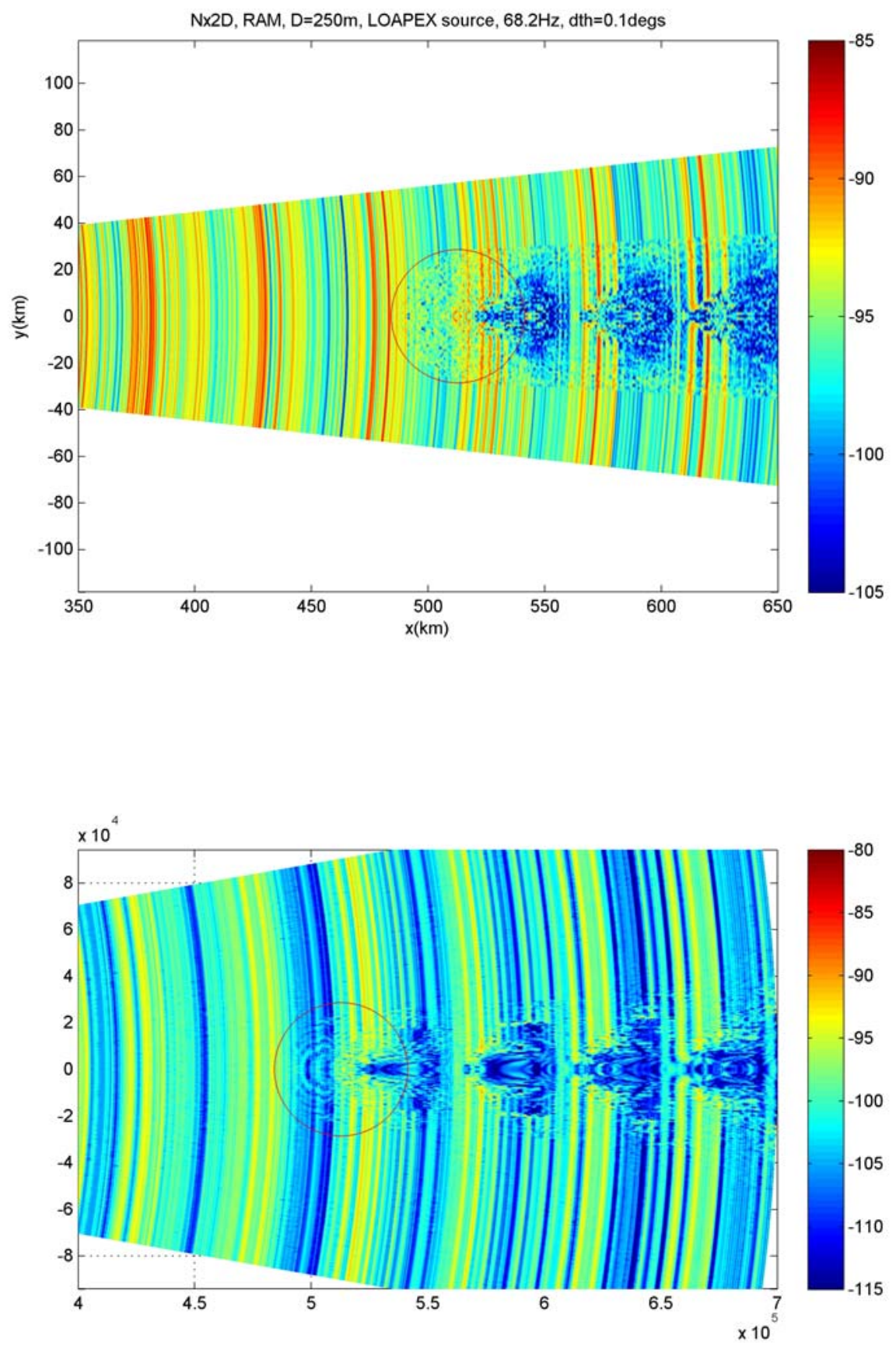

Figure 5-50: TL on a horizontal plane at a depth of 250m from the Nx2D (RAM, top panel) and the 3D model (3D spectral coupled-mode model, bottom panel). 

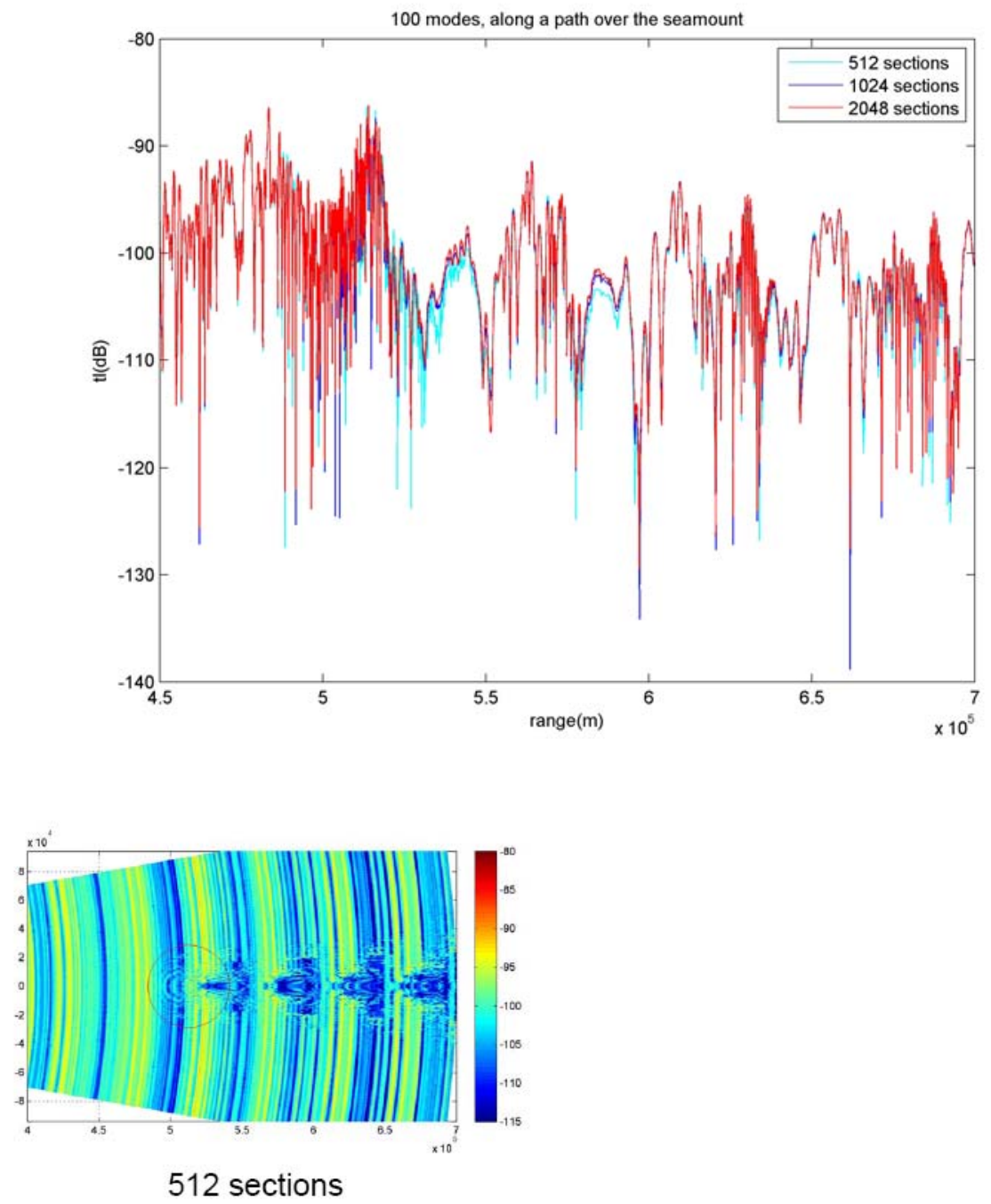

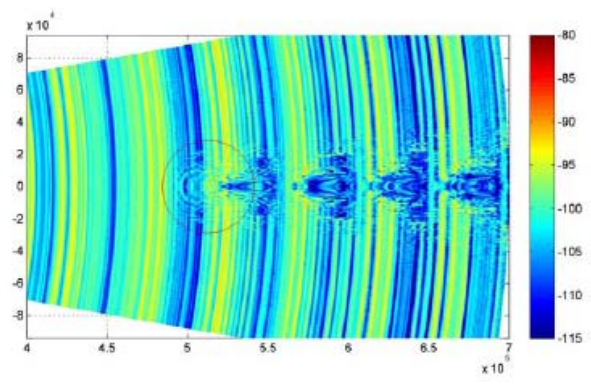

1024 sections

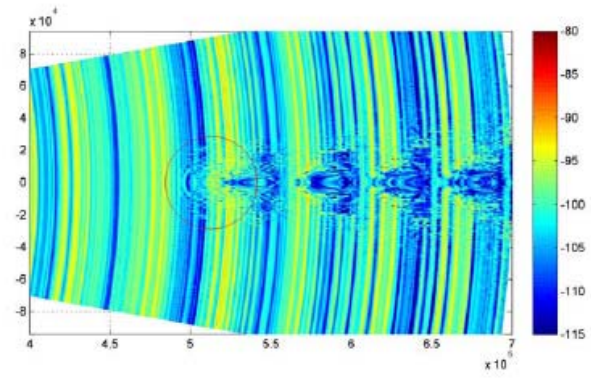

2048 sections

Figure 5-51: TL using the 3D spectral coupled-mode model with various range steps (or the number of sections). 

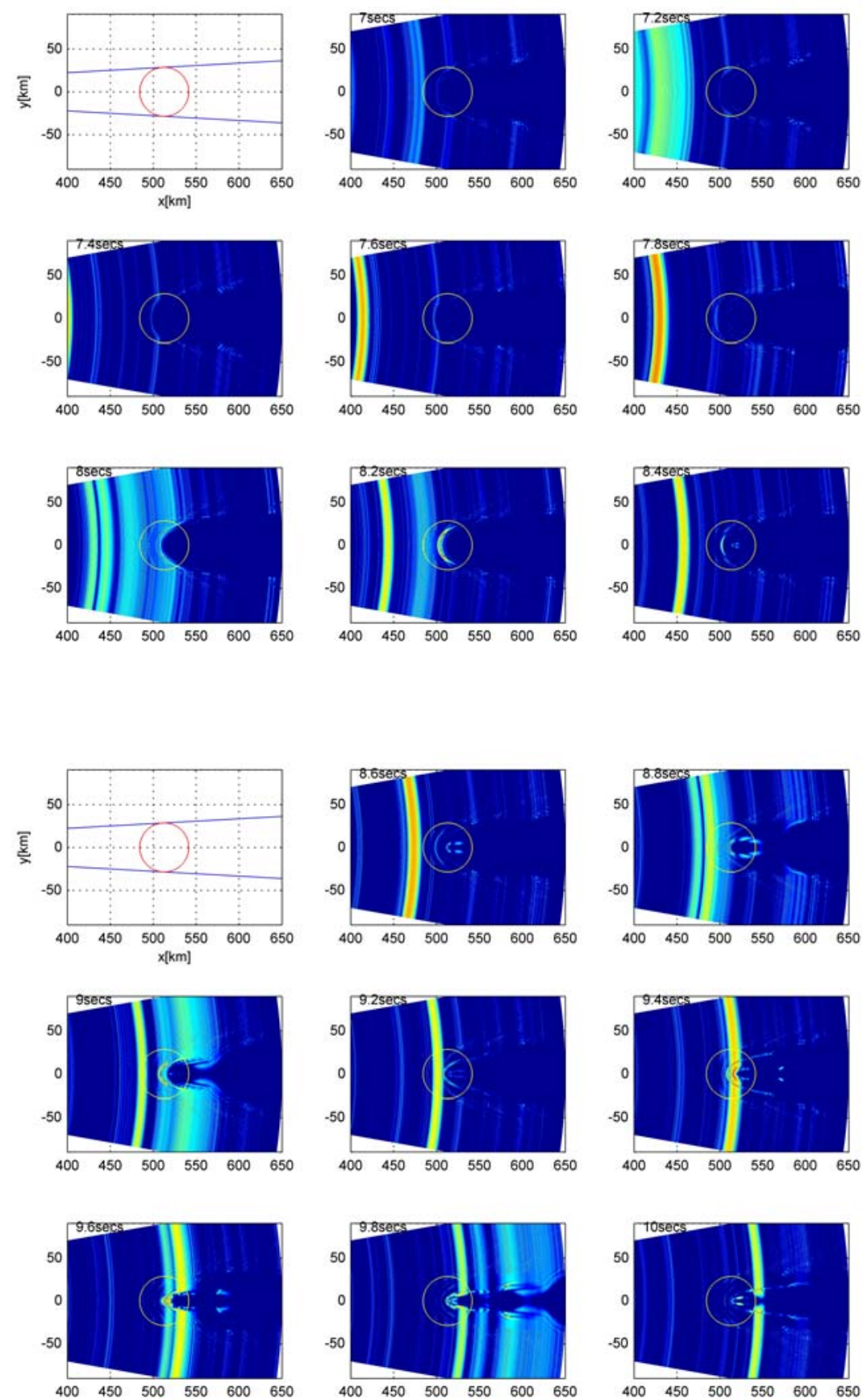

Figure 5-52: Computed pulse arrivals for a broadband source with a center frequency of $15 \mathrm{~Hz}$ source at a depth of $200 \mathrm{~m}$ (7.0 8.4 seconds). 

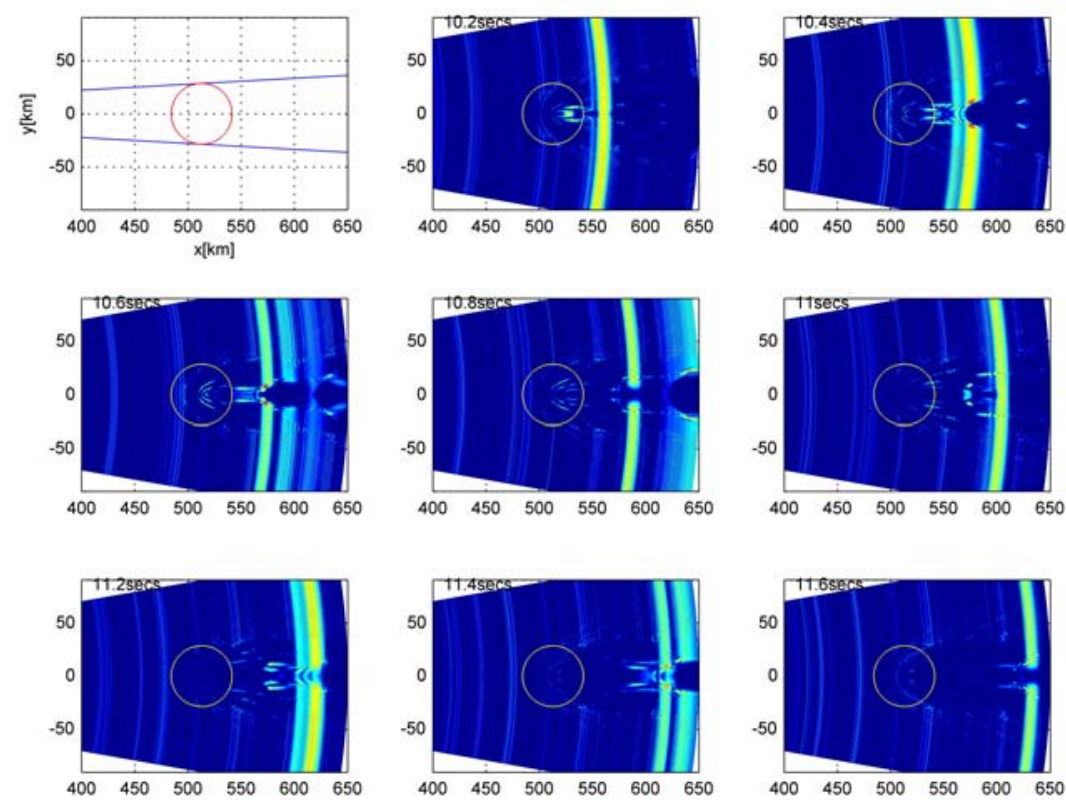

Figure 5-52: Computed pulse arrivals for a broadband source with a center frequency of $15 \mathrm{~Hz}$ source at a depth of 200m (10.2 11.6 seconds). 

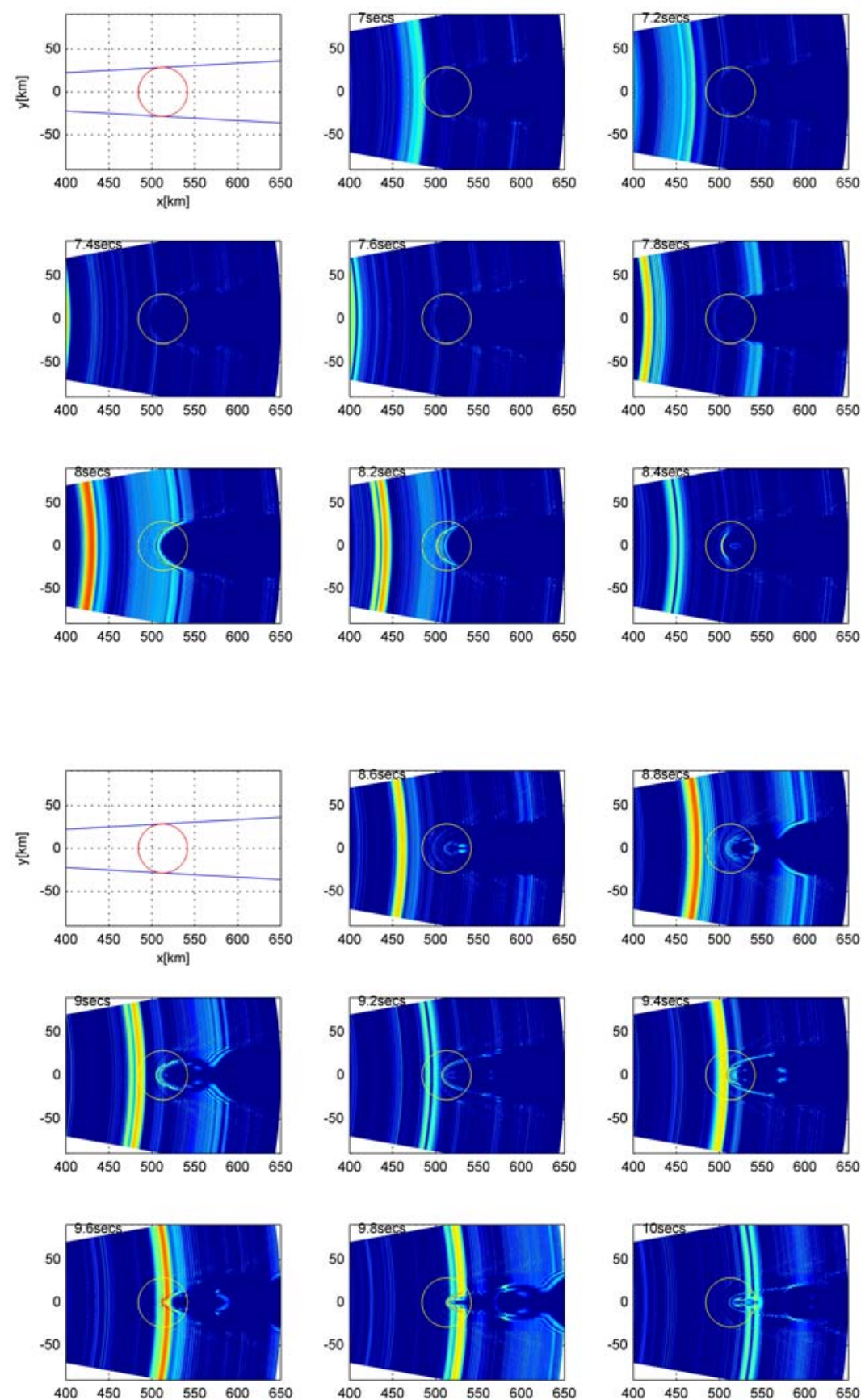

Figure 5-53: Computed pulse arrivals for a broadband source with a center frequency of $15 \mathrm{~Hz}$ source at a depth of 500m (7.0 8.4 seconds). 

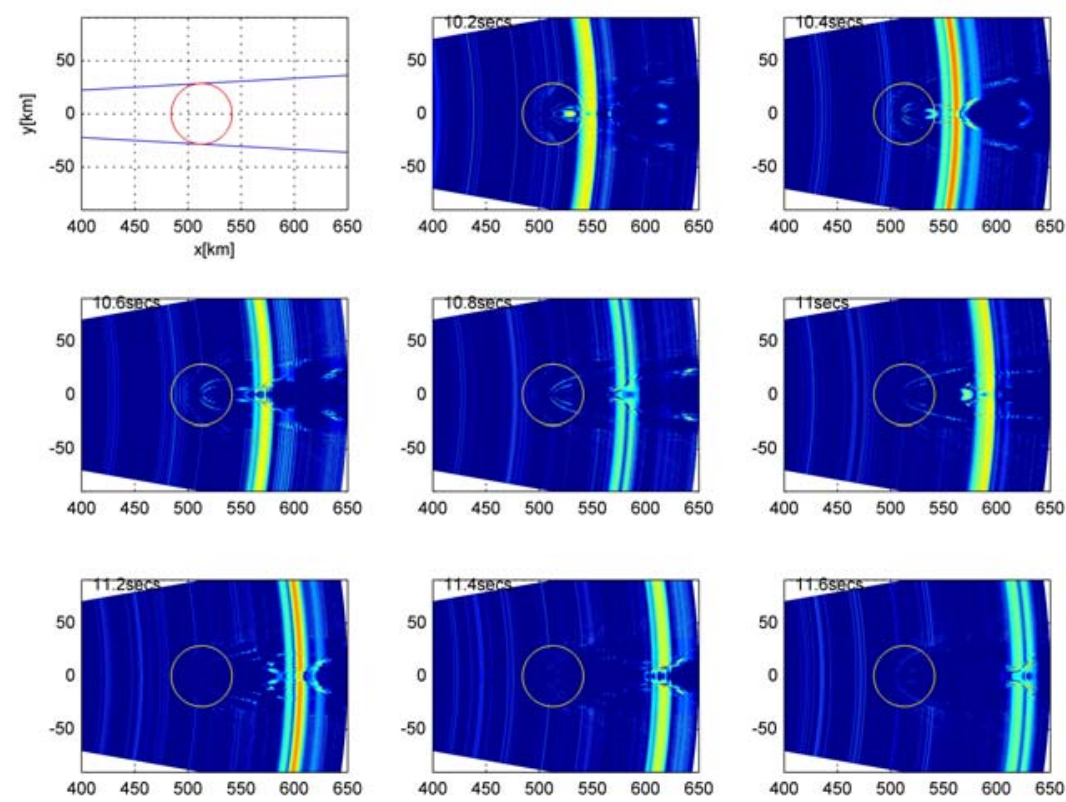

Figure 5-53: (continued) Computed pulse arrivals for a broadband source with a center frequency of $15 \mathrm{~Hz}$ source at a depth of 500m (10.2 11.6 seconds). 


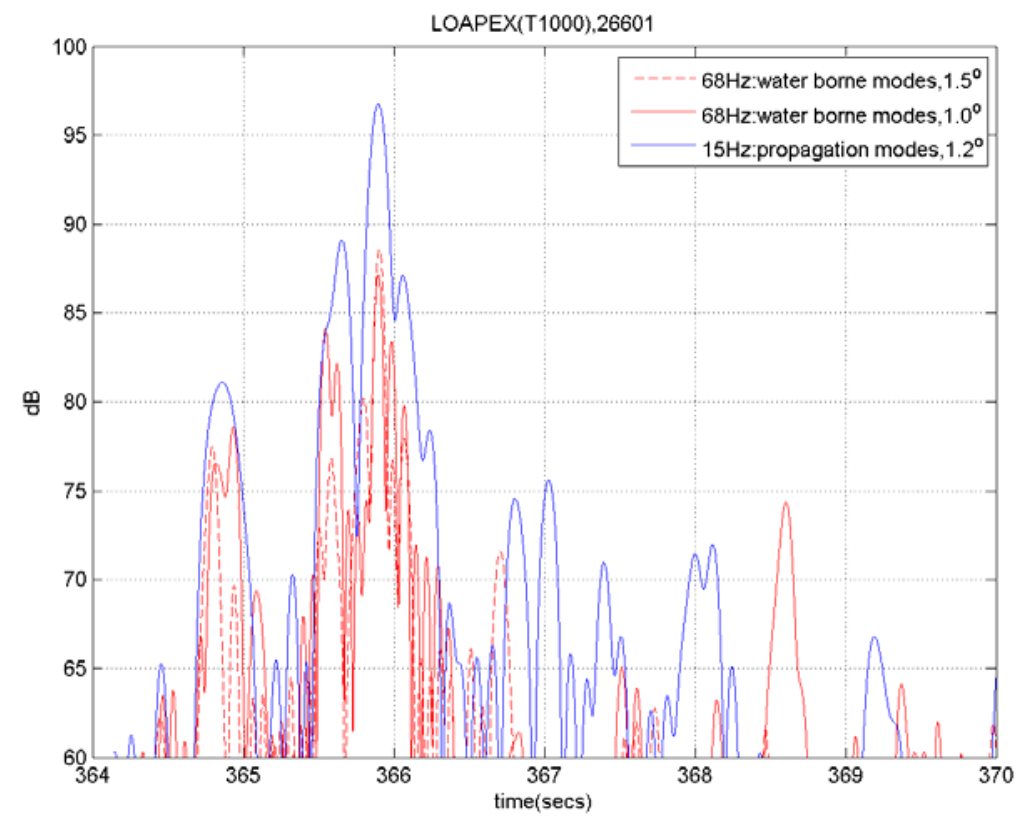

Figure 5-54: Comparison of pulse arrivals between two broadband pulses with a center frequency of $68.2 \mathrm{~Hz}$ LOAPEX source (with water-borne modes only) and $15 \mathrm{~Hz}$ source (with propagation modes) for 26601 . The angles denote the location of the 26601 with respect to the peak of the seamount.

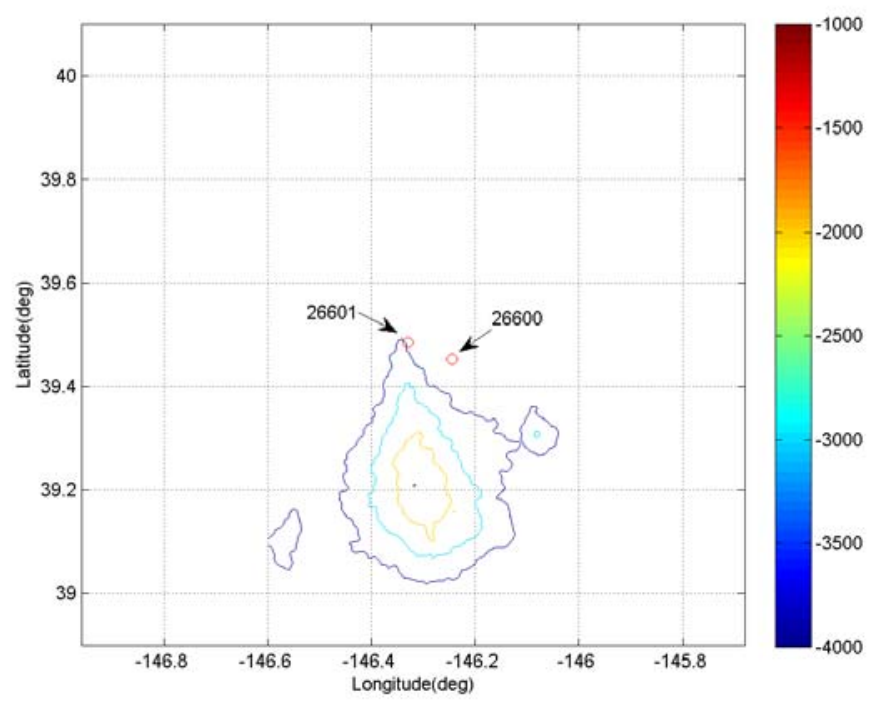

Figure 5-55: Kermit-Roosevelt seamount and the location of receiver for 26601 (Longitude: 146.329506, Latitude: 39.484648). 

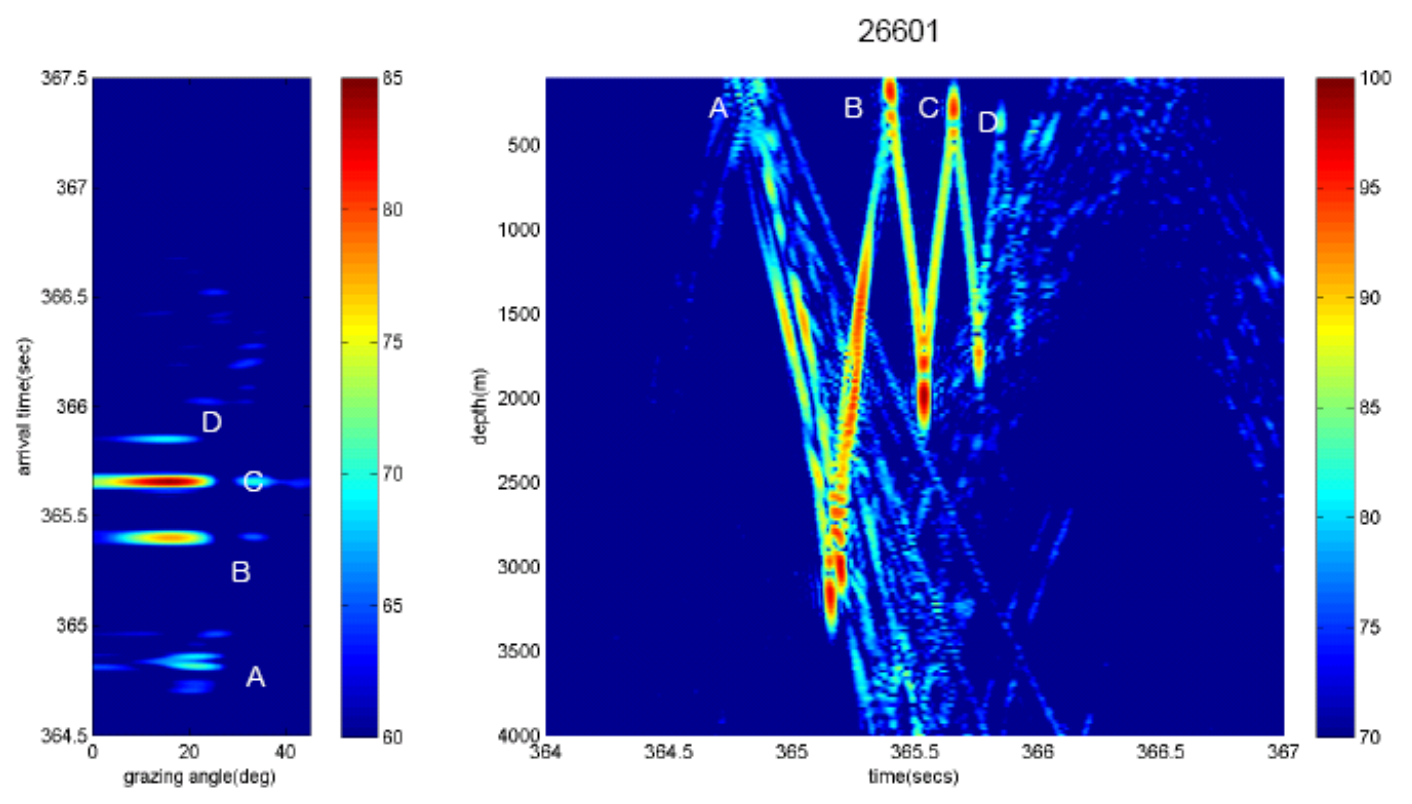

Figure 5-56: Pulse arrivals for 26601 (LOAPEX) using 2D PE. Range-dependent bathymetry and the sound speed fields from objective analysis are used for the simulation. Beamformed pulse arrivals at a depth of $300 \mathrm{~m}$ (left panel) and the depth stacked wave fronts (right panel) are shown.

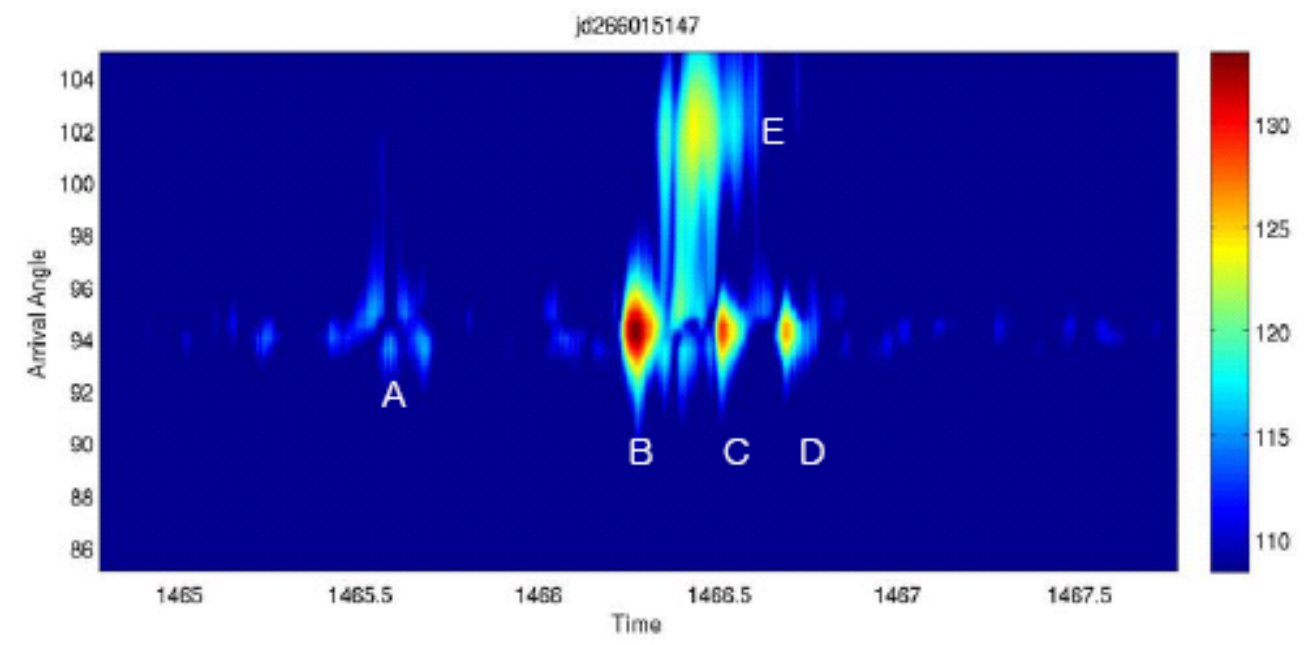

Figure 5-57: Measured pulse arrivals in the BASSEX experiment for 26601. E appears to be a horizontal refracted ray by the Kermit-Roosevelt seamount. 


\section{Chapter 6}

\section{Conclusion}

In this thesis, the reconciliation between the measured broadband pulses from the BASSEX experiment and the simulated pulses using various two-dimensional (2D) and three-dimensional (3D) theoretical sound propagation models was carried out to investigate the physical characteristics of the sound propagation around seamounts.

The rays and the pulse arrivals from the $2 \mathrm{D}$ ray tracing method and the $2 \mathrm{D} \mathrm{PE}$ showed the clear shadow and convergence zones formed behind the seamount. The modal amplitude obtained by the modal decomposition method for the 2D PE pressure fields showed the strong mode coupling due to the seamount; the mode cut-off at up-slope sound propagation, and mode repopulation at down-slope propagation can be explained by the energy (or modal amplitude) dissipation and transfer.

The shadow and convergence zones behind the seamounts were matched well between the experiment data and the 2D and 3D sound propagation models; however, the reconciliation of the broadband pulses behind the seamount was more challenging because of the complicated physical phenomena due to the sloped bathymetric effects as well as the uncertainties from the geoacoustic properties and sound speed fields. In particular, the application of the 3D sound propagation model is more limited due to the low computational efficiency.

The broadband pulses using the 2D parabolic equation (PE) for the SPICEX source 2 were successfully matched with the measurement in the BASSEX experiment, in particular, within the convergence zones in which the refracted rays appear. However, it was difficult to reconcile the reflected rays in the shadow zones and the transition regions between the shadow and convergence zone because the geoacoustic properties and local bathymetry play an important role in the reflection. These complicated reflections are related to the ray chaos, which needs a statistical approach and can be a challenging 
problem in future.

Through the reconciliation, the increase of computational efficiency of the 3D spectral coupled-mode model (W. Luo, PhD Thesis, MIT, 2007) was explored using parallel computing to realize the broadband pulses with strong azimuthal coupling around a seamount. The realized 3D broadband pulses showed the clear shadow and convergence zones as well as the wider disturbed (shadow) zone than in the Nx2D calculation because of the horizontal refraction. The size of the shadow zone is one of significant concerns on the sound propagation around a seamount.

However, due to the limited computational efficiency, we mainly considered the $15 \mathrm{~Hz}$ broadband source in the 3D broadband pulse realization. The broadband pulses using a higher frequency source, $68.2 \mathrm{~Hz}$, were computed with the limited vertical modes. Hence, although the primary pulse arrivals are consistent with the detected pulses in the BASSEX experiment, the horizontal refracted rays could not be resolved.

To verify the horizontal refraction, the increase in computational efficiency of the 3D spectral coupled-mode model is required to handle the higher frequency source such as $68.2 \mathrm{~Hz}$ with wider bandwidth. The higher efficiency can be achieved by using faster and more CPUs with parallel computing, or introducing some approximations. Since the enormous computer system can be limited and costly, some approximations can be essential to the higher efficiency. In the thesis, several approximation methods were explored: the truncation of normal modes, the sub-sampling of azimuthal modes, and the real-value argument of the Hankel and Bessel functions. However, these approximation methods can be applied to limited regions, in particular, far from the seamount. It is worth noting that using a perturbation of the horizontal wavenumber and modal shape is a promising way to reduce the computational time.

The Kermit-Roosevelt seamount was modeled by a simple conical seamount for the 3D model. However, we found that the horizontal refracted rays are axis-asymmetric through the simulation of horizontal refracted ray using the horizontal ray method with the adiabatic assumption. This axis-asymmetry can be a possible explanation for why the horizontal refracted rays were detected only at the left side of the seamount. 
This implies that the sophisticated bathymetry based on the real bathymetry measurement with range-dependent sound speed needs to be explored. In addition, we need a sound propagation model which can handle the sophisticated problem and is fast enough to obtain the broadband pulse in a reasonable time. The $3 \mathrm{D} \mathrm{PE}$ can be a candidate; however, the model also needs improvement to be applied to long-range sound propagation and broadband pulses for a higher frequency source.

In this work, for the preparation of the simulation, we examined the construction of a sound speed field database using the objective analysis, based on the sound speed profiles from the BASSEX experiment, World Ocean Atlas (WOA) 2005, and CTD casts. The simulated pulse arrivals were compared with those from the experiment, showing the increased correlation between the pulse arrivals; however, it is difficult to conclude on the validity of the sound speed database because of the limited measured data, and the random timing error of the DAQ system in the experiment. Since the precise sound speed fields covering the entire area are essential for the sound propagation model, in particular, the $3 \mathrm{D}$ sound propagation model, more efforts are required to obtain the precise sound speed fields. 


\section{Appendix A}

\section{Specifications of acoustic sources in the BASSEX}

\section{experiment}

Table A-1: $\quad$ M-sequence signal parameters of the SPICEX sources [54]

\begin{tabular}{|c|c|c|c|}
\hline HLF-5 Acoustic sources & S1 & $\mathrm{S} 2$ & \\
\hline Source Depth & $773.5^{1}$ & $738.0^{1}$ & $\mathrm{~m}$ \\
\hline Source level & 192 & 192 & $\mathrm{~dB}$ re $1 \mu \mathrm{Pa}$ at $1 \mathrm{~m}$ \\
\hline Center Frequency & 250 & 250 & $\mathrm{~Hz}$ \\
\hline Cycles/digit & 2 & 2 & \\
\hline Digit length & 12.000 & 12.000 & ms \\
\hline Sequence length(L) & 1023 & 1023 & digits(degree 10) \\
\hline Sequence period & 12.2760 & 12.2760 & $\mathrm{~S}$ \\
\hline Sequence law[octal] & 2033 & 3471 & \\
\hline Artifact location & 531 & 474 & digit \\
\hline $\begin{array}{c}\text { Sequence } \\
\text { initialization[octal] }\end{array}$ & 1000 & 1000 & \\
\hline Phase modulation angle & $89.209215^{\circ}$ & $89.209215^{\circ}$ & \\
\hline Longitude & 34.324333 & 34.88912 & $\operatorname{deg} N$ \\
\hline Latitude & -143.01708 & -148.408033 & $\operatorname{deg} E$ \\
\hline
\end{tabular}

1: The designed source depth was $750 \mathrm{~m}$ for both sources; the measured source depths were found based on the position records by the installed acoustic transducers on the sea floor around sources [54]. 
Table A-2: $\quad$ M-sequence signal parameters of the Kauai sources [54]

\begin{tabular}{|c|c|c|}
\hline Source Depth & 825 & $\mathrm{~m}$ \\
\hline Source level & 195 & $\mathrm{~dB}$ re $1 \mu \mathrm{Pa}$ at $1 \mathrm{~m}$ \\
\hline Center Frequency & 75 & $\mathrm{~Hz}$ \\
\hline Cycles/digit & 26.6667 & $\mathrm{~ms}$ \\
\hline Digit length & 1023 & digits(degree 10) \\
\hline Sequence length(L) & 27.2800 & $\mathrm{~s}$ \\
\hline Sequence period & 3471 & \\
\hline Sequence law[octal] & 474 & \\
\hline Artifact location & 1000 & \\
\hline Sequence & & \\
\hline initialization[octal] & $89.209215^{\circ}$ & \\
\hline Phase modulation angle & 44 & deg N \\
\hline Sequence repetitons & 1200.3200 & deg E \\
\hline Transmission duration & 22.349156 & \\
\hline Longitude & -159.569924 & \\
\hline Latitude & & \\
\hline
\end{tabular}


Table A-3: M-sequence signal parameters of the LOAPEX Sources [54]

\begin{tabular}{|c|c|c|c|}
\hline Source Depth & 800 & 350 & $\mathrm{~m}$ \\
\hline Source level & 195 & 194 & $\mathrm{~dB}$ re $1 \mu \mathrm{Pa}$ at $1 \mathrm{~m}$ \\
\hline Center Frequency & 75 & 68 & $\mathrm{~Hz}$ \\
\hline Cycles/digit & 2 & 2 & \\
\hline Digit length & 26.6667 & 29.4118 & $\mathrm{~ms}$ \\
\hline Sequence length(L) & 1023 & 1023 & Digits(degree 10) \\
\hline Sequence period & 27.2800 & 30.0882 & $\mathrm{~S}$ \\
\hline Sequence law[octal] & 2033 & 2033 & \\
\hline Artifact location & 531 & 531 & digit \\
\hline $\begin{array}{c}\text { Sequence } \\
\text { initialization[octal] }\end{array}$ & 1000 & 1000 & \\
\hline Phase modulation angle & $89.209215^{\circ}$ & $89.209215^{\circ}$ & \\
\hline \multicolumn{4}{|c|}{ Sequence repetitions transmitted } \\
\hline 20 minutes & 44 & 40 & \\
\hline 80 minutes & 176 & 160 & \\
\hline
\end{tabular}

\begin{tabular}{|c|c|c|}
\hline Station & Latitude [deg N] & Longitude [deg E] \\
\hline T50 & 33.513590 & -138.208350 \\
\hline T250 & 33.869780 & -140.322990 \\
\hline T500 & 34.248840 & -142.882500 \\
\hline T1000 & 34.864170 & -148.280130 \\
\hline T1600 & 35.285610 & -154.949970 \\
\hline T2300 & 35.312730 & -162.647970 \\
\hline T3200 & 34.631820 & -172.472870 \\
\hline
\end{tabular}




\section{Appendix B}

\section{Matched filtered pressure}

Let us define the source pressure in $\mu \mathrm{Pa}$ as:

$$
s(t)=A q(t) \text { or } S(f)=A Q(f),
$$

where $q(t)$ and $Q(f)$ are a Fourier transform pair and satisfy unit energy such as

$$
\int|q(t)|^{2} d t=\int|Q(f)|^{2} d f=1
$$

Then the pressure at the receiver can be expressed as follows:

$$
P(f)=A Q(f) G\left(r \mid r_{o}, f\right)
$$

The matched filter is derived by finding a linear filter which gives a maximum SNR, and the resultant filter is simply the time reversed version of a source signal, $q(t)$, which has no unit.

The matched filtered pressure becomes

$$
\begin{aligned}
p_{\text {mf }}\left(\tau_{\max }\right) & =p(\tau) * q^{*}(-\tau)=\int p(t) q^{*}(t-\tau) d t \\
& =\int A Q(f) Q^{*}(f) G\left(r \mid r_{o}, f\right) d f \\
& =\int A|Q(f)|^{2} G\left(r \mid r_{o}, f\right) d f
\end{aligned}
$$


where $\tau_{\max }=\underset{\tau}{\arg \max } p_{m f}$.

The matched filtered pressure in decibel is $10 \log _{10}\left|p_{m f}\right|^{2}=20 \log _{10}\left|p_{m f}\right| \mathrm{dB}$ re $1 \mu \mathrm{Pa}$

The matched filtered pressure for the source is

$$
p_{m f}\left(\tau_{\max }\right)=s(\tau) * q^{*}(-\tau)=\int A q(t) q^{*}(t-\tau) d t=\int A|q(t)|^{2} d t=A
$$

In decibel, $20 \log _{10}\left(p_{m f}\right)=20 \log _{10} A \mathrm{~dB}$ re $1 \mu \mathrm{Pa}$.

For the BASSEX experiment, two correction factors are needed to obtain the matched filtered pressure shown above:

i) a factor for unit energy of the reference signal, $1 / \sqrt{\int|q(t)|^{2} d t}$,

ii) unit conversion from the original data to physical data in micro-pascal ( $\mu \mathrm{Pa})$ with the following gain:

pressure $(d B$ re $1 \mu P a)=20 \log _{10}\left(x_{\text {original }}\right)+24.35$.

Then time-averaged power can be acquired by the summation of the squared of matched filtered pressure in time domain:

$$
\begin{gathered}
P=\frac{1}{T} \int_{0}^{T}\left|p_{m f}(t)\right|^{2} d t \\
10 \log _{10} P \quad(d B r e \mu P a)=10 \log _{10} \int_{0}^{T}\left|p_{m f}(t)\right|^{2} d t-10 \log _{10} T .
\end{gathered}
$$




\section{Appendix C}

\section{XBT output file}

\#\#\# Sound Velocity Profile /SCG.PROC/CRUISE.DATA/SVPROFILES/KRUS03RR.svp.20

\#\#\# Created by /scgscg/bin/DOsvp

\#\#\# by scg on rv-revelle date: Mon Aug 16 01:36:12 GMT 2004

\#\#\# XBT file name: /SCG.PROC/CRUISE.DATA/XBT/tf_00022.edf

\#\#\# Date of Launch : 08/16/04

\#\#\# Time of Launch $\quad$ : 01:12:36

\#\#\# Sequence \# : $\quad 22$

\#\#\# Latitude $\quad$ : 2321.1797 N (SPS)

\#\#\# Longitude : $15952.3376 \mathrm{~W}$

\#\#\# Serial \# $\quad$ : 08919

\#\#\# Probe Type $\quad$ : Fast Deep (Original**)

\#\#\# Terminal Depth $\quad$ : 1000 m

\#\#\# Depth Coefficient $1: \quad-0.00182$

\#\#\# Depth Coefficient 2 : 6.39

\#\#\# Raw Data Filename $\quad$ : TF_00022.RDF

\#\#\# Display Units : METRIC

\#\#\# Salinity used is: 34.8

\#\#\# Salinity table used: /scgscg/src/bin/SVPROFILES/files/salinity.tbl

$0.0 \quad 1539.5$

$21.1 \quad 1539.7$

$31.3 \quad 1539.6$

$45.9 \quad 1539.3$

$54.8 \quad 1534.6$

$63.1 \quad 1533.1$

$70.7 \quad 1530.8$

$77.0 \quad 1530.1$ 
$80.9 \quad 1529.9$

$96.7 \quad 1527.8$

$102.4 \quad 1527.1$

$105.6 \quad 1526.9$

$113.8 \quad 1525.8$

$120.1 \quad 1525.5$

$125.2 \quad 1524.9$

$136.5 \quad 1522.8$

$139.7 \quad 1521.9$

$147.3 \quad 1520.8$

$151.7 \quad 1520.5$

$959.5 \quad 1481.6$

$978.1 \quad 1481.8$

$1000.2 \quad 1482.0$

\# Carter Table Area 52

$1500.0 \quad 1485.0$

$2500.0 \quad 1499.0$

$3500.0 \quad 1514.0$

$4500.0 \quad 1534.0$

$5500.0 \quad 1551.0$

$6500.0 \quad 1571.0$

$7500.0 \quad 1589.0$

$8500.0 \quad 1609.0$

$9500.0 \quad 1629.0$

$10500.0 \quad 1648.0$

\#11500,1667

\#12500,1687 


\section{Appendix D}

\section{Modal decomposition method: modal spectrum of the Parabolic Equation pressure field}

\section{D.1 Theory}

The pressure field given by a parabolic equation (PE) solver, $P_{P E}(r, z)$, can be written as follows:

$$
P_{P E}(r, z ; \varpi)=\frac{1}{\sqrt{r}} \Psi_{P E}(r, z ; \varpi) e^{j k_{0} r}
$$

where $k_{0}$ is a reference wave number, and $\Psi_{P E}$ is governed by

$$
2 i k_{0} \frac{\partial \Psi_{P E}}{\partial r}+\frac{\partial^{2} \Psi_{P E}}{\partial z^{2}}+k_{0}{ }^{2}\left[n^{2}(r, z)-1\right] \Psi_{P E}=0
$$

Suppose $\Phi_{m}(r, z ; \varpi)$ is a local mode at range $r$, then the pressure field from the PE can be expressed as a summation of the product of the amplitude of local mode and the modal shape:

$$
P_{P E}(r, z ; \varpi)=\sum_{m} P_{m}(r ; \varpi) \Phi_{m}(r, z ; \varpi)
$$

If we use the orthonomality of the local mode, $\Phi_{m}(r, z ; \varpi)$ as follows: 


$$
\int_{0}^{D} \frac{\Phi_{m}^{2}(z)}{\rho(z)} d z=1
$$

then the amplitude at each mode in the above summation can be obtained by:

$$
P_{m}(r, \varpi)=\int_{0}^{D} P_{P E}(r ; \varpi) \frac{\Phi_{m}(r, z ; \varpi)}{\rho(z)} d z
$$

Substituting Eq. (D.1) into Eq. (D.5), we obtain:

$$
P_{m}(r ; \varpi)=\left[\int_{0}^{D} \frac{e^{j k_{0} r}}{\sqrt{r}} \Psi_{P E}(r, z ; \varpi) \frac{\Phi_{m}(r, z ; \varpi)}{\rho(z)} d z\right]=A_{m}(r ; \varpi) \frac{e^{j k_{0} r}}{\sqrt{r}}
$$

If the source spectrum is $S(\omega)$, then a pulse is given by a Fourier transform:

$$
\begin{aligned}
p(r, z, t) & =\frac{1}{2 \pi} \int_{-\bar{\omega}_{\max }}^{\sigma_{\max }} S(\omega) P_{P E}(r, z, \varpi) e^{-j \omega t} d \omega \\
& =\frac{1}{2 \pi} \int_{-\omega_{\max }}^{\omega_{\max }} S(\omega)\left(\sum_{m} P_{m}(r, \omega) \Phi_{m}(r, z, \omega)\right) e^{-j \omega t} d \omega \\
& =\frac{1}{2 \pi} \int_{-\omega_{\max }}^{\omega_{\max }} S(\omega)\left(\sum_{m} A_{m}(r, \omega) \frac{e^{j k_{0} r}}{\sqrt{r}} \Phi_{m}(r, z, \omega)\right) e^{-j \omega t} d \omega \\
& =\sum_{m} \frac{1}{2 \pi} \int_{-\omega_{\max }}^{\omega_{\max }} S(\omega) A_{m}(r, \omega) \frac{e^{j k_{0} r}}{\sqrt{r}} \Phi_{m}(r, z, \omega) e^{-j \omega t} d \omega \\
& =\sum_{m} p_{m}(r, z, t)
\end{aligned}
$$

where a modal pulse at mode $m, p_{m}(r, z, t)$, describes: 


$$
p_{m}(r, z, t)=\frac{1}{2 \pi} \int_{-\varpi_{\max }}^{\sigma_{\max }} S(\omega)\left[A_{m}(r, \varpi) \frac{e^{j k_{0} r}}{\sqrt{r}}\right] \Phi_{m}(r, z, \varpi) e^{-j \varpi t} d \varpi
$$

In following sections, the modal decomposition method is applied to problems which have weak and strong mode coupling due to upslope and downslope propagation. The problems are adapted from [3].

\section{D.2 Weak coupling}

The environment, as shown in Fig. D-1, consists of a mild upslope section $\left(0.7^{\circ}\right)$ up to 10 $\mathrm{km}$, a $5 \mathrm{~km}$ flat section, and a steeper downslope $\left(1.4^{\circ}\right)$ section. The bathymetry and a range-independent SVP are shown in Fig. D-1. The detailed environmental input is given in [3]. In addition, Fig. D-1 shows the modal shapes along the section, and Fig. D-2 shows a transmission loss plot using the 2D PE for a $50 \mathrm{~Hz}$ source frequency.

For the $50 \mathrm{~Hz}$ source, we have four propagation modes at the source, and two modes in the shallow section of $80 \mathrm{~m}$ depth. However, with the source depth of $100 \mathrm{~m}$, only modes 1 and 3 among the 4 modes are excited at the starting field, which provides two-mode interference in Fig. D-2. At a range of $7 \mathrm{~km}$, mode 3 cuts off and leaks into the bottom, and, in the shallow part after the modal cut-off, there is a weak interference structure demonstrating energy transfer into mode 2.

The modal amplitude obtained by the modal decomposition method is shown in Fig. D-3. The two modes 1 and 3 are excited with high amplitude at the starting field, and the modal cut-off takes place with the abrupt change of the modal amplitude of mode 3 at a range of $7 \mathrm{~km}$. However, there is no significant change in the amplitude of mode 2 (< $3 \%$ ), which claims that the adiabatic approximation can be valid with the weak mode coupling. 


\section{D.3 Strong coupling}

To demonstrate the strong mode coupling, we increase bottom slope to $17^{\circ}$ as shown in Fig. D-4. Figure D-5 shows transmission loss computed by the 2D PE. The initial field consists of modes 1 and 3 only; however, mode 3 cuts off during upslope propagation. In contrast to the weak coupling problem, on the shelf, there is a two-mode interference showing energy transfer into mode 2. Modal amplitude in Fig. D-6 demonstrates the energy transfer to mode 2 and the strong mode coupling.
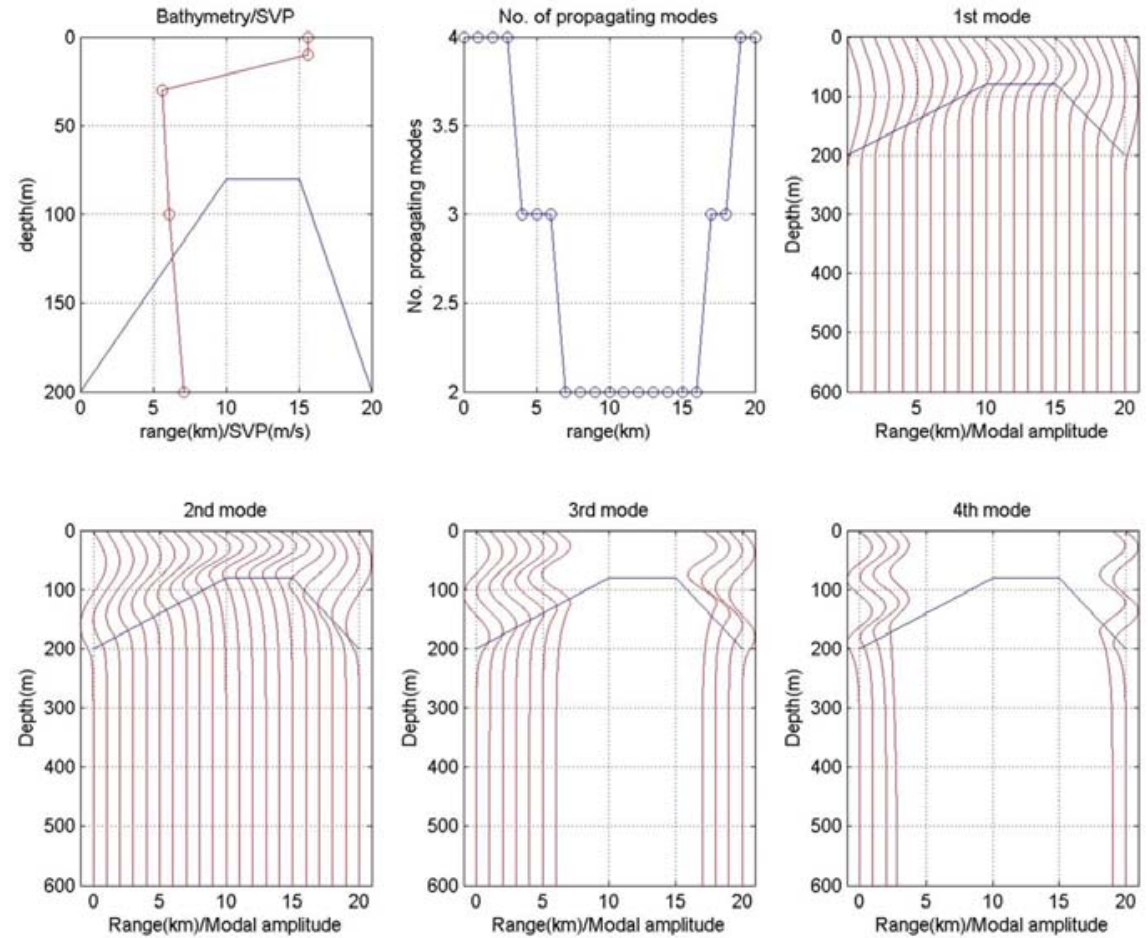

Figure D-1: Bathymetry and SVP (top leftmost), number of propagation modes (top middle), and modal shapes for $1-4^{\text {th }}$ mode versus range. 


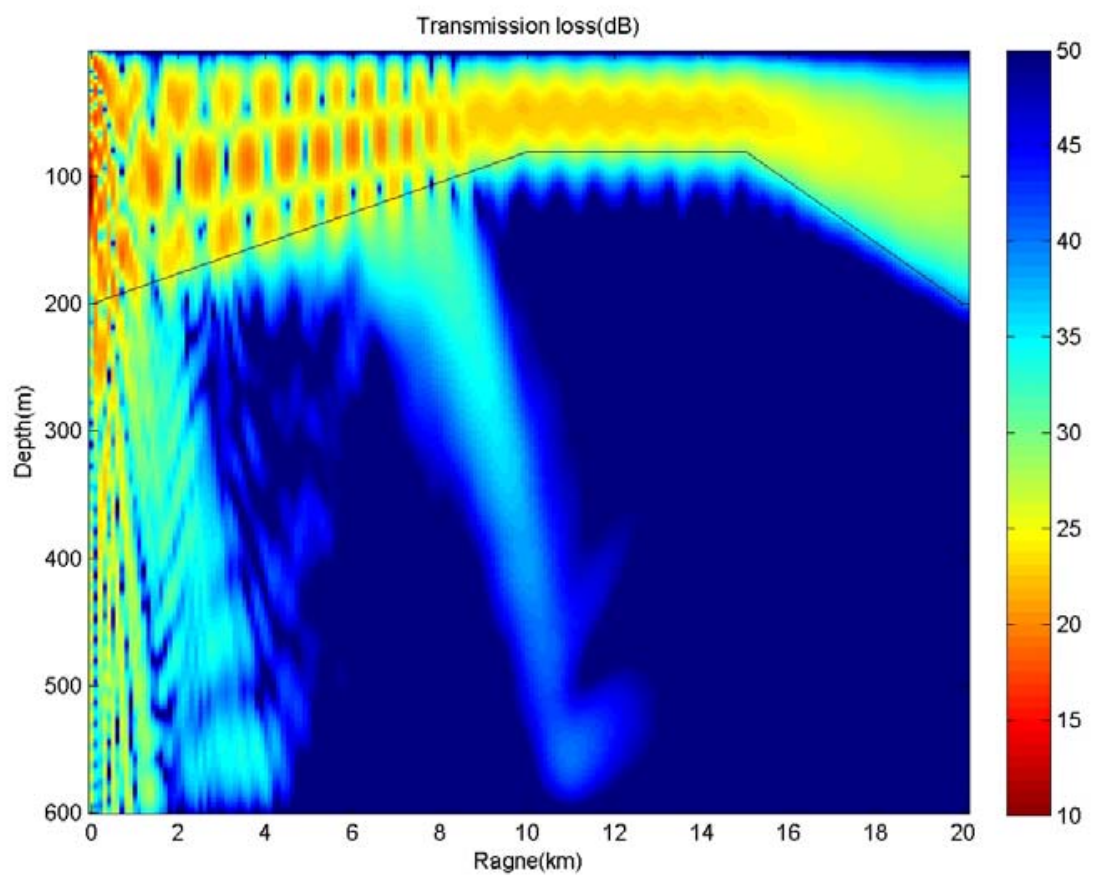

Figure D-2: Transmission loss of the PE. Mode 2 leaks into the bottom at a range of $7 \mathrm{~km}$.
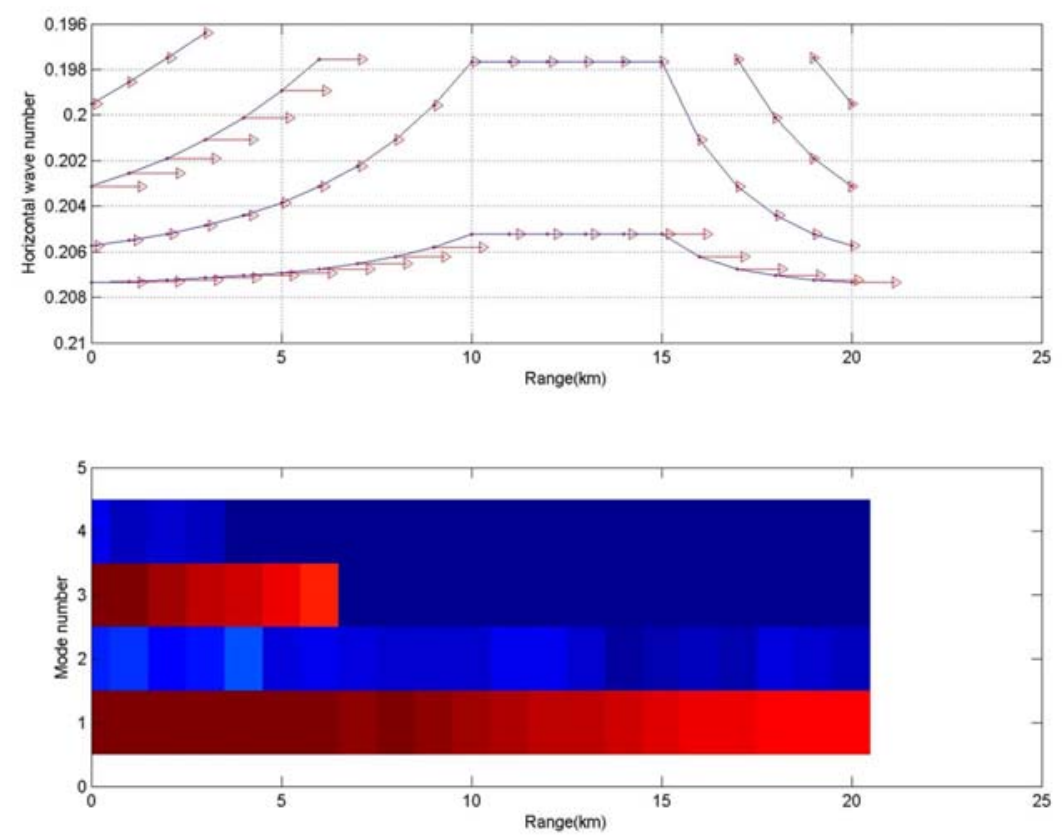

Figure D-3: Modal amplitude and wavenumber versus range. Only modes 1 and 3 are excited at a starting field, and the mode 3 cuts off at a range of $7 \mathrm{~km}$. The modal amplitude shows negligible coupling between modes. 

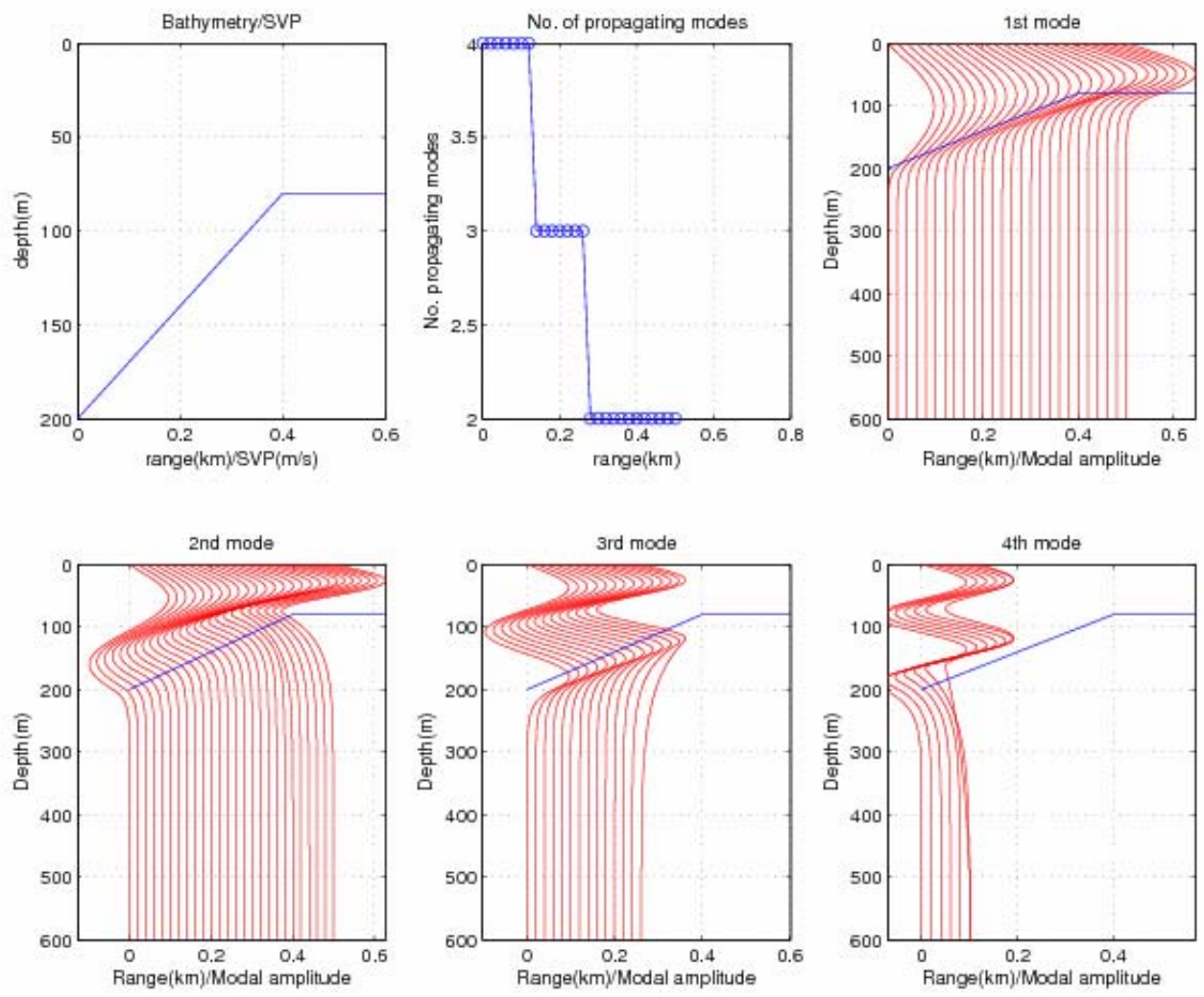

Figure D-4: Bathymetry and SVP (top leftmost), number of propagation modes (top middle), and modal shapes for $1-4^{\text {th }}$ mode versus range. 


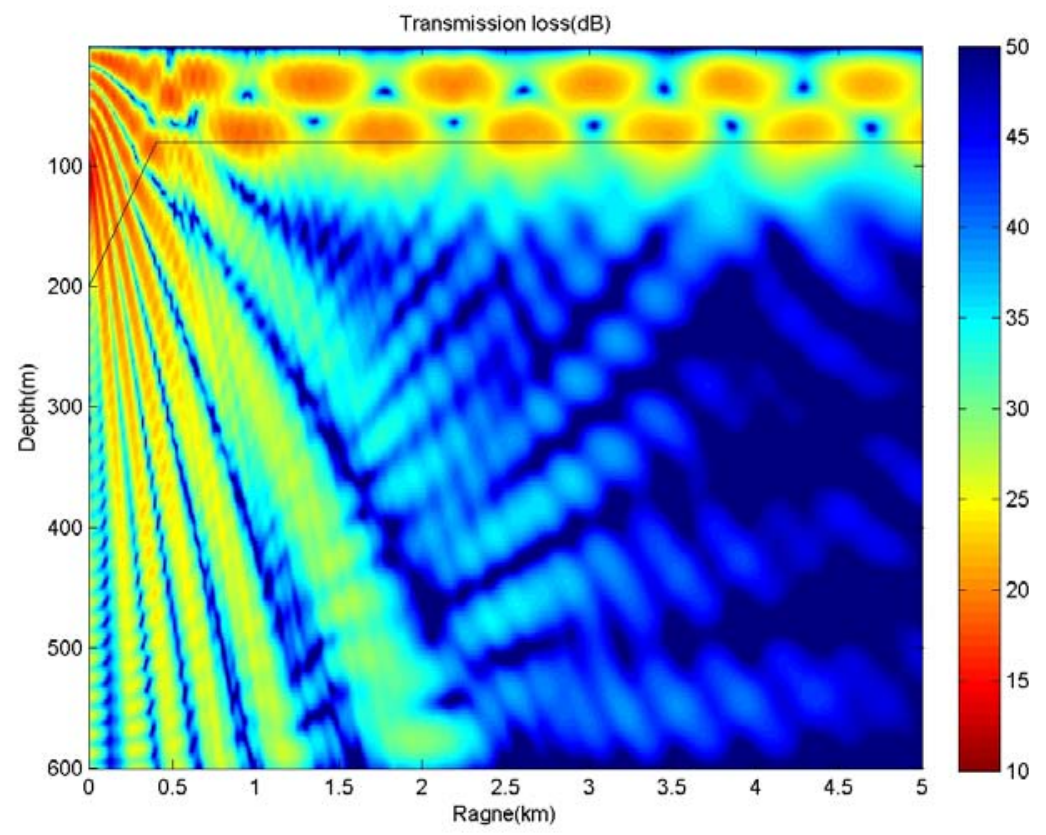

Figure D-5: Transmission loss of the PE
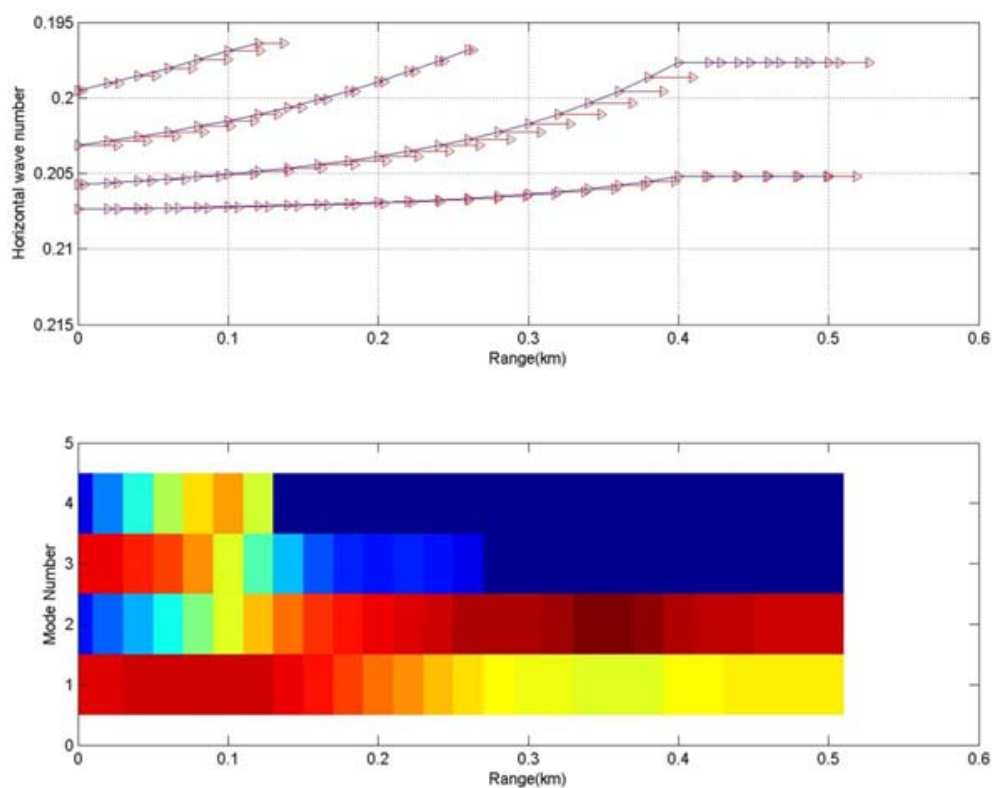

Figure D-6: Modal amplitude and wavenumber versus range. Only modes 1 and 3 are excited at a starting field, and the mode 3 cuts off during upslope propagation at a range of $100 \mathrm{~m}$. After the modal cut-off, energy transfer to mode 2 is quite clear, demonstrating the strong mode coupling. 


\section{References}

1. E. C. Shang and Y. Y. Yang. Acoustic Travel Time Computation Based on PE Solution. Journal of Computational Acoustics, 1(1):91 100,1993

2. E. C. Shang, Y. Y. Yang, T. M. Georges. Dispersion and Repopulation of HeardAscension Modes. J. Acoust. Soc. Am., 96(4):2371 2379, 1994

3. F. B. Jensen and H. Schmidt. Spectral Decomposition of PE Fields in a Wedge-Shaped Ocean. Progress in Underwater Acoustics, edited by H. Merklinger (Plenum, New York), pp.557 564,1987 


\section{Bibliography}

[1] John Northop. Underwater sound propagation across the Hawaiian Arch. J. Acoust. Soc. Am., 48(1):417-418, 1970.

[2] David A. Nutile and Albert N. Guthrie. Acoustic shadowing by seamounts. J. Acoust. Soc. Am.,66(6):1813-1817, 1979.

[3] Gordon R. Ebbeson and R. Glenn Turner. Sound propagation over Dickins. Seamount in the Northeast Pacific Ocean. J. Acoust. Soc. Am., 73(1):143-152, 1983.

[4] N. Ross Champan and Gordon R. Ebbeson. Acoustic shadowing by an isolated seamount. J. Acoust. Soc. Am., 73(6):1979-1984, 1983.

[5] H. Medwin and R. Spaulding. The seamounts as a diffracting body in BottomInteracting Ocean Acoustics. Edited by W.A. Kuperman and F. B. Jensen, Plenum, 1980.

[6] W. A. Kuperman, Michael B. Porter, and John S. Perkins, and Richard B. Evans. Rapid computation of acoustic fields in three-dimensional ocean environments. J. Acoust. Soc. Am., 89(1):125-133, 1991.

[7] Gerassimos A. Athanassoulis and Aristides M. Prospathopoulos. Three dimensional acoustic scattering of a source-generated filed from a cylindrical island. J. Acoust. Soc. Am., 100(1):206-218, 1996.

[8] Michael. I. Taroukadis. A coupled mode formulation for the solution in water in the presence of a conical seamount. Journal of Computaional Acoustics, 4:101-121, 1996.

[9] Jeremie Eskenazi. A computer model for sound propagation around conical seamounts. Matster's thesis, Massachusetts Institute of Technology, 2001.

[10]Wenyu Luo, Three-dimensional propagation and scattering around a conical seamount. PhD thesis, Massachusetts Institute of Technology, 2007. 
[11] Michael D. Collins. The Time-domain solution of the wide-angle parabolic equation including the effects of sediment dispersion. J. Acoust. Soc. Am., 84: 2114-2125, 1988.

[12] Michael D. Collins. A Split-step Pade solution for the parabolic equation metod. $J$. Acoust. Soc. Am., 93:1736-1742, 1993.

[13] Michael D. Collins, R. J. Cederberg, David B. King, and Stanley A. Chin-Bing. Comparison of algorithms for solving parabolic wave equations. J. Acoust. Soc. Am., 100(1):178-182, 1996.

[14] Michael D. Collins and Stanley A. Chin-Bing. Three-dimensional parabolic equation model that includes the effects of rough boundaries. J. Acoust. Soc. Am., 87(3):1104-1109, 1990.

[15] Ding Lee and Martin H. Schultz, Numerical Ocean Acoustic Propagation in Three Dimensions, World Scientific, 1995, ISBN 981022303X.

[16] Ding Lee, George Botseas, and William L. Siegmann. Examination of threedimensional effects using a propagation model with azimuth-coupling capability(FOR3D). J. Acoust. Soc. Am., 91(6):3192-3202, 1992.

[17] John A. Fawcett. Modeling three-dimensional propagation in an oceanic wedge using parabolic equation methods. J. Acoust. Soc. Am., 93(5):2627-2631, 1993.

[18] C. H. Harrison. Acoustic shadow zones in the horizontal plane. J. Acoust. Soc. Am., 65(1):51-56, 1979.

[19] C. H. Harrison. Three-dimensional ray paths in basins, troughs, and near seamounts by use of ray invariants. J. Acoust. Soc. Am., 62:1382-1388, 1977.

[20] R. Doolittle, A. Tolstoy and M. Buckingham. Experimental confirmation of horizontal refraction of $\mathrm{cw}$ acoustic radiation from a point source in a wedge-shaped ocean environment. J. Acoust. Soc. Am.,83(6):2117-2125, 1988. 
[21] Michael Buckingham. Theory of acoustic propagation around a conical seamount. $J$. Acoust. Soc. Am.,80(1):265-277, 1986.

[22] K. D. Heaney, W. A. Kuperman, and B. E. McDonald. Perth-Bermuda sound propagation(1960): Adiabatic mode interpretation, J. Acoust. Soc. Am., 90(5):2586-2594, 1991.

[23] B. Edward Mcdonald, Michael D. Collins, W. A. Kuperman and Kevin D. Heaney. Comparison of data and model predictions for Heard Island acoustic transmissions. $J$. Acoust. Soc. Am.,96(4):2357-2370, 1994.

[24] Kevin B. Smith, Chris W. Miller, Anthony F. D’Agostino, Brian Sperry, James H. Miller and Gopu R. Potty. Three-dimensional propagation effects near the mid-Atlantic Bight Shelf break(L). J. Acoust. Soc. Am.,112(2):373-376, 2002.

[25] D. E. Weston. Horizontal refraction in a three-dimensional medium of variable stratification. Proc. Phys. Soc. Lond., 78:46-52, 1961.

[26] Walter Munk and F. Zachariasen. Refraction of sound by islands and seamounts. $J$. Atmos. Oceanic Technol., 8:554-574, 1991.

[27] John S. Perkins and Ralph N. Baer. An Approximation to the three-dimensional parabolic-equation method for acoustic propagation. J. Acoust. Soc. Am., 72(2):515-522, 1982.

[28] Finn B. Jensen, William A. Kuperman, Michael B. Porter, and Henrik Schmidt. Computational Ocean Acoustics. Springer-Verlag New York, Inc., 2000.

[29] Water Munk, Peter Worceter, and Carl Wunsch. Ocean Acoustic Tomography. Cambridge University Press, 1995

[30] L. M. Brekhovskikh and Yu. P. Lysanov. Fundamentals of Ocean Acoustics. Springer-Verlag, 2003. 
[31] Joseph Sikora III. Sound propagation around underwater seamounts. Master's thesis, Massachusetts Institute of Technology, 2005.

[32] Peter F. Worceter and James A. Mercer. North Pacific Acoustic Laboratory. 2004

[33] Kevin D. Heaney. BASSEX preliminary report. 2004

[34] James Mercer and Bruce Howe. LOAPEX cruise plan. 2004

[35] V. A. Del Grosso. New equation for the speed of sound in natural waters (with comparisons to other equations). J. Acoust. Soc. Am.,56(4):1084-1091, 1974.

[36] NOAA. World Ocean Atlas 2005. 2005

[37] Brian D. Dushaw. Worldwide Sounc Speed, Temperature, Salinity, and Buoyancy from the NOAA World Ocean Atlas 2005. http://staff.washington.edu/dushaw/WOA/

[38] Brian D. Dushaw, Peter F. Worceter, Bruce D. Cornuelle, and Bruce M. Howe. On equations for the speed of sound in water. J. Acoust. Soc. Am.,93(1):255-275, 1993.

[39] John L. Spiesberger and Kurt Metzger. A new algorithm for sound speed in seawater. J. Acoust. Soc. Am.,89(6):2677-2688, 1991.

[40] James B. Bowlin, John L. Spiesberger, Thimothy F. Duda, and Lee F. Freitag. Ocean Acoustical Ray-tracing Software RAY. Woods Hole Oceanographic Institution, 1992.

[41] Vasa Associates. SEALAB

[42] Michael D. Vera, Kevin D. Heaney, and The NPAL Group. The effect of bottom interaction on transmissions from North Pacific Acoustic Laboratory Kauai source. $J$. Acoust. Soc. Am.,117(3):1624-1634, 1991.

[43] Peter F. Worceter, Bruce D. Cornuelle, Matthew A. Dzieciuch, Walter H. Munk, 
Bruce M. Howe, James A. Mercer, Robert C. Spindel, John A. Colosi, Kurt Metzger, Theodore G. Bridsall, and Arhtur B. Baggeroer. A test of basin-scale acoustic thermometry using a large aperture vertical array at 3250-km range in the eastern North Pacific Ocean. J. Acoust. Soc. Am.,105(6):3185-3201, 1999.

[44] E. C. Shang and Y. Y. Yang. Acoustic Travel Time Computation Based on PE Solution. Journal of Computational Acoustics, 1(1):91-100, 1993.

[45] E. C. Shang, Y. Y. Yang, T. M. Georges. Dispersion and Repopulation of HeardAscension Modes. J. Acoust. Soc. Am., 96(4):2371 2379, 1994

[46] F. B. Jensen and H. Schmidt. Spectral Decomposition of PE Fields in a WedgeShaped Ocean. Progress in Underwater Acoustics: 557 564 edited by H. Merklinger, Plenum, 1987.

[47] John A. Colosi, Stanley M. Flatte, and Charls Bracher. Internal-wave effects on $1000 \mathrm{~km}$ oceanic acoustic pulse propagation: Simulation and comparison with experiment. J. Acoust. Soc.Am.,96(1):452-468, 1994.

[48] Ching-Sang Chiu and Yves Desaubies. A planetary wave analysis using the acoustic and conventional arrays in the 1981 Ocean Tomography Experiment. J. Phys. Oceanogr., 17:1270-1287,1987.

[49] A. E. Newhall, J. F. Lynch, C. S. Chiu and J. R. Daugherty. Improvements in Threedimensional raytracing codes for underwater acoustics. Computation Acoustics, Vol.1:169-185, IMACS Prcoceedings, 1990.

[50] E. F. Carter and A. R. Robinson. Analysis models for the estimation of oceanic fields. J. Atmos. and Oceanic Tech., 4(1):49-74, 1987.

[51] Frédéric Sturm and John A. Fawcett. On the use of higher-order aimuthal schemes in 3-D PE modeling. J. Acoust. Soc. Am., 113(6):3134-3145, 2003.

[52] Frédéric Sturm. Numerical Study of broadband sound pulse propagation in three- 
dimensional oceanic waveguides. J. Acoust. Soc. Am., 117(3):1058-1079, 2005.

[53] Joseph Sikora III. Sound propagation around underwater seamounts. PhD thesis, Massachusetts Institute of Technology, 2009.

[54] Michael B. Porter, Finn B. Jensen and Carlo M. Ferla. The problem of energy conservation in one-way model. J. Acoust. Soc. Am.,89(3):1058-1067, 1991.

[55] C. T. Tindle, L. M. O’Driscoll, and C. J. Higham. Coupled mode perturbation theory of range dependence. J. Acoust. Soc. Am.,108(1):76-83, 2000.

[56] Michael B. Porter, Finn B. Jensen, and Carlo M. Ferla. The problem of energy conservation in one-way models. J. Acoust. Soc. Am.,89(3):1058-1067, 1991.

[57] Jianguo Yan and Kang K. Yen. A derivation of three-dimensional ray equations in ellipsoidal coordintes. J. Acoust. Soc. Am.,97(3):1538-1544, 199.

[58] Carl Wunsch. Discrete Inverse and State Estimation Problems. Cambridge University Press, 2006

[59] Pierre F. J. Lermusiaux and A. R. Robinson. Data assimilation via error subspace statistical estimation. Part I: Theory and Schemes. Monthly Weather Review, 127(8), 1385-1407, 1999

[60] Pierre F. J. Lermusiaux. Data assimilation via error subspace statistical estimation. Part II: Mid-Atlantic Bight shelfbreak front simulations and ESSE validation. Monthly Weather Review, 127(8), 1408-1432, 1999

[61] Pierre F. J. Lermusiaux, Ching-Sang Chiu, Glen G. Gawarkiewicz, Phil Abbot, Allan R. Robinson, Robert N. Miller, Patrick J. Haley, Wayne G. Leslie, Sharanya J. Majumdar, Alex Pang and Francois Lekien. Quantifying Uncertainties in Ocean Predictions. Oceanography, Special issue on "Advances in Computational Oceanography”, T. Paluszkiewicz and S. Harper (Office of Naval Research), Eds., Vol. 19, 1, 92-105, 2006 
[62] XBT User's guide: http://www.sippican.com/contentmgr/showdetails.php/id/312

[63] C. M. Ferla, M. B. Porter and F. B. Jensen. C-SNAP: Coupled SACLANTCEN normal mode propagation loss model. SACLANTCEN SM-274.

[64] Open MPI: http://www.open-mpi.org/

[65] MPICH2: http://www.mcs.anl.gov/research/projects/mpich2/

[66] pMatlab http://www.ll.mit.edu/mission/isr/pmatlab/pmatlab.html

[67] Hanh Kim and Julia Mullen. Introduction to parallel programming and pMatlab v0.7. MIT Lincoln Laboratory

[68] Condor: http://www.cs.wisc.edu/condor/

[69] C. T. Tindle, L. M. O’Driscoll, and C. J. Higham. Coupled perturbed modes over a sloping penetrable bottom. J. Acoust. Soc. Am., 108(1):76-83, 2000.

[70] C. J. Higham and C. T. Tindle. Coupled perturbed modes over a sloping penetrable bottom. J. Acoust. Soc. Am., 114(6):3119-3124, 2003.

[71] Kevin D. Heaney and W. A. Kuperman. Frequency interpolation technique for broadband parabolic equation calculations. J. Computational Acoustics, 7(1):27-38, 1999

[72] GTOPO30: http://edc.usgs.gov/products/elevation/gtopo30/gtopo30.html

[73] G. Brooke, D. Thomson, and G. Ebbeson. PECan: A Canadian parabolic equation model for underwater sound propagation. J. Computational Acoustics, 9(1):69-100, 2001

[74] Michael D. Collins and Richard B. Evans. A two-way parabolic equation for acoustic backscattering in the ocean. J. Acoust. Soc. Am.,91(3):1357-1368, 1992. 